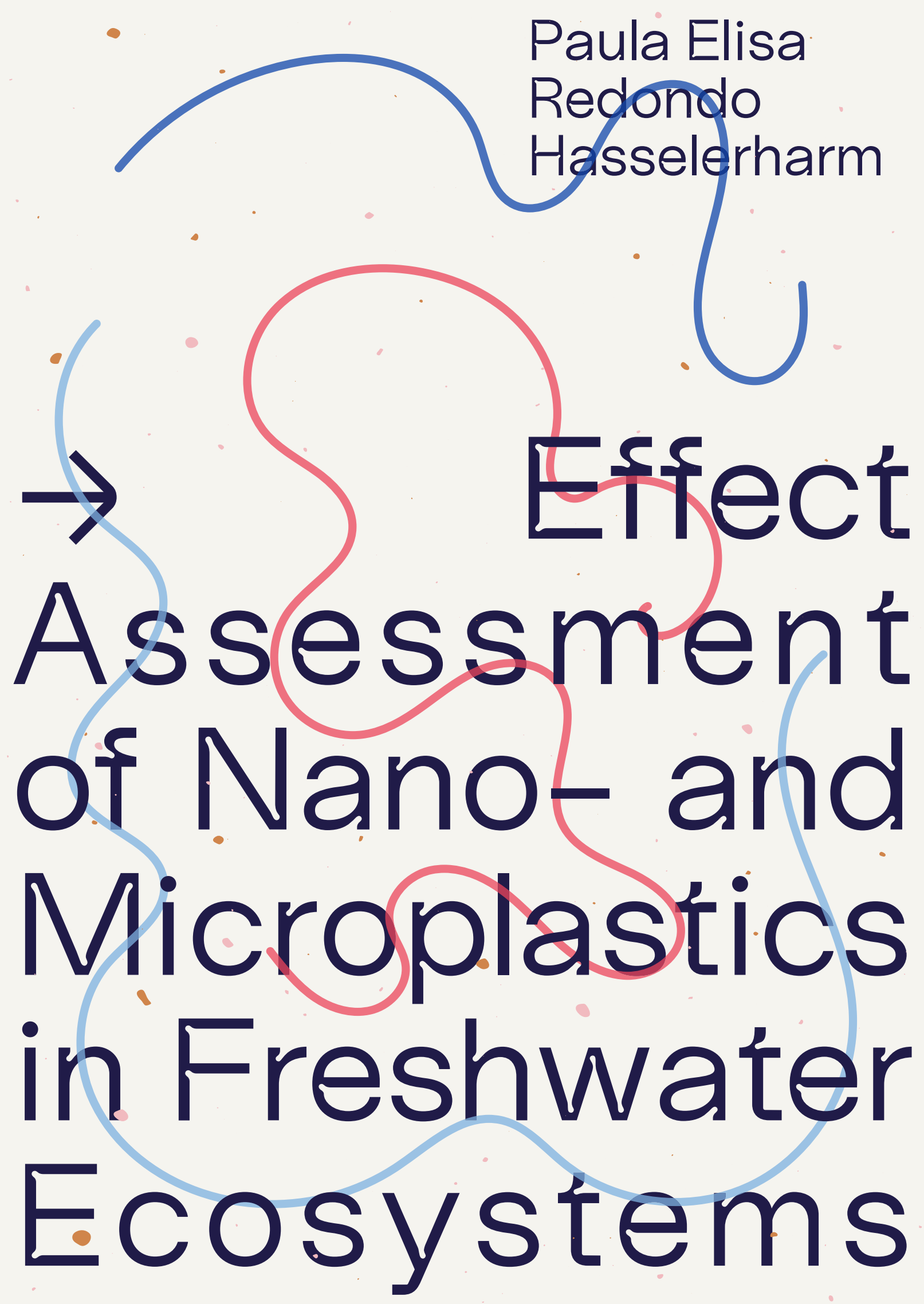




\section{Propositions:}

1. Microplastics pose a risk to benthic macroinvertebrates. (this thesis).

2. The effects of nano- and microplastics on the growth and mortality of benthic macroinvertebrates depend more on the characteristics of the species than on plastic particle properties. (this thesis).

3. When assessing the human health risks of chemicals in drinking water, not only single chemicals but also chemical mixtures should be considered.

4. The scientific community would greatly benefit from a stronger training on a single concept: statistical significance.

5. Scientists and science communicators have the role of reducing the distance between perceived and actual risk.

6. Plastic isn't waste until we waste it.

7. The people make the place, but the food and the weather make the people.

Propositions belonging to the thesis, entitled

Effect Assessment of Nano- and Microplastics in Freshwater Ecosystems

Paula Elisa Redondo Hasselerharm

Wageningen, 16 December 2020 



\section{Effect Assessment of Nano- and Microplastics in Freshwater Ecosystems}


Thesis committee

\section{Promotor}

Prof. Dr Albert A. Koelmans

Professor of Aquatic Ecology and Water Quality Management

Wageningen University \& Research

\section{Co-promotor}

Dr Edwin T.H.M. Peeters

Associate Professor, Aquatic Ecology and Water Quality Management Group

Wageningen University \& Research

\section{Other members:}

Prof. Dr Thom Kuyper, Wageningen University \& Research

Prof. Dr Willie Peijnenburg, Leiden University

Dr Gert Everaert, Vlaams Instituut voor de Zee, Oostende, Belgium

Dr Ellen Besseling, Waterschap Rijn en IJssel, Doetinchem

This research was conducted under the auspices of the Graduate School for SocioEconomic and Natural Sciences of the Environment (SENSE). 


\title{
Effect Assessment of Nano- and \\ Microplastics in Freshwater Ecosystems
}

\author{
Paula Elisa Redondo Hasselerharm
}

\section{Thesis}

submitted in fulfilment of the requirements for the degree of doctor at Wageningen University

by the authority of the Rector Magnificus,

Prof. Dr A.P.J. Mol, in the presence of the

Thesis Committee appointed by the Academic Board to be defended in public

on Wednesday 16 December 2020

at 1:30 p.m. in the Aula. 
Paula Elisa Redondo Hasselerharm

Effect Assessment of Nano- and Microplastics in Freshwater Ecosystems 320 pages

$\mathrm{PhD}$ thesis, Wageningen University, Wageningen, The Netherlands (2020) With references, with summary in English

ISBN 978-94-6395-594-2

https://doi.org/10.18174/533220 
A mis padres, Gonny y Paco. 



\section{Table of Contents}

Chapter 1 General Introduction …........................................... 9

Chapter 2 Microplastic Effect Thresholds for Freshwater Benthic

Macroinvertebrates

Chapter 3 Ingestion and Chronic Effects of Car Tire Tread Particles on Freshwater Benthic Macroinvertebrates

Chapter 4 Uptake and Effects of Metal-Doped Nanoplastics on Gammarus pulex

Chapter 5 Nano- and Microplastics Affect the Composition of Freshwater Benthic Communities in the Long Term

Chapter 6 Assessing the Toxicity of Nanoplastics using In Vitro Bioassays with Relevance for Human Health

Chapter 7 Quality Criteria for Microplastic Effect Studies in the Context of Risk Assessment: A Critical Review

Chapter 8 Microplastic Effect Assessment in the Context of Risk:

A Synthesis

Appendices 149

References 261

Summary 299

Acknowledgements 305

Curriculum vitae 309 

Chapter 1

General Introduction 



\section{General Introduction}

1.1. The problem of plastic pollution. The release of plastic waste into the environment has become one of the major water quality problems of the Anthropocene. Plastic is a cheap, durable and versatile material with innumerable applications and benefits for society. ${ }^{1}$ Since the 1950 s, the world plastic production has exponentially increased, reaching about 360 million tons in 2018. ${ }^{2,3}$ This tremendous increase was followed by an increase in the concentration of plastic waste detected in the open ocean. ${ }^{2}$ Plastic waste has not only accumulated in the open ocean, but also in freshwater and terrestrial systems, shorelines and even in the deep sea. ${ }^{4}$ From the plastics generated in $2015,9 \%$ were recycled, $12 \%$ were incinerated and $79 \%$ accumulated in landfills or were released into the environment. ${ }^{5}$ As plastic is a very persistent material and constitutes $10 \%$ of the discarded waste and $50-80 \%$ of marine litter, effective waste management measures may help to avoid plastic release into the environment. ${ }^{4-6}$ If preventive measures are not taken and the current plastic production trend continues, 12,000 million tons of plastic waste are expected to be found in the environment by $2050 .{ }^{5}$ As claimed by a great number of scientists in the last three decades, plastic pollution could potentially impact biota and human health. ${ }^{6-10}$ For these reasons, there is an urgent need to evaluate the environmental and human risks of plastic debris. ${ }^{11}$

1.2. Plastic classes by size, source, chemical composition, shape and density. With the term "plastic" I refer to all polymers, including thermoplastics, elastomers and synthetic fibres, as suggested by the scientific community. ${ }^{12}$ Plastics can be classified by their size, source, chemical composition, shape and density. ${ }^{13}$ When it comes to size, plastics can be defined as nanoplastics (NP), with a size smaller than $0.1 \mu \mathrm{m}$; microplastics (MP), with a size between $0.1 \mu \mathrm{m}-5 \mathrm{~mm}$; and macroplastics, with a size larger than $5 \mathrm{~mm} .{ }^{14}$ By source, plastics detected in the environment can be divided into primary and secondary plastics. ${ }^{14}$ Primary plastics are intentionally made with a specific size for cosmetical, medical, pharmaceutical, and industrial purposes. ${ }^{14-17}$ Secondary plastics are formed due to the fragmentation of larger plastics via physical and biological processes. ${ }^{18-20}$ The chemical composition of plastics includes the polymer type and the additives, which will provide each plastic product with specific properties depending on its function. From the plastics produced in 2018, almost $30 \%$ were made of polyethylene (PE), which is used to produce bags, food packaging, etc. ${ }^{3}$ The second most commonly used polymer was polypropylene (PP), followed by polyvinyl-chloride (PVC), polyurethane (PUR), polyethylene-terephthalate (PET) and polystyrene (PS). ${ }^{3}$ By shape, plastics are commonly categorized as fragments, fibres, spheres, films, pellets, foams and flakes. ${ }^{21}$ Fragmentation processes occurring in the environment will also play a role in the final chemical composition and shape of plastics. Finally, plastics are often divided between 
positively and negatively buoyant if their density is lower or higher than the local water density, respectively. ${ }^{22}$ If no other environmental factors play a role in the buoyancy of the plastics (i.e., biofilm formation, vertical mixing due to wind, etc.), positively buoyant plastics will float, while negatively buoyant plastics will sink. ${ }^{22}$

1.3. Fate and abundance of $N P$ and $M P$ in aquatic systems. Main pathways for the entrance of primary plastics in freshwater systems are: wastewater treatment plant (WWTP) effluents, soil erosion and runoff, shipping activities and atmospheric deposition. ${ }^{23,24}$ Pathways for primary plastics in marine systems include: freshwaters, which release between 1.2 and 2.4 million tons of plastic every year globally, soil erosion and runoff, shipping activities and atmospheric deposition. ${ }^{24,25}$ The fate of plastics depends on their properties (size, shape, chemical composition, density), but also on the type of ecosystem, the biota present and the weather conditions. ${ }^{26-28}$ These properties will affect the sedimentation rates of individual particles and the formation of aggregates, which might also enhance their sedimentation. ${ }^{23}$ When it comes to the ecosystem type, the fate of plastics in freshwaters differs between lentic (lakes, ponds, ditches and canals) and lotic (rivers and streams) systems. ${ }^{23}$ The principal difference among them is the higher flow velocity in lotic systems compared to lentic systems, which will affect the sedimentation processes. ${ }^{23}$ Ditches may have an intermediate position: they flow slowly in general, but may exhibit strong flow in times of heavy rainfall leading to transport of sediment to other systems. Therefore, lentic systems and sedimentation areas in lotic systems can act as a sink for plastics due to their longer water residence times. ${ }^{23,27}$ In marine ecosystems, vertical transport will be determined by plastic buoyancy, while horizontal transport will be driven by surface winds, creating regions of convergence (the 5 subtropical gyres) and divergence. ${ }^{22,23}$ The biota present in an environmental system may ingest plastic particles and transport them to another location, or may increase the density of plastics due to the formation of a biofilm on their surface, which will increase their sedimentation rates. ${ }^{23}$ The weather conditions will affect the mixing of water and the resuspension of plastics from sediments in freshwaters, and the accumulation of plastics in the oceanic gyres and beach sediments due to surface currents..$^{23,29,30}$

Environmental concentrations of MP found for the aquatic environment strongly depend on the sampling and polymer identification methods used. ${ }^{31}$ Sieves or nets used during the sampling have mesh sizes between 20 and $333 \mu \mathrm{m}$ and polymer identification techniques have detection limits between 1 and $1000 \mu \mathrm{m} .{ }^{32}$ Consequently, NP and submicron MP $(<1 \mu \mathrm{m})$ are generally not included in the analysis. ${ }^{32}$ Moreover, commonly used polymer identification methods are not able to detect polymers with high black carbon content, such as particles released from tires, which are expected to highly contribute to MP pollution. ${ }^{14,33}$ For these reasons, MP abundance in the aquatic environment is probably underestimated. Following recent systematic reviews, the highest MP concentrations in freshwaters have been found in WWTP, being the highest ever detected MP concentration found in a WWTP in Denmark, which contained 442,393 particles/1 (in numbers) or $29.55 \mathrm{mg} / \mathrm{g}$ of water (in mass). ${ }^{34,35}$ The highest MP number concentrations detected in surface waters were found in the Snake River in the USA, with 
concentrations raging up to 5,405 particles/1. ${ }^{36,37}$ Of the measured MP, $79.4 \%$ were smaller than $500 \mu \mathrm{m}$, despite sizes below $100 \mu \mathrm{m}$ were not analysed due to the sampling method used. ${ }^{36}$ In freshwater sediments, the highest MP number concentrations have been found in sediments of the Wen-Rui Tang river in China, which ranged up to 74,800 particles/kg of sediment dw. ${ }^{38}$ Sediment samples taken from various locations within this river contained between $73 \%$ and $95 \%$ of MP with sizes below $300 \mu \mathrm{m} .{ }^{38}$ This indicates that smaller MP fractions might be more abundant than the bigger ones. Most common polymer types sampled in freshwaters are PP, PE and PS and most abundant shapes are fragments, fibres, films, foams, pellets and spheres. ${ }^{35,38}$ In the marine environment, the highest MP number concentrations were found in beach sediments in Asia, reaching 80,000 particles $/ \mathrm{m}^{2} .{ }^{39,40}$ Highest MP number concentrations reported in marine sediments were detected in a Belgian harbour, where 390 particles/kg sediment dry weight (dw) were found. ${ }^{41}$ When it comes to NP, only one recent study was able to detect PVC, PET, PS and PE NP in the North Atlantic Subtropical Gyre. ${ }^{42}$ When comparing freshwater with marine abundance data, freshwaters seem to contain much higher MP concentrations.

1.4. Interactions between NP and MP and aquatic biota. As plastics are ubiquitous in the environment, biota will likely encounter them and interact with them. ${ }^{43}$ The nature of this interaction will depend on the properties of the NP and MP, (size, shape, chemical composition, density), the species traits, and the environmental conditions in which the interaction occurs, as the formation of biofilms can increase the size and density of the plastic particles and modify their shape. ${ }^{44,45}$ The interaction of NP and MP with the exposed organism can be internal if particle properties and species traits allow for the ingestion of the NP and MP. ${ }^{46}$ Also, NP and MP may externally interact with biota by for instance adhering to the surface of the exposed organisms. ${ }^{43,47-53}$

In the field, MP have been detected in the digestive tract of invertebrates, amphibians, fish, reptiles, birds, and mammals. ${ }^{54-60}$ All of these organisms do probably contain NP as well; however, they cannot be detected with current techniques due to their small size. Hence, the NP fraction in biota from the field is still unknown. ${ }^{32}$ In the laboratory, ingestion of NP and MP has mostly been studied for fish and invertebrates. ${ }^{61-67}$ After ingestion, NP and MP could be transported along the digestive tract until excretion or could accumulate in the gut, the digestive gland, the liver, and in oil storage droplets. ${ }^{68-}$ ${ }^{70}$ Adverse effects of NP and MP have been reported at sub-organismal, individual and population levels once critical effect threshold concentrations were exceeded. ${ }^{40}$ At the sub-organismal level, NP and MP have been found to increase oxygen consumption, cause inflammation, genotoxicity, neurotoxicity, oxidative stress, gut dysbiosis, alter the ionic exchange and the enzymatic activity. ${ }^{69-78}$ At the individual level, NP and MP have been found to affect survival, growth, feeding, reproduction, emergence, mobility, and the embryonic development. ${ }^{66,78-86}$ Effects of NP at population levels have never been studied, while MP effects at population levels have only been studied for marine invertebrates during a 3-months period, which were found to reduce their number and biomass, and for the freshwater cladoceran Daphnia magna for 3 weeks, which was found to reduce its biomass. ${ }^{87-89}$ The mechanisms causing adverse effects of NP and MP on 
biota are still unclear, but several studies have attributed these effects to a physical damage caused by the blockage of the food passage, which could lead to a feeling of satiation, or a reduced feeding in the presence of NP and MP. ${ }^{83,86,90}$ Other studies have suggested that the effects found could be caused by specific NP and MP properties, such as the surface groups, or the leaching of toxic chemicals. ${ }^{83,90-92}$ The external interaction of NP and MP has also been considered as a potential mechanism explaining the detection of adverse effects in aquatic species. For example, the reduction of the photosynthetic capacity of Skeletonema costatum was related with the adsorption and aggregation of MP to the surface of this diatom. ${ }^{47}$ Also, the reduction in the feeding rate of Hydra attenuata was linked to the adhesion of MP to the tentacles of this hydrozoan. ${ }^{52}$ It must be noted that the quality of the data varied widely across the studies cited here, which affects the weight of the evidence of the acclaimed mechanisms.

1.5. Environmental risks of NP and MP. To characterize an environmental risk of NP and $\mathrm{MP}$, exposure and effects need to be assessed. Exposure assessments focus on quantifying the environmental concentrations of a specific stressor, while effect assessments describe the relationship between exposure concentrations and the effects caused on the endpoints studied. ${ }^{93}$ To conduct an environmental risk assessment for NP and MP, a tiered approach can be followed, as commonly done for chemicals. ${ }^{11}$ Tiers 1 and 2 consist on the development of single species test batteries with NP and MP and the comparison of the generated data with ecotoxicity data from literature, respectively. ${ }^{93}$ Tier 3 englobes the performance of field experiments to study the effects of NP and MP at population levels, which is extremely relevant, as the detection of negative effects within this level could trigger responses that can affect the whole community and because the effects found are closer to what will happen in nature than those detected in laboratory studies. ${ }^{93}$ The Predicted No Effect Concentration (PNEC) will be calculated based on the outcome of the effect assessment in each tier after dividing it by an assessment factor (AF), which is a numerical value used to address the uncertainties in the extrapolation of experimental data to the relevant environmental exposure situation. Then, the PNEC will be compared with the Predicted Environmental Concentrations (PECs) or the Measured Environmental Concentrations (MECs) derived from the exposure assessment. ${ }^{94}$

To date, few studies have attempted to evaluate the risks of NP and/or MP in freshwater and marine ecosystems. ${ }^{40,95-97}$ These studies created Species Sensitivity Distributions (SSD), which are cumulative probability distributions of threshold effect concentrations for a stressor, obtained from single species tests. ${ }^{98}$ This tool is often used in risk assessment and belongs to the previously defined tier 2 . It is used to calculate the concentration where $5 \%$ of the species would be affected in an ecosystem, the so called $\mathrm{HC}_{5}$, and to derive maximum allowable concentrations of a stressor. ${ }^{97,99}$ All of these studies used available literature effect data and expressed the need to reduce the uncertainties of the used data and to improve the quality of future SSDs. ${ }^{40,95-97}$

1.6. Implications for human health. Humans can be exposed to NP and MP through drinking water, food, dermal contact and inhalation. ${ }^{100} \mathrm{MP}$ have been detected in single 
use and reusable plastic and glass water bottles and in treated and untreated tap water. ${ }^{35}$ Humans can directly ingest MP through drinking water but also through their diet, as MP have been detected in seafood, chicken gizzards, honey, sugar, beer, and salt. ${ }^{101-105}$ Humans could also accidentally ingest MP via personal care products that are known to contain MP, such as toothpastes and facial scrubs. ${ }^{17,106}$ After ingestion, NP and small MP could potentially enter the intestinal mucus, or could translocate to the circulatory system, as shown for rats exposed to NP and small MP. ${ }^{107-109}$ Although dermal contact with MP from clothes and cosmetic products is part of our daily life, MP uptake through the dermis is not very plausible due to their big size. ${ }^{100}$ However, NP could potentially penetrate human skin, as dermal uptake has been proven in mice exposed to gold nanoparticles. ${ }^{110}$ Indoor and outdoor atmospheric fallout of synthetic fibres and fragments has been widely demonstrated for MP. ${ }^{111-114}$ Airborne MP could be inhaled by humans and be trapped by lung tissues, ${ }^{115}$ as demonstrated by Pauly et al. (1998), ${ }^{116}$ who detected the presence of MP fibres in $87 \%$ of the human lung tissues evaluated. ${ }^{116}$ In addition, MP inhalation could cause lung inflammation, as demonstrated for airborne tire particles with sizes smaller than $10 \mu \mathrm{m} .{ }^{117} \mathrm{NP}$ and MP cell internalization has been demonstrated in in vitro tests with human cells, although it remains unclear whether these would occur in vivo. ${ }^{18,119}$ Inside cells, MP do not bind to membranes and could potentially interact with intercellular structures. ${ }^{118}$ While MP have been found to cause cytotoxicity and oxidative stress in human cells, ${ }^{120,121}$ NP have been found to cause genotoxicity, cytotoxicity, oxidative stress, inflammation and morphological changes. ${ }^{19,120}$ Nowadays, there is a scientific debate about the contribution of each of these pathways to NP and MP incorporation by humans. ${ }^{122}$ Some studies consider that particle settling during food consumption or contamination by food packaging might contribute more to NP and MP ingestion by humans than the NP and MP already present in food. ${ }^{113,123}$ A recent study estimated that the annual MP consumption through food in the USA ranged from 39000 to 52000 particles/person/year, while MP inhalation ranged between 35000 and 69000 particles/person/year. ${ }^{124}$ In addition, MP intake via bottled water and via tap water could increase MP consumption in another 90000 or 4000 particles/person/year, respectively. ${ }^{124}$

1.7. Knowledge gaps and challenges to assess the risks of plastics. Since scientists started raising concerns about the potential impacts of plastic pollution on aquatic biota, a wide number of studies has been published assessing the ecotoxicity of plastics. Still, 77\% of the ecotoxicological studies published until the end of 2017 focused on marine organisms, while only $23 \%$ used freshwater organisms. ${ }^{125}$ For this reason, and because freshwaters are the main pathways for the entrance of MP to marine systems and contain higher MP concentrations than marine systems, there was an urgent need to evaluate the effects of MP on freshwater species when this thesis started. In freshwaters, assessing the effects of MP on benthic species seemed particularly important, as MP can accumulate in sediments and reach relatively high concentrations. ${ }^{38,40}$ Also, available data at the start of the thesis consisted of multiple MP types tested under very different conditions, making the comparability among the outcomes of the published studies very complicated. For an adequate assessment of the risk of a specific particle or chemical compound, the use of standardized tests to compare results among species is required. ${ }^{11,93}$ In addition, MP were 
generally tested under unrealistic conditions, for short time periods, using only one MP type (one polymer, one size, one shape) and one very high concentration. ${ }^{126}$ The properties of the tested MP (e.g., size, shape, polymer type) were in general not well characterized and the effects observed could almost never be attributed to a particle effect only, as the removal or characterization of chemical additives or chemicals adsorbed to the MP was not considered. ${ }^{11}$ Finally, MP uptake has been rarely quantified in exposure assessments with aquatic biota and the methods used consisted mostly of the visual identification of MP, which can lead to mistakes due to subjectivity or the leaching of the fluorophore to tissues. ${ }^{127}$ When it comes to NP, only few studies had evaluated their uptake and effects at the time the present research started and even nowadays the available data is still scarce. Nevertheless, it has been stated that effects of NP might be worse than those caused by MP due to their smaller size and higher surface area, which makes them more susceptible to be incorporated by cells and makes them capable of binding chemicals more effectively. ${ }^{128}$ Accordingly, evaluating the risks of NP on freshwater systems should also be prioritized.

Besides the environmental risks of NP and MP, the potential impacts of NP and MP on human health needed to be urgently assessed. Until the start of this thesis, most studies had focused on the internalization and translocation of NP and MP and their cytotoxic and oxidative stress in human cells, using very high unrealistic concentrations. ${ }^{12}$ As it occurred in laboratory tests with aquatic species, no standard methods exist to assess the effects of NP and MP on humans and studies done to date generally included one high NP or MP concentration, from which effect thresholds could not be derived and where the realism was lacking. Consequently, it is worth considering to what extent standard in vitro tests commonly used for the screening of effects caused by chemicals in freshwater samples could be applied for NP and MP. Moreover, environmentally realistic concentrations need to be tested, as well as environmentally realistic matrixes which are known to contain NP and MP, such as WWTP effluents.

1.8. Aim of this thesis. The general objective of this thesis is to assess threshold effect concentrations of NP and MP in freshwater systems, and to provide guidance on how such effects should be assessed in the context of risk assessment. To accomplish this, we formulated seven main research questions:

1. To what extent do standardized low-tier single species tests allow for the detection of effect thresholds for NP and MP at the individual level?

2. How to measure ingested, accumulated and egested number concentrations of NP and MP, maximum ingestible sizes and how to calculate trophic transfer factors (TTFs) to compare results across studies?

3. To what extent do high-tier outdoor tests allow for the detection of effect thresholds of NP and MP at the community level? 
4. To what extent can in vitro tests with relevance for human health be used to detect effects of NP?

5. What could be improved in effect tests when it comes to quality assurance?

6. Which effect mechanisms can be considered as demonstrated when strict quality criteria are applied to the literature on MP studies reported to date?

7. Can we provide an estimate of the ecological risks of MP using the data generated in this thesis and literature data, and which further recommendations can be made?

In order to answer the first research question, we assess the effects of polystyrene MP fragments (Chapter 2), MP made from car tires (Chapter 3) and metal-doped NP on freshwater benthic macroinvertebrates with different living and feeding strategies with the aim of measuring their individual effect thresholds. To achieve this aim, standardized 28-days single species tests were performed. In Chapter 2, pre-washed polystyrene MP fragments with sizes between 20 and $500 \mu \mathrm{m}$ were mixed with natural sediment at concentrations ranging from 0 to $40 \% \mathrm{MP}$ in sediment dw, including one environmentally relevant concentration. Species selected were the amphipods Gammarus pulex and Hyalella azteca, the isopod Asellus aquaticus, the worms Lumbriculus variegatus and Tubifex spp. and the bivalve Sphaerium corneum. Endpoints assessed included survival, growth and feeding activity of G. pulex, H. Azteca, A. aquaticus and Tubifex spp., reproduction, growth and feeding activity of $L$. variegatus and survival and growth of $S$. corneum. In Chapter 3, MP were made by scraping the first $2 \mathrm{~cm}$ of five used tires and grinding them until obtaining a size distribution of 10 to $585 \mu \mathrm{m}$. Main constituents of the MP were quantified with thermo-gravimetric analysis and gas chromatography - mass spectrometry (GC-MS). Six MP concentrations ranging from 0 to $10 \%$ in sediment $\mathrm{dw}$ were tested to evaluate the survival and growth of G. pulex. A. aquaticus, L. variegatus and Tubifex spp.. In Chapter 4, the freshwater amphipod G. pulex was exposed to 228 $\mathrm{nm}$ irregularly shaped palladium-doped NP via natural sediment at six concentrations raging from 0 to $3 \%$ in sediment $\mathrm{dw}$, with the aim of assessing their chronic effects on survival and growth.

To tackle the second research question, in Chapter 2 we analysed the ingestion, retention and egestion of MP for G. pulex and H. azteca using $\mu$-Fourier Transformed Spectroscopy and provided maximum ingestible sizes and trophic transfer factors (TTFs) for G. pulex. In Chapter 3, a method to quantify tread particles in organisms and faeces was developed using image analysis, as the ingestion, accumulation and egestion of tread particles by organisms had never been studied before. We also tested the resistance of tread particles to the digestion's fluids used, measured the maximum ingestible sizes and calculated TTFs for G. pulex, which were compared with the TTF values obtained for G. pulex in Chapter 2. In Chapter 4, NP concentrations were quantified based on palladium concentrations measured with inductively coupled plasma mass spectrometry (ICP-MS) in the body of the exposed organisms and in the faecal pellets excreted during a 24 hours post-exposure depuration period. In addition, palladium-doped NP concentrations were 
measured in sediments and water to demonstrate the accuracy of the dosing and to quantify the resuspension of NP from the sediment.

To answer the third research question, in Chapter 5 we evaluate the effects of a range of $\mathrm{NP}$ or MP concentrations on the recolonization of a freshwater benthic macroinvertebrate community after 3- and 15-months of exposure via natural sediment using an outdoor ditch as experimental system. Hence, trays containing sediment and NP or MP at five concentrations between 0 and $5 \%$ plastic in sediment $\mathrm{dw}$, including two environmentally realistic concentrations, were embedded at the bottom of the ditch containing a stable donor community. Spherical PS NP with an average size of $96 \mathrm{~nm}$ and PS MP fragments with sizes ranging from 20 to $516 \mu \mathrm{m}$ were used for the NP and MP treatments, respectively. The donor community was allowed to colonize the trays and after 3 and 15 months, trays were retrieved, and species were identified and counted. Effects were assessed on the community composition, population sizes and species diversity. In this chapter, we present the first long-term community effect thresholds for freshwater benthic macroinvertebrates exposed to NP and MP and compare them with environmental concentrations measured in freshwater sediments.

With the aim of answering the fourth research question, in Chapter 6 we explore the potential use of in vitro toxicity tests to evaluate the risks of NP on human health, in this case with and without chemical mixtures originating from WWTP effluent and surface water samples. Therefore, we evaluated the genotoxicity of two sizes of spherical PS NP $(50 \mathrm{~nm}$ and $500 \mathrm{~nm})$ at four environmentally relevant concentrations $(0,2.5,25$ and 250 $\mu \mathrm{g} / \mathrm{l})$ in three matrices using the Ames fluctuation test, which has the purpose of detecting base-pair and frameshift mutations in the genome of Salmonella typhimurium with and without metabolic activation. We thereby assess the genotoxicity of the environmental matrices, NP alone, NP in the presence of chemicals extracted from surface water, and NP the presence of chemicals extracted from WWTP effluent. Finally, we provide recommendations to increase the relevance of in vitro tests for the assessment of NP risks for human health.

Following recently developed quality assessment methods for papers studying the abundance of MP in biota and water samples, ${ }^{31,35}$ we address the fifth research question in Chapter 7 by critically reviewing 105 papers that report MP effects on aquatic biota. To this aim, 20 Quality assurance/Quality control (QA/QC) criteria were defined within four main categories: particle characterization, experimental design, applicability for risk assessment and ecological relevance. Based on our own analysis and practical experience learnt in Chapters 2, 3, and 5, a guidance protocol for testing ecotoxicological effects of MP for aquatic species is consequently provided. In addition, with the aim of detecting knowledge gaps within effect studies with MP, we give an overview of the characteristics of the reviewed studies with respect to the size, shape and polymer type of the MP used, the tested species, the duration of the exposure, the endpoints studied and use or not of effect thresholds to report the results. 
In addition, the answer to the sixth research question is also included in Chapter 7, where demonstrated and suggested effects and effect mechanisms reported in the 105 reviewed papers are summarized and discussed, with the results of the quality evaluation applied to assess the overall weight of evidence regarding ecologically relevant effects with applicability for risk assessment. Scores from the technical part of the QA/QC assessment (particle characterization and experimental design) were used to assess the relative credibility of adverse effects reported.

Finally, to answer the seventh research question, in Chapter $8 \mathrm{I}$ bring all the answers to the research questions together and develop an ecological risk assessment for MP using the data generated in Chapters 2, 3 and $\mathbf{5}$ and data taken from the literature. Following the tiered approach, I first compare MP effect thresholds obtained in Chapters $\mathbf{2}$ and $\mathbf{3}$ with environmental concentrations of the corresponding MP types measured in freshwater sediments (tier 1). Then, two SSDs are created, one for water exposure data and one for sediment exposure data using the effect thresholds obtained in Chapters $\mathbf{2}$ and $\mathbf{3}$ and MP effect thresholds taken from the literature (tier 2). Here, a $\mathrm{HC}_{5}$ is calculated. For tier 3 , I use the community effect thresholds obtained in Chapter $\mathbf{5}$ and compare them with field measured concentrations. Finally, the implications of the results obtained in this thesis are discussed and recommendations for future research are provided. 


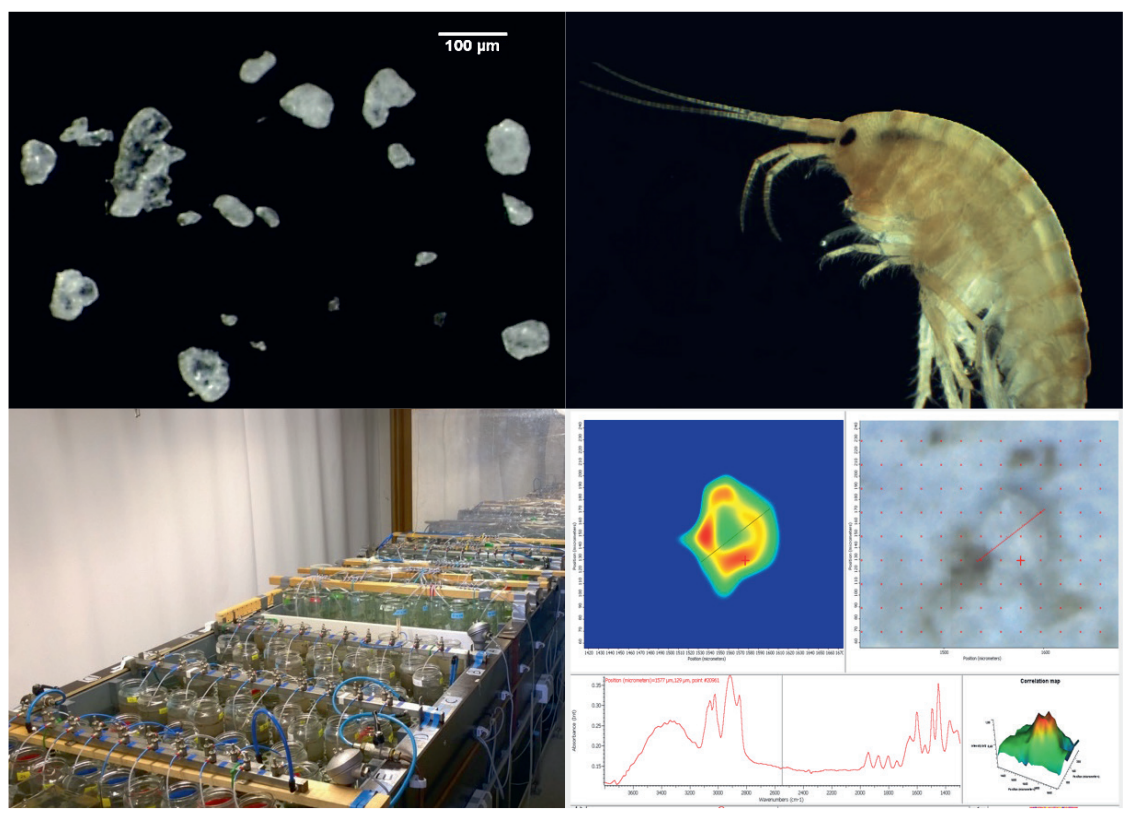




\section{Chapter 2}

\section{Microplastic Effect Thresholds for Freshwater Benthic Macroinvertebrates}

Published as: Redondo-Hasselerharm, P.E.; Falahudin, D.; Peeters, E.T.H.M.; Koelmans, A.A. Microplastic Effect Thresholds for Freshwater Benthic Macroinvertebrates. Environ. Sci. Technol. 2018. 52, 2278-2286. 


\begin{abstract}
Now that microplastics have been detected in lakes, rivers, and estuaries all over the globe, evaluating their effects on biota has become an urgent research priority. This is the first study that aims at determining the effect thresholds for a battery of six freshwater benthic macroinvertebrates with different species traits, using a wide range of microplastic concentrations. Standardized 28 days single species bioassays were performed under environmentally relevant exposure conditions using polystyrene microplastics $(20-500 \mu \mathrm{m})$ mixed with sediment at concentrations ranging from 0 to $40 \%$ sediment dry weight ( $\mathrm{dw}$ ). Microplastics caused no effects on the survival of Gammarus pulex, Hyalella azteca, Asellus aquaticus, Sphaerium corneum, and Tubifex spp. and no effects were found on the reproduction of Lumbriculus variegatus. No significant differences in growth were found for $H$. azteca, A. aquaticus, S. corneum, L. variegatus, and Tubifex spp. However, G. pulex showed a significant reduction in growth $\left(\mathrm{EC}_{10}=\right.$ $1.07 \%$ sediment $\mathrm{dw}$ ) and microplastic uptake was proportional with microplastic concentrations in sediment. These results indicate that although the risks of environmentally realistic concentrations of microplastics may be low, they still may affect the biodiversity and the functioning of aquatic communities which after all also depend on the sensitive species.
\end{abstract}




\section{Introduction}

Microplastics (MP), defined as plastic particles with a size $<5 \mathrm{~mm},{ }^{7}$ have been detected in both terrestial and aquatic ecosystems. ${ }^{24}$ While their abundance and distribution in the marine environment have been found to be of great importance and have been covered already for a decade, their presence in terrestial and freshwater ecosystems is only recognized more recently. ${ }^{24,26,129}$ Nevertheless, a wide range of MP has been identified at different concentrations in water and sediment samples from lakes, rivers and estuaries all over the globe. ${ }^{24,26,129}$

Key factors influencing the fate and transport of MP in freshwater systems are the type of aquatic system, as well as the climate conditions and plastic sources in the area. ${ }^{23,26,27}$ Moreover, MP properties such as size, density or shape, have a direct effect on the processes of biofouling and aggregation, affecting the sedimentation and resuspension of particles and, thus, the abundance of MP in the water column and sediments. ${ }^{23,26,27}$ In fact, particle size has been found to strongly affect the presence of MP hotspots along river sediments, indicating that sediments can act as a sink for MP. ${ }^{27}$ Recent data shows that the Rhine river contains the highest MP concentrations detected in all freshwater bodies studied. Concentrations up to 4000 particles $/ \mathrm{kg}$ or $1 \mathrm{~g} / \mathrm{kg}(\mathrm{dw})$ were recorded in the German Rhine river shore sediments, with the smallest MP fraction $(63-630 \mu \mathrm{m})$ being the most abundant in numbers. ${ }^{130}$ In the Dutch area, up to 4900 particles/kg (dw) were accounted in the suspended particulate matter, in which $30 \%$ of the particles had a size between $10-300 \mu \mathrm{m}$ and $70 \%$ were bigger. ${ }^{131}$

Understanding the interaction between MP and biota in freshwater systems has been identified as a high priority research need ${ }^{26}$ and there is a general agreement on the idea that an effect assessment should be performed to evaluate the risk of exposure to MP. ${ }^{11}$ This is especially important in the case of freshwater benthic organisms, that seem to have a higher risk of exposure due to the sinking of MP onto sediments. ${ }^{27}$ Previous studies have indeed demonstrated that MP are taken up from sediments by freshwater species $^{46,83,132-135}$ and that the capacity of freshwater invertebrates to ingest MP depends on their feeding type. ${ }^{46}$ Moreover, this uptake was related to a decrease in the growth of Gammarus fossarum exposed to polymethylmethacrylate (PMMA) and polyhydroxybutyrate (PHB) MP. ${ }^{133}$ Also, a reduction in the growth and reproduction of Hyalella azteca was found after the exposure to polyethylene (PE) MP. ${ }^{83}$ However, no effects were reported on the survival, molting, metabolism and feeding activity of Gammarus pulex after the uptake of polyethylene terephthalate (PET) MP. ${ }^{135}$ MP uptake did not cause any effects on the marine isopod Idotea emarginata ${ }^{136}$ but did cause weight loss and a reduced feeding activity on the marine lugworm Arenicola marina.$^{92,137,138}$ No or limited effects have also been found for other marine invertebrates. ${ }^{81,139}$ This suggests that benthic macroinvertebrates are affected by the presence of MP but also that the susceptibility could be species specific. 
Current studies have mainly focused on the ingestion of MP; ${ }^{129}$ however, the quality, reliability and usability of the few ecological effect data published have been put into question. ${ }^{140,141}$ The use of non-standardized laboratory bioassays and unrealistic exposure scenarios hinders the understanding of the risks associated with MP. ${ }^{11,140,141}$ Furthermore, it remains unclear if adverse effects are caused by a physical impact of the particles themselves, by chemical toxicity or by a combination of both. ${ }^{24}$ Moreover, an effect assessment for MP should aim at detecting the effect thresholds for traditional endpoints in ecotoxicology (i.e., $\mathrm{LC}_{50}$ or $\mathrm{EC}_{50}$ ), ${ }^{140}$ and for this, sufficient doses and replication are needed in order to fit dose-response models, which are commonly used in chemical risk assessment. $^{11}$

In the present study we aim at determining the effect thresholds for a battery of freshwater benthic macroinvertebrates with different species-specific traits, using a wide range of MP concentrations. Standardized 28 days single species bioassays were performed under environmentally relevant exposure conditions using polystyrene (PS) MP (20 - $500 \mu \mathrm{m})$ mixed with sediment at concentrations ranging from 0 to $40 \%$ plastic in sediment $\mathrm{dw}$. We did not aim to assess chemical effects, as this has been dealt with in many earlier studies, e.g. Besseling et al. (2013; 2017), Browne et al. (2013), ${ }_{137,138,142}$ and because it has been argued recently that chemical risks of MP should be separated from risks associated with physical effects. ${ }^{11}$ We are not aware of earlier studies systematically assessing MP effect thresholds for a range of organisms. Effects of PS MP on mortality and growth were assessed for six benthic freshwater macroinvertebrates: Gammarus pulex, Hyalella azteca, Asellus aquaticus, Sphaerium corneum, Lumbriculus variegatus and Tubifex spp.. Effects of PS MP on feeding activity was also assessed as feeding rate for G. pulex, $H$. azteca and A. aquaticus and as egestion rate for Tubifex spp. and L. variegatus. Moreover, for G. pulex and H. azteca, the presence of PS MP in the faecal pellets and in their bodies after 24 hours defeacation was asessed in order to investigate if the differences in the effects caused by the exposure to MP on both species were related with their ingestion and egestion mechanisms. For all endpoints, environmentally relevant exposure conditions were simulated by using natural sediments and by including the highest concentration found in a freshwater sediment. PS, ground to a wide and environmentally relevant range of sizes and shapes, was considered as a fair approximation to assess the physical effects of 'environmental MP'. ${ }^{130}$ After all, PS density matches that of the average environmental MP ${ }^{20,27}$ (see calculation in Table A2.1) and polymer density has limited impact on physical effects. Any potential additives present were removed from the MP to eliminate any ambiguity concerning what caused the effect of the particles.

\section{Materials and Methods}

Microplastics. Irregular PS fragments were provided in a powdered form by Axalta Coating Systems GMBH (Cologne, Germany). Particle size distribution (PSD), measured with a Mastersizer 3000 (Malvern Instruments), revealed an unimodal distribution spanning from 20 to $500 \mu \mathrm{m}$, with a modus centred at $229 \mu \mathrm{m}$ in volume and $36 \mu \mathrm{m}$ in 
number (Figure A2.1). To remove additives present, if any, the MP were washed with methanol three times, shaken, filtered with a $20 \mu \mathrm{m}$ metal sieve and dried for at least 2 days in a fume hood at room temperature. Polymer identity and purity were confirmed with FTIR spectrometry (Nicolet iN10, ThermoFisher) and particle shape was confirmed with an Olympus SZX10 stereomicroscope (Figure A2.2).

Test organisms. Species selected were the amphipods Gammarus pulex (Linnaeus, 1758) and Hyalella azteca (Saussure, 1858), the isopod Asellus aquaticus (Linnaeus, 1758), the bivalve Sphaerium corneum (Linnaeus, 1758), and the worms Lumbriculus variegatus (Müller, 1774) and Tubifex spp. (Lamark, 1816). These species are common members of freshwater communities, are often used in laboratory experiments and differ in their living and feeding strategies, as well as in their sensitivity to environmental pollutants. ${ }^{93,143,144}$ G. pulex, H. azteca and A. aquaticus are regarded as being mainly shredders while $S$. corneum is classified as a facultative suspension feeder. $S$. corneum is an epibenthic species that lives and feeds on the sediment, while G. pulex and H. azteca are also active swimmers. L. variegatus and Tubifex spp. are both endobenthic deposit feeders, with $L$. variegatus regarded as a bulk feeder while Tubifex spp. exhibits selectivity in its feeding behaviour. ${ }^{145}$

Following previously published procedures, ${ }^{144,146}$ G. pulex, A. aquaticus and S. corneum were collected from an unpolluted brook (Heelsum, The Netherlands), a ditch (Heteren, The Netherlands) and a pond (Renkum, The Netherlands), respectively. H. azteca and $L$. variegatus were obtained from Wageningen Environmental Research (Wageningen, The Netherlands) and Tubifex spp. were obtained from a local pet shop. Prior to the experiments, organisms were acclimatized for two weeks in aerated buckets with copperfree Dutch Standard Water (DSW) inside a water bath at $16 \pm 1{ }^{\circ} \mathrm{C}$ while maintaining a 12:12 light:dark cycle. During the acclimatization, G. pulex, A. aquaticus and H. azteca were fed with dry poplar leaves that were collected in the field and $S$. corneum, $L$. variegatus and Tubifex spp. were fed with TetraMin ${ }^{\circledR}$ fish food pellets.

Sediments. Freshwater sediments were collected from a non-contaminated ditch in Veenkampen (Wageningen, The Netherlands) using a standard dip net. Background concentrations of $\Sigma \mathrm{PAH}$ and $\Sigma \mathrm{PCBs}$ were factors of $>8$ and $>70$ below toxicity thresholds, ${ }^{144}$ whereas heavy metals were below negligible risk levels according to Dutch sediment quality criteria (Table A2.2). Sediments were passed over a $2 \mathrm{~mm}$ sieve, homogenized and placed in a freezer to kill any organisms present and to preserve the Total Organic Matter (TOM) content. Prior to the experiments, sediments were thawed and homogenized again. Four representative subsamples were taken to determine the $\%$ TOM through loss on ignition $\left(3 \mathrm{~h}, 550{ }^{\circ} \mathrm{C}\right)$, which was $31.6 \% \pm 3.5(\mathrm{n}=4)$.

Experimental design. Bioassay experimental units consisted of $750 \mathrm{ml}$ glass beakers filled with 211 grams of wet sediment and $300 \mathrm{ml}$ of copper-free DSW. Polystyene MP were added to the sediment to obtain eight final uniform concentrations of $0,0.1,1,5,10,20$, 30 and $40 \%$ plastic weight in the total sediment mixture. 
Concentrations ranged from environmentally relevant ( 0 - $1 \%$ plastic weight in sediment $\mathrm{dw}$ ) to very high concentrations, to evaluate dose-response relations and to maximize the chance of accurately detecting the effect threshold. ${ }^{11}$ Four replicates of each concentration were made, except for $H$. Azteca, for which only three replicates were made and for which the concentration of 5\% was excluded. Beakers with the suspension of sediment and PS were manually shaken to overcome the energy barrier to settling due to the surface tension (if any), after which particles settled within $48 \mathrm{~h}$. Two weeks prior to the start of the experiment, beakers were placed in a water bath at a constant temperature of $16 \pm 1{ }^{\circ} \mathrm{C}$ and accomodated with aeration.

At the start of the experiment, 11 randomly selected individuals were placed in their corresponding beakers. The size range of $G$. pulex, A. aquaticus and $S$. corneum was between 4 and $7 \mathrm{~mm}$ and for $H$. azteca between 1 and $3 \mathrm{~mm}$. Active adult worms with an average wet weight of 3.2 and $12.4 \mathrm{mg}$ per worm were selected for Tubifex spp. and $L$. variegatus, respectively. The starting length and weight of 44 randomly selected individuals from the initial population were assessed. During the experiments, 2 poplar leaves discs with a diameter of $3 \mathrm{~cm}$ were supplied to the beakers of G. pulex, A. aquaticus and $H$. azteca at day 0 and 14. Poplar leaves discs were previously conditioned with DSW for 3 days. For $S$. corneum, a TetraMin ${ }^{\circledR}$ suspension of $0.5 \mathrm{mg}$ per individual per day was added every 3 days. No additional food was needed for L. variegatus and Tubifex spp. due to the high organic matter content of the sediment. Dissolved oxygen (DO), $\mathrm{pH}$, temperature, conductivity, and $\mathrm{NH}_{3}$ were measured in at least one replica of each concentration at day $0,3,7,14,17,21,24$ and 28. To keep water levels constant, DSW was added weekly until the end of the experiment. Water quality variables remained constant in all beakers along the experiment (Table A3.3), except for the treatments with Tubifex spp. and L. variegatus, where $\mathrm{pH}$ approached values below the recommended limits (6 - 9) at day 14 and 24, respectively. ${ }^{147}$ This was solved by replacing $100 \mathrm{ml}$ from the surface water layer in the bioassay by fresh DSW. On average, the measured temperature was $16 \pm 0.3{ }^{\circ} \mathrm{C}$, $\mathrm{pH}$ was $7.3 \pm 0.5$, oxygen concentration was $8.9 \pm 0.2 \mathrm{mg} / 1$ and conductivity was $477 \pm 45 \mu \mathrm{S} / \mathrm{cm}$. Unionised levels of ammonia decreased along the experiment for all species, reaching an average of $0.002 \pm 0.001 \mathrm{mg} \mathrm{NH} / \mathrm{L}$ at the end of the experiment. All unionised ammonia levels were always below the $\mathrm{LC}_{50}$ values available for these species. ${ }^{143,148-150}$

Mortality and growth. After 28 days, each system was sieved and the surviving organisms were collected, counted and transferred to clean DSW to depurate their gut for $24 \mathrm{~h}$. Thereafter, G. pulex, A. aquaticus and S. corneum were preserved in ethanol $80 \%$ and their length was measured: shell lenght of $S$. corneum, body lengh of A. aquaticus, and head capsule (HD in $\mathrm{mm}$ ) of G. pulex, from which total length (TL) was calculated as TL $=-2.07+9.82$ HD. ${ }^{151}$ Growth was determined as the difference in mean length (in $\mathrm{mm}$ ) of the animals in each replicate at the end minus the mean length from 44 animals at the start of the experiment. For H. azteca, L. variegatus and Tubifex spp., growth was measured as a difference in dry weight (in $\mathrm{mg}$ ) of the population at the start and at the end of the experiment. 
Feeding activity of G. pulex, H. azteca, A. aquaticus, L. variegatus and Tubifex spp.

Feeding rates - The feeding rate (mg dw leaf/organism/d) of G. pulex, H. azteca and $A$. aquaticus was calculated from the loss of the added poplar leaves using the following equation: ${ }^{152}$

$\mathrm{FR}=\frac{((L 1 \times C l)-L 2)}{\left(\frac{L i 1+L i 2}{2}\right) \times t}$

where L1 is the initial and L2 the final dry weight of the Populus sp. disc (mg), Li1 and $\mathrm{Li} 2$ are the numbers of living organisms at the start and at the end of the experiment (Li1=11 individuals), $\mathrm{Cl}$ is the leaching-decomposition correction factor, calculated by dividing the initial dry weight by the final dry weight of the leaves in the control sample; and $t$ is the incubation time (days).

Egestion rates. The egestion rate of L. variegatus and Tubifex spp. was assessed in a separate 15-day bioassay experiment following Leppanen and Kukkonen (1998) ${ }^{153}$ assuming that the weight of the faecal pellets represents the feeding rate of worms. ${ }^{154}$ Another batch of experimental systems was made following the same procedure as for the mortality and growth tests but now only three concentrations were prepared $(0,0.1$ and $40 \%$ ) in quadruplicate. After two weeks of acclimatization (with aeration, in a water bath at $16 \pm 0.3{ }^{\circ} \mathrm{C}$, with $12: 12$ light:dark cycle) to promote settling of particles, five active worms were added to these bioassays. When all organisms appeared to be buried in the sediment, a sand layer of $2 \mathrm{~mm}$ thickness and with a particle size between $0.5-1$ $\mathrm{mm}$ was added. The egested pellets of the organisms were collected with a pipette at day 2, 5, 7, 12, and 15 for L. variegatus and at day 1, 5, 7, 12, and 15 for Tubifex spp.. The collected faecal pellets were kept at $5{ }^{\circ} \mathrm{C}$ until they were filtered with GF/F $0.7 \mu \mathrm{m}$ glass filters and dried at $60{ }^{\circ} \mathrm{C}$ for 48 hours and weighed. ${ }^{23}$ At the end of the experiment, worms were gathered and placed in clean DSW water to clear their gut content for $24 \mathrm{~h}$. Finally, wet weight (ww) and dry weight ( $\mathrm{dw}$ ) (heating at $60^{\circ} \mathrm{C}$ for 48 hours) were determined per replicate. The egestion rate was calculated as the amount of faeces produced per worm per hour (mg dw per worm per h). No mortality occurred during the 15 days experiment.

Ingestion, retention and egestion of polystyrene microplastic by G. pulex and H. azteca. The presence of MP in bodies of G. pulex and H. azteca and in their faecal pellets was checked at the end of the 28-day bioassays. Samples were digested with $30 \% \mathrm{H}_{2} \mathrm{O}_{2}$ and incubated at $60^{\circ} \mathrm{C}$ in a water bath for $48 \mathrm{~h}^{155}$ following a protocol modified from Claessens et al. (2013). Afterwards, samples were filtered through $25 \mathrm{~mm}$ Anodisc inorganic filter membranes of $0.2 \mu \mathrm{m}$ pore size, which were dried in an oven at $50{ }^{\circ} \mathrm{C}$ for at least 48 hours and analysed with a $\mu$-Fourier Transform Infrared Spectrophotometer ( $\mu$-FTIR; Nicolet iN10, ThermoFisher) with a single MCT detector and ultra-fast stage. Following Mintenig et al. (2016), ${ }^{32}$ four pre-determined and equally sized chemical maps covering one third of the total filter area were made using an aperture size of $50 \times 50 \mu \mathrm{m}$ and mapping stage step sizes of $20 \mu \mathrm{m}$. A correlation map between the analysed area and the spectra from the original PS MP sample was made with the OMNIC PICTA Software to 
determine identity, number and size of the ingested and excreted particles. The number of MP in the body of the organisms (retained MP) and the number of particles found in their faecal pellets (egested MP) were calculated for each treatment replicate. Organisms and faecal pellets from the $1 \%$ treatment, as well as one replica of the $30 \%$ treatment, were used to optimize the extraction of MP and, therefore, their data were omitted from further analysis. Organisms from four control beakers (exposed to sediment without PS MP) were used as blanks and were also checked for the presence of any MP, which were considered as contamination. The numbers of particles found in controls for the retained and egested particles were subtracted from the numbers of particles found in each replica of each PS treatment, and were 0.10 and 0.6 particles per organism, respectively.

Ingestion and egestion data were expressed on a MP particle number as well as on a weight basis, per unit of weight of organism and sediment. MP number concentrations were directly taken from the FTIR mapping data. Weight based data require a number to weight conversion, for which we used an approximated volume of the particles, and the default density of PS $\left(1.05 \mathrm{~g} / \mathrm{cm}^{3}\right) .{ }^{156}$ The approximate volumes (length $\mathrm{x}$ width $\mathrm{x}$ depth) of the PS MP fragments were calculated as follows. First it was assumed that the particles would prefer a flat position on the filter, such that their length and width dimensions directly measurable from the 2D map top view, each are larger than the third (depth) dimension, which is not observable from the 2D maps. This unknown third dimension was assumed to be the smallest and was thus approximated as half of the second dimension. We emphasise that this method is not accurate for individual particles, but becomes robust when it concerns larger numbers of our irregularly shaped particles where the distribution of the third (depth) dimension can be assumed to be symmetrical. The dry weight ( $\mathrm{dw}$ in $\mathrm{mg}$ ) of the organisms of G. pulex was estimated based on their lengh ( $\mathrm{L}$ in $\mathrm{mm}$ ) as $\mathrm{dw}=0.00321 \times \mathrm{L}^{2.8309} .{ }^{157}$ The number of particles per gram of sediment was calculated from the mass of PS per dose, PS density and the measured particle volume distribution (Figure A2.2).

Data analysis. Survival data as quantal data were analysed using Generalized Linear Model (GLMs) with a binomial distribution and probit model. ${ }^{158}$ One-way Analysis of Variance (ANOVA) and regression analysis were used to evaluate the effects of increasing MP concentrations in sediment on the growth, feeding rate and MP retention and egestion. Normality of the residuals and homogeneity of variances were tested with a Shapiro-Wilk Normality test and Levene's test, respectively. Repeated measures (RM) ANOVA was used to determine the effects on the egestion rate over time. All statistical data analyses were conducted using SPSS 23 (IBM Corp., NY, USA). When a significant effect on an endpoint was found, a four parameter log-logistic dose-response model was fitted; ${ }^{159}$ 


$$
f(x, b, c, d, e)=c+\frac{d-c}{1+\left(\frac{x}{e}\right)^{b}}
$$

where $\mathrm{f}(\mathrm{x}, \mathrm{b}, \mathrm{c}, \mathrm{d}, \mathrm{e})$ is the bioassay response variable, $\mathrm{x}$ is the MP concentration, $e$ is the median effect dose $\left(\mathrm{EC}_{50}\right)$ and $\mathrm{b}, \mathrm{c}, \mathrm{d}$ are fitting parameters. In case parameter $\mathrm{c}$ was zero, Eq. 2 was reduced to a three-parameter model. ${ }^{159}$ The SD of the $\mathrm{EC}_{50}$ was calculated as the $95 \%$ confidence interval (CI95) divided by 1.96 , where CI95 was calculated according to Draper and Smith (1981). ${ }^{146,160}$ The $\mathrm{EC}_{10}$ was calculated by solving the parameterized response model for a $10 \%$ effect dose.

\section{Results and Discussion}

Mortality. Mortality of L. variegatus could not be determined due to their reproduction by fragmentation during the experiment. As the average number of surviving worms per replicate in controls was increased by a factor of at least 1.8 , the reproduction factor could be calculated as the number of worms at the end of the experiment divided by the number of worms at the beginning of the experiment. ${ }^{147}$ Survival for the other species was higher than $80 \%$ in controls, except for G. pulex, for which the average survival was $66 \%$.

Chronic exposure to PS MP concentrations up to $40 \%$ in sediment dw caused no significant mortality in G. pulex, A. aquaticus, S. corneum, H. azteca and Tubifex spp. (Figure 2.1A-E) and no significant effects were found on the reproduction of $L$. variegatus (Figure 2.1F). Same lack of effects on mortality has been reported in earlier studies with benthic macroinvertebrates exposed to MP. For instance, survival of the freshwater amphipods G. pulex and H. azteca were not affected by the exposure to PET and PE MP, respectively ${ }^{83,135}$ Furthermore, no mortality was reported for the marine isopod Idotea emarginata exposed to PE, PS and polyamide (PA) MP and the marine lugworm $A$. marina exposed to PE MP. ${ }^{138}$

Growth. The effect of PS MP concentrations on the growth of the organisms was assessed as a difference in length (in $\mathrm{mm}$ ) for G. pulex, A. aquaticus and S. corneum, and as a difference in dry weight (in $\mathrm{mg}$ ) for $H$. azteca, Tubifex spp. and L. variegatus (Figure 2.2). One-way ANOVA and regression analysis showed no relation between PS MP concentrations in sediment and the growth of A. aquaticus, S. corneum, H. azteca L. variegatus and Tubifex spp. However, individuals of G. pulex exposed to sediment containing high MP concentrations (from 10 to $40 \%$ ) showed a significant reduction in size compared to controls (ANOVA, $P=0.002$ ). The fit of the log-logistic model (Eq. 2) was highly significant $\left(P=2.27 \times 10^{-4}\right)$ and resulted in an $\mathrm{EC}_{50}$ value of $3.57 \%$ sediment $\mathrm{dw}( \pm 3.22)$ and an $\mathrm{EC}_{10}$ value of $1.07 \%$ (Figure A2.3). 


$$
\text { A }
$$
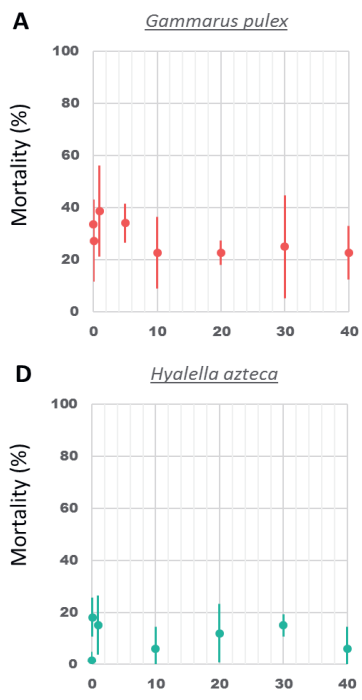

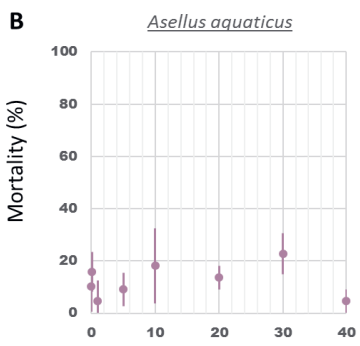

E

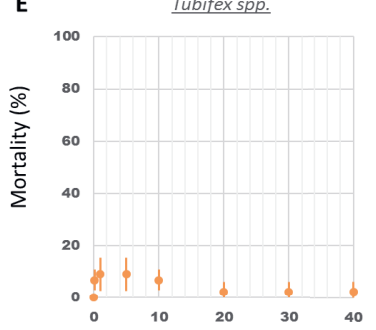

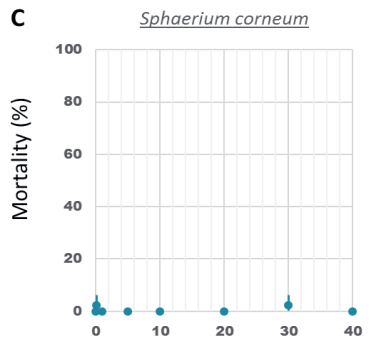

$\mathbf{F}$

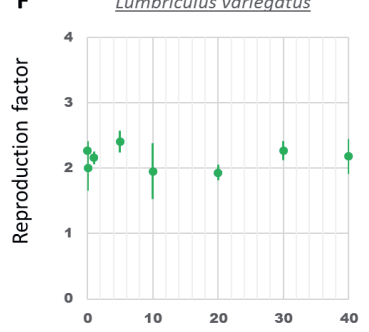

Polystyrene Microplastic Concentration (\% dw in sediment)

Figure 2.1. Mean mortality ( $\pm S D$.) for G. pulex (A), A. aquaticus (B), S. corneum (C), H. azteca (D), Tubifex spp. (E); and Reproduction factor of $L$. variegatus (F) after a 28-day exposure to PS MP concentrations ranging from 0 to $40 \%$ in sediment $d w$.

The CI95 of 3.22 in the $\mathrm{EC}_{50}$ value reflects the rather high variability among replicates. These outcomes reveal that a chronic exposure to PS MP results in a species specific and dose-dependent effect of PS MP on the growth of the benthic macroinvertebrates tested. However, while the growth of G. pulex was significantly reduced with increasing PS MP dose in sediment, the growth of the five other organisms was not altered by the presence of these particles at concentrations up to $40 \%$ plastic in sediment dw. Hence, the $\mathrm{EC}_{10}$ values for these species are higher than $40 \%$ plastic in sediment dw. Growth inhibition of G. pulex by a chronic PS MP exposure from sediment has not been reported before. However, chronic exposure of a closely related freshwater shrimp, G. fossarum, to polymethylmethacrylate (PMMA) and polyhydroxy-butyrate (PHB) MP in water caused a decrease in growth at a concentration of 100,000 MP particles per individual with a similar size range. ${ }^{133} \mathrm{H}$. azteca, another amphipod in the present study, showed no reduction in growth after a 28-day exposure to PS MP concentration up to $40 \%$ in sediment. In contrast, a previous study showed a decrease in the growth of $H$. azteca after a 28-day exposure to PE MP in water at concentrations of 5000 and 10,000 PE MP particles per $\mathrm{ml} .{ }^{83}$ Such differences between study outcomes may relate to (a) differences in the exposure medium, as the presence of natural particles seems to reduce the ingestion of MP in freshwater invertebrates, ${ }^{46}$ and (b) to a higher bioavailability of particles in suspension as compared to particles mixed in the sediment as in the present bioassays. No effects were found on growth for the marine isopod I. emarginata exposed to PE, PS and PA MP in water, ${ }^{136}$ while weight loss of the marine lugworm A. marina was reported at concentrations of $7.4 \% \mathrm{PS} \mathrm{MP}^{137}$ and $>5 \%$ uPVC MP in sediment $\mathrm{dw} .{ }^{92}$ 

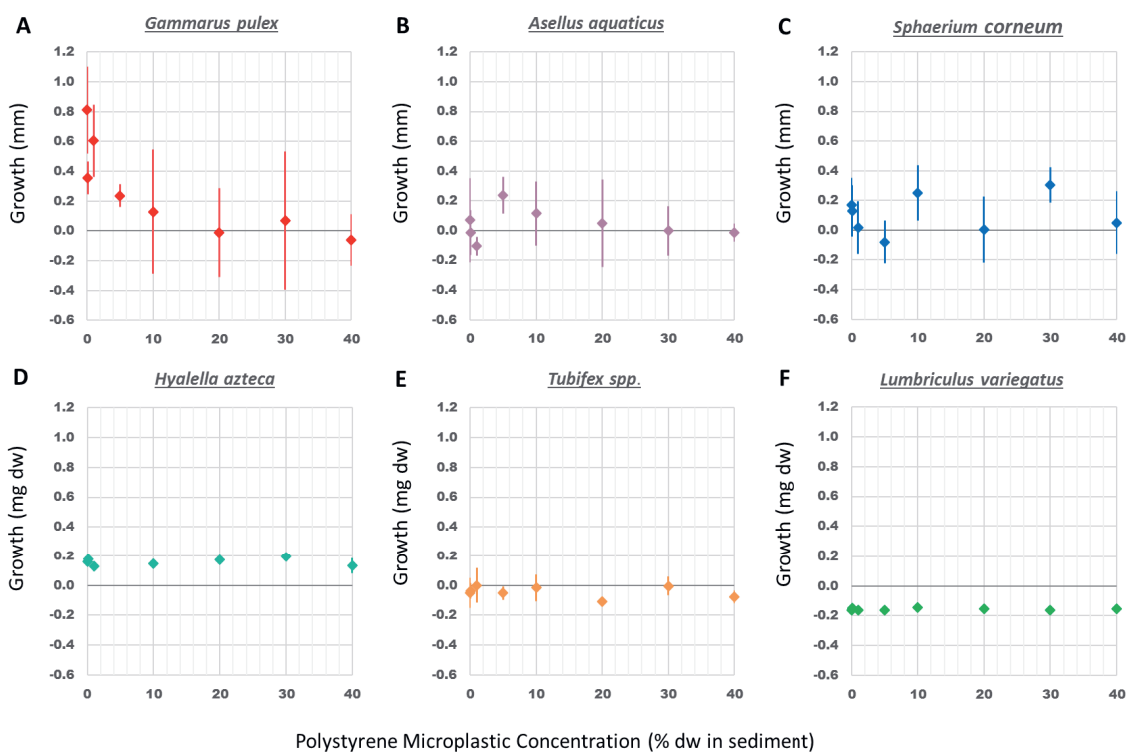

Figure 2.2 Mean growth ( $\pm S D$.) as length (in mm) of G. pulex (A), A. aquaticus (B), S. corneum (C); and as dry weight (in $\mathrm{mg}$ ) of H. azteca (D), Tubifex spp. (E) and L. variegatus (F) after a 28-day exposure to PS MP concentrations ranging from 0 to $40 \%$ in sediment $d w$.

Feeding activity. Feeding rate of G. pulex and H. azteca was calculated as the dry weight (in mg) of Populus sp. leaves consumed per organism per day (Figure A2.4). No differences were found on the feeding activity of $G$. pulex and H. azteca after a 28-day exposure to PS MP concentrations up to $40 \%$ in sediment dw (Figure A2.4). These results are in accordance with Weber et al. (2018), where no effects on the feeding activity of $G$. pulex were found after an exposure to PET MP in water. ${ }^{135}$ These findings indicate that weight loss of G. pulex was probably not caused by a reduction in the consumption of Populus sp. leaves during the experiment and that the presence of MP in the sediment did not alter the feeding rate of these benthic amphipods. Similarly, while the growth of $G$. fossarum was reduced after a 28-day exposure to PMMA and PHB particles, the feeding rate was also unaffected. ${ }^{133}$

The egestion rate of L. variegatus and Tubifex spp. was assessed as the dry weight of the faeces egested (in mg) per organism per hour over a 15-day period (Figure A2.5). At the end of the experiment, all L. variegatus and Tubifex spp. survived and no reproduction was observed in the additional $15-\mathrm{d}$ period experiment. The egestion rate of $L$. variegatus increased during the first week of exposure and then decreased until the end of experiment, while the egestion rate for Tubifex spp. increased over time until the end of experiment (Figure A2.5). The average egestion rates of L. variegatus and Tubifex spp. were 0.43 and $0.32 \mathrm{mg}$ dry faeces per $\mathrm{mg}$ dry organism per $\mathrm{h}$, respectively, and this difference was significant along the sampling time (RM ANOVA; $P<0.05$ ). However, MP exposure had no negative effects on the egestion rate of the worms and the interactive effect between MP exposure and sampling time was also not significant. 
Ingestion, Retention and Egestion of Microplastic. At the end of the 28-day exposure to PS MP, organisms of G. pulex and H. azteca were allowed to clean their gut for $24 \mathrm{~h}$. Remaining faecal pellets as well as the body of the organisms were checked for MP, separately. No MP were found in the body nor in the faecal pellets of H. azteca at any concentration, indicating that these organisms did not ingest microplastic particles in the size range of 20 to $500 \mu \mathrm{m}$ PS. This is consistent with the lack of effect found for this species in the present 28-day exposure test.

In contrast to $H$. azteca, PS MP were found at all concentrations in the body of G. pulex, as well as in their faecal pellets after a 24-hour depuration time. Size frequency distribution of the MP found in the body of all organisms $(n=191)$ ranged from 22 to $165 \mu \mathrm{m}$, with an average size of $61 \mu \mathrm{m}$ (Figure A2.6). The size frequency distribution of the microplastics found in the faeces $(n=840)$ ranged from 16 to $165 \mu \mathrm{m}$, with an average size of $57 \mu \mathrm{m}$ (Figure A2.6). MP with a size $>165 \mu \mathrm{m}$ accounted for only $<0.01 \%$ of the total amount (in number) and were considered to originate from an external source of MP (i.e., particles attached to the external body of the organisms) and were removed from the analysis. The total amount of ingested particles (retained + egested) $(n=1031)$ ranged from 16 to $165 \mu \mathrm{m}$, with an average size of $58 \mu \mathrm{m}$ (Figure A2.6)

A linear regression revealed a significant, positive relation between the number of MP inside the body of $G$. pulex and the number of MP in the sediment exposure medium (Linear Regression; $\mathrm{n}=23 ; P=6.65 \times 10^{-8}$; Figure $2.3 \mathrm{~A}$ ). One of the concentrations was designated as an outlier (Iglewics and Hoaglin's robust test) which was not taken into account in the subsequent determination of the regression parameters. A linear relationship was also found when mass-based concentrations were used (Linear Regression; $\mathrm{n}=23 ; P=3.97 \times 10^{-7}$; Figure 2.3B).
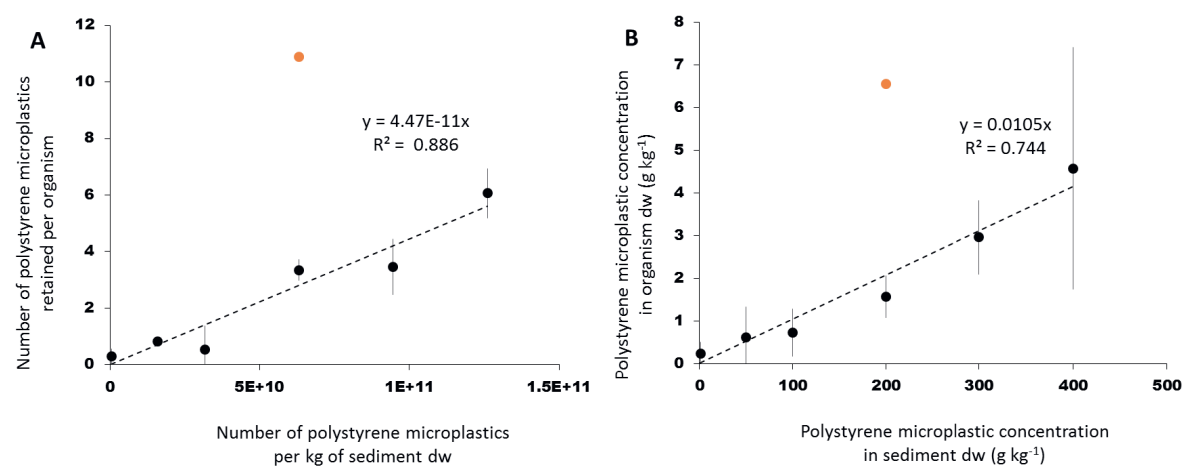

Figure 2.3. Mean PS MP concentration $(n=4)$ per individual of G. pulex $( \pm S D)$ as a function of the PS $M P$ concentrations in sediment, as: (A) number of PS MP retained per organism by number of PS MP per $\mathrm{kg}$ of sediment $\mathrm{dw}$; (B) $\mathrm{g} / \mathrm{kg}$ of PS MP retained per organism $\mathrm{d} w \mathrm{by} \mathrm{g} / \mathrm{kg}$ of PS MP per sediment $d w$. Linear regressions were based on the individual datapoints $(n=22)$ with omission of one suspected outlier (orange marker). 
There was also a significant, positive relation between the number of PS MP in the faeces egested by $G$. pulex and the number of PS MP in sediment (Linear Regression; $\mathrm{n}=23 ; P$ $=6.63 \times 10^{-6}$; Figure A2.7A). Similarly, the weight of PS MP egested per organism dw (g $/ \mathrm{kg})$ also increased linearly with the weight of the PS MP in sediment $\mathrm{dw}(\mathrm{g} / \mathrm{kg})$ (Linear Regression; $\mathrm{n}=23 ; P=4.9 \times 10^{-7}$; Figure A2.7B).

These data show that up to a concentration of $40 \%$, uptake by G. pulex (Figure 2.3) is proportional to the concentration in the sediment, either expressed as number or as mass. Given the demonstrated proportionality between exposure and uptake, the slope of the line in Figs 2.3A and B can be interpreted as trophic transfer factors (TTF) with a value of $(4.47 \pm 0.35) \times 10^{-11}\left(\mathrm{TTF}_{\text {number}}\right.$; Figure 2.3A) and $(10.5 \pm 1.3) \times 10^{-3}\left(\mathrm{TTF}_{\text {mass }}\right.$; Figure 2.3B). The TTF represents the ratio of the MP concentration in the organism and that in the sediment exposure medium, which appears to be constant up to $40 \%$ sediment $\mathrm{dw}$. These TTF values are low, which can be explained by the fact that only a limited part of the size range in the sediment is actually taken up, that is, the TTFs mechanistically reflect transfer and size selection. When corrected for the $165-500 \mu \mathrm{m}$ bio-unavailable fraction, pure estimates of net transfer are obtained, being TTF $=(5.16 \pm 0.40) \times 10^{-11}$ (number) and $0.028 \pm 0.0036$ (mass). As previously stated for other freshwater amphipods exposed to MP, our results indicate that growth reduction of G. pulex was a sub-lethal effect caused by a lower ability of these organisms to assimilate food due to the ingestion of PS MP, ${ }^{83,133}$ as well as by the gut blockage by these particles due to a longer excretion time needed to depurate their gut. ${ }^{83}$ Therefore, the observed constancy and magnitude of TTF may still change over time. Based on MP excretion studies performed with other freshwater amphipods exposed to different MP types, ${ }^{83,133}$ G. pulex is expected to be able to completely depurate if enough time is given and if the ingestion of particles concludes. These findings indicate that MP uptake is size-dependent and that shape might affect the ability of organisms to excrete them. This is in accordance with previous studies showing that MP uptake by freshwater invertebrates is size-specific and feeding type dependent and that irregularly shaped MP need a significant longer clearance time in comparison to spherical MP. ${ }^{46,83}$ Moreover, the high mobility of G. pulex ${ }^{161}$ could have increased MP uptake in comparison to the other epibenthic species, revealing the importance of species specific traits in the effects of MP on benthic invertebrates.

\section{General discussion}

We showed that for a range of freshwater species with different traits exposed to PS MP in sediment under the same environmentally relevant conditions, no effect was found for five out of six species even at extremely high concentrations ( $40 \%$ sediment $\mathrm{dw})$. Only for one of the species, G. pulex, a significant reduction in growth was found, which is likely to be explained by the demonstrated size-selective uptake of PS MP and their slow excretion, leading to a depletion of energy reserves as found earlier for marine worms as a result of MP ingestion..$^{92}$ As mentioned earlier, our wide range of PS particle sizes and shapes can be considered as a fair approximation of environmental MP when it concerns their physical effects. Field measured concentrations in freshwater sediments, ${ }^{24,26,129-131}$ 
although often provisional due to methodological limitations, are far below the calculated $\mathrm{EC}_{10}$ or $\mathrm{EC}_{50}$ effect threshold values for $G$. pulex. This means that extrapolating these results to the environment leads to small chances of such physical effects, and consequently low current risk for the benthic community of freshwater systems. However, MP concentrations are expected to increase in the environment, ${ }^{162}$ which implies that effects are not unthinkable in the future. Finding high effect thresholds for most species does not rule out risks on the level of biodiversity or on the community functioning, as these also depend on the performance of the most sensitive species, here G. pulex. In fact, G. pulex plays a key role in the processing of coarse particulate organic matter in streams, ${ }^{163}$ is an important prey for fish, ${ }^{164}$ and its feeding inhibition has shown to alter the benthic macroinvertebrate community, ${ }^{152}$ which means that responses at community and ecosystem levels could occur over time. Eventually, the combination of effect threshold data in species sensitivity distributions may represent a more refined approach as part of a higher tier in the assessment of physical effects of MP. ${ }^{11}$ Moreover, for G. pulex we demonstrated ingestion to be proportional to dose and we introduced the concept of TTF accumulation factors for MP, which may be useful in exposure assessments. If the observed ingestion behaviour would be confirmed to be general among benthic invertebrates, uptake and exposure models may rely on using constant ingestion rates or steady state TTFs for a wide range of MP concentrations in sediments.

\section{Acknowledgements}

This study was funded by the Dutch Technology Foundation TTW, project no. 13940. We acknowledge additional support from KWR; IMARES; NVWA; RIKILT; the Dutch Ministry of Infrastructure and the Environment; the Dutch Ministry of Health, Welfare and Sport; Wageningen Food \& Biobased Research; STOWA; RIWA; and water boards Hoogheemraadschap van Delfland, Zuiderzeeland, Rijn en IJssel, Vechtstromen, Scheldestromen, Aa en Maas, de Dommel, and Rivierenland. We would like to thank John Beijer and Jos Sewalt for their practical assistance and advice, Svenja Mintenig for her help in the development of the $\mu$-FTIR analysis protocol and Lyke Stuurman and Dianneke van Wijk for performing the first pilot tests. 



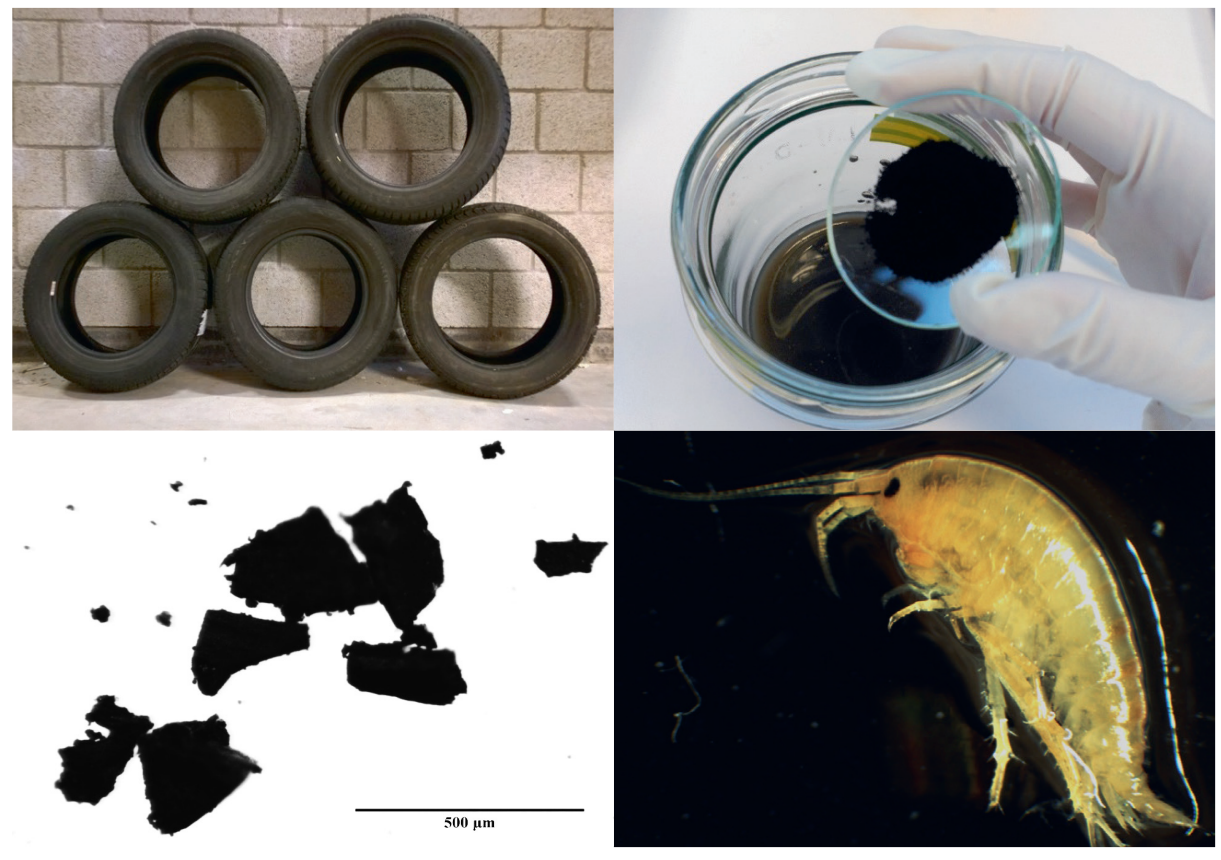




\section{Chapter 3}

\section{Ingestion and Chronic Effects of Car Tire Tread Particles on Freshwater Benthic Macroinvertebrates}

Published as: Redondo-Hasselerharm, P.E.; De Ruijter, V.N.; Mintenig S.M.; Verschoor, A.; Koelmans, A.A. Ingestion and Chronic Effects of Car Tire Tread Particles on Freshwater Benthic Macroinvertebrates. Environ. Sci. Technol. 2018. $52,13986-13994$. 


\begin{abstract}
Micronized particles released from car tires have been found to contribute substantially to microplastic pollution, triggering the need to evaluate their effects on biota. In the present study, four freshwater benthic macroinvertebrates were exposed for 28 days to tread particles (TP; $10-586 \mu \mathrm{m}$ ) made from used car tires at concentrations of $0,0.1,0.3$, 1, 3 and $10 \%$ sediment dry weight. No adverse effects were found on the survival, growth and feeding rate of Gammarus pulex and Asellus aquaticus, the survival and growth of Tubifex spp., and the number of worms and growth of Lumbriculus variegatus. A method to quantify TP numbers inside biota was developed and here applied to G. pulex. In bodies and faeces of $G$. pulex exposed to $10 \%$ car tire TP, averages of 2.5 and 4 tread particles per organism were found, respectively. Chemical analysis showed that, although car tire TP had a high intrinsic zinc content, only small fractions of the heavy metals present were bioavailable. PAHs in the TP-sediment mixtures also remained below existing toxicity thresholds. This combination of results suggests that real in situ effects of TP and TPassociated contaminants when dispersed in sediments are probably lower than those reported after forced leaching of contaminants from car tire particles.
\end{abstract}




\section{Introduction}

During the past decade, extensive research has been conducted to evaluate the emissions and environmental concentrations of microplastics (MP) worldwide. A few studies considered micronized particles released from car tires as part of MP pollution and concluded that they constitute a significant global source of MP. ${ }^{14,165,166}$ This fact, together with the concerns raised by governmental institutions and the general public about potential adverse effects of particles released from car tires, brings out the need to quantify the amount of car tire particles in the environment and to evaluate their bioavailability and effects on biota. ${ }^{11,165,167,168}$

Depending on the generation process and their composition, different car tire particle types are formed. Tread particles (TP) originate from the grinding or abrasion of a tire tread, ${ }^{169}$ which include the finely crushed rubber particles made from old car tires that are commonly used in synthetic turf fields. ${ }^{167}$ Tire wear particles (TWP) can be released into the environment as a result of the mechanical abrasion of car tires with the road surface. ${ }^{170}$ Although rubber is the main constituent of car tires, sulphur and zinc oxide are added during the vulcanisation process, black carbon or silica are added as fillers, and oil is added to increase the wet grip performance. ${ }^{165}$ Besides these general additives, tires can contain other additives depending on their specific properties defined by the application. ${ }^{11,165}$ In aquatic systems, chemicals may leach out into the aqueous phase at different rates depending on the environmental conditions (temperature, $\mathrm{pH}$ and salinity) and the composition and size of the particles. ${ }^{11,171,172}$ For instance, total zinc content has been found to be three times higher in TP than in TWP. ${ }^{169}$ Different release rates can lead to differences in chemical bioavailability and to complex mixture effects.

TWP released from car tires and old tire TP used as infill in artificial turfs are the most important sources for micronized rubber particles in the environment. ${ }^{165}$ Whereas MP detection methods have evolved considerably over the past decade, ${ }^{32}$ the methodology used to quantify the amount of car tire particles in the environment is still limited. Car tire particle concentrations in water and sediments are estimated based on chemical markers, such as benzothiazoles or zinc, or the rubber type, ${ }^{171}$ markers that are unable to distinguish between TP and TWP, and may include chemicals from other environmental sources. ${ }^{111}$ Concentrations in biota have never been measured, probably because their levels are below the detection limits. No laboratory studies on the ingestion of TWP or $\mathrm{TP}$ are available either. Car tire particle concentrations have been measured in surface waters $(0.09-6.3 \mathrm{mg} / \mathrm{l})$ and sediments $(0.3-155 \mathrm{~g} / \mathrm{kg}$ dry weight $) .{ }^{173-178}$ This indicates that part of the car tire particles entering the surface water may sink to the sediment compartment due to their higher density. ${ }^{27}$

To date, effect studies done on aquatic biota have mostly focused on the evaluation of the effects of car tire leachates, which were often extracted under conditions forcing chemical release, such as high temperatures and low $\mathrm{pH}$ values. ${ }^{171}$ Some of the leachates were prepared using whole tires, ${ }^{179,180}$ while others were extracted from TP or TWP, ${ }^{172,181-183}$ 
which seem to be more toxic, probably due to a faster release of chemicals from smaller particles. ${ }^{171}$ Although studying the effects of elutriates extracted from car tire particles may be useful for screening level assessments, evaluating the effects of car tire particles in water or sediment under natural conditions is more environmentally realistic. ${ }^{184}$ For instance, earlier studies showed adverse effects of car tire TP on the development of Rana sylvatica larvae at a concentration of $83.8 \mathrm{~g} / \mathrm{kg}$ of sediment dry weight. ${ }^{185}$ In contrast, no effects of $10 \mathrm{~g}$ of tire and road wear particles (TRWP) per kg of sediment dry weight were found for the amphipod Hyalella azteca and the larvae midge Chironomus dilutus. ${ }^{184}$

Knowing that sediments accumulate settling car tire particles, their bioavailability and effects on benthic macroinvertebrates should be evaluated. Moreover, for a proper assessment of the risks of car tire TP, not only environmentally realistic conditions and concentrations should be used, but also a systematic setup should be followed in order to ensure the comparability among species. In the present study, chronic effects of car tire TP were evaluated for four freshwater benthic macroinvertebrates: the amphipod Gammarus pulex, the isopod Asellus aquaticus and the worms Tubifex spp. and Lumbriculus variegatus. We used a standardized setup, previously used to evaluate the effects of polystyrene (PS) MP on the same species (Chapter 2). ${ }^{186}$ Effects were assessed using a wide range of environmentally relevant concentrations of car tire TP, under environmentally realistic conditions. Additionally, for the first time, tread particle ingestion and egestion were investigated. This was done for G. pulex by quantifying the number of particles in their body and in faeces at the end of the experiment. Here, $G$. pulex was used as a model invertebrate species for ingestion, as it was demonstrated their ability to ingest MP in an earlier work (Chapter 2). ${ }^{186}$ We developed a method in order to be able to assess TP particles inside organisms, which included testing the resistance of TP rubber materials to animal tissue digestion fluids and developing an image analysis approach for quantifying ingested TP particles.

\section{Material and Methods}

Preparation of the car tire tread particles. With the purpose of mimicking an environmentally relevant scenario of car tire TP exposure, five second hand tires of various brands were bought in Supervelg (Drunen, the Netherlands) (Table A3.1). Using a metal grater, milimiter sized particles were scrapped from the first $2 \mathrm{~cm}$ of each tire. After freezing the particles with liquid nitrogen to prevent them from burning, they were ground and sieved over a $500 \mu \mathrm{m}$ sieve in an Ultra Centrifugal Mill ZM 1000 (Retsch, Germany). A mixture was made by the combination of the five car tires particles at equal weight proportions and this mixture was sieved again over a $500 \mu \mathrm{m}$ sieve to guarantee any bigger particles being removed.

Characterization of the car tire tread particles. The particle size distribution (PSD) of the car tire TP mixture was measured by laser diffraction using a Mastersizer 3000 (Malvern Instruments), which is capable of measuring particle sizes between $0.01-3500 \mu \mathrm{m}$. Particle shape was examined under an Olympus SZX10 stereomicroscope. Main 
constituents of the car tire TP were quantified using thermogravimetric analysis (TGA/DSC 3+, Mettler Toledo). Upon heating the sample, the mass loss was determined allowing to distinguish between (i) volatile substances (e.g. plasticizers, that vaporize between $\left.30-300{ }^{\circ} \mathrm{C}\right)$, (ii) the actual polymer $\left(300-600{ }^{\circ} \mathrm{C}\right)$, (iii) black carbon $(600-$ $850^{\circ} \mathrm{C}$ ), and the residue, which is composed of (iv) inorganic fillers (e.g. zinc oxide). ${ }^{187,188}$ For the combustion of black carbon the gas was switched from nitrogen to air $\left(50 \mathrm{ml} \mathrm{min}{ }^{-}\right.$ ${ }^{1}$, see Table A3.2 in the Appendix for instrumental settings). While heating from 300 to $600{ }^{\circ} \mathrm{C}$, the evolved gases were trapped to further characterize comprised polymers. This was done by coupling a cartridge filled with a hydrophilic-lipophilic balanced polymer (HLB, Oasis Water Corporation, Massachusetts, USA) directly to the TGA. Trapped decomposition products were extracted by flushing with $2 \mathrm{ml}$ of Dichlormethane (DCM, Honeywell Research Chemicals, USA) of which $2 \mu$ were injected manually in a gas chromatograph coupled to a mass spectrometer (GC-MS, Agilent Technologies, Table A3.2). Characteristic mass spectra of the decomposition products (pyrolysates) of polymers typically used in tire production were taken from literature (Table A3.3). ${ }^{189,190}$ Their presence was used to identify polymers incorporated in the car tire TP. Finally, the total amount of zinc was quantified. The inorganic residues $(120 \mathrm{mg})$ were exposed to microwave acid extraction using 13\% nitric acid (Merck, Suprapur), heated under pressure and kept at temperatures between $133-163{ }^{\circ} \mathrm{C}$ for 30 minutes. Subsequently, the sample was filtered and the total amount of zinc was determined using Inductively Coupled Plasma Mass Spectrometry (ICP-MS) (X Series 2, Thermo Fisher Scientific) (Table A3.2).

Sediments. Freshwater sediments were collected with a standard dip net at Veenkampen (Wageningen, The Netherlands) in December 2016. Previous studies have shown that PAH background concentrations at this location are below toxicity thresholds. ${ }^{144,191,192}$ Sediments were sieved, homogenized and placed in a freezer at $-20^{\circ} \mathrm{C}$. Prior to the experiments, sediments were thawed and thoroughly homogenized again, and four representative subsamples were taken to determine the percentage of Total Organic Matter (TOM) through loss on ignition $\left(3 \mathrm{~h}, 550{ }^{\circ} \mathrm{C}\right)$, which was $40 \pm 0.8 \%(\mathrm{n}=4)$. All data are depicted with mean \pm standard deviation, unless otherwise stated.

Test organisms. Following previous procedures (Chapter 2), ${ }^{144,186}$ G. pulex and A. aquaticus were collected from a relatively unpolluted brook (Heelsum, The Netherlands) and ditch (Heteren, The Netherlands). L. variegatus were obtained from Wageningen Environmental Research (Wageningen, The Netherlands) and Tubifex spp. were purchased at a local pet shop. Prior to the experiments, organisms were acclimatized for one week in aerated buckets with copper-free Dutch Standard Water (DSW) inside a water bath at $16 \pm 1{ }^{\circ} \mathrm{C}$ while maintaining a 12:12 light:dark cycle. During the acclimatization, G. pulex and A. aquaticus were fed with dry poplar leaves that were collected in the field and Tubifex spp. and L. variegatus were fed with TetraMin ${ }^{\circledR}$ fish food pellets. 
Experimental design. Sediments were spiked to achieve the concentrations: 0, 0.1, 0.3, 1, 3 and $10 \%$ of car tire TP dry weight in the total sediment mixture. These concentrations correspond to $0,1.0,3.0,10,30$ and $100 \mathrm{~g} / \mathrm{kg}$ respectively, which are within the range of measured car tire wear and tear particle concentrations in sediments. ${ }^{165}$ Each experimental unit consisted of a $750 \mathrm{ml}$ glass beaker filled with $211 \mathrm{~g}$ of the corresponding car tire TP - sediment mixture. For each concentration, 3 replicas were made. Two weeks prior to the start of the experiment, beakers were placed in a water bath at a constant temperature of $16 \pm 1{ }^{\circ} \mathrm{C}$ and aerated. Then, 11 randomly selected individuals from the corresponding species were placed in the experimental units. The starting length of 33 randomly selected individuals from the initial population was assessed as body lengh for A.aquaticus, and head capsule (HD in $\mathrm{mm}$ ) for G. pulex, from which total length (TL) was calculated as $\mathrm{TL}=-2.07+9.82 \mathrm{HD} .{ }^{151}$ The average size of $G$. pulex and A. aquaticus was $4.6 \pm 0.8 \mathrm{~mm}$ $(\mathrm{n}=33)$ and $4.5 \pm 0.5 \mathrm{~mm}(\mathrm{n}=33)$, respectively. The starting dry weight of 33 active adult worms from the initial population of Tubifex spp. and L. variegatus was determined. The average dry weight per worm was $0.42 \pm 0.05 \mathrm{mg}(\mathrm{n}=33)$ for Tubifex spp. and $1 \pm$ $0.08 \mathrm{mg}(\mathrm{n}=33)$ for L. variegatus. During the experiments, 2 poplar leaves discs with a diameter of $3 \mathrm{~cm}$ were supplied to the beakers of G. pulex and A. aquaticus at day 0 and 14. Poplar leaves discs were previously conditioned with DSW for 3 days. No additional food was needed for Tubifex spp. and L. variegatus due to the high organic matter content of the sediment. Temperature, dissolved oxygen (DO), $\mathrm{pH}$ and $\mathrm{NH}_{3}$ were measured in all beakers once a week, while conductivity (EC) was measured only at the start and at the end of the experiment. To keep water levels constant, DSW was added weekly until the end of the experiment. Water quality variables remained constant in all beakers along the experiment (Table A3.4). Unionised levels of ammonia decreased along the experiment for all species, reaching an average of $0.03 \pm 0.01 \mathrm{mg} \mathrm{NH}_{3} / \mathrm{L}(\mathrm{n}=12)$ at the end of the experiment. All unionised ammonia levels were always below the $\mathrm{LC}_{50}$ values available for these species. ${ }^{143,149,193}$

Analysis of heavy metals and PAHs in sediments mixed with car tire tread particles. Especially heavy metals and polycyclic aromatic hydrocarbons (PAH) are relevant in explaining potential chemical effects from sediments polluted with TP. ${ }^{171,194,195}$ Therefore, sediments with TP mixed from all treatments were analysed for heavy metals and PAHs at $\mathrm{t}=0$. Two extra beakers were prepared in the same way and at the same time as the experimental units. After the 2 week acclimatization period, sediments from the two duplicates were mixed and freeze dried. The total amount of Zinc (Zn), Sulphur (S), Cadmium (Cd), Chromium (Cr), Copper (Cu), Nickel (Ni), and Lead (Pb) were analysed using microwave acid extraction with Inductively Coupled Plasma Atomic Emission Spectroscopy (ICP-AES) and ICP-MS after destruction with $\mathrm{HNO}_{3}-\mathrm{HCl} .{ }^{196}$ Additionally, the sediment-TP mixtures were extracted with $0.01 \mathrm{M} \mathrm{CaCl}_{2}$ to determine the mildly extractable concentrations as a proxy for bioavailable metal concentrations. ${ }^{197}$ Following earlier procedures, ${ }^{144}$ PAHs were extracted from the sediment-TP mixtures using accelerated solvent extraction (ASE) and analysed by High Performance Liquid Chromatography (HPLC) after sample clean-up. Clean-up recoveries for $14 \mathrm{PAH}$ were 
$91 \pm 6 \%$, and ranged from 78 to $98 \%$. Concentrations were corrected for blanks and recoveries. For further details on PAH analysis, the reader is referred to the Appendix.

Effects on Survival, growth and feeding rate: After 28 days, the content of each experimental unit was sieved over a $0.35 \mathrm{~mm}$ sieve. Surviving organisms were collected, counted and transferred to clean DSW to depurate their gut for 24 hours, following procedures from previous MP ingestion studies..$^{53,134,137}$ The number of worms per replicate was used as endpoint for L. variegatus instead of survival, as they reproduced by fragmentation during the experiments. ${ }^{198}$ G. pulex and A. aquaticus were preserved in $70 \%$ ethanol until their length was measured, which was done in the same way as for the starting population. Growth was determined as the difference in mean length (in $\mathrm{mm}$ ) of the animals in each replicate at the end minus the mean length from 33 animals at the start of the experiment.The growth of Tubifex spp. and L. variegatus was determined as biomass increase per replicate by subtracting the average dry weight of the starting population from the average dry weight of the populations at the end of the exposure test. Feeding rate (mg dry weight leaf/organism/d) of G. pulex and A. aquaticus was calculated from the loss of the added poplar leaves using the equation from Maltby et al. (2002), described in the Appendix. ${ }^{152}$

Resistance of car tire TP to $\mathrm{H}_{2} \mathrm{O}_{2}$ and ingestion by G. pulex.

Surviving individuals of G. pulex from controls (TP concentration of $0 \%$ sediment dry weight) and the two highest exposure concentrations ( 3 and 10\% car tire TP in sediment dry weight), as well as the faeces excreted by these organisms during the 24-hour defeacation period, were analysed for the presence of car tire TP using $30 \% \mathrm{H}_{2} \mathrm{O}_{2}$ to purify the biota samples.

Resistance of car tire TP to $\mathrm{H}_{2} \mathrm{O}_{2}$. Prior to the purification of the samples, the resistance of car tire TP to $30 \% \mathrm{H}_{2} \mathrm{O}_{2}$ was tested. For this, 80 car tire TP cut from the scrapped sample were distributed in 8 porcelain cups and dried in an oven at $40{ }^{\circ} \mathrm{C}$ for $72 \mathrm{~h}$. The mean dry weight of the particles from each cup $(n=10)$ was measured with a Cubis ${ }^{\circledR}$ Micro balance (Sartorius, Germany). Pictures of each particle were taken with a CMEX camera (Euromex, The Netherlands) under an Olympus SZX10 stereomicroscope and the mean particle area $(n=10)$ was measured using ImageJ Software. Four groups of ten particles were added to glass beakers containing $30 \% \mathrm{H}_{2} \mathrm{O}_{2}$ and the other four were added to glass beakers containing Milli-Q water. All glass beakers were placed in a New Brunswick Scientific G25 shaking incubator at $45^{\circ} \mathrm{C}$ and $80 \mathrm{rpm}$ for 24 hours. After this period, all particles were flushed with water and dried in an oven at $40^{\circ} \mathrm{C}$ for $72 \mathrm{~h}$. Finally, the mean weight of the particles was measured again and new pictures were taken to calculate the mean particle area.

Ingestion of TP by G. pulex. Following the protocol by Löder et al. (2017) with modifications, ${ }^{199}$ bodies and faeces of $G$. pulex were added to $10 \mathrm{ml}$ of $30 \% \mathrm{H}_{2} \mathrm{O}_{2}$ and placed in a New Brunswick Scientific G25 shaking incubator at $45^{\circ} \mathrm{C}$ and $80 \mathrm{rpm}$ for 24 
hours. The presence of car tire TP in bodies and faeces was studied separatelly, whereas bodies and faeces from each individual replica were pooled and treated together. A subsequent chitinase step was needed for the body samples to remove all chitine leftovers. For this, to remove the $30 \% \mathrm{H}_{2} \mathrm{O}_{2}$, each sample was filtered through a stainless steel filter with a mesh size of $10 \mu \mathrm{m}$. The residues on the filter were rinsed with $15 \mathrm{ml}$ of phosphatebuffered saline (PBS) solution (pH 5) into a beaker in which $1 \mathrm{ml}$ of chitinase (EC 3.2.1.14, ASA Spezialenzyme GmbH, Wolfenbüttel, Germany) was added. Samples were placed in a New Brunswick Scientific G25 shaking incubator at $37{ }^{\circ} \mathrm{C}$ and $80 \mathrm{rpm}$ for 5 days. Finally, samples were filtered through $25 \mathrm{~mm}$ aluminium oxide filters (Anodisc, Whatman, UK) with a pore size of $0.2 \mu \mathrm{m}$, which were dried in an oven at $45^{\circ} \mathrm{C}$ for at least 48 hours. Note that Raman or Fourier Transform Infrared Spectroscopy (FTIR) used to identify MP in biota, such as in Redondo-Hasselerharm et al. (2018) (Chapter 2), are not applicable to black particles due to high IR absorption. ${ }^{200,201}$ Therefore, all black particles found on each of the filters were photographed with a CMEX camera (Euromex, The Netherlands) under an Olympus SZX10 stereomicroscope. Particle size was measured using image analysis software (ImageJ). Only particles within the size range of the original TP mixture were accounted. Finally, the number of black particles within the size range of $10-586 \mu \mathrm{m}$ found in each filter was divided by the number of surviving individuals in the corresponding replicate at the end of the experiment to obtain the number of particles per organism. Blanks were included $(n=3)$ to correct for contamination by particles within the targeted size range. Size frequency of the particles found in bodies and faeces of G. pulex at concentrations 3 and $10 \%$ were analysed after measuring their length (in $\mu \mathrm{m}$ ) in ImageJ. Following our previously published approach (Chapter 2), ${ }^{186}$ the number of car tire TP per gram of sediment was calculated from the mass of car tire TP per dose, TP density and the measured particle volume distribution.

Statistical analysis. Data analysis was done in SPSS 23 (IBM Corp., NY). Generalized Linear Models (GLMs) were applied to study the effects of car tire TP on all endpoints using the log-transformed concentration as covariate. GLMs were selected based on the data distribution of each endpoint. One-way ANOVA $(\mathrm{p}<0.05)$ were conducted to determine the effects of car tire TP on the number of worms of $L$. variegatus, the growth of G. pulex, A. aquaticus, Tubifex spp., and L. variegatus, and the feeding rate of G. pulex and $A$. aquaticus. One-way ANOVA was also used to study differences in the number of car tire TP found in bodies and faeces of G. pulex at zero concentration (i.e. the blanks) and the two highest concentrations. Residuals were tested for normality using ShapiroWilk test $(\mathrm{p}>0.05)$ and visualized with a Q-Q plot. Variances were tested for homogeneity using Levene's test $(\mathrm{p}>0.05)$. Post hoc multiple comparisons were done using Tukey's and Bonferroni tests. If the assumption of homogeneity of variances was violated, one-way Welch ANOVA $(\mathrm{p}<0.05)$ was conducted. An independent t-test was applied to compare the average dry weight $(\mathrm{mg})$ and the average area $\left(\mathrm{mm}^{2}\right)$ of the particles before and after the $\mathrm{H}_{2} \mathrm{O}_{2}$ and the $\mathrm{H}_{2} \mathrm{O}$ treatments. The difference in dry weight and area between the particles before and after each treatment was compared between treatments as well. 


\section{Results and Discussion}

Characterization of car tire TP. Particle size distribution of the car tire TP mixture showed an unimodal distribution spanning from approximately $10 \mu \mathrm{m}$ to $586 \mu \mathrm{m}$ with a modus centred at $239 \mu \mathrm{m}$ by volume (Figure A3.1A) and $10.5 \mu \mathrm{m}$ by number of particles (Figure A3.1B). This size distribution included previously reported size ranges for TWP and TP. ${ }^{169,202}$ Particle shape was found to be generally angulated (Figure A3.2), as described by Kreider et al. (2010) for TP. ${ }^{169}$

TGA analysis revealed that the tire mixture contained volatile substances (7\%), polymeric substances (52.4\%), black carbon (6.5\%) and inorganic fillers (34.1\%) (Figure A3.3). Car tire TP were also analysed individually, revealing a similar composition for all tires used (Figure A3.3). Thus, further analyses were only conducted for the car tire TP mixture. The MS data were screened for the presence of decomposition products of polymers typically used during tire production ${ }^{189,203}$ after which methyl-butadiene and dipentene, butadiene and styrene were confirmed (Figure A3.4). That implies that the car tire TP consisted of blends of polyisoprene and styrene butadiene rubber (SBR) (Table A3.3). Benzothiazole (indicator $\mathrm{m} / \mathrm{z} 135$ ), used as a vulcanisator during the tire production, was identified too (Figure A3.4). The total amount of zinc, determined from the inorganic tire residues $(16.58 \mathrm{~g} / \mathrm{kg})$, was $5.65 \mathrm{~g}$ zinc $/ \mathrm{kg}$ tire TP mixture.

Analysis of heavy metals and PAHs in sediments mixed with car tire tread particles.

Metal analysis. Concentrations of $\mathrm{Zn}, \mathrm{Cd}, \mathrm{Cr}, \mathrm{Cu}, \mathrm{Ni}, \mathrm{Pb}$ and $\mathrm{S}$ in the sediment- $\mathrm{TP}$ mixtures did not vary among treatments, except for $\mathrm{Zn}$ (Table A3.5). This implies that the added TP did not contain sufficient quantities of these elements to cause a measurable change in overall concentrations, except for $\mathrm{Zn}$. The total concentration of $\mathrm{Zn}$ in sediments was linearly correlated $\left(\mathrm{R}^{2}=0.99\right)$ with the nominal concentration of car tire TP in sediment (Figure A3.5). The slope of this line represents the $\mathrm{Zn}$ added with every extra $1 \%$ of TP, which translates into a tire TP mixture $\mathrm{Zn}$ content of $6.54 \pm 0.37 \mathrm{~g} / \mathrm{kg}$. This is only slightly different from the value of 5.65 mentioned above, which is explained from the different digestion and analytical method used. The linearity illustrates the accuracy of the dosing and the mixing. The data show that by adding TP up to $10 \%$ dry weight, the sediment background $\mathrm{Zn}$ concentration of $75 \mathrm{mg} / \mathrm{kg}$ was increased almost tenfold to $735 \mathrm{mg} / \mathrm{kg}$. The $\mathrm{CaCl}_{2}$ extractable (bioavailable) concentrations of $\mathrm{Zn}$, however, were a factor of 1000 times lower than the total amount (Table A3.5), and in fact were below the detection limit and remained at least a factor 30 below the $\mathrm{LC}_{50}$ values for Tubifex spp. and L. variegatus $\left(990.1 \mu \mathrm{g} / \mathrm{kg}\right.$ and $2954 \mu \mathrm{g} / \mathrm{kg}$, respectively). ${ }^{204,205} \mathrm{As}$ for the other $\mathrm{CaCl}_{2}$ extractable elements, only $\mathrm{S}, \mathrm{Cr}$ and $\mathrm{Ni}$ were detected. They also did not increase with increasing car tire TP concentrations in sediment and also remained at non-toxic concentrations. These chemical data already show that TP elutriate tests are not likely to represent ecologically relevant results as they do not account for the limited bioavailability of metals in the sediment mixture. ${ }^{172,181}$ 
PAH analysis. PAH concentrations did not increase with increasing car tire TP concentrations in sediment (Table A3.6), and PAH concentrations in controls (TP concentration of $0 \%$ sediment dry weight) were similar to previously reported $\mathrm{PAH}$ concentrations for the same sediment. ${ }^{144}$ Therefore, we conclude that PAHs did not leach from the car tire TP to the sediment and did not contribute to the PAH concentration in the systems. This is in agreement with previous studies that also reported a low contribution of TP and TWP to PAH concentrations to the environment. ${ }^{169,206}$ PAHs are not easily extracted even under extreme environmental conditions and its bioavailability is expected to be low. ${ }^{11,169}$ The Sum of PAHs ( $\sum$ PAH) for all sediment-TP mixtures was at least 2 times lower than the probable effect concentration (PEC) reported by MacDonald et al. (2000), which is $22.8 \mathrm{mg} / \mathrm{kg}$ dry weight. ${ }^{207}$ Note that outlying PAH concentrations were observed for the lowest TP treatment $(0.1 \%)$ with factor 10 higher numbers than those for all other treatments and those previously reported for the same sediment. ${ }^{144}$ We have no conclusive explanation for the outlier but an incidental contamination may have played a role.

Effects on survival, growth and feeding rate. Survival in controls (TP concentration of $0 \%$ sediment dry weight) was on average $79 \%, 73 \%$ and $84 \%$ for G. pulex, A. aquaticus and Tubifex spp., respectively. Data analysis with GLM revealed no significant relationship between the survival of $G$. pulex, A. aquaticus and Tubifex spp. and increasing car tire TP concentrations in sediment $\left(\mathrm{GLM} ; P_{\text {G.pulex }}=0.063 ; P_{\text {A.aquaticus }}=\right.$ 0.654; $P_{\text {Tubifexsp. }}=0.692$ ) (Figure 3.1). For L. variegatus, there was no significant relationship between the number of worms and increasing car tire TP concentrations $\left(\mathrm{GLM} ; P_{\text {L.variegatus }}=0.380\right)$ and no significant differences were found between car tire TP concentrations in sediment and the number of worms at the end of the experiment $\left(\right.$ ANOVA; $P_{\text {L.variegatus }}=0.084$ ) (Figure 3.1). No significant relationship between the growth of G. pulex, A. aquaticus, Tubifex spp. and L. variegatus and increasing car tire $\mathrm{TP}$ concentrations in sediment was found (GLM, $P_{\text {G.pulex }}=0.554 ; P_{\text {A.aquaticus }}=0.470$; $\left.P_{\text {Tubifex spp. }}=0.160 ; P_{\text {L.variegatus }}=0.262\right)$. No significant differences between car tire TP concentration in sediment and growth were found for G. pulex, A. aquaticus, Tubifex spp. and $L$. variegatus (Welch, $P_{G \text {. pulex }}=0.334$; ANOVA $P_{\text {A.aquaticus }}=0.143 ; P_{\text {Tubifexspp. }}=0.054$; $\left.P_{\text {L.variegatus }}=0.441\right)($ Figure 3.2). Mean feeding rates were $0.098 \pm 0.023(\mathrm{n}=3) \mathrm{mg} \mathrm{dw}$ per organism per day and $0.089 \pm 0.029(\mathrm{n}=3) \mathrm{mg} \mathrm{dw}$ per organism per day for $G$. pulex and $A$. aquaticus, respectively (Figure A3.6). There was no significant relationship between the feeding rate of $G$. pulex and A. aquaticus and increasing car tire TP concentrations in sediment $\left(\mathrm{GLM} ; P_{\text {G.pulex }}=0.520 ; P_{\text {A. aquaticus }}=0.336\right)$ and no significant differences between car tire TP concentration and feeding rate were found for $G$. pulex and $A$. aquaticus (ANOVA; $P_{G . p u l e x}=0.26 ; p_{\text {A.aquaticus }}=0.595$ ). No adverse effects were found on the survival and growth of G. pulex, A. aquaticus and Tubifex spp., and the number of worms and growth of $L$. variegatus. This means that neither the particles themselves nor any of the associated chemicals were toxic at TP concentrations up to $10 \%$ sediment dry weight, which complies to the low chemical bioavailability discussed in the previous section. Interestingly, there seems to be a trend towards significance (GLM; $P$ $=0.063$ ) between the survival of $G$. pulex and car tire TP concentrations in sediment. 
However, this could have been caused by the incidental high PAH level at $0.1 \%$ and lower survival observed for this treatment.

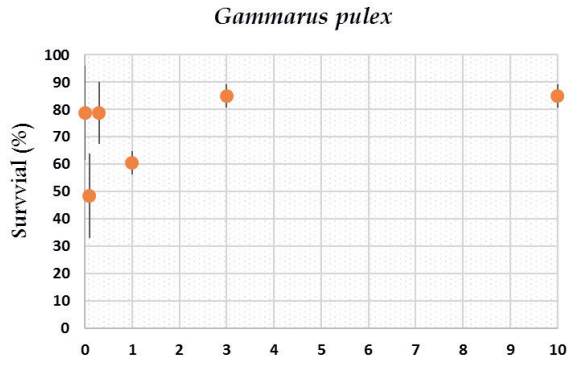

Car tire TP concentration (\% DW in sediment)

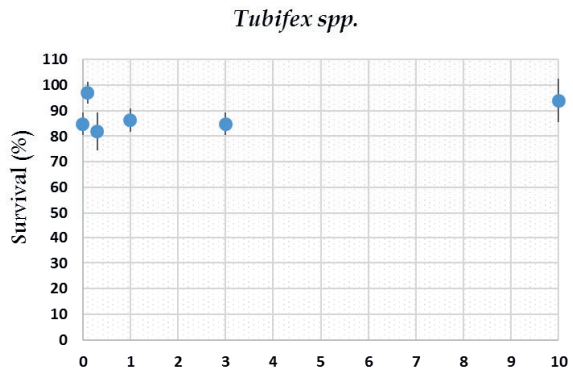

Car tire TP concentration (\% DW in sediment)

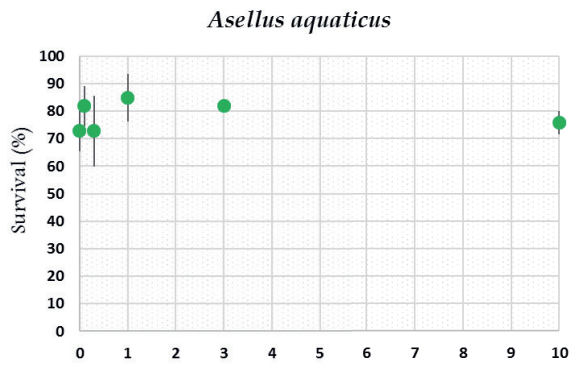

Car tire TP concentration (\% DW in sediment)

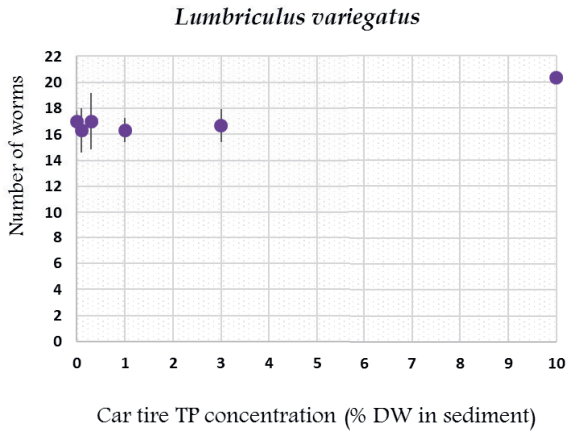

Figure 3.1. Survival of G. pulex, A. aquaticus and Tubifex spp. and number of worms of L. variegatus after 28 days of exposure to car tire TP at increasing concentrations in sediment. Error bars are mean $\pm S D n$ $=3$, except for treatment $1 \%$ of Tubifex spp., where $n=2$.

Quantifcation of car tire TP in body and faeces of G. pulex.

Resistance of car tire TP to $\mathrm{H}_{2} \mathrm{O}_{2}$. Mean dry weight (in $\mathrm{mg}$ ) and area (in $\mathrm{mm}^{2}$ ) of the car tire tread particles before and after their addition to $\mathrm{H}_{2} \mathrm{O}_{2}$ and $\mathrm{H}_{2} \mathrm{O}$ for 24 hours are shown in Table A3.7. No significant differences were found between the mean dry weight (mg) from all particles before and after the $\mathrm{H}_{2} \mathrm{O}_{2}$ and $\mathrm{H}_{2} \mathrm{O}$ treatments (Independent t-test; $P_{\mathrm{H}_{2} \mathrm{O} 2}$ $\left.=0.995 ; P_{\mathrm{H} 2 \mathrm{O}}=0.955\right)$. No significant differences were found between the mean area $\left(\mathrm{mm}^{2}\right)$ from all particles before and after the $\mathrm{H}_{2} \mathrm{O}_{2}$ and $\mathrm{H}_{2} \mathrm{O}$ treatments (Independent $\mathrm{t}$ test; $\left.P_{\mathrm{H} 2 \mathrm{O} 2}=0.968 ; P_{\mathrm{H} 2 \mathrm{O}}=0.974\right)$. Furthermore, the difference in area and weight before and after each treatment was not statistically different between $\mathrm{H}_{2} \mathrm{O}_{2}$ and $\mathrm{H}_{2} \mathrm{O}$ treatments (Independent t-test, $P_{\text {weight }}=0.168 ; P_{\text {area }}=0.385$ ). These results indicate that the treatment with $\mathrm{H}_{2} \mathrm{O}_{2}$ did not affect mass and area of the TP, and thus is not expected to affect the number of car tire TP found in the body and faeces of G. pulex. 


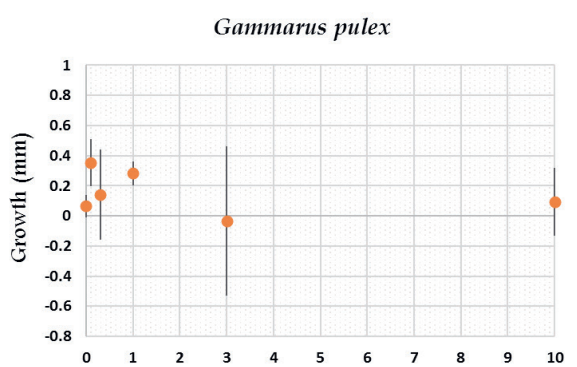

Car tire TP concentration (\% DW in sediment)

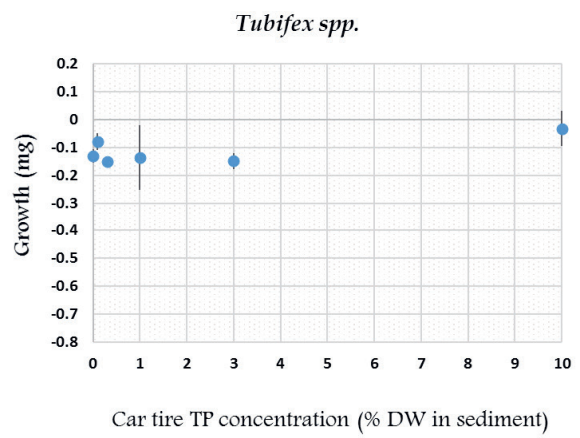

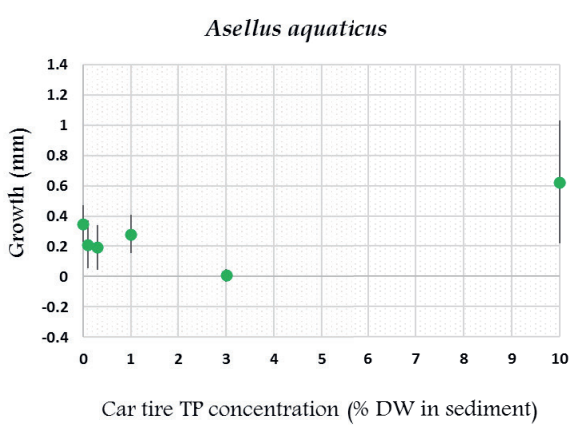

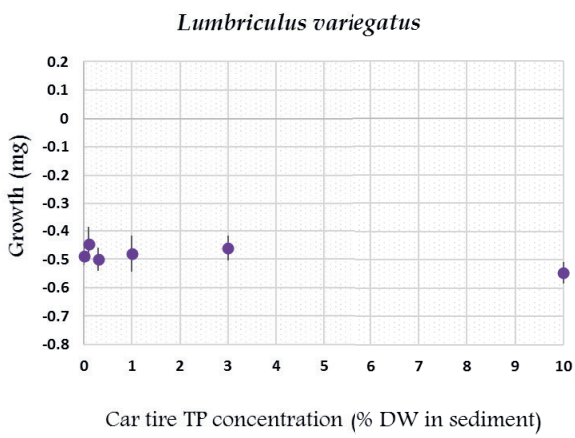

Figure 3.2. Growth of G. pulex, A. aquaticus, Tubifex spp. and L. variegatus after 28 days of exposure to car tire TP at increasing concentrations in sediment. Error bars are mean $\pm S D n=3$, except for treatment $1 \%$ of Tubifex spp., where $n=2$.

Ingestion of TP by G. pulex. One-way ANOVA revealed significant differences in the number of black particles with a size range of $10-586 \mu \mathrm{m}$ found per organism in the body and faeces of G. pulex exposed to the control treatment (TP concentration of $0 \%$ sediment $\mathrm{dw}$ ) and $10 \%$ car tire TP in sediment (ANOVA; $P_{\text {Body }}=0.008 ; P_{\text {Faeces }}=0.001$ ) (Figure 3.3). Significant differences in the number of particles with the same characteristics were also found between organisms of G. pulex exposed to 3 and $10 \%$ car tire TP in sediment dry weight (ANOVA; $P_{\text {Body }}=0.037 ; P_{\text {Faeces }}=0.003$ ) (Figure 3 ). After correcting for the number of black particles with a size range of $10-586 \mu \mathrm{m}$ in controls (TP concentration of $0 \%$ sediment dry weight), considering them as contamination of the samples, an average of 2.5 and 4 car tire TP per organism were found in bodies and faeces of $G$. pulex exposed to $10 \%$ car tire TP in sediment, respectively. Size frequency of the particles found in bodies of $G$. pulex ranged from 14 to $272 \mu \mathrm{m}$, with an average size of $66 \mu \mathrm{m}$ (Figure A3.7). Size frequency of the particles found in faeces of G. pulex ranged from 14 to $555 \mu \mathrm{m}$ and had a mean size of $65 \mu \mathrm{m}$ (Figure A3.8). Although the average particle size found in bodies and faeces of $G$. pulex were similar to the ones reported for PS MP, the upper size range was higher for car tire TP than for PS MP (Chapter 2). ${ }^{186}$ 
When calculating the number of car tire TP at $10 \%$ sediment dry weight, using the average density given by Verschoor et al. (2017) for TWP $\left(1.20 \mathrm{~g} / \mathrm{cm}^{3}\right)$, a value of $5.28 \times 10^{8}$ is obtained. ${ }^{208}$ If we calculate the Trophic Transfer Factor (TTF) ${ }^{209}$ for car tire TP at $10 \%$ sediment dry weight as the number concentration in G. pulex divided by the number of car tire TP in the sediment, we get a TTF of $4.7 \times 10^{-9}$. In an earlier study (Chapter 2), ${ }^{186}$ we reported a TTF of $(4.47 \pm 0.35) \times 10^{-11}$ for PS MP retained by G. pulex. This indicates that the TTF for car tire TP is approximately 100 times higher than the TTF of PS MP. A total of 1.4 and 5.9 PS MP per organism were retained and egested, respectively, at $10 \%$ PS MP in sediment dry weight (Chapter 2). ${ }^{186}$ This indicates that, although the number of car tire TP retained by G. pulex was higher than the number of retained PS MP, the total number of PS MP ingested was similar $(1.4+5.9=7.3)$ as the total number of car tire TP ingested $(2.5+4=6.5)$. When comparing this value with the number of PS MP found in the sediment at the same dose of $10 \%$, which was $3.15 \times 10^{10} \mathrm{PS} \mathrm{MP}$, we realize that at the same percentage, a lower number of car tire TP are found in the sediment. This is due to the higher density of the car tire TP, as well as the presence of a higher number of smaller particles $(10-20 \mu \mathrm{m})$ in the car tire TP mixture in comparison to the PS MP used before (Chapter 2). ${ }^{186}$

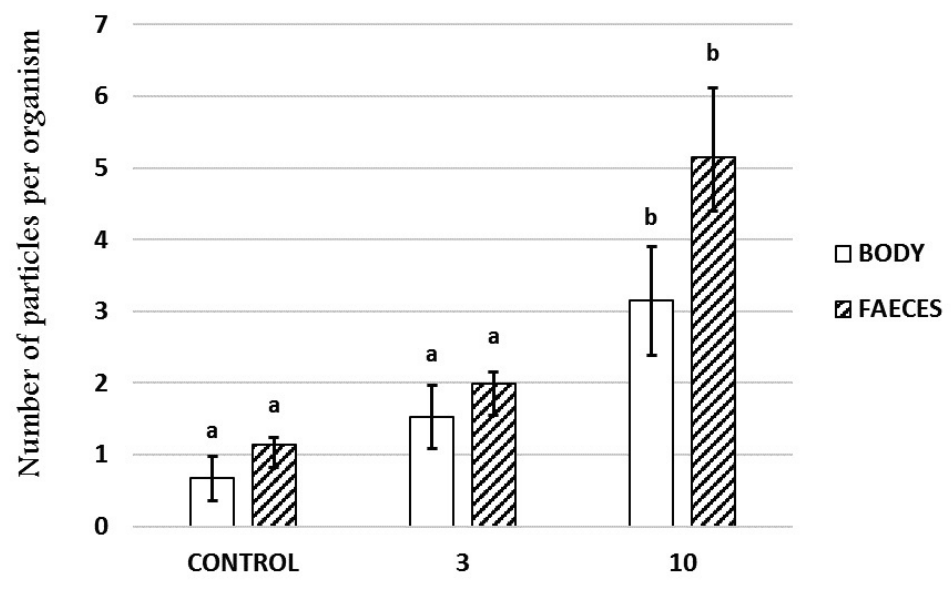

Car tire TP concentration (\% DW in sediment)

Figure 3.3. Number of black particles with a size between 10 and $500 \mu \mathrm{m}$ found per organism in the body (white column) and faeces (stripped column) of G. pulex after the exposure to 0, 3 and 10\% car tire TP in sediment dry weight (dw) for 28 days. Error bars are mean $\pm S D n=3$. 


\section{General Discussion and Implications}

Our study showed that car tire TP, including chemicals associated with this material, did not negatively affect four freshwater benthic invertebrates, even at concentrations of $10 \%$ sediment dry weight. This implies that car tire TP effects can be more mild or even absent under ecologically relevant conditions than suggested in elutriate tests. ${ }^{172,179,182}$ As the maximum Predicted Environmental Concentrations (PEC) in sediments range from 0.3 to $155 \mathrm{~g} / \mathrm{kg}$ dry weight, ${ }^{171}$ we can conclude that car tire TP pose a low risk to freshwater benthic invertebrates. This is in agreement with previous studies evaluating the effects of TRWP mixed with sediments on aquatic organisms. ${ }^{181,184}$ However, potential long-term effects caused by the slow release and gradual environmental increase of bioavailable zinc and other substances caused by ageing of rubber particles are not expressed by these experiments. For G. pulex, the ingestion of car tire TP was demonstrated after a 28-day exposure to $10 \%$ car tire TP in sediment. An average of 2.5 and 4 car tire TP was found in bodies and faeces of $G$. pulex at this concentration, respectively. This ingestion did not lead to negative effects on its survival, growth or feeding rate. In contrast, in an earlier work, the ingestion of another particle type (PS MP) was found to cause a reduction in the growth of the same species, using the same methodology (Chapter 2). ${ }^{186}$ In both cases, particles ingested by G. pulex were found to have a similar average size (57 vs. 66 $\mu \mathrm{m}$ ) (Chapter 2). ${ }^{186}$ This demonstrates that implications of particles probably may be case-specific and that the probably multi-causal mechanisms underlying such effects need further attention.

\section{Acknowledgements}

This study was funded by the Dutch Technology Foundation TTW, project no. 13940. We acknowledge additional support from KWR; IMARES; NVWA; RIKILT; The Dutch Ministry of Infrastructure and the Environment; The Dutch Ministry of Health, Welfare and Sport; Wageningen Food \& Biobased Research; STOWA; RIWA; and water boards Hoogheemraadschap van Delfland, Zuiderzeeland, Rijn en IJssel, Vechtstromen, Scheldestromen, Aa en Maas, de Dommel, and Rivierenland. We gratefully acknowledge Frits Gillissen, John Beijer, Marlies Vollebregt and Guus Frissen for their technical assistance; Patrick Bäuerlein and Claudia Kooijman (KWR Watercycle Research Institute) for their contribution to the characterization of the car tire TP; and Edwin Peeters for his advice on the statistical analysis. 



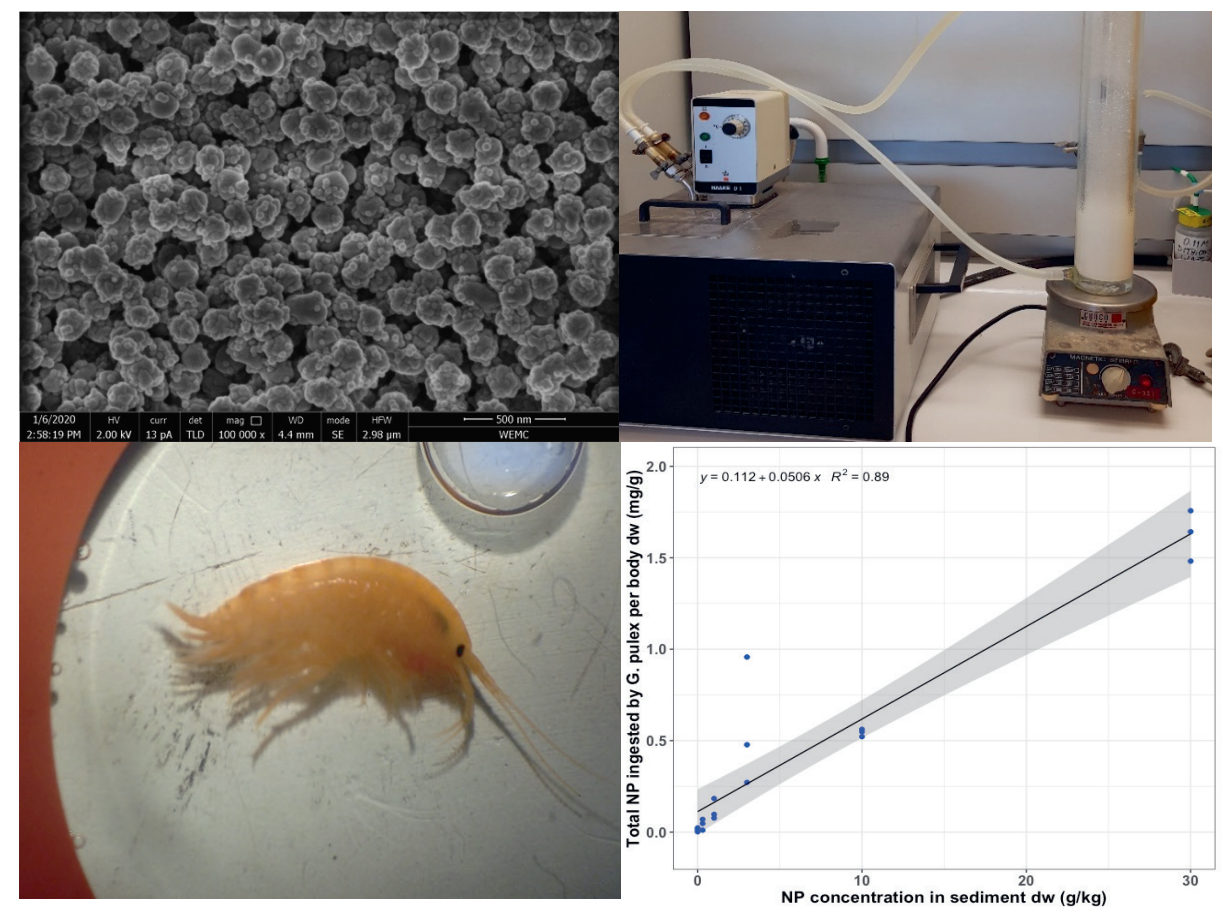




\section{Chapter 4}

\section{Uptake and Effects of Metal-doped Nanoplastics on Gammarus pulex}

In preparation: Redondo-Hasselerharm, P.E.; Mitrano, D.; Koelmans, A.A. Uptake and Effects of Metal-doped Nanoplastics on Gammarus pulex. 


\begin{abstract}
Because of the difficulty of measuring nanoplastics (NP), the use of NP doped with rare earth metals has been proposed as a promising approach to detect NP in environmental media and biota. In the present study, the freshwater amphipod Gammarus pulex was exposed to palladium (Pd)-doped NP with a bumpy surface via natural sediment at six spiking concentrations $(0,0.3,1,3,10$ and $30 \mathrm{~g}$ plastic/ $\mathrm{kg}$ of sediment dry weight) with the aim of assessing their uptake and chronic effects using 28 days standardized single species toxicity tests. NP concentrations were quantified based on Pd concentrations measured on digests of the exposed organisms and faecal pellets excreted during a postexposure 24 hours depuration period with ICP-MS. Additionally, NP concentrations were measured in sediments and water to demonstrate the accuracy of the dosing and to quantify the resuspension of NP from the sediment caused by the organisms. A significant positive linear relationship between the uptake of NP by G. pulex and the concentration of NP in sediments was observed, yet no statistically significant effects were found on the survival or growth of G. pulex. A biodynamic model fitted well to the data and suggested bioaccumulation would occur in two kinetic compartments, the major one being reversible with rapid depuration to clean medium. Model fitting yielded a mass based trophic transfer factor (TTF), conceptually similar to the traditional biota sediment accumulation factor, for NP in the gut of 0.031 . This value is close to a TTF value of 0.025 that was obtained for much larger microplastic in a previous study. Mechanistically, this suggests that ingestion of plastic is limited by the volume of the diverse mixture of plastic particles. We demonstrate that metal-doping provides opportunities for precise quantification of NP accumulation and exposure in fate and effect studies, which can be a clear benefit for NP risk assessment.
\end{abstract}




\section{Introduction}

Plastic accumulation in the environment has been of great societal, political and scientific concern in the last decade due to its ubiquity and ability to persist for long time periods. ${ }^{4}$ Once plastics are released into the environment, they go through physical and biological degradation processes, resulting in the formation of both microplastics (MP; $<5 \mathrm{~mm}$ ) and nanoplastics $(\mathrm{NP} ;<1 \mu \mathrm{m}){ }^{4,13}$ Moreover, some pharmaceutical and cosmetic products contain NP and MP, which can also enter marine, freshwater and terrestrial ecosystems. ${ }^{166,210}$ Environmental concentrations of MP have been measured in water, sediment and biota samples in all habitats worldwide. ${ }^{35,37}$ In contrast, NP concentrations in environmental matrices are still generally unquantified, as the sampling methods and identification techniques available for particulate plastic generally have detection limits $>1 \mu \mathrm{m} .{ }^{32}$ The difficulty to detect NP is one of the major challenges in assessing their proliferation and risk. Several methods have been proposed to detect them, even in natural and complex matrices, such as the use of crossflow ultrafiltration coupled with field flow fractionation and pyrolysis gas chromatography-mass spectrometry. ${ }^{32,42}$ However, to date only one study has been able to detect NP of various polymer types in surface water samples taken at the North Atlantic Subtropical Gyre. ${ }^{42}$ Because of the difficulties in applying NP analytical methods to complex environmental matrices, no study has ever measured NP concentrations in field sediments and biota samples. ${ }^{211} \mathrm{NP}$ abundance in environmental ecosystems is expected to be high due to the aforementioned fragmentation of larger plastics, which are found to degrade into NP after long-term exposures to visible and UV light. ${ }^{12,19,20,210,212} \mathrm{NP}$ are predicted to be particularly abundant in freshwater sediments due to their retention caused by a fast heteroaggregation with natural solids, thereby posing an exposure route to benthic biota. ${ }^{27,129}$

The challenges for detecting NP in the environment limit the evaluation of their exposure, but also the assessment of their effects and the risks they pose to biota and to human health ${ }^{31,32,211}$ Although the effects of NP on aquatic biota have been broadly investigated for exposures in aqueous media, the mechanisms behind these effects are unclear due to the aforementioned analytical difficulties, which hamper the determination of uptake by biota. ${ }^{125,126}$ To date, many studies have used fluorescently-labelled plastics to assess biological uptake of NP. ${ }^{67,213,214}$ Some of these studies observed specific tissues or the whole body of the organisms under a fluorescent microscope, while others measured the fluorescent particles in the remaining solution with a fluorescence spectrophotometer after digesting the samples. ${ }^{67,213,214}$ The suitability of fluorescently-labelled NP to assess ingestion was recently questioned, as Catarino et al. (2019) and Schür et al. (2019) demonstrated that the fluorescent dye can leach out of the NP and reach biological tissues without the plastic. ${ }^{127,215}$ In addition, cell auto-fluorescence is often not taken into account by studies assessing the ingestion of fluorescent plastic particles. ${ }^{127}$ Consequently, the use of fluorescently-labelled NP to assess biological uptake could lead to misinterpretation of the results. ${ }^{127}$ Another method has been proposed to track NP in complex matrices, which consists of the use of metal-doped plastics, which can be measured accurately with sensitive analytical techniques such as inductively coupled plasma mass spectrometry 
(ICP-MS). ${ }^{216}$ This approach has also been successfully applied to study the fate and behaviour of NP in complex environmental systems. ${ }^{217,218}$

Aquatic organisms are able to actively ingest NP or adsorb them to their surfaces and transfer them into higher trophic levels. ${ }^{12,126}$ The effects of NP on aquatic biota have raised particular concerns over the last years due to their small size, which allows them to be taken up by cells, affecting biota on a cellular level. ${ }^{12,126}$ In fact, a recent review concluded that NP caused more adverse effects on aquatic organisms than MP. ${ }^{126} \mathrm{NP}$ have been found to cause deleterious effects on aquatic biota at the individual level, affecting their growth, reproduction, mobility and feeding, and at the sub-organismal level, causing oxidative stress and affecting their gene expression and immune system among other effects. ${ }^{84,85,219-222}$ Studies assessing the effects of NP generally use pristine and smooth spherical particles. ${ }^{126}$ However, irregularly shaped NP could occur more often in nature due to their formation through fragmentation and degradation of MP. ${ }^{211}$ Hence, testing irregularly shaped NP under more realistic environmental conditions should be a priority, as effects of NP might be shape-dependent as it occurs for MP (Chapter 7). ${ }^{223}$ In contrast to the abundant literature data on NP effects on aquatic biota upon aquatic exposure, the effects of NP under sediment exposure conditions have only been studied for a few organisms. Sediment assessments come with different complexities than the aqueous ones, because exposure conditions are different. Moreover, the few studies published used particle mixtures as exposures, rendering it impossible to distinguish between NP and MP effects. ${ }^{76,88,224}$ To date, we are aware of two articles that have evaluated the effects of NP only on freshwater benthic species using sediment exposure conditions (Chapter 5). .25,226 $^{2}$

The limited information with respect to effects of irregularly shaped NP on freshwater benthic species, in combination with the susceptibility of their habitat to pollution with $\mathrm{NP}$, urgently calls for investigation. Moreover, due to the complexity and inaccuracy of the existing methods for quantifying NP in sediments and biota samples, the use of metaldoped NP as tracers in complex matrices needs to be explored. ${ }^{211}$ In this study, we used metal-doped NP to study the uptake and effects on the survival and growth of the freshwater benthic macroinvertebrate Gammarus pulex using 28 days standardized single species sediments toxicity tests. Individuals of $G$. pulex were exposed to $228 \mathrm{~nm}$ polystyrene NP with a bumpy surface containing a palladium $(\mathrm{Pd})$ core at concentrations ranging from 0 to $30 \mathrm{~g} / \mathrm{kg} \mathrm{NP}$ in sediment dry weight (dw). We measured the concentration of $\mathrm{Pd}$ in the body of the exposed organisms and in the faecal pellets excreted during a 24-hour depuration period after the chronic exposure as a method to assess NP uptake and bioaccumulation. Concentrations of Pd in sediments were quantified at the start of the experiment and in water samples at the start and at the end of the experiment to show the accuracy of the dosing. Data interpretation was assisted by biodynamic modelling of the bioaccumulation of NP by G. pulex. For comparison, MP accumulation data obtained from an earlier experiment were modelled as well (Chapter 2). ${ }^{186}$ 


\section{Material and Methods}

Nanoplastics. A solution containing metal-doped NP with a bumpy surface were synthesized according to our previous work. ${ }^{227}$ Briefly, the NP consisted of a polyacrylonitrile core with Pd doping $(0.27 \%$ by weight $)$ and a polystyrene shell, resulting in a rough (bumpy) outer surface of the particles. The Pd tracer was chemically entrapped in the core, and with the addition of the shell, it was demonstrated that minimal leaching of the metal from the NP occurred over time in a variety of environmental and biological conditions. ${ }^{227}$ The z-average size (nm) was measured by dynamic light scattering (DLS) with a Zetasizer (Nano ZS, Malvern instruments), the shape was confirmed by scanning electron microscopy (SEM) imaging and the solid content was measured with Thermogravimetric Analysis. Average particle size was $227.6 \pm 1.47 \mathrm{~nm}(\mathrm{n}=3)$ as measured by DLS, SEM images confirmed bumpy surface of the particles (Figure A4.1), and the dry content was $8.55 \%$.

Chemical control. Styrene and acrylonitrile are volatile compounds that can be toxic to aquatic organisms when effect threshold concentrations are exceeded.228,229 To remove any potential remains of these chemicals from the particle synthesis process, the solution was purged with clean air for 10 hours at $30^{\circ} \mathrm{C}$ with an airflow of $5 \mathrm{~L} / \mathrm{h}$. This purging duration, together with subsequent aeration for 2 weeks, was a priori designed to cause $>$ 99.99\% removal based on air flow, the chemicals Henry's Law constants and an assumed chemical equilibrium between these chemicals in solution and bubbles rising over the height of the water column. Nevertheless, some traces may have remained, and other chemicals present in the solution could also potentially affect the tested organisms (i.e., surfactants SDS and KPE). To address these potential effects from co-exposure to the chemicals involved in the NP synthesis, we calculated if the chemical concentrations in the experimental design were below known effect threshold concentrations (provided in the Appendix; Table A4.2, Figures A4.3, A4.4).

Test organisms. We selected the amphipod Gammarus pulex as test organism because of its key role in aquatic ecosystems and its demonstrated sensitivity to MP (Chapter 2). ${ }^{163,164,186}$ Following previous procedures conducted in our laboratories (Chapters 2 and 3), ${ }^{144,186,230}$ G. pulex were collected from a non-contaminated ${ }^{144}$ brook in Heelsum, The Netherlands, in June 2019. Once in the lab, individuals were sorted by their narrow body size, excluding the smallest and largest for use in the NP exposure tests. Organisms were acclimatized in aerated buckets with copper-free Dutch Standard Water (DSW) in a water bath at $15.5 \pm 1{ }^{\circ} \mathrm{C}$ while maintaining a 12:12 light:dark cycle. During the acclimatization period (14 days), organisms were fed with field dry poplar leaves.

Sediments. Following previous studies conducted in our laboratory (Chapters 2 and 3), ${ }^{186,220,230}$ sediments were sampled from an unpolluted ${ }^{144}$ ditch in Veenkampen (Wageningen, The Netherlands) with a standard dip and and sieved over a $2 \mathrm{~mm}$ sieve. Sediments in the containers were allowed to settle overnight and the overlying water was removed the morning after. Remaining sediments were homogenized with a hand drill 
and stored in a freezer to kill any living organisms and to preserve the sediment. Directly before the start of the experiments, sediments were unfrozen and re-homogenized. Four representative sediment subsamples were taken to determine the Total Organic Matter (TOM) content, using the loss on ignition method $\left(3 \mathrm{~h}, 550^{\circ} \mathrm{C}\right)$, which was $39.95 \pm 0.92$ $\%$.

Experimental design. A total of 11 individuals of $G$. pulex per experimental unit were exposed to NP at concentrations of $0,0.03,0.1,0.3,1$ and $3 \%$ of plastic in sediment dw, which corresponds to $0,0.3,1,3,10$ and $30 \mathrm{~g}$ of plastic per $\mathrm{kg}$ of sediment $\mathrm{dw}$. For each concentration, five replicas were prepared, from which three were used for the exposure assessment and two were used to verify the concentration (in mass) of NP in sediment and water at the start of the experiment $(t=0)$. Experimental units consisted of $750 \mathrm{ml}$ glass beakers containing $184.21 \mathrm{~g}$ of wet sediment, spiked with the corresponding NP concentrations and a $350 \mathrm{ml}$ copper-free DSW layer. To avoid NP homo-aggregation and assure homogeneous mixing during the preparation of the amendments, the plastic solutions were added drop by drop to sediment contained in a $2 \mathrm{~L}$ glass beaker placed inside an ultrasonic waterbath. At the same time, the sediment was vigorously mixed with a stainless steel hand mixer (Chapter 5). ${ }^{220,226}$ Sediment amendments were prepared per concentration and were then divided into replicate beakers, to assure replicates to be as identical as possible. After the addition of the sediment, beakers were allowed to settle for 24 hours and subsequently DSW water was slowly added to avoid resuspension of the particles into the water phase. Finally, beakers were randomly placed in a water bath and acclimatized at $15.5 \pm 1{ }^{\circ} \mathrm{C}$ with a 12:12 light:dark cycle for two weeks prior to the start of the experiment. After the acclimatization, 11 organisms were randomly introduced into each of the beakers. In addition, another 66 randomly selected organisms were preserved in $70 \%$ ethanol to assess the length of the starting population. Organisms were fed with two $3 \mathrm{~cm}$ poplar leaf discs at days 0 and 14, which were previously soaked in DSW for 3 days. Aeration was supplied to the beakers and the top water layer was carefully renewed weekly in all beakers to keep the water levels constant. Temperature, dissolved oxygen, $\mathrm{pH}$, electroconductivity, and ammonia levels $\left(\mathrm{NH}_{3}\right)$ were measured once a week in one replicate per exposure concentration. The mean $( \pm \mathrm{SD})$ temperature, dissolved oxygen, $\mathrm{pH}$, electroconductivity and ammonia levels $\left(\mathrm{NH}_{3}\right)$ along the experiment were $16.0 \pm 0.17^{\circ} \mathrm{C}$, $9.5 \pm 0.32 \mathrm{mg} / \mathrm{l}, 7.8 \pm 0.12,535 \pm 33.7 \mu \mathrm{S} / \mathrm{cm}$ and $0.87 \pm 0.98 \mathrm{mg} / \mathrm{l}$, respectively.

Effects on survival and growth. At the end of the experiment, beakers were sieved over a $0.35 \mathrm{~mm}$ sieve and gently washed with tap water. Surviving individuals were counted, rinsed and transferred to glass beakers containing $30 \mathrm{ml}$ of clean DSW, where they were allowed to depurate their gut content for 24 hours. Thereafter, G. pulex were placed in $70 \%$ ethanol until their length and that of the starting population was measured under an Olympus SZX10 stereomicroscope. For this, the head capsule (HD) size was measured and the total length (TL) was calculated following the equation: $\mathrm{TL}=-2.07+9.82 \mathrm{HD} .{ }^{151}$ The growth was then calculated as the difference in the mean TL of the exposed organisms per replicate minus the mean TL of the starting population. The average size of the starting population was $5.37 \pm 0.91 \mathrm{~mm}(\mathrm{n}=66)$. 
Palladium analysis of the metal-doped NP in biota, faeces, sediment and water samples. After measuring the length of the exposed G. pulex, organisms were dried in an oven at $40^{\circ} \mathrm{C}$ for 24 hours and weighted per experimental unit. Nanoplastic body burden was assessed by total $\mathrm{Pd}$ concentration as a group per replica, as $\mathrm{mg} \mathrm{Pd} / \mathrm{mg} \mathrm{dw}$ G. pulex. Glass beakers containing $30 \mathrm{ml}$ DSW with the faecal pellets depurated after the 28-day exposure for 24 hours were freeze dried prior to the analysis of the Pd concentrations as $\mu \mathrm{g} \mathrm{Pd} / \mathrm{ml}$ DSW.

For the analysis of Pd in sediments, at $\mathrm{t}=0$ beakers were taken out of the waterbath and the overlying water was carefully removed with a syringe. All sediments in their original beakers and $60 \mathrm{ml}$ of the removed water from concentrations 0,1 and $3 \% \mathrm{NP}$ per sediment $\mathrm{dw}$ placed in glass beakers were stored in the freezer until further analysis. In addition, $60 \mathrm{ml}$ of water was taken from the three replicate beakers at concentrations 0 and $3 \%$ at $\mathrm{t}$ $=28 \mathrm{~d}$ before sieving them in order to analyse whether Pd concentrations in water (as $\mu \mathrm{g}$ $\mathrm{Pd} / \mathrm{ml} \mathrm{DSW}$ ) increased over the exposure to G. pulex. Sediment samples of all concentrations were freeze dried and homogenized with a stainless-steel spoon prior to analysis. A total of $300 \mathrm{mg}$ of dry sediment were weighed per replicate and $\mathrm{Pd}$ concentrations were measured as $\mu \mathrm{g} \mathrm{Pd} / \mathrm{mg} \mathrm{dw}$ of sediment.

All samples underwent microwave acid digestion prior to analysis by ICP-MS. Biota and sediment samples were placed into Teflon digestion vessels with $6 \mathrm{ml}$ of concentrated $\mathrm{HNO}_{3}$ and $2 \mathrm{ml} \mathrm{HCl}$. For the faeces and water samples, glass beakers were washed with the $\mathrm{HNO}_{3}$ and $\mathrm{HCl}$, mixed with a pipette and added to the Teflon tubes. Immediately after, tubes were closed and left overnight at room temperature $\left(20 \pm 1^{\circ} \mathrm{C}\right)$. The morning after, tubes were introduced into a microwave (CEM MARS 6) to allow the first digestion step to take place $\left(200^{\circ} \mathrm{C}\right.$ for $\left.60 \mathrm{~min}\right)$. Once the first digestion step was completed, the sample was taken out and allowed to cool to room temperature. Then, two $0.75 \mathrm{ml}$ aliquots of $\mathrm{H}_{2} \mathrm{O}_{2}$ and $3.25 \mathrm{ml}$ ultrapure water were added to the sample with a second round of microwave digestion $\left(175^{\circ} \mathrm{C}\right.$ for $\left.15 \mathrm{~min}\right)$. Samples were then transferred into a $50 \mathrm{ml}$ digiprep tube. The volume of the sample was made up to $50 \mathrm{ml}$ by adding ultrapure water. Pd concentration in the samples was measured using High Resolution ICP-MS (Thermo Scientific, Element2). Control samples for every digestion matrix were performed, with two replicate samples of the NP stock solution and two replicate samples of a dissolved Pd standard $(200 \mu \mathrm{g} / \mathrm{l})$ spiked into the matrix. The Pd variation between replicates in the three runs was $2.5 \%$ for NP stock solution and $1.5 \%$ for the Pd stock standards. Recovery tests were performed using two Pd spiked sediment samples and one Pd spiked G. pulex sample. Recovery of Pd from the spiked samples were $100.3 \pm 0.6 \%$ and $102 \%$ from the sediment and G. pulex, respectively.

Statistical analysis. Analysis of the data was done in SPSS 23 (IBM Corp., NY). Generalized Linear Models (GLMs) were used to study the effects of the NP on the survival of the tested organisms. One-way ANOVA $(p<0.05)$ were used to study the effects of the NP on the growth of the tested organisms. The normality of the residuals was first checked with the Shapiro-Wilk test $(p>0.05)$ and visualized on a Q-Q plot. 
Homogeneity of the variances was tested using Levene's test $(\mathrm{p}>0.05)$ and post hoc multiple comparisons were done using Tukey's and Bonferroni tests. If the assumption of homogeneity of variances was violated, a one-way Welch ANOVA ( $p<0.05)$ was conducted. Kruskal-Wallis test was used when data were not normally distributed. Linear regressions were fit for the $\mathrm{Pd}$ concentrations measured in the plastic-sediment mixtures at $\mathrm{t}=0$ as a function of the NP concentrations in sediment. Linear regressions were fit for the NP concentrations based on the measured Pd in bodies and faeces of G. pulex at the end of the experiment and after a 24-hour gut depuration period as a function of the NP concentrations in the sediment. All data are depicted with average \pm standard deviation, unless otherwise stated.

Biodynamic modelling of the bioaccumulation of Pd-doped nanoplastics by $G$. pulex. The bioaccumulation of NP by $G$. pulex between $\mathrm{t}=0$ and $\mathrm{t}=28$ days was modelled as a function of NP dose and time (Equation 1) using a traditional first order two compartment model allowing for irreversible uptake in the body and reversible transport to and from the gut, under constant exposure conditions: 231,232

$$
C_{G . P u l e x, t}=C_{S E D}\left(F_{B} k_{u p} t+\left(1-F_{B}\right) \frac{k_{u p}}{k_{\text {elim }}}\left[1-e^{-k_{\text {elim }} \times t}\right]\right)
$$

For the subsequent 1-day depuration phase, i.e., $\mathrm{t}>28$ days (Equation 2) was used:

$$
C_{G . P u l e x, t-28}=C_{G . P u l e x, t=28} \times e^{-k_{\text {elim }} \times(t-28)}
$$

Here, $C_{G . P u l e x}$ is the measured NP concentration in G. pulex $(\mu \mathrm{g} / \mathrm{kg}), C_{S E D}$ is the measured exposure NP concentration in sediment $(\mu \mathrm{g} / \mathrm{kg}), k_{u p}$ is the uptake rate constant $\left(\mu \mathrm{g} \times \mathrm{kg}^{-1}\right.$

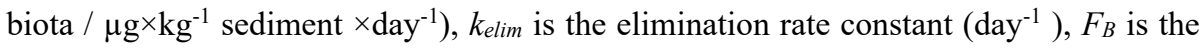
poorly or irreversible fraction accumulating in the body (dimensionless) and $t$ is exposure time (days). Note that the compartments are kinetically defined and that referring to the reversible and irreversible particle reservoirs as 'gut' versus 'body' formally is a matter of interpretation. The model was fitted to the experimental data by optimizing the parameters $k_{u p}, k_{\text {elim }}$ and $F_{B}$ using a weighted relative least squares criterion.

\section{Results and Discussion}

NP in sediment and water samples. The concentration of Pd in the NP stock solution was $3.022 \pm 0.077(\mathrm{n}=6) \mathrm{g} / \mathrm{kg}$, which means that NP contained $0.302 \mathrm{wt} \% \mathrm{Pd}$. The average measured background Pd concentration in sediment was $2.620 \mathrm{mg} / \mathrm{kg}(\mathrm{n}=2)$, which is close to the value of the intercept $(\alpha=2.259 \pm 0.1365)$ (Figure 4.1). At $t=0$, measured Pd concentrations in sediment were proportional to the nominal NP doses $\left(\mathrm{R}^{2}=1, \mathrm{n}=\right.$ 12), with a slope corresponding to a Pd content of $3.009 \pm 0.0105 \mathrm{~g} / \mathrm{kg}$ (Figure 4.1). These values of 3.022 and 3.009 are identical within error limits, confirming the adequate and representative addition, mixing and analysis of the Pd-doped NP in the sediment matrix (Figure 4.1) (Linear Regression (LR), $P=2.2 \times 10^{-16}$ ). 


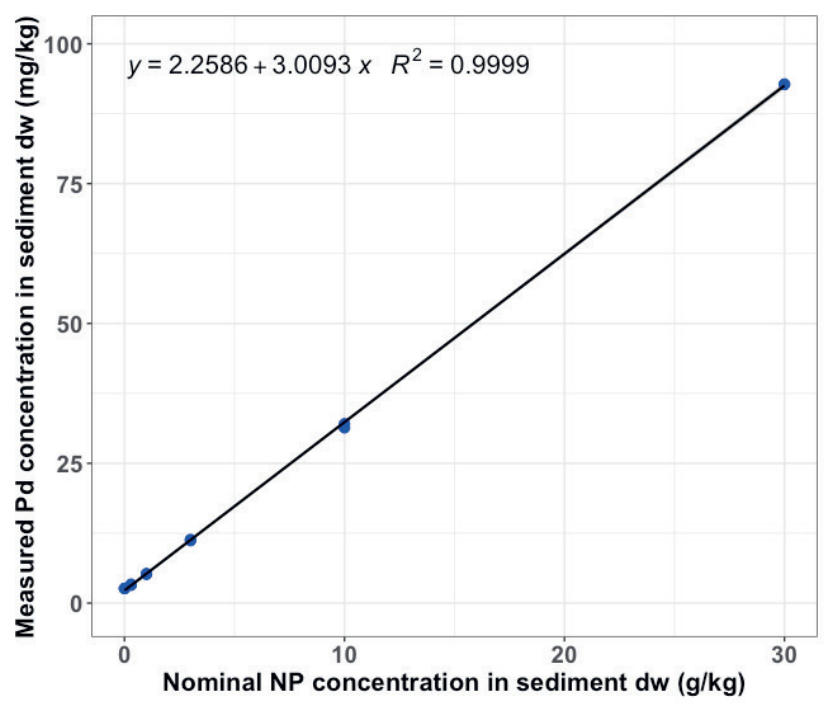

Figure 4.1. Measured Pd concentrations in sediment $d w(\mathrm{mg} / \mathrm{kg})$ at $t=0$ as a function of the nominal NP concentrations in sediment $d w(\mathrm{~g} / \mathrm{kg})$. The linear regression is based on 12 individual data points.

Following the same conversion between $\mathrm{Pd}$ and NP, average measured NP concentrations in water at $\mathrm{t}=0$ were $0.251 \pm 0.056 \mathrm{mg} / \mathrm{l}$ and $1.390 \pm 0.037(\mathrm{n}=2) \mathrm{mg} / \mathrm{l}$ for the nominal $\mathrm{NP}$ spiking concentrations of 10 and $30 \mathrm{~g} / \mathrm{kg}$ in sediment $\mathrm{dw}$, respectively. Knowing the concentration of NP in the overlying water, we calculated the proportion of NP which were resuspended from the sediment at $\mathrm{t}=0$, which was $0.07 \%$. We hypothesize that this resuspension was a result of either the system preparation procedure or the aeration of the beakers during the acclimatization. At $\mathrm{t}=28 \mathrm{~d}$, the measured NP concentration in the overlying water was $19.83 \pm 3.94(\mathrm{n}=3) \mathrm{mg} / \mathrm{l}$ for the nominal NP concentration of 30 $\mathrm{g} / \mathrm{kg}$ in sediment $\mathrm{dw}$. At $\mathrm{t}=28 \mathrm{~d}, 1 \%$ of the NP was found to be suspended, which suggests that the exposures of NP in the sediment were close to their nominal spiking values.

Ingestion of NP by G. pulex. Pd concentrations were measured in the body of the surviving organisms after the 28 days of exposure to the NP and a posterior 24-hour defaecation period in DSW. Additionally, Pd concentrations in the excreted faeces were measured. There was a linear relationship (LR, $P=3.46 \times 10^{-3}$ ) between the concentration of NP measured in the body of $G$. pulex $(\mathrm{mg} / \mathrm{g})$ and the nominal NP concentration in sediment $\mathrm{dw}(\mathrm{g} / \mathrm{kg})$ (Figure 4.2A). A significant positive linear relationship (LR, $P=1.49 \times 10^{-7}$ ) was found between the measured NP concentrations in faeces of G. pulex and the nominal NP concentrations in sediment (Figure 4.2B). Because NP were measured in the body and the egested faeces, the total ingested NP can be calculated as the sum of these components. For this total ingested NP, a highly significant positive linear relationship with dose is obtained (LR, $P=4.37 \times 10^{-9}$ ) with a multiple R-squared of 0.89 (Figure $4.2 \mathrm{C}$ ). As the datapoints have incremental intervals, we also provide the log-transformed version of Figure 4.2 (Figure A4.2). 

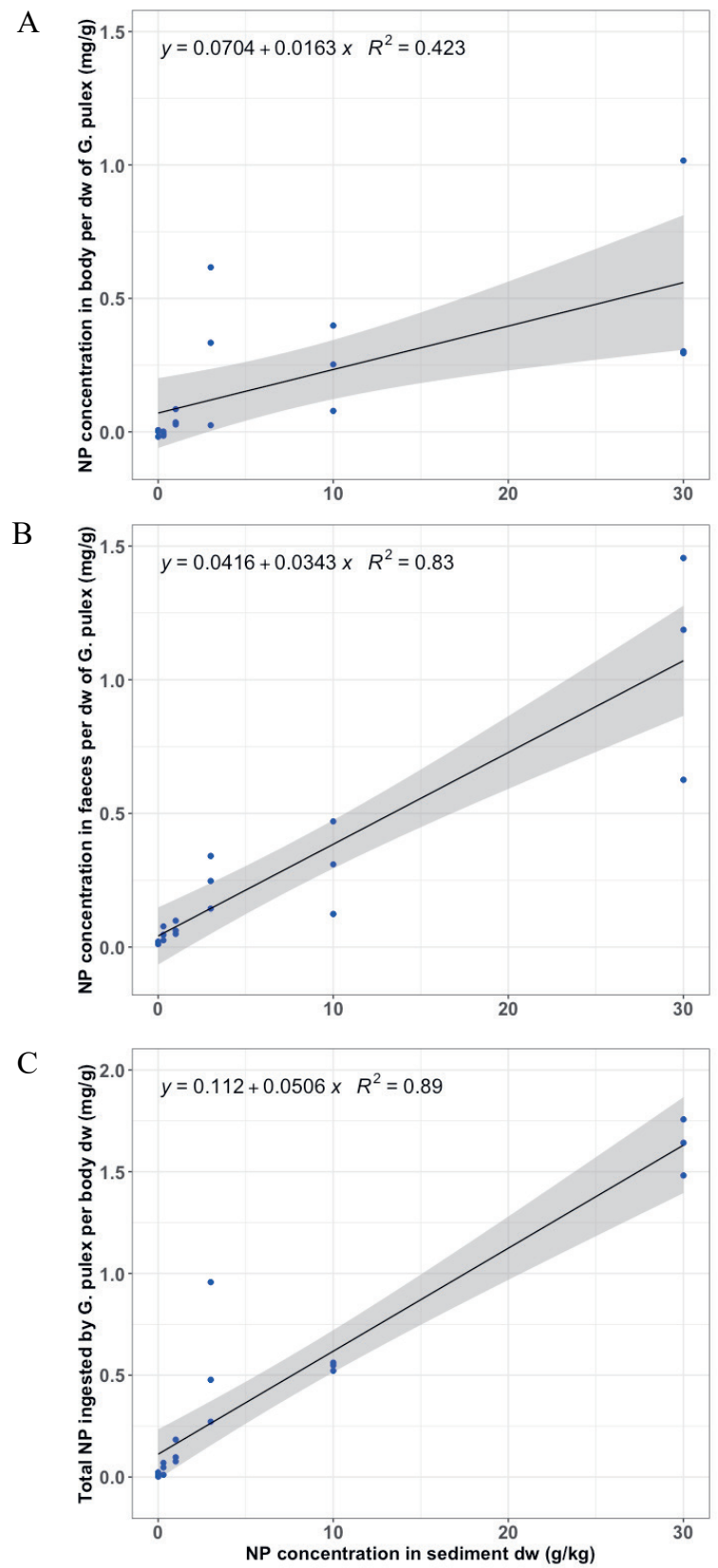

Figure 4.2. NP concentration measured in A) the body of G. pulex $(\mathrm{mg} / \mathrm{g})$, B) faeces of G. pulex per body weight $(\mathrm{mg} / \mathrm{g})$ and C) total NP ingested by G. pulex $(\mathrm{mg} / \mathrm{g})$ per body dw after summing up the concentration of NP in bodies and faeces; after 28 days of exposure to NP concentrations in sediment $d w(\mathrm{~g} / \mathrm{kg})$. The linear regressions are based on 18 individual data points. 
A summary of the NP concentrations measured in body and faeces of G. pulex per body $\mathrm{dw}$, alone and combined as the total NP ingested, can be found in Table A4.1. Interestingly, the relative errors $(n=3)$ were substantially smaller for the total NP ingested in the highest two concentrations ( 10 and $30 \mathrm{~g} / \mathrm{kg}$ sediment $\mathrm{dw}$ ) compared to the errors in the separate body and faeces concentrations. This indicates that defaecation as well as body burden had higher variation than the total ingestion. The average proportion of NP defecated by $G$. pulex was $58.62 \pm 23.15(\mathrm{n}=12)$, including the four highest concentrations $(1-30 \mathrm{~g} / \mathrm{kg})$. The lowest exposure concentration $(0.3 \mathrm{~g} / \mathrm{kg})$ was excluded from the dataset as measurement values were below the ICP-MS detection limit.

Biodynamic modelling of the bioaccumulation of NP by $G$. pulex. Mass balance calculations of NP in sediment, water and biota showed that after 28 days of exposure, less than $1 \%$ of NP mass was lost from the sediment. This demonstrates that the model assumption of constant exposure concentration was met. We fit the first order bioaccumulation model (Eqs. 1 and 2) with three parameters (p) to four triplicate concentrations at two time points i.e. $(\mathrm{p}=3, \mathrm{n}=24)$. The two lowest doses $(0$ and 0.3 $\mathrm{g} / \mathrm{kg}$ ) were omitted as $\mathrm{Pd}$ concentration measurements were below the detection limit. The fit to the experimental data was always within 1 SD (Figure 4.3), which confirmed the absence of dose dependency and was highly significant (ANOVA, $P=1.7 \times 10^{-77}$ ). Model parameter optimization yielded an uptake rate constant $\left(k_{u p}\right)$ of $0.076 \mu \mathrm{g} \times \mathrm{kg}^{-1}$ biota / $\mu \mathrm{g} \times \mathrm{kg}^{-1}$ sediment $\times$ day $^{-1}$ and an elimination rate constant $\left(k_{\text {elim }}\right)$ of 2.44 day $^{-1}$. Steady state concentrations in the reversible (gut) compartment were reached within 2 to 3 days (Figure 4.3). Subsequently, uptake in the irreversible (body) compartment steadily increased, suggesting that accumulation would have continued beyond the time frame of our experimental set-up. After 28 days, depuration rapidly removed part of the total accumulated NP from the organism, but a poorly reversible fraction remained (Figure 4.3 insert), which illustrates the necessity of distinguishing between these two compartments. A fraction of only $0.96 \%\left(F_{B}=0.0096\right)$ of NP ingested were estimated to transfer from the gut into the irreversible (body) compartment. However, even this small fraction eventually leads to a considerable body burden after 28 days due to the lack of (measurable) depuration from that reservoir.

Redondo-Hasselerharm et al. (2018) defined a MP steady state trophic transfer factor (TTF) for the body of G. pulex as $T T F_{B O D Y}=C_{G . p u l e x, b o d y} / C_{S E D}\left(\right.$ Chapter 2). ${ }^{186}$ This TTF relates to the mass concentration of MP remaining in the organisms after gut depuration, divided by the MP mass concentration in the sediment after 28 days. The value for the $T T F_{B O D Y}$ for NP in the present study was $0.020 \mu \mathrm{g} \times \mathrm{kg}^{-1}$ biota $/ \mu \mathrm{g} \times \mathrm{kg}^{-1}$ sediment $\mathrm{dw}$ (Table 4.1). Similar to $T T F_{B O D Y}$, an apparent TTF for the reversible (gut) compartment can be calculated as $T T F_{G U T}=C_{G \text {.pulex,faeces }} / C_{S E D}=k_{\text {up }} / k_{\text {elim }}$, which was $0.031 \mu \mathrm{g} \times \mathrm{kg}^{-1}$ biota $/ \mu \mathrm{g} \times \mathrm{kg}^{-1}$ sediment dw. Consequently, the comparison between the modelled $T T F_{G U T}$ and $T T F_{B O D Y}$ reveals that after 28 days, $60.2 \%(100 \times 0.031 /[0.031+0.020])$ of all ingested NP reside in the gut, compared to $39.8 \%$ in the body (Table 4.1 ). These percentages derived from the model are consistent with the $58.62 \pm 23.15 \%$ observed to be defecated from the gut in the experimental work. 


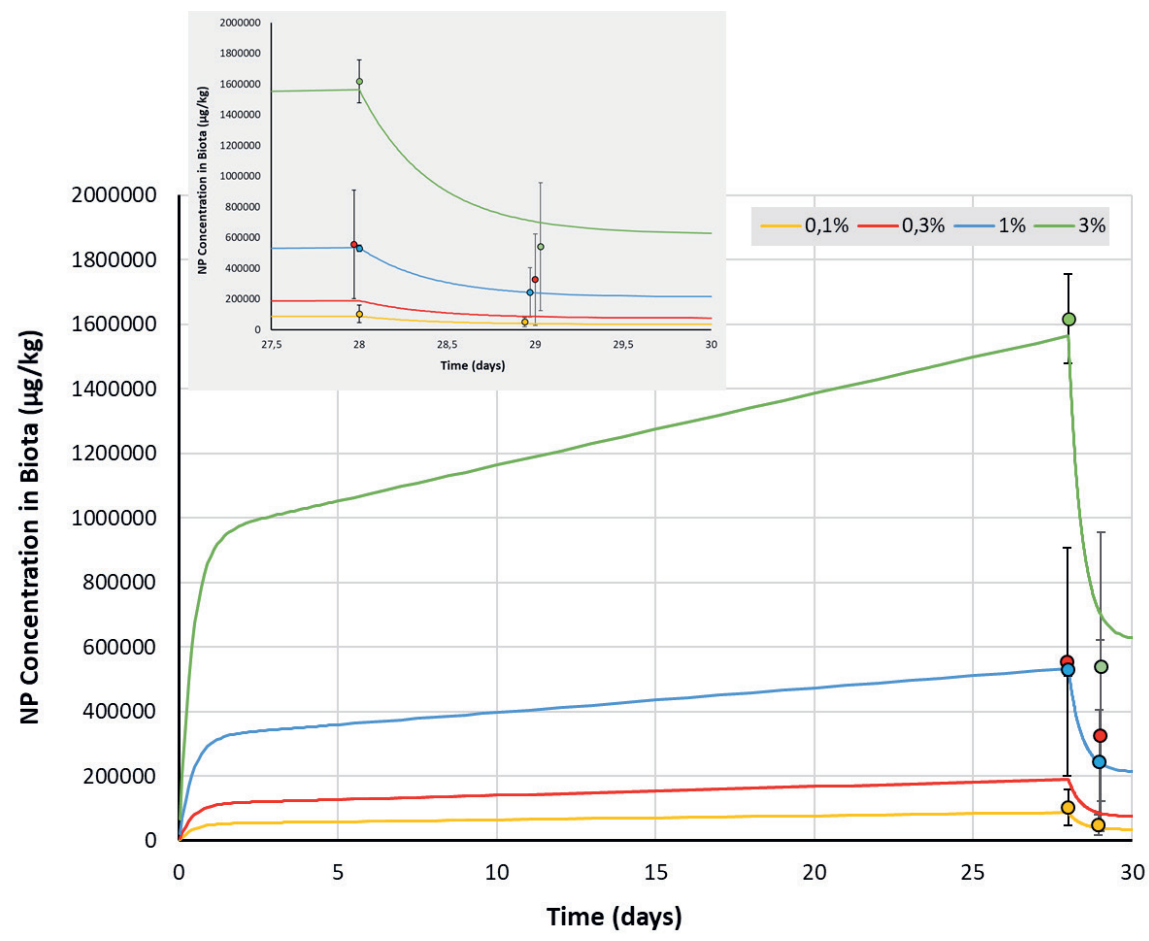

Figure 4.3. Measured (datapoints) and modelled (curves) uptake of Pd-doped NP by G. pulex over 28 days of exposure to sediment amended with Pd-doped NP, followed by 1 day of depuration in clean medium (insert). Data on measured NP concentrations ( \pm 1 SD) after depuration (see insert) after 29 days were set apart for 0.05 day for better visibility of the datapoints on the $x$ axis.

Comparison of accumulation kinetics and trophic transfer factors between NP and MP. We have previously derived bioaccumulation data for MP using the same sediment, test organism and experimental design as used in this present experiment. This calls for a comparison between the two datasets, which we here provide by applying the same modelling approach to the MP data (details provided in the Appendix; Figure A4.5). The kinetic parameters, 28-day $T T F_{G U T}$ and 28-day $T T F_{T O T A L}$ agree with parameters estimated for NP within a factor of only two (Table 4.1). This is striking considering the large size difference between the two test particles $(0.23 \mu \mathrm{m}$ NP versus $20-165 \mu \mathrm{m} \mathrm{MP})$. This would suggest a random ingestion of particles, dominated by species traits rather than particle properties. It has been hypothesized that satiation in combination with dilution of food is one of the main demonstrated adverse effect mechanisms upon ingestion of small plastic particles (Chapter 7). ${ }^{223}$ This mechanism would imply that the mass-based $T T F_{G U T}$ values would be similar regardless of actual particle size, as long as particles would be ingestible (Chapter 7). ${ }^{223,233}$ The current finding that NP and MP with large size differences yield very similar $T T F_{G U T}$ under the same exposure conditions supports 
this hypothesis. We emphasize that this value only applies to the 28-day time point. $T T F_{B O D Y}$ is likely to increase further after 28 days, but the current data do not allow us to speculate on the actual trend. With a value of 0.092 , the $T T F_{B O D Y}$, reflecting accumulation in the irreversible fraction, was 4.5 times larger for MP compared to NP. This can be explained from MP particles being trapped in the gut, as opposed to NP particles that would easily be defecated with other organic matter-based gut contents.

Table 4.1. Bioaccumulation kinetic parameters and sizes of apparent accumulation reservoirs for nanoplastic (present study) and microplastic (remodelled from Redondo-Hasselerharm et al., 2018) (Chapter 2) ${ }^{186}$ in Gammarus pulex.

\begin{tabular}{|c|c|c|c|}
\hline Parameter & Nanoplastic & Microplastic & Unit \\
\hline Uptake rate constant $\left(k_{u p}\right)$ & 0.076 & 0.044 & $\begin{array}{l}\mu \mathrm{g} \times \mathrm{kg}^{-1} \text { biota } / \mu \mathrm{g} \times \mathrm{kg}^{-1} \\
\text { sediment } \times \mathrm{day}^{-1}\end{array}$ \\
\hline Elimination rate constant $\left(k_{\text {elim }}\right)$ & 2.44 & 4.61 & day $^{-1}$ \\
\hline Irreversible (stored) fraction $\left(F_{B}\right)$ & 0.96 & 2.79 & $\%$ \\
\hline TTFBODY $^{\text {(a) }}$ & 0.020 & 0.092 & $\begin{array}{l}{[\mathrm{mg} / \mathrm{kg} \text { organism }] /} \\
{[\mathrm{mg} / \mathrm{kg} \text { sediment }]}\end{array}$ \\
\hline $\mathrm{TTF}_{\mathrm{GUT}}{ }^{(\mathrm{a})}$ & 0.031 & 0.025 & $\begin{array}{l}{[\mathrm{mg} / \mathrm{kg} \text { organism }] /} \\
{[\mathrm{mg} / \mathrm{kg} \text { sediment }]}\end{array}$ \\
\hline TTF $_{\text {TOTAL }}{ }^{(a)}$ & 0.051 & 0.116 & $\begin{array}{l}{[\mathrm{mg} / \mathrm{kg} \text { organism }] /} \\
{[\mathrm{mg} / \mathrm{kg} \text { sediment }]}\end{array}$ \\
\hline Percentage in body ${ }^{\text {(a) }}$ & 39.8 & 78.7 & $\%$ \\
\hline Percentage in gut ${ }^{(a)}$ & 60.2 & 21.3 & $\%$ \\
\hline
\end{tabular}

(a) These TTF values and percentages are conditional; they depend on exposure time.

Effects on survival and growth. The average survival in controls was $86.4 \% \pm 9.5(\mathrm{n}=6)$. The exposure to NP had no significant effects on the survival of G. pulex (GLM, $P=$ 0.577). No significant differences on the growth of $G$. pulex were found among the NP concentrations ranging up to $30 \mathrm{~g} / \mathrm{kg}$ sediment $\mathrm{dw}$, nor among chemical concentrations in the chemical solution (Figure 4.4).

To date, hardly anything is known about NP. ${ }^{13}$ Heinlaan et al. (2020) assessed the effects of 26 and $100 \mathrm{~nm}$ PS NP on the midge larvae Chironomus riparius and the ostracod Heterocypris incongruens. ${ }^{225}$ No significant effects were found on the survival of $C$. riparius after 48 hours of exposure and no significant effects were found on the survival and growth of $H$. incongruens after 6 days of exposure. ${ }^{225}$ In Redondo-Hasselerharm et al. (2020), the effects of $96 \mathrm{~nm}$ PS NP were evaluated on a freshwater benthic community using outdoor tests (Chapter 5). ${ }^{226}$ While after 3 months of exposure, no effects were found on the community composition, a reduction in the abundance of Naididae worms was observed after 15 months of exposure at a concentration of 5\% plastic per sediment $\mathrm{dw}$ (Chapter 5). ${ }^{226}$ The absence of shorter term effects (up to 3 months) of NP on freshwater benthic organisms found in previous papers is therefore in accordance with the results obtained in this study. 

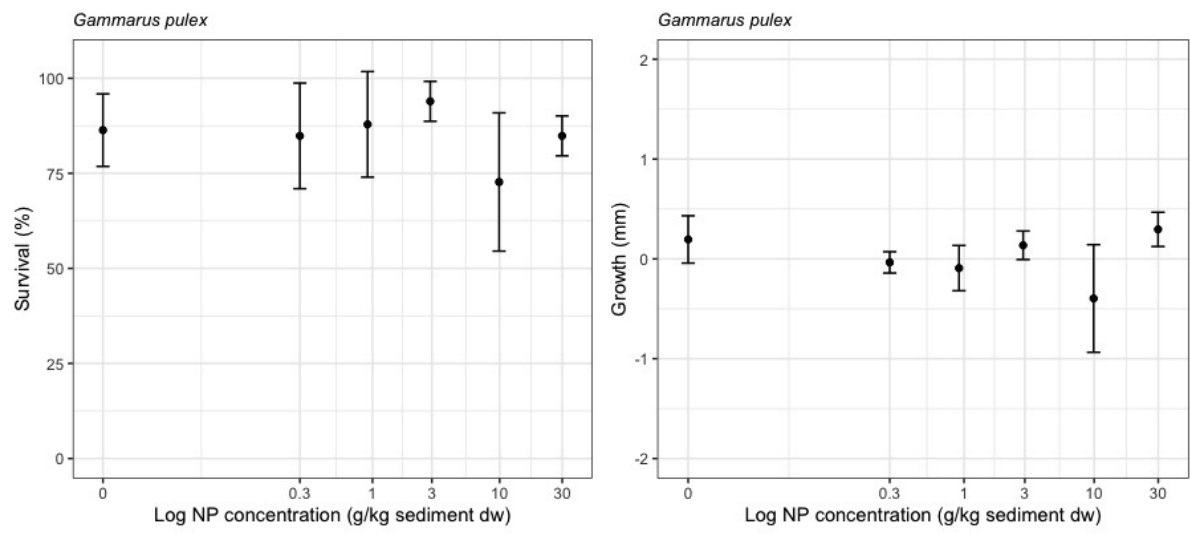

Figure 4.4. Survival (\%) and growth as length ( $\mathrm{mm}$ ) of Gammarus pulex as a function of the Log $N P$ concentration ( $g / k g$ sediment $d w)$. Error bars are mean $\pm S D(n=3)$.

Previous studies have stated that NP might pose a greater risk compared to MP due to their smaller size, as they are more prone to getting lodged in small body structures. ${ }^{53,67}$ However, Redondo-Hasselerharm et al. (2018) found significant adverse effects on the growth of G. pulex after a 28-day sediment exposure to irregularly shaped PS MP (size range: $20-500 \mu \mathrm{m})$, with a median effect dose $\left(\mathrm{EC}_{50}\right)$ of $3.57 \pm 3.22 \%$ (Chapter 2$) .{ }^{186}$ The $\mathrm{TTF}_{\mathrm{GUT}}$ mass value found for the PS MP was 0.025 , which is very close to the TTF mass value found in the present study. However, the difference in effects found for both particle types may be related with the higher $T T F_{B O D Y}$ found for the PS MP $(4.5 \mathrm{x}$ higher, see Table 4.1), which may reflect particles being trapped in the gut, leading to hindered passage of food or other functions.

\section{Conclusion and Prospect.}

We used Pd-doped PS NP with a bumpy surface to assess uptake and effects on the freshwater benthic amphipod G. pulex using single species tests with natural sediments. Bioaccumulation was demonstrated, but no effects of the NP were found on the survival and growth of G. pulex at concentrations up to $30 \mathrm{~g} / \mathrm{kg}$ of sediment dw. Therefore, the No observed Effect Concentration (NOEC) was equal to $30 \mathrm{~g} / \mathrm{kg}$ of NP per sediment $\mathrm{dw}$. The model particles were used as a proxy for environmental NP, and we demonstrated that metal-doping enabled us to accurately measure the NP in various highly complex environmental matrices such as natural water, sediment and biota. This was very advantageous, since currently direct NP analysis would not have been possible and thus we would have only been able to report effects on the organisms. However, here we were able to conduct a mass balance between all environmental and biological compartments, as well as quantify NP uptake and depuration rates. 


\section{Acknowledgments}

We thank the Chemical Biological Soil Laboratory (CBLB) of Wageningen University and Research for performing the analysis of the metal-doped NP. We also thank B.F. Arlina, J. Beijer and F. Gillissen for their practical assistance. This study was funded by the Dutch Technology Foundation TTW, project no. 13940. We acknowledge additional support from KWR; WMR; NVWA; RIKILT; the Dutch Ministry of Infrastructure and the Environment; the Dutch Ministry of Health, Welfare and Sport; Wageningen Food and Biobased Research; STOWA; RIWA; and water boards Hoogheemraadschap van Delfland, Zuiderzeeland, Rijn en IJssel, Vechtstromen, Scheldestromen, Aa en Maas, de Dommel, and Rivierenland. 


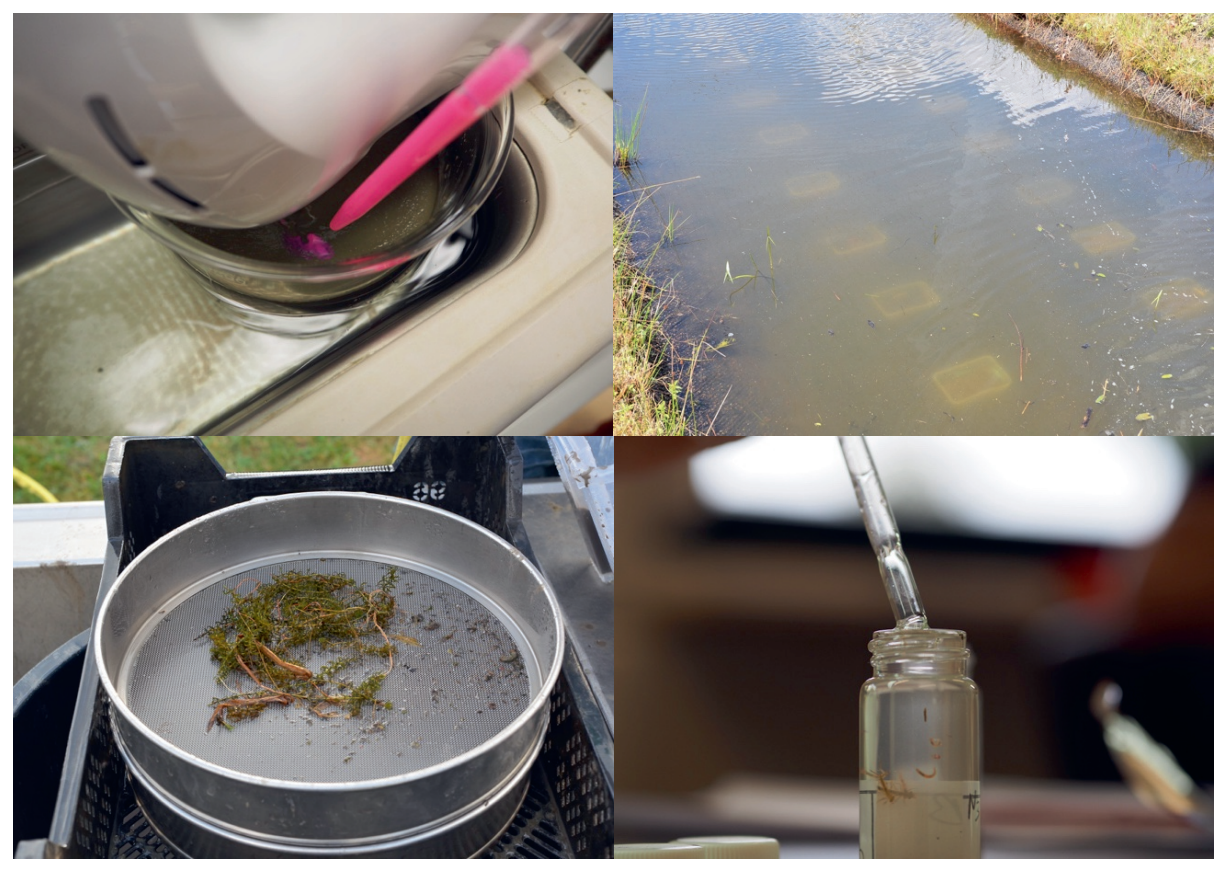




\section{Chapter 5}

\section{Nano- and Microplastics Affect the Composition of Freshwater Benthic Communities in the Long Term}

Published as: Redondo-Hasselerharm, P.E.; Gort, G.; Peeters, E.T.H.M.; Koelmans, A.A. Nano- and Microplastics Affect the Composition of Freshwater Benthic Communities in the Long Term. Science Advances, 2020, 6: eaay4054 


\begin{abstract}
Given the societal concern about the presence of nano- and microplastics in the environment, our nescience with respect to in situ effects is disturbing. Data on long term implications under ecologically realistic conditions are particularly important for the risk assessment of nano- and microplastics. Here, we evaluate the long term (up to 15 months) effects of five concentrations of nano- and microplastics on the natural recolonization of sediments by a macroinvertebrate community. Effects were assessed on the community composition, population sizes and species diversity. Nano- and microplastics adversely affected the abundance of macroinvertebrates after 15 months, which was caused by a reduction in the number of Naididae at the highest concentration (5\% plastic per sediment dry weight). For some other taxa, smaller but still significant positive effects were found over time, altogether demonstrating that nano- and microplastics affected the community composition.
\end{abstract}




\section{Introduction}

Nanoplastics (NP), with a size smaller than $0.1 \mu \mathrm{m}$, and microplastics (MP), with a size between $0.1 \mu \mathrm{m}$ and $5 \mathrm{~mm}$, comprise the smallest particle fraction of plastic debris globally. ${ }^{13}$ Although the accumulation of NP and MP is currently a major concern, ${ }^{13}$ studies addressing their effects on single species are scarce and nothing is known about their long-term effects at the community level. ${ }^{13,234,235}$ Freshwaters are particularly affected as sediments are known to accumulate NP and MP due to the vicinity of sources and due to aggregation and biofouling processes and subsequent settling, which create hotspot areas that might pose a risk for benthic organisms. ${ }^{27}$

The ability of freshwater benthic macroinvertebrates to ingest MP depends on species characteristics such as their feeding habit or developmental stage (Chapter 2), ${ }^{46,186}$ as well as on plastic particle properties and environmental conditions. ${ }^{45}$ Single-species laboratory studies have found that the ingestion of MP by freshwater benthic macroinvertebrates can cause adverse effects, ${ }^{40,133,236}$ which also seems to differ among species. For instance, a reduction in the growth of the amphipod Gammarus pulex was found after a 28-day exposure to polystyrene (PS) MP, while five other benthic macroinvertebrates were not affected under the same experimental conditions (Chapter 2). ${ }^{186}$ Over time, these differences in sensitivity to MP particles may lead to changes in the community structure, triggering disproportionate responses. ${ }^{237}$ For instance, reductions in the abundance of shredders such as the amphipod G. pulex, have shown to affect detritus processing. ${ }^{238}$ Consequently, changes in benthic community structure can have negative consequences for the functioning of ecosystems. ${ }^{238}$ However, single species laboratory tests cannot offer the ecological realism required to detect such ecological implications. After all, they lack the ecological processes that drive community change in the long term, such as community interactions, temperature and light variations, flow dynamics, seasonality, aging, and reproduction. Therefore, the effects of MP should be evaluated under field conditions and for much longer time periods to take all these processes into account.

The aim of this study was to evaluate the effects of NP and MP on a benthic macroinvertebrate community located in an outdoor experimental ditch, for a long exposure time of up to 15 months. Trays containing natural sediment mixed with NP or MP at concentrations of $0,0.005,0.05,0.5$ and $5 \%$ plastic per sediment dry weight were embedded in the sediment of a ditch that contained a well-characterized donor community. This community is typical for standing water systems such as ditches, canals, ponds and lakes. Deposition and accumulation of NP and MP may occur in such systems, rendering their benthic communities to be particularly exposed to these particles. Spherical PS NP with an average size of $96.3 \pm 1.85 \mathrm{~nm}$ and irregular PS MP fragments with sizes ranging from 20 to $516 \mu \mathrm{m}$ were used for the NP and MP treatments, respectively. Each NP and MP concentration was prepared in quadruplicate, and concentrations were selected on the basis of measured environmental concentrations in the Rhine river shore sediments, which were up to $1 \mathrm{~g} / \mathrm{kg}(0.1 \%$ plastic per sediment dry 
weight). ${ }^{130}$ The two lowest concentrations used in the present study $(0.005$ and $0.05 \%)$ can therefore be considered environmentally realistic. ${ }^{130}$ After 3 and 15 months of colonization, trays were retrieved and species were identified and counted. The contribution of plastic type, exposure time and concentration plus the interaction of time and concentration, but also by block (spatial variation) and the interaction of block with type of the plastic particles, were evaluated for the effect on abundance of benthic macroinvertebrates, number of taxa, Shannon diversity index $(H)$ and the number of individuals of 21 taxa for both NP and MP treatments separately. We provide long-term community effect thresholds for freshwater benthic macroinvertebrates and compare them with environmental concentrations measured in freshwater sediments.

\section{Material and Methods}

$N P$ and $M P$. Following earlier studies,${ }^{220,239}$ spherical carboxylated PS NP were provided by the Food and Biobased Department of Wageningen University (The Netherlands). Zaverage size (nm) was measured with a Zetasizer (Nano ZS, Malvern instruments) and was $96.3 \pm 1.85 \mathrm{~nm}(\mathrm{n}=3)$ for particles $100 \mathrm{x}$ diluted in Milli-Q water. NP were synthesised from styrene monomers using 4,4'-Azobis (4-cyanopentanoic acid) as initiator and sodium dodecyl sulphate (SDS) as surfactant. The dye Rhodamine B methacrylate was provided by the Physical Chemistry and Soft Matter Department of Wageningen University (The Netherlands) and added during the synthesis. The final solution contained $41.91 \%$ dry weight of NP, $1.1 \%$ wet weight of SDS and $0.4 \%$ wet weight of Rhodamine B methacrylate, which was covalently bound to the polymer, preventing it to leach out. Repeated addition of initiator and other aspects of the experimental design were tuned to achieve near-complete polymerization, leaving low concentrations of styrene monomer and SDS used. It was calculated what would be the eventual styrene and SDS concentrations during exposure in the experimental ditch based on added sediment-bound masses of these compounds and an assessment of subsequent desorption, dispersion and dilution (calculation provided in the SI). Concentrations were at least a factor 29 lower than the short term and long term effect thresholds for these chemicals provided by the European Chemical Agency (ECHA). ${ }^{228,240}$

Irregular PS MP fragments were obtained from Axalta Coating Systems GMBH (Cologne, Germany). MP particle size distribution was measured with a Mastersizer 3000 (Malvern Instruments) and ranged from 20 to $516 \mu \mathrm{m}$, with an average size of $227.7 \pm$ $6.01(\mathrm{n}=4)$ in volume $\%$ and $32.7 \pm 0.98(\mathrm{n}=4)$ in number $\%$. Following earlier procedures (Chapter 2), ${ }^{186,220}$ MP were thoroughly washed with methanol to remove organic chemicals associated with the MP, if any. PS was chosen because its density matches that of the average environmental MP (Chapter 2) $)^{20,186}$ and because it's one of the most abundant polymer types found in freshwater systems. ${ }^{130,241}$ MP size and shape ranges used in this study can also be considered environmentally relevant. ${ }^{130,241}$

Plastic - sediment mixtures. Sediments were sampled from an adjacent ditch with similar characteristics using a standard dip net. Sediments were passed through a $2 \mathrm{~mm}$ sieve, 
homogenized with a hand drill, collected in containers and frozen at $-20{ }^{\circ} \mathrm{C}$ in order to kill any remaining living organisms. Plastic was added to the sediment to achieve concentrations of $0,0.005,0.05,0.5$ and $5 \%$ plastic in sediment dry weight. Such a wide range also is needed to assess community level effect thresholds from dose-response relationships. ${ }^{11}$ Knowing that environmental plastic concentrations are likely to increase exponentially, ${ }^{13,97}$ the higher ends of the concentration range used are likely to be realistic in the future and, therefore, its potential effects should be included in prospective risk assessments. ${ }^{11,45}$

To promote the formation of homogeneously dispersed hetero-aggregates of the NP with the sediment particles as would occur in nature, ${ }^{242}$ batches of $1.5 \mathrm{~L} \mathrm{NP}$-sediment mixtures were first made in the laboratory while stirring vigorously. NP were added drop by drop with a glass pipette to sediment contained in a $2 \mathrm{~L}$ glass beaker inside an ultrasonic bath while using an electrical stainless-steel hand mixer at full speed. Once in the field, these batches were added to a cement mill together with clean sediment and mixed for 30 minutes until a homogeneous NP-sediment mixture was created. To prepare MP-sediment mixtures, MP were added in a powder form directly to the cement mill containing the clean sediment. For each concentration, the plastic-sediment mixture was spread over eight thoroughly pre-rinsed consumer-grade polypropylene trays ( 28 by 19 by $14 \mathrm{~cm}$ ), creating a sediment layer of $5 \mathrm{~cm}$. Four of these eight trays were exposed to the donor community for 3 months and the other four were exposed for 15 months.

Experimental design. Experiments were conducted from July 2016 until September 2017 in a ditch located at Sinderhoeve, an experimental field station of Wageningen University (The Netherlands). The ditch is $40 \mathrm{~m}$ long, $3.3 \mathrm{~m}$ wide on the surface and $1.6 \mathrm{~m}$ at the bottom, and $0.5 \mathrm{~m}$ deep. One week before the start of the experiment, rooted macrophytes were removed from the ditch and reduced to a discontinuous central strip to facilitate the placement of the trays (Figure A5.1). At the start of the experiment, a total of 80 trays (two plastic types $\mathrm{x}$ two time points $\mathrm{x}$ five concentrations $\mathrm{x}$ four replicas) were distributed along the experimental ditch (Figure A5.1). Following a randomized complete block design, the ditch was divided in four blocks of $10 \mathrm{~m}$ long (block A, 0 to $10 \mathrm{~m}$, block B, 10 to $20 \mathrm{~m}$, block C, 20 to $30 \mathrm{~m}$, block D, 30 to $40 \mathrm{~m}$ ). Each block was then divided in northern line and southern line, leaving the discontinuous central strip of macrophytes between them. One replica of each treatment was assigned to each block, having replicas A, B, C, and D for each treatment. Within each block, the corresponding replicas of each treatment were randomly embedded in the sediment of the ditch by submerging the trays manually from a movable platform above the ditch. This way, any potential alteration of the system was avoided.

After 3 and 15 months from the start of the experiment, one replica of each plastic concentration was retrieved at each block. To prevent the resuspension of the sediment during the retrieval, a thoroughly prewashed polyethylene plastic sheet of 50 by $50 \mathrm{~cm}$ was first placed on top of the sediment layer. Immediately after, the tray was covered with a lid and carefully lifted up to the water surface. Trays were then sieved over a $0.5 \mathrm{~mm}$ 
sieve and flushed with water until sediments were removed. The remaining sample was placed in polypropylene trays, and organisms were sorted, fixed in 70 to $80 \%$ ethanol and stored upon identification. Rooted macrophytes and overhanging branches were gathered from each tray, dried at $60{ }^{\circ} \mathrm{C}$ for 24 hours and measured to obtain an estimate of macrophyte biomass (in milligrams). At the start of the experiment and after 3 and 15 months, the macroinvertebrate composition of the donor system (i.e., outside the trays) was assessed by taking transects between blocks A-B and C-D. For this, a standard dip net was swept over the sediment from one bank to the other, using a platform to avoid other disturbances in the ditch. Once in the laboratory, organisms from trays and transects were identified to the lowest possible taxonomic levels by certified biologists specialized in benthic invertebrate identification.

The colonization ratio was calculated as the number of individuals $/ \mathrm{m}^{2}$ and taxa in trays retrieved after 3 months divided by the number of individuals $/ \mathrm{m}^{2}$ and taxa in the donor system at the start of the experiment. Colonization ratios in terms of number of individuals $/ \mathrm{m}^{2}$ were always higher than one because of the different sampling methods used, which underestimated the number of individuals $/ \mathrm{m}^{2}$ of the endo-benthic taxa in the donor community. In addition, reference community ratios were calculated as the number of individuals $/ \mathrm{m}^{2}$ and taxa in trays retrieved after 3 and 15 months by the number of individuals $/ \mathrm{m}^{2}$ and taxa in trays in the donor system at the same time points. Colonization ratios for the number of individuals $/ \mathrm{m}^{2}$ and taxa are presented in Table 5.1. Reference community ratios for the number of individuals $/ \mathrm{m}^{2}$ and taxa are presented in Table A5.1.

Table 5.1. Colonization ratios based on the number of individuals $/ m 2$ and based on taxa. Colonization ratio for number of individuals was calculated as the number of individuals $/ m 2$ found in trays retrieved after 3 months divided by the number of individuals $/ m 2$ found in the donor system at the start of the experiment. For taxa, this was performed similarly, i.e., the number of taxa in trays after 3 months divided by the number of taxa at start. Means $\pm S D$ correspond to $n=4$, except for 0.05 and $0.5 \%$ (3 months), where $n=3$; and transects, where $n=2$.

\begin{tabular}{|c|c|c|c|c|c|c|c|c|}
\hline & \multicolumn{4}{|c|}{$\mathbf{N P}$} & \multicolumn{4}{|c|}{ MP } \\
\hline & \multicolumn{2}{|c|}{ Individuals $/ \mathbf{m}^{2}$} & \multicolumn{2}{|c|}{ Taxa } & \multicolumn{2}{|c|}{ Individuals $/ \mathbf{m}^{2}$} & \multicolumn{2}{|c|}{ Taxa } \\
\hline & Mean & SD & Mean & SD & Mean & SD & Mean & SD \\
\hline $\mathbf{0}$ & 4.35 & 1.14 & 0.87 & 0.11 & 3.51 & 1.03 & 0.86 & 0.12 \\
\hline 0.005 & 4.19 & 1.38 & 0.82 & 0.18 & 5.14 & 1.17 & 0.88 & 0.13 \\
\hline 0.05 & 5.23 & 0.99 & 0.86 & 0.06 & 5.41 & 1.52 & 0.72 & 0.15 \\
\hline 0.5 & 4.03 & 0.96 & 0.76 & 0.09 & 4.90 & 1.27 & 0.79 & 0.02 \\
\hline 5 & 4.69 & 1.02 & 0.73 & 0.09 & 3.51 & 0.47 & 0.74 & 0.11 \\
\hline
\end{tabular}


Temperature $\left({ }^{\circ} \mathrm{C}\right)$, dissolved oxygen (DO) $(\mathrm{mg} / \mathrm{l}), \mathrm{pH}$ and electro-conductivity (EC) $(\mu \mathrm{S} / \mathrm{cm})$ were measured with a Multi 3630 IDS (WTW) along the 15 months experiment. Additionally, water samples were taken from three locations in the ditch to analyse $\mathrm{NH}_{4}$ $(\mathrm{mg} / \mathrm{l})$, nitrate $(\mathrm{mg} / \mathrm{l})$, total nitrogen $(\mathrm{mgN} / \mathrm{l})$ and total phosphorus $(\mathrm{mgP} / \mathrm{l})$ content. In the first 3 months, measurements were taken at nine time points, to cover the potential changes in the water quality variables derived from the macrophytes' growth along the ditch. After this period, measurements were taken once every 3 months. A summary of the water quality variables measured throughout the experiment is shown in Table A5.2.

Nominal versus actual NP and MP exposure. Three representative sediment samples were taken from the plastic-sediment mixtures added to the trays (including the control) at time zero. In addition, a representative sediment subsample was taken from each individual tray after its removal from the system and before the sieving. Total organic matter (TOM) content was analysed in these sediment samples using loss on ignition $\left(3 \mathrm{~h}, 550{ }^{\circ} \mathrm{C}\right)$ to determine the plastic content through thermal degradation. By subtracting the \%TOM obtained in controls from those in the treatment trays, nominal plastic concentrations could be verified in the mixtures as the thermal degradation of PS occurs below $550{ }^{\circ} \mathrm{C} .{ }^{243}$ The small relative error and good agreement with nominal concentrations for the four most accurately measured doses (see the 0.5 and 5\% data for NP and MP in Figure 5.1) demonstrate the homogeneity and accuracy of the preparation of the plastic-sediment mixtures added to the trays, and confirm that no losses occurred. Furthermore, the slopes of the linear regressions between measured and nominal NP and MP concentrations in sediment after 15 months of exposure had slopes of $0.960 \pm 0.037(\mathrm{n}=16)(\mathrm{MP})$ and $0.993 \pm 0.040(\mathrm{n}=16)(\mathrm{NP})$ in ordinal scale (Figure 5.1). The linearity and the slopes being virtually equal to 1 further illustrate the accuracy of the preparation of the plasticsediment mixtures added to the trays.

Statistical analyses. A linear model for the Shannon index and the weight of the macrophytes and generalized linear models (GLM) for total abundance and for abundance of 21 individual taxa were fit using a Poisson distribution with log link function and an extra scale parameter to account for overdispersion. In all linear and generalized linear models, the response was explained by the factors type of plastic, time and concentration, and the interaction of time and concentration but also by block and the interaction of block with type of plastic. The results from this model fitting were presented as analysis of variance tables and analysis of deviance tables, showing per plastic type the main effects and interaction of time and concentration, but also the effect of the block factor and its interaction with type of plastic and an overall comparison between plastic types. Besides the overall comparison between plastic types, means from each concentration were compared between plastic types, which are shown in the lower row of the analysis of variance or deviance tables. The hypothesis tests in the tables were performed using type II model comparisons. Tukey multiple comparison tests were used to compare the effects of NP or MP concentrations per time point when the $P$ value for the effect of plastic concentration or plastic concentration in interaction with time was $<0.05$. As 


\section{Chapter 5}
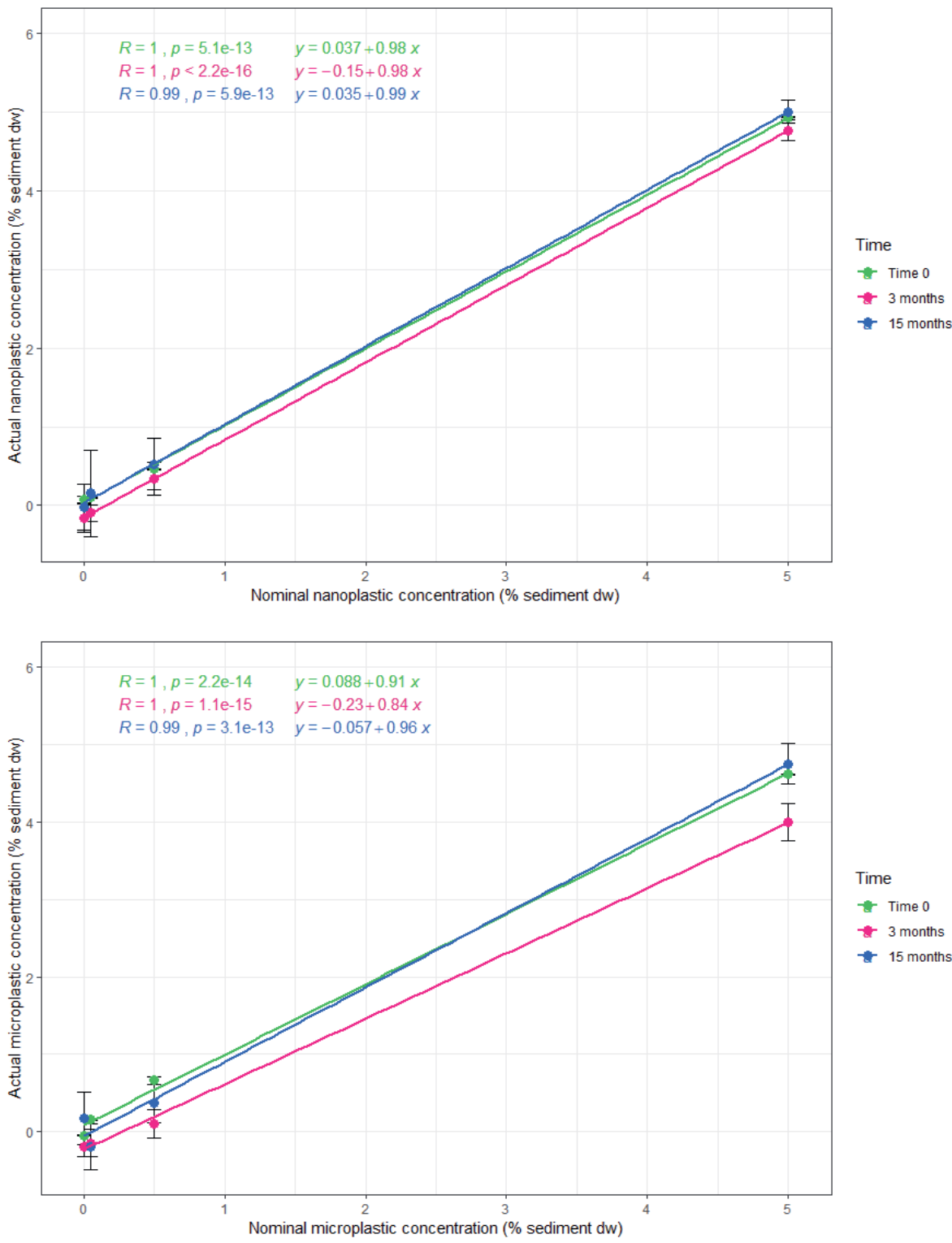

Figure 5.1. NP (top) and MP (bottom) concentrations measured in the plastic-sediment mixtures at time zero, and in trays retrieved after 3 and 15 months after subtracting the \%TOM in controls from the measured \%TOM in trays as a function of the nominal NP concentration (top) and the nominal MP concentration (bottom) (as \% sediment dry weight). For the starting concentration, average $\pm S D$. $(n=3)$ was based on three samples taken from the initial concentrations prepared.Values for 3 and 15 months represent the average $\pm S D .(n=4)$, which correspond to each of the four treatment replicas distributed along the ditch. 
macrophyte dry weight appeared to highly depend on time for both NP and MP (ANOVA; $\left.P_{\text {time }}<0.001\right)$, macrophytes were not included in the analyses. Taxa with very low numbers of individuals per tray were also omitted from the analysis. Linear regressions were fit for the \% TOM content measured in the plastic-sediment mixtures added to the trays at time zero and in trays retrieved after 3 and 15 months as a function of the nominal concentrations added. All statistical analysis and graphs were performed in R (version 3.5.2, R Development Core Team) and packages emmeans, car and ggplot2 were used.

\section{Results}

$N P$ and MP effects on the abundance and diversity of the benthic macroinvertebrate community. NP and MP concentrations had significant negative effects on the total abundance of macroinvertebrates, which is the sum of all individuals of all taxa found in trays (GLM; $\mathrm{NP}_{\text {conc }} P=0.04, \mathrm{MP}_{\text {conc }} P=0.03$ ) (Figure 5.2). Multiple comparison analysis performed for each time point revealed no significant differences among concentrations after 3 months exposure for both NP and MP. After 15 months, however, the abundance of macroinvertebrates at the highest NP concentration (5\%) was significantly lower than at the second highest concentration $(0.5 \%)$ and the lowest concentration $(0.005 \%)$ (Tukey; NP15 $5 / 0.5 P=0.03$, NP15 $5_{5 / 0.005} P=0.002$ ). After 15 months, the abundance of macroinvertebrates at the highest MP concentration (5\%) was significantly lower than the second highest MP concentration $(0.5 \%)$ (Tukey; MP15 $\left.5_{5 / 0.5} P=0.02\right)$. In contrast to these results, NP and MP concentrations did not affect the number of taxa (Figure 5.3) (GLM; $\left.\mathrm{NP}_{\text {conc }} P=0.34, \mathrm{MP}_{\text {conc }} P=0.31\right)$, nor the Shannon diversity index $(H)\left(\mathrm{GLM} ; \mathrm{NP}_{\text {conc }} P=\right.$ $0.56, \mathrm{MP}_{\text {conc }} P=0.57$ ) (Figure 5.4).

When categorizing the number of benthic macroinvertebrates found in trays by class (Figure A4.2), it appears that this reduction in macroinvertebrate abundance at the highest $\mathrm{NP}$ and MP concentrations is mainly caused by the class Clitellata, which mostly consisted of Naididae worms (Tables A5.3, A5.4). Again here, both NP and MP concentrations had a significant negative effect on the abundance of this family of worms (GLM; $\mathrm{NP}_{\text {conc }} P=0.008, \mathrm{MP}_{\text {conc }} P=0.008$ ) (Figure 5.5). Just like for the macroinvertebrate abundance, the number of Naididae did not differ among concentrations after 3 months exposure for both NP and MP. After 15 months, the number of Naididae at the highest NP concentration (5\%) was significantly lower than the second highest concentration $(0.5 \%)$ and the lowest concentration $(0.005 \%)$ (Tukey; NP155/0.5 $P$ $\left.=0.04, \mathrm{NP} 15_{5 / 0.005} P=0.001\right)$. After 15 months, the number of Naididae at the highest MP concentration (5\%) was significantly lower than the second highest concentration (0.5\%) (Tukey; MP15 $\left.5_{5 / 0.5} P=0.01\right)$. 

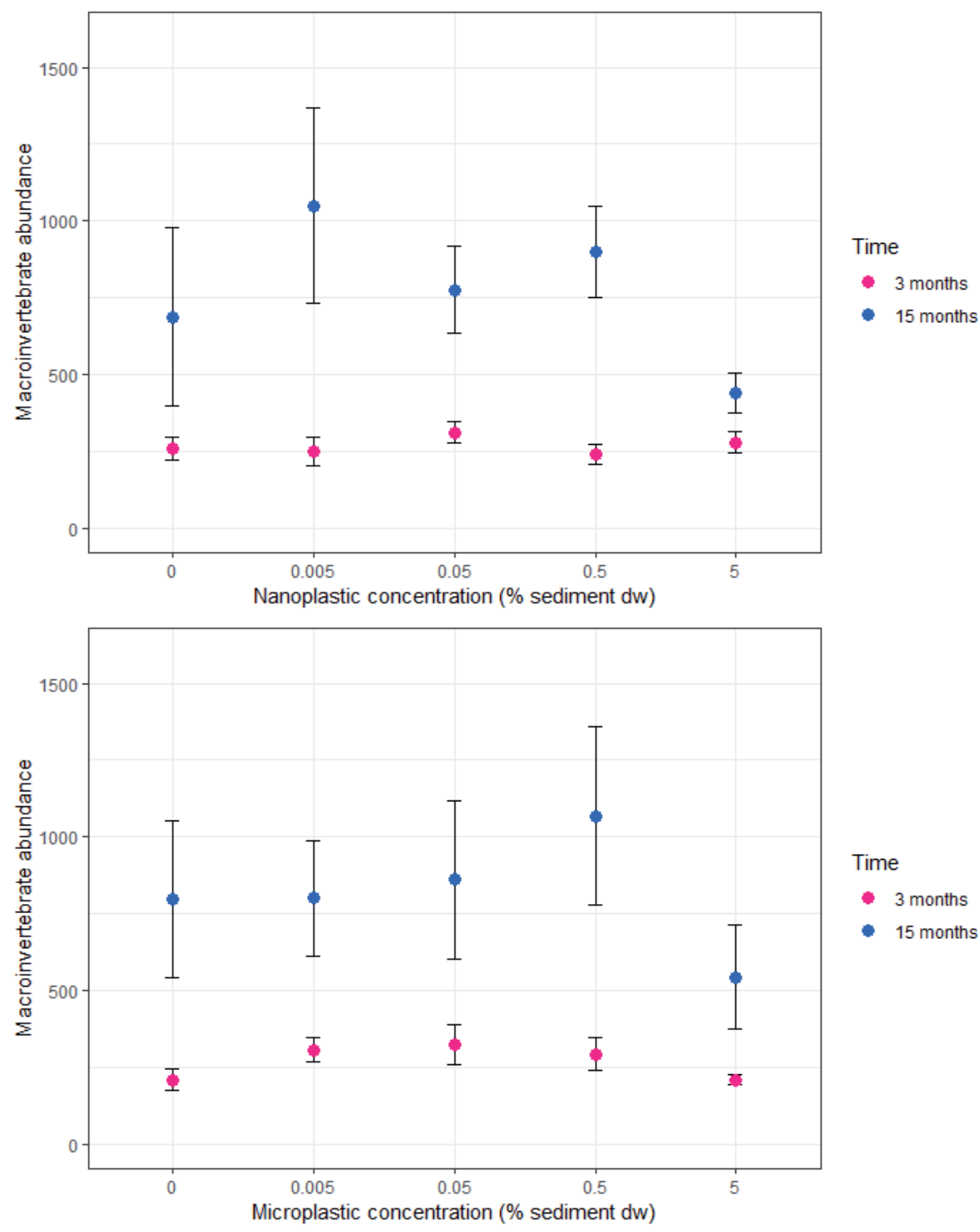

Figure 5.2. Total number of macroinvertebrates found in trays retrieved after 3 and 15 months with increasing NP (top) and MP (bottom) concentrations (as \% sediment dry weight (dw)). Error bars are means $\pm S E, n=4$, except for MP treatments 0.05 and $0.5 \%$ retrieved after 3 months and 0 and $5 \%$ retrieved after 15 months, where $n=3$. 


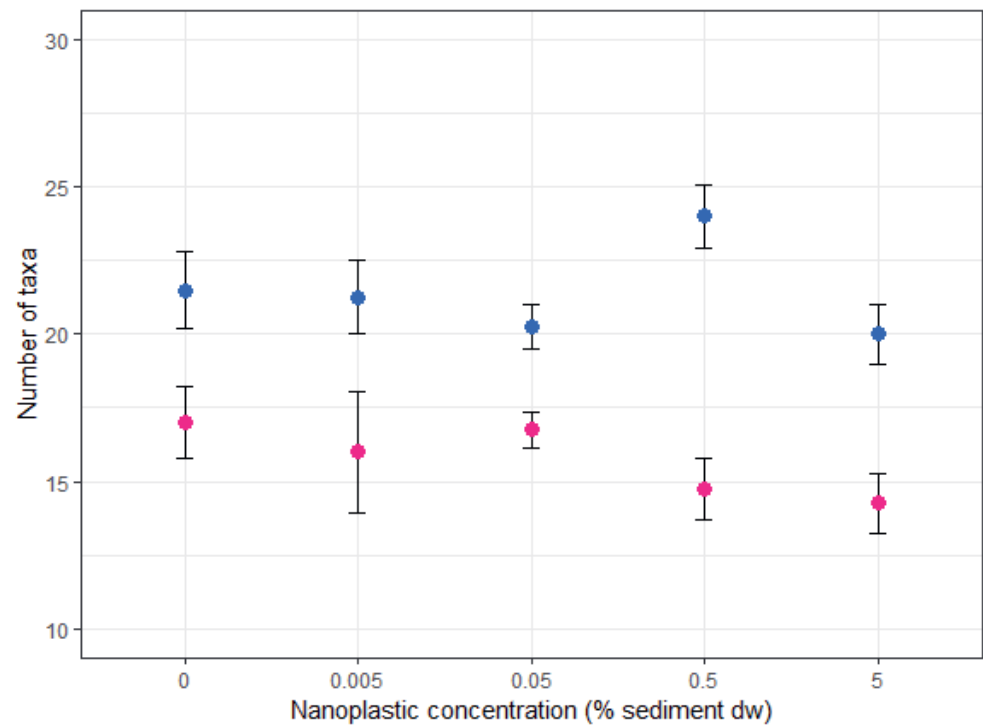

Time

- 3 months

15 months

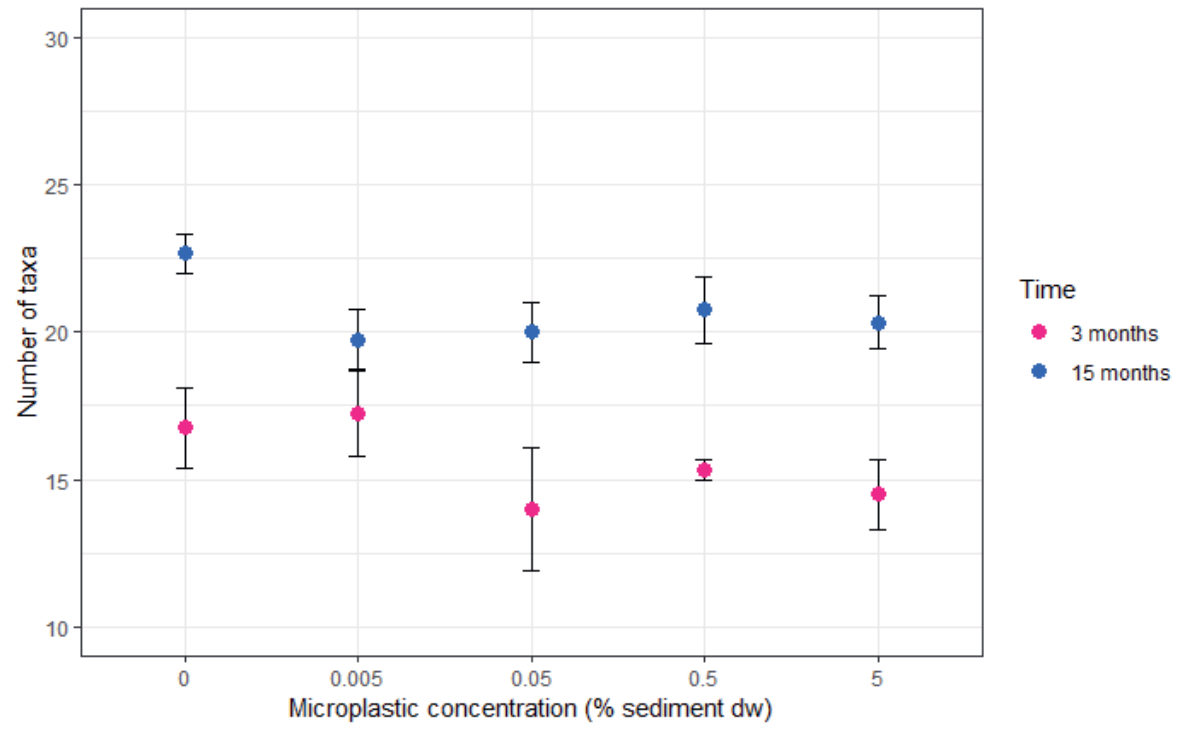

Figure 5.3. Total number of taxa found in trays retrieved after 3 and 15 months with increasing NP (top) and MP (bottom) concentrations (as \% sediment dry weight $(d w)$ ). Error bars are means $\pm S E, n=4$, except for MP treatments 0.05 and $0.5 \%$ retrieved after 3 months and 0 and $5 \%$ retrieved after 15 months, where $n=3$. 


\section{Chapter 5}
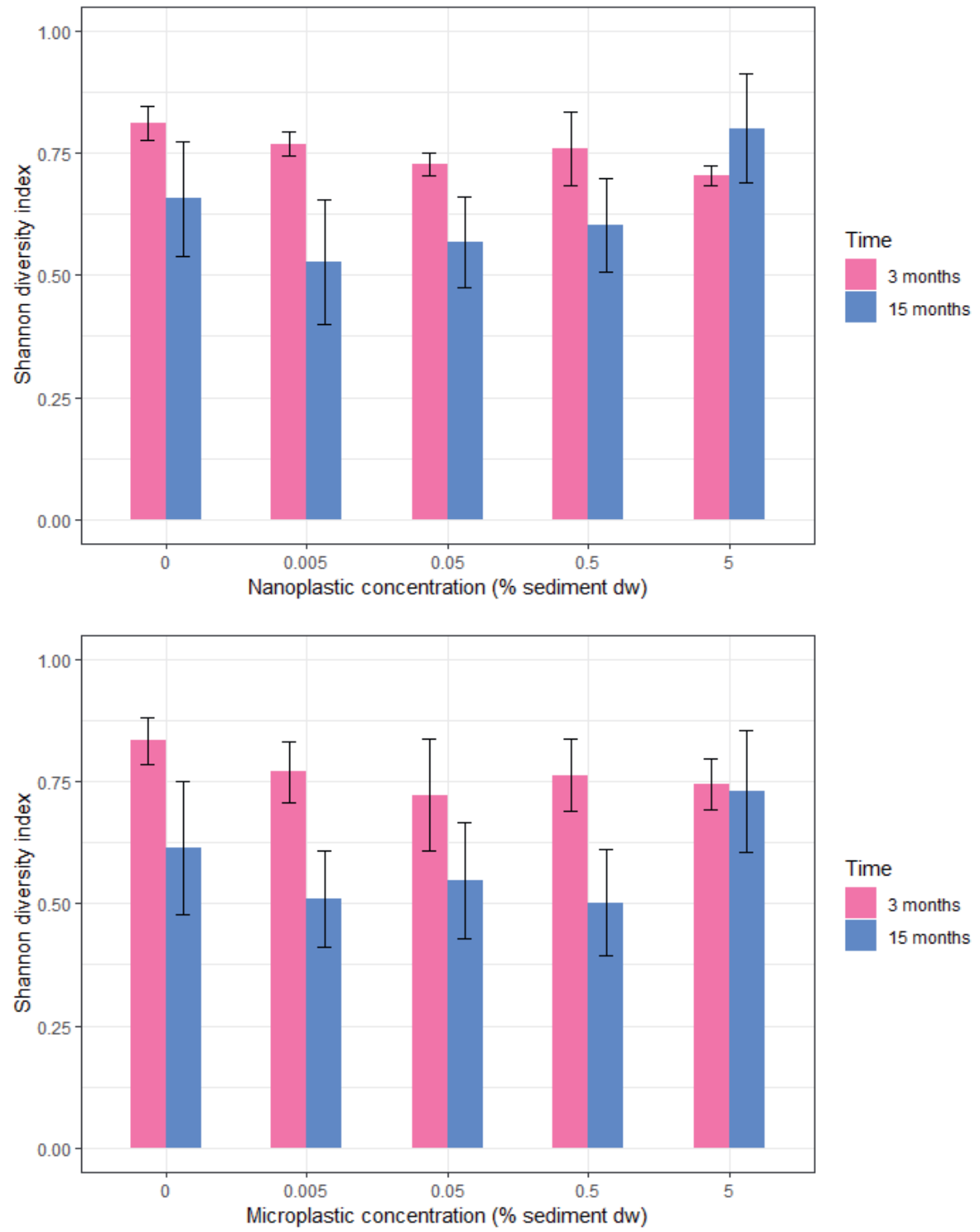

Figure 5.4. Shannon diversity index in trays containing NP (top) and MP (bottom) at five concentrations $(0,0.005,0.05,0.5$ and $5 \%$ plastic per sediment dry weight) after 3 and 15 months. Error bars are means $\pm S E, n=4$, except for MP treatments 0.05 and $0.5 \%$ retrieved after 3 months and 0 and $5 \%$ retrieved after 15 months, where $n=3$. 

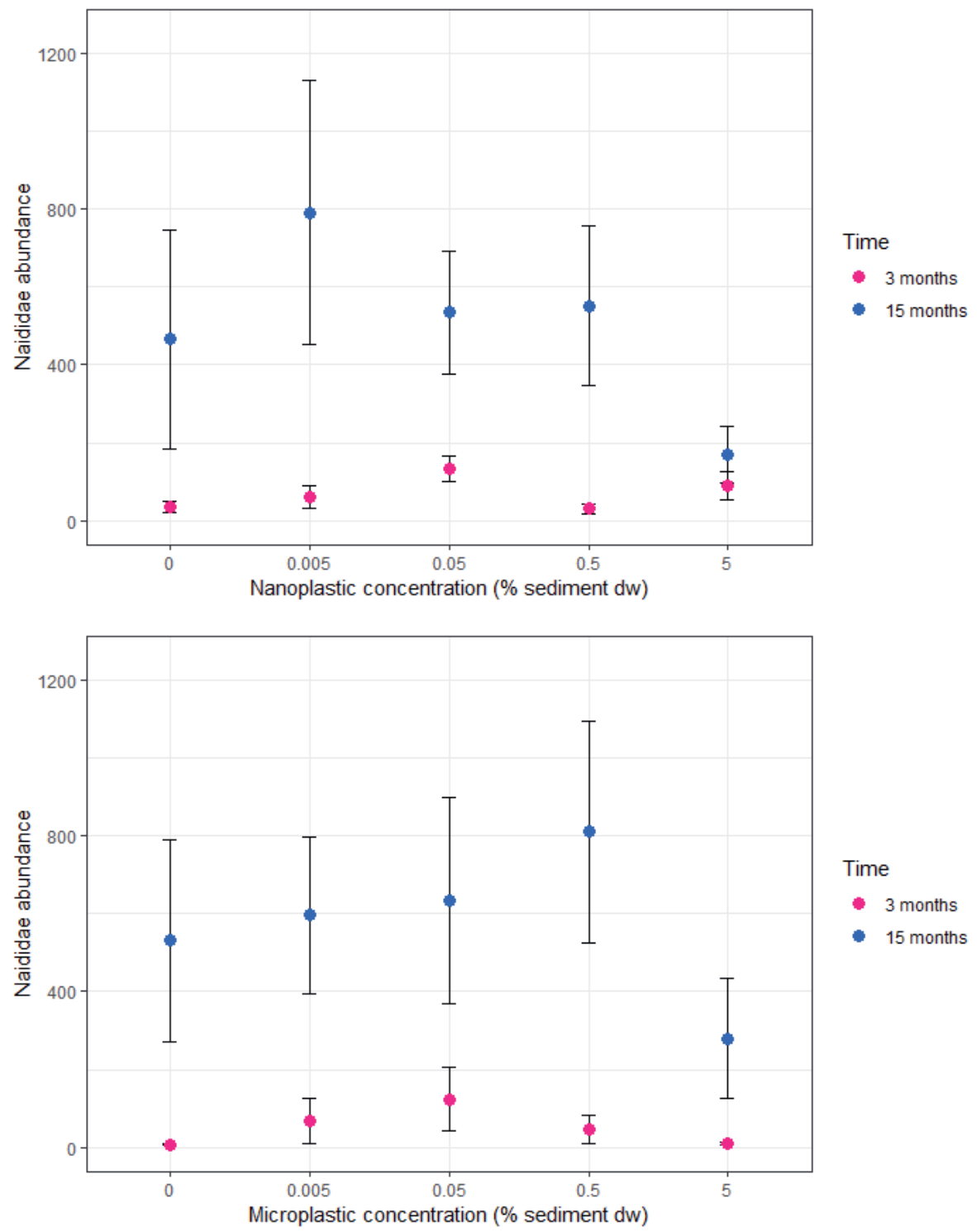

Figure 5.5. Number of individuals from the family Naididae found in trays retrieved after 3 and 15 months with increasing NP (top) and MP (bottom) concentrations (as \% sediment dry weight). Error bars are means $\pm S E, n=4$, except for MP treatments 0.05 and $0.5 \%$ retrieved after 3 months and 0 and $5 \%$ retrieved after 15 months, where $n=3$. 
Besides Naididae, NP concentration had a significant positive effect on the number of Valvata over time (GLM; $\mathrm{NP}_{\text {conc }} P=0.02$ ) (Figure A5.2). Tukey multiple comparisons test showed however no significant differences among NP concentrations per time points. NP had also a significant positive effect on the number of Orthocladiinae (GLM; NP conc $P=0.02$ ) (Figure A5.3). As for Valvata, no significant differences among concentrations were found per time point. MP had a significant positive effect on the number of individuals of Hippeutis complanatus (GLM; $\mathrm{MP}_{\text {conc }} P=0.03$ ) and Gyraulus albus (GLM; $\mathrm{MP}_{\text {conc }} P=0.002$ ) (Figures A5.4, A5.5). Again, no significant differences among $\mathrm{NP}$ and MP concentrations were found per time point. For the 16 other taxa analysed, no effects of NP and MP concentrations were found.

The overall effects of NP on the abundance of macroinvertebrates, the number of taxa, the Shannon Diversity Index $(H)$ and the abundance of Naididae did not differ significantly from those for MP. However, when comparing the means between the two plastic types per concentration-time combination in one hypothesis test, a significant

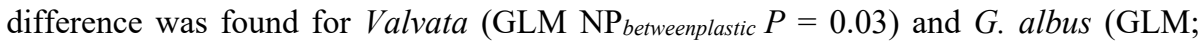
$\mathrm{NP}_{\text {betweenplastic }} P=0.006$ ). For Orthocladiinae and $H$. complanatus the difference in effects between plastic types had $P$ values of 0.08 and 0.05 , respectively.

\section{Discussion}

After 15 months, the total abundance of macroinvertebrates, the number of taxa and the number of Naididae worms were significantly higher than after 3 months for both NP and MP treatments, confirming the colonization of the trays over time as intended. In contrast, the Shannon Diversity Index $(H)$ significantly decreased over time for both NP and MP treatments, probably due to a higher abundance of the family Naididae, which dominated all trays except for the ones with the highest NP and MP concentration (5\%). A higher diversity at the highest NP and MP concentration (5\%) can be observed (Figure 5.3), although effects of NP and MP on the Shannon diversity index $(H)$ were not statistically significant. It is possible that a decrease in the abundance of only one taxon i.e., the Naididae, might not have been sufficient in this period of time to obtain statistically significant effects on the Shannon diversity index $(\mathrm{H})$, given that all other species affect the index as well. It cannot be ruled out that effects on diversity would become significant after a prolonged exposure. The spatial variation (block) had a significant influence on the total abundance of macroinvertebrates, the number of Naididae and the Shannon diversity index $(H)$, revealing that the distribution of the organisms along the ditch was not entirely homogeneous. This, however, is considered part of the targeted ecological realism of the experimental design.

Despite the influences of time and spatial variation (block) on the total abundance of macroinvertebrates and the abundance of Naididae worms, effects of NP and MP particles were detectable. Community effects for other inert particles, such as activated carbon and multi-walled carbon nanotubes, have been previously detected using a similar setup. ${ }^{192,244}$ For instance, a lower abundance of Lumbriculidae worms and Pisidiidae clams was found 
after 15 months of exposure to activated carbon via natural sediment. ${ }^{192}$ To the best of our knowledge, this is the first time that effects of NP and MP are demonstrated in a setting with such a high level of natural ecological variability (i.e. diurnal and seasonal variation, spatial variation) and for an exposure time longer than 3 months. To our knowledge, community effects have only been reported for MP in one earlier study, which exposed a marine benthic community to $80 \mu \mathrm{g} / 1$ of polylactic acid and high density polyethylene MP for 3 months using outdoor mesocosms. ${ }^{89} \mathrm{MP}$ affected the abundance of periwinkles Littorina sp. and isopods Idotea balthica, and the biomass of the clam Scrobicularia plana and the lugworm Arenicola marina. ${ }^{89}$ In the present study, differences were observed over time, especially for the Naididae worms, where the abundance increase allowed distinguishing differences among treatments. The number of Naididae increased by a factor of 13 (from 37 to 466) and 70 (from 8 to 531) in the NP and MP controls, respectively, while it only increased by a factor of 2 (from 90 to 160) and 30 (from 9 to 279) at the highest concentration in a period of 1 year. For the other taxa affected by the exposure to NP and MP, differences between 3 and 15 months were much smaller, and their abundance was always below 40 individuals per tray, which makes the conclusions less evident than for Naididae.

The detected community effects of NP and MP could affect ecosystem functions. For instance, the burrowing activity of worms causes mixing of particles and chemicals in the sediment top layer, facilitates the oxidation of organic matter and reduces minerals in the sediment thereby mobilizing nutrients and sulphide bound heavy metals from the sediment back to the water layer. ${ }^{245,246}$ In addition, worms are an easy and nutritious prey for fish and other benthic invertebrates in the system. ${ }^{245}$ This implies that these functions could be impaired due to the reduction in the abundance of Naididae worms observed here.

It has been hypothesized that for NP different and probably more severe effects may be anticipated than for MP, due to a higher chance of translocation, systemic uptake and subsequent particle toxicity effects. ${ }^{100,247}$ For MP, mainly physical effect modes of action have been suggested. ${ }^{13,45}$ The effects of NP on the abundance of macroinvertebrates, the number of taxa, the Shannon diversity index $(H)$ and the abundance of Naididae did not differ significantly from those for MP. The similarity observed here relates to the effect thresholds and to the identity of the primarily affected species, i.e., worms. We have no conclusive explanation for this similarity; however, plausible explanations can be provided. For instance, upon aging, biofouling, encapsulation and aggregation of the smallest particles in the sediment, ${ }^{248,249}$ they could lose behaviours that specifically relate to the sub-micrometre scale, rendering them more similar to larger MP particles. Formation of hetero-aggregates between the NP and sediment particles could strongly reduce differences in bioavailability, uptake and particle-specific effects, such that only the general effect of loss of habitat quality due to dilution of food remains. Accordingly, the simultaneous presence of natural particles is essential when evaluating the effects of NP and MP on benthic macroinvertebrates (Chapter 2). ${ }^{45,186,250}$ 
As mentioned, this study was designed to detect community level impacts, and therefore we are not able to demonstrate the exact mechanism that caused the lower abundance of Naididae worms. MP ingestion has been previously demonstrated for Tubifex worms, which belong to the family Naididae. ${ }^{134}$ In a study by Hurley et al. (2017), Tubifex worms were able to ingest MP fragments with a size between 50 and $4500 \mu \mathrm{m}$ contained in natural sediment, and were found to retain MP for longer time periods than other sediment components. ${ }^{134}$ A reduction in food intake due to the dilution of organic matter in the sediment, together with the uptake and longer retention of MP by the Naididae worms, could have caused a depletion of energy reserves over time, as previously found in laboratory tests for other benthic invertebrates (Chapter 2). ${ }^{92,133,186}$ For these worms, this energy depletion might have taken longer than for other benthic invertebrates, as the exposure of Tubifex worms to the same PS MP fragments used in the present study using standard chronic laboratory bioassays, did not cause any effects on their survival, growth nor feeding activity (Chapter 2). ${ }^{186}$ Therefore, exposure time seems to be an important factor to take into account when evaluating the ecologically relevant effects of MP. Standard laboratory tests might not be sufficient to detect NP and MP effects for all organisms. When it comes to NP, filter feeders were found to be able to ingest NP particles alone or as aggregates with natural particles. ${ }^{251}$ Aggregates were more likely to be ingested than NP alone, leading to a reduction in species feeding activity.

\section{Implications}

The exposure of a benthic community to NP and MP for 15 months led to a lower total abundance of macroinvertebrates, which was correlated with a lower number of Naididae worms. The number of Naididae found in trays after 3 months was low, probably due to a low colonization of the systems, and did not significantly differ among concentrations. In contrast, after 15 months of exposure, which included the growth season and was five time longer, the number of Naididae significantly increased in all treatments, except for the highest MP concentration (5\%), where the number of Naididae was significantly lower in comparison to lower concentrations. Next to the overall pattern in macroinvertebrate and Naididae abundances, individual differences were also found for $\mathrm{NP}$ and MP. In contrast to Naididae, differences among treatments per time point were not detectable for these taxa, probably due to the low number of individuals found in trays ( $<40$ individuals per tray), which makes the conclusions less evident than for Naididae. While the overall effects of NP on the abundance of macroinvertebrates and Naididae did not differ significantly from those for MP, significant differences between NP and MP were found for the gastropods Valvata and G. albus. In the case of the dipteran Orthocladiinae and the gastropod $H$. complanatus, although only one plastic type had a significant effect on their abundance, the difference in effects between plastic types was not statistically significant, with $P$ values of 0.08 and 0.05 , respectively.

Our present study does not aim for a full-fledged risk assessment; however, it is insightful to provide a provisional comparison between some of the higher concentrations reported for natural sediments, and the long-term effect threshold concentrations found here. Our 
effect threshold concentrations have weight $\%$ as measurement unit, and we thus use environmental data with the same unit. For shoreline sediments of the Rhine river, MP concentrations have been reported to range up to $0.1 \%$ plastic per sediment dry weight, which we found to be the highest reported mass based concentration, to date. ${ }^{130}$ The most abundant particle sizes found in the Rhine River sediments were $<630 \mu \mathrm{m}$, which matches the most abundant sizes within the range of the MP used in the present study (20 $-516 \mu \mathrm{m}$ ), and thus implies that the comparison is not obscured by size differences. The no observed effect concentration and the lowest observed effect concentration detected in our present study for NP and MP were $0.5 \%$ and $5 \%$ plastic/sediment dry weight, respectively. This means that our two environmentally realistic concentrations of 0.005 and $0.05 \%$ did not cause a community effect even after 15 months of exposure. These concentrations are, however, expected to rise and perhaps may already occur at hot spot locations.

When it comes to NP concentrations in freshwater sediments, no data are yet available due to the present limitations in detecting them. ${ }^{32}$ Only one recent study by Ter Halle et al. (2017) was able to demonstrate the presence of NP in a real environment. ${ }^{42}$ Therefore, environmental concentrations of NP still need to be quantified, although they are expected to be at least as abundant as larger plastic particles. ${ }^{252}$. In the present study, the same community effect thresholds are found for NP and MP, which is in accordance with the results obtained by Besseling et al. (2019) after the elaboration of Species Sensitivity Distributions for the exposure to NP and MP via the water phase. ${ }^{40}$ They reported $\mathrm{HC}_{5}$ values for NP and MP to be similar, i.e. 5.4 and $1.67 \mu \mathrm{g} / 1$ with overlapping $95 \%$ confidence intervals. Although relevant because NP and MP concentrations are expected to increase in the near future due to ongoing emissions and fragmentation, ${ }^{13}$ community effect thresholds found in this study were far higher than the highest concentrations reported for freshwater sediments thus far. Nevertheless, given the wide recognition of increasing exposures, ${ }^{97}$ the here detected ecological effects should be taken into account in future risk assessments of NP and MP.

\section{Acknowledgements}

This study was funded by the Dutch Technology Foundation TTW, project no. 13940. We acknowledge additional support from KWR; WMR; NVWA; RIKILT; the Dutch Ministry of Infrastructure and the Environment; the Dutch Ministry of Health, Welfare and Sport; Wageningen Food and Biobased Research; STOWA; RIWA; and water boards Hoogheemraadschap van Delfland, Zuiderzeeland, Rijn en IJssel, Vechtstromen, Scheldestromen, Aa en Maas, de Dommel, and Rivierenland. We thank M.C. Boerwinkel, D. Belgers, I. Roessink, J. Beijer, F. Gillissen, S. Mintenig, M. Kooi, N. H. Mohammed Nor, Noël Diepens, R. van Wijngaarden, W. Dimmers and J. van Smeden for helping during the field and laboratory works. We also thank J. Sprakel, R. Simonsz and F. Alvarado Chacón for providing the NP. 


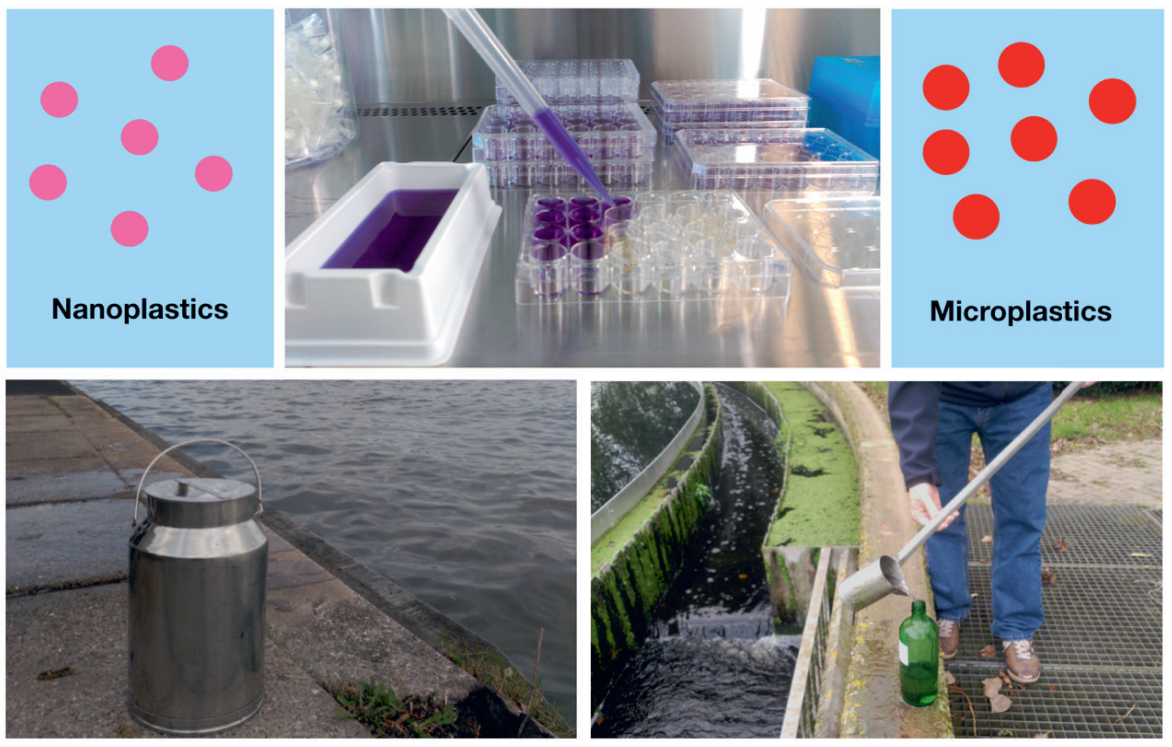




\section{Chapter 6}

\section{Assessing the Toxicity of Nanoplastics using In Vitro Bioassays with Relevance for Human Health}

Submitted as: Redondo-Hasselerharm, P.E.; Baken, K.A.; Pap, L.; Mintenig, S.M.; Bäuerlein, P.S.; van Wezel, A.P.; Koelmans, A.A. Assessing the toxicity of nanoplastics using in vitro bioassays with relevance for human health. Environmental Toxicology and Chemistry. 


\begin{abstract}
Accumulation of nano- and microplastics in aquatic systems is currently a major concern due to their potential threat to aquatic organisms and human health. Although in vitro studies with plastic particles are relatively scarce, a few demonstrated genotoxic effects of nano- and microplastics on biota and human cells, but only at very high concentrations. In the present study, we evaluated the genotoxicity of two nanoplastic sizes (50 and 500 $\mathrm{nm})$ at four concentrations $(0,2.5,25$ and $250 \mu \mathrm{g} / \mathrm{l})$ alone and in combination with chemicals extracted from surface water and wastewater treatment plant (WWTP) effluent using the Ames fluctuation test. No significant genotoxic effects of any nanoplastic size were found in the absence of extracted chemicals. The interaction between medium and high $50 \mathrm{~nm}$ nanoplastic concentrations and the high $500 \mathrm{~nm}$ nanoplastic concentration with surface water chemicals caused significant genotoxicity in the bacterial strain TA98 without metabolic activation. In the bacterial strain TA100 with metabolic activation, significant genotoxicity was found for the high $500 \mathrm{~nm}$ nanoplastic concentration in the presence of surface water chemicals and for the medium $500 \mathrm{~nm}$ nanoplastic concentration in the presence of WWTP effluent chemicals. Although cytotoxicity of the solvent and the WWTP effluent extract may have influenced some test results, overall, our results hint at adverse mixture effects with regards to mutagenicity of nanoplastics with chemicals from surface waters as present in the environment.
\end{abstract}




\section{Introduction}

Accumulation of nano- and microplastics in aquatic systems is currently a major concern due to their potential threat to aquatic organisms and human health. ${ }^{13,125,234}$ Microplastics (MP), with a size smaller than $5 \mathrm{~mm},{ }^{7}$ have been detected in freshwater systems worldwide. ${ }^{13}$ Although wastewater treatment plant (WWTP) effluents have been found to contain significantly higher concentrations compared to other freshwater sources, ${ }^{33,35}$ WWTP concentration data still are scarce. ${ }^{253}$ Nanoplastics (NP), with a size below 1000 $\mathrm{nm}$ in at least one dimension, ${ }^{21,254}$ are an even more difficult target for current detection techniques due to their small size. ${ }^{253}$ To date, no NP occurrence data in freshwater systems have thus been published. ${ }^{12}$ However, due to the ongoing fragmentation of plastic particles $^{212}$ and their direct spillage from different industrial sectors and release from many products, ${ }^{210} \mathrm{NP}$ number concentrations in the environment are expected to be considerable. Besides the chemical additives incorporated during their synthesis, NP and MP will also adsorb hydrophobic organic chemicals (HOCs) and metals present in surrounding media. ${ }^{255,256}$ The transfer of these HOCs from ingested MP into aquatic organisms is expected to be negligible compared to the transfer of HOCs sorbed to ingested natural food or prey items. ${ }^{257,258}$ However, the hazard associated with such transfer may be more relevant for nano-sized polymer particles, which are known to exhibit higher sorption affinities for $\mathrm{HOCs}^{259}$ and may be subject to systemic uptake. Such chemical transfer might be relevant at sites where NP and chemicals accumulate, such as WWTP influents and effluents.

NP can be easily taken up by aquatic organisms via food or water ${ }^{12,46,115}$ and can be transferred into cells, organs and tissues. ${ }^{12,70,77,260-263}$ A large number of in vivo studies has in fact demonstrated that NP can cause adverse effects on the individual and subindividual levels for many aquatic organisms at relatively high concentrations alone, or in combination with adsorbed chemicals. ${ }^{12,264}$ Humans are also exposed to NP via food and drinking water, as well as through inhalation and dermal contact, ${ }^{12,115}$ as recently highlighted by the WHO. ${ }^{265}$ Traditionally, in vitro toxicity testing approaches have played an important role in elucidating mechanisms of chemical toxicity and in providing toxicity data relevant for environmental media. ${ }^{266,267}$ However, there is little information on the applicability of such tests for assessing effects of NP. A few in vitro studies on NP so far have demonstrated that they can induce immune responses, inflammation or oxidative stress. ${ }^{12,120,268,269}$ Only few studies have focused on evaluating the genotoxic effects of NP. ${ }^{270-274}$ No significant genotoxicity was found for the plant Vicia faba exposed to $5 \mu \mathrm{m}$ PS MP, while the exposure of $V$. faba to $100 \mathrm{~nm}$ PS NP had significant dose-dependent genotoxic effects. ${ }^{270}$ When it comes to human genotoxic effects, PS NP were found to cause significant genotoxic effects on the human pulmonary epithelium and fibroblast foreskin cells, lymphocytes and macrophages. ${ }^{271-273}$ However, the NP concentrations at which genotoxic effects were found in these studies were higher than what can be considered environmentally relevant. 
Here, we further explore the potential of in vitro toxicity testing to inform risk assessment of NP, in this case with and without chemical mixtures originating from environmental media such as surface water and wastewater. We evaluated the genotoxicity of $50 \mathrm{~nm}$ and $500 \mathrm{~nm} \mathrm{NP}$ at four concentrations $(0,2.5,25$ and $250 \mu \mathrm{g} / \mathrm{l})$ in three matrices using the Ames fluctuation test, to detect base-pair and frameshift mutations in the genome of two Salmonella typhimurium strains with and without metabolic activation. ${ }^{275}$ The Ames fluctuation test has been previously used for other nanoparticles, such as ultra-fine particles (UFPs), diesel exhaust particles (DEPs) or metal nanoparticles, ${ }^{276-278}$ next to its common use to test environmental mixtures of synthetic chemicals. ${ }^{279,280}$ Matrices consisted of a DMSO solvent control, a surface water extract, and a WWTP effluent extract, the latter extracts providing established proxies for contaminated environmental media in which NP are known to reside. ${ }^{281,282}$ We thereby assessed the genotoxicity of a) the environmental matrices, b) two sizes of NP alone, c) NP in the presence of chemicals from surface water, and d) NP in the presence of chemicals from WWTP effluent. Finally, we provide recommendations to increase the relevance of in vitro tests for the risk assessment of NP.

\section{Material and Methods}

Environmental matrices. Surface water and WWTP extracts were prepared as proxies for chemical contamination in these waters. ${ }^{282,283}$ To this end, $32 \mathrm{~L}$ of surface water was sampled from the Lekkanaal at Nieuwegein (The Netherlands) and placed in a $20 \mathrm{~L}$ clean stainless-steel container and twelve cleaned $1 \mathrm{~L}$ glass bottles. In addition, $4 \mathrm{~L}$ of effluent water were taken from the WWTP in Nieuwegein and placed in four cleaned $1 \mathrm{~L}$ glass bottles. Both surface water and WWTP effluent samples were stored at $4{ }^{\circ} \mathrm{C}$ in the dark for a maximum time of one week before extraction. Following previously published procedures, ${ }^{281,284}$ surface water was extracted by solid phase extraction (SPE) (200 mg OASIS ${ }^{\circ}$ HLB $5 \mathrm{cc}$ LP Glass cartridge, Waters Corporation, Milford, USA) and concentrated 10,000 times in dimethyl sulfoxide (DMSO), which were then distributed into $141 \mathrm{ml}$ conical glass vials with $80 \mu \mathrm{l}$ each. Since undiluted WTTP effluent was expected to be cytotoxic to the Ames test bacteria, an extract of surface water enriched with WWTP effluent (referred to as 'WWTP effluent extract' hereafter) was prepared. To this end, $75 \%$ surface water combined with $25 \%$ WWTP effluent was extracted by SPE and again concentrated 10,000 times in DMSO and distributed into $141 \mathrm{ml}$ conical glass vials with $80 \mu \mathrm{l}$ each. In addition, $141 \mathrm{ml}$ conical glass vials were prepared containing $80 \mu \mathrm{l}$ of DMSO as a solvent control. All $1 \mathrm{ml}$ conical glass vials were stored at $-20{ }^{\circ} \mathrm{C}$ until use.

Nanoplastics. Spherical carboxylated PS NP with nominal sizes of $50 \mathrm{~nm}$ and $500 \mathrm{~nm}$ were purchased from Polysciences Inc, (Warrington, PA, USA). Both stock solutions, with a concentration of $25 \mathrm{mg} / \mathrm{ml}$, were diluted 100, 1,000 or 10,000 times in Milli-Q water. Then, $4 \mu \mathrm{l}$ of the corresponding dilution was added using a microliter syringe with a cemented needle to the $1 \mathrm{ml}$ conical glass vials already containing $80 \mu \mathrm{l}$ of surface water extract, WWTP effluent water extract or DMSO solvent control. For the treatment 
without NP, $4 \mu 1$ of Milli-Q water was added to the extract. Samples were kept overnight at $4{ }^{\circ} \mathrm{C}$, vortexed and incubated at $37^{\circ} \mathrm{C}$ for a few minutes before use. Based on nominal concentrations as provided by the supplier, exposure number concentrations were $3.64 \times 10^{10}, 3.64 \times 10^{11}$ and $3.64 \times 10^{12} 50 \mathrm{~nm} \mathrm{NP}$ particles/ 1 and $3.64 \times 10^{7}, 3.64 \times 10^{8}$ and $3.64 \times 10^{9} 500 \mathrm{~nm} \mathrm{NP}$ particles/1 for weight concentrations of 2.5, 25 and $250 \mu \mathrm{g} / \mathrm{l}$, respectively.

Ames fluctuation test. The Ames fluctuation test was performed as described previously with minor modifications. ${ }^{281}$ S. typhimurium T98 and T100 strains, both with and without metabolic activation by $\mathrm{S} 9$ enzyme mix, were obtained from Xenometrix $\mathrm{GmbH}$ (Allschwil, Switzerland) and used instead of TAmix. Bacteria were exposed to the NP in DMSO, surface water extract, or WWTP effluent extract in a final concentration of $0,2.5$, 25 and $250 \mu \mathrm{g} / \mathrm{l}$. A negative control (NC), consisting of DMSO, and positive controls (PC), being $20 \mathrm{mg} / \mathrm{l}$ 4-nitroquinoline $\mathrm{N}$-oxide and $500 \mu \mathrm{g} / \mathrm{ml}$ 4-nitro-o-phenylenediamine for TA98-S9, 5 and $20 \mu \mathrm{g} / \mathrm{ml}$ 2-aminoanthracene for TA98+S9 and TA100+S9 respectively, and $12.5 \mu \mathrm{g} / \mathrm{ml}$ nitrofurantoin for TA100-S9, were included. Besides genotoxicity, cytotoxicity was tested to identify potential artifacts caused by effects on cell survival and growth. ${ }^{281}$ The exposure duration of the cells to each treatment was 48 hours for the evaluation of the genotoxicity and 3 hours for the evaluation of the cytotoxicity. The Ames fluctuation test was performed twice in independent experiments based on identical test conditions in two consecutive weeks $(\mathrm{n}=2)$. All samples were tested three times in each test condition in each independent experiment. A procedure control for the extraction was not included, and genotoxic effects related to the extraction procedure can thus not be excluded. However, these effects are unlikely, since background genotoxic responses would then be observed in all samples processed, which was not the case.

Data analysis. The triplicate Ames test measurements were averaged. To fulfil the quality criteria, test results were considered valid if the $\mathrm{NC}$ yielded between 0 and 10 yellow wells and the PC yielded in $\geq 25$ yellow wells. ${ }^{285}$ A sample was considered cytotoxic when the Optical Density (OD) at $595 \mathrm{~nm}$ was significantly different (with $95 \%$ confidence) from the corresponding control, following a $t$-distribution. A sample was considered genotoxic when the average number of yellow wells was significantly different (with 95\% confidence) from the corresponding control, following a $\chi^{2}$ distribution, in at least one test condition (TA98 or TA100 with or without metabolic activation) in two independent tests. 


\section{Results and Discussion}

The NC and PC always met the quality criteria (Figures A6.1, A6.2). The sections below will describe the statistically significant genotoxic and cytotoxic effects found for bacterial strains TA98 \pm S9 and TA100 \pm S9 exposed to each treatment. Summaries of the statistically significant genotoxic and cytotoxic effects are shown in Tables $6.1-6.3$. A more detailed description of the results can be found in the Appendix. A summary of the overall results is shown in Table A6.1.

Effects of the environmental matrices. The surface water matrix without NP was significantly genotoxic in TA100+S9 in both independent experiments in comparison to the NC (Table 6.2) (Figure A6.2). However, compared to the DMSO matrix without NP the surface water matrix without NP was significantly genotoxic in TA100+S9 only in the second experiment (Table 6.2) (Figure A6.2). This could be due to the significant cytotoxicity found for the DMSO matrix in TA100+S9, which could have hindered the detection of significant genotoxic effects (Table 6.1). As the genotoxicity of surface water was not proven significantly in both independent experiments for the rest of the strains in comparison to the $\mathrm{NC}$, we cannot unambiguously conclude that the surface water matrix was on itself genotoxic to TA98 \pm S9 and TA100-S9 (Table 2). For the same reason, the DMSO and WWTP effluent matrices cannot be considered significantly genotoxic to any bacterial strain in comparison to the NC (Tables 6.1, 6.3).

Table 6.1. Summary of the statistically significant genotoxic and cytotoxic effects (marked with black stripes) for the DMSO control without NP compared to the NC and for three $50 \mathrm{~nm}$ and $500 \mathrm{~nm} N P$ concentrations in DMSO compared to the DMSO control without NP. The upper and lower half per box represent the first and second experiment, respectively, both calculated as the average of three measurements. Detailed results can be found in the Appendix.

\begin{tabular}{|c|c|c|c|c|c|c|c|c|}
\hline & & & & $50 \mathrm{~nm}$ & & & $500 \mathrm{~nm}$ & \\
\hline Endpoint & Strain & Control & $2.5 \mu \mathrm{g} / \mathrm{L}$ & $25 \mathrm{\mu g} / \mathrm{L}$ & $250 \mu \mathrm{g} / \mathrm{L}$ & $2.5 \mu \mathrm{g} / \mathrm{L}$ & $25 \mu \mathrm{g} / \mathrm{L}$ & $250 \mu \mathrm{g} / \mathrm{L}$ \\
\hline \multirow{4}{*}{ Genotoxicity } & TA98 -S9 & & & & & $\overline{l>}$ & & $\bar{\gamma}$ \\
\hline & TA98 + S9 & & & & & & & \\
\hline & TA100 -S9 & $\bar{\nabla}$ & & & & & & \\
\hline & TA100 +S9 & $\overline{7}$ & . IIIII & 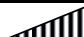 & & . IIII & & \\
\hline \multirow{4}{*}{ Cytotoxicity } & TA98 -S9 & $\pi$ & $\overline{7}$ & & & 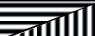 & & \\
\hline & TA98 + S9 & m. & & & & & & \\
\hline & TA100 - S9 & . & & & & & & \\
\hline & TA100 +S9 & हाাा & & & & & & \\
\hline
\end{tabular}



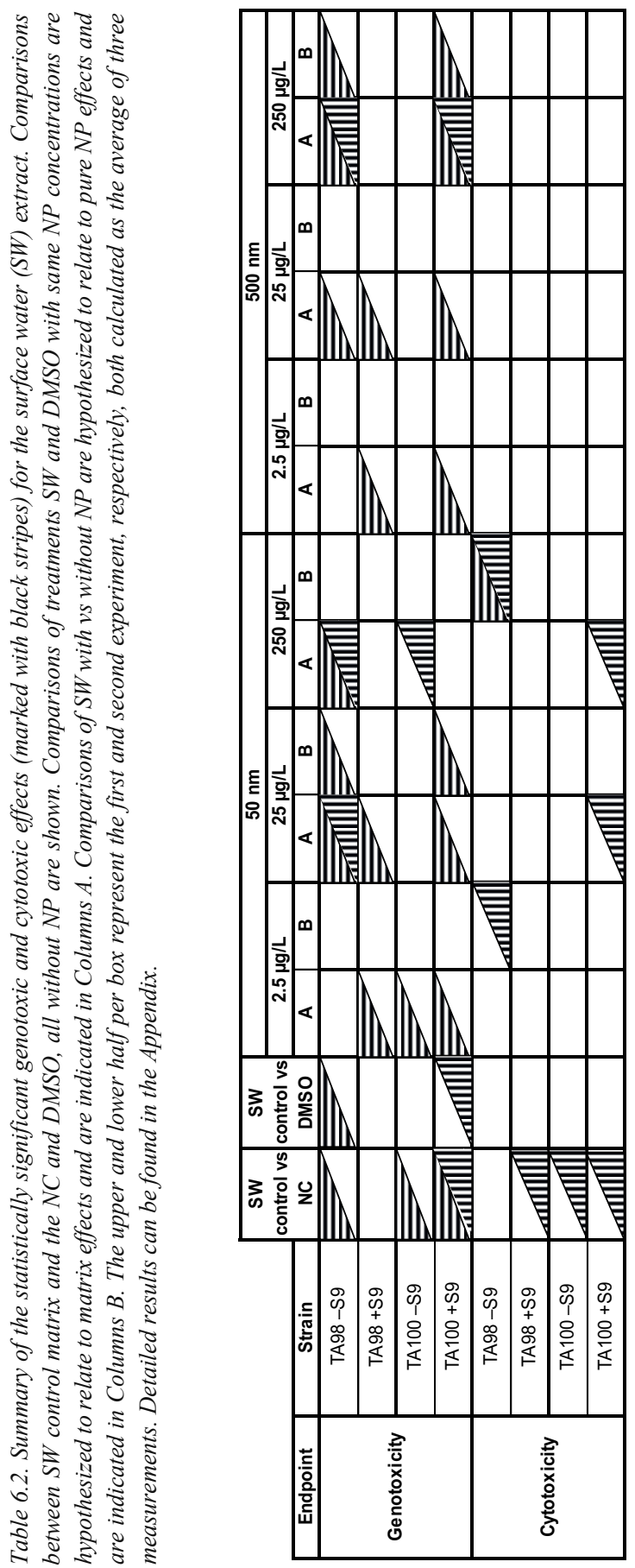

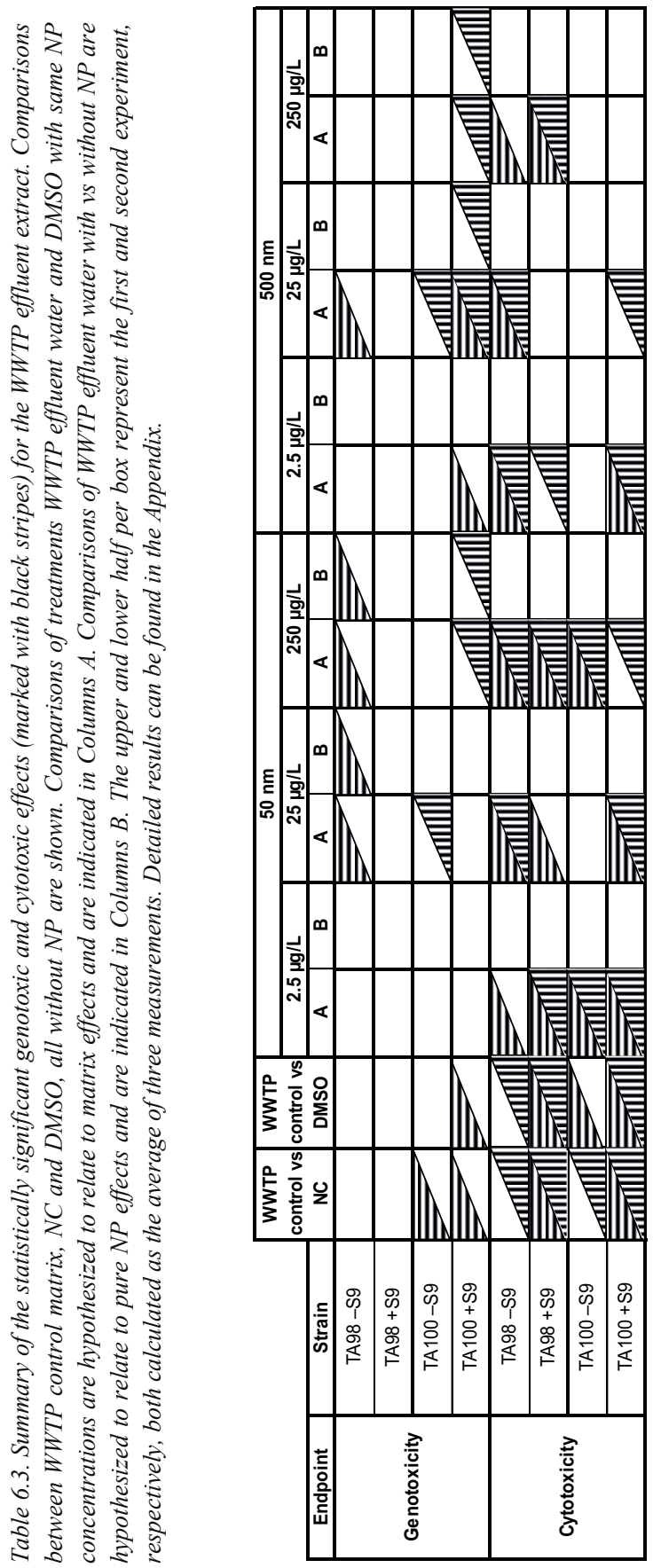
In some cases, the absence of significant genotoxic effects in one of the two independent experiments may be explained by a significant cytotoxicity in the same experiment. This occurred in TA100-S9 exposed to the surface water matrix in comparison to the NC (Table 6.2) and in TA98+S9 and TA100+S9 exposed to the WWTP effluent matrix in comparison to the NC and DMSO (Table 6.3). A detailed chemical characterization of the surface water and WWTP effluent extracts was beyond scope of this study, so we cannot confirm the presence or absence of potentially genotoxic chemicals in these extracts. In previous studies, surface water extracts prepared from water samples taken at the same location did show genotoxicity in TA98 $\pm \mathrm{S} 9,{ }^{282}$ and mutagenicity of WWTP effluent has been reported as well. ${ }^{286}$ The lack of reproducible genotoxic responses of surface water in other strains than TA100+S9 and of WWTP in the present study may be explained by temporal fluctuations in waste and surface water quality and responses around the limit of detection of the test, since $80 \mu$ of extract was used here instead of $100 \mu \mathrm{l}$ used in previous studies.

Effects of nanoplastics. No significant genotoxic effects of $50 \mathrm{~nm}$ NP or $500 \mathrm{~nm} \mathrm{NP}$ in DMSO were found at concentrations of 2.5, 25 and $250 \mu \mathrm{g} / \mathrm{l}$ in any of the strains in both independent experiments when compared to the DMSO matrix without NP or MP (Table 6.1). Cytotoxicity occurred for TA98-S9 at the lowest $500 \mathrm{~nm}$ NP concentration in both experiments and could have caused the absence of genotoxic effects in the second experiment (Table 6.1). A possible explanation for this absence of effects relates to the specific types of genotoxicity, i.e., base pair and frameshift mutations, that are measured in the Ames fluctuation test. PS NP showed other types of genotoxicity (e.g. double strand breaks and micronuclei) in previous experiments, ${ }^{270-273}$ which will not be visible in the Ames fluctuation test. In addition, NP used in the present study are not exactly the same as the ones used in previous studies, and they differ in characteristics such as the average size, the surface properties or the manufacturer. Also, the cell types used in the present tests differ from those studies in previous studies. It could be possible that the specific NP used in this study do not cause base pair or frameshift mutations in Salmonella bacteria. A last explanation could be our aim of using lower NP concentrations than the ones used in previous studies showing genotoxicity in other cell types, ${ }^{270,272,273}$ which may have caused genotoxic responses below the detection limit of the Ames fluctuation test.

Effects of nanoplastics in the presence of surface water chemicals. Significant genotoxicity was found in both independent experiments at the medium and high $50 \mathrm{~nm}$ $\mathrm{NP}$ concentrations in surface water in TA98-S9, as well as at the high $500 \mathrm{~nm} \mathrm{NP}$ concentration in surface water in TA98-S9 and TA100+S9 (Table 6.2). These significant adverse effects are only found when compared to DMSO with the same NP concentration, not when compared to the surface water matrix without NP. Only at the highest $50 \mathrm{~nm}$ NP concentration in TA98-S9, significant cytotoxicity occurred in both independent experiments, which could have masked the genotoxic effect of the highest $50 \mathrm{~nm} \mathrm{NP}$ concentration in surface water in comparison to surface water without NP (Table 6.2). As the surface water matrix without NP was not significantly genotoxic in TA98-S9 in comparison to the DMSO matrix without NP, it seems that the presence of medium and 
high $50 \mathrm{~nm}$ NP and high $500 \mathrm{~nm}$ NP concentrations in the surface water enhanced the genotoxicity of surface water. In the bacterial strain TA100+S9, however, the surface water matrix without NP was already significantly genotoxic in comparison to the DMSO matrix, although cytotoxicity was observed for the DMSO matrix without NP, which may have resulted in lower test responses, and therefore, significance was more easily reached in this sample.

In a previous study by Paget et al. (2015), carboxylated PS NP of $50 \mathrm{~nm}$ did not cause significant genotoxicity in human Calu-3 lung epithelial cells, while significant genotoxicity was found in THP-1 macrophages at concentrations of $1,000 \mu \mathrm{g} / 1$ and $100,000 \mu \mathrm{g} / 1 .^{271}$ This difference in effects between cell types was explained by the much stronger uptake of NP by the macrophages in comparison to the human Calu-3 lung epithelial cells. ${ }^{271}$ Nanoparticle internalization by S. typhimurium TA98 strain cells with and without metabolic activation has been previously demonstrated for metal oxide nanoparticles. ${ }^{278,287} \mathrm{ZnO}$ and $\mathrm{TiO} 2$ nanoparticles were taken up after 60 minutes of exposure in a dose-dependent manner from 8 to $8,000 \mu \mathrm{g} / 1 .{ }^{278}$ The uptake of $500 \mathrm{~nm} \mathrm{NP}$ has never been studied for S. typhimurium, but the internalization of $500 \mathrm{~nm}$ particles has been demonstrated for the murine melanoma cell line B16-F10. ${ }^{262}$ Although in the present study the uptake of NP was not evaluated, we argue that uptake of $50 \mathrm{~nm} \mathrm{NP}$ at concentrations 25 and $250 \mu \mathrm{g} / \mathrm{l}$, which are the medium and high concentrations used in the present study, could have occurred for the S. typhimurium TA98 strain. Uptake of the lower concentration could have also occurred, but the level of genotoxicity might have been too low to be detected in the Ames fluctuation test. These NP may transfer chemicals sorbed from surface water into $S$. typhimurium TA98 strain cells, thereby stimulating a genotoxic effect of the surface water that could not be observed in the absence of NP. The same might have occurred with the highest 500 NP concentration, which might have been taken up by $S$. typhimurium together with the sorbed chemicals from the surface water, increasing the chemical concentration inside the bacteria and causing genotoxic effects.

Effects of nanoplastics in the presence of WWTP effluent chemicals. Significant genotoxicity was found in TA100+S9 exposed to the medium $500 \mathrm{~nm}$ NP concentration in WWTP effluent water in both experiments in comparison to the DMSO with the same NP concentration (Table 6.3). Again, cytotoxicity was observed for the DMSO matrix without NP in TA100+S9, which may have resulted in lower test responses, and therefore, significance was more easily reached. No significant genotoxic effects of NP in WWTP effluent water were found at concentrations of $2.5,25$ and $250 \mu \mathrm{g} / 1$ in TA98 \pm S9 and TA100-S9 in both independent experiments when compared to the DMSO with the same NP concentrations or the WWTP effluent matrix without NP (Table 6.3). The results of the treatments with NP in combination with WWTP effluent were however strongly influenced by the growth inhibition in all strains (Table 6.3). This may have masked potential genotoxic effects, and therefore no conclusion can be drawn with respect to synergistic effects of NP and constituents of WWTP effluent. 


\section{General Discussion and Conclusions}

This first scoping study was designed to explore the potential genotoxicity of $50 \mathrm{~nm}$ and $500 \mathrm{NP}$ at four concentrations, alone and in combination with a chemical mixture as extracted from surface water and WWTP effluent samples using the Ames fluctuation test. We found no significant genotoxic effects of NP alone in any of the strains tested, with or without metabolic activation. In contrast to our results, several studies have demonstrated the genotoxic capacity of NP. ${ }^{270-273}$ These differences might depend on the type of test used, as the Ames fluctuation test is designed to detect base-pair and frameshift mutations, the cell types and the NP studied. Moreover, the concentrations of $\mathrm{NP}$ at which genotoxic responses have been found in previous studies are higher than the concentrations selected in the present study. Therefore, following our results, we can conclude that NP at lower and thus more realistic concentrations are not expected to cause base pair and frameshift mutations.

For the bacterial strain TA98 without metabolic activation, a significant genotoxicity was found at the medium and high $50 \mathrm{~nm} \mathrm{NP}$ concentrations and at the highest $500 \mathrm{~nm} \mathrm{NP}$ concentration in combination with surface water chemicals. NP might have been taken up by $S$. typhimurium together with the chemicals from the surface water, increasing chemical concentrations inside the bacteria and triggering frameshift mutations. We found that this effect disappeared in the presence of metabolic enzymes, which may be either explained by metabolic transformation of surface water chemicals causing frameshift mutations, or the aggregation of NP, including the sorbed chemicals, with the proteins of the S9 mix, causing reduced bioavailability of the chemicals and reduced mutagenicity. We can conclude that at low concentrations of $50 \mathrm{~nm}$ NP in combination with surface water chemicals will not necessarily cause frameshift mutations in mammals. For the bacterial strain TA100 with metabolic activation, significant genotoxicity was found at the medium $500 \mathrm{~nm}$ NP concentration in the presence of WWTP effluent and at the high $500 \mathrm{~nm}$ NP concentration in the presence of surface water extracts in comparison to the DMSO with the same NP concentrations. These effects might have been influenced by the cytotoxicity observed for the DMSO solvent control in this bacterial stain, which may have resulted in lower test responses, and therefore, significance was more easily reached. Therefore, we cannot conclude with certainty that environmentally realistic concentrations of $500 \mathrm{~nm}$ NP in combination with chemicals have a synergistic mutagenic effect in the TA100 test strain as well.

Although in the Ames fluctuation test the evaluation of the cytotoxicity of NP is only meant to explain the absence of genotoxicity, a significant cytotoxicity was found in TA98 without metabolic activation in both independent experiments at the lowest $500 \mathrm{~nm}$ NP concentration. In the presence of surface water chemicals, significant cytotoxicity was found in the same strain TA98 without metabolic activation when exposed to the highest $50 \mathrm{~nm}$ NP concentration as compared to surface water without NP, revealing a potential cytotoxic effect of $50 \mathrm{~nm}$ NP with adsorbed chemicals. In the presence of WWTP effluent 
chemicals, all treatments were strongly influenced by the growth inhibition in all strains, which was due to the use of a matrix which appeared to be cytotoxic in itself.

The sensitivity of the Ames fluctuation test to evaluate nanoparticle mutagenicity has been questioned by Clift et al. (2013). ${ }^{287}$ In the present study, we observed that several genotoxicity results were consistent among the two independent experiments. Nevertheless, in some cases the genotoxic results from both independent experiments differed. This could be explained by a response around the detection limit. Also, the sorption of chemicals to the NP and to the walls of the well plates and aggregation of NP could differ between replicates in a medium without a surfactant, leading to different responses among replicates. Therefore, the assessment of the behaviour of the NP in the tested medium and the use of a higher number of replicates might be necessary in order to perform an accurate evaluation of the risks of NP when using the Ames fluctuation test or other in vitro tests. For future risk assessments, including at least one environmentally realistic concentration in the range of the tested concentrations is recommendable to evaluate current risks.

\section{Acknowledgements}

This study was funded by the Dutch Technology Foundation TTW, project no. 13940. We acknowledge additional support from KWR, WMR, NVWA, RIKILT, the Dutch Ministry of Infrastructure and the Environment, the Dutch Ministry of Health, Welfare and Sport, Wageningen Food \& Biobased Research; STOWA, RIWA, and water boards Hoogheemraadschap van Delfland, Zuiderzeeland, Rijn en IJssel, Vechtstromen, Scheldestromen, Aa en Maas, de Dommel, and Rivierenland. Also, the Joint Research Program of the Dutch water companies (BTO) is acknowledged for additional support. We gratefully acknowledge René van Doorn from KWR Watercycle Research Institute for performing the chemical extraction. 



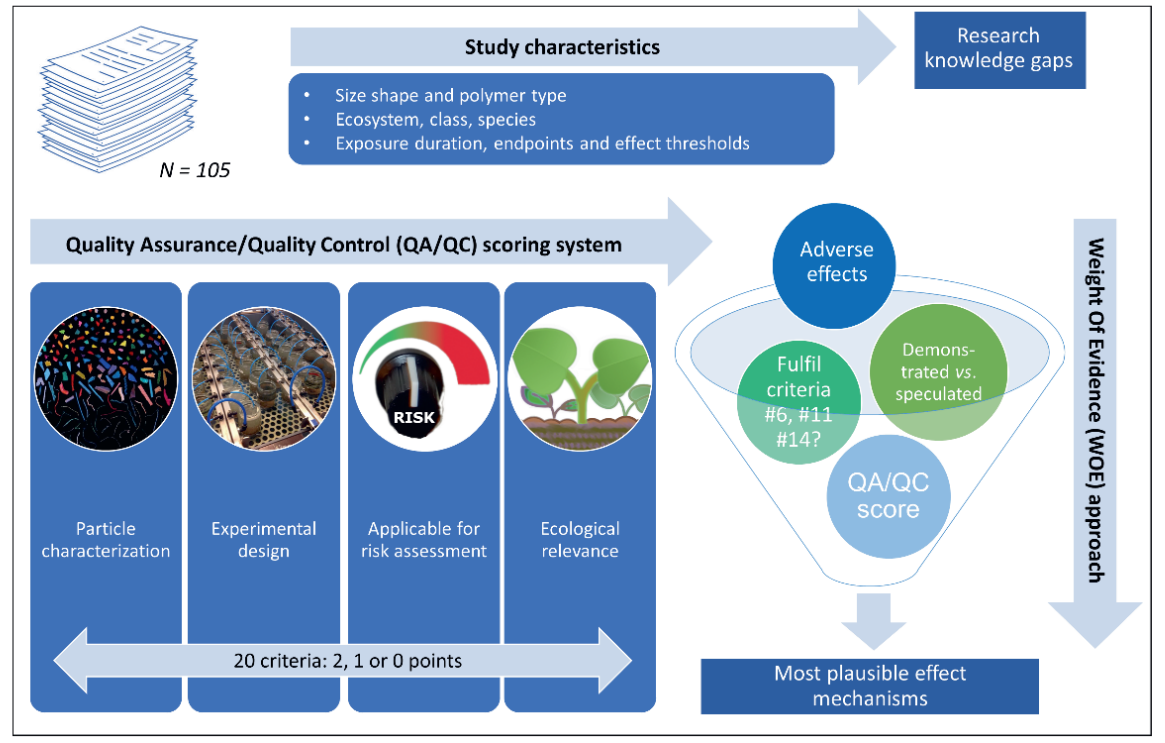




\section{Chapter 7}

\section{Quality Criteria for Microplastic Effect Studies in the Context of Risk Assessment: A Critical Review}

Published as: De Ruijter, V.N. *; Redondo-Hasselerharm, P.E.*; Gouin, T.; Koelmans, A.A. Quality criteria for microplastic effect studies in the context of risk assessment: A critical review. Environmental Science and Technology,

54: 11692-11705 (2020).

* Equal contribution 


\begin{abstract}
In the literature, there is widespread consensus that methods in plastic research need improvement. Current limitations in quality assurance and harmonization prevent progress in our understanding of what the true effects of microplastic (MP) in the environment are. Following the recent development of quality assessment methods for studies reporting concentrations in biota and water samples, we propose a method to assess the quality of MP effect studies. We reviewed $105 \mathrm{MP}$ effect studies with aquatic biota, provided a systematic overview of their characteristics, developed 20 quality criteria in four main criteria categories (particle characterization, experimental design, applicability in risk assessment, and ecological relevance), propose a protocol for future effect studies with particles, and, finally, used all the information to define the weight of evidence with respect to demonstrated effect mechanisms. On average, studies scored $44.6 \%$ (range $20-77.5 \%$ ) of the maximum score. No study scored positively on all criteria, reconfirming the urgent need for better quality assurance. Most urgent recommendations for improvement relate to avoiding and verifying background contamination, and to improving the environmental relevance of exposure conditions. The majority of the studies (86.7\%) evaluated on particle characteristics properly, nonetheless, it should be underlined that by failing to provide characteristics of the particles, an entire experiment can become irreproducible. Studies addressed environmentally realistic polymer types fairly well; however, there was a mismatch between sizes tested and those targeted when analysing MP in environmental samples. In far too many instances, studies suggest and speculate mechanisms that are poorly supported by the design and reporting of data in the study. This represents a problem for decision-makers and needs to be minimized in future research. In their papers, authors frame 10 effects mechanisms as 'suggested', whereas 7 of them are framed as 'demonstrated'. When accounting for the quality of the studies according to our assessment, three of these mechanisms remained. These are inhibition of food assimilation and/or decreased nutritional value of food, internal physical damage and external physical damage. We recommend that risk assessment addresses these mechanisms with higher priority.
\end{abstract}




\section{Introduction}

In the last decade, the body of literature addressing the occurrence and impacts of plastic debris has substantially increased. ${ }^{288}$ Particular attention has been given to microplastic particles (MP), generally defined as plastic particles $1 \mu \mathrm{m}-5 \mathrm{~mm}^{7,21,289-292}$ which have been detected at a wide range of concentrations in various aquatic systems, from remote marine to coastal zone and estuarine areas, as well as in freshwater lakes and rivers. ${ }^{40,96,293,294}$ Their ubiquity in aquatic systems and their small size has resulted in concerns regarding their effects on aquatic biota for which ingestion has been observed at all levels of biological organization. ${ }^{125,295,296}$

Characterizing and quantifying the environmental fate and transport of MP requires insight into the influence of various environmental processes and pathways. ${ }^{27,40,44}$ The release of MP into the environment can occur either directly, such as via primary emissions from products during their manufacture and consumer-use life cycle, or alternatively, can be generated from the degradation and fragmentation of mismanaged plastic waste, commonly referred to as secondary MP, which results in a heterogeneous mixture of particle types, shapes, and sizes released to the environment. ${ }^{297}$ It is generally agreed that secondary sources represent the dominant source of MP. ${ }^{298}$ Primary sources are estimated to contribute between 15 and $31 \%$ of all plastic in the environment. ${ }^{166}$

To assess the ecological risk associated with exposure to MP, there is a need to develop robust toxicological dose-response relationships, which can effectively relate environmentally relevant exposures with effects. ${ }^{45}$ Because of the heterogeneous presence of MP in the environment of varying concentrations of shapes, sizes, and polymer composition, there is a need to better understand effect mechanisms and the key factors triggering them. For instance, effects observed following exposure to MP on an organism can either be initiated due to sorption of the particles on the external surface of the organism or due to other mechanisms of action being triggered following their ingestion. ${ }^{125}$ Effects following exposure to MP, both external and internal, have been assessed in laboratory studies for a wide range of species (Chapter 2). ${ }^{47,50,52,53,186}$ The ingestion and/or adsorption of MPs has been suggested to cause adverse effects on toxicological endpoints at various levels of biological organization, generally observed in laboratory test systems at relatively high exposure concentrations (Chapter 2). ${ }^{47,52,53,186,299}$ Furthermore, experimental work has suggested that effects of MPs can occur at the community level (e.g. biodiversity, species composition) (Chapter 5), ${ }^{89,226}$ population level (e.g., abundance) ${ }^{87}$ individual level (e.g. survival, reproduction, growth, feeding, emergence, embryonic development, mobility, and physiology) (Chapter 2), ${ }^{47,53,186,236}$ or sub-organismal level (e.g., inflammation, reduced lysosomal stability in the digestive gland, reduced antioxidant capacity, DNA damage, neurotoxicity, oxidative damage, gut dysbiosis and alteration of the genetic expression, the ionic exchange and 
enzymatic activity). ${ }^{69,90,96,288,299-302}$ Several studies have speculated that elevated MP concentrations can cause physical damage (i.e., blockage of food passage), leading to a feeling of satiation and a reduced feeding. ${ }^{80,133,303}$ Some studies have attributed the effects to specific properties of the polymer composition, such as the availability of functional surface groups, ${ }^{83,304}$ while other studies have assigned effects of MP to the leaching of chemical additives and plasticizers or other hydrophobic organic pollutants. ${ }^{89,90,305-307} \mathrm{~A}$ limitation identified for studies testing ecotoxicological effects, however, is a lack of consistency and standardization of test methods necessary to characterize dose-response relationships for specific endpoints. Particularly problematic is the need for standard methods in relation to the dosing of particulates, such as MP, an issue that can result in ambiguous results and considerable speculation regarding the proposed mechanisms of action representative of ecologically relevant exposures. ${ }^{45,308}$ Consequently, the weight of the evidence supportive of a quantitative risk assessment for MP remains unclear. Recent reviews have discussed the evidence regarding the occurrence of MP effects and the underlying effect mechanisms. ${ }^{126,309,310}$ However, in their evaluations of the literature, the quality of studies was not taken into account, possibly leading to biased assessments. While these reviews underline that the quality of effect studies should improve and call for more ecologically and environmentally relevant exposure systems in order to better assess the effect of MP on the environment, we argue that the quality of studies should be assessed first, in order to be able to discard unreliable data.

A fundamental element of assessing ecological risk is the availability of a suite of standardized test systems and analytical tools and methods, which enable the application of dose-response relationships relating environmental exposure to effect threshold concentrations that are consistent and of sufficient quality. ${ }^{311-313}$ This also applies to the relatively young field of MP risk assessment, where many studies have emphasized the need to improve the quality of data needed to inform risks assessment(s). ${ }^{31,35,96,140,314-318}$ Efforts to assess the quality of data emerging from studies reporting on exposure concentrations of MPs in biota and in surface and drinking water, adopting methods similar to the existing Klimisch and CRED approaches, ${ }^{311,312}$ have recently been developed and applied. ${ }^{31,35}$ Whereas these systems and aspects of these systems start to be adopted and recommended in the literature, ${ }^{265,319-325}$ currently, a similar evaluation method for assessing the quality of MP effect studies is lacking.

The aim of the present study is to critically review the literature reporting on ecotoxicological effects of MP on aquatic biota, emphasizing quality assurance aspects of studies, and assessing the weight of the evidence (WOE) the studies provide with respect to the effect mechanisms that they report. This is done by first developing a quantitative evaluation method for effect studies and methods employed to assess effects of MP on aquatic biota. The evaluation method is subsequently applied retrospectively to the reviewed studies. Average scores per evaluation criterion are used to prioritize and provide guidance with respect to the analytical and test system protocol that would benefit most from refinement. Based on our analysis, a guidance protocol for testing ecotoxicological effects of MP for aquatic species is provided. Demonstrated and 
suggested effect mechanisms reported in the reviewed papers are summarized and discussed, with the results of the quality evaluation applied as a method to assess the overall weight of evidence regarding probable ecologically relevant effects of MP.

\section{Methods}

Literature search. Literature was retrieved from the database from the systematic review underlying the SAPEA report. ${ }^{288}$ In addition, an extensive literature search accessing the Natural Science Collection database available at ProQuest ${ }^{\circledR}$ was performed for ecotoxicological effect studies with MP until November 2019. The following search strings were used: (effect OR impact OR endpoint OR toxicity) AND (growth OR feeding OR consumption OR survival OR mortality OR behaviour OR behaviour OR stress OR response(s) OR activity OR reproduction OR inhibition) AND (microplastic(s) OR microbead OR polyethylene (PE) OR polystyrene (PS) OR polyamide (PA) OR polypropylene (PP) OR polyvinyl chloride (PVC)) AND (aquatic OR freshwater OR marine OR estuarine) NOT (chemicals OR additives). Studies were only included when at least one type of MP tested had a diameter between $1 \mu \mathrm{m}$ and $5 \mathrm{~mm}$. To enable interpretation of particle effects, studies explicitly aiming to study effects of plasticassociated chemicals, or aiming to solely study accumulation, ingestion and/or egestion of MP were excluded from the analysis.

Assessment of general study characteristics. A total of 10 characteristics were extracted from each paper and summarized (Table A7.1): Size, Shape, Polymer type, Ecosystem (fresh, marine, estuarine), Taxonomy categories (Class, Species), Exposure duration, Endpoints studied, Endpoints affected and Effect threshold when reported (as either LCx, ECx, LOEC or NOEC). When a size range was used, the upper and lower size ranges are noted, however, if an average size was provided together with the range, the average is also recorded. In instances when the average was not given, it is assumed that the particles are uniformly distributed between the upper and lower size limit and that the average can be estimated accordingly. For shapes, the terms "beads" and "spheres" are assumed to be the same and are combined in a single category. As the definition of "irregular" is ambiguous and could include any non-regular shape, it is included as a separate category.

For the analysis of the taxonomic groups we followed De Sá et al. (2018), ${ }^{125}$ where classes polychaeta and clitellata are combined in the category "annelida", classes bivalvia and gasteropoda are combined in the category "mollusca", classes anthozoa and hydrozoa are combined in the category "cnidaria", classes branchiopoda, hexanauplia and monogononta are combined in the category "small crustacea", class malacostraca is renamed "large crustacea" and class actinopterygii is renamed "fish". ${ }^{25}$ Additionally, classes gammaproteobacteria and cyanophyceae are combined in the category "bacteria", classes bacillariophyceae, chlorophyceae, trebouxiophyceae, dinophyceae and mediophyceae are combined in the category "microalgae" and class liliopsida is renamed "macrophyte". 
Quantitative quality assessment. All of the 105 reviewed studies are evaluated based on 20 Quality assurance/Quality control (QA/QC) criteria in the following categories: particle characterization, experimental design, applicability for risk assessment, and ecological relevance. These categories are consistent with the principles of sound ecotoxicology proposed by Harris et al. (2014), which represent fundamental elements for ensuring quality and reproducibility and are thus critical when designing, applying and reporting ecotoxicological effect studies for MP. ${ }^{326}$ A summary of the 20 QA/QC criteria is shown in Table 7.1 and a detailed motivation for each criterion is provided in the Appendix (methods continued). Building on the methods developed by both Hermsen et al. (2018) and Koelmans et al. (2019), each criterion is assigned a score of either 2 (adequate), 1 (adequate with restrictions) or 0 (inadequate) points (Table A7.2). ${ }^{31,35}$ All studies collated as part of this literature review are independently assessed by three of the authors, with scores subsequently tabulated and discussed to reach consensus, sometimes leading to adjustments of the original formulation of a criterion to decrease potential ambiguities. The scores per individual study can be found in the Supporting Information of the publication in Environmental Science and Technology.

Consistent with the approach adopted in previous method evaluation papers, ${ }^{31,35}$ we emphasize that the scores assigned for each study should not be perceived as a judgement indicative of the relative value of a study, i.e. a paper scoring low on a certain criterion could still provide valuable and reliable information regarding other potential insights. Problem formulation is therefore an important element to understand, in that depending on the purpose of an effect study the results may or may not help to inform the decisionmaking process with respect to assessing risk. A WOE may be assembled, for instance, regarding an effect mechanism, but the mechanism may not necessarily be ecologically relevant (see Appendix, criterion 13, Endpoints, p11, methods continued). The primary objective of the evaluation criteria developed and applied in this study is directed at providing insight regarding those aspects of MP ecotoxicological effect studies that could be improved in future studies in order to better inform the application of a quantitative environmental risk assessment. The evaluation criteria, however, also provide the opportunity to assess the current WOE of effect mechanisms.

Analysis of perceived versus demonstrated mechanisms explaining adverse effects. Authors' conclusions with respect to observed adverse effects and the mechanisms explaining them are summarized in the Appendix (Table A7.3). In instances where the discussion and conclusions included ambiguous terms, such as, 'may', 'could', 'can', 'would', 'postulate', 'suggest', 'might', 'potentially', 'most likely', 'imply' the reported mechanisms are classified under the category 'suggested'. If the discussion and/or conclusion used more definitive terminology, such as, 'demonstrate', 'observe', 'indicate', 'induce', 'provide', and 'evidence', the reported mechanisms are classified under the category 'demonstrated'. When a combination of both ambiguous and definitive terminology are used in the same sentence to describe an effect mechanism, the mechanism is considered as 'suggested'. Terms that imply a mechanism to be either 'demonstrated' or 'speculated' are reported in italic, whereas key words indicating the 
mechanism category are reported in bold. Finally, in addition to classifying effect mechanisms as either "suggested' or 'demonstrated', specific categories based on the modes of actions proposed by authors are recorded and numbered accordingly.

Table 7.1. Summary of specific guidance proposed towards the adoption of Standardized Protocol for testing the effects of MP in aquatic test systems for the purposes of strengthening the quality of data generated with respect to quality assurancelquality control (QA/QC) criteria. A detailed motivation for each criterion is provided in the Appendix (see methods continued).

\section{GUIDANCE TO INCREASE THE TECHNICAL QUALITY OF EFFECT TESTS (1 - 12)}

\section{Particle characterization}

1. Particle size

2. Particle shape

3. Polymer type

4. Source of MP

5. Data reporting

\section{Experimental design}

6. Chemical purity

7. Laboratory preparation

8. Verification of background contamination

9. Verification of exposure

10. Homogeneity of exposure
Size is a crucial factor explaining effects of MP and thus should be reported. If a range of sizes is used; a full (i.e., $\geq 10$ bins) size distribution is measured and reported. If a single size is used, that size is measured with an indication of measurement error and reported.

Shape is a crucial factor explaining effects of MP and thus should be measured and reported. Shapes are measured with high resolution picture and reported.

Polymer type can be a factor explaining effects of MP and thus should be reported. Polymer identity confirmed with e.g., FTIR, Raman spectroscopy or similar methods.

Specification on where MP stock or solution is bought and/or how it is selfmade maximizes reproducibility and thus should be reported. The origin and/or production of MP in own laboratory is reported in detail.

Unambiguous units are required to ensure reproducibility of the experiment and to make it possible to compare data across experiments. MP concentrations are reported as mass as well as number concentration.

In order to test particle toxicity, the toxicity of other chemicals in solution or mixture should be ruled out. This includes additives present in MPs, chemicals associated with food particles and surfactants (e.g., Tween). Chemical effects other than from the polymer or solution/mixtures are ruled out. MPs are cleaned with organic solvent.

MP contamination arising from the laboratory (air, water and materials) should be minimized.

- All materials used (equipment, tools, work surfaces and clothing) should be free of MP. All materials used are thoroughly washed with high quality water (e.g., Milli-Q water).

- Measures are taken to prevent MP contamination from air.

- Cotton lab coats were used to avoid microfibre contamination.

MP contamination of the exposure systems in the laboratory should be assessed. Level of contamination evaluated and quantified, e.g., with FTIR, Raman or similar method.

Not only the nominal concentration should be mentioned. The exposure concentration should be measured. Measurement of exposure concentration and evidence that at least $80 \%$ of the nominal concentration throughout the test is maintained.

Verification of homogeneity is crucial for the MP characterization and the assessment of bioavailability.

- Water as medium: Picture or measurement of MP in water that demonstrated well mixed or dispersion in solution.

- Sediment as medium: Description of method used to obtain homogenous exposure. 
Exposure of the organism to MP should be verified by measurement. $\begin{array}{ll}\text { Exposure of the organism to MP is measured quantitatively with e.g., FTIR } \\ \text { assessment } & \text { or Raman. In case MPs are ingested additionally a digestion step is included }\end{array}$ (see criteria 9 and 10 Hermsen et al. 2018) (1) $^{31}$

12. Replication For statistical rigor in detecting effect thresholds (e.g., EC 50 or $\mathrm{EC}_{10}$ ), sufficient replicates should be tested. 3 or more replicates

\section{GUIDANCE TO INCREASE THE APPLICABILITY IN ECOLOGICAL RISK ASSESSMENT (13-20)}

\section{Applicable for Risk assessment}

13. Endpoints

14. Presence of natural (food) particles

15. Reporting of effect thresholds

16. Quality of dose-response relationship

\section{Ecological relevance}

17. Concentration range tested

18. Aging and biofouling

19. Diversity of MP tested

20. Exposure time
Endpoints should be considered that inform ecologically relevant population level risk assessment and clearly reported. Endpoints taken at the community (e.g., bacteria and algae) or individual level (e.g., survival, mortality, growth, development, reproduction).

The exposure conditions should be environmentally relevant. Natural particles (at least food) are added to avoid force feeding of MP. Criterion not applicable to algae or bacteria and hence these studies receive 2 points. To enable PEC/PNEC types of comparisons, the effect threshold should be assessed with error of uncertainty using dose- response relationships. Effect thresholds are reported as $\mathrm{L}(\mathrm{E}) \mathrm{C}_{\mathrm{x}}$ with error or uncertainty intervals. For statistical rigor in detecting effect thresholds (e.g., EC $\mathrm{E}_{50}, \mathrm{EC}_{10}$ ), sufficient doses should be tested, including a treatment control, covering the full shape of the effect curve and emphasizing the slope for parameter estimation. Multiple doses, at least 6 , including a treatment control.

Concentrations should be motivated (with a reference in the appropriate unit) from measured environmental concentrations (MEC). More than 1 environmentally relevant concentration was used within the range tested. Aging and biofouling is what occurs in the environment and could affect the uptake of MP; therefore, it is crucial to consider this for an ecological relevant experiment. MP particles should have undergone process to make them more environmentally realistic, accounting for biofouling. Additionally, pictures of altered particles are provided. In the environment, MPs have a wide variety of shapes and sizes. This needs to be taken into account for environmentally relevant effect assessment. A wide range of sizes (order of magnitude), shapes and densities is used, thereby approaching the diversity of environmental microplastic. It is crucial to use appropriate exposure times to allow for the detection of adverse effects.

- Bacteria and phytoplankton: 1 week or longer

- Zooplankton: 21 days or longer

- Benthic invertebrates: 28 days or longer

- Fish: 3 months or longer

- Macrophytes: 28 days or longer

\section{Results and discussion}

\section{Study characteristics}

Characteristics of the tested microplastics: size, shape, polymer type.

Size. A total of 178 different MP sizes have been tested in the 105 reviewed papers. The cumulative distribution illustrates that about $75 \%$ of studies tested the effects for MP $<$ $100 \mu \mathrm{m}$, or 'small MPs' 35,265 (Figure 7.1), with approximately 30\% of MP having sizes 
$<10 \mu \mathrm{m}$. Of the 178 sizes tested, 58.4\% corresponded to a size range, while $41.6 \%$ consisted of one size only. Moreover, $16.3 \%$ of the tested MP included a size range greater than one order of magnitude.

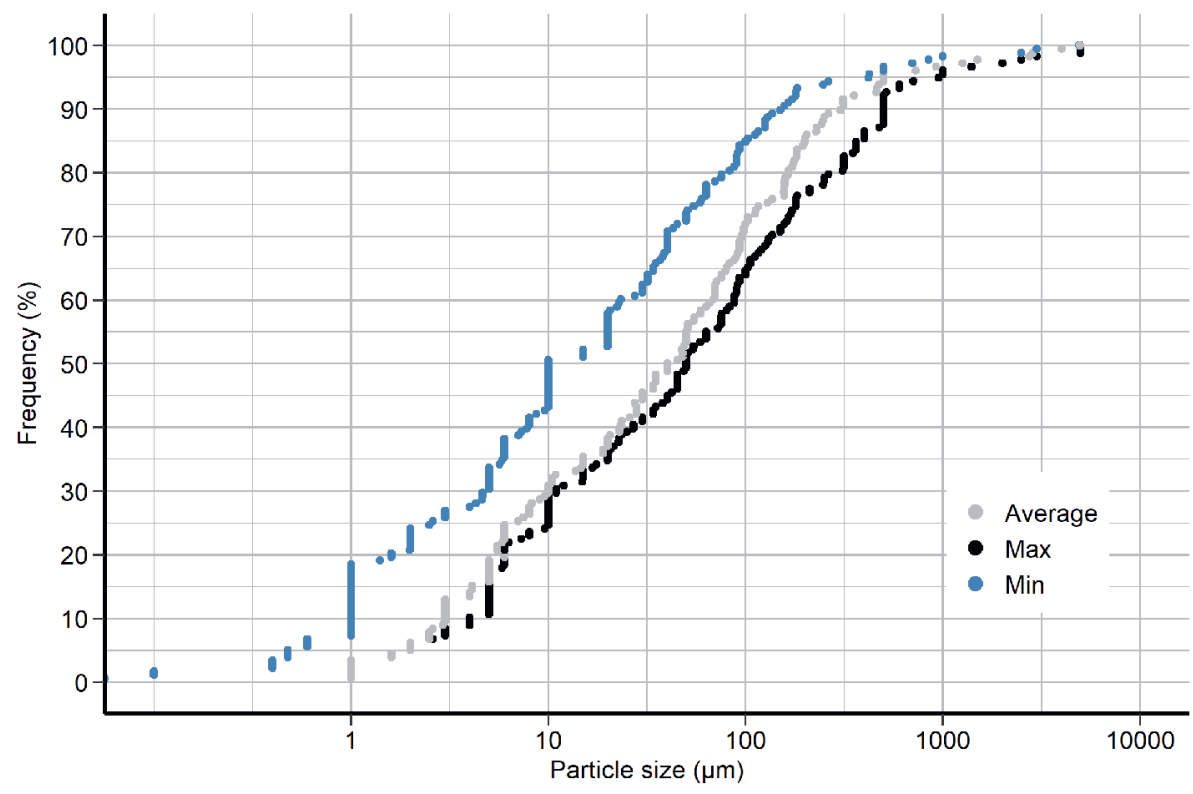

Figure 7.1. Cumulative frequency distributions for MP particle sizes used in effect tests for aquatic biota. The majority of studies tested a size range, which implies that separate cumulative distributions can be plotted for the minimum (Min), the maximum (Max) and the average size tested across studies.

Species-specific traits, such as size selective ingestion of MP have been demonstrated for aquatic organisms (Chapter 3). ${ }^{46,53,230,303}$ Size selectivity can potentially help understanding effect mechanisms that influence the toxicological response of an organism. Mechanistic insight, however, can only be demonstrated when an appropriate range of particles sizes is used. Therefore, when evaluating the effects of MP of only one size, the most detrimental sizes for a specific species may not be included in the analysis, resulting in an underestimation of actual effects across the MP size range. Furthermore, it can be assumed that effects of MP of a certain size will differ in the presence of other sizes of MP, since there can be complex particle-particle interactions that may influence exposure as well as complex organism-particle interactions that can be difficult to account for when limiting testing to one size or narrow size range distributions. The observation that effects testing of MP to date is dominated by particles $<100 \mu \mathrm{m}$ (Figure 7.1) implies that comparisons between MP sizes used in effect studies and sizes of MP found in the environment are difficult to be made, particularly since the detection of MP $<100 \mu \mathrm{m}$ represents an ongoing analytical challenge. ${ }^{35}$ Nevertheless, we recommend the use of MP size distributions that are appropriate for the species being tested, which can potentially add greater insight between adverse effects and organism-particle interactions. 
Shape. The shapes of MP reported in the 105 studies are dominated by spheres/beads, followed by irregular MP, fragments and fibres (Figure 7.2A). We assume that most of the studies reporting the use of irregular MP have tested either fragments, films, foams and sheets, or a combination thereof. Consequently, characterizing MP into distinct categories includes a subjective, qualitative, element that is difficult to enable differentiation, but which could result in greater refinement of shapes divided into more categories that would provide opportunities for better mechanistic understanding.

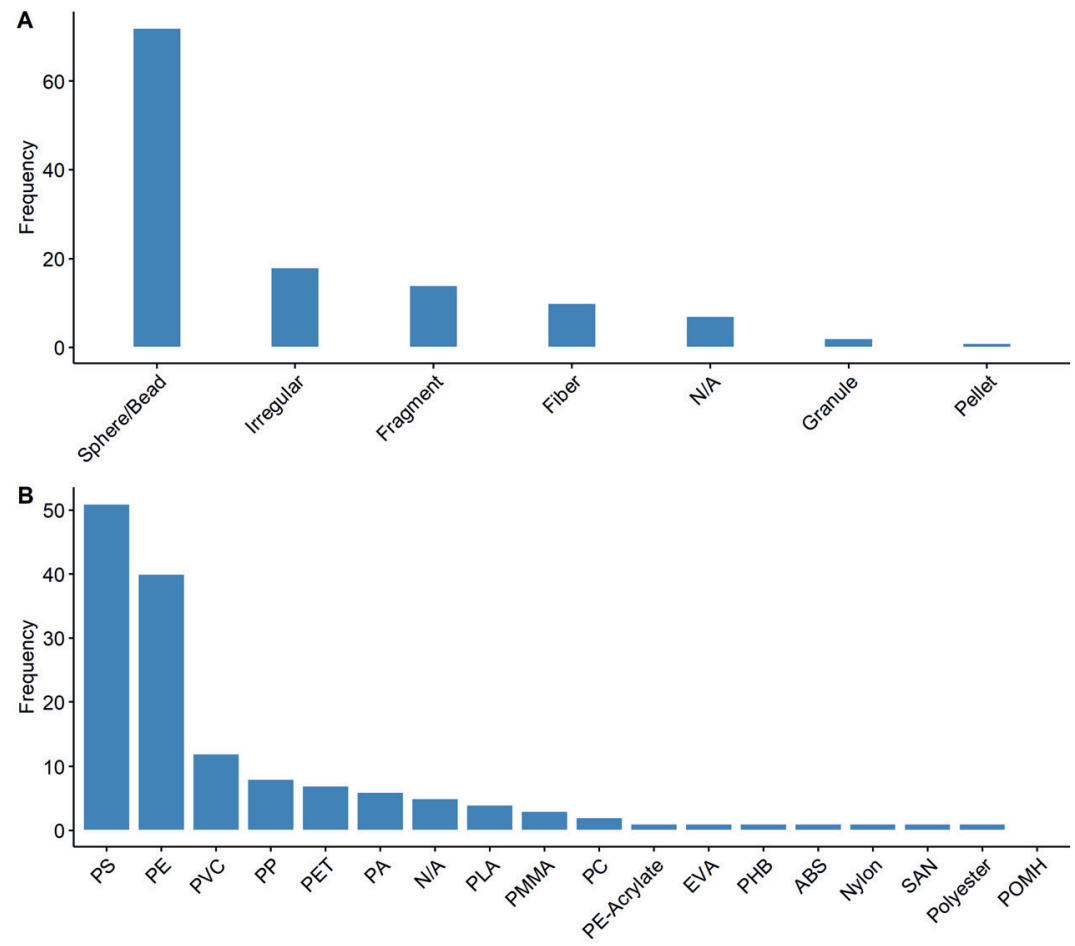

Figure 7.2. Number of studies reporting a particular shape (A) or polymer type (B) for the MP used in the exposure tests (from a total of 124 records for shapes and 145 records for polymer types). $P S=$ polystyrene, $P E=$ polyethylene,$P V C=$ polyvinyl chloride,$P P=$ polypropylene, $P E T=$ terephthalate,$P A=$ polyamide, $N / A=$ not analysed,$P L A=$ polylactic acid,$P M M A=$ poly (methyl methacrylate) $P C=$ polycarbonate, $P E-$ Acrylate $=$ polyethylene-acrylate, $E V A=$ ethylene-vinyl acetate, $P H B=$ Polyhydroxybutyrate, $A B S=$ Acrylonitrile butadiene styrene, $S A N=$ Styrene acrylonitrile resin, $P O M H=$ Polyoxymethylene homopolymer.

When comparing the shapes used in different effect studies with those shapes commonly observed in environmental samples, there is considerable inconsistency. While $58.1 \%$ of effect studies have tested MP spheres/beads, this category only represents $6.5 \%$ of the MP detected in water and sediment samples. ${ }^{297}$ In contrast, only $8.1 \%$ of the tested MP in effects studies were fibres, although they are the most abundant shape category detected in water and sediment, typically representing about half of MP detected. ${ }^{297}$ Therefore, the 
use of fibres in effects studies represents a significant opportunity for advancing quantitative data for the purposes of assessing environmental risks.

An important factor to consider in future studies is how the shape of MP might influence their ingestion and egestion by aquatic organisms, ${ }^{83,327,328}$ which can potentially influence their relative toxicity. Thus, the use of shapes representative of those detected in the environment has the potential to benefit both the ecological relevance and mechanistic understanding of risks associated with MP commonly encountered in the environment.

Polymer type. The most common polymer types used in the 105 effect studies reviewed were PS and PE. Together they represent $62.3 \%$ of the MP types tested (Figure 7.2B). The use of these two polymers is relatively consistent with the polymer types typically observed in the environment, whereby the three most commonly detected polymers in surface waters are PS, PE, and PP. ${ }^{35}$ In effect studies, however, the inclusion of PP is limited to only $5.5 \%$ of MPs tested. Given that the polymer type can influence the fate of MP in both the test system and ecosystem, depending on its density, surface chemistry, degree of crystallinity, and presence of chemical additives and plasticizers, it is important to include as much detail as possible with respect to the polymer composition. ${ }^{44,45}$ Consistent with the need to advance the effects testing and mechanistic understanding of MP with respect to size and shape, as discussed above, there is also a need to strengthen understanding of the influence that the polymer composition may represent towards an observed adverse effect on various species. Insight regarding the relationships between size, shape and polymer composition is important for advancing environmental risk assessment and helping to inform the decision-making process.

Exposed organisms, exposure duration, endpoints studied, and effect thresholds reported.

The organisms tested in the 105 studies evaluated consist of $52.4 \%$ marine, $42.9 \%$ freshwater and $4.8 \%$ estuarine species. The most abundant organisms studied are small crustaceans (which belong to the zooplankton category), followed by molluscs and fish (Figure 7.3A). The most common exposure durations used were: 24 h, 96 h, 240 h (10 days), $336 \mathrm{~h}$ (14 days), $504 \mathrm{~h}$ (21 days) and $672 \mathrm{~h}$ (28 days) (Figure A7.2). The exposure durations generally correspond to the recommended exposure durations of standard ecotoxicity test guidelines for chemicals, implying that exposure durations are also closely linked to standard effect endpoints, such as mortality, growth, and reproduction. However, there is literature indicating that effects of MP can be time dependent (Chapter 5) $226,329,330$ and standard test protocol guidelines applicable for chemicals may not be applicable for the effect testing of MPs. Nevertheless, chronic effects testing of MP adopting longer study durations does not appear to be well represented, with only $18 \%$ of studies using an exposure time $>28$ days, and $<2 \%$ (i.e., 2 papers) with exposure times above three months. Consequently, it is recommended that future effects testing include greater emphasis on assessing longer term effects. 

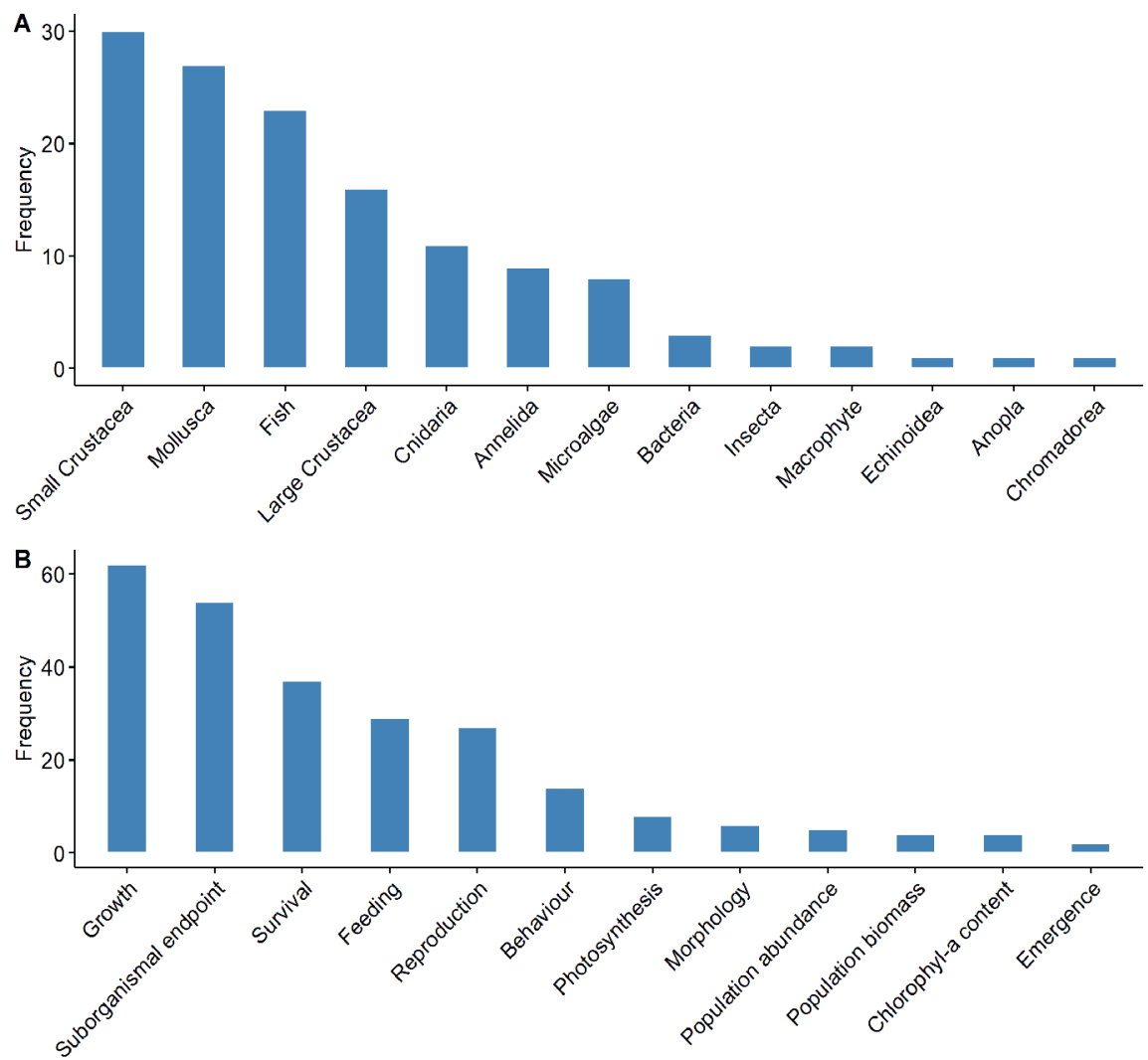

Figure 7.3. Number of studies evaluating the effects of MP on organisms of a certain taxonomic group (A) and on a particular endpoint (B) (from a total of 134 records for organisms and 252 records for endpoints).

Effects of MP on growth are observed to be the most often studied (25.4\%), followed by sub-organismal endpoints $(21.4 \%)$, survival (14.7\%), feeding (11.5\%), and reproduction (9.9\%) (Figure 7.3B). Population-level endpoints correspond to only $<4 \%$ of the total endpoints studied. From the 105 papers, only about $10 \%$ reported effect thresholds (as either $\mathrm{LC}_{\mathrm{x}}, \mathrm{EC}_{\mathrm{x}}, \mathrm{LOEC}$ or NOEC). Of all the studies providing effect thresholds, $33.3 \%$ report them as number concentration (i.e., particles/1), 50\% as weight concentration (i.e., $\mathrm{mg} / \mathrm{l}$ ), and $16.7 \%$ in both units. In order to assess the environmental risks of MP, effect thresholds are fundamental, preferably in both units, which will also further enable comparisons between studies for use in developing quantitative WOE with respect to effects and risks.

\section{Quality assessment}

The results of the scoring based on the quantitative quality assessment proposed in this study imply that substantial improvements can be made in how MP effect studies are designed and conducted (Figures 7.4 and 7.5). As previously stated, the scores obtained 
should not be interpreted as an absolute value of judgment, but as a guide for identifying and prioritizing study-design components that would benefit most in improvement for the purposes of assessing environmental risks. Consequently, we suggest that those studies with relatively high scores represent the most reliable and useful in the context of risk assessment (Figure 7.5). Individual studies, however, often had other objectives, which were not necessarily consistent with information needed to support an assessment of risk. It is important, therefore, to assess each of the specific criteria and to compare them with other studies rather than simply evaluating the studies based on how they rank on their total score. The first subset of criteria (criteria 1 -12) enable the evaluation of the general technical quality of an effect test study. Here, the average score across all studies is 11.3 (range 5 to 18), of a maximum possible score of 24. In this first subset there are no studies for which positive scores on all quality criteria is assigned. The second set of criteria (criteria 13 - 20) relates to the relevance of the papers for their use in environmental risk assessment. For these criteria, the average score across all studies evaluated is 6.6 (range 0 to 14) of a maximum potential score of 16. Again, no studies had positive scores for each of the ecological relevance quality criteria defined. Finally, the total scores combine both the technical quality and ecological relevance evaluation criteria, whereby the total score can be used as part of a quantitative WOE approach in the context of risk assessment. The average total score is 17.8 (range 8 to 31 ), from a maximum possible score of 40, indicating that results from effect studies assessing MP are often not fully reliable and/or reproducible. All studies included in this review were assigned a criterion value of 0 in at least one criterion, implying that important $\mathrm{QA} / \mathrm{QC}$ criteria are consistently poorly addressed in the design and reporting of MP effect studies. With respect to the general technical quality of the effect studies evaluated, $34.8 \%$ of the criteria in studies are assigned a value of 0 , whereas $50.1 \%$ of studies receive the same poor-quality score with respect to their ecological relevance. Average scores per criterion ranged from 0.06 to 1.79 (Figure 7.4). Those criteria that are typically evaluated high across all studies include the reporting of the source of the MP, the use of replicates, reporting on ecologically relevant endpoints and the inclusion of food particles within the test study. A more detailed evaluation of each category is provided below.

Particle characterization. The category with the highest average score is "particle characterization" (Figure 7.4). Overall, the majority of studies evaluated are observed to provide satisfactory reporting on particle characteristics (scores $>1$ ). Only a limited number of studies (13.3\%) fail to report on either one of these specifics. Improvements, however, are suggested, such as related to efforts towards the confirmation of size, shape and polymer type, as opposed to simply relying on information from the manufacturer. Nonetheless, by failing to provide characteristics of the particles, an entire experiment can become irreproducible. Lastly, it should be noted that approximately $60.0 \%$ of studies either don't report a concentration or limit reporting to a mass or number concentration, which further complicates comparison across studies. It is thus suggested that with relatively limited resource towards addressing the shortcomings identified, substantial improvements can be realized within this quality criteria category.. 


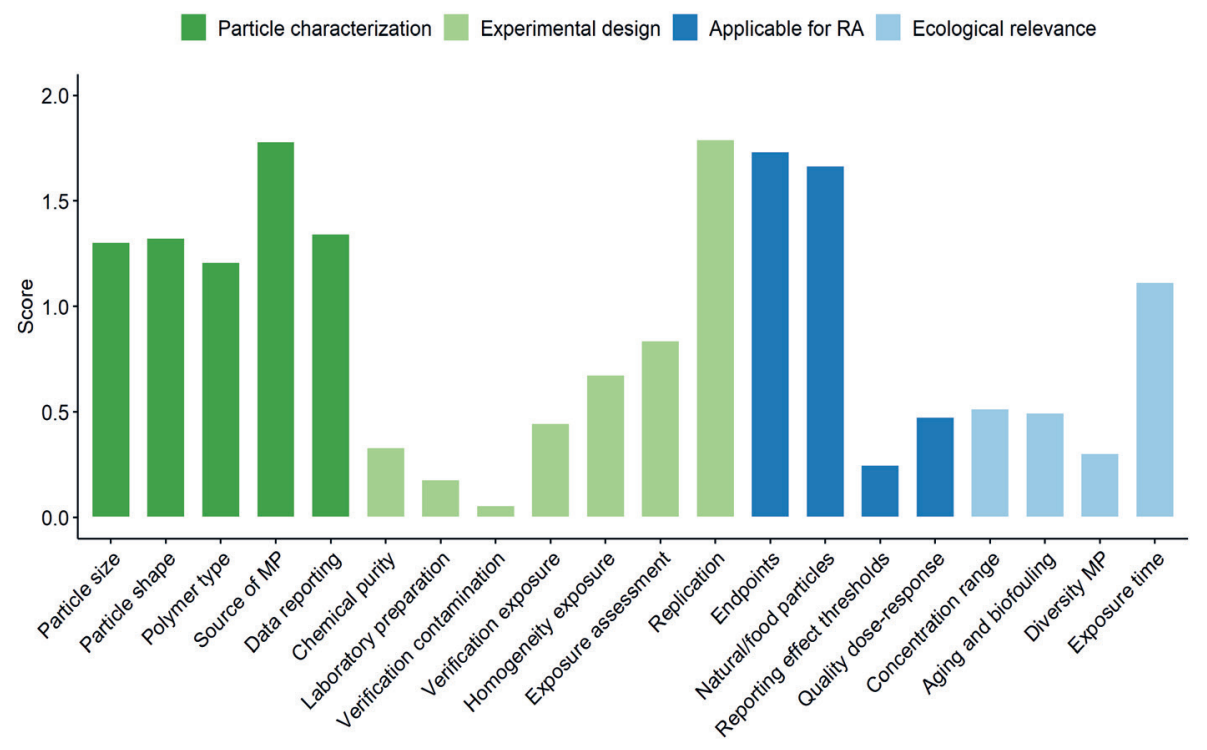

Figure 7.4. QA/QC quantitative system scores from $n=105$ studies. Average scores per criterion with categories "particle characterization", "experimental design", "applicable for RA" and "ecological relevance". Each criterion is assigned a score of either 2 (adequate), 1 (adequate with restrictions) or 0 (inadequate) points.

Experimental design. As a general observation, the majority of studies scored poorly within the category of experimental design (Figure 7.4). Concern is particularly apparent with respect to the quality evaluation criteria of "laboratory preparation" and "verification of background contamination", with average scores of 0.18 and 0.06 , respectively. While MP are often said to be ubiquitous in the environment, including indoor (laboratory) air, ${ }^{288}$ only $3.8 \%$ of the reviewed studies thoroughly report how they minimized potential contamination arising from air, water and all materials used during the experiment. Additionally, only $4.8 \%$ of the reviewed papers verified the background contamination (visually).

Only a few, $6.7 \%$, of the evaluated studies included a protocol specifically used to preclean MP with an organic solvent. Additionally, 20\% of studies took measures to ensure chemical purity. For instance, Karami et al. (2017) and Romano et al. (2018) measured certain chemical contaminants associated with the MP,331,332 however, this still does not exclude chemical effects from experimental results. Some studies include a solvent control, but do not account for chemical contaminants that might be present in the MP themselves. ${ }^{135}$ Importantly, the majority of studies (73.3\%) do not mention the potential for chemical contaminants influencing observed adverse effects, making it difficult to disentangle particle toxicity from a potential chemical toxicity. 


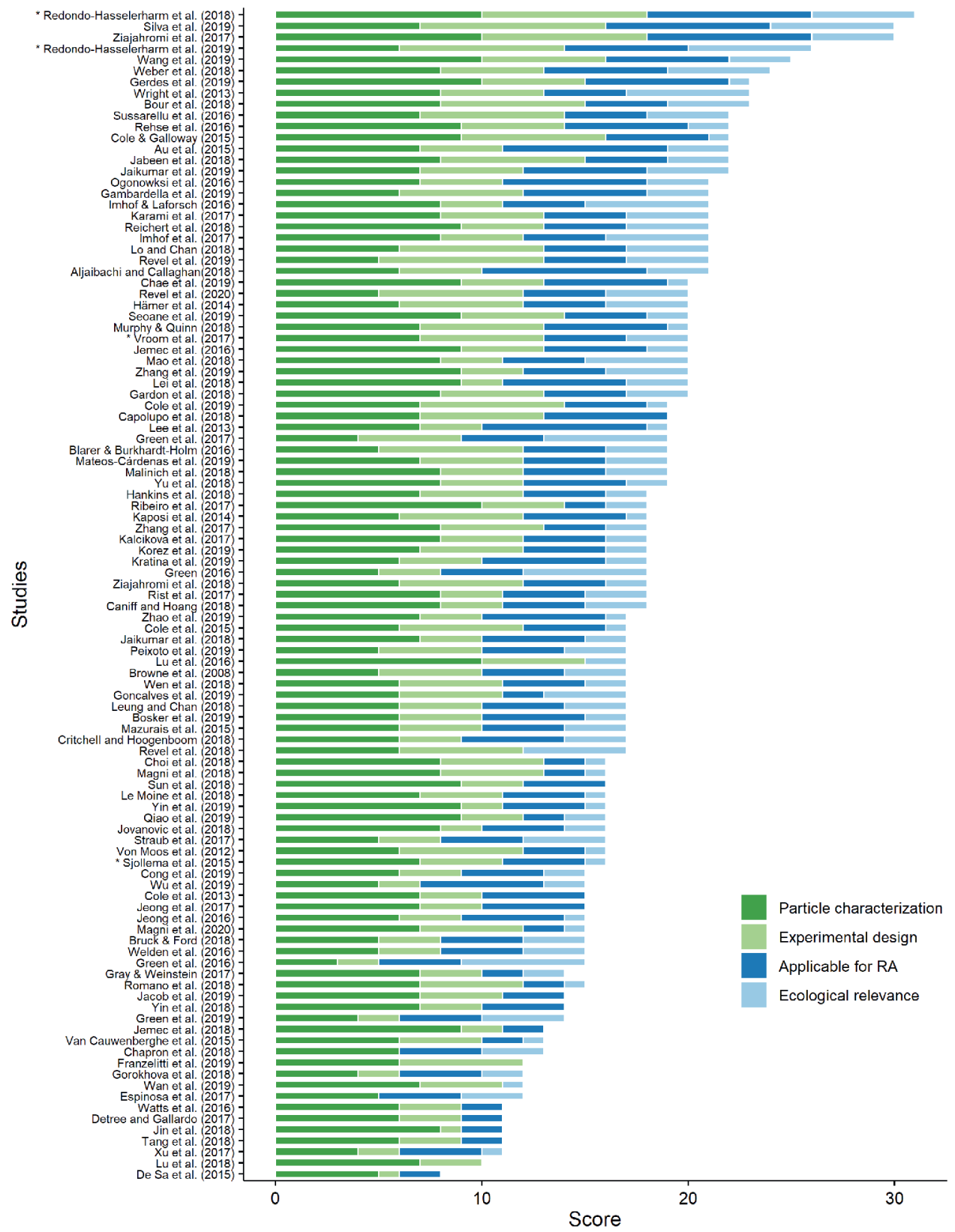

Figure 7.5. QA/QC quantitative system scores from $n=105$ studies. Scores per study with categories "particle characterization", "experimental design", "applicable for RA" and "ecological relevance". *Studies with involvement of 1 or more of the authors of the present paper. See Table A7.3 in the Appendix of Chapter 7 for the detailed scores. 
The criteria "verification of exposure" and "homogeneity of exposure" also are observed to score low, with average scores of 0.45 and $0.68(n=105)$, respectively. These criteria are critical for enabling the reproducibility of study results, which further increase the uncertainty associated with reported effect thresholds. Finally, the criterion "exposure assessment" (average value of 0.84 ) is generally unsatisfactory in the studies evaluated. While most studies (78.1\%) include a description verifying that MP have been ingested by test organisms, verification is often (72.4\%) demonstrated in either a separate experiment, qualitatively, visually or without a digestion step.

While it is acknowledged that the resources needed to address the shortcomings identified with the criteria falling under the category of 'experimental design' are likely to be high, failing to address the various criterion results in studies with greater uncertainties and which thus fail to add value to broader scientific understanding as well as for strengthening opportunities to assess environmental risk. It is therefore prudent to carefully consider experimental design in future effect studies, with the development and application of standard test protocols applicable to MP identified as an urgent need to better guide researchers.

Applicability to risk assessment. An important implication of data reported from ecotoxicity effects studies is their role in assessing environmental risks. Consequently, suggestions for improvement made under this category are perceived to have implications for the regulatory decision-making process. Results from the studies evaluated under the criteria related to applicability for risk assessment imply the need for improvements to "reporting of effect thresholds" and "quality of dose-response relationship", where average scores of 0.25 and 0.48 were assessed, respectively. As mentioned above, a limited number of studies (10.5\%) are observed to explicitly report on effect thresholds with an indication of error. Moreover, only $30.5 \%$ of the 105 studies include a sufficient number of concentration doses to ensure statistical rigor in detecting these effect thresholds. The majority $(86.7 \%)$ of reported endpoints for MP effects, however, are informative to the risk assessment process, with $84.8 \%$ including a source of food to avoid the artefact of force-feeding MP to test organisms.

Ecological relevance. Apart from the criterion "exposure time', which shows an average score of 1.11 and was thus evaluated as satisfactory among the 105 studies, all other criteria in this category score low. The criterion "diversity of MP", with an average of 0.30 , is of particular concern. Only $33 \%$ of the studies included at least one environmentally realistic concentration, raising concerns regarding the relationship between laboratory-based observations of adverse effects and ecological risks. Most studies (71.4\%) assessed the effects of MP using a single MP type or MP with a limited range of characteristics. Only one study used a mixture in their experiment representative of environmental exposure. ${ }^{333}$ Only two studies included the influence of biofouling when assessing the effects of MP, subsequently characterizing the microbiology of the biofilm. ${ }^{333,334}$ 


\section{Weight of evidence for mechanisms explaining adverse effects of microplastic to aquatic biota}

Currently, the knowledge on effect mechanisms for MP is limited and there is a need to increase mechanistic understanding of toxicological modes-of-action. ${ }^{11,335,336}$ Criterion \#11 "exposure assessment of organism" aims at improving the strategic design of effect testing that might enable results to differentiate between intrinsic physicochemical properties of the MP themselves and how those interact with species-specific biological and physiological traits to influence an observed adverse effect (see Appendix, methods continued). Acknowledging that MP represent a complex mixture of particles (shape, size and type), incorporating strategies that enable effect-assessment to move from a 'substance-based' approach to a 'mechanism-based' approach may add considerable value in assessing environmental risk, not just for MP but for any other particle-stressor organisms may encounter. ${ }^{335,336}$ Knowledge on effect mechanisms will enhance the strategic application of species sensitivity distributions for distinct categories of effects. Finally, advancing scientific understanding of particle effect mechanisms, such as those associated with exposure to MP, will aid in the development of effect models. ${ }^{337}$

Given the importance of advancing the scientific weight-of-evidence with respect to the effect mechanisms following exposure to MP, each of the 105 studies is reviewed with respect to the mechanisms that authors used to explain the adverse effects they observed. The analysis is based on four considerations. Firstly, we verified whether authors refer to the mechanisms they described using terms such as 'suggested' versus 'demonstrated' (see Table A7.3 in the Appendix). If authors themselves described a mechanism as 'demonstrated', the WOE is perceived to be stronger. Secondly, the frequency of reporting certain mechanisms was assessed (Table 7.2). The more often a mechanism is reported in the literature, the stronger the perceived WOE can be considered to be, in that consistency between studies in relation to observed effect mechanisms is assumed. Third, the relative strength of the WOE supportive of an effect mechanism is further scrutinized based on the criteria \#6 "chemical purity", \#14 "addition of food" and most importantly \#11 "exposure assessment of organism". While all 20 criteria are crucial in order to ensure quality and reproducibility of data from effect studies, the latter three criteria are specifically important in order to successfully assess the mechanisms behind adverse effects. Fourth and finally, the scores from the QA/QC assessment are used to assess the relative credibility of effect mechanisms reported.

Suggested versus demonstrated mechanisms for adverse effects. From the 105 studies evaluated in this review, 10 separate effect mechanisms are identified as 'suggested', whereas 7 mechanisms are identified to be 'demonstrated', the latter including: 1) inhibited food assimilation and/or decreased nutritional value 2) internal physical damage, 3) external physical damage, 4) oxidative stress, 5) disturbance of essential processes that affect physiology, 6) adjustment of energy metabolism to cope with MP and 7) microbial imbalance (Table 7.2). Three additional mechanisms are reported as speculated only: 8) leaching of additives or chemicals, 9) (cellular) stress and effects of 
10) surface properties. While 100 times studies describe an effect mechanism as "suggested", only 34 times studies describe an effect mechanism as "demonstrated". The most frequently suggested mechanisms are "inhibited food assimilation and/or decreased nutritional value" and "internal physical damage" with a frequency of 32 and 20 suggested occurrences, respectively. However, it is notable that only 9 and 7 studies have reported these mechanisms as demonstrated, respectively.

Table 7.2. Tiered weight of evidence (WOE) approach for effect mechanisms reported in 105 studies, by number of studies that (a) frame a mechanism as 'suggested', (b) frame a mechanism as 'demonstrated', (c) fulfil the three quality assurance criteria (score $>0$ ) considered most relevant to identify effect mechanisms (\#6, \#11, \#14), and (d) average score according to QA/QC of studies that fulfilled those three quality assurance criteria.

\begin{tabular}{|c|c|c|c|c|c|}
\hline \# & $\begin{array}{l}\text { Description of } \\
\text { mechanism } \\
\text { explaining } \\
\text { adverse effect }\end{array}$ & Suggested $^{\mathrm{a}}$ & Demonstrated $^{b}$ & $\begin{array}{c}\text { Number of } \\
\text { studies that } \\
\text { fulfil criteria } \\
\# 6, \# 11 \text { and } \\
\# 14^{c}\end{array}$ & $\begin{array}{c}\text { Average score } \\
\text { of studies that } \\
\text { fulfil criteria } \\
\# 6,11 \text { and } 14 \\
\text { QA/QC }\end{array}$ \\
\hline 1 & $\begin{array}{l}\text { Inhibited food } \\
\text { assimilation and/or } \\
\text { decreased } \\
\text { nutritional value }\end{array}$ & 32 & 9 & 5 & 21.4 \\
\hline 2 & $\begin{array}{l}\text { Internal physical } \\
\text { damage }\end{array}$ & 20 & 7 & 3 & 21.0 \\
\hline 3 & $\begin{array}{l}\text { External physical } \\
\text { damage }\end{array}$ & 8 & 4 & 2 & 24.0 \\
\hline 4 & Oxidative stress & 6 & 8 & 1 & 16.0 \\
\hline 5 & $\begin{array}{l}\text { Disturbance of } \\
\text { essential processes } \\
\text { that affect } \\
\text { physiology }\end{array}$ & 8 & 3 & 0 & - \\
\hline 6 & $\begin{array}{l}\text { Adjustment of } \\
\text { energy metabolism } \\
\text { to cope with MP }\end{array}$ & 1 & 2 & 0 & - \\
\hline 7 & Microbial imbalance & 2 & 1 & 0 & - \\
\hline 8 & $\begin{array}{l}\text { Leaching additives } \\
\text { or chemicals }\end{array}$ & 14 & 0 & - & - \\
\hline 9 & (Cellular) stress & 8 & 0 & - & - \\
\hline \multirow[t]{2}{*}{10} & $\begin{array}{l}\text { Effects of surface } \\
\text { properties }\end{array}$ & 2 & 0 & - & - \\
\hline & Total & 100 & 34 & 11 & \\
\hline
\end{tabular}


1) Inhibited food assimilation and/or decreased nutritional value. Within the studies that report on "inhibited food assimilation and/or decreased nutritional value" as demonstrated, there are 5 studies that meet the crucial criteria "chemical purity", "addition of food" and "exposure assessment of organism" and have therefore reliably concluded on the demonstrated effect explaining the adverse effect, scoring 21.4 points QA/QC on average (Chapter 2). ${ }^{52,186,213,304,306}$ For instance, Blarer and Burkhardt-Holm (2016) visually quantified the presence of PA fibres in the digestive tract of Gammarus fossarum and showed inhibition of food assimilation. ${ }^{304}$

2) Internal physical damage. Of the 7 studies that report on the demonstrated mechanism of "internal physical damage", there are 3 studies that also comply with the aforementioned crucial criteria (\#6, \#11 and \#14) (Chapter 2). ${ }^{75,186,338}$ The studies by Redondo-Hasselerharm et al. (2018) (Chapter 2), Qiao et al. (2019) and Von Moos et al. (2012) are assigned a score of 31, 16 and 16 in the QA/QC assessment, respectively. $75,186,338$ Wang et al. (2019), scored relatively high with 25 points. ${ }^{339}$ Moreover, they were able to verify the exposure of MP to organisms, and also avoided potential systemdependent artefacts by including a protocol for adding food during their experiments. ${ }^{339}$ However, they do not include measures to ensure chemical purity, resulting in some caution when interpreting the mechanism as 'demonstrated'. ${ }^{339}$

3) External physical damage. Although not one of the most often speculated (8 times), the mechanism "external damage," is concluded to be demonstrated in 4 studies. ${ }^{299,340-342}$ Among these, there are 2 studies that fulfilled the crucial criteria (\#6,\#11 and \#14). The one with the highest QA/QC score is Ziajahromi et al. (2017) with 30 points, who observed malformations on the carapace of Ceriodaphnia dubia. ${ }^{345}$ Additionally, with a score of 18, Kalčíková et al. (2017) showed that microbeads with sharp edges affected the root growth and reduced viability of root cells of Lemna minor. ${ }^{340}$ This study qualitatively assessed the adsorption of MP onto root surface and took measures to ensure chemical purity.

4) Oxidative stress. Oxidative stress has frequently been framed as a demonstrated mechanism for the effects observed ( 8 times). There is, however only one study that complied with the three criteria crucial to reliably assess a demonstrated mechanism (i.e. \#6, \#11, \#14). Qiao et al. (2019) observed inflammation and oxidative stress in the gut of Danio rerio. ${ }^{338}$ Besides qualitatively assessing MP in the gut, they also took measures to ensure chemical purity, and fish were fed daily. This study however, scored relatively low on QA/QC (16 points), rendering the results less reliable. Oxidative stress is a molecular mechanism and can be defined as an imbalance in the production of free radicals and the ability of organisms to deal with them. ${ }^{301}$ As oxidative stress is also an endpoint, it is likely that it has often been considered as demonstrated. Moreover, oxidative stress is one of the most commonly measured biomarkers. ${ }^{335,343}$ It is, however, not clear if oxidative stress is a response to another MP toxicity mechanism or that the MP toxicity directly works at the molecular level. ${ }^{302,336}$ Elucidating on this aspect will aid in choosing relevant endpoints to use within risk assessment frameworks. ${ }^{336}$ 
5) Disturbance of essential processes that affect physiology. The mechanism "disturbance of essential processes that affect physiology" is claimed to be demonstrated 3 times. ${ }^{344-}$ ${ }^{346}$ No studies, however, comply with the criteria to credibly ascertain the demonstrated mechanism.

6) Adjustment of energy metabolism to cope with MP. While the mechanism "adjustment of energy metabolism to cope with MP" is suggested once, it is reported as 'demonstrated' two times. ${ }^{49,77}$ Seoane et al. (2019) showed that MP caused a slight decrease in the growth rate of the marine diatom Chaetoceros neogracile, but also a significant decrease in the esterase activity and the lipid reserves of MP-exposed cells. ${ }^{49}$ While scoring relatively well on the overall QA/QC scores (20 points), this study did not take any measures to ensure chemical purity, rendering the result less reliable. Additionally, Watts et al. (2016) showed that crabs were able to overcome minor effects on ion exchange by minor physiological regulation, however did not meet criteria $\# 6$ and $\# 14 .{ }^{77}$

7) Microbial imbalance. Two studies speculate that adverse effects are caused by microbial activity or the presence of bacteria on the MP. ${ }^{89,307}$ Additionally, there is one that has framed this mechanism as demonstrated. ${ }^{72}$ However, no measures were taken to ensure chemical purity or assess MP exposure to the organisms. ${ }^{72}$

8) Leaching of additives or chemicals. In 14 studies, leaching of additives or adsorbed chemicals from MP was speculated to be an explanation for the observed effect of MP; however, this mechanism has never been framed as demonstrated. Demonstrating this mechanism can be achieved by simply washing MP with organic solvent thoroughly and repeatedly, subsequently enabling to distinguish particle from chemical toxicity of MP. Interestingly, Cole et al. (2019) only suggested that leaching of chemicals could have played a role, i.e., not claiming the mechanism to be demonstrated. However, they received maximum score of 2 on this criterion (\#6), meaning that in our view they adequately addressed the issue actually rendering the mechanism to be demonstrated. ${ }^{347}$

9) Cellular stress. As "cellular stress" is a broad term, hard to specify and hence not easily measurable, it is likely that for this reason it has never been framed as a demonstrated mechanism.

10) Effects of surface properties. Only two studies speculate that adverse effects measured in their studies are due to the surface properties of MP. ${ }^{83,304}$ No study, however, claims to have demonstrated an effect of surface properties.

Overall final WOE assessment of mechanisms explaining adverse effects of MP - When comparing the demonstrated mechanisms according to studies it is apparent that "inhibited food assimilation and/or decreased nutritional value" has been demonstrated most often with relatively high overall QA/QC scores (average $=21.4$ ). Most importantly 5 out of 9 studies comply with the crucial criteria to reliably assess a mechanism, making it a plausible mechanism to explain adverse effects with high overall WOE. 
Additionally, the mechanism "internal physical damage" has a relatively high overall WOE. Of the 7 studies that managed to demonstrate this mechanism, 3 fulfilled the crucial criteria (\#6, \#11, \#14) with an average score of 21.0 points. While the mechanism "external physical damage" has been demonstrated less often, effects have been measured with higher reliability than for other demonstrated mechanisms. The 2 out of 4 studies that comply with the crucial criteria to reliably assess a mechanism, score an average of $24 \mathrm{QA} / \mathrm{QC}$ points, thus also making it a plausible and high WOE mechanism explaining adverse effects.

\section{Perspective and outlook}

Research on the effects of MPs on biota in aquatic and other environmental compartments is a relatively new discipline in the environmental sciences. As a result, approaches to assess these effects vary widely across research groups, with both the nature of effects testing and analytical methods developing rapidly over time. Here, we evaluate the quality of 105 studies that report on the ecotoxicological effects of MPs for aquatic biota. The evaluation includes studies of organisms at various functional groups, such as phytoplankton, macrophytes, zooplankton, benthic invertebrates, and fish. The evaluation criteria developed as part of the evaluation can be used as guidance towards best practices to assess exposure, effects and effect threshold concentrations for MPs, and can provide a quantitative quality assessment of studies reporting adverse effects of MPs on aquatic organisms. Lastly, we summarize and discuss the characteristics of the tests that have been performed thus far (e.g., particle size ranges, concentrations, polymer types, particle shapes, species, endpoints, test duration) in order to detect knowledge gaps within effect studies, and use information gained from the review of the literature to assess the WOE with respect to the effect mechanisms most likely influenced by exposure to MPs.

When adopting strict quality criteria, an overall lack of reliability is observed in the studies evaluated in this review, particularly for how data from available effect studies can be used to help inform the risk assessment process. This is partly related to technical shortcomings in the experimental design, such as not ensuring chemical purity, prevention and verification of MP contamination in the laboratory, and partly to limitations in the relevance of studies, for instance when studies do not use ecologically relevant particles or testing conditions. This implies that based on the current state-ofthe-science, the WOE for ecological effects is very limited and the environmental risk of MPs is difficult to assess. The lack of clear evidence for ecological effects in nature due to relatively poor-quality effects studies available for the risk assessment process is worrying, particularly given concerns raised by the public and decision-makers to provide a quantitative assessment of the risks for MPs. The purpose of the present study is therefore to provide timely guidance on best practices needed to improve and standardize effects testing protocols. This includes the need for access to standardized test methods using reference MPs that can be used between research groups in an effort to strengthen both replication and inter- and intra-laboratory reproducibility. We recommend that at least one of these reference materials is an environmentally realistic mixture of particles, 
i.e., having a realistic range of sizes, shapes, densities and ages. This way, organisms themselves select the fraction from the mixture that is bioavailable and relevant for them. This would mimic the situation in nature better than tests with single type materials. The adoption of standardized test methods and use of environmentally relevant reference materials would help reduce uncertainties inherent in the effects data and strengthen both environmental risk assessment and mechanistic understanding of the ecotoxicity of MP.

Based on our review of study characteristics, it appears that particle type 'fibres' and polymer type 'polypropylene' are understudied in effect studies. Ideally, the MP tested should be as realistic as possible, thus representing a broad range of sizes, shapes, densities and polymer types. The ecological relevance of tests should be increased by extending exposure times, as chronic tests are rarely performed. In order for effect tests to be more informative for risk assessment, the reporting of thresholds effect concentrations should be made more accurate and explicit, preferably as either $\mathrm{LC}_{\mathrm{x}}, \mathrm{EC}_{\mathrm{x}}$, LOEC or NOEC values, with the use of both mass and particle unit concentrations.

Based on the evaluation of the WOE pertaining to effect mechanisms associated with exposure to MPs, we observe that the WOE is strongest for the mechanisms related to 'inhibition of food assimilation and/or decreased nutritional value', 'internal physical damage' and for the mechanism 'external physical damage'. To increase the WOE of ecological effects and effect mechanisms we recommend that the guidance provided in this evaluation study be used to develop studies that explore the mechanistic nature of both MPs and generic particle effects on aquatic organisms more broadly.

\section{Acknowledgements}

The authors acknowledge funding by CEFIC-LRI. P.R.H acknowledges funding by the Dutch Technology Foundation NWO-TTW (Project 13940). A.A.K. acknowledges funding by Wageningen University and Research. T.G. acknowledges funding by ECETOC. 




\section{Chapter 8}

Microplastic Effect Assessment in the Context of Risk: A Synthesis 

8.1. Introduction. The release of plastic waste into the environment and its transport and accumulation along all habitats globally have raised concerns regarding the impacts plastic could have on biota and humans. ${ }^{4,7,13,26}$ Plastics, including thermoplastics, elastomers and synthetic fibres, are produced with different chemical compositions, sizes, shapes and densities, depending on their purpose. ${ }^{14,166}$ Once they enter the environment, plastic properties will be modified by physical and biological processes, such as wind, UV radiation or the formation of biofilms. ${ }^{44}$ Plastics are commonly classified by their size as nanoplastics (NP), microplastics (MP) and macroplastics. Following the general consensus established to define engineered nanomaterials, NP are sometimes defined as plastics with a size $<100 \mathrm{~nm} .{ }^{348,349}$ However, other studies defined NP based on the conventional units of size, being NP all plastics with sizes in the nanometre scale (1 $1000 \mathrm{~nm}){ }^{21,254} \mathrm{MP}$ are plastics with sizes $<5 \mathrm{~mm}$, with a lower size limit determined by the chosen NP definition, and macroplastics comprise all plastics with sizes $>5 \mathrm{~mm}$. ${ }^{7,13}$ Since the first scientists alerted about the great amount of plastic detected in the open ocean, a wide number of studies have been published addressing the fate, sources, abundance and effects of nano- and microplastics (NMP) in the marine environment. ${ }^{6,350}$ Nevertheless, in recent years, a few studies highlighted the need to fill the same knowledge gaps for freshwater ecosystems, as they constitute major sources for the entrance of plastic in the marine environment, and also can act as a sink for NMP and thereby affect freshwater organisms. ${ }^{26,129}$ In freshwaters, concentrations of MP have been quantified in water, sediment and biota samples, while NP concentrations are still not known, as sampling and analysing them is extremely difficult. ${ }^{32} \mathrm{NP}$ concentrations in freshwaters are however expected to be high due to the fragmentation of MP over time, as demonstrated in laboratory studies. ${ }^{19,212}$ The fate and abundance of NMP depend on the ecosystem type, the climate conditions and the sources, as well as on plastic properties. ${ }^{27,28}$ MP number concentrations in surface waters and freshwater sediments vary strongly among ecosystems and within ecosystems, ranging up to 5,405 particles/1 for surface water and 74,800 particles/ $\mathrm{kg}$ sediment dry weight (dw). ${ }^{36-38} \mathrm{MP}$ mass concentrations are rarely provided, with the highest concentrations reported being 563 $\mathrm{g} / \mathrm{km}^{2}$ for surface waters and $1 \mathrm{~g} / \mathrm{kg} \mathrm{dw}$ for sediment. ${ }^{37,130,351}$ When including small macroplastics as well as MP, mass concentrations detected in sediments range up to 36.23 $\mathrm{g} / \mathrm{kg}$ in sediment dw. ${ }^{352}$ 
In the environment, biota will likely encounter plastics and interact with them internally or externally, depending on the particle properties and species traits. ${ }^{43}$ It has been demonstrated that the exposure of aquatic organisms to NMP can lead to adverse effects at sub-organismal, individual and population levels once critical effect threshold concentrations are exceeded. ${ }^{69-78}$ The mechanisms behind these effects have been rarely demonstrated, but several studies have attributed them to internal or external physical damages, reduced feeding, and specific NMP properties (i.e., surface groups, chemical leachates). ${ }^{83,86,90-92}$ Because MP are ubiquitous in freshwater environments while knowing that they can cause adverse effects on the surrounding biota, there is an urgent need to assess their environmental risks. ${ }^{11}$ The same applies for NP, even though their concentrations in freshwaters are not known and can only be estimated with models. ${ }^{40}$

To characterize the risks of NMP in the freshwater environment, exposure and effect assessments need to be conducted. ${ }^{11}$ In retrospective exposure assessment, NMP concentrations are measured in the environment. For this, several sampling and detection protocols have been developed to measure MP abundance in water and sediments. ${ }^{35,37}$ Alternatively, for prospective exposure assessments, models can be used. ${ }^{97,353}$ In the effect assessment, the relationship between exposure concentrations and threshold-effect concentrations is studied for specific endpoints using laboratory and outdoor tests. ${ }^{93}$ Data on effects of MP in the literature include a wide variety of polymer types, sizes and shapes, tested under several approaches, hindering the comparability of the data obtained, and resulting in a poor understanding of MP effects. ${ }^{140}$ Therefore, in order to characterize the risks of NMP in freshwater ecosystems, standardized tests need first to be performed to measure effect-threshold concentrations. It has been proposed that a tiered approach may be adequate to assess the effects of NMP, where effect-threshold concentrations are measured at the individual level in lower tiers and at the community level in higher tiers. ${ }^{11}$ These tiers are related with the biological levels of organization that are relevant in environmental risk assessment, which are the individual, population and ecosystem biological levels (Figure 8.1). Individual effect thresholds are measured for the individual and population levels, while community effect thresholds are determined at the ecosystem level. In addition, the accumulation of NMP in the tested organisms should be analysed after the exposure when possible to unravel effect mechanisms, maximum ingestible sizes and potentially transferable concentrations to higher trophic levels. Moreover, a guidance protocol should be developed for MP effect studies, emphasizing on their quality assurance, and applied to the current literature to assess the weight of evidence of the effects and mechanisms reported.

Finally, although this thesis is mainly focused on the environmental risks of NMP, human risks of NP also need to be urgently assessed. Humans are exposed to NMP via drinking water, food, inhalation and dermal contact and the few in vitro studies available have demonstrated the existence of severe effects of NP on human cell lines, such as the induction of immune responses, inflammation or oxidative stress. ${ }^{100}$ As it occurs with the literature on the ecotoxicological effects of NMP, no standard methods exist to assess effects on human health, and studies generally included only one high concentration, from 
which effect thresholds cannot be derived and where the realism was lacking. Consequently, standard in vitro bioassays commonly used for the screening of humanlinked effects caused by chemicals in freshwater samples should be explored as tools to assess threshold-effect concentrations for NP.

\section{ECOLOGICAL RELEVANCE}

\begin{tabular}{|c|c|c|c|}
\hline \multirow[t]{4}{*}{ Subindividual } & Individual & Population & Ecosystem \\
\hline & Mortality & Fecundity & Community composition \\
\hline & Growth & Offspring viability & Population sizes \\
\hline & Feeding activity & Larval development & Species diversity \\
\hline
\end{tabular}

Figure 8.1. Levels of biological organization for which threshold-effect concentrations can be measured. Those biological levels with relevance in an environmental risk assessment are highlighted in colours. Examples of endpoints that can be assessed at these levels are given (adapted from SAPEA et al. (2019)). ${ }^{13}$

The overall objective of this thesis was to assess the effects of NMP in freshwater ecosystems within a risk assessment context. To this aim, we formulated the following research questions: 1) To what extent do standardized low-tier single species tests allow for the detection of effect thresholds for NP and MP at the individual level? 2) How to measure NP and MP uptake? 3) To what extent do high-tier outdoor tests allow for the detection of effect thresholds of NP and MP at the community level? 4) To what extent can in vitro tests with relevance for human health be used to detect effects of NP? 5) What could be improved in effect tests when it comes to quality assurance? 6) Which effect mechanisms can be considered as demonstrated when strict quality criteria are applied to the literature on MP studies reported to date? 7) Can we provide an estimate of the ecological risks of MP using the data generated in this thesis and literature data?

In this final chapter (Chapter 8), based on our achievements I will answer to these aims and research questions as originally outlined in Chapter 1. This is done by providing guidance and recommendations for the use of risk assessment tools to evaluate the effects of NP and MP, including: standardized single species tests to detect individual effect thresholds (section 8.2), outdoor experiments to detect community effect thresholds (section 8.3), and tests in vitro with relevance for human health (section 8.4). I will discuss the advantages of the quantitative scoring system developed to assess the quality of MP studies and will reflect on the demonstrated mechanisms explaining the effects assessed (section 8.5). Lastly, we will perform a tiered effect assessment of MP for freshwaters using the data generated in this thesis and data taken from the literature (section 8.6). The risk of MP will be characterized for freshwater sediments by comparing Measured Environmental Concentrations (MECs) obtained from literature with the calculated Predicted No Effect Concentrations (PNECs) in each of the tiers. 
8.2. Guidance for single species standardized testing with NMP. For the characterization of the environmental risks of NMP, their effect thresholds need to be assessed on biota at different ecological levels. ${ }^{11}$ Individual effects thresholds are determined in the laboratory using single species tests with organisms of various taxonomic groups with relevance for a specific ecosystem. ${ }^{93}$ To answer the first two research questions listed in Chapter 1 To what extent do standardized low-tier single species tests allow for the detection of effect thresholds for NP and MP at the individual level?; and, How to measure NMP uptake?, we designed and conducted single species tests with freshwater benthic macroinvertebrates exposed to NMP via sediment to measure their individual threshold-effect concentrations. Benthic macroinvertebrates were exposed to: (a) polystyrene (PS) MP fragments with a size range between 20 and $500 \mu \mathrm{m}$ at 8 concentrations from 0 to $40 \%$ in sediment dw (Chapter 2); (b) MP made by scraping and grinding second-hand tires with a final size distribution of 10 to $585 \mu \mathrm{m}$ at 6 concentrations between 0 to $30 \%$ in sediment $\mathrm{dw}$ (Chapter 3); and (c) raspberry-shaped PS NP with a palladium-core and an average size of $227.6 \pm 1.47 \mathrm{~nm}$ at 6 concentrations ranging from 0 to $3 \%$ in sediment $\mathrm{dw}$ (Chapter 4). The tested benthic macroinvertebrates included the amphipod Gammarus pulex, who was exposed to all NMP; the isopod Asellus aquaticus, the worm Lumbriculus variegatus and the worm Tubifex spp., who were exposed to both MP types; and the amphipod Hyalella azteca and the bivalve Sphaerium corneum, who were exposed to the PS MP only. As no guidelines for the testing of NMP were available at the start of this thesis, several considerations were taken during the assessment of the effect thresholds to increase the reliability of the results and facilitate their use in risk assessment. These are described below:

Experimental design and standardization. To adequately assess concentration-response relationships of any stressor, the use of a sufficient number of doses is recommended (Chapter 7). ${ }^{223}$ However, most effect studies from the literature include less than five plastic doses in their experimental design, leading to a lack of statistical rigor in the effect thresholds reported (Chapter 7). ${ }^{223}$ This is particularly important when no effects are found and No Observed Effect Concentrations (NOEC) are derived, as the use of a few concentrations and/or only low concentrations may not reflect well the real sensitivity of the species to the stressor in a risk assessment. In addition, the number of replicates in the experimental design should suffice to increase the statistical rigor, but also to compensate for the uncertainties derived from potential inhomogeneous particle exposures along the water column or sediments. The probability of the exposed organisms to encounter NMP in the system, potentially leading to the appearance of adverse effects, will depend on the properties of the plastic (density, size, shape), on exposure conditions (e.g., use of aeration, shaking, biofilm formation) and the species traits (e.g., feeding behaviour, bioturbation). ${ }^{45}$ One major current problem faced by risk assessors is the difficulty of comparing the results across studies testing NMP. The available effect thresholds reported in the literature belong to plastic particles of different sizes, shapes and polymer types, which have been tested under diverse conditions. To allow for the comparability of the results among species, standardized tests should be performed. In this thesis, we always used $350 \mathrm{ml}$ glass beakers as bioassays, Dutch Standard Water, sediment sampled at the 
same location, equal test conditions (water temperature, aeration systems light:dark cycle) and the same bioassay acclimatization (14 days) and exposure durations (28 days). Future studies should ideally aim at testing a battery of organisms under the same conditions to detect the most sensitive species and therefore evaluate the risks of the tested NMP. However, only very few studies to date have tested more than two species with the same plastic material under the same test conditions (Chapter 7). ${ }^{181,184,223,354-356}$

Particle characterization and composition. Plastic particle properties have been found to influence their chances of being ingested and the appearance of adverse effects after their exposure. ${ }^{45}$ Therefore, a proper characterization of the size, shape and polymer type of the tested plastic material is key to unravel the effects of NMP. In this thesis, we measured the Particle Size Distribution (PSD) of the MP and NP. To characterize the shape, pictures of the NP and MP were taken with a scanning electron microscope (SEM) and a stereomicroscope, respectively. Polymer identity was confirmed with Thermogravimetric analysis (TGA) for NP and the MP released from car tires and with $\mu$-Fourier Transform Infrared spectrometry (FTIR) for the PS MP. In addition to the characterization of the tested material, it is essential to guarantee the use of chemically clean plastic when the aim is to assess the particle effects. Chemical additives such as flame retardants, plasticizers or fluorescent markers, are often added during the synthesis of plastics to provide them with specific functions. ${ }^{357}$ Chemicals can also be present in the solution where particles are contained to keep them in suspension, such as surfactants, or due to an incomplete synthesis of the polymers during the polymerization, such as styrene monomers. When using plastic particles in powder form, which are big enough to not homo-aggregate in a solution, cleaning the particles with a solvent to remove the potential presence of chemicals is a good solution to disentangle particle from chemical effects. In Chapter 2, MP were washed with methanol three times, shaken, filtered with a $20 \mu \mathrm{m}$ metal sieve, rinsed with Milli-Q water and dried at room temperature. Removing chemicals for NP or MP in solution can for instance be reached using dialysis purification steps, ultrafiltration or by purging the solution with clean air. ${ }^{127,219,220,225,239}$ When removing all chemicals from the NMP is not feasible, leachates or added chemical controls are sometimes used to discern among particle and chemical effects or the unlikeliness of chemical effects can be assessed by calculation. In Chapter 4, the NP solution was purged with clean air and then aerated during the acclimatization to promote the release of the volatile chemicals. The MP made from tires used in Chapter $\mathbf{3}$ were not cleaned and the original material was tested, as our aim was to assess the combination of the particle and chemical effects and compare them with previous studies testing chemical leachates only, extracted under extreme conditions. In this case, all constituents were quantified using TGA and a gas chromatograph coupled to a mass spectrometer (GC-MS).

Environmental realism. The need of using environmentally realistic concentrations in the tests with NMP has been emphasized by several scientists, which is indeed very valuable to understand the effects at current concentrations. ${ }^{140,141}$ Including higher concentrations is important as well, as some organisms are able to tolerate MP weight concentrations up 
to $70 \%$ in sediment, and because environmental concentrations are expected to increase in the future. ${ }^{11,333}$ In the case of NP, realistic concentrations are still unknown due to the size limits of the available polymer detection techniques. However, Kooi and Koelmans (2019) demonstrated that MP size distributions are fairly constant and showed how this can be used to extrapolate across size classes. ${ }^{297}$ We can therefore expect that environmental mass and number concentrations of NP will be at least equal to those reported for MP. Besides the environmentally realism of the concentrations used, the NMP used in the tests do not often reflect well the plastic particle mixtures that are found in nature. Most effect studies use a small sub-set of all possible plastic particle characteristics (i.e. one size), while environmental plastic particles consist of mixtures of different sizes, shapes and polymer types (Chapter 7). ${ }^{223}$ Using a limited size, shape or polymer type may provide valuable information on how specific properties can influence uptake and effects, and the selection of these properties will in the end be linked with the purpose of the study. However, using environmentally realistic MP with a diversity of particles that approximates distributions for the physicochemical properties of MP, will enable better environmental realism in MP effects testing. Moreover, the properties of the tested plastic materials in laboratory studies seem to strongly mismatch with the plastics found in the environment (Chapter 7). ${ }^{12,126,223}$ For instance, while $58.1 \%$ of the effect studies with MP used spherical particles in their tests, spheres only represent a $6.5 \%$ of the MP samples in water and sediments (Chapter 7). ${ }^{223}$ In Chapter 2, we used PS, whose density matches that of the average environmental plastic, ${ }^{186}$ ground to a wide range of environmentally relevant sizes and shapes, as one of our aims was to study the physical effects of the MP on the affected species (ingestion, excretion and bioaccumulation) and to assess the maximum ingestible sizes and trophic transfer factors (TTFs) for a specific polymer type. In Chapter 3, we made MP from five second-hand tires of various brands ground to a wide range of sizes and shapes. Future effect studies should aim at using diverse and environmentally realistic MP mixtures, as done by Binelli et al. (2020), who tested MP collected from lakes. ${ }^{358}$ The use of more environmentally realistic NP in effects studies is more challenging, as the commercially available NP are typically polymerized with one size and a spherical shape. ${ }^{12}$ Obtaining a range of sizes and shapes requires the self-polymerization of the NP or their formation via the degradation of bigger plastic particles. To our knowledge, only one study has evaluated the effects of NP produced from the abiotic degradation of MP. ${ }^{219}$ In Chapter 4, we used raspberry-shaped NP which were designed with the purpose to increase the environmentally realism of the particles. ${ }^{227}$ Finally, in the environment, NMP will undergo abiotic and biotic processes that modify their physical properties and affect their uptake and effects. ${ }^{359}$ Therefore, the use of environmentally relevant test conditions, such as natural water and sediment or long system acclimatization periods before the exposure, will increase the ecological relevance of the study, and the results found in the laboratory tests will be closer to what will happen in the field due to the incorporation of naturally occurring factors. In all our single species tests we used natural sediment, and the NMP were embedded in sediments for 14 days prior to the start of the exposure, allowing for the formation of biofilms. 
Exposure assessment. In order to understand the mechanisms behind the detected adverse effects for NMP, it is essential to measure their uptake by the exposed organisms. Most studies assessing both uptake and effects have done it in separate experiments, often under different conditions (e.g., different exposure times, various food conditions). Even though this provides information with respect to the ability of the organisms to ingest NMP, the test conditions and the species' developmental stage may influence their uptake. ${ }^{46,359}$ For instance, Ziajahromi et al. (2018) found that polyethylene MP with a size range of $100-$ $126 \mu \mathrm{m}$ significantly affected the emergence of Chironomus tepperi after 10 days of exposure. ${ }^{80}$ However, they assessed the ingestion of these MP after 5 days of exposure only, finding no uptake, and therefore suggested that the exposed organisms might have been able to ingest them in the last 5 days due to their increase in size. ${ }^{80}$ This was based on the findings of Scherer et al. (2017), who found a relationship between the life stage and the sizes of the MP ingested for a similar species, Chironomus riparius. ${ }^{46}$ For this reason, our experiments were designed with the purpose of quantifying the NMP inside the body of the exposed organisms at the end of the 28-day exposure, after a 24 hours gut depuration period. We measured the concentrations of NMP in the body of the organisms, as well as in the faeces, in order to discern among the retained and the excreted NMP.

Various methods are used in the literature to detect NMP in biological samples, which mainly depend on the size of the particles and the biological matrix of the species. Some methods are based on the visual detection of fluorescently-labelled NMP using a fluorescent microscope, which can lead to misinterpretation of the results due to leaching of the fluorescent dye from NMP to the biological tissues. ${ }^{127}$ Non-fluorescent MP are often visually sorted under a microscope after purifying the samples, which may leave out the small and light-coloured MP and include inorganic particles that have not been removed well during the purification process. ${ }^{31}$ As suggested in the critical review on analytical methods assessing MP in biota samples by Hermsen et al. (2018), without formal evidence of polymer identity using $\mu$-FTIR spectroscopy or RAMAN, a particle cannot be reported as being a MP. ${ }^{31}$ Thus, to accurately assess the MP ingested by the exposed biota, these techniques should be applied when possible. In Chapter 2, $\mu$-FTIR spectroscopy was used to quantify the number and sizes of the PS MP in the body and faeces of $G$. pulex. For Chapter 3, $\mu$-FTIR spectroscopy could not be used due to the high IR absorption of the TP, and the particles were visually assessed in the body and faeces of $G$. pulex under a stereomicroscope. In both cases, organisms exposed to all concentrations were previously purified and the measured concentrations in controls were subtracted from the rest of the concentrations to account for potential contamination and the presence of inorganic particles in the visually analysed particles. Quantification of NP concentrations in complex matrices remains a challenge, as $\mu$-FTIR spectroscopy or RAMAN have detection limits $>1 \mu \mathrm{m} .{ }^{32}$ A recent method has however been developed to measure NP in complex matrices, which is based on the use of metal-doped NP, and can therefore be measured with ICP-MS. ${ }^{216,227}$ This has been successfully applied for waste water samples as well as biological samples. ${ }^{216,227}$ We applied this method in Chapter 4 to measure the concentration of palladium-doped NP in the body and faeces of G. pulex. 
When NMP concentrations in the exposure medium and in the biota after gut depuration are measured, operationally defined trophic transfer factors (TTFs) or bioaccumulation factors (BAFs) can be calculated by dividing the concentration of the NMP in the biota by the concentration of the NMP in the exposure medium (Chapters $\mathbf{2}$ - 4). ${ }^{186,230,360,361}$ These factors allow for the comparison among different plastic particles and species, revealing which NMP are more easily bioaccumulated and which organisms will transfer more NMP to higher trophic levels. For instance, the TTFs for G. pulex exposed to PS NP and PS MP via sediment were 0.031 and $0.025 \mu \mathrm{g} \times \mathrm{kg}-1$ biota $/ \mu \mathrm{g} \times \mathrm{kg}-1$ sediment $\mathrm{dw}$, respectively, revealing that both NMP were similarly incorporated and accumulated on a mass basis (Chapter 4). Verifying actual NMP concentrations in the exposure medium when possible is highly recommended to demonstrate the accuracy of the dosing and to obtain solid factors. ${ }^{140}$ Moreover, reporting the results in both number and mass concentrations will facilitate the comparison across studies. ${ }^{45}$ While the actual PS MP concentrations were not measured due to the laborious methods needed to quantify MP in sediments, actual concentrations of the MP made from tires and PS NP in sediments were rapidly assessed by analysing the zinc and palladium concentrations in sediments, respectively, at the start of the exposure (Chapters 3 and 4). In both cases, the linearity of the measurements demonstrated the accuracy of the dosing.

8.3 Opportunities to use outdoor tests to detect NMP effect thresholds at the community level. In the effect assessment of NMP, higher-tier approaches consist of the determination of community effect thresholds using semi-field experiments. ${ }^{11}$ For this, a pelagic or benthic community is exposed to a range of NMP doses via water or sediment, respectively, using outdoor mesocosms. At the beginning of this research, community effect thresholds had never been measured for NMP, and therefore we framed a third research question: How to perform high-tier outdoor tests to detect effect thresholds of NMP at the community level? To address it, we followed previous studies assessing community effect thresholds for other inert particles, such as activated carbon and multiwalled carbon nanotubes. ${ }^{192,244}$ Experiments consisted on the long-term exposure of a well-established donor benthic invertebrate community inhabiting a semi-artificial outdoor ditch to trays containing PS NP or PS MP at five concentrations ranging from 0 to 5\% plastic/sediment dw (Chapter 5). ${ }^{226}$ Organisms were allowed to colonize the trays, and four replicates per treatment were retrieved after 3 months, while four others were retrieved after 15 months, in order to evaluate the effects of NMP on the community composition, population sizes and species diversity.

As done for the detection of individual effect thresholds for NMP, a sufficient number of doses, including environmentally realistic concentrations as well as higher ones, is needed to measure dose-response relationships at a community level. Outdoor experiments are ecologically relevant (Figure 8.1), which is one of the advantages of performing high-tier tests. The environmental variables will influence the detection of effect thresholds, and therefore, the use of a sufficient number of replicates is particularly important to account for the variation given by the environmental factors. Moreover, to account for the spatial variation in the outdoor system, the use of a randomized block design is recommended, 
which should be studied in the statistical analysis as a separate factor. In our experiments, we studied the effect of time as well in the obtained results and found clear time-related effects. Colonization ratios ${ }^{1}$ were between 4 and 5.4 for the individuals $/ \mathrm{m}^{2}$ and 0.72 and 0.88 for the taxa, confirming a high tray colonization over this period. However, no effects on the community were found after 3 months of exposure to NP or MP. One year later, a statistically significant and substantial effect on benthic macroinvertebrate abundance was found for the highest NP and MP concentrations. Time was a key factor influencing the detection of community effect thresholds and outdoor tests should thus last long enough to allow for the appearance of such effects. Despite the influence of spatial variation and time on the total abundance of macroinvertebrates, effects of the NMP were detectable, which illustrates the effectivity of the developed method to assess their community effect thresholds.

A substantial reduction in the number of macroinvertebrates was found after 15 months of exposure, which was mainly caused by the reduction of the most abundant family of species. Other taxa were slightly affected, but their abundance was always below 40 individuals per tray, which shows that a sufficient abundance is required in order to detect highly significant community effect thresholds. The growth of the populations cannot however be controlled in these tests, and it will mainly depend on the donor community, the ecosystem and the climate conditions. This is one of the challenges of using long-term outdoor experiments, together with the difficulties of identifying the mechanisms behind the effects found, as these become more complex when predator-prey interactions are considered over more than one generation. Finally, verifying the concentrations of NMP at the start and at the end of the exposure is needed when using open experimental units, such as trays, which will increase the ecologically realism of the study even more in contrast to closed experimental units.

Since the start of this thesis, several critical reviews and reports have claimed the lack of available data with respect to the ecological impacts of NMP. ${ }^{13,235}$ Nevertheless, no other community effect thresholds have been published for NP or MP than those reported in Redondo-Hasselerharm et al. (2020) (Chapter 5). ${ }^{226}$ Only in Green (2016) and Green et al. (2017), the effects of MP were evaluated over 60 and 50 days, respectively, on two marine bivalves and their associated benthic communities. ${ }^{88,89}$ For this, polypropylene buckets were filled with intact sediment cores collected from the field and placed onto an outdoor table with flow-through seawater. ${ }^{88,89}$ Five replicas per dose were made, adequately covering the variations per concentration; however, only three MP doses were tested, including the control, impeding the determination of reliable community effect thresholds. Although some environmental realism was considered by using natural sediment and seawater and by locating the trays outside the laboratory where they could be affected by the climate conditions, the exposed organisms were still confined in the buckets. As explained by the authors in the paper, these systems were a good compromise between the highly controlled laboratory systems and field experiments. ${ }^{88,89}$

\footnotetext{
${ }^{1}$ Colonization ratios were defined as the number of individuals $/ \mathrm{m}^{2}$ (or taxa) in trays retrieved after 3 months divided by the number of individuals $/ \mathrm{m}^{2}$ (or taxa) in the donor system at the start of the experiment.
} 
8.4. Opportunities to use in vitro assays to inform the risk assessment of NP. Humans can be exposed to NMP and little is known about the potential impacts on human health. ${ }^{13,115,265}$ Several studies have been published where the effects of NMP have been evaluated using in vitro bioassays. Although these are commonly used as a screening tool to monitor water quality, their results can be used in risk assessment. ${ }^{362}$ They are able to detect the effect of all active known and unknown chemicals in a sample, can account for mixture effects and are animal-protective. However, as it occurs for the laboratory and outdoor effect tests, no standard methods exist to assess the effects of NMP on humans. For this reason, we formulated the fourth research question: To what extent can in vitro tests with relevance for human health be used to detect effects of NP?

To answer this research question, in Chapter 6 we evaluated the genotoxicity of nanoplastic particles of two sizes $(50$ and $500 \mathrm{~nm})$ at four concentrations $(0,2.5,25$ and $250 \mu \mathrm{g} / \mathrm{l})$ alone and in combination with chemicals extracted from surface water and wastewater treatment plant (WWTP) effluent water using the Ames fluctuation test. This test is a cost effective alternative to mammalian genotoxic testing (e.g., micronucleus and comet assay), designed to detect base-pair and frameshift mutations in the genome of two Salmonella typhimurium strains with and without metabolic activation. ${ }^{275}$ No significant genotoxic effects of any NP size were detected in the absence of extracted chemicals. In contrast, adverse mixture effects were found with regards to mutagenicity for NP in combination with chemicals from surface waters. To date, other types of genotoxicity (i.e. double strand breaks and micronuclei) have been demonstrated for NP using in vitro bioassays with relevance for human health. ${ }^{271-273}$ These could not be visible in the Ames fluctuation test, which is only able to detect base pair and frameshift mutations. ${ }^{275}$ Therefore, each in vitro test is very specific on an endpoint, meaning that a battery of tests should be done to cover all types of genotoxicity. Additionally, environmental NP are highly diverse with respect to sizes, shapes, polymer types and surface characteristics. This means that NP characteristics might influence the effects found.

The Ames fluctuation test has been previously conducted to test the genotoxicity of other nanoparticle types, such as multi-walled carbon nanotubes (MWCNTs), diesel exhaust nanoparticles (DEPs), ultra-fine nanoparticles (UFPs) or metal nanoparticles. ${ }^{276-278,363}$ However, the suitability and sensitivity of this test to evaluate nanoparticle mutagenicity has been put into question. ${ }^{287}$ Clift et al. (2013) showed that various nanoparticles were able to penetrate $S$. typhimurium without inducing a significant mutagenicity through the Ames test. ${ }^{287}$ In contrast, some of these nanoparticles induced a significant mutagenic effect using an in vitro mammalian cell system, suggesting that the Ames test may not be a good indicator for nanoparticle mutagenicity. ${ }^{287}$ Based on their outcomes, NP uptake by $S$. typhimurium does not seem to be directly related with mutagenic effects in the Ames test. ${ }^{287}$ In Chapter 6, we found a lack of reproducible genotoxic responses in the two independent experiments conducted with NP in combination with surface and WWTP effluent water, which might be caused by temporal fluctuations in surface and wastewater or by having responses around the detection limit. The sorption of chemicals to the NP and the well-plates walls, as well as the aggregation of the NP could differ among 
replicates. For this reason, studying the behaviour of the NP in the tested medium and the use of a larger number of replicas might be needed to increase the accuracy of the test results. This will compromise the number of doses that can be tested, reducing the statistical rigor in detecting effect thresholds for the evaluation of NP risks. In summary, the Ames fluctuation test does not seem to be a suitable bioassay to assess the risks of NP alone and together with chemicals extracted from surface water and WWTP effluent.

8.5. A quantitative scoring system to evaluate the quality of MP effect studies and to identify demonstrated mechanisms for MP effects. Since the beginning of this thesis, an increasing number of studies has been published reporting the effects of MP on aquatic biota using single species tests. Figure 8.2 shows the number of papers reporting effects of MP and/or NP on aquatic species, with or without sorbed contaminants, additives, metals or other engineered particles, published since the first one available in 2008. The figure was made using 305 papers assessed in recent reviews (Chapter 7). ${ }^{13,125,126}$ The number of publications reporting effects of MP and NP is exponentially increasing and is about $2 \mathrm{x}$ higher for MP than for NP. Even though the number of papers reporting effects of MP on aquatic organisms is increasing over time, the usability of the results in risk assessment is still low, as there is a lack of consistency and standardization among the test methods followed due to the absence of guidelines for the assessment of MP effect thresholds. Moreover, even though some studies are able to demonstrate mechanisms behind the effects found, most of them suggest mechanisms that are poorly supported by the experimental design or the results obtained. This has led to considerable speculation regarding to the effects and effect mechanisms reported, which represents a problem for risk assessment and decision-makers. To address these issues, we framed the two final research questions: What could be improved in effect tests when it comes to quality assurance?; and, Which effect mechanisms can be considered as demonstrated when strict quality criteria are applied to the literature?

To answer them, in Chapter 7, ${ }^{223}$ we first assessed the quality of the data from studies reporting effects of MP, adopting similar existing methods (e.g., Klimisch and CRED approaches), ${ }^{311,312}$ which were recently applied to address the quality of exposure concentrations of MP in biota and surface water. For this, we designed a scoring system based on 20 quality criteria (QA/QC) included in four categories and applied it to $105 \mathrm{MP}$ effect studies. The results obtained allowed us to identify which categories had been addressed best and which ones need to be revised by future studies in order to conduct reliable ecotoxicity tests. Many studies to date have stated the need to harmonize the methods and to improve the quality of MP exposure and effect studies. In fact, since the publication of the quality criteria on the analysis of MP in biota samples by Hermsen et al. (2018), several publications have considered it to elaborate the experimental design and ensure the quality of the study. ${ }^{364-366}$ This indicates that guidelines addressing the quality of the literature are acknowledged by the scientific community and that future approaches may be more consistent across research groups, leading to a better understanding of MP effects on aquatic organisms. 


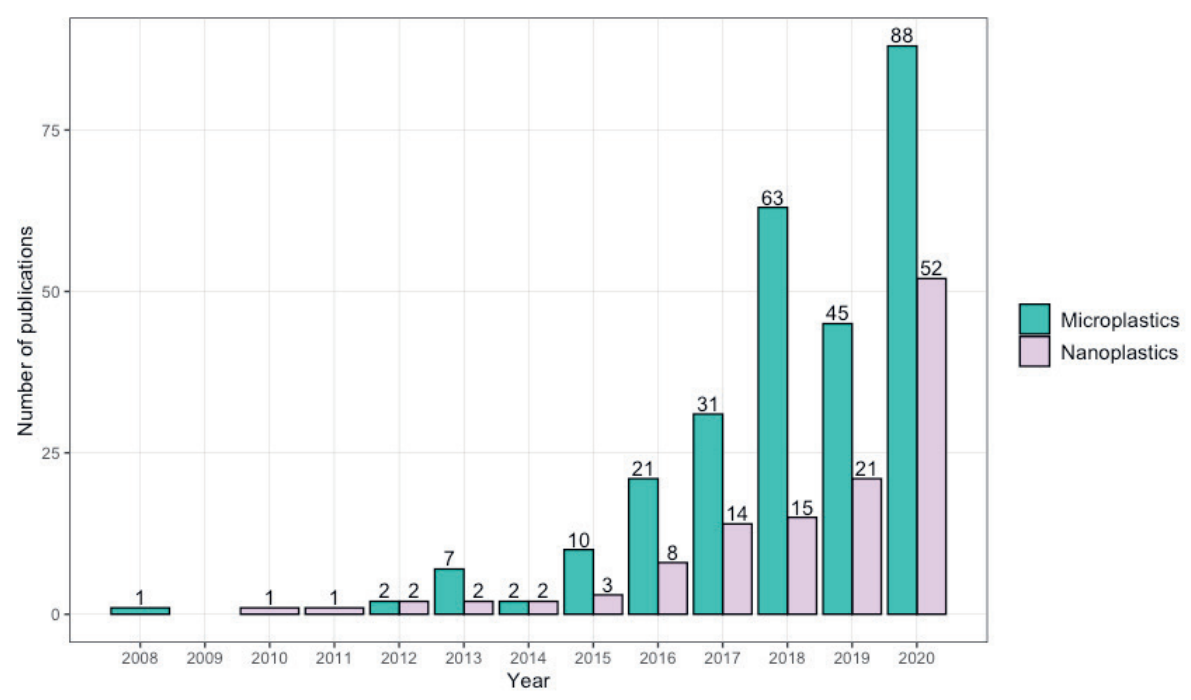

Figure 8.2. Number of publications per year reporting effects of micro- and nanoplastics on aquatic biota, alone or with sorbed contaminants, additives, metals or other engineered particles. Papers of the year 2020 were collected for the first half of 2020 and were multiplied by 2. A total of 305 publications were used, from which 16 tested both particle types.

Next to the scoring, we noted the demonstrated and suggested effect mechanisms reported by the 105 reviewed papers. There were 10 effects mechanisms identified as suggested and 3 additional ones reported as speculated only. While these studies described an effect mechanism as suggested 100 times, they only claimed a demonstrated effect mechanism in 34 occasions. This shows that the experimental design of a great number of these papers did not aim to explain the adverse effects observed. Then, scores obtained in three crucial quality criteria were used as a quantitative weight of evidence (WOE) approach to assess the relative strength of an effect mechanism. The criteria were chemical purity, addition of food, and exposure assessment of the test organisms. Finally, the total QA/QC score obtained by each of the studies was used to assess the relative credibility of mechanisms underlying adverse effects reported. Outcomes of Chapter 7 revealed that the WOE was strongest for the following mechanisms: inhibition of food assimilation and/or decreased nutritional value of food, internal physical damage and external physical damage.

Although this is the first quality criteria developed based on a quantitative scoring system for MP effect studies, other critical reviews have addressed their flaws and have suggested approaches for improving their protocols. ${ }^{140,141}$ Also, several recent reviews performed meta-analysis with literature data and discussed the evidences regarding the occurrence of MP effects and the underlying effect mechanisms. ${ }^{126,309,310,367}$ However, the quality of the studies was not taken into account, which could potentially bias the results obtained. For instance, Bucci et al. (2020) stated that the most evident mechanism underlying growth reduction was MP ingestion, which lead to a lower food assimilation. ${ }^{309}$ Similarly, a decreased reproductive output was attributed to a reduced feeding, affecting the energy 
allocation for reproduction. ${ }^{309}$ These conclusions are in agreement with the mechanisms identified in Chapter 7 following a WOE approach. ${ }^{223}$ Another described mechanism was the presence of chemicals in the MP, which could affect the endocrine system and increase organism's reproduction. ${ }^{309}$ Nevertheless, without taking the quality criteria of chemical purity into account, it is unclear whether the studies using this mechanism to explain an effect discerned between particle and chemical effects. In fact, based on the results of our scoring, only $20 \%$ of the studies ensured chemical purity (Chapter 7). ${ }^{223}$ Finally, Kögel et al. (2020) summarized nine determining factors behind MP toxicity on aquatic biota based on the literature, which are concentration, particle size, polymer type, polymer condition (shape, weathering, surface modifications), food availability, exposure time, background contamination, species, sex and developmental stage. ${ }^{126}$ These are directly related with the effects and effects mechanisms found for MP and should be addressed by future studies in their experimental design in order to have a better understanding on the toxicity of MP on aquatic organisms.

8.6. Current risks of MP in freshwater sediments. In Chapters $\mathbf{2}$ - 5, we determined the individual and community effect thresholds for freshwater benthic invertebrates exposed to MP via sediment. In this section, the results obtained in previous chapters are used together with literature data to conduct an effect assessment for MP in freshwater sediments following the tiered approach (Figure 8.3). The tiered approach is commonly used to assess the effects of chemicals but also can be applied to evaluate MP effects. ${ }^{11}$ Due to the absence of guidelines to assess the risks of MP, the EFSA guidance on tiered risk assessment for chemicals was followed. ${ }^{368}$ In a tiered effect assessment, Predicted No Effect Concentrations (PNECs) are calculated for the different levels of ecological relevance and complexity. Then, PNECs are compared with Predicted Environmental Concentrations (PECs), which are calculated using mathematical models, or Measured Environmental Concentrations (MECs), which are calculated using field concentrations measured for the ecosystem under consideration, or reported in the literature for similar ecosystems, with the PEC- or MEC- to PNEC ratio used as an indicator of risk. ${ }^{40,94}$ 


\section{Tiered Effect Assessment}

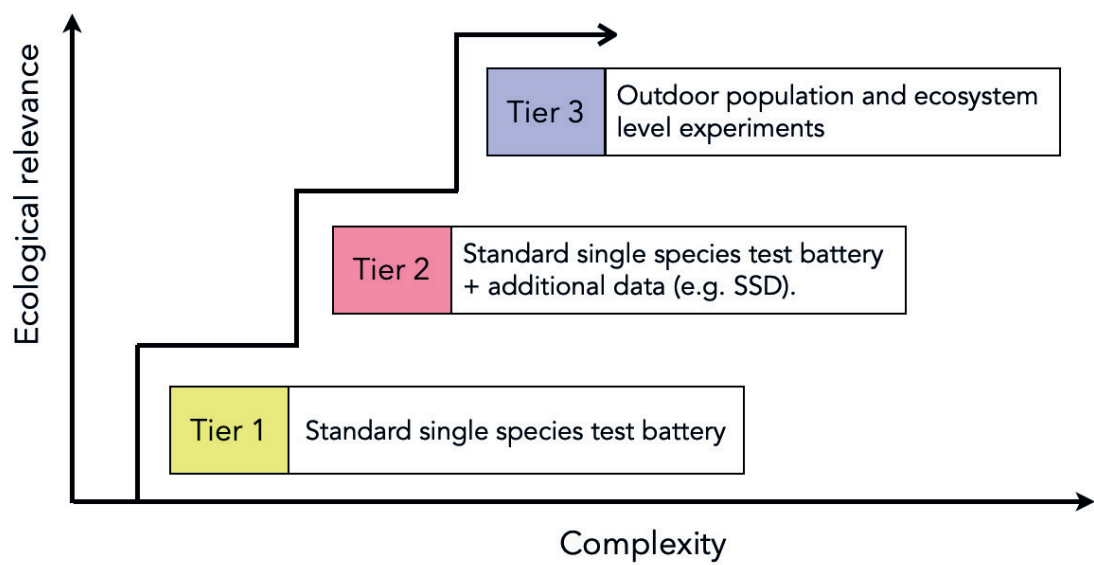

Figure 8.3. Schematic overview of a tiered approach in an environmental risk assessment. In each tier, a predicted no effect concentration (PNECs) is calculated, which can then be compared with predicted environmental concentrations (PECs) or measured environmental concentrations (MECs) to assess the risks (adapted from Diepens et al., 2014). ${ }^{369}$

8.6.1. Exposure assessment: MECs in freshwater sediments. To calculate measured environmental concentrations (MECs) in freshwater sediments for the risk characterization of MP, we compiled the number concentrations (particles $/ \mathrm{kg} \mathrm{dw}$ ) for 20 water bodies reported by eleven peer-reviewed studies (Table A8.1). Then, to solve the non-alignment of the MP concentration data given by the studies that targeted different MP size ranges, data were rescaled following the methodology described in Koelmans et al. (2020). ${ }^{233}$ For each of the reported size ranges, the number concentration was rescaled to match a common range of 1 to $5000 \mu \mathrm{m}$. For the risk characterization, the minimum, maximum and mean MECs reported for these 20 water bodies are plotted as a cumulative frequency distribution and compared with the PNECs obtained in each tier (Figure A8.1).

8.6.2. Tier 1: Effect assessment using standard single species tests. In tier 1, thresholdeffect concentrations are measured for a variety of species belonging to different taxonomic groups using standard single species tests. To calculate the risk characterization ratio (RCR), the MEC is divided by the PNEC derived after applying the corresponding assessment factor (AF). ${ }^{93,368}$ In Chapter 2, we tested six freshwater benthic invertebrates: G. pulex, H. azteca, A. aquaticus, Tubifex spp., and L. variegatus, and $S$. corneum. ${ }^{186}$ Using the same experimental design, Clokey et al. (2020) tested three additional freshwater benthic species: Procambarus acutus, Pacifastacus leniusculus and Potamopyrgus antipodarum. ${ }^{370}$ Finally, two freshwater macrophytes were tested for the same sediment and MP mixture: Myriophyllum spicatum and Elodea spp. From this total of eleven species, only G. pulex showed a reduction in growth when exposed to PS MP fragments with a size range between 20 and $500 \mu \mathrm{m}$, with an $\mathrm{EC}_{10}$ of $3.37 \times 10^{9}$ particles/kg dw, which corresponded to $1.07 \%$ sediment $\mathrm{dw}$ (Chapter 2). To calculate 
the PNEC based on this effect threshold in tier 1, an AF of 10 was chosen following Diepens et al. (2015). ${ }^{93}$ A PNEC of $3.37 \times 10^{8}$ particles $/ \mathrm{kg}$ dw, which corresponded to $0.107 \%$ sediment $\mathrm{dw}$ was thus obtained for tier 1 . With the aim of comparing the obtained PNEC with the rescaled MECs, the PNEC was rescaled for the bioavailable MP fraction following Koelmans et al. (2020). For this, we used the size of the biggest MP ingested by G. pulex in Chapter 2, which was $165 \mu \mathrm{m}$. The rescaled to $1-165 \mu \mathrm{m}$ PNEC was $7.77 \times 10^{11}$ particles $/ \mathrm{kg} \mathrm{dw}$. When visually comparing the rescaled PNEC with the rescaled MECs, we observe that all MECs are below the PNEC obtained in tier 1 (Figure A8.2). Additionally, the RCRs calculated for all water bodies are below 1, indicating no immediate risk of MP to freshwater sediments at tier 1.

8.6.3. Tier 2: Effect assessment using Species Sensitivity Distributions. In tier 2, the ecotoxicity data generated in tier 1 and additional ecotoxicity data taken from the literature are combined in Species Sensitivity Distributions (SSDs). In these SSDs, a $\mathrm{HC}_{5}$ value is calculated, which is the value where $5 \%$ of the species would be affected in an ecosystem. A PNEC is then derived from the $\mathrm{HC}_{5}$ value after applying the corresponding $\mathrm{AF}$, which can be then compared with the MEC to assess the RCR. ${ }^{93,371}$ To construct the $\mathrm{SSD}$, we used the data from tier 1 in combination with literature chronic effect threshold concentrations for freshwater benthic species exposed to MP via sediments. A summary of the data used in the SSD is provided in Table A8.2. Only chronic exposures ( $>10 \mathrm{~d})$ were used and the ecotoxicological endpoints selected were survival, growth, reproduction and emergence. Following Adam et al. (2019), LOECs were extracted only if no $\mathrm{LC}_{\mathrm{x}}$ or $\mathrm{EC}_{\mathrm{x}}$ values were reported and the highest NOEC was used only if no other endpoints were provided. Dose descriptors $\mathrm{EC}_{\mathrm{x}}$ and LOECs were converted to NOECs using an AF of 2, according to the ECHA guidelines. ${ }^{371}$ Again, following Koelmans et al. (2020), to develop an SSD consistent with the rescaling concept applied for MECs, effect thresholds were converted into volume equivalent threshold-effect concentrations for environmental MP, which were meant to solve the non-alignment with respect to the sizes, shapes and densities of the MP used in the tests. Then, we calculated actual exposure concentrations using the maximum ingestible sizes for the tested freshwater benthic species (Table A8.3). As for some of the species no data on MP ingestion was given in the studies reporting the effect thresholds, we defined them based on maximum ingestible food sizes or mouth opening size obtained from the literature. For the macrophytes, the bio-unavailable fraction was assumed to be negligible. ${ }^{233}$ The bivalve $S$. corneum was excluded from the SSD because the maximum ingestible particle and food sizes found in the literature were always $<20 \mu \mathrm{m}$, which was the smallest MP used in the effect test. ${ }^{43,372}$ The SSD with the rescaled threshold-effect concentrations plotted as a cumulative distribution is shown in Figure 8.4. 


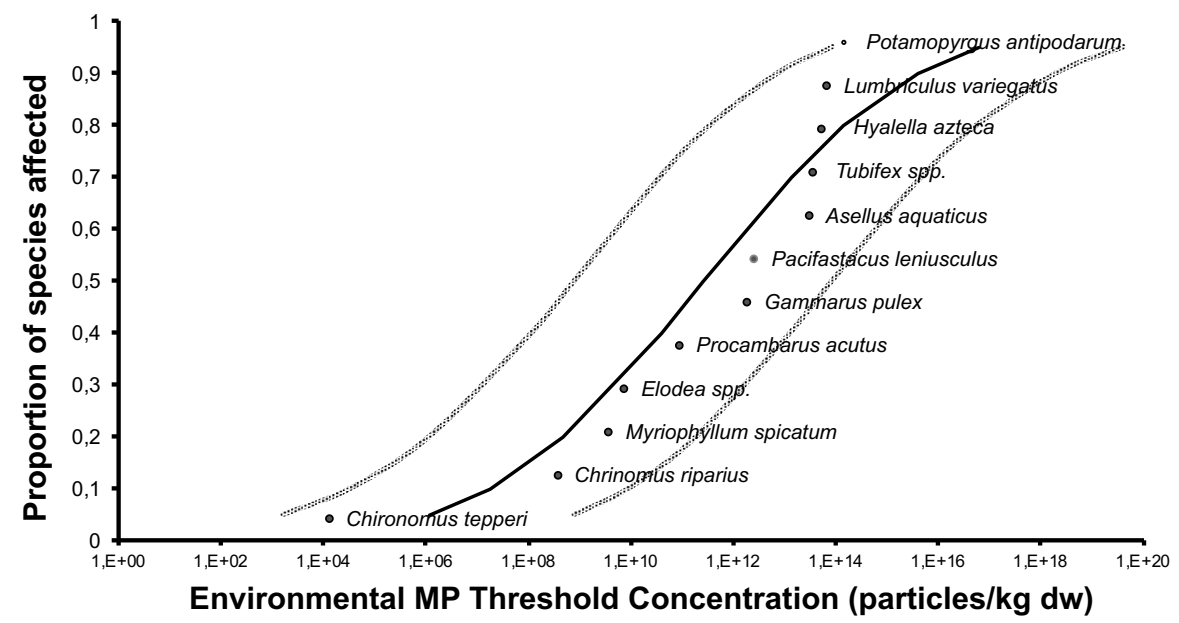

Figure 8.4. Species Sensitivity Distribution (SSD) for freshwater benthic species exposed to microplastics via sediment using single species tests $\left(R^{2}=0.844 ; H C_{5}=1.13 \times 10^{6}\right.$ particles $\left./ \mathrm{kg} \mathrm{dw}\right)$. Values are corrected for the bioavailability and polydispersity of the microplastics. Grey curves relate to $95 \%$ CI.

The $\mathrm{HC}_{5}$ obtained from the SSD is $1.13 \times 10^{6}$ particles $/ \mathrm{kg} \mathrm{dw}$, with the $95 \%$ low and high confidence interval $(\mathrm{CI})$ boundaries being $1.61 \times 10^{3}$ and $8.01 \times 10^{8}$ particles $/ \mathrm{kg} \mathrm{dw}$, respectively. Following Diepens et al. (2015), we addressed the remaining uncertainty in the $\mathrm{HC}_{5}$ caused by the wide $\mathrm{CI}$ by applying an $\mathrm{AF}$ of 4 and obtained a tier 2 PNEC of $2.84 \times 10^{5}$ particles $/ \mathrm{kg} \mathrm{dw}$, with $4.01 \times 10^{2}$ and $2.00 \times 10^{8}$ particles $/ \mathrm{kg} \mathrm{dw}$ as the low and high CI. Most sensitive organisms to MP pollution seem to be diptera larvae from the genus Chrironomus, which were severely affected at low MP concentrations, ${ }^{80,236}$ and the macrophytes M. spicatum and Elodea spp., which can be affected by the whole MP size continuum, ${ }^{220}$ which increases their sensitivity. The least sensitive organisms are the snail $P$. antipodarum, the amphipod $H$. azteca and the worms L. variegatus and Tubifex spp. To assess the risk of MP in tier 2, the MECs as well as the PNEC and low 95\% CI are plotted in Figure 8.5. When looking at the maximum MECs reported and using the PNEC based on the median $\mathrm{HC}_{5}$, we observe that MECs exceed the maximum measured PNECs for three water bodies. These water bodies are the Wen-Rui Tang River in China, ${ }^{38}$ the Amsterdam Canals in The Netherlands, ${ }^{131}$ and the Elbe River in Germany, ${ }^{373}$ for which RCR of $1.64,1.38$ and 1.14 are calculated with the maximum MECs reported, respectively. The RCR in the Wen-Rui Tang River is above 1, indicating that there is a risk. Therefore, we can conclude that MP pose a risk for the benthic biota living and feeding in freshwater sediments of $15 \%$ of the water bodies studied, according to this risk assessment approach. In addition, it should be noted that all MECs exceed the lower $95 \%$ CI of the PNEC (Figure 8.5), indicating that we cannot assure that any location could not experience a risk. 


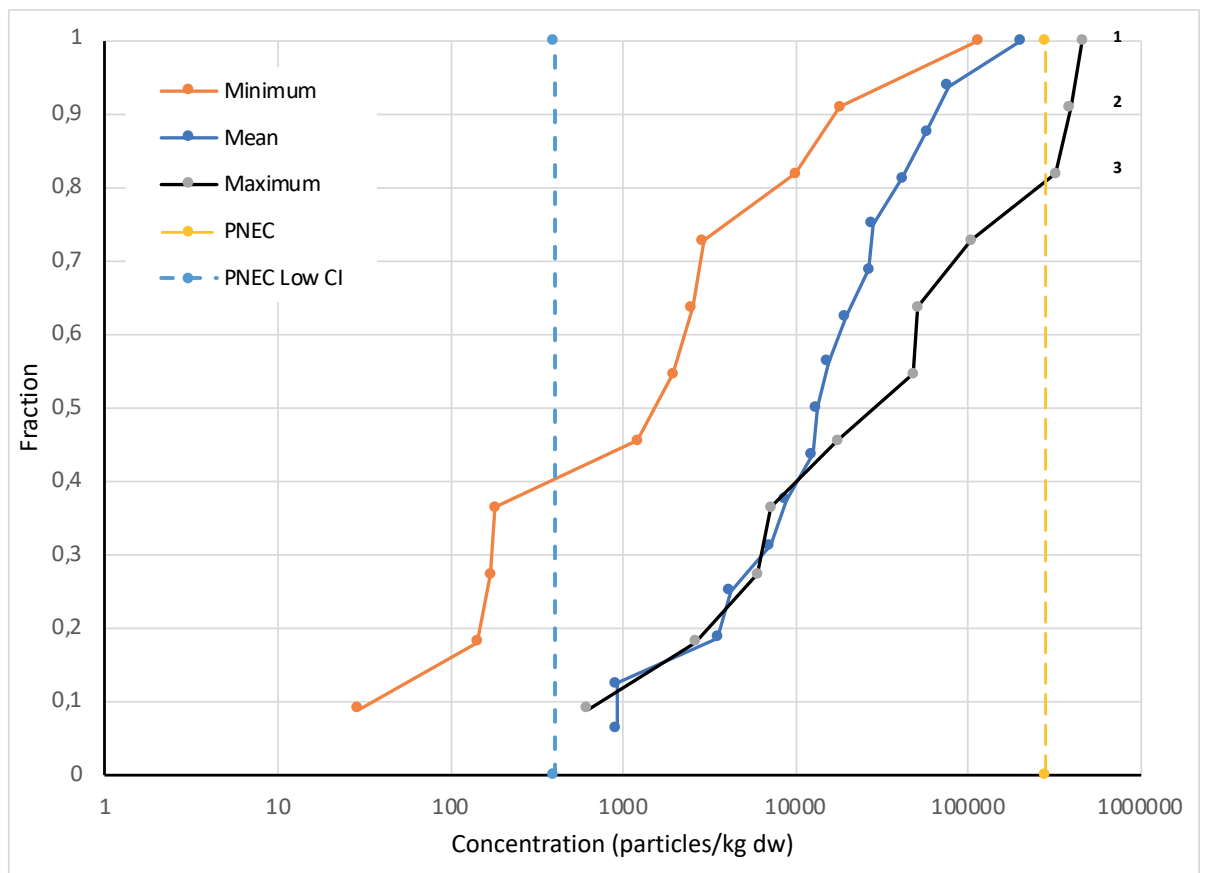

Figure 8.5. Cumulative frequency distribution of rescaled to 1-5000 $\mu$ m measured exposure concentrations (MECs) in 20 water bodies from Europe, Asia and Africa compared to the predicted no effect concentration (PNEC) obtained in Tier 2 based on the HCs calculated from a species sensitivity distribution (SSD) for freshwater benthic species. MECs are based on the minimum, maximum and mean concentrations reported. Labels: 1) Wen-Rui Tang River in China; 2) Amsterdam Canals in The Netherlands; 3) Elbe River in Germany.

This is the first SSD made for freshwater benthic biota exposed to sediment-MP mixtures. It includes twelve benthic species of various taxonomic groups (five crustaceans, two annelids, two insect larvae, two macrophytes, and one mollusc), meeting the minimum requirement of ten species set by the European Chemicals Agency (Table A8.2). ${ }^{371}$ Current data available on MP effect thresholds measured for freshwater benthic biota and MECs of MP in freshwater sediments are limited, making the conclusions of the observed risks assessment based on a tier 2 PNEC preliminary. Moreover, the rescaling concepts applied here, ${ }^{233}$ are provisional and need further validation with empirical data. To obtain a robust SSD for sediment-dwelling species exposed to MP, effect thresholds should be measured on a larger number of species and for the species already present in the SSD exposed to other MP types. Finally, MP concentrations should be measured in a wider number of freshwater ecosystems globally to have a better view on current environmental concentrations of MP. This is particularly important due to the demonstrated risks in this preliminary SSD. 
To date, 5 studies have evaluated the risks of NP and/or MP in marine and/or freshwater ecosystems by constructing SSDs with data available in the literature. ${ }^{40,95-97}$ Everaert et al. (2018) performed a risk assessment for marine biota exposed to MP (size: $0.001-5$ $\mathrm{mm}) ;{ }^{97}$ Burns \& Boxal (2018) and VKM (2019) combined marine and freshwater effect thresholds from organisms exposed to MP (size: $10 \mu \mathrm{m}-5 \mathrm{~mm}$ ) to increase statistical power due to the low number of data available to build separate SSDs $;{ }^{96,374}$ Besseling et al. (2019) also combined marine and freshwater ecotoxicity data for the same reason but made two separate SSDs based on the size of the particles: one for NP (size: $1-100 \mathrm{~nm}$ ) and one for MP (size: $1 \mu \mathrm{m}-5 \mathrm{~mm}) ;{ }^{40}$ Adam et al. (2019) created an SSD for freshwater data combining NP and MP in the same SSD. ${ }^{95}$ These studies also stated the preliminary character of their SSDs due to the low number of data available and expressed the need to improve the quality of the data. It is important to mention that, although the taxonomic groups included in the published SSDs for NP and MP belonged to pelagic and benthic species, they included water exposure data only. While Burns \& Boxal (2018) and Everaert et al. (2018) found no risks of MP at current measured and predicted environmental concentrations, ${ }^{96,97}$ Besseling et al. (2019), Adam et al. (2019) and VKM (2019) found indications of risks at some locations where MP concentrations were measured. ${ }^{40,95,374}$ These differences may be explained by the improvement of the methods used to detect MP in water samples, which has allowed recent publications to quantify MP with smaller particle sizes, which often seem to be present in high numbers. ${ }^{95,374}$ Besseling et al. (2019) stated that risks of MP could occur at near-shore marine surface waters ${ }^{40}$ Adam et al. (2019) showed $0.12 \%$ of the global MECs had a RCR $>1,{ }^{95}$ while VKM (2019) demonstrated that the RCR was above 1 in $5 \%$ of the sampled locations, including marine and freshwater ecosystems. ${ }^{374}$ Therefore, based on tier 2 PNECs, risks of MP in marine and freshwater ecosystems cannot be excluded.

8.6.4. Tier 3: Effect assessment based on outdoor ecosystem level tests. In tier 3, semifield experiments are carried out to measure effect thresholds at the community level. A PNEC is then derived after applying the corresponding AF and compared to MECs. The only study published to date assessing effect thresholds for freshwater benthic communities is Redondo-Hasselerharm et al. (2020) (Chapter 5). ${ }^{226}$ Here, a mass based NOEC of $0.5 \%$ of MP per sediment dw was obtained after exposing a freshwater benthic community to PS MP fragments via sediment for 15 months. ${ }^{226}$ Following RedondoHasselerharm et al. (2018) (Chapter 2), a mass based NOEC was calculated using the mass of PS MP per dose, the density of PS $\left(1.05 \mathrm{~g} / \mathrm{cm}^{3}\right)$ and the measured particle volume distribution. A number-based NOEC of $1.58 \times 10^{9}$ particles $/ \mathrm{kg}$ dw was thus obtained. According to the Water Framework Directive (WFD) ${ }^{375}$, we used an AF of 5 to account for the variation in the NOEC, obtaining a tier 3 PNEC of $3.15 \times 10^{8}$ particles $/ \mathrm{kg} \mathrm{dw}$. No rescaling was applied to this value because ingestion was not assessed for the community. When plotting the MECs together with the tier 3 PNEC, we observe that the PNEC is much higher than the maximum MP concentrations detected in freshwater sediments (Figure A8.2). Thus, we can conclude that MP do not pose a risk for freshwater benthic communities at current environmental concentrations. 
8.6.5. Tiered risk assessment for nano- and microplastic: Limitations and recommendations. Following the outcomes of tier 1 in the environmental risk assessment provided in the previous section, we could conclude that MP are not expected to cause significant effects on benthic species via a sediment exposure (Figure A8.2). However, the predicted no effect concentration (PNEC) calculated in tier 1 is based on the results of eleven species exposed to the same PS MP under equal laboratory conditions. Therefore, even though tier 1 is a good first step to understand what effects PS MP could have on a particular freshwater ecosystem, we should aim at increasing the environmental relevance of the risk assessment by moving on to a higher tier (Figure 8.3). In tier 2, the data from tier 1 are compared with individual effect thresholds reported for other benthic species using a Species Sensitivity Distribution (SSD). In contrast of the results obtained in tier 1, in tier 2 we observe that MP could pose a risk for the most sensitive benthic species in highly polluted freshwater sediments (Figure 8.5). Nevertheless, it should be noted that the diptera larvae Chironomus tepperi is the only species below the $\mathrm{HC}_{5}$ value in the SSD (Figure 8.4.), which would thus be affected by the MP exposure in these highly polluted ecosystems. ${ }^{80}$ This data point (C. tepperi) is actually based on the results of one study only where significant adverse effects were found for the diptera larvae when exposed to low PE MP concentrations. ${ }^{80}$ In this paper by Ziajahromi et al. (2018), ${ }^{80}$ which scored 18 out of 40 in our QA/QC (Chapter 7), ${ }^{223}$ the chemical purity of the MP was not addressed and the measured effect threshold was based on one MP concentration only. ${ }^{80}$ Therefore, we should be careful when claiming that MP could pose a risk for freshwater benthic species and consider these outcomes as preliminary. To improve the accuracy of the measured $\mathrm{HC}_{5}$, individual effect thresholds need to be measured for a larger number of benthic organisms and for the species already present in the SSD exposed to other MP types, ideally following a QA/QC to ensure the reliability of the data. For NP, as mentioned earlier, insufficient data is available for such an assessment.

Future laboratory studies should also aim at unravelling the mechanisms behind the effects found. In de Ruijter et al. (2020) (Chapter 7), ${ }^{223}$ we observed that three mechanisms were the most evident after applying a weight of evidence approach. ${ }^{223}$ One of them, which was the inhibition of food assimilation and/or decreased nutritional value of food, was used to explain the reduction in growth found for G. pulex in RedondoHasselerharm et al. (2018) (Chapter 2). ${ }^{186}$ In Chapter 4, G. pulex of a similar size range were exposed to metal-doped NP using the same experimental design and the same sediment as in Chapter 2 and no effects of NP on the growth of G. pulex were found, as opposed to the MP. We modelled the bioaccumulation of MP and NP in G. pulex and observed that both particle types yielded very similar mass-based trophic transfer factors for the gut at the end of the 28 days of exposure. However, the mass-based trophic transfer factor for the body of G. pulex, reflecting accumulation in the irreversible fraction, was 4.5 times higher for the MP than for the NP. This suggests that MP might be trapped more easily in the gut in comparison to NP. Therefore, determining the mechanisms and factors behind the effects observed in laboratory tests with NP and MP should be considered by upcoming studies. 
When looking at the results of tier 3, where the ecological relevance of the data increases (Figure 8.3), we observe that the PNEC derived from the community effect threshold for MP was several orders of magnitude above the MECs in freshwater sediments (Figure A8.2). In this case, we conclude that MP do not seem to pose a risk for freshwater benthic communities at current concentrations. To date, no other study assessed the effects of MP on a freshwater benthic community and the results of tier 3 are only based on RedondoHasselerharm et al. (2020) (Chapter 5) ${ }^{226}$. Here, the total number of Chironomini, which is the tribe where the genus Chironomus belongs to, was generally low after 3 and 15 months (averages $<30$ organisms per system), hindering the detection of statistically significant effects. Future studies should aim at evaluating community effect thresholds for MP using outdoor ecosystems to have a better understanding of the ecological risks of MP. Short term laboratory-controlled systems are useful to learn about the effects and effect mechanisms of MP on biota; however, they neglect relevant factors such as time, which affects the ecological interactions that occur in real habitats and the evolution of multiple generations. Moreover, in the single species tests performed in the laboratory, organisms are confined to a small environment where they are continually exposed to the MP. In contrast, in open outdoor tests as those used in Redondo-Hasselerharm et al. (2020) (Chapter 5), organisms are able to enter and leave the trays, better mimicking what would occur in real ecosystems. Therefore, future efforts should be put on evaluating the risks of MP at tier 3, with a focus on the species that have already shown to be sensitive to the presence of MP in sediments.

8.7. Concluding remarks. Plastic production has exponentially increased since the $1950 \mathrm{~s}$, leading to an augmented presence of plastic waste in marine and freshwater ecosystems over time. ${ }^{2,376}$ In a recent study, a MP-time curve was made by using the sedimentary record from an urban lake in China. ${ }^{376}$ They found that MP abundance increased from 741 to 7707 particles/kg over the past 60 years. ${ }^{376}$ Nowadays, plastic production is still increasing and, therefore, MP concentrations are expected to also be higher in the future. ${ }^{3}$ For this reason, MECs need to be measured in a wider range of freshwater bodies around the globe to have a broader overview on current environmental MP concentrations, but also predicted environmental concentrations should be estimated using mathematical model to assess prospective environmental risks of MP. In their risk assessment, for instance, Everaert et al. (2018) predicted a 50-fold increase in the total MP mass in the ocean between 2010 and $2100 .{ }^{97}$ Similar predicted environmental concentrations should be calculated for freshwater sediments and compared with the predicted no effect concentrations derived in this thesis. The results of this preliminary freshwater sediment risk assessment for MP, as those MP risk assessments already available in the literature for marine and surface waters, ${ }^{40,95-97,374}$ could be used by public authorities to establish permissible exposure limits to guarantee the wellbeing of the ecosystems in the future. Finally, the issue of nanoplastic remains one of the largest knowledge gaps, which calls for urgent attention. 


Appendices 



\section{Appendix of Chapter 2}

Table A2.1. Calculation of the weighted average density for environmental microplastics based on data provided by Andrady et al. (2011).

Table A2.2. Background elemental concentrations of $\mathrm{Zn}, \mathrm{Cd}, \mathrm{Cr}, \mathrm{Cu}, \mathrm{Ni}$ and $\mathrm{Pb}$ in the Veenkampen sediment using a) Extraction with $\mathrm{HNO}_{3}-\mathrm{HCl}$; b) Extraction with $0.01 \mathrm{M} \mathrm{CaCl}_{2}$, compared to the Dutch Sediment Quality Criteria (SQC) based on Target values.

Table A2.3. Water Quality Parameters (Mean \pm SD.).

Figure A2.1. Particle Size Distribution $(\mathrm{n}=3)$ of the original microplastic mixture in: a) Volume \%; ) Number $\%$.

Figure A2.2. Light microscope pictures of the irregularly shaped particles (Olympus SZX10 Stereomicroscope).

Figure A2.3. Growth of G. pulex as a function of polystyrene microplastic dose.

Figure A2.4. Mean feeding rate $( \pm \mathrm{SD})$ as $\mathrm{mg} d \mathrm{~d}$ of Populus sp. leaves consumed per organism per day during the 28-day exposure to PS microplastic concentrations ranging from 0 to $40 \%$ in sediment (dw) for a) G. pulex; b) H. Azteca; c) A. aquaticus.

Figure A2.5. Mean egestion rate $( \pm \mathrm{SD})$ as $\mathrm{mg} \mathrm{dw}$ of faeces egested per organism per day during a 15-d exposure to PS microplastic concentrations ranging from 0 to $40 \%$ in sediment (dw) for a) Tubifex spp; $b$ ) L. variegatus.

Figure A2.6. Size Frequency of a) Retained Microplastics; b) Egested Microplastics.

Figure A2.7. Mean microplastic concentration $(\mathrm{n}=4)$ per individual of $G$. pulex $( \pm \mathrm{SD})$ at increasing microplastic concentrations in sediment as a) number of microplastics egested per organism by number of microplastics per $\mathrm{kg}$ of sediment (dw); b) $\mathrm{g} \mathrm{kg}^{-1}$ of microplastics egested per organism dw by $\mathrm{g} \mathrm{kg}^{-1}$ of microplastics per sediment (dw). 
Table A2.1. Calculation of the weighted average density for environmental microplastics based on data provided by Andrady et al. (2011). ${ }^{20}$

a) Calculation assuming a density of $1 \mathrm{~g} / \mathrm{cm}^{3}$ for the rest fraction of $6 \%$.

Plastic type Density $\quad$ Fraction $\quad$ Weighted
$\left(\mathrm{g} / \mathrm{cm}^{3}\right)^{\mathrm{a}}$
Produced $^{\mathrm{a}}$
$\left(\mathrm{g} / \mathrm{cm}^{3}\right)$

\begin{tabular}{|c|cc|c|}
\hline LDPE & 0.92 & 0.21 & 0.1932 \\
HDPE & 0.94 & 0.17 & 0.1598 \\
PP & 0.84 & 0.24 & 0.2016 \\
PS & 1.05 & 0.06 & 0.063 \\
PET & 1.37 & 0.07 & 0.0959 \\
PVC & 1.38 & 0.19 & 0.2622 \\
REST* & 1 & 0.06 & 0.06 \\
\hline \multicolumn{2}{|l}{ WEIGHTED AVERAGE DENSITY } & & $\mathbf{1 . 0 3 5 7}$ \\
\hline
\end{tabular}

${ }^{\text {a }}$ according to Table 1 in Andrady et al. $(2011)^{20}$

${ }^{\mathrm{b}}$ for the REST fraction of $6 \%$ a density of $1 \mathrm{~g} / \mathrm{cm}^{3}$ was assumed

c the weighted average density is the average density of environmental microplastic, assuming all produced plastic types contribute to microplastic with weights equal to their production fraction 


\section{Table A2.1, continued}

b) Calculation neglecting the unknown rest fraction by scaling the sum of the fractions for the known polymers to $100 \%$

\section{Plastic type Density $\quad$ Fraction $\quad$ Weighted}

\begin{tabular}{|c|c|c|c|}
\hline & $\left.\mathrm{g} / \mathrm{cm}^{3}\right)^{\mathrm{a}}$ & Produced $^{\mathrm{a}}$ & $\left(\mathrm{g} / \mathrm{cm}^{3}\right)$ \\
\hline LDPE & 0.92 & 0.21 & 0.1932 \\
\hline HDPE & 0.94 & 0.17 & 0.1598 \\
\hline PP & 0.84 & 0.24 & 0.2016 \\
\hline PS & 1.05 & 0.06 & 0.063 \\
\hline PET & 1.37 & 0.07 & 0.0959 \\
\hline PVC & 1.38 & 0.19 & 0.2622 \\
\hline \multicolumn{3}{|c|}{ WEIGHTED AVERAGE DENSITY ${ }^{\mathrm{b}}$} & 1.0380 \\
\hline
\end{tabular}

a according to Table 1 in Andrady et al. $(2011)^{20}$

$\mathrm{b}$ the weighted average density is the average density of environmental microplastic, assuming all produced plastic types contribute to microplastic with weights equal to their production fraction 
Table A2.2. Background elemental concentrations of $\mathrm{Zn}, \mathrm{Cd}, \mathrm{Cr}, \mathrm{Cu}, \mathrm{Ni}$ and $\mathrm{Pb}$ in the Veenkampen sediment using A) Extraction with $\mathrm{HNO}_{3}-\mathrm{HCl}$; B) Extraction with $0.01 \mathrm{M}$ $\mathrm{CaCl}_{2}$, compared to the Dutch Sediment Quality Criteria (SQC) based on Target values.

a)

\begin{tabular}{|c|c|c|c|c|c|c|}
\hline & $\begin{array}{c}\mathrm{Zn} \\
{[\mathrm{mg} / \mathrm{kg}]}\end{array}$ & $\begin{array}{c}\text { Cd } \\
{[\mathrm{mg} / \mathrm{kg}]}\end{array}$ & $\begin{array}{c}\mathrm{Cr} \\
{[\mathrm{mg} / \mathrm{kg}]}\end{array}$ & $\begin{array}{c}\mathrm{Cu} \\
{[\mathrm{mg} / \mathrm{kg}]}\end{array}$ & $\begin{array}{c}\mathrm{Ni} \\
{[\mathrm{mg} / \mathrm{kg}]}\end{array}$ & $\begin{array}{c}\mathbf{P b} \\
{[\mathbf{m g} / \mathbf{k g}} \\
]\end{array}$ \\
\hline Detection limit & 5 & 0.05 & 0.8 & 3 & 1.6 & 0.3 \\
\hline $\begin{array}{l}\text { Veenkampen } \\
\text { sediment }\end{array}$ & 75 & 0.47 & 47.5 & 26 & 31.7 & 31.9 \\
\hline Dutch SQC $^{a}$ & 140 & 0.8 & 100 & 36 & 35 & 85 \\
\hline \multicolumn{7}{|l|}{ b) } \\
\hline & $\begin{array}{c}\mathrm{Zn} \\
{[\mathrm{mg} / \mathrm{kg}]}\end{array}$ & $\begin{array}{c}\text { Cd } \\
{[\mathrm{mg} / \mathrm{kg}]}\end{array}$ & $\begin{array}{c}\mathrm{Cr} \\
{[\mathrm{mg} / \mathrm{kg}]}\end{array}$ & $\begin{array}{c}\mathrm{Cu} \\
{[\mathrm{mg} / \mathrm{kg}]}\end{array}$ & $\begin{array}{c}\mathrm{Ni} \\
{[\mathrm{mg} / \mathrm{kg}]}\end{array}$ & $\begin{array}{c}\mathrm{Pb} \\
{[\mathrm{mg} / \mathrm{kg}]}\end{array}$ \\
\hline Detection limit & 300 & 3 & 5 & 400 & 6 & 20 \\
\hline $\begin{array}{l}\text { Veenkampen } \\
\text { sediment }\end{array}$ & 11 & 1 & 14 & 6 & 16 & 0 \\
\hline Dutch SQC a & 140 & 0.8 & 100 & 36 & 35 & 85 \\
\hline
\end{tabular}

a Values giving an indication of the benchmark for environmental quality in the long term on the assumption of negligible risks to the ecosystem (background concentration of metals (Cb) presented in Table 6.2 in Lijzen et al., 2001). ${ }^{377}$ 
Table A2.3. Water Quality Parameters (Mean \pm SD)

\begin{tabular}{lccccc}
\hline Species & $\mathbf{p H}$ & $\begin{array}{c}\mathbf{O}_{\mathbf{2}} \\
{[\mathbf{m g} / \mathbf{l}]}\end{array}$ & $\begin{array}{c}\mathbf{E C} \\
{[\mathbf{\mu S} / \mathbf{m}]}\end{array}$ & $\begin{array}{c}\mathbf{T} \\
{\left[{ }^{\circ} \mathbf{C}\right]}\end{array}$ & $\begin{array}{c}\mathbf{N H}_{3} \\
{[\mathbf{m g N} / \mathbf{l}]}\end{array}$ \\
\hline G. pulex & $7.7 \pm 0.1$ & $8.8 \pm 0.1$ & $474 \pm 17$ & $16.2 \pm 0.06$ & $0.04 \pm 0.01$ \\
H. azteca & $7.4 \pm 0.1$ & $9.2 \pm 0.2$ & $409 \pm 15$ & $16.0 \pm 0.04$ & $0.04 \pm 0.01$ \\
A. aquaticus & $7.3 \pm 0.1$ & $9.0 \pm 0.1$ & $562 \pm 110$ & $15.8 \pm 0.09$ & n.a. \\
S. corneum & $7.3 \pm 0.2$ & $8.8 \pm 0.2$ & $473 \pm 16$ & $16.7 \pm 0.08$ & $0.02 \pm 0.003$ \\
L. variegatus & $7.2 \pm 0.1$ & $8.7 \pm 0.2$ & $478 \pm 28$ & $15.8 \pm 0.04$ & n.a. \\
Tubifex spp. & $7.1 \pm 0.1$ & $8.8 \pm 0.2$ & $466 \pm 25$ & $15.9 \pm 0.04$ & n.a. \\
\hline n.a.= not analysed & & & & &
\end{tabular}



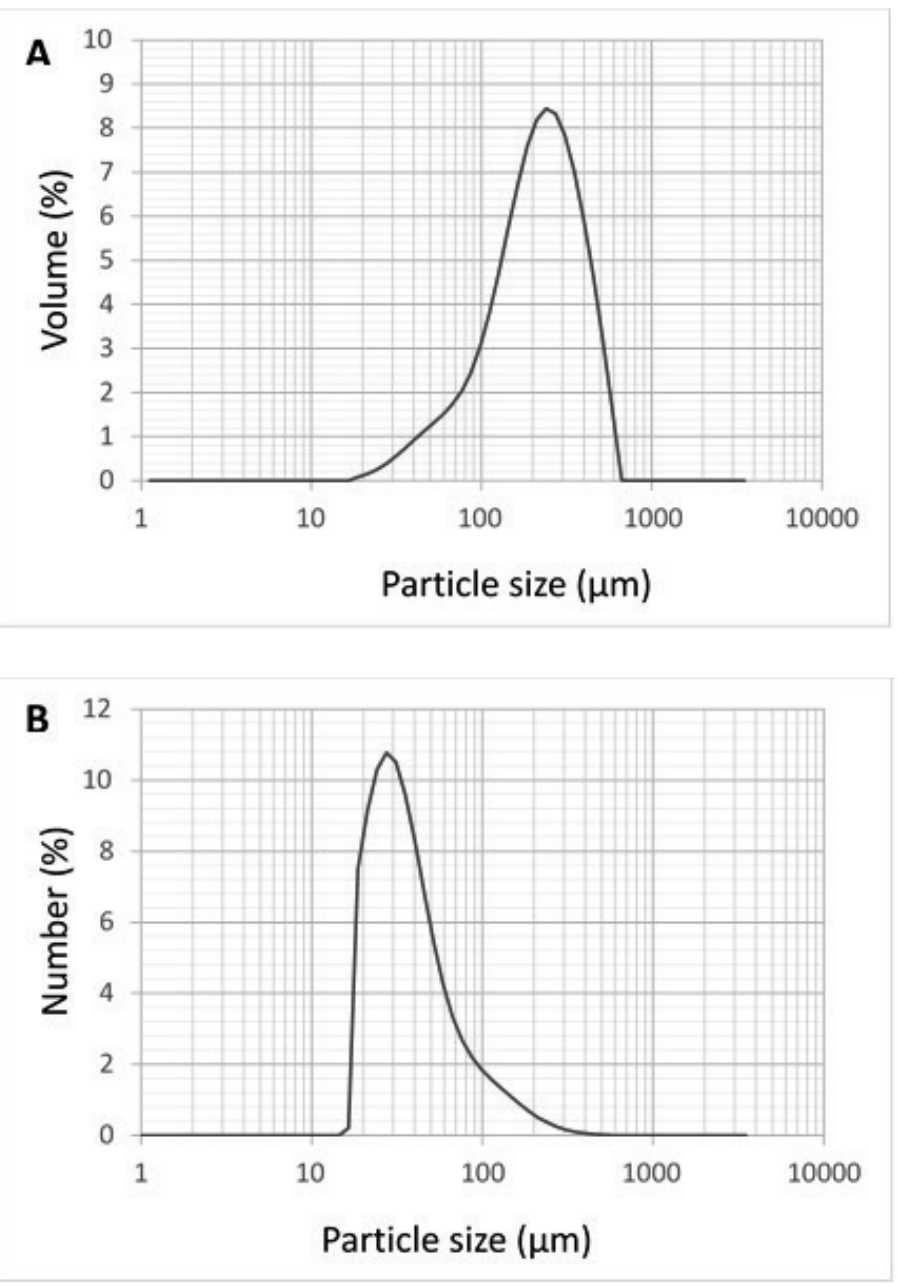

Figure A2.1. Particle Size Distribution $(\mathrm{n}=3)$ of the original microplastic mixture in: a) Volume \%; b) Number \%. 

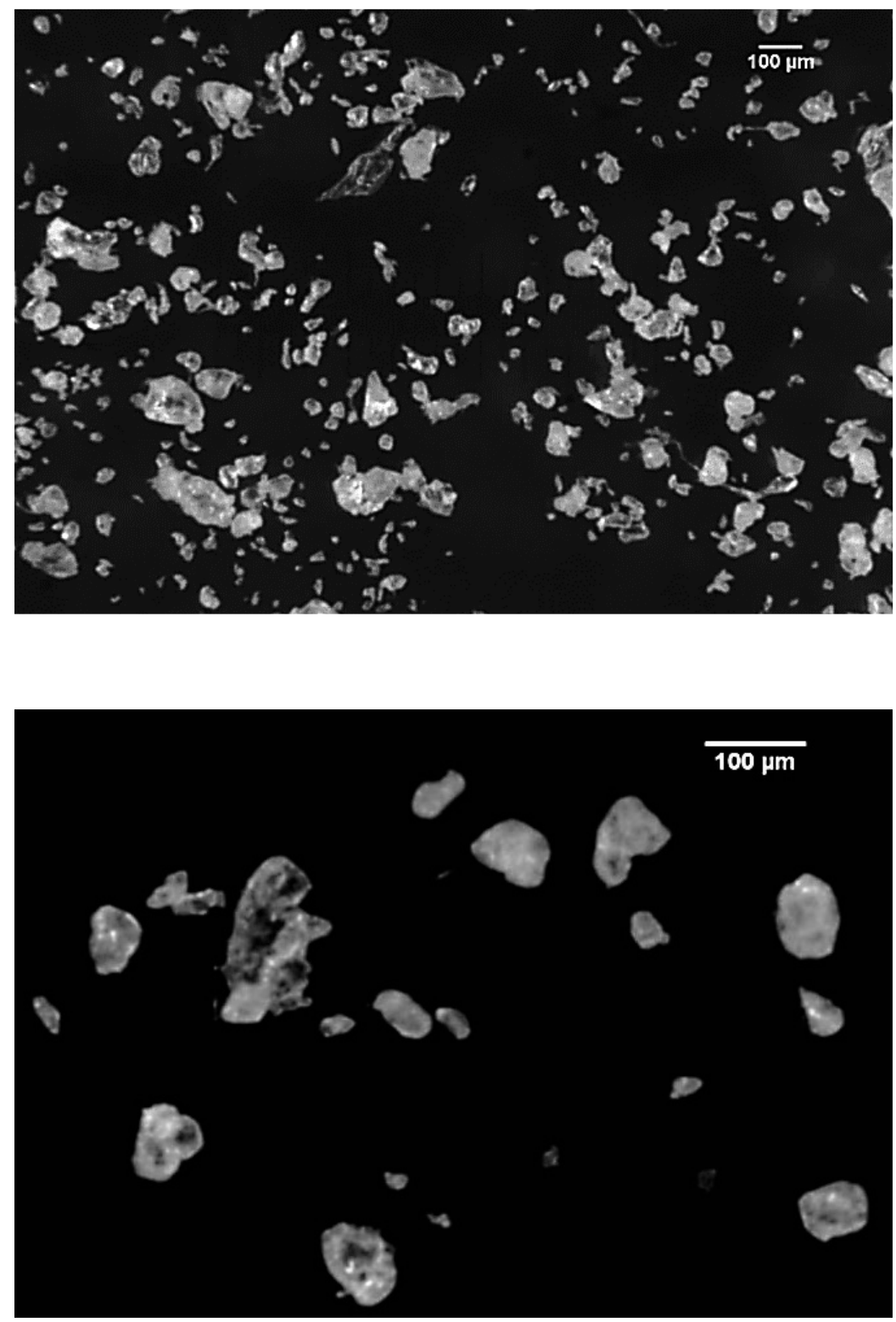

Figure A2.2. Light microscope pictures of the irregularly shaped particles (Olympus SZX10 Stereomicroscope). 


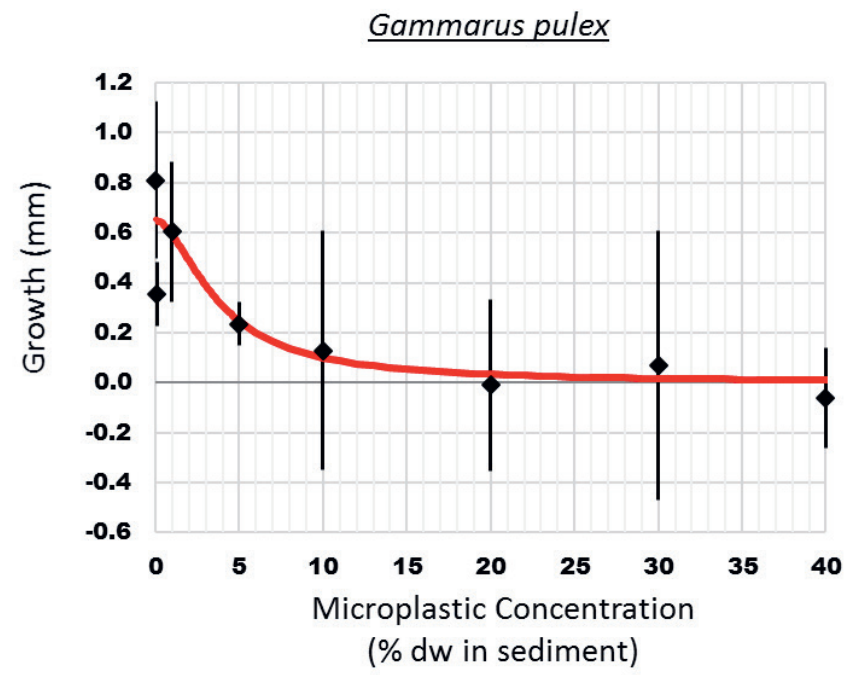

Figure A2.3. Growth of G. pulex as a function of polystyrene microplastic dose. The red curve relates to the best fit of the log-logistic response model (Eq. 2 in the main manuscript). The $50 \%$ effect $\left(\mathrm{EC}_{50}\right)$ is fitted at a dose of $3.57 \% \mathrm{dw}$. The $\mathrm{EC}_{10}$ was obtained by solving Eq 2 for the dose at $10 \%$ of the observed effect $(1.07 \% \mathrm{dw})$. 
a) Gammarus pulex

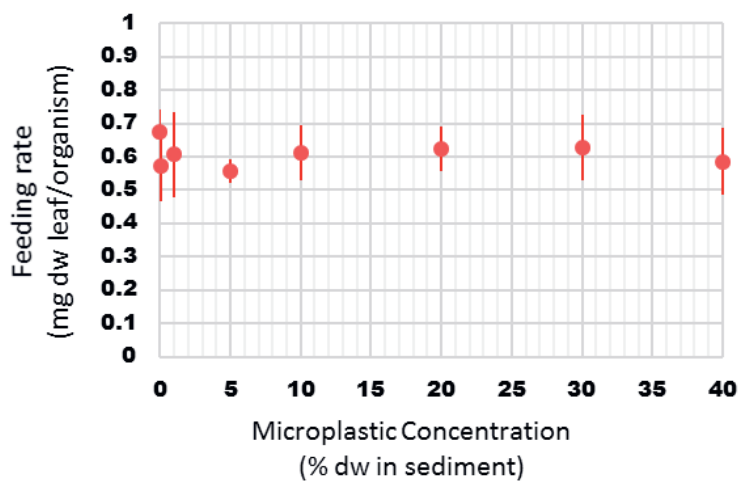

b) Hyalella azteca

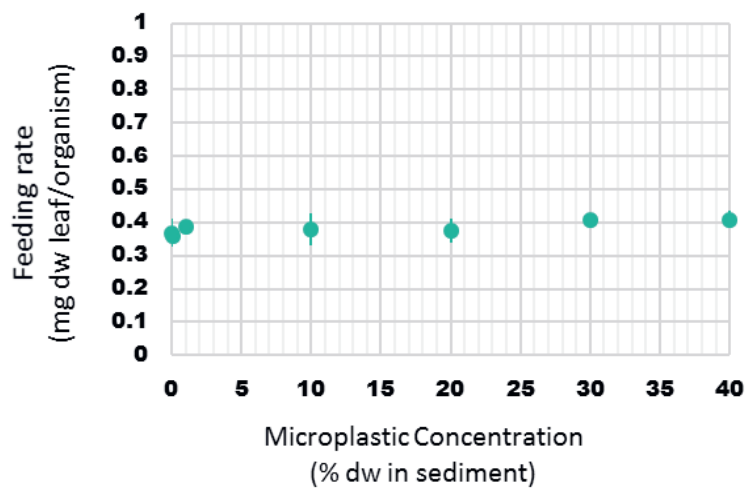

c) Asellus aquaticus

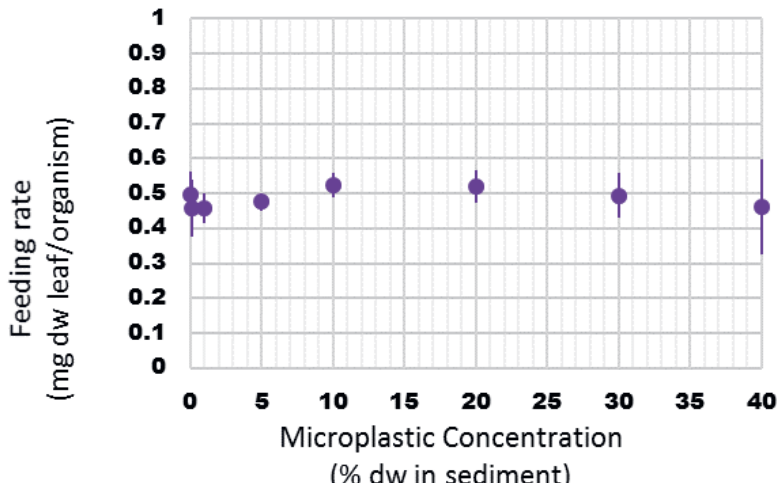

Figure A2.4. Mean feeding rate $( \pm \mathrm{SD})$ as $\mathrm{mg} \mathrm{dw}$ of Populus spp. leaves consumed per organism per day during the 28-day exposure to PS microplastic concentrations ranging from 0 to $40 \%$ in sediment (dw) for a) G. pulex; b) H. Azteca; c) A. aquaticus. 
a) Tubifex spp.

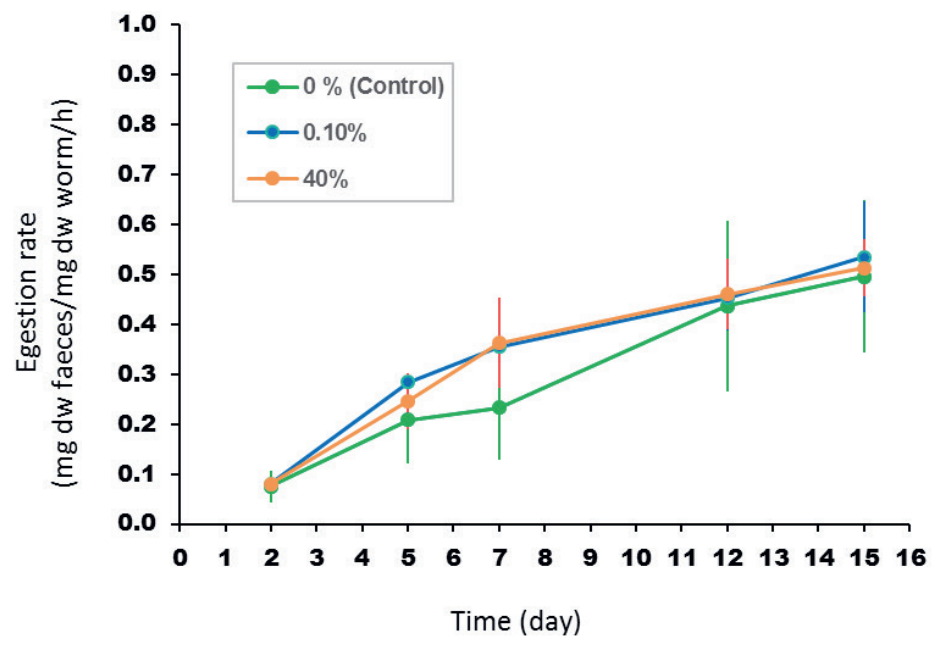

b) Lumbriculus variegatus

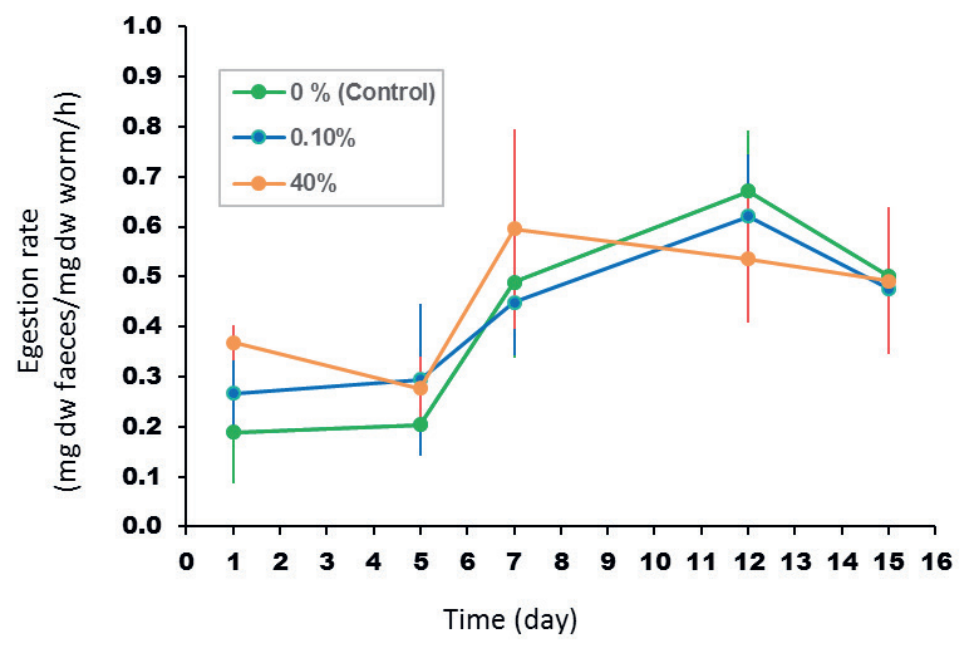

Figure A2 .5. Mean egestion rate $( \pm \mathrm{SD})$ as $\mathrm{mg} \mathrm{dw}$ of faeces egested per organism per day during a 15-d exposure to PS microplastic concentrations ranging from 0 to $40 \%$ in sediment (dw) for a) Tubifex spp; b) L. variegatus. 
a)

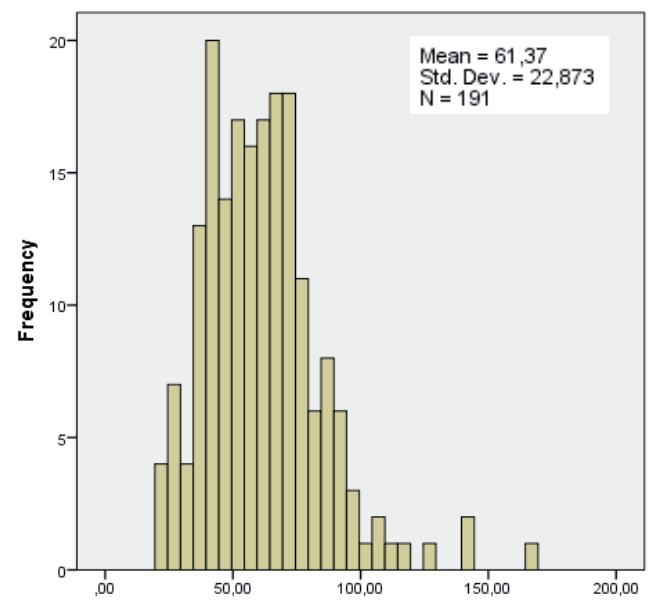

b)

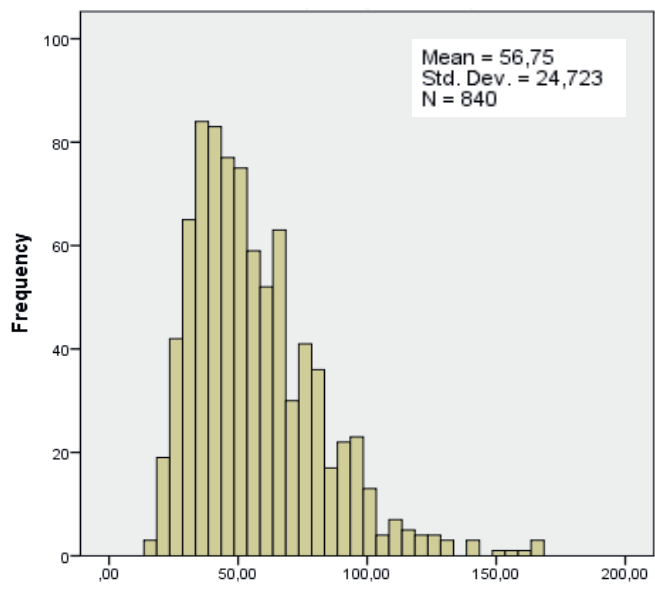

c)

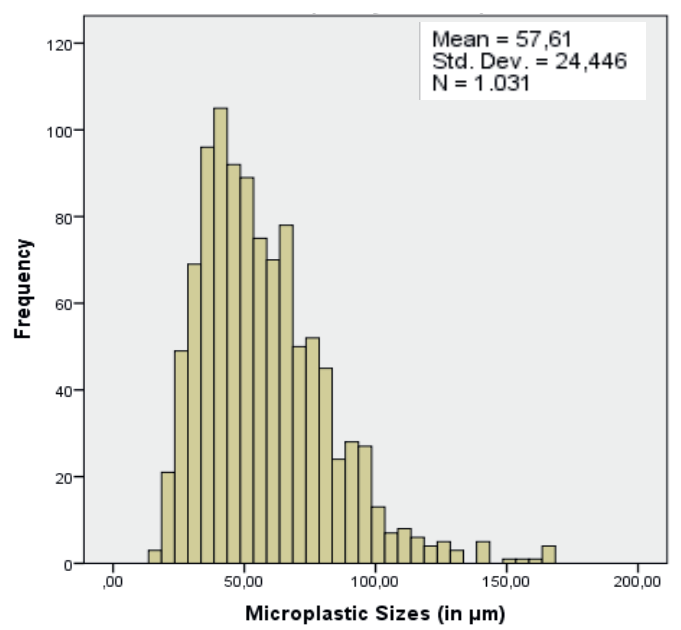

Figure A2.6. Size Frequency of a) retained microplastics; b) egested microplastics; c) total ingested microplastics (sum of $a$ and $b$ ). 
a)

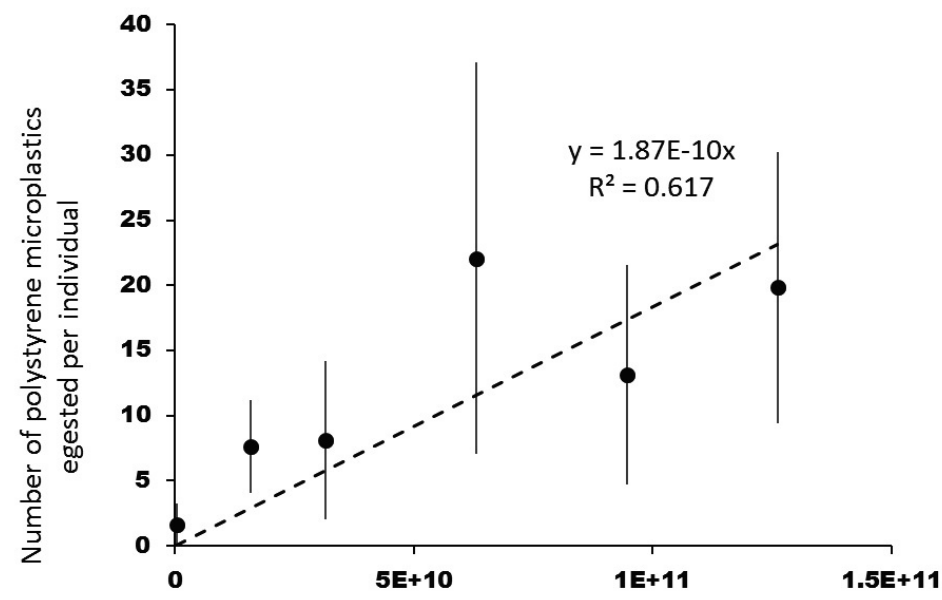

Number of polystyrene microplastics per kg of sediment

b)

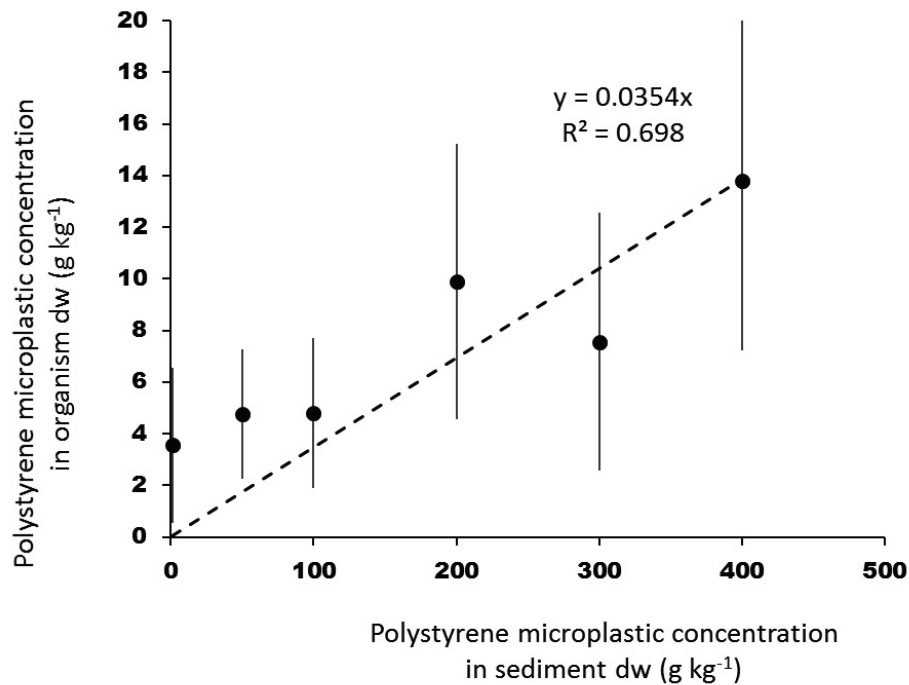

Figure A2 .7. Mean microplastic concentration $(\mathrm{n}=4)$ per individual of $G$. pulex $( \pm \mathrm{SD})$ at increasing microplastic concentrations in sediment as a) number of microplastics egested per organism by number of microplastics per $\mathrm{kg}$ of sediment (dw); b) $\mathrm{g} \mathrm{kg}^{-1}$ of microplastics egested per organism $\mathrm{dw}$ by $\mathrm{g} \mathrm{kg}^{-1}$ of microplastics per sediment (dw). 


\section{Appendix of Chapter 3}

Table A3.1. Car tires used for the manufacturing of TP.

Table A3.2. TGA, GC-MS and ICP-MS instrumental settings to analyse car tire TP.

Table A3.3. Polymer pyrolysates detected in the used car tire tread particles by TGA coupled to GC-MS.

Table A3.4. Mean $( \pm \mathrm{SD})$ temperature $\left({ }^{\circ} \mathrm{C}\right), \mathrm{pH}, \mathrm{DO}(\%)$ and $\mathrm{EC}(\mu \mathrm{S} / \mathrm{cm})$ in each treatment for all species tested.

Table A3.5. Measurements of $\mathrm{Zn}, \mathrm{S}, \mathrm{Cd}, \mathrm{Cr}, \mathrm{Cu}, \mathrm{Ni}$ and $\mathrm{Pb}$ concentrations at six car tire $\mathrm{TP}$ concentrations in sediment $\mathrm{dw}(0,0.1,0.3,1.0,3.0$ and $10 \%)$ using: a) extraction with $\mathrm{HNO}_{3}-\mathrm{HCL}$; b) extraction with $0.01 \mathrm{M} \mathrm{CaCl}_{2}$

Table A3.6. Concentration of PAHs (in $\mathrm{mg} / \mathrm{kg}$ ) at six car tire TP concentrations $(0,0.1,0.3,1.0,3.0$ and 10 $\%$ ) in sediment $\mathrm{dw}$.

Table A3.7. Mean dry weight (in $\mathrm{mg}$ ) and area (in $\mathrm{mm}^{2}$ ) of ten car tire tread particles per replicate (in quadruplicate) before and after their addition to $\mathrm{H}_{2} \mathrm{O}_{2}$ and $\mathrm{H}_{2} \mathrm{O}$ for 24 hours.

Figure A3.1. Particle size distribution of the car tire TP determined by a) volume of particles b) the number of particles.

Figure A3.2. Pictures of the car tire TP mixture taken with a CMEX camera (Euromex, The Netherlands) under an Olympus SZX10 Stereomicroscope.

Figure A3.3. Weight loss (\%) of individual car tire TP and their mixture using thermogravimetric analysis.

Figure A3.4. Pyrogram of the analysed car tire TP represented by the total ion current.

Figure A3.5. Nominal Zinc ( $\mathrm{Zn})$ concentration in sediment (X-Axis) against measured Zinc (Zn) concentration in TP-sediment mixtures (Y-Axis).

Figure A3.6. Feeding rate (mg dw leaf/organism/d) of G. pulex and A. aquaticus after 28 days of exposure to car tire $\mathrm{TP}$ at increasing concentrations in sediment.

Figure A3.7. Size frequency of the total number of car tire tread particles measured in the body of G. pulex at concentrations 3 and $10 \%$.

Figure A3.8. Size frequency of the total number of car tire tread particles measured in faeces of G. pulex at concentrations 3 and $10 \%$.

PAHs analysis

Feeding rate 
Table A3.1. Car tires used for the manufacturing of TP.

\begin{tabular}{clllll}
\hline Nr & Brand & Tire & Type $^{\mathbf{a}}$ & DOT-code $^{\mathbf{b}}$ & $\begin{array}{l}\text { Sidewall- } \\
\text { markings }\end{array}$ \\
\hline 1 & Goodyear & Ultragrip 7 & $\mathrm{w}$ & H30F 2FOR & 205/55 R16 91 T \\
2 & Michelin & Energy Radial & $\mathrm{s}$ & DU8X 2201 & $175 / 65$ R14 82 T \\
3 & Dunlop & SP Sport 2000 & $\mathrm{s}$ & K5 5F 12W & $195 / 55$ R15 85 V \\
4 & Dunlop & SP Sport 07 & s & N5HR JC1R & $175 / 70$ R14 84 T \\
5 & Continental & ContiEco & s & CNU4 PVB9 & $195 / 50$ R15 82 T \\
\hline
\end{tabular}

${ }^{a} w=$ winter tire $s=$ summer tire

${ }^{b}$ DOT $=$ Department of transportation; DOT-codes provide information on production date and location. 
Table A3.2. TGA, GC-MS and ICP-MS instrumental settings to analyse car tire TP.

\begin{tabular}{|c|c|c|}
\hline \multirow[t]{5}{*}{$T G A$} & Instrument & TGA/DSC 3+, Mettler Toledo \\
\hline & Temperature program & 30 to $850{ }^{\circ} \mathrm{C}$, at a constant heating rate of $20{ }^{\circ} \mathrm{C}$ \\
\hline & Purge gas & 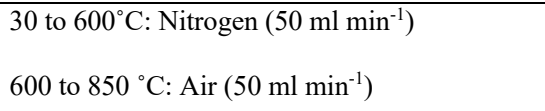 \\
\hline & Sample mass & Approximately $3 \mathrm{mg}$ \\
\hline & Sample holder (cup) & Aluminium oxide, $70 \mu 1$ \\
\hline \multirow[t]{7}{*}{$G C$} & Instrument & GC (7820A), Agilent Technologies \\
\hline & Sample injection & Manual, $2 \mu 1$ \\
\hline & Injector & Split-splitless \\
\hline & Mode & Split ratio 7:1 \\
\hline & Temperature & 250 \\
\hline & Flow & $1 \mathrm{ml} \mathrm{min}-1$ \\
\hline & Temperature program & $50 \rightarrow 300$ at 8.5 degrees min- 1 \\
\hline \multirow[t]{4}{*}{$M S$} & Instrument & MS (5977B), Agilent Technologies \\
\hline & Ionization energy & $70 \mathrm{eV}$ \\
\hline & Scan rate & 8.6 scans sec- 1 \\
\hline & Mass range & $35-330$ \\
\hline \multirow[t]{8}{*}{$I C P-M S$} & Instrument & X Series 2, Thermo Fisher Scientific \\
\hline & Forwarded Power & $1200 \mathrm{~W}$ \\
\hline & Nebuliser Gas Flow & $0.9 \mathrm{~L}$ min- 1 \\
\hline & Auxiliary Gas Flow & $0.8 \mathrm{~L}$ min- 1 \\
\hline & Cool Gas Flow & $13.0 \mathrm{~L}$ min-1 \\
\hline & CCT Gas & $\mathrm{He} / \mathrm{H}$ Mixture (with 7\% H) \\
\hline & CCT Gas Flow & $5.2 \mathrm{ml} \mathrm{min}-1$ \\
\hline & Dwell Time & $100 \mathrm{~ms}$ \\
\hline
\end{tabular}


Table A3.3. Polymer pyrolysates detected in the used car tire tread particles by TGA coupled to GC-MS.

\begin{tabular}{|c|c|c|c|}
\hline Original polymer type ${ }^{(a)}$ & Pyrolysate $^{(\mathbf{b})}$ & $\begin{array}{l}\text { Target ion } \\
\text { (qualifiers) }\end{array}$ & $\begin{array}{l}\text { Present retention } \\
\text { time }^{(c)}\end{array}$ \\
\hline \multirow[t]{2}{*}{ Polyisoprene } & Methyl-butadiene (monomer) & $67(68,53,39)$ & $6.8 \mathrm{~min}$ \\
\hline & Dipentene (dimer) & $68(93,136)$ & $6.8 \mathrm{~min}$ \\
\hline Styrene butadiene rubber & Butadiene (monomer) & $39(54,53)$ & $4.1 \mathrm{~min}$ \\
\hline (SBR) & Styrene (monomer) & $104(103,78,51)$ & $4.3 \mathrm{~min}$ \\
\hline $\begin{array}{l}\text { Benzothiazole } \\
\text { (vulcanisator) }\end{array}$ & Benzothiazole (vulcanisator) & $135(108)$ & $10.9 \mathrm{~min}$ \\
\hline
\end{tabular}

(a) Polymers used during tire production ${ }^{189}$.

(b) Pyrolysates of the polymers used during tire production (i.e. indicated under (a), ${ }^{189}$, identified in the present study.

(c) GC-MS retention times assessed in the present study, see also Fig S4. 
Table A3.4. Mean $( \pm \mathrm{SD})$ temperature $\left({ }^{\circ} \mathrm{C}\right), \mathrm{pH}, \mathrm{DO}(\%)$ and $\mathrm{EC}(\mu \mathrm{S} / \mathrm{cm})$ in each treatment for all species tested. All parameters were measured in the 3 replicates at 5 time points along the experiment, except for EC, which was only measured at the start and at the end of the experiment.

\begin{tabular}{|c|c|c|c|c|c|c|}
\hline G. pulex & $0 \%$ & $0.1 \%$ & $0.3 \%$ & $1 \%$ & $3 \%$ & $10 \%$ \\
\hline $\mathrm{T}\left({ }^{\circ} \mathrm{C}\right)$ & $15.6 \pm 0.06$ & $15.6 \pm 0.05$ & $15.6 \pm 0.07$ & $15.6 \pm 0.05$ & $15.6 \pm 0.06$ & $15.6 \pm 0.04$ \\
\hline $\mathrm{pH}$ & $8.14 \pm 0.08$ & $8.15 \pm 0.13$ & $8.15 \pm 0.12$ & $8.24 \pm 0.08$ & $8.23 \pm 0.08$ & $8.43 \pm 0.1$ \\
\hline DO (\%) & $96.2 \pm 2.67$ & $96.1 \pm 2.70$ & $97.2 \pm 1.04$ & $97.6 \pm 1.29$ & $96.1 \pm 1.44$ & $96.7 \pm 0.71$ \\
\hline $\mathrm{EC}(\mu \mathrm{S} / \mathrm{cm})$ & $694.8 \pm 79.9$ & $700.7 \pm 91.6$ & $677.8 \pm 144.6$ & $744.7 \pm 114.9$ & $762.8 \pm 135.4$ & $623.3 \pm 78.9$ \\
\hline A. aquaticus & $0 \%$ & $0.1 \%$ & $0.3 \%$ & $1 \%$ & $3 \%$ & $10 \%$ \\
\hline $\mathrm{T}\left({ }^{\circ} \mathrm{C}\right)$ & $15.6 \pm 0.04$ & $15.6 \pm 0.04$ & $15.6 \pm 0.03$ & $15.6 \pm 0.06$ & $15.6 \pm 0.06$ & $15.6 \pm 0.03$ \\
\hline $\mathrm{pH}$ & $8.14 \pm 0.07$ & $8.13 \pm 0.14$ & $8.14 \pm 0.08$ & $8.23 \pm 0.07$ & $8.29 \pm 0.08$ & $8.3 \pm 0.1$ \\
\hline DO (mg/l) & $96.9 \pm 0.77$ & $96.5 \pm 1.25$ & $96.6 \pm 0.92$ & $96.4 \pm 1.71$ & $97.1 \pm 1.07$ & $93.6 \pm 7.78$ \\
\hline $\mathrm{EC}(\mu \mathrm{S} / \mathrm{cm})$ & $640.0 \pm 62.4$ & $782.3 \pm 152.0$ & $615.2 \pm 42.2$ & $851.7 \pm 162.0$ & $700.8 \pm 70.1$ & $724.7 \pm 139.4$ \\
\hline Tubifex spp. & $0 \%$ & $0.1 \%$ & $0.3 \%$ & $1 \%$ & $3 \%$ & $10 \%$ \\
\hline $\mathrm{T}\left({ }^{\circ} \mathrm{C}\right)$ & $15.6 \pm 0.09$ & $15.6 \pm 0.09$ & $15.7 \pm 0.04$ & $15.6 \pm 0.06$ & $15.6 \pm 0.06$ & $15.6 \pm 0.05$ \\
\hline $\mathrm{pH}$ & $8.13 \pm 0.14$ & $8.10 \pm 0.16$ & $8.10 \pm 0.16$ & $8.17 \pm 0.14$ & $8.31 \pm 0.08$ & $8.42 \pm 0.14$ \\
\hline DO (mg/l) & $96.5 \pm 2.6$ & $97.0 \pm 0.83$ & $97.0 \pm 0.93$ & $93.8 \pm 9.14$ & $96.3 \pm 1.49$ & $96.9 \pm 0.96$ \\
\hline $\mathrm{EC}(\mu \mathrm{S} / \mathrm{cm})$ & $669.8 \pm 72.0$ & $639.3 \pm 50.3$ & $622.8 \pm 48.2$ & $596.7 \pm 41.9$ & $624.0 \pm 64.0$ & $674.8 \pm 82.8$ \\
\hline L. variegatus & $0 \%$ & $0.1 \%$ & $0.3 \%$ & $1 \%$ & $3 \%$ & $10 \%$ \\
\hline $\mathrm{T}\left({ }^{\circ} \mathrm{C}\right)$ & $15.6 \pm 0.09$ & $15.7 \pm 0.31$ & $15.6 \pm 0.04$ & $15.6 \pm 0.06$ & $15.6 \pm 0.08$ & $15.6 \pm 0.05$ \\
\hline $\mathrm{pH}$ & $8.20 \pm 0.10$ & $8.12 \pm 0.08$ & $8.20 \pm 0.12$ & $8.19 \pm 0.09$ & $8.29 \pm 0.13$ & $8.45 \pm 0.12$ \\
\hline DO (mg/l) & $97.0 \pm 0.84$ & $96.2 \pm 1.23$ & $96.6 \pm 1.59$ & $97.5 \pm 0.81$ & $96.1 \pm 3.00$ & $96.6 \pm 1.23$ \\
\hline $\mathrm{EC}(\mu \mathrm{S} / \mathrm{cm})$ & $674.0 \pm 91.4$ & $607.7 \pm 34.9$ & $657.7 \pm 84.3$ & $612.3 \pm 44.7$ & $639.7 \pm 65.2$ & $640.1 \pm 80.0$ \\
\hline
\end{tabular}


Table A3.5. Measurements of $\mathrm{Zn}, \mathrm{S}, \mathrm{Cd}, \mathrm{Cr}, \mathrm{Cu}, \mathrm{Ni}$ and $\mathrm{Pb}$ concentrations at six car tire TP concentrations in sediment $\mathrm{dw}(0,0.1,0.3,1.0,3.0$ and $10 \%)$ using:

a) Extraction with $\mathrm{HNO}_{3}-\mathrm{HCL}$.

\begin{tabular}{lccccccc}
\hline Treatment & $\begin{array}{c}\mathrm{Zn} \\
{[\mathrm{mg} / \mathrm{kg}]}\end{array}$ & $\begin{array}{c}\mathrm{S} \\
{[\mathrm{mg} / \mathrm{kg}]}\end{array}$ & $\begin{array}{c}\mathrm{Cd} \\
{[\mathrm{mg} / \mathrm{kg}]}\end{array}$ & $\begin{array}{c}\mathrm{Cr} \\
{[\mathrm{mg} / \mathrm{kg}]}\end{array}$ & $\begin{array}{c}\mathrm{Cu} \\
{[\mathrm{mg} / \mathrm{kg}]}\end{array}$ & $\begin{array}{c}\mathrm{Ni} \\
{[\mathrm{mg} / \mathrm{kg}]}\end{array}$ & $\begin{array}{c}\mathrm{Pb} \\
{[\mathrm{mg} / \mathrm{kg}]}\end{array}$ \\
\hline Detection limit & 5 & 30 & 0.05 & 0.8 & 3 & 1.6 & 0.3 \\
$0 \%$ & 75 & 13711 & 0.47 & 47.5 & 26 & 31.7 & 31.9 \\
$0.10 \%$ & 84 & 14441 & 0.53 & 49.1 & 26 & 33.1 & 33.4 \\
$0.30 \%$ & 143 & 14267 & 0.49 & 46.8 & 25 & 31.3 & 32.1 \\
$1.0 \%$ & 97 & 14292 & 0.53 & 47.5 & 25 & 31.8 & 33.0 \\
$3.0 \%$ & 277 & 14086 & 0.52 & 47.0 & 26 & 31.9 & 32.5 \\
$10 \%$ & 735 & 13253 & 0.54 & 42.1 & 24 & 27.7 & 29.1 \\
\hline
\end{tabular}

b) Extraction with $0.01 \mathrm{M} \mathrm{CaCl}_{2}$.

\begin{tabular}{lccccccc}
\hline Treatment & $\begin{array}{c}\mathrm{Zn} \\
{[\mathrm{mg} / \mathrm{kg}]}\end{array}$ & $\begin{array}{c}\mathrm{S} \\
{[\mathrm{mg} / \mathrm{kg}]}\end{array}$ & $\begin{array}{c}\mathrm{Cd} \\
{[\mu \mathrm{g} / \mathrm{kg}]}\end{array}$ & $\begin{array}{c}\mathrm{Cr} \\
{[\mu \mathrm{g} / \mathrm{kg}]}\end{array}$ & $\begin{array}{c}\mathrm{Cu} \\
{[\mu \mathrm{g} / \mathrm{kg}]}\end{array}$ & $\begin{array}{c}\mathrm{Ni} \\
{[\mu \mathrm{g} / \mathrm{kg}]}\end{array}$ & $\begin{array}{c}\mathrm{Pb} \\
{[\mu \mathrm{g} / \mathrm{kg}]}\end{array}$ \\
\hline Detection limit & 300 & 0.6 & 3 & 5 & 400 & 6 & 20 \\
$0 \%$ & & & & & & & \\
$0.10 \%$ & n.d. & 251 & n.d. & 14 & n.d. & 16 & n.d. \\
$0.30 \%$ & n.d & 254 & n.d. & 13 & n.d. & 16 & n.d. \\
$1 \%$ & n.d & 261 & n.d. & 13 & n.d. & 17 & n.d. \\
$3 \%$ & n.d & 206 & n.d. & 12 & n.d. & 16 & n.d. \\
$10 \%$ & n.d & 218 & n.d. & 12 & n.d. & 17 & n.d. \\
\hline & n.d & 170 & n.d. & 13 & n.d. & 16 & n.d. \\
\hline
\end{tabular}

n.d.: lower than detection limit 
Table A3.6. Concentration of PAHs (in $\mathrm{mg} / \mathrm{kg}$ ) at six car tire TP concentrations $(0,0.1$, $0.3,1.0,3.0$ and $10 \%$ ) in sediment dw.

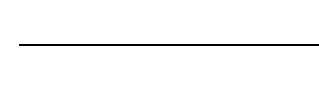

Fluorene

Phenanthrene

Anthracene

Fluoranthene

Pyrene

B(a)anthracene

Chrysene

B(e)pyrene

$\mathrm{B}(\mathrm{b})$ fluoranthene

$\mathrm{B}(\mathrm{k})$ fluoranthene

B(a)pyrene

$\mathrm{Db}(\mathrm{ah})$ anthracene

B(ghi)perylene

Ind(123)pyrene

$\Sigma \mathrm{PAH}$
$0 \%$

0.45

0.12

1.81

1.40

0.78

0.74

0.67

0.94

0.39

0.75

0.52

0.09

0.62

9.29

$143.84 *$
$0.1 \%$

$8.30 *$

$13.07^{*}$

$0.3 \%$

$4.06^{*}$

$29.48 *$

$22.73 *$

$12.82 *$

$14.96^{*}$

$6.37 *$

$9.12 *$

$4.19 *$

$7.83 *$

4.51 *

$0.95 *$

$5.45^{*}$

0.79

0.39

0.40

0.48

0.09

0.13

1.38

1.24

2.04

1.65

0.60

0.95

0.85

0.74

0.47

0.65

0.27

0.51

0.36

0.05

0.37

7.82

10.83
$3 \%$

$10 \%$

* Outlier 
Table A3.7. Mean dry weight (in $\mathrm{mg}$ ) and area (in $\mathrm{mm}^{2}$ ) of ten car tire tread particles per replicate (in quadruplicate) before and after their addition to $\mathrm{H}_{2} \mathrm{O}_{2}$ and $\mathrm{H}_{2} \mathrm{O}$ for 24 hours.

\begin{tabular}{|c|c|c|c|c|c|c|c|}
\hline \multirow[t]{2}{*}{ Treatment } & \multirow[t]{2}{*}{ Replica } & \multicolumn{3}{|c|}{ Dry Weight (mg) } & \multicolumn{3}{|c|}{ Area $\left(\mathbf{m m}^{2}\right)$} \\
\hline & & Before & After & Difference & Before & After & Difference \\
\hline \multirow{4}{*}{$\mathrm{H}_{2} \mathrm{O}_{2}$} & 1 & 11,592 & 11,643 & $-0,051$ & 6,244 & 6,343 & $-0,099$ \\
\hline & 2 & 15,466 & 15,34 & 0,126 & 6,208 & 6,109 & 0,098 \\
\hline & 3 & 14,79 & 14,823 & $-0,033$ & 7,733 & 7,755 & $-0,022$ \\
\hline & 4 & 11,296 & 11,301 & $-0,005$ & 5,481 & 5,571 & $-0,089$ \\
\hline \multirow{4}{*}{$\mathrm{H}_{2} \mathrm{O}$} & 1 & 13,665 & 13,601 & 0,064 & 6,398 & 6,374 & 0,024 \\
\hline & 2 & 16,509 & 16,382 & 0,127 & 6,718 & 6,706 & 0,012 \\
\hline & 3 & 14,259 & 14,22 & 0,039 & 6,672 & 6,683 & $-0,011$ \\
\hline & 4 & 11,868 & 11,784 & 0,084 & 5,359 & 5,322 & 0,037 \\
\hline
\end{tabular}




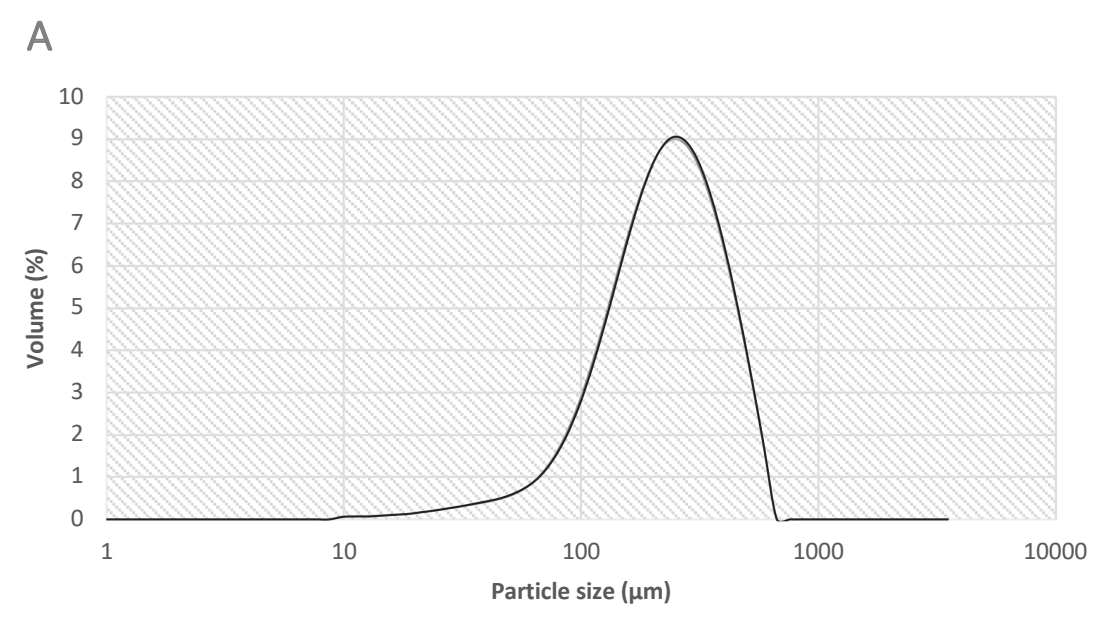

\section{B}

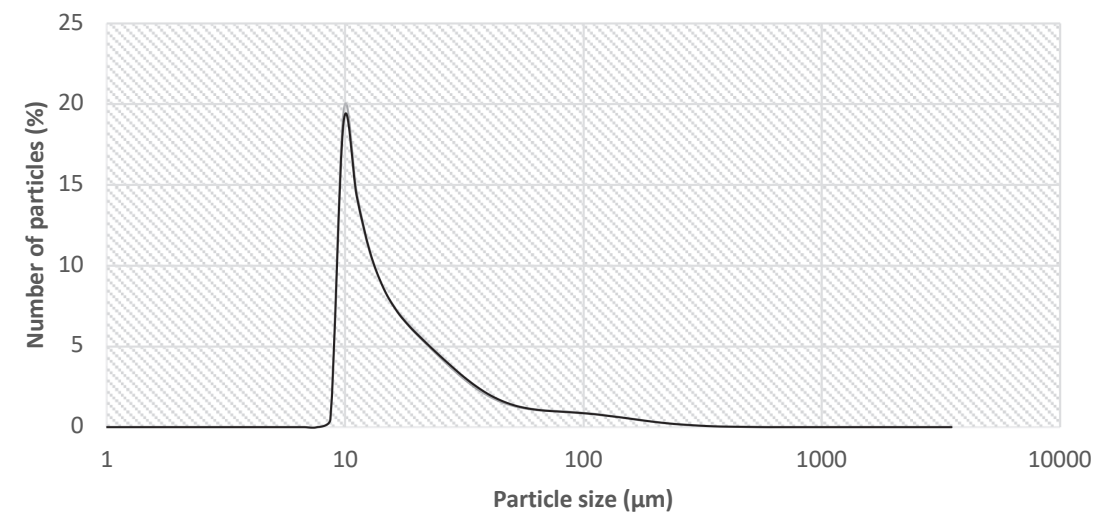

Figure A3.1. Particle size distribution of the car tire TP determined by a) volume of particles b) the number of particles. Both graphs contain three repeated measurements. 


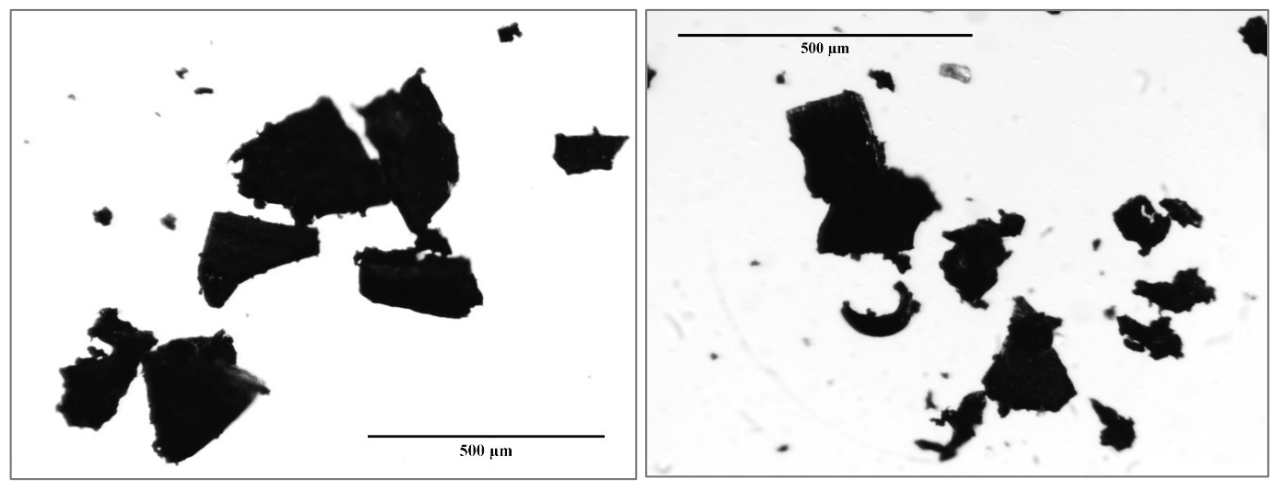

Figure A3.2. Pictures of the car tire TP mixture taken with a CMEX camera (Euromex, The Netherlands) under an Olympus SZX10 Stereomicroscope. 


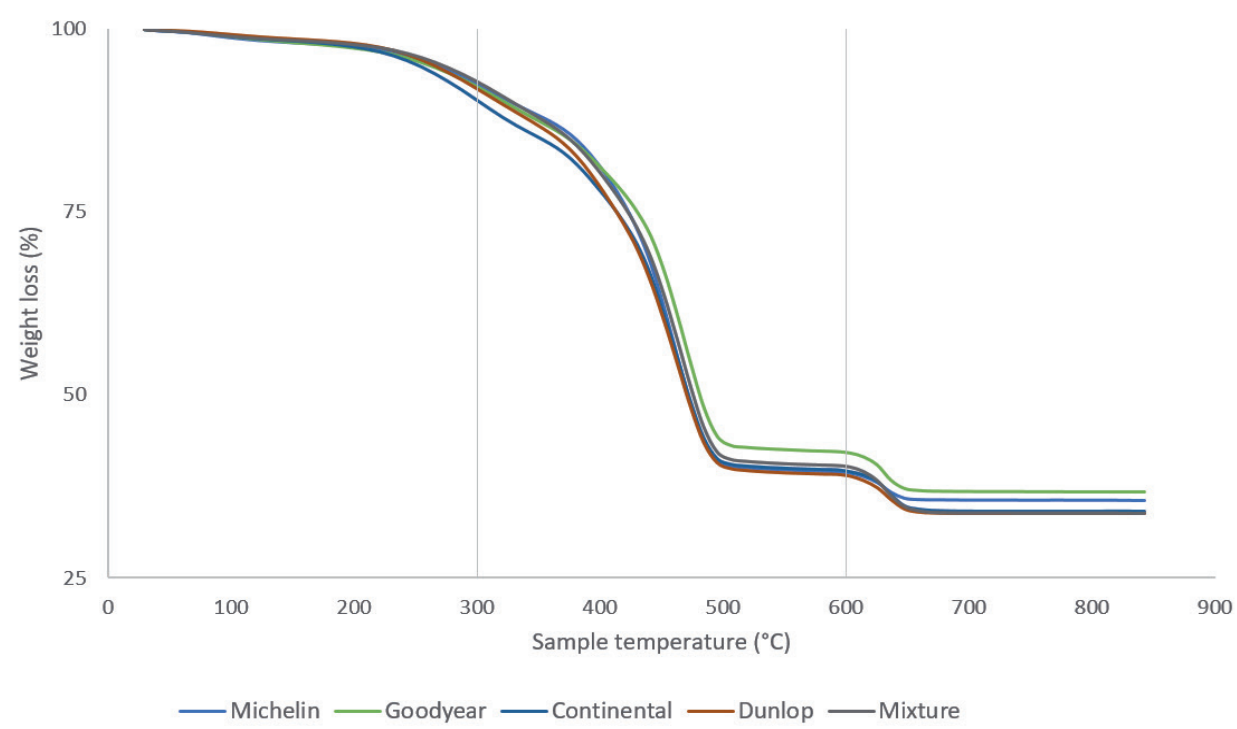

Figure A3.3. Weight loss (\%) of individual car tire TP and their mixture using thermogravimetric analysis to distinguish between (i) volatile substances (that vaporize between $\left.30-300^{\circ} \mathrm{C}\right)$, (ii) the actual polymer $\left(300-600^{\circ} \mathrm{C}\right)$, (iii) black carbon $\left(600-850^{\circ} \mathrm{C}\right)$, and the residual (iv) inorganic fillers. 


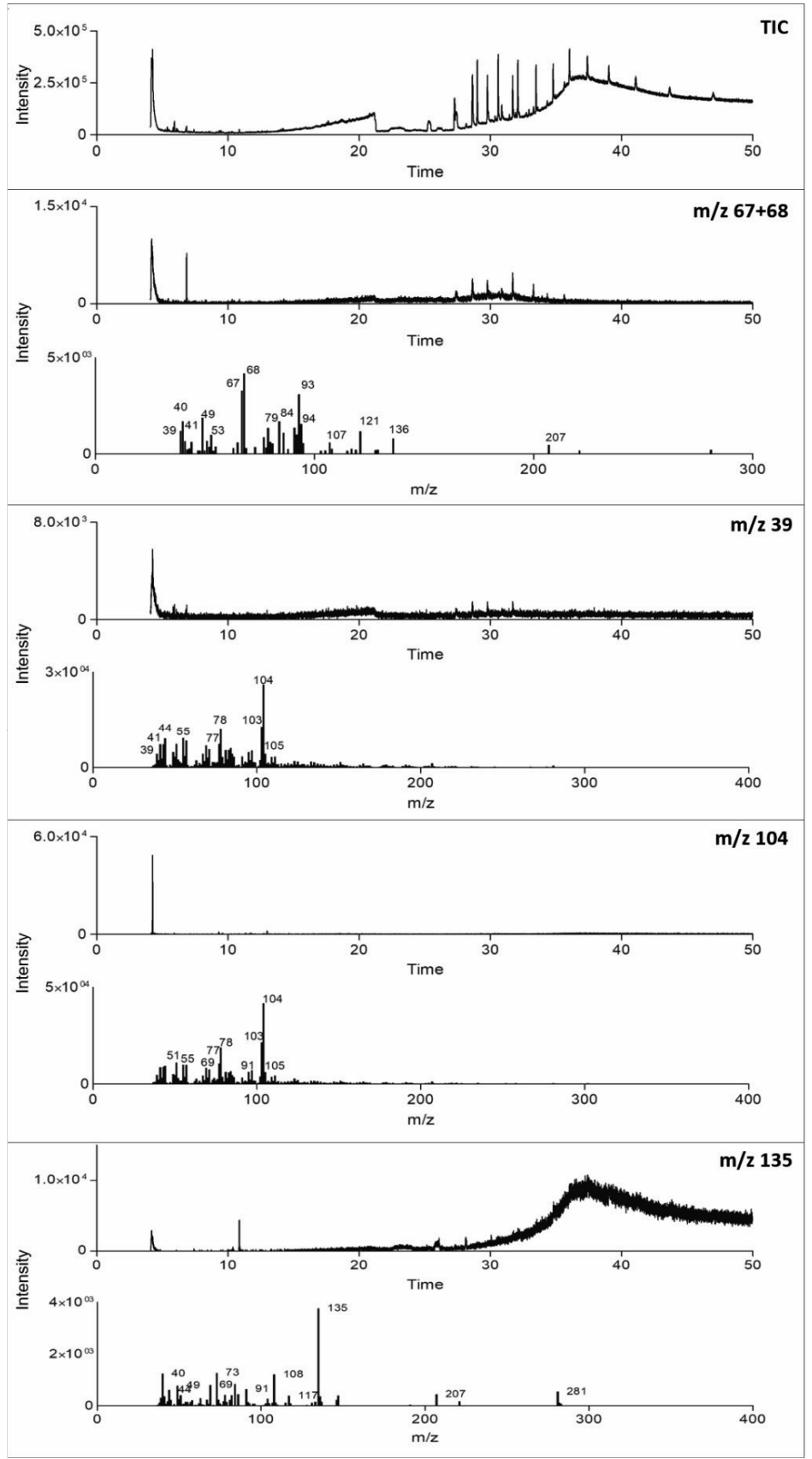

Figure A3.4. Pyrogram of the analysed car tire TP represented by the total ion current (Total ion chromatogram, TIC, $1^{\text {st }}$ Panel). Second - fifth panel: Ion chromatograms and mass spectra of identified polymer pyrolysates (see Table A3.3). 


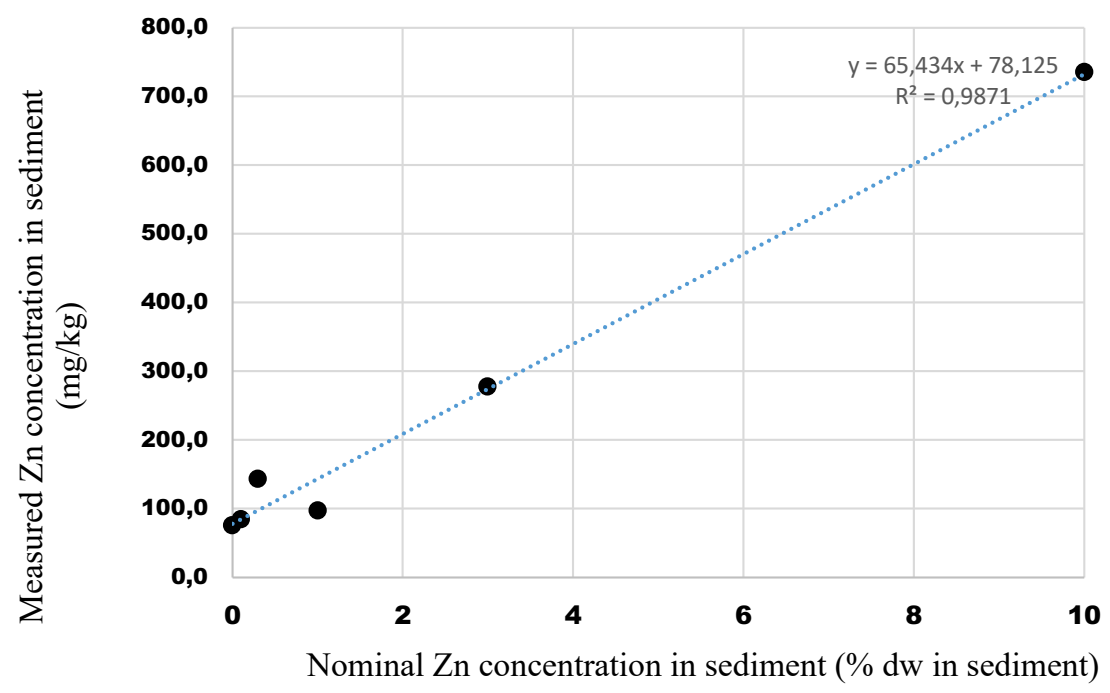

Figure A3.5. Nominal Zinc (Zn) concentration in sediment (X-Axis) against measured Zinc (Zn) concentration in TP-sediment mixtures (Y-Axis). 


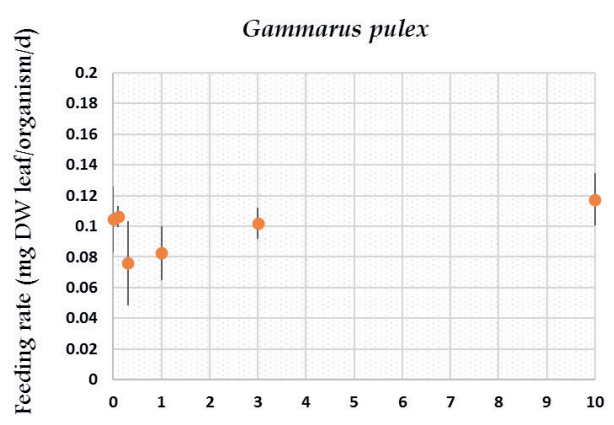

Car tire TP concentration (\% DW in sediment)

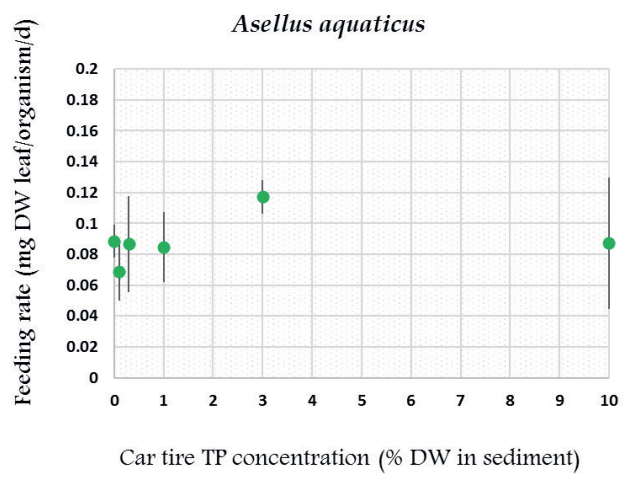

Figure A3.6. Feeding rate (mg dw leaf/organism/d) of G. pulex and A. aquaticus after 28 days of exposure to car tire TP at increasing concentrations in sediment. Error bars are mean $\pm \mathrm{SD} \mathrm{n}=3$. 


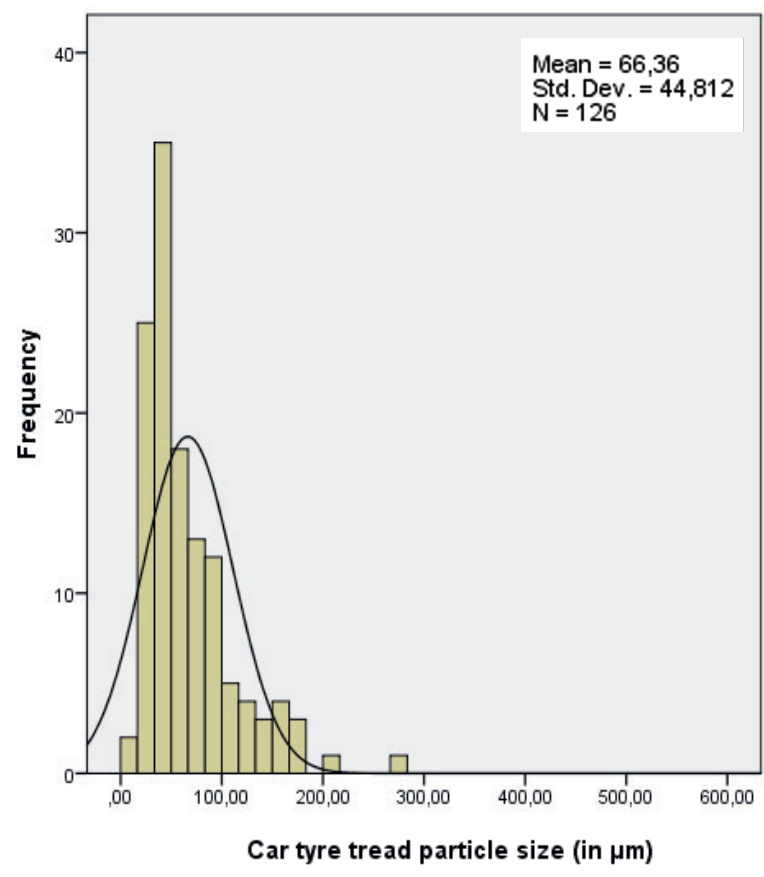

Figure A3.7. Size frequency of the total number of car tire tread particles measured in the body of $G$. pulex at concentrations 3 and $10 \%$. 


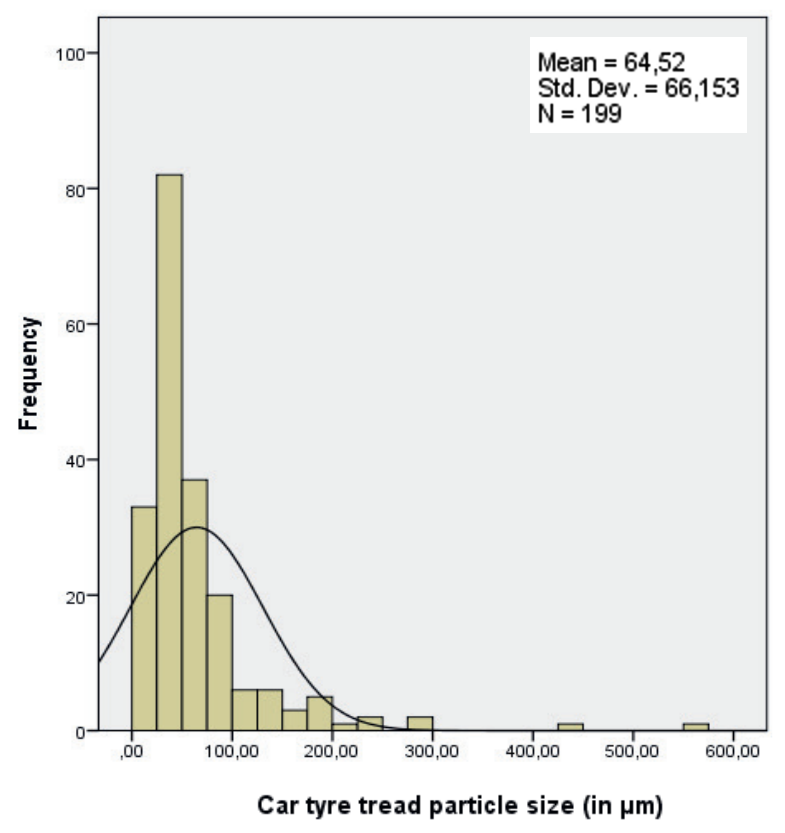

Figure A3.8. Size frequency of the total number of car tire tread particles measured in faeces of $G$. pulex at concentrations 3 and $10 \%$. 
PAHs were extracted from the sediment-TP mixtures using accelerated solvent extraction (ASE) with a mixture of $\mathrm{n}$-hexane and acetone $(1: 1, \mathrm{v} / \mathrm{v})$ at $100{ }^{\circ} \mathrm{C}$, heat time $5 \mathrm{~min}$., static time 5 min., 2 cycles. The extractions were passed through a $10 \%$ aluminium oxide column to remove polar components The solution was concentrated on a modified Kuderna-Danish apparatus followed by evaporation to $1 \mathrm{ml}$ under a gentle flow of nitrogen. Subsequently the sample was eluted with $30 \mathrm{ml}$ of hexane over a column with $4 \mathrm{~g}$ of aluminium oxide (10\% water), followed by evaporation to $1 \mathrm{ml}$ under a gentle flow of nitrogen.. Samples were exchanged to acetonitrile, after which 2-methylchrysene was added as an internal standard. PAHs were analysed on a Agilent 1100 High Performance Liquid Chromatograph (HPLC) equipped with $250 \times 4.6 \mathrm{~mm}$ Vydac guard analytical reverse-phase C18 columns (201GD54T and 201TP54), with methanol/water as mobile phase. After each run, the columns were rinsed with acetonitrile. PAHs were detected on an HP 1100 multiwavelength fluorescence detector.

Feeding rate ${ }^{152}$

$\mathrm{FR}=\frac{((L 1 \times C l)-L 2)}{\left(\frac{L i 1+L i 2}{2}\right) \times t}$

where L1 is the initial and L2 the final dry weight of the Populus sp. disc (mg), Li1 and $\mathrm{Li} 2$ are the numbers of living organisms at the start and at the end of the experiment (Li1=11 individuals), $\mathrm{Cl}$ is the leaching-decomposition correction factor, calculated by dividing the initial dry weight by the final dry weight of the leaves in the control sample; and $t$ is the incubation time (days). 


\section{Appendix of Chapter 4}

Table A4.1. Mean \pm SD nanoplastic concentration per body dw of Gammarus pulex $(\mathrm{mg} / \mathrm{g})$ in body, faeces and the sum of both; and nanoplastic concentration in body and faeces with respect to the total ingested nanoplastics (\%) at nanoplastic concentrations in sediment of $0.3,1,3,10$ and $30 \mathrm{~g} / \mathrm{kg}$ of sediment.

Figure A4.1. Images of the Pd-doped NP taken under a FEI Magellan 400 scanning electron spectroscope illustrating the raspberry-like shape of the particles.

Figure A4.2. Log NP concentration measured in A) the body of G. pulex (mg/g), B) faeces of G. pulex per body weight $(\mathrm{mg} / \mathrm{g})$ and $\mathrm{C}$ ) total NP ingested by G. pulex $(\mathrm{mg} / \mathrm{g})$ per body dw after summing up the concentration of NP in bodies and faeces; after 28 days of exposure to Log NP concentrations in sediment $\mathrm{dw}(\mathrm{g} / \mathrm{kg})$.

Assessment of the likeliness of effects caused by chemical residues from nanoplastic synthesis

Table A4.2. Numbers calculated for the different steps in the assessment of the likeliness of effects from the background chemicals originating from NP synthesis.

Figure A4.3. Styrene removal by gas purging.

Figure A4.4. Styrene removal during acclimatization.

Figure A4.5. Measured and modelled uptake of MP by G. pulex over 28 days of exposure to sediment amended with MP, followed by 1 day of depuration in clean medium. 
Table A4.1. Mean \pm SD nanoplastic concentration per body $d w$ of Gammarus pulex (mg/g) in body, faeces and the sum of both (grey); and nanoplastic concentration in body and faeces with respect to the total ingested nanoplastics (\%) (blue) at nanoplastic concentrations in sediment of $0.3,1,3,10$ and $30 \mathrm{~g} / \mathrm{kg}$ of sediment.

\begin{tabular}{|c|c|c|c|c|c|}
\hline & \multicolumn{2}{|c|}{$\begin{array}{c}\text { NP concentration per body dw of } \\
\text { Gammarus pulex } \mathbf{( m g / g )}\end{array}$} & $\begin{array}{c}\text { \%of NP in body and faeces } \\
\text { of the total ingested NP }\end{array}$ \\
\hline $\begin{array}{c}\text { NP } \\
\text { concentration } \\
\text { in sediment } \\
\mathbf{( g / k g )}\end{array}$ & BODY & FAECES & TOTAL & BODY & FAECES \\
\hline $\mathbf{0 . 3}$ & Nean \pm SD & Mean \pm SD & Mean \pm SD & Mean & Mean \\
\hline $\mathbf{1}$ & $0.053 \pm 0.031$ & $0.054 \pm 0.026$ & $0.107 \pm 0.057$ & 39.57 & - \\
\hline $\mathbf{3}$ & $0.328 \pm 0.300$ & $0.228 \pm 0.098$ & $0.556 \pm 0.351$ & 47.79 & 52.21 \\
\hline $\mathbf{1 0}$ & $0.246 \pm 0.160$ & $0.285 \pm 0.173$ & $0.532 \pm 0.020$ & 45.18 & 54.82 \\
\hline $\mathbf{3 0}$ & $0.541 \pm 0.415$ & $1.073 \pm 0.423$ & $1.614 \pm 0.138$ & 32.99 & 67.00 \\
\hline Average $\%$ & & & & $41.38 \pm \mathbf{2 3 . 1 5}$ & $\mathbf{5 8 . 6 2} \pm \mathbf{2 3 . 1 5}$ \\
\hline
\end{tabular}




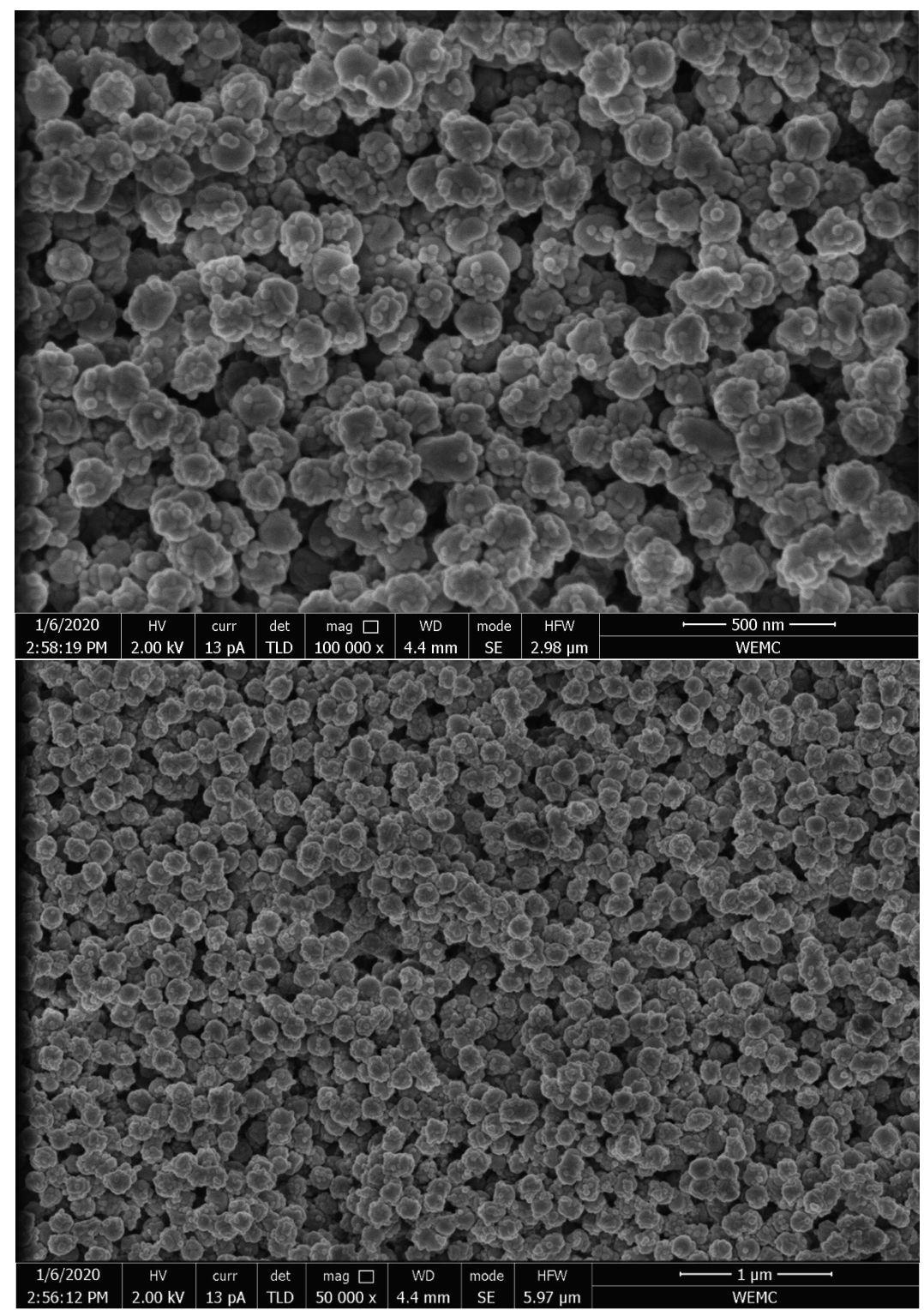

Figure A4.1. Images of the Pd-doped NP taken under a FEI Magellan 400 scanning electron spectroscope $(50,000 \mathrm{x}$ and $100,000 \mathrm{x}$ magnification) illustrating the raspberry-like shape of the particles. 
A

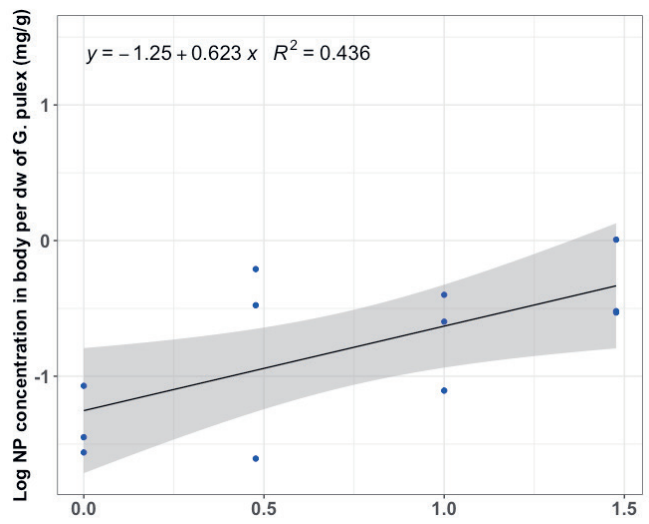

B

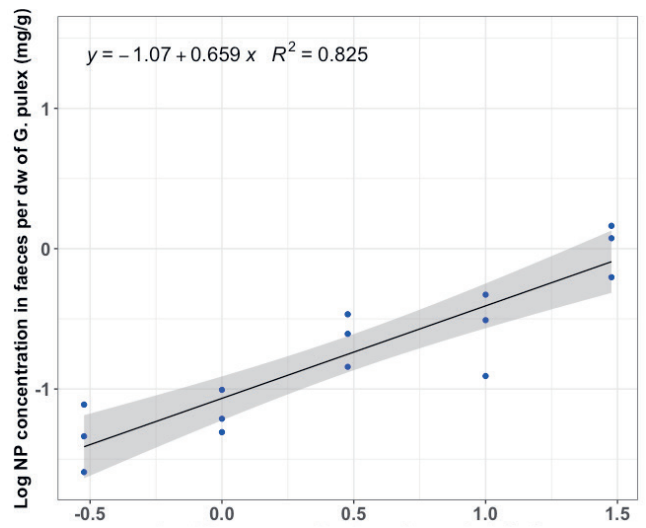

$\mathrm{C}$

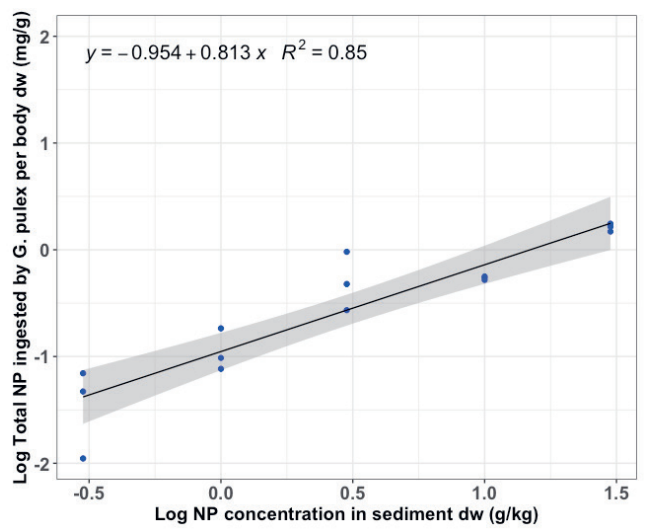

Figure A4.2. Log NP concentration measured in A) the body of G. pulex (mg/g), B) faeces of G. pulex per body weight $(\mathrm{mg} / \mathrm{g})$ and, C) total NP ingested by G. pulex $(\mathrm{mg} / \mathrm{g})$ per body dw after summing up the concentration of NP in bodies and faeces; after 28 days exposure to Log NP concentrations in sediment $\mathrm{dw}(\mathrm{g} / \mathrm{kg})$. Linear regressions $\left(P_{\text {body }}=1.94 \times 10^{-2} ; P_{\text {faeces }}=2.87 \times 10^{-6}\right.$; $P_{\text {Total }}=1.02 \times 10^{-6}$ ) are based on 12 individual data points for the body due to the loss of the control and lowest concentration values after log-transforming the data; and 15 individual data points for the faeces and total NP ingested, due to the loss of the control values after the log-transformation. 


\section{Assessment of the likeliness of effects caused by chemical residues from nanoplastic synthesis}

The concentrations of the chemicals used in the synthesis of Pd-doped NPs change due to (incomplete) polymerization, dilution upon transfer of the spiked volume to the bioassay systems, sorption to sediment, and volatilization due to purging, either prior to the experiment, or during acclimatization prior to exposure. The concentrations during all of these steps are summarized in Table 4.2. Ultimately, margins of exposure (MOE) were larger than 1 for all chemicals.

Table 4.2. Numbers calculated for the different steps in the assessment of the likeliness of effects from the background chemicals originating from NP synthesis.

\begin{tabular}{|c|c|c|c|c|c|c|c|c|c|}
\hline $\mathbf{1}$ & $\mathbf{2}$ & $\mathbf{3}$ & $\mathbf{4}$ & $\mathbf{5}$ & $\mathbf{6}$ & $\mathbf{7}$ & $\mathbf{8}$ & $\mathbf{9}$ & $\mathbf{1 0}$ \\
\hline Chemical & Weight & Yield & $\mathbf{C}_{\text {total }}$ & $\mathbf{C}_{\text {residual }}$ & $\mathbf{C}_{\text {bioassay,TOT }}$ & $\mathbf{K p}$ & $\mathbf{C}_{\text {bioassay,total }}$ & $\mathbf{C}_{\text {crit }}$ & MOE \\
\hline Water & $\mathrm{g}$ & $\mathbf{6}$ & $\mathrm{g} / \mathrm{l}$ & $\mathrm{g} / \mathrm{l}$ & $\mathrm{g} / \mathrm{l}$ & $1 / \mathrm{kg}$ & $\mathrm{mg} / \mathrm{l}$ & $\mathrm{mg} / \mathrm{l}$ & $(-)$ \\
\hline Acrylonitrile & 50 & 95 & 79.69 & 3.98 & $9,42 \times 10^{-02}$ & 5 & 73.6 & 2.00 & $\begin{array}{c}913.5 \\
(\mathrm{p})\end{array}$ \\
\hline Styrene & 22.5 & 95 & 35.86 & 1.79 & $4,24 \times 10^{-02}$ & 50 & 11.1 & 1.90 & $\begin{array}{c}1 \times 10^{18} \\
(\mathrm{p})\end{array}$ \\
\hline $\mathrm{DVB}$ & 1.18 & 95 & 1.88 & 0.09 & $2,22 \times 10^{-03}$ & 778.1 & $4,98 \times 10^{-02}$ & 0.69 & 13.85 \\
\hline $\mathrm{SDS}$ & 2.46 & 90 & 3.92 & 0.39 & $9,27 \times 10^{-03}$ & 2700.0 & $6,08 \times 10^{-02}$ & 1.8 & 29.60 \\
\hline $\mathrm{KPS}$ & 3.30 & 95 & 5.26 & 0.26 & $6,22 \times 10^{-03}$ & 1.0 & 5.89 & 92 & 15.63 \\
\hline $\mathrm{KPE}$ & 1.50 & 90 & 2.39 & 0.24 & $5,65 \times 10^{-03}$ & 2700 & $3.71 \times 10^{-02}$ & $0.18^{*}$ & 4.85 \\
\hline $\mathrm{K}_{2} \mathrm{PdCl}$ & & & & & & & & \\
\hline
\end{tabular}

1. Chemical abbreviations: $\mathrm{DVB}=$ divinylbenzene. $\mathrm{SDS}=$ sodiumdodecylsulphate, $\mathrm{KPS}=$ potassium persulphate, $\mathrm{KPE}=$ poly(ethyleneglycol)4-nonlpheyl 3-sulfopropylether potassium salt. $\mathrm{K}_{2} \mathrm{PdCl}_{4}=$ Potassium tetrachloropalladate(II).

2. Weight used in the synthesis of Pd-doped NP.

3. The polymerization and encapsulation of monomer, intitiator and surfactants is virtually complete. Still conservative yields $<100 \%$ were used in order to obtain a worst case assessment of chemical effects.

4. Original aqueous concentration prior to polymerization, i.e. at start, the concentration of acrylonitrile is $50 / 627,43=79.69 \mathrm{~g} / 1$

5. The residual concentration after polymerization taking the yield into account.

6. Concentration in the bioassay, calculated from the spiked volume of the NP disperson and the water volume in the bioassay.

7. The sediment to water partition coefficient (literature value)

8. The aqueous concentration during the bioassay, assuming equilibration with sediment, calculated from the volume of water, the mass of sediment, $\mathrm{m}$ the $\mathrm{Kp}$ and the quantity of added chemical: $\mathrm{C}_{\text {bioassay,TOT }}=\frac{\text { Cresidual }}{1+[S E D] * K p} \quad$ with $[\mathrm{SED}]$ is the mass to liquid ration of the sediment $(\mathrm{kg} / \mathrm{l})$ concentration of the sediment.

9. The threshold effect concentreation for chemical toxicity, based on literature values. For KPE no threshold effect concentration could be found. For this chemical we used a worst case scenario and set the threeshold value at $10 \%$ of that for the other surfactant, SDS. 
10. The Margin of exposure (MOE), calculated as $\mathrm{MOE}=\mathrm{C}_{\text {crit }} / \mathrm{C}_{\text {bioassay,total }}$. An MOE larger than 1 means that no chemical effect can occur. For the volatile chemicals acrylonitrile

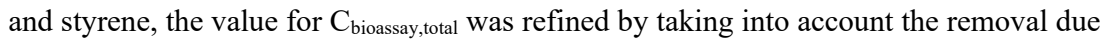
to purging the systems prior to exposure and during the acclimitization period (Figs S3 and S4). The effect purging was calculated using: $[\mathrm{C}]=[\mathrm{Co}] * e^{-\frac{F x H x t}{V}}$ with $\mathrm{C}$ and $\mathrm{C} 0$ are chemical concentrations at start $(\mathrm{Co})$ and during purging $(\mathrm{C}), \mathrm{F}$ is flow rate $(\mathrm{L} / \mathrm{h}), \mathrm{H}$ is Henry's law constant, $\mathrm{t}$ is time ( $\mathrm{h}$ ) and $\mathrm{V}$ is water volume.

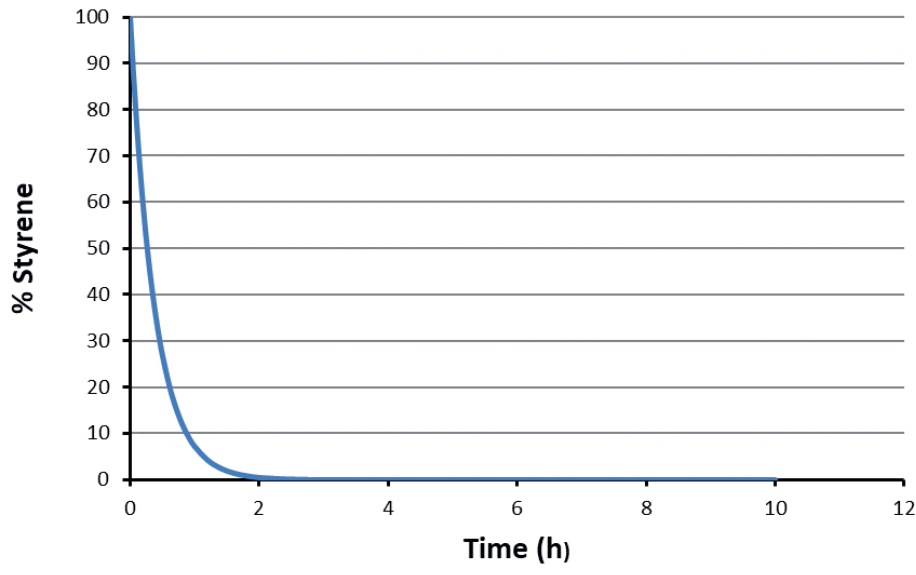

Figure A4.3. In 2-3 hours, any styrene left in the water is removed completely by gas purging.

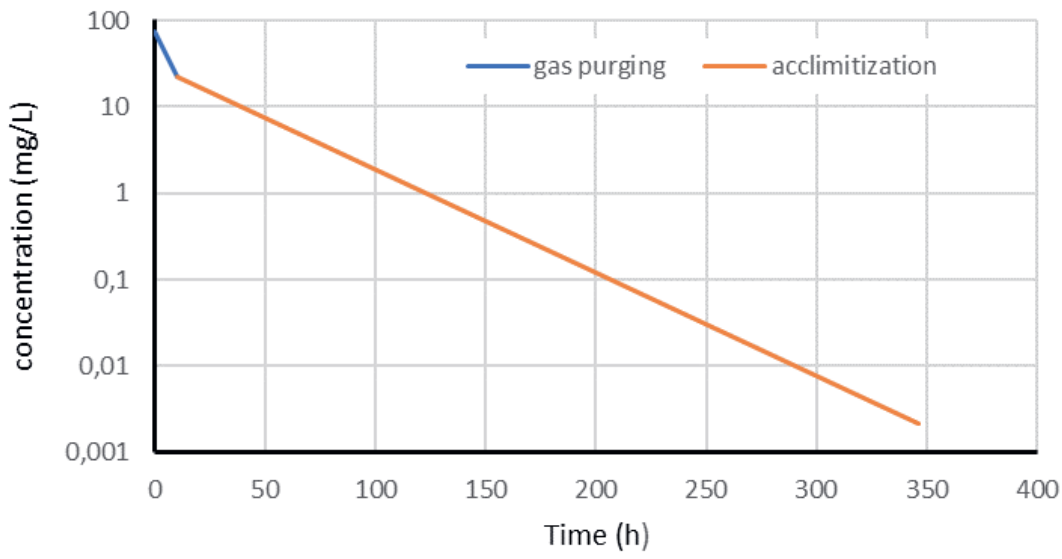

Figure A4.4. In 8 hours of gas purging (blue line), followed by two weeks of acclimatization under continuous aeration prior to the experiment (orange line), the initial acrylonitrile concentration decreases with 4 to 5 orders of magnitude. 


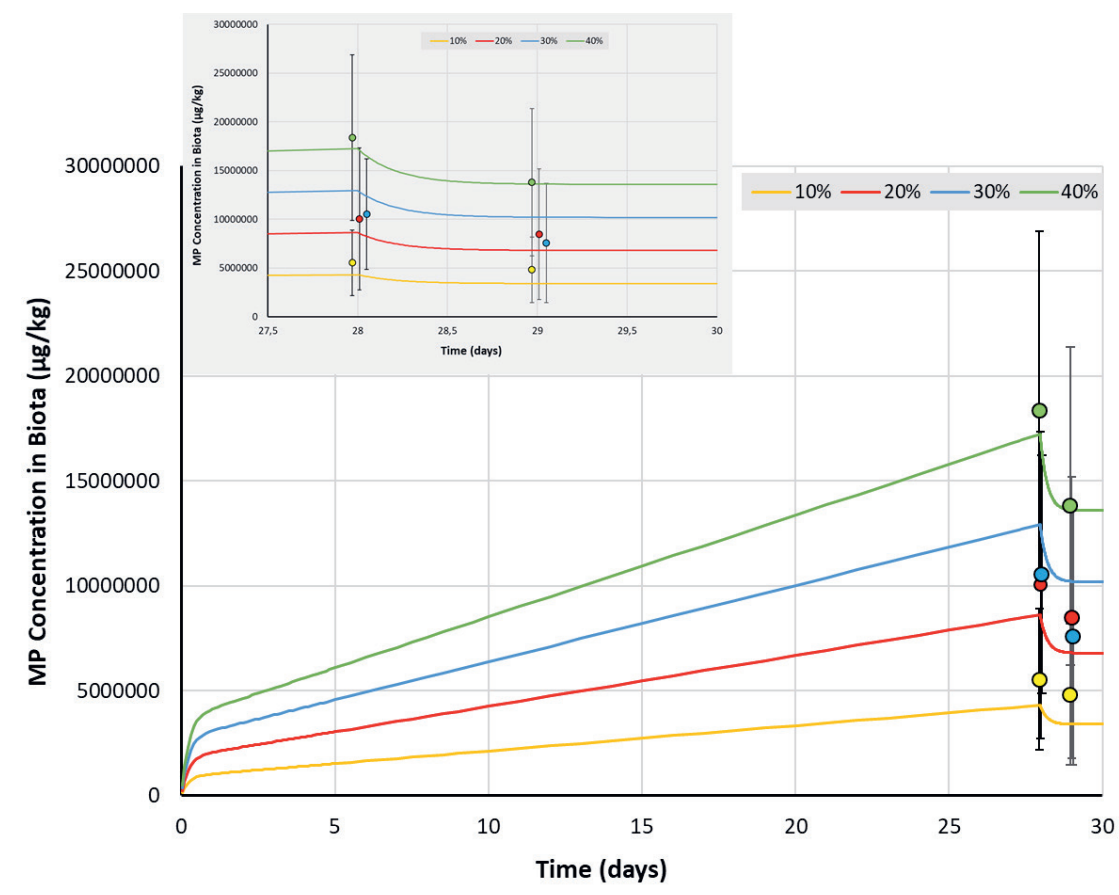

Figure A4.5. Measured and modelled (Eqs. 1 and 2) uptake of MP by G. pulex over 28 days of exposure to sediment amended with MP, followed by 1 day of depuration in clean medium (insert). Data on measured NP concentrations ( $\pm 1 \mathrm{SD}$ ) after depuration (see insert) after 29 days were set apart for 0.05 day for better visibility of the datapoints on the $\mathrm{x}$-axis. Data from RedondoHasselerharm et al. (2018) (Chapter 2). ${ }^{186}$ The model was highly significant $\left(P=4.4 \times 10^{-99}\right)$. 


\section{Appendix of Chapter 5}

Calculations of styrene and SDS concentrations in the experimental ditch

Table A5.1. Mean abundance $( \pm \mathrm{SD})$ per taxon in transects and NP trays.

Table A5.2. Mean abundance $( \pm \mathrm{SD})$ per taxon in transects and MP trays.

Table A5.3. Reference community ratio based on the number of individuals $/ \mathrm{m}^{2}$ and based on taxa.

Table A5.4. Temperature $\left({ }^{\circ} \mathrm{C}\right)$, dissolved oxygen $(\mathrm{mg} / \mathrm{l}), \mathrm{pH}$, electro-conductivity $(\mu \mathrm{S} / \mathrm{cm})$ measured at two locations (meters 10 and 30) in the experimental ditch at the start of the experiment (0) and after 1,2,3,5, $6,7,11,12,24,36,48$ and 60 weeks.

Figure A5.1. Number of individuals per class found in trays retrieved after 3 months (M3) and 15 months (M15) for nanoplastics (upper panel) and microplastics (lower panel) treatments.

Figure A5.2. Valvata abundance in trays retrieved after 3 and 15 months with increasing NP (upper panel) and MP (lower panel) concentrations (as \% sediment dry weight).

Figure A5.3. Orthocladiinae abundance in trays retrieved after 3 and 15 months with increasing NP (upper panel) and MP (lower panel) concentrations (as \% sediment dry weight).

Figure A5.4. Hippeutis complanatus abundance in trays retrieved after 3 and 15 months with increasing NP (upper panel) and MP (lower panel) concentrations (as \% sediment dry weight).

Figure A5.5. Gyraulus albus abundance in trays retrieved after 3 and 15 months with increasing NP (upper panel) and MP (lower panel) concentrations (as \% sediment dry weight). 
Following principles published previously, ${ }^{220}$ here we calculate the background concentrations of styrene monomer and SDS that can be expected in the ditches based on (I) the dimensions, i.e. weights and volumes of sediment and water compartments in the ditch, (II) quantities of styrene and SDS added to the ditch via the addition of nanopolystyrene dispersion, (III) well-established principles of sediment water partitioning. Subsequently, the resulting concentrations are compared to known effect thresholds for these chemicals.

I. Weights and volumes of sediment and water in the ditch

Water volume of the ditch $=[(132 \times 0.5)+(64 \times 0.5)] / 2=49 \mathrm{~m}^{3}=49000 \mathrm{~L}$

Sediment volume of the ditch (without sediment in trays) $=64 \times 0.25=16 \mathrm{~m}^{3}=16000 \mathrm{~L}$ Total weight of the sediment in the ditch $=16 \times 1600=25600 \mathrm{~kg}$.

II. Quantities of styrene and SDS added to the ditches, via the embedded trays.

a) Styrene

Volume of Nano-PS spike solution in 1 highest dose $(5 \%$ PS) tray $=0.16883 \mathrm{~L}$

The $2 \mathrm{~L}$ of Nano-PS spike solution contained at most (styrene at start minus polystyrene at end) $=\sim 900-838.9 \mathrm{~g}=61.1 \mathrm{~g}$ un-polymerized styrene monomer left. This means that in 1 tray with $0.16883 \mathrm{~L}$ Nano-PS spike solution added, we have $0.16883 \times 61.1 / 2=5.158$ g styrene.

There are 8 of such trays with maximum dose, 8 with 10x lower dose, 8 with 100x, and 8 with $1000 \mathrm{x}$ lower dose. Therefore, the total quantity of styrene added to the entire ditch is:

$8 \times(1+0.1+0.01+0.001) \times 5.158=45.844$ g styrene.

As styrene is a volatile chemical, the actual concentration will be (much) lower. However, here we use this original concentration as a worst-case scenario.

b) $S D S$

The 2 L of Nano-PS spike solution contained $\sim 23.3 \mathrm{~g}$ dissolved SDS. This means that in 1 tray with 0.16883 L Nano-PS spike solution added, we have $0.16883 \times 23.3 / 2=1.9669$ g SDS.

There are 8 of such trays with maximum dose, 8 with 10x lower dose, 8 with 100x, and 8 with $1000 \mathrm{x}$ lower dose. Therefore, the total quantity of styrene added to the entire ditch is: 
$8 \times(1+0.1+0.01+0.001) \times 1.9669=17.482 \mathrm{~g} \mathrm{SDS}$.

III. Realistic calculation of styrene and SDS concentration in the water of the ditch taking into account steady state sorption to the sediment

General method to calculate sediment-water partitioning

It is assumed that the total concentration of chemical in the water (CT) is split in a truly dissolved concentration $(\mathrm{Cw})$ and a part which is sorbed to the sediment. Then the following mass balance equation holds:

$\mathrm{CT}=\mathrm{Cw}+\mathrm{Csed} \times[\mathrm{SED}]$

In which $\mathrm{CT}$ is the total concentration of chemical in the ditch $\left(\mathrm{gxL}^{-1}\right), \mathrm{Cw}$ is the free aqueous concentration $\left(\mathrm{gxL}^{-1}\right)$, Csed is the chemical concentration sorbed in sediment $\left(\mathrm{mgxkg}^{-1}\right)$ and $[\mathrm{SED}]$ is the concentration of sediment in the system $\left(\mathrm{kgxL}^{-1}\right)$.

Due to sediment water exchange from the trays, turbulent and diffusional mixing, a steady state can be assumed. At equilibrium, Csed is related to $\mathrm{Cw}$, via:

Csed $=\mathrm{Kd} \times \mathrm{Cw}$

in which $\mathrm{Kd}$ is the sediment-water distribution coefficient for the chemical $\left(\mathrm{Lxkg}^{-1}\right)$. Combination of equations [1] and [2] yields

$\mathrm{CT}=\mathrm{Cw} \times(1+\mathrm{Kd} \times[\mathrm{SED}])$

which now can be solved for $\mathrm{Cw}$ if [SED] is known:

$\mathrm{Cw}=\mathrm{CT} /(1+\mathrm{Kd} \times[\mathrm{SED}])$

\section{Calculation for $S D S$}

In the ditch, the sediment to water solid to liquid ratio $[\mathrm{SED}]$ is $[\mathrm{SED}]=25600 / 49000=$ $0.52245 \mathrm{~kg} / 1$

For SDS the total concentration CT is CT $=17.482 / 49000=0.000356837 \mathrm{~g} / \mathrm{l}=0.356837$ $\mathrm{mg} / \mathrm{l}$

A literature value for the $\mathrm{Kd}$ for sediment in freshwater ${ }^{378}$ is $2700 \mathrm{lxkg}^{-1}$ 
Therefore, for SDS: $\mathrm{Cw} \approx 0.356837 /(1+2700 \times 0.52245)=0.00025279 \mathrm{mgxL}^{-1}=\mathbf{0 . 2 5}$ $\boldsymbol{\mu g x l}^{-1}$

\section{Calculation for Styrene}

Styrene is volatile and can be expected to steadily dissipate from the ditches to reach zero concentration. Still, we here provide a calculation to estimate the aqueous phase concentration in case styrene would be conservative.

For styrene the total concentration CT is CT $=45.844 / 49000=0.00093559 \mathrm{~g} / 1=0.93559$ $\mathrm{mg} / \mathrm{l}$

A literature value for the Koc for sediment is $10^{2.96} \mathrm{xkg}^{-1} 228$

With a \%TOM of about 5 to $10 \%$ (see Table A5.1), this would give a Kp of Kp $=50$ 1/kg.

Therefore, for styrene $\mathrm{Cw} \approx 0.93559 /(1+50 \times 0.52245)=0.0345 \mathrm{mgxL}^{-1}=\mathbf{3 4 . 4 9} \boldsymbol{\mu g x l ^ { - 1 }}$

As mentioned, this is a worst-case concentration due to the fact that styrene is volatile and will have reached (much) lower concentration.

Effect thresholds for SDS and styrene

Short term $\mathrm{LC}_{50}$ and $\mathrm{EC}_{50}$ values for aquatic invertebrates with SDS are between 1.20 and $14.40 \mathrm{mg} / \mathrm{l}^{240} \mathrm{NOEC}$ values from chronic toxicity tests of aquatic invertebrates with SDS are between 0.88 and $5.76 \mathrm{mg} /$ l. $^{240}$

Short term $\mathrm{LC}_{50}$ and $\mathrm{EC}_{50}$ values for aquatic invertebrates with styrene are between 4.7 and $9.5 \mathrm{mg} / 1 .{ }^{228}$ The NOEC value from chronic toxicity tests of aquatic invertebrates with styrene is $1.01 \mathrm{mg} / \mathrm{l}^{228}$

Concentrations calculated above thus are at least a factor 29 lower than the short term and long-term effect thresholds for these chemicals provided by European Chemical Agency (ECHA). 228,240

This means that these chemicals are not expected to have contributed to the community effects found in this study. 


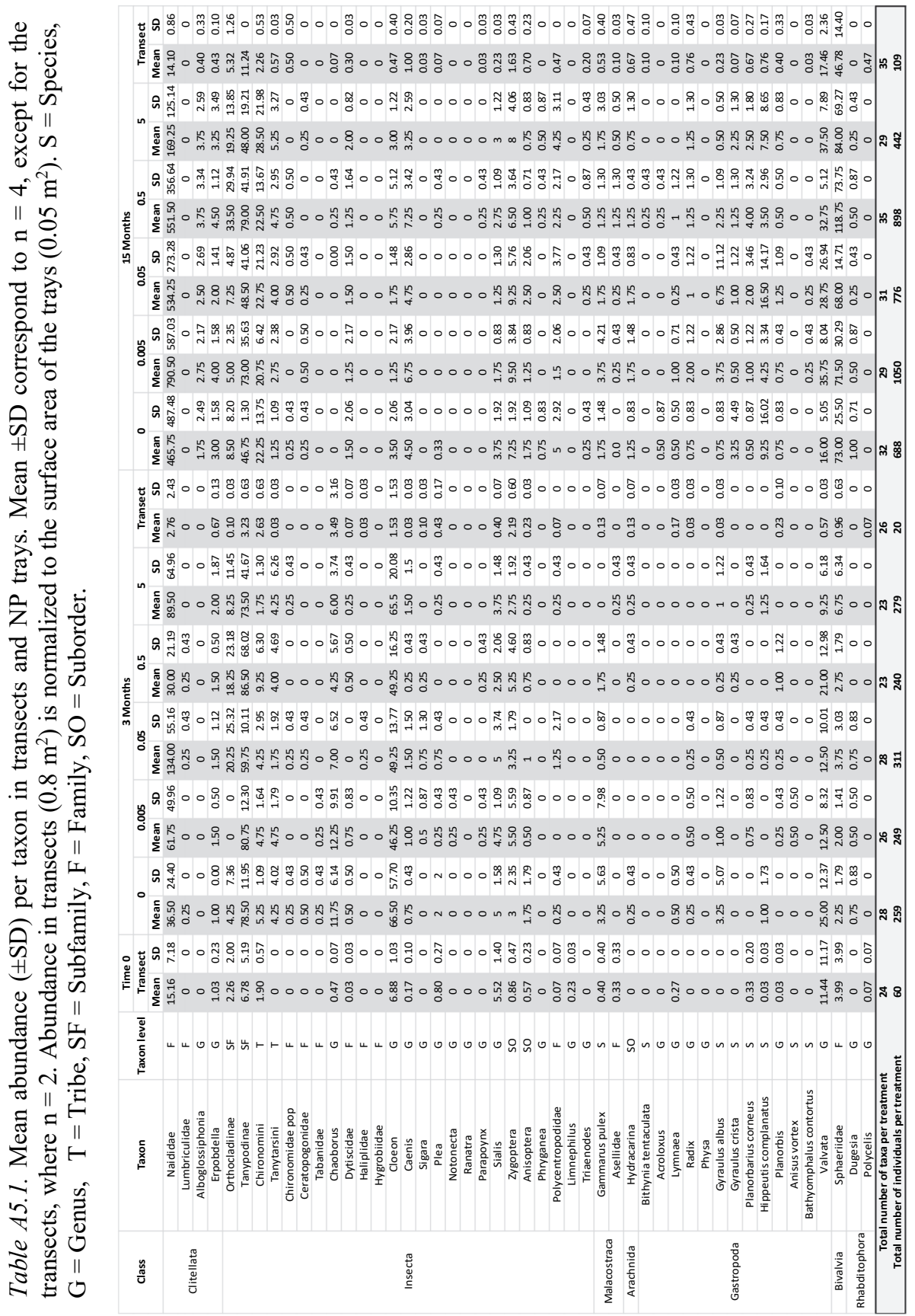




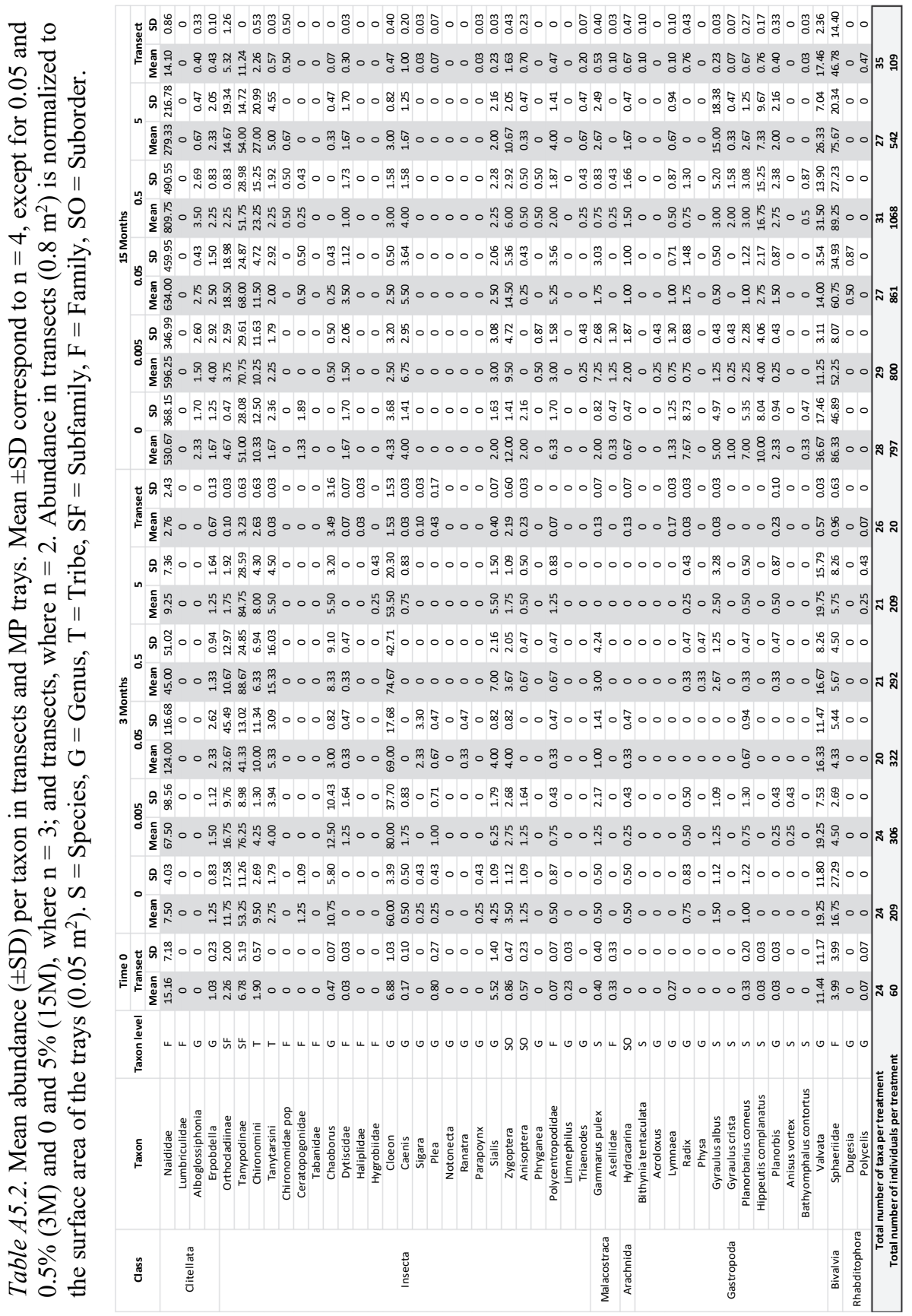


Table A5.3. Reference community ratio based on the number of individuals $/ \mathrm{m}^{2}$ and based on taxa. Ratio for number of individuals was calculated as number of individuals $/ \mathrm{m}^{2}$ found in trays retrieved after 3 months (3M) and 15 months (15M) divided by the number of individuals $/ \mathrm{m}^{2}$ found in the donor system at the same time points. For taxa, this was done similarly, i.e. number of taxa in trays after 3 months (3M) and 15 months (15M), divided by the number of taxa in the donor system at the same time points. Mean $\pm \mathrm{SD}$ correspond to $n=4$, except for 0.05 and $0.5 \%(3 \mathrm{M})$, where $n=3$; and transects, where $n$ $=2$.

\begin{tabular}{|c|c|c|c|c|c|c|c|c|}
\hline & \multicolumn{4}{|c|}{ Nanoplastics 3M } & \multicolumn{4}{|c|}{ Microplastics 3M } \\
\hline & \multicolumn{2}{|c|}{ Individuals $/ \mathbf{m}^{2}$} & \multicolumn{2}{|c|}{ Taxa } & \multicolumn{2}{|c|}{ Individuals $/ \mathbf{m}^{2}$} & \multicolumn{2}{|c|}{ Taxa } \\
\hline & Mean & SD & Mean & SD & Mean & SD & Mean & SD \\
\hline $\mathbf{0}$ & 12.79 & 3.34 & 0.77 & 0.10 & 10.32 & 3.04 & 0.76 & 0.11 \\
\hline 0.005 & 12.31 & 4.05 & 0.73 & 0.16 & 15.11 & 3.44 & 0.78 & 0.11 \\
\hline 0.05 & 15.37 & 2.93 & 0.76 & 0.05 & 15.92 & 4.47 & 0.64 & 0.13 \\
\hline 0.5 & 11.86 & 2.82 & 0.67 & 0.08 & 14.42 & 3.73 & 0.70 & 0.02 \\
\hline 5 & 13.78 & 2.99 & 0.65 & 0.08 & 10.33 & 1.38 & 0.66 & 0.09 \\
\hline
\end{tabular}

\begin{tabular}{|c|c|c|c|c|c|c|c|c|}
\hline & \multicolumn{4}{|c|}{ Nanoplastics 15M } & \multicolumn{4}{|c|}{ Microplastics 15M } \\
\hline & \multicolumn{2}{|c|}{ Individuals $/ \mathbf{m}^{2}$} & \multicolumn{2}{|c|}{ Taxa } & \multicolumn{2}{|c|}{ Individuals $/ \mathbf{m}^{2}$} & \multicolumn{2}{|c|}{ Taxa } \\
\hline & Mean & SD & Mean & SD & Mean & SD & Mean & SD \\
\hline 0 & 6.36 & 4.65 & 0.73 & 0.08 & 7.37 & 3.32 & 0.77 & 0.03 \\
\hline 0.005 & 9.70 & 5.09 & 0.72 & 0.07 & 7.40 & 3.03 & 0.67 & 0.06 \\
\hline 0.05 & 7.17 & 2.26 & 0.69 & 0.04 & 6.35 & 4.11 & 0.68 & 0.06 \\
\hline 0.5 & 8.30 & 2.37 & 0.81 & 0.06 & 8.59 & 4.65 & 0.70 & 0.07 \\
\hline 5 & 4.08 & 1.04 & 0.68 & 0.06 & 5.01 & 2.21 & 0.69 & 0.04 \\
\hline
\end{tabular}


Table A5.4. Temperature $(\mathrm{T})\left({ }^{\circ} \mathrm{C}\right)$, dissolved oxygen (DO) $(\mathrm{mg} / \mathrm{l}), \mathrm{pH}$, electroconductivity $(\mathrm{EC})(\mu \mathrm{S} / \mathrm{cm})$ measured at two locations (meters 10 and 30) in the experimental ditch at the start of the experiment (0) and after 1, 2, 3, 5, 6, 7, 11, 12, 24, 36,48 and 60 weeks. $\mathrm{NH}_{4}(\mathrm{mg} / \mathrm{l}), \mathrm{NO}_{3}(\mathrm{mg} / \mathrm{l})$, Total Nitrogen $(\mathrm{N})(\mathrm{mgN} / \mathrm{l})$ and Total Phosphorus $(\mathrm{P})(\mathrm{mgP} / \mathrm{l})$ analysed from water samples taken at three different locations (meters 10,20 and 30) in the experimental ditch at the start of the experiment (0) and after $1,2,3,5,12,48$ and 60 weeks.

\begin{tabular}{|c|c|c|c|c|c|c|c|c|c|c|c|c|c|c|}
\hline Week & & 0 & 1 & 2 & 3 & 5 & 6 & 7 & 11 & 12 & 24 & 36 & 48 & 60 \\
\hline \multirow[t]{2}{*}{$\mathrm{T}\left({ }^{\circ} \mathrm{C}\right)$} & $10 \mathrm{~m}$ & 20.6 & 22.3 & 25.9 & 24.9 & 21.3 & 24.5 & 24.0 & 22.8 & 20 & 7.0 & 13.6 & 21.3 & 14 \\
\hline & $30 \mathrm{~m}$ & 20.7 & 22.6 & 26.9 & 24.8 & 21.3 & 23.2 & 24.1 & 22.0 & 20.1 & 6.8 & 13.5 & 21.3 & 14 \\
\hline \multirow{2}{*}{$\begin{array}{c}\text { DO } \\
(\mathrm{mg} / \mathrm{l})\end{array}$} & $10 \mathrm{~m}$ & 8.0 & 8.5 & 7.5 & 11.5 & 13.8 & 13.8 & 9.6 & 12.8 & 13.1 & 12.4 & 12.5 & 13.7 & 8.4 \\
\hline & $30 \mathrm{~m}$ & 7.6 & 8.2 & 7.1 & 9.8 & 12.2 & 11.8 & 8.4 & 11.9 & 12.1 & 12.1 & 12.3 & 12.7 & 7.0 \\
\hline \multirow[t]{2}{*}{ pH } & $10 \mathrm{~m}$ & 6.9 & 7.8 & 7.2 & 8.1 & 9.7 & 9.9 & 9.1 & 9.4 & 9.4 & 7.9 & 9.3 & 9.3 & 8.0 \\
\hline & $30 \mathrm{~m}$ & 7.0 & 7.7 & 7.1 & 7.5 & 9.4 & 9.5 & 8.9 & 9.3 & 9.3 & 7.5 & 9.0 & 9.0 & 8.0 \\
\hline \multirow{2}{*}{$\begin{array}{c}\mathrm{EC} \\
(\mu S / \mathrm{cm})\end{array}$} & $10 \mathrm{~m}$ & 94 & 98 & 101 & 94 & 77 & 86 & 78 & 81 & 79 & 90 & 80 & 80 & 104 \\
\hline & $30 \mathrm{~m}$ & 94 & 98 & 101 & 96 & 73 & 79 & 78 & 80 & 79 & 92 & 80 & 80 & 104 \\
\hline \multirow{3}{*}{$\begin{array}{c}\mathrm{NH}_{4} \\
(\mathrm{mg} / \mathrm{l})\end{array}$} & $10 \mathrm{~m}$ & 0.15 & 0.10 & 0.24 & & 0.08 & & & & 0.08 & & & 0.07 & 0.05 \\
\hline & $20 \mathrm{~m}$ & 0.07 & 0.12 & 0.24 & n.a. & 0.12 & n.a. & n.a. & n.a & 0.05 & n.a. & n.a. & 0.10 & 0.06 \\
\hline & $30 \mathrm{~m}$ & 0.10 & 0.14 & 0.31 & & 0.13 & & & & 0.08 & & & 0.07 & 0.03 \\
\hline \multirow{3}{*}{$\begin{array}{c}\mathrm{NO}_{3} \\
(\mathrm{mg} / \mathrm{l})\end{array}$} & $10 \mathrm{~m}$ & 0.01 & 0.02 & 0.06 & & 0.02 & & & & 0.02 & & & 0.08 & 0.05 \\
\hline & $20 \mathrm{~m}$ & 0.01 & 0.02 & 0.04 & n.a. & 0.02 & n.a. & n.a. & n.a. & 0.01 & n.a. & n.a. & 0.01 & 0.01 \\
\hline & $30 \mathrm{~m}$ & 0.01 & 0.02 & 0.05 & & 0.02 & & & & 0.01 & & & 0.01 & 0.00 \\
\hline \multirow{3}{*}{$\begin{array}{r}\text { Total N } \\
(\mathrm{mgN} / \mathrm{l})\end{array}$} & $10 \mathrm{~m}$ & 0.56 & 0.61 & 0.95 & & 0.97 & & & & 0.39 & & & 0.26 & 3.02 \\
\hline & $20 \mathrm{~m}$ & 0.52 & 0.41 & 0.86 & n.a. & 0.35 & n.a. & n.a. & n.a. & 0.33 & n.a. & n.a. & 0.72 & 1.91 \\
\hline & $30 \mathrm{~m}$ & 0.59 & 0.41 & 0.99 & & 0.67 & & & & 0.58 & & & 0.63 & 2.56 \\
\hline \multirow{3}{*}{$\begin{array}{l}\text { Total P } \\
(\mathrm{mgP} / \mathrm{l})\end{array}$} & $10 \mathrm{~m}$ & 0.07 & 0.05 & 0.05 & & 0.05 & & & & 0.04 & & & 0.03 & 0.14 \\
\hline & $20 \mathrm{~m}$ & 0.06 & 0.05 & 0.06 & n.a. & 0.03 & n.a. & n.a. & n.a. & 0.03 & n.a. & n.a. & 0.03 & 0.15 \\
\hline & $30 \mathrm{~m}$ & 0.06 & 0.05 & 0.05 & & 0.18 & & & & 0.03 & & & 0.04 & 0.36 \\
\hline
\end{tabular}



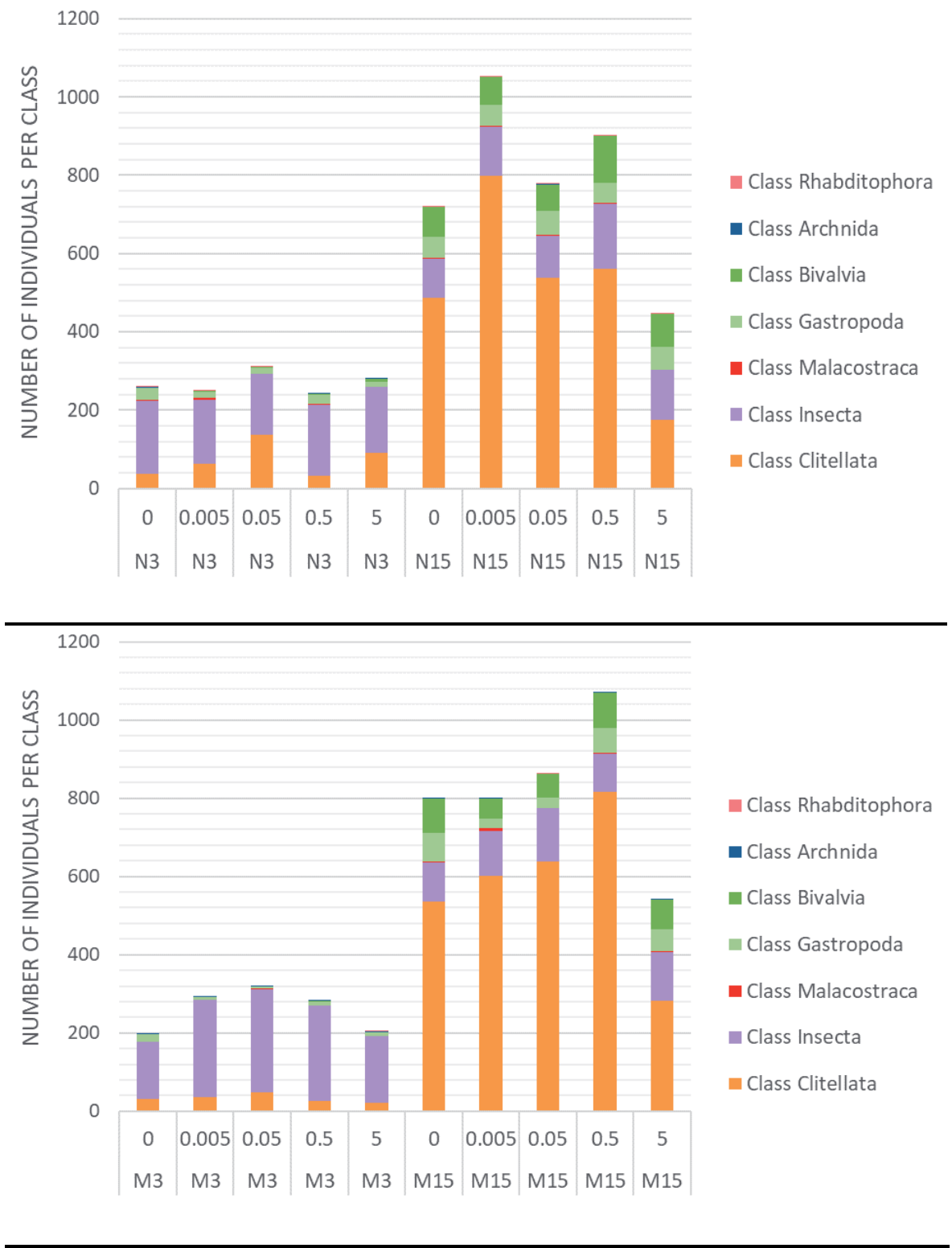

Figure A5.1. Number of individuals per class found in trays retrieved after 3 months (M3) and 15 months (M15) for nanoplastics (upper panel) and microplastics (lower panel) treatments. Colours in the legend represent the different classes of invertebrates found in trays. 


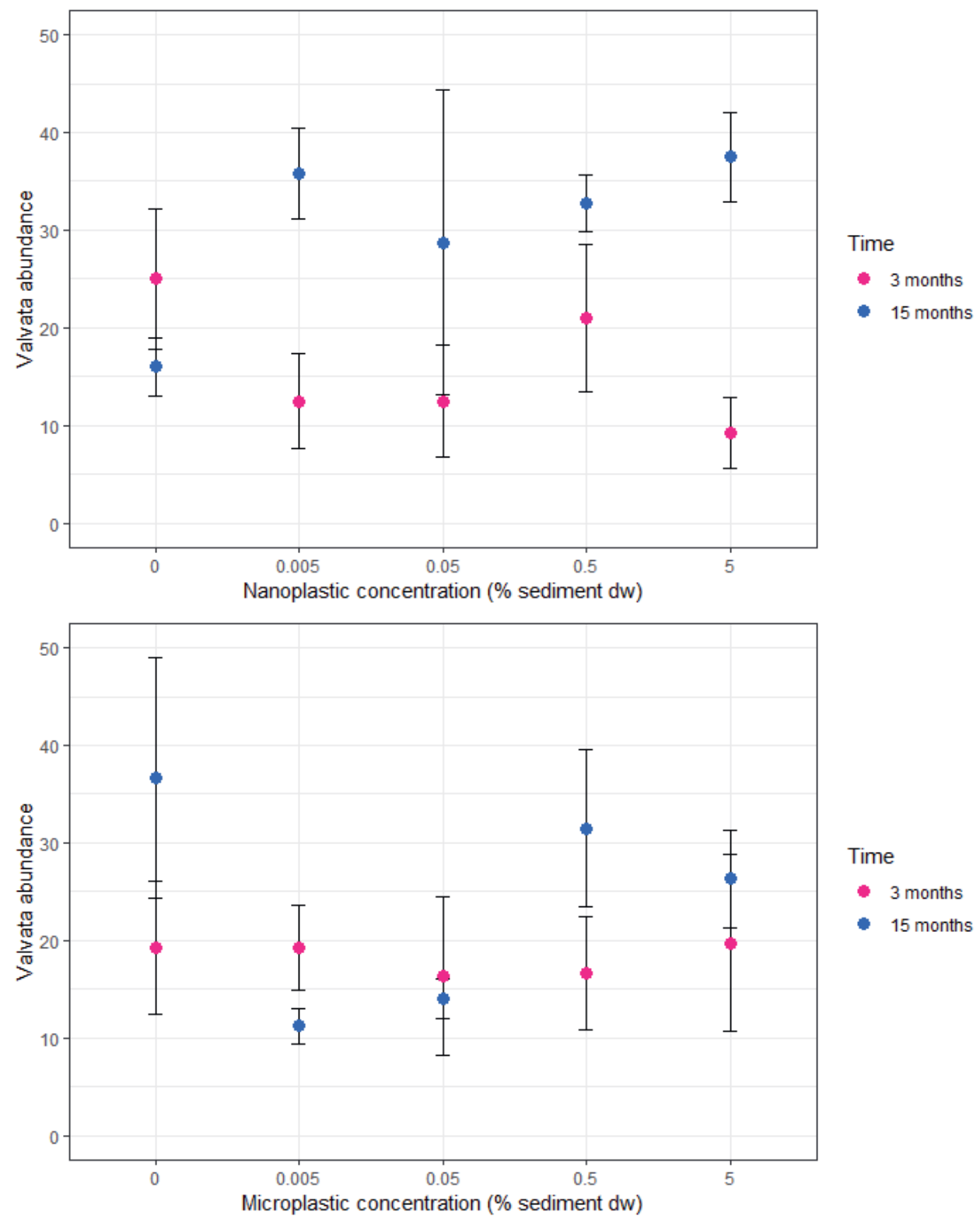

Figure A5.2. Valvata abundance in trays retrieved after 3 and 15 months with increasing NP (upper panel) and MP (lower panel) concentrations (as \% sediment dry weight). Error bars are mean $\pm \mathrm{SE} n=4$, except for MP treatments 0.05 and $0.5 \%$ retrieved after 3 months and 0 and $5 \%$ retrieved after 15 months, where $n=3$. 


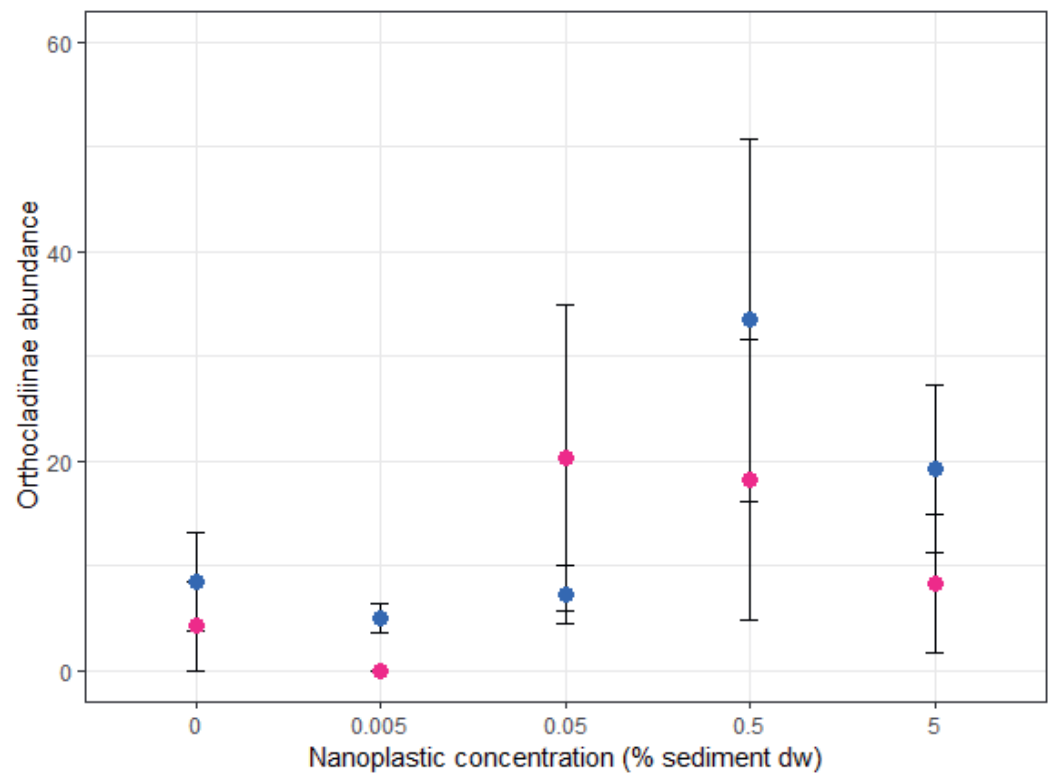

Time

- 3 months

- 15 months

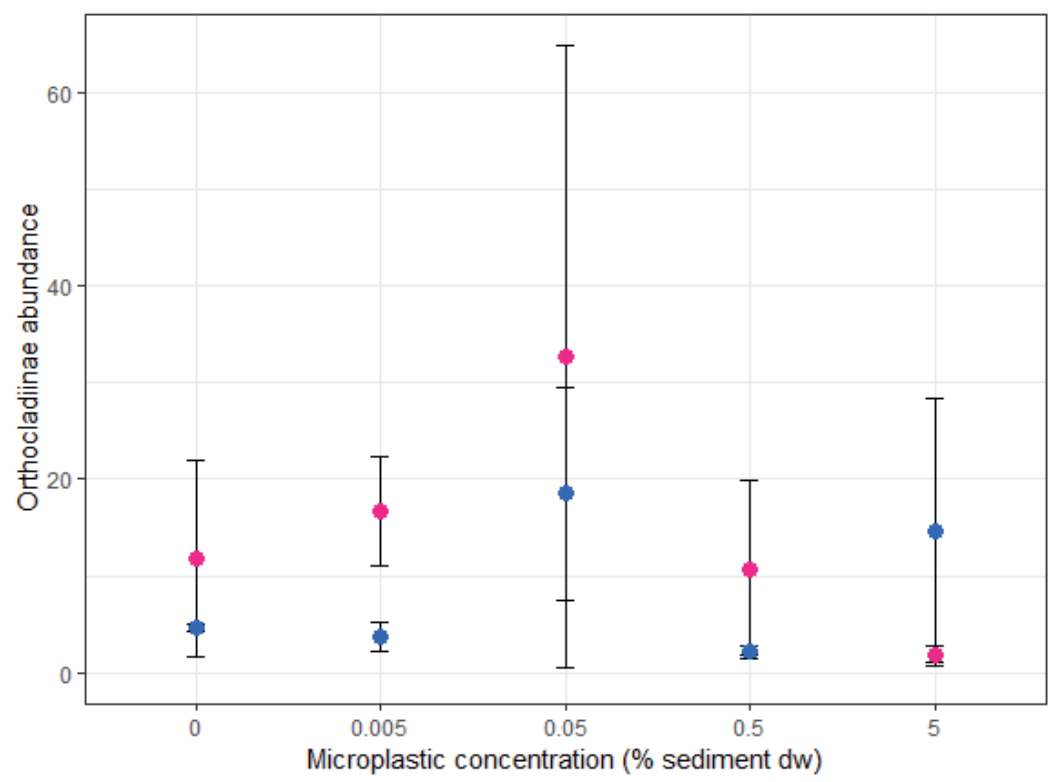

Time

- 3 months

- 15 months

Figure A5.3. Orthocladiinae abundance in trays retrieved after 3 and 15 months with increasing NP (upper panel) and MP (lower panel) concentrations (as \% sediment dry weight). Error bars are mean $\pm \mathrm{SE} n=4$, except for MP treatments 0.05 and $0.5 \%$ retrieved after 3 months and 0 and $5 \%$ retrieved after 15 months, where $n=3$. 

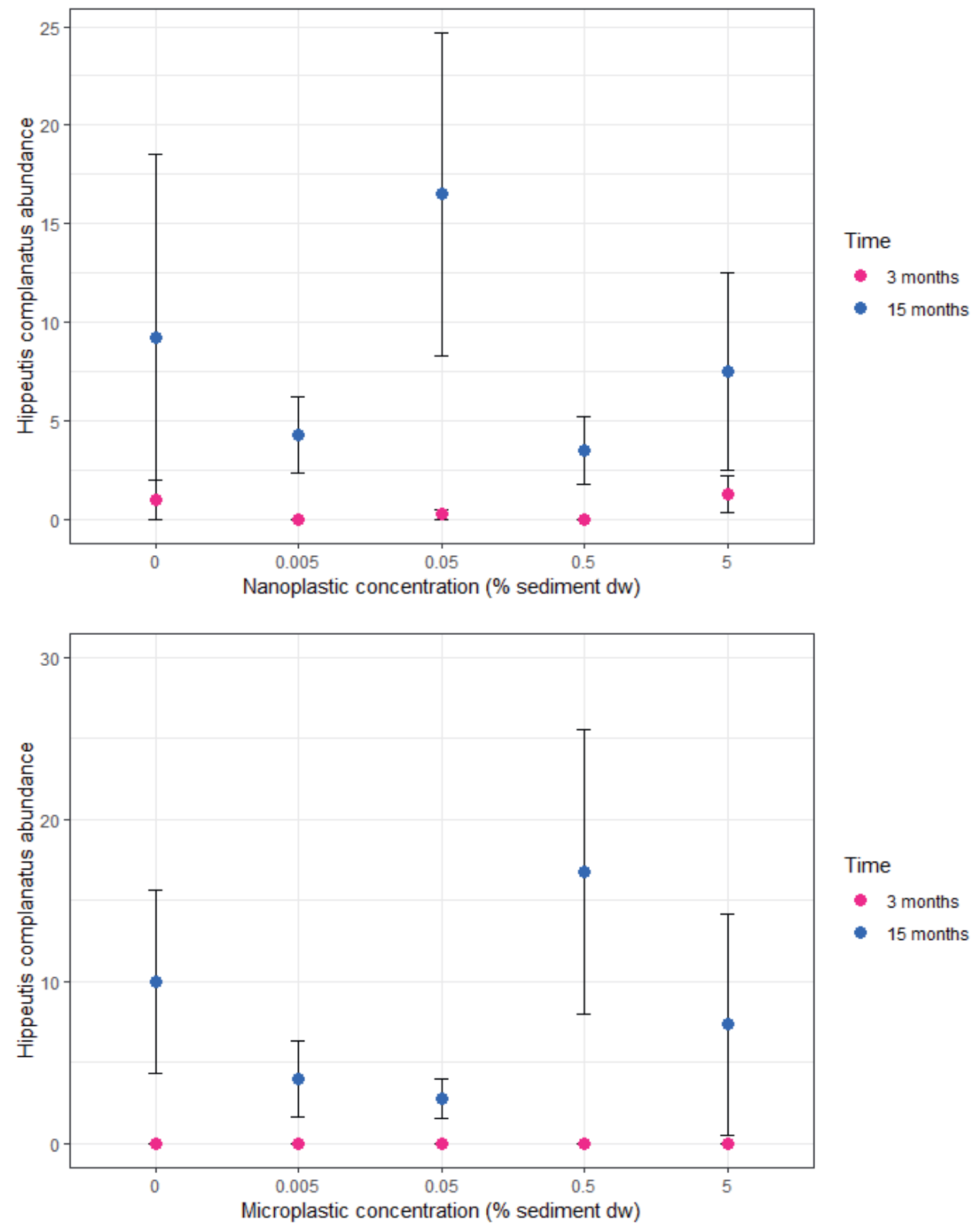

Figure A5.4. Hippeutis complanatus abundance in trays retrieved after 3 and 15 months with increasing NP (upper panel) and MP (lower panel) concentrations (as \% sediment dry weight). Error bars are mean $\pm \mathrm{SE} n=4$, except for MP treatments 0.05 and $0.5 \%$ retrieved after 3 months and 0 and $5 \%$ retrieved after 15 months, where $n=3$. 


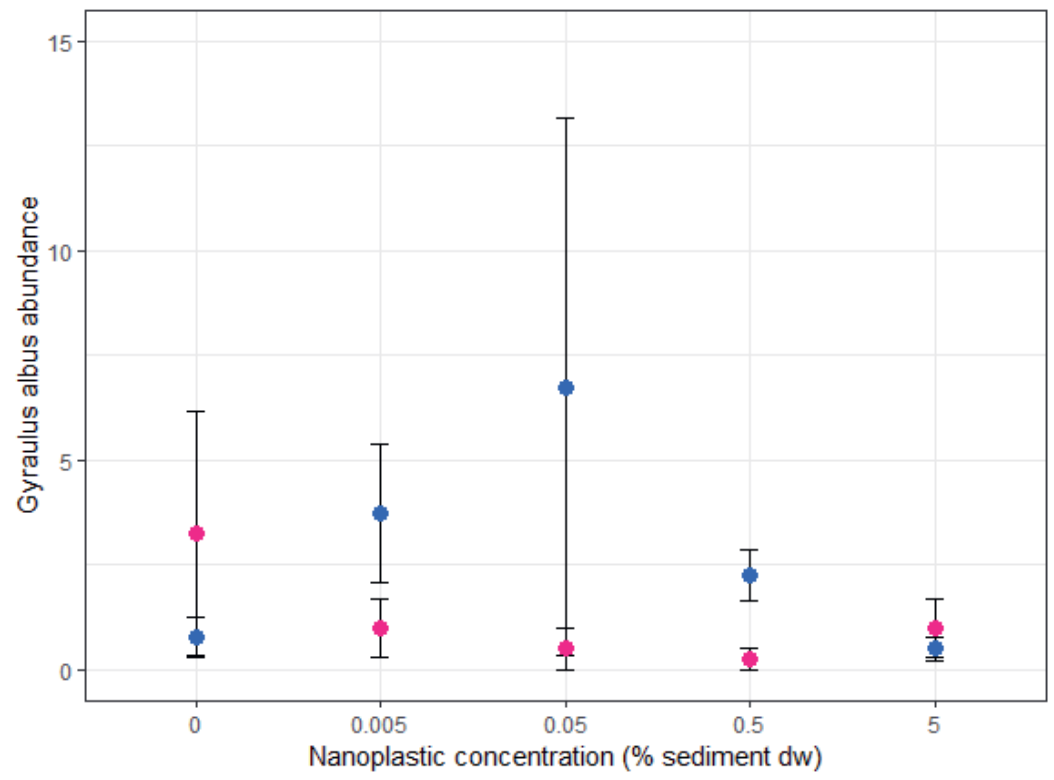

Time

- 3 months

- 15 months

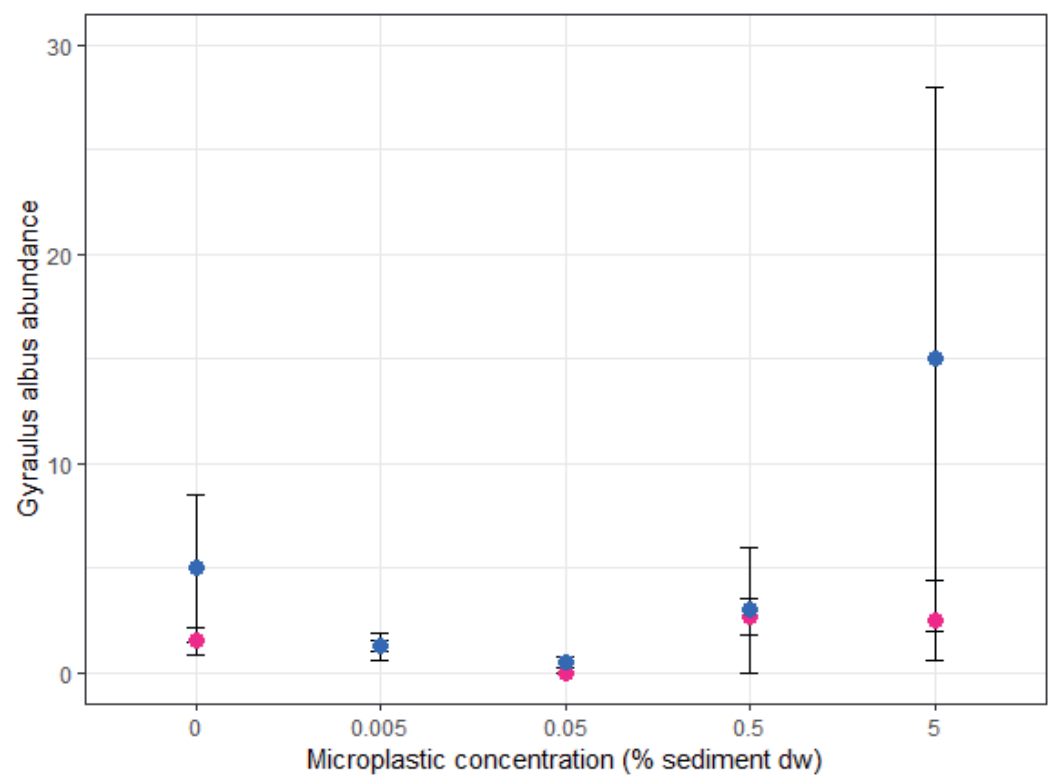

Time

- 3 months

15 months

Figure A5.5. Gyraulus albus abundance in trays retrieved after 3 and 15 months with increasing NP (upper panel) and MP (lower panel) concentrations (as \% sediment dry weight). Error bars are mean $\pm \mathrm{SE} n=4$, except for MP treatments 0.05 and $0.5 \%$ retrieved after 3 months and 0 and $5 \%$ retrieved after 15 months, where $n=3$. 

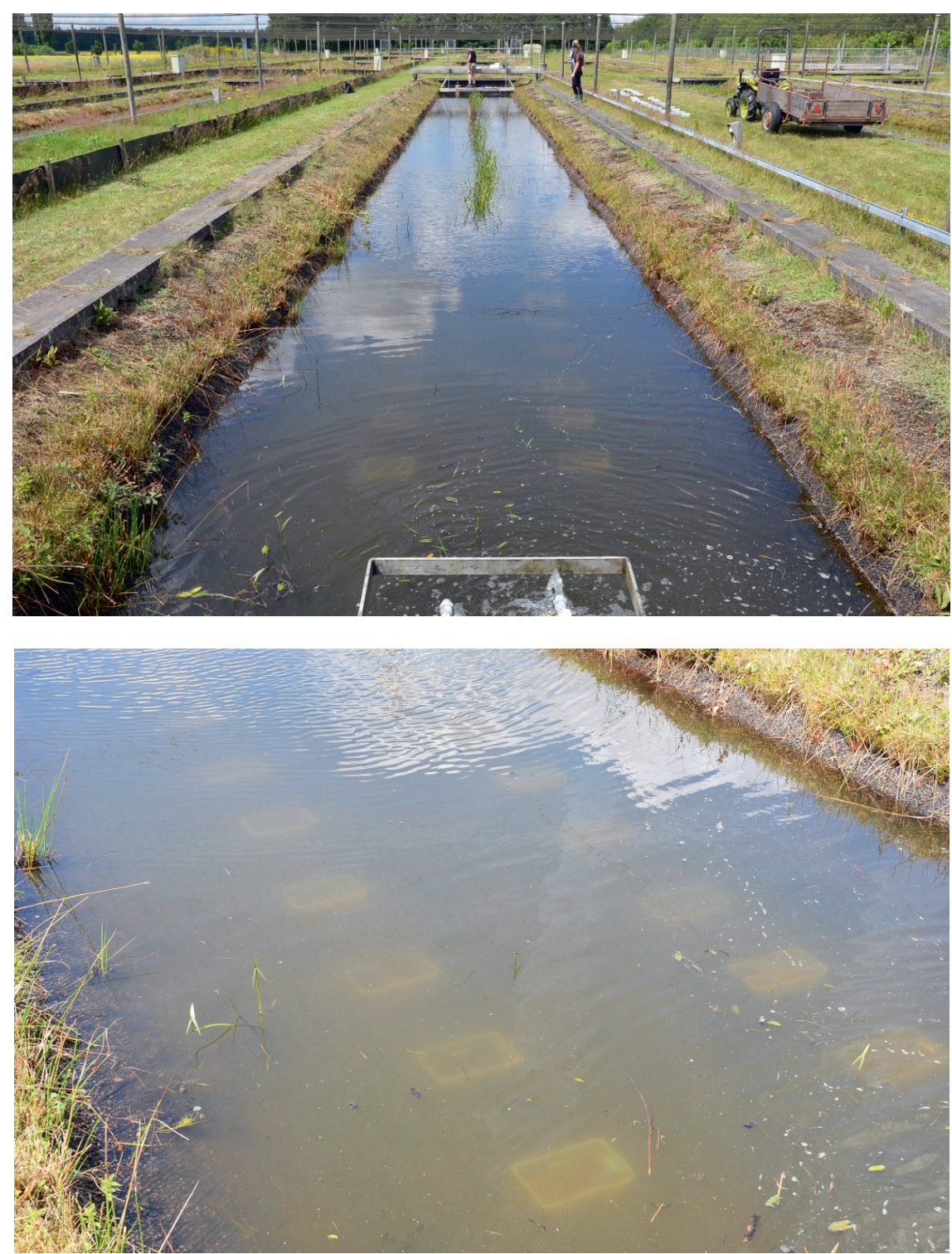

Figure A5.6. Pictures of the experimental ditch with the trays embedded in the sediment. Photo Credit: Albert A. Koelmans, Wageningen University. 


\section{Appendix of Chapter 6}

Effects of the environmental matrices

Effects of nanoplastics

Effects of nanoplastics in the presence of surface water chemicals

Effects of nanoplastics in the presence of WWTP effluent chemicals

Table A6.1. Summary of the significant genotoxic and cytotoxic effects found.

Figure A6.1. Ames fluctuation test results with TA98-S9 and TA98+S9 in DMSO, Surface water (SW) and SW + WWTP effluent water (WWTP) extracts for both experiments.

Figure A6.2. Ames fluctuation test results with TA100-S9 and TA100+S9 in DMSO, Surface water (SW) and SW + WWTP effluent water (WWTP) extracts for both experiments.

Figure A6.3. Ames fluctuation test results with TA98-S9 and TA98+S9 in DMSO with increasing NP concentrations $(2.5,25$ and $250 \mu \mathrm{g} / \mathrm{l})$ for both experiments.

Figure A6.4. Ames fluctuation test results with TA100-S9 and TA100+S9 in DMSO with increasing NP concentrations $(2.5,25$ and $250 \mu \mathrm{g} / \mathrm{l})$ for both experiments.

Figure A6.5. Ames fluctuation test results of TA98-S9 in DMSO and surface water (SW) with increasing NP concentrations $(2.5,25$ and $250 \mu \mathrm{g} / 1)$.

Figure A6.6. Ames fluctuation test results of TA98+S9 in DMSO and surface water (SW) with increasing NP concentrations $(2.5,25$ and $250 \mu \mathrm{g} / \mathrm{l})$.

Figure A6.7. Ames fluctuation test results of TA100-S9 in DMSO and surface water (SW) with increasing NP concentrations $(2.5,25$ and $250 \mu \mathrm{g} / \mathrm{l})$.

Figure A6.8. Ames fluctuation test results of TA100+S9 in DMSO and surface water (SW) with increasing NP concentrations $(2.5,25$ and $250 \mu \mathrm{g} / \mathrm{l})$.

Figure A6.9. Ames fluctuation test results of TA98-S9 in DMSO and WWTP effluent water with increasing NP concentrations $(2.5,25$ and $250 \mu \mathrm{g} / 1)$.

Figure A6.10. Ames fluctuation test results of TA98+S9 in DMSO and WWTP effluent water with increasing NP concentrations $(2.5,25$ and $250 \mu \mathrm{g} / \mathrm{l})$.

Figure A6.11. Ames fluctuation test results of TA100-S9 in DMSO and WWTP effluent water with increasing NP concentrations $(2.5,25$ and $250 \mu \mathrm{g} / \mathrm{l})$.

Figure A6.12. Ames fluctuation test results of TA100-S9 in DMSO and WWTP effluent water with increasing NP concentrations $(2.5,25$ and $250 \mu \mathrm{g} / \mathrm{l})$. 


\section{Effects of the environmental matrices}

The DMSO matrix without NP was genotoxic in TA100-S9 $\left(\chi^{2} ; P=3.12 \times 10^{-10}\right)$ and TA100+S9 $\left(\chi^{2} ; P=0.049\right)$ in the first experiment in comparison to the NC (Figure A6.2, $\mathrm{SI})$. The DMSO matrix without NP was cytotoxic for all strains in the second experiment (t-test; $\left.P_{\mathrm{TA} 98-\mathrm{S} 9}=2.75 \times 10^{-3}, P_{\mathrm{TA} 98+\mathrm{S} 9}=0.01, P_{\mathrm{TA} 100-\mathrm{S} 9}=3.26 \times 10^{-4}, P_{\mathrm{TA} 100+\mathrm{S} 9}=3.77 \times 10^{-3}\right)$, and in TA100+S9 also in the first experiment (t-test; $P=0.02)$ in comparison to the NC (Table A6.1). The SW matrix without NP was genotoxic in TA98-S9 $\left(\chi^{2} ; P=0.01\right)$ (Figure A6.1) and TA100-S9 $\left(\chi^{2} ; P=4.84 \times 10^{-11}\right)$ (Figure A6.2) in the first experiment and in TA100+S9 in both experiments $\left(\chi^{2} ; P_{\mathrm{EXP} 1}=6.34 \times 10^{-3} ; P_{\mathrm{EXP} 2}=1.38 \times 10^{-2}\right)$ (Figure A6.2) in comparison to the NC. When compared to the DMSO matrix without NP, the SW matrix without NP was genotoxic in TA98-S9 $\left(\chi^{2} ; P=4.7 \times 10^{-3}\right)$ (Figure A6.1) in the first experiment and in TA100+S9 $\left(\chi^{2} ; P=0.01\right)$ (Figure A6.2) in the second experiment. The SW matrix without $\mathrm{NP}$ was cytotoxic in TA98+S9 and TA100 \pm S9 only in the second experiment when compared to the NC (t-test; $\left.P_{\mathrm{TA} 98+\mathrm{S} 9}=0.02, P_{\mathrm{TA} 100-\mathrm{S} 9}=2.11 \times 10^{-3}, P_{\mathrm{TA} 98+\mathrm{S} 9}=0.01\right)$ and was never cytotoxic when compared to the DMSO matrix without NP (Table A6.1). The WWTP effluent water matrix without NP was genotoxic for TA100-S9 $\left(\chi^{2} ; P=0.04\right)$ and TA100+S9 $\left(\chi^{2} ; P=9.67 \times 10^{-5}\right)$ in the first experiment in comparison to the NC and only for TA100+S9 $\left(\chi^{2} ; P=0.02\right)$ when compared to the DMSO matrix without NP (Figure A6.2). The WWTP effluent water matrix without NP was cytotoxic in both experiments in TA98+S9 (t-test; $\left.P_{\mathrm{EXP} 1}=1.75 \times 10^{-3}, P_{\mathrm{EXP} 2}=1.43 \times 10^{-3}\right)($ Figure A6.1) and TA100+S9 (t-test; $\left.P_{\mathrm{EXP} 1}=3.76 \times 10^{-4}, P_{\mathrm{EXP} 2}=2.12 \times 10^{-5}\right)$ in comparison to the NC (Figure A6.2). The WWTP effluent water matrix without NP was cytotoxic in TA98-S9 only in the second experiment (t-test; $P=5.99 \times 10^{-5}$ ) and in TA100-S9 only in the first experiment (t-test; $P$ $\left.=7.07 \times 10^{-5}\right)$ in comparison to the NC (Table A6.1). When compared to the DMSO matrix without NP, the cytotoxicity in TA98-S9 (t-test; $\left.P=1.69 \times 10^{-4}\right)$, TA98+S9 $\left(\mathrm{t}-\right.$ test $; P_{\text {EXP1 }}=$ $\left.2.47 \times 10^{-3}, P_{\text {EXP2 }}=3.04 \times 10^{-3}\right)$ and TA100+S9 (t-test; $\left.P_{\text {EXP1 }}=2.47 \times 10^{-3} ; P_{\text {EXP2 }}=1.79 \times 10^{-4}\right)$ remained the same, while in TA100-S9 the first experiment was significant (t-test; $P=$ 0.01 ) and the second was not significant anymore (Table A6.1).

\section{Effects of nanoplastics}

Significant genotoxicity was found only in the second experiment in TA100+S9 when exposed to the two lowest NP concentrations $\left(\chi^{2} ; P_{2.5 \mu \mathrm{g} / 1}=4.3 \times 10^{-5}, P_{25 \mu \mathrm{g} / 1}=1.7 \times 10^{-3}\right)$ and the lowest $500 \mathrm{~nm}$ NP concentration $\left(\chi^{2} ; P_{2.5 \mu \mathrm{g} / 1}=0.01\right)$ in comparison to DMSO without NP (Figure A6.4). Moreover, significant genotoxicity was found in TA98-S9 in the first experiment at the lowest and highest $500 \mathrm{~nm}$ NP concentrations $\left(\chi^{2} ; P_{2.5 \mu \mathrm{g} / 1}=0.01, P_{250 \mu \mathrm{g} / 1}\right.$ $=0.02$ ) (Figure. S5.3). Significant cytotoxicity was found in TA98-S9 at the lowest 50 $\mathrm{nm}$ NP concentration in the first experiment (t-test; $\left.P_{\mathrm{TA} 98-\mathrm{S} 9}=0.04\right)$ and at the lowest 500 $\mathrm{nm}$ NP concentration in both experiments $\left(\mathrm{t}\right.$-test; $\left.P_{\mathrm{EXP} 1}=0.03, P_{\mathrm{EXP} 2}=0.04\right)$ in comparison to the DMSO matrix without NP (Table A6.1). 


\section{Effects of nanoplastics in the presence of surface water chemicals}

$50 \mathrm{~nm}$ nanoplastics. Medium and high $50 \mathrm{~nm}$ NP concentrations in SW had significant genotoxic effects on TA98-S9 in comparison to DMSO with the same NP concentration in the first experiment $\left(\chi^{2} ; P_{25 \mu \mathrm{g} / 1}=3.61 \times 10^{-6}, P_{250 \mu \mathrm{g} / 1}=1.46 \times 10^{-3}\right)$ and in the second experiment $\left(\chi^{2} ; P_{25 \mu \mathrm{g} / \mathrm{l}}=2.53 \times 10^{-3}, P_{250 \mu \mathrm{g} / \mathrm{l}}=1.97 \times 10^{-3}\right)$ (Figure A6.5). When compared to SW without NP, only the medium $50 \mathrm{~nm} \mathrm{NP}$ concentration in the first experiment remained significant $\left(\chi^{2} ; P=0.01\right.$ ) (Figure A6.5). Low and medium $50 \mathrm{~nm} \mathrm{NP}$ concentrations in SW had significant genotoxic effects on TA98+S9 $\left(\chi^{2} ; P_{2.5 \mu \mathrm{g} / 1}=\right.$ $\left.4.50 \times 10^{-3}, P_{25 \mu \mathrm{g} / 1}=4.91 \times 10^{-3}\right)\left(\right.$ Figure A6.6) and TA100+S9 $\left(\chi^{2} ; P_{2.5 \mu \mathrm{g} / 1}=8.85 \times 10^{-4}, P_{25}\right.$ $\left.{ }_{\mu \mathrm{g} / 1}=2.56 \times 10^{-4}\right)($ Figure A6.8) with respect to DMSO with the same $50 \mathrm{~nm} \mathrm{NP}$ concentration, but only in e first experiment. When compared to SW without NP, significant genotoxicity was found only in TA100+S9 at the medium $50 \mathrm{~nm} \mathrm{NP}$ concentration in the first experiment $\left(\chi^{2} ; P=0.01\right)$ (Figure A6.8). In TA100-S9, significant genotoxicity was found at the lowest $50 \mathrm{~nm}$ NP concentration in the first experiment $\left(\chi^{2} ; P=4.26 \times 10^{-5}\right)$ and at the highest in the second experiment $\left(\chi^{2} ; P=0.03\right)$ in comparison to DMSO with the same concentrations, while no significant genotoxic effects were found in comparison to SW without NP (Figure A6.7). Significant cytotoxicity was found in TA100+S9 at the medium and high $50 \mathrm{~nm}$ NP concentration in the second experiment (t-test; $\left.P_{25 \mu \mathrm{g} / 1}=0.01, P_{250 \mu \mathrm{g} / 1}=0.01\right)$ in comparison to DMSO with the same NP concentration (Table A6.1).

$500 \mathrm{~nm}$ nanoplastics. Medium and high $500 \mathrm{~nm}$ NP concentrations had significant genotoxic effects on TA98-S9 in the first experiment $\left(\chi^{2} ; P_{25 \mu \mathrm{g} / \mathrm{l}}=8.22 \times 10^{-5}, P_{250 \mu \mathrm{g} / \mathrm{l}}=\right.$ $\left.1.22 \times 10^{-4}\right)$ and only the high $500 \mathrm{~nm}$ NP concentration had significant genotoxic effects on TA98-S9 in the second experiment $\left(\chi^{2} ; P=5.88 \times 10^{-3}\right)$ in comparison to DMSO with the same NP concentration (Figure A6.5). Low and medium $500 \mathrm{~nm}$ NP concentrations in $\mathrm{SW}$ had significant genotoxic effects on TA98+S9 $\left(\chi^{2} ; P_{2.5 \mu \mathrm{g} / 1}=2.50 \times 10^{-3} ; P_{25 \mu \mathrm{g} / 1}=\right.$ 0.02 ) in comparison to DMSO with the same NP concentration, but only in the first experiment (Figure A6.6). Low and high $500 \mathrm{~nm}$ NP concentrations in SW were genotoxic to TA100+S9 in comparison to DMSO with the same NP concentration in the first experiment $\left(\chi^{2} ; P_{2.5 \mu \mathrm{g} / 1}=8.85 \times 10^{-4}, P_{250 \mu \mathrm{g} / 1}=6.20 \times 10^{-5}\right)$ and only the highest $500 \mathrm{~nm}$ NP concentration in SW was also genotoxic to TA100+S9 in the second experiment $\left(\chi^{2}\right.$; $\left.P_{250 \mu \mathrm{g} / 1}=6.20 \times 10^{-3}\right)($ Figure A6.8). For $500 \mathrm{~nm} \mathrm{NP}$, only the high concentration was genotoxic for TA98-S9 $\left(\chi^{2} ; P=6.35 \times 10^{-4}\right)\left(\right.$ Figure A6.5) and TA100+S9 $\left(\chi^{2} ; P=0.01\right)$ (Figure A6.8) in the first experiment in comparison to SW without NP. Significant cytotoxicity occurred in TA98-S9 in the second experiment at the low $500 \mathrm{~nm} \mathrm{NP}$ concentration (t-test; $P=2.86 \times 10^{-3}$ ) and in both experiments at the high $500 \mathrm{~nm} \mathrm{NP}$ concentration (t-test; $P_{\mathrm{EXP} 1}=0.04, P_{\mathrm{EXP} 2}=0.02$ ) in comparison to the $\mathrm{SW}$ matrix without NP. (Table A6.1). 
Effects of nanoplastics in the presence of WWTP effluent chemicals.

$50 \mathrm{~nm}$ nanoplastics. Medium and high $50 \mathrm{~nm}$ NP concentrations in WWTP effluent water had a significant genotoxic effect on TA98-S9 in comparison to DMSO with the same NP concentration $\left(\chi^{2} ; P_{25 \mu \mathrm{g} / 1}=1.38 \times 10^{-3}, P_{250 \mu \mathrm{g} / 1}=4.91 \times 10^{-3}\right)$, but only in the first experiment (Figure A6.9). Both treatments remained significantly genotoxic in comparison to WWTP effluent water without NP $\left(\chi^{2} ; P_{25 \mu \mathrm{g} / 1}=0.04, P_{250 \mu \mathrm{g} / 1}=0.02\right)$ (Figure A6.9). No significant genotoxic effects were found for TA98+S9 at any $50 \mathrm{~nm} \mathrm{NP}$ concentration (Figure A6.10). Significant genotoxic effects were found in the second experiment for TA100-S9 at the medium $50 \mathrm{~nm}$ NP concentration $\left(\chi^{2} ; P=1.65 \times 10^{-4}\right)$ (Figure A6.11) and for TA100+S9 at the high $50 \mathrm{~nm} \mathrm{NP}$ concentration $\left(\chi^{2} ; P=6.20 \times 10^{-}\right.$ ${ }^{5}$ ) (Figure A6.12) in comparison to DMSO with the same NP concentration. When compared to WWTP effluent water without NP, only the genotoxicity in TA100+S9 at the high $50 \mathrm{~nm} \mathrm{NP}$ concentration remained significant $\left(\chi^{2} ; P=1.37 \times 10^{-4}\right)$ (Figure A6.12). Significant cytotoxic effects were found at the lowest $50 \mathrm{~nm}$ NP concentration in WWTP effluent water in both experiments for TA98+S9 (t-test; $P_{\mathrm{EXP} 1}=6.75 \times 10^{-3}, P_{\mathrm{EXP} 2}=$ $\left.8.70 \times 10^{-3}\right)$, TA100-S9 (t-test; $\left.P_{\mathrm{EXP} 1}=0.01, P_{\mathrm{EXP} 2}=2.19 \times 10^{-3}\right)$ and TA $100+\mathrm{S} 9$ (t-test; $P_{\mathrm{EXP} 1}$ $\left.=2.31 \times 10^{-3} ; P_{\mathrm{EXP} 2}=2.53 \times 10^{-5}\right)$ in comparison to DMSO with the same NP concentration (Table A6.1). Significant cytotoxic effects were found at the medium $50 \mathrm{~nm} \mathrm{NP}$ concentration in WWTP effluent water in both experiments for TA98-S9 (t-test; $P_{\text {EXP1 }}=$ $\left.1.80 \times 10^{-3}, P_{\mathrm{EXP} 2}=2.23 \times 10^{-4}\right)$ and TA100+S9 (t-test; $\left.P_{\mathrm{EXP} 1}=0.01, P_{\mathrm{EXP} 2}=3.88 \times 10^{-4}\right)$ in comparison to DMSO with the same NP concentration (Table A6.1). Significant cytotoxic effects were found at the highest $50 \mathrm{~nm}$ NP concentration in WWTP effluent water in both experiments for TA98-S9 (t-test; $P_{\mathrm{EXP} 1}=9.32 \times 10^{-3}, P_{\mathrm{EXP} 2}=4.34 \times 10^{-4}$ ), TA98+S9 (t-test; $\left.P_{\mathrm{EXP} 1}=0.02, P_{\mathrm{EXP} 2}=0.046\right)$ and TA100-S9 (t-test; $P_{\mathrm{EXP} 1}=2.24 \times 10^{-4}$, $\left.P_{\text {EXP2 }}=0.03\right)$ in comparison to DMSO with the same NP concentration (Table A6.1).

$50 \mathrm{~nm}$ nanoplastics. Significant genotoxic effects were found at the medium $500 \mathrm{~nm} \mathrm{MP}$ concentration in TA98-S9 in the first experiment $\left(\chi^{2} ; P=0.04\right)$ (Figure A6.9) and in TA100-S9 in the second experiment $\left(\chi^{2} ; P=2.94 \times 10^{-3}\right)$ (Figure A6.11) in comparison to DMSO with the same NP concentration. No significant genotoxic effects were found for TA98+S9 and TA100+S9 at any $500 \mathrm{~nm}$ NP concentration (Figures A6.10, A6.12). Significant cytotoxic effects were found at the lowest $500 \mathrm{~nm}$ NP concentration in WWTP effluent water in both experiments for TA98-S9 (t-test; $P_{\mathrm{EXP} 1}=0.03, P_{\mathrm{EXP} 2}=2.45 \times 10^{-3}$ ) and TA100+S9 (t-test; $\left.P_{\mathrm{EXP} 1}=0.01 ; P_{\mathrm{EXP} 2}=0.04\right)$ in comparison to DMSO with the same NP concentration (Table A6.1). Significant cytotoxic effects were found at the medium $500 \mathrm{~nm}$ NP concentration in WWTP effluent water in both experiments for TA98-S9 (ttest; $\left.P_{\mathrm{EXP} 1}=1.16 \times 10^{-3}, P_{\mathrm{EXP} 2}=3.70 \times 10^{-4}\right)$, and at the highest $500 \mathrm{~nm}$ NP concentration in WWTP effluent water in both experiments for TA98+S9 (t-test; $P_{\mathrm{EXP} 1}=0.02 ; P_{\mathrm{EXP} 2}=$ $8.90 \times 10^{-3}$ ) in comparison to DMSO with the same NP concentration (Table A6.1). 
Table A6.1. Summary of the significant genotoxic and cytotoxic effects found.

\begin{tabular}{|c|c|c|c|c|}
\hline & TA98-S9 & TA98+S9 & TA100-S9 & TA100+S9 \\
\hline DMSO $^{\text {(a) }}$ & $-/(-)$ & $-/(-)$ & $+/(-)$ & $(+) /(-)$ \\
\hline $\mathbf{S W}^{\text {(a) }}$ & $+/-$ & $-/(-)$ & $+/(-)$ & $+/(+)$ \\
\hline $\mathbf{S W}^{(\mathbf{b})}$ & $+/-$ & $-/-$ & $-/-$ & $-/+$ \\
\hline WWTP ${ }^{\text {(a) }}$ & $-/(-)$ & $(-) /(-)$ & $+/(-)$ & $(+) /(-)$ \\
\hline WWTP $^{(\mathbf{b})}$ & $-/(-)$ & $(-) /(-)$ & $(-) /-$ & $(+) /(-)$ \\
\hline $50 \mathrm{~nm}$ NP $2.5 \mu \mathrm{g} / \mathrm{I}^{(\mathrm{b})}$ & $(-) /-$ & $-/-$ & $-/-$ & $-/+$ \\
\hline $50 \mathrm{~nm} \mathrm{NP} 25 \mu \mathrm{g} / \mathrm{l}^{(\mathrm{b})}$ & $-/-$ & $-/-$ & $-/-$ & $-/+$ \\
\hline 50 nm NP $250 \mu \mathrm{g} / \mathrm{l}^{(\mathrm{b})}$ & $-/-$ & $-/-$ & $-/-$ & $-/-$ \\
\hline $50 \mathrm{~nm} \mathrm{NP} 2.5 \mu \mathrm{g} / \mathrm{l}+\mathrm{SW}^{(\mathrm{c})}$ & $-/-$ & $+/-$ & $+/-$ & $+/-$ \\
\hline $50 \mathrm{~nm}$ NP $25 \mu \mathrm{g} / \mathrm{l}+\mathrm{SW}^{(\mathrm{c})}$ & $+/+$ & $+/-$ & $-/-$ & $+/(-)$ \\
\hline $50 \mathrm{~nm}$ NP $250 \mu \mathrm{g} / \mathrm{l}+\mathrm{SW}^{(\mathrm{c})}$ & $+/+$ & $-/-$ & $-/+$ & $-/(-)$ \\
\hline $50 \mathrm{~nm}$ NP $2.5 \mu \mathrm{g} / \mathrm{l}+\mathrm{SW}^{(\mathrm{d})}$ & $-/(-)$ & $-/-$ & $-/-$ & $-/-$ \\
\hline 50 nm NP $25 \mu \mathrm{g} / \mathrm{l}+\mathrm{SW}^{(\mathrm{d})}$ & $+/-$ & $-/-$ & $-/-$ & $+/-$ \\
\hline 50 nm NP $250 \mu \mathrm{g} / \mathrm{l}+\mathrm{SW}^{(\mathrm{d})}$ & $(-) /(-)$ & $-/-$ & $-/-$ & $-/-$ \\
\hline $50 \mathrm{~nm}$ NP $2.5 \mu \mathrm{g} / \mathrm{l}+\mathrm{WWTP}{ }^{(\mathrm{c})}$ & $(-) /-$ & $(-) /(-)$ & $(-) /(-)$ & $(-) /(-)$ \\
\hline $50 \mathrm{~nm} \mathrm{NP} 25 \mu \mathrm{g} / \mathrm{l}+\mathrm{WWTP}{ }^{(c)}$ & $(+) /(-)$ & $(-) /-$ & $-/+$ & $(-) /(-)$ \\
\hline 50 nm NP $250 \mu \mathrm{g} / \mathrm{l}+$ WWTP ${ }^{(c)}$ & $(+) /(-)$ & $(-) /(-)$ & $(-) /(-)$ & $(-) /(+)$ \\
\hline $50 \mathrm{~nm}$ NP $2.5 \mu \mathrm{g} / \mathrm{l}+\mathrm{WWTP}{ }^{(\mathrm{e})}$ & $-/-$ & $-/-$ & $-/-$ & $-/-$ \\
\hline 50 nm NP $25 \mu \mathrm{g} / \mathrm{l}+\mathrm{WWTP}^{(\mathrm{e})}$ & $+/-$ & $-/-$ & $-/-$ & $-/-$ \\
\hline $50 \mathrm{~nm}$ NP $250 \mu \mathrm{g} / \mathrm{l}+\mathrm{WWTP}{ }^{(\mathrm{e})}$ & $+/-$ & $-/-$ & $-/-$ & $-/+$ \\
\hline $500 \mathrm{~nm}$ NP $2.5 \mu \mathrm{g} / \mathrm{I}^{(\mathrm{b})}$ & $(+) /(-)$ & $-/-$ & $-/-$ & $-/+$ \\
\hline 500 nm NP $25 \mu \mathrm{g} / \mathbf{l}^{(b)}$ & $-/-$ & $-/-$ & $-/-$ & $-/-$ \\
\hline $500 \mathrm{~nm}$ NP $250 \mu \mathrm{g} / \mathrm{l}^{(\mathrm{b})}$ & $+/-$ & $-/-$ & $-/-$ & $-/-$ \\
\hline $500 \mathrm{~nm}$ NP $2.5 \mu \mathrm{g} / \mathrm{l}+\mathrm{SW}^{(\mathrm{c})}$ & $-/-$ & $+/-$ & $-/-$ & $+/-$ \\
\hline $500 \mathrm{~nm} \mathrm{NP} 25 \mu \mathrm{g} / \mathrm{l}+\mathrm{SW}^{(\mathrm{c})}$ & $+/-$ & $+/-$ & $-/-$ & $+/-$ \\
\hline 500 nm NP $250 \mu \mathrm{g} / \mathrm{l}+\mathrm{SW}^{(\mathrm{c})}$ & $+/+$ & $-/-$ & $-/-$ & $+/+$ \\
\hline 500 nm NP $2.5 \mu \mathrm{g} / \mathrm{l}+\mathrm{SW}^{(\mathrm{d})}$ & $-/-$ & $-/-$ & $-/-$ & $-/-$ \\
\hline 500 nm NP $25 \mu \mathrm{g} / \mathrm{l}+\mathrm{SW}^{(\mathrm{d})}$ & $-/-$ & $-/-$ & $-/-$ & $-/-$ \\
\hline $500 \mathrm{~nm} \mathrm{NP} 250 \mu \mathrm{g} / \mathrm{l}+\mathrm{SW}^{(\mathrm{d})}$ & $+/-$ & $-/-$ & $-/-$ & $+/-$ \\
\hline $500 \mathrm{~nm}$ NP $2.5 \mu \mathrm{g} / \mathrm{l}+\mathrm{WWTP}{ }^{(\mathrm{c})}$ & $(-) /(-)$ & $-/(-)$ & $-/-$ & $(+) /(-)$ \\
\hline 500 nm NP $25 \mu \mathrm{g} / \mathrm{l}+\mathrm{WWTP}{ }^{(c)}$ & $(+) /(-)$ & $-/-$ & $-/+$ & $+/(+)$ \\
\hline 500 nm NP $250 \mu \mathrm{g} / \mathrm{l}+\mathrm{WWTP}{ }^{(\mathrm{c})}$ & $(-) /-$ & $(-) /(-)$ & $-/-$ & $-/+$ \\
\hline 500 nm NP $2.5 \mu \mathrm{g} / \mathrm{l}+\mathrm{WWTP}^{(\mathrm{e})}$ & $-/-$ & $-/-$ & $-/-$ & $-/-$ \\
\hline 500 nm NP $25 \mu \mathrm{g} / \mathrm{l}+\mathrm{WWTP}^{(\mathrm{e})}$ & $-/-$ & $-/-$ & $-/-$ & $-/+$ \\
\hline 500 nm NP $250 \mu \mathrm{g} / \mathrm{l}+$ WWTP ${ }^{(e)}$ & $-/-$ & $-/-$ & $-/-$ & $-/+$ \\
\hline
\end{tabular}

(a) In comparison to the $\mathrm{NC}$

(b) In comparison to DMSO matrix without NP

(c) In comparison with DMSO and the same NP concentration

(d) In comparison to SW matrix without NP

(e) In comparison to WWTP effluent matrix without NP

+ indicates a significant positive response, - a negative response, $(+)$ a possible false statistically significant positive response due to cytotoxicity and $(-)$ a possible false negative response due to cytotoxicity. 
TA98 - S9

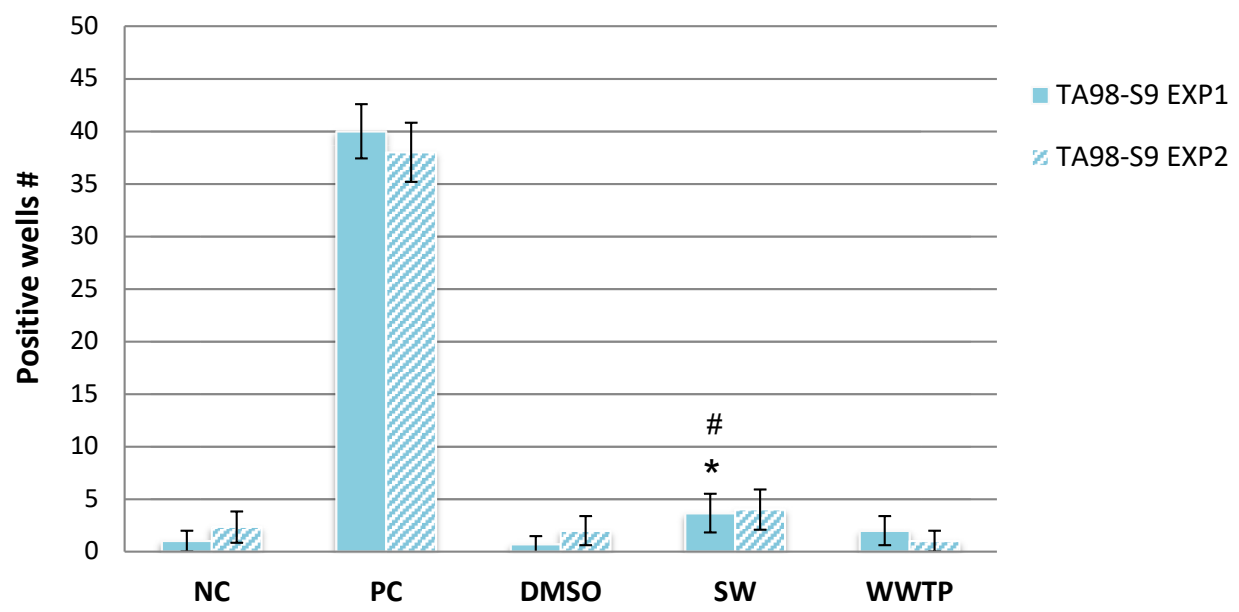

TA98 + S9

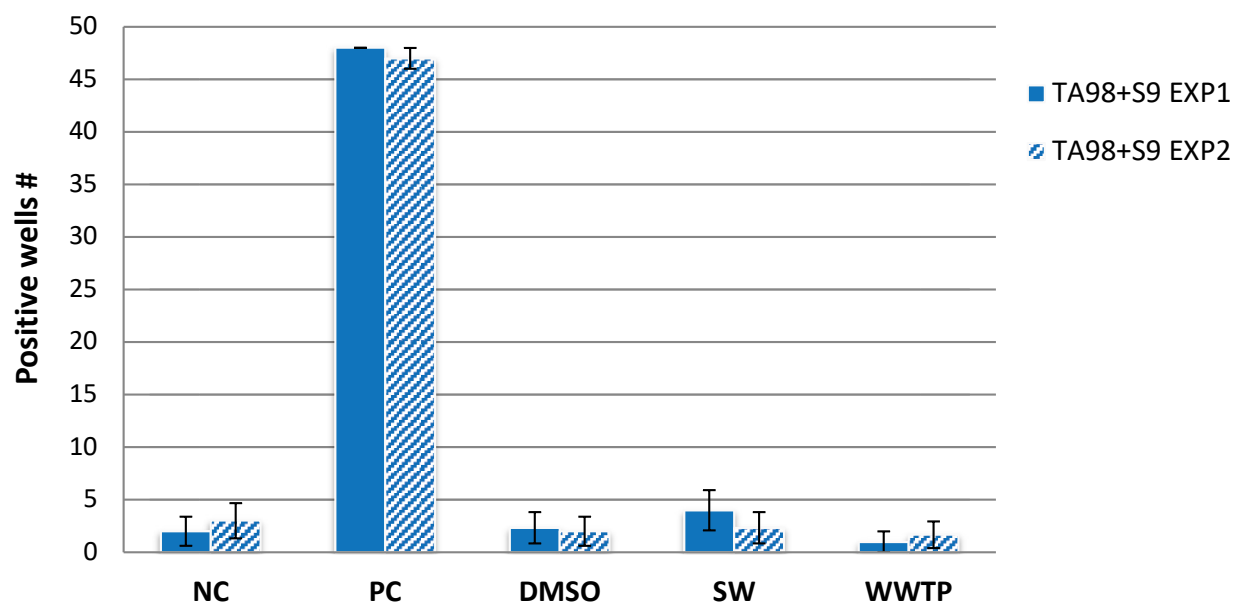

Figure A6.1. Ames fluctuation test results with TA98-S9 (upper panel) and TA98+S9 (lower panel) in DMSO, Surface water (SW) and SW + WWTP effluent water (WWTP) extracts for both experiments (EXP1 and EXP2). Results of a negative control (NC) and a positive control (PC) are also shown. Bars represent average values $(n=3)$ for two independent experiments \pm standard deviations. Asterisks show significant differences between the environmental matrices and the NC in each of the experiments. Hashes show significant differences between the SW and WWTP effluent water matrices and the DMSO matrix in each of the experiments. 


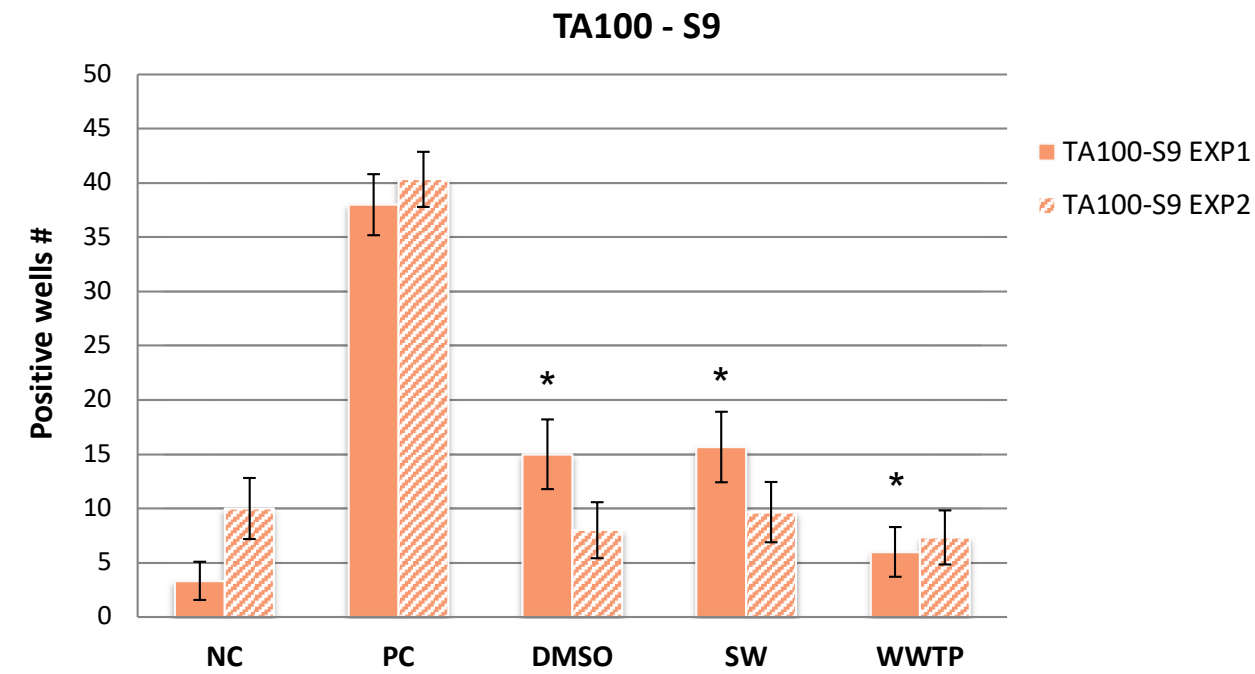

TA100 + S9

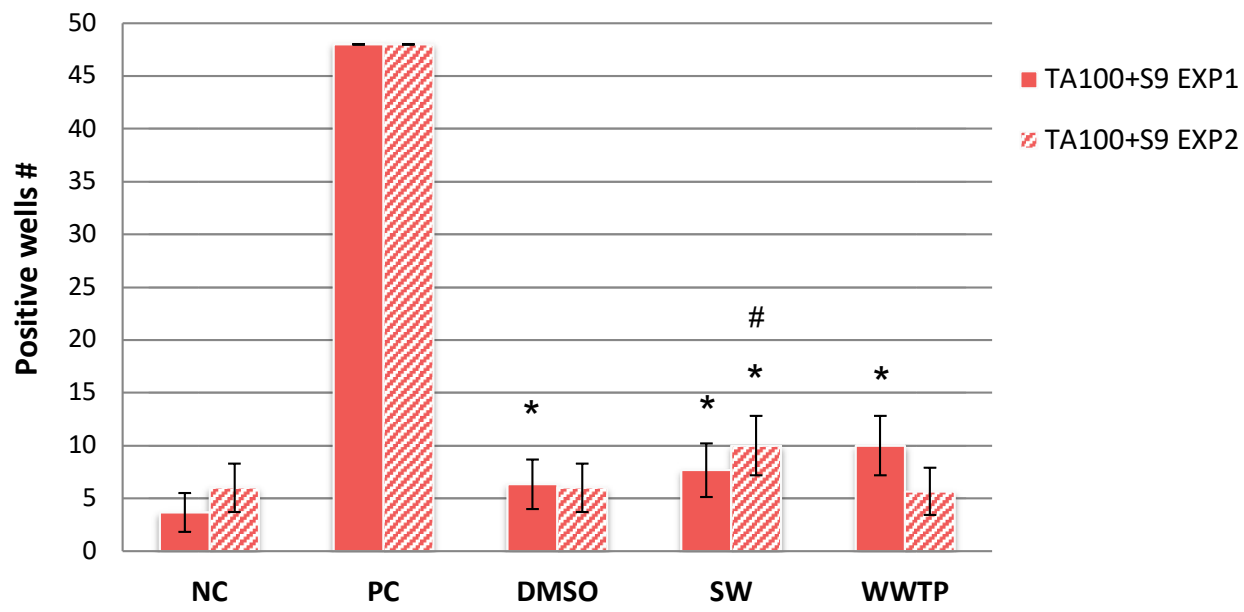

Figure A6.2. Ames fluctuation test results with TA100-S9 (upper panel) and TA100+S9 (lower panel) in DMSO, Surface water (SW) and SW + WWTP effluent water (WWTP) extracts for both experiments (EXP1 and EXP2). Results of a negative control (NC) and a positive control (PC) are also shown. Bars represent average values $(n=3)$ for two independent experiments \pm standard deviations. Asterisks show significant differences between the environmental matrices and the NC in each of the experiment. Hashes show significant differences between the SW and WWTP effluent water matrices and the DMSO matrix in each of the experiments. 
TA98 - S9

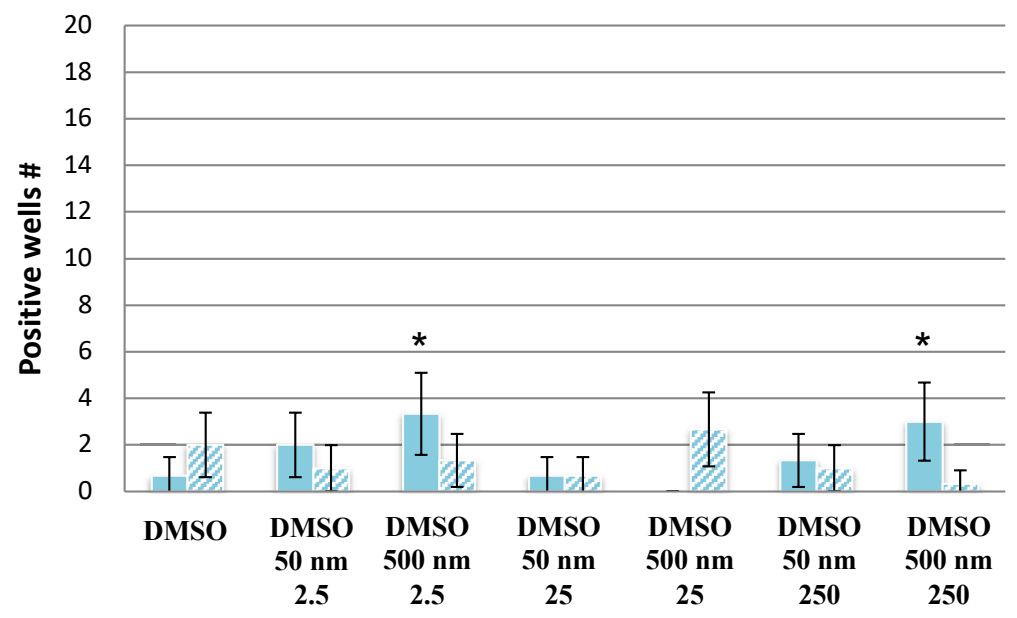

TA98-S9 EXP1

TA98-S9 EXP2

TA98 + S9

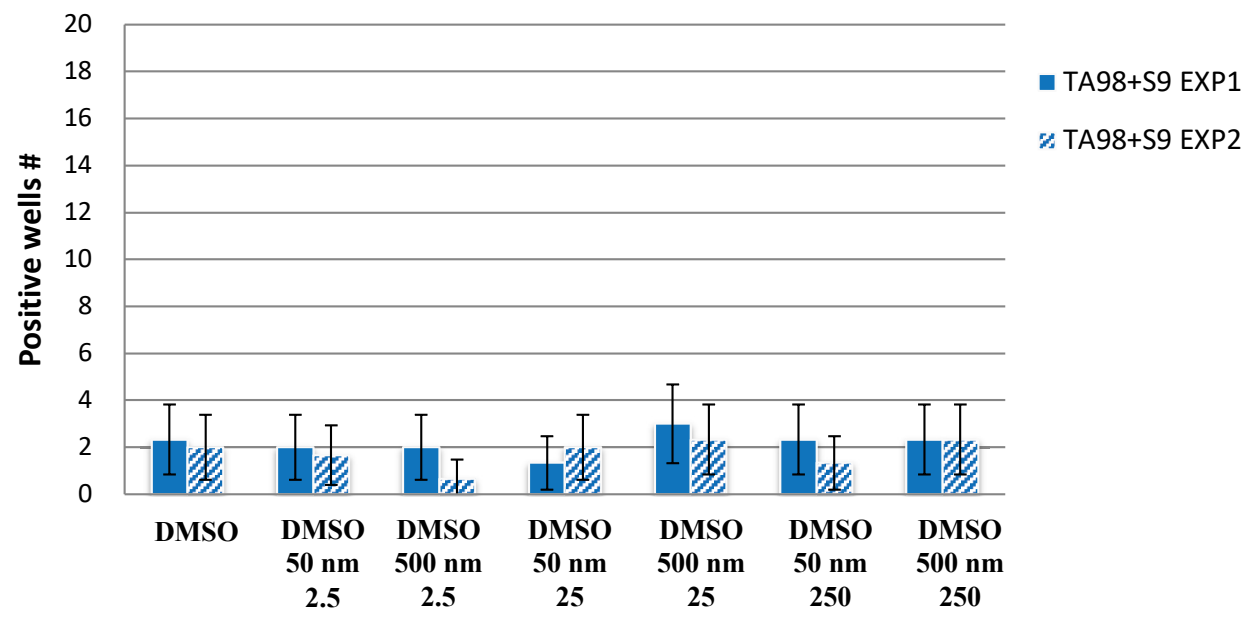

Figure A6.3. Ames fluctuation test results with TA98-S9 (upper panel) and TA98+S9 (lower panel) in DMSO with increasing NP or MP concentrations $(2.5,25$ and $250 \mu \mathrm{g} / \mathrm{l})$ for both experiments (EXP1 and EXP2). Bars represent average values of two experiments \pm standard deviations $(n=3)$. Asterisks show significant differences between the treatments with NP concentrations and the DMSO matrix without NP in each of the experiments. No positive wells were found for the medium $500 \mathrm{~nm}$ NP concentration in DMSO in TA98-S9 in the first experiment. 


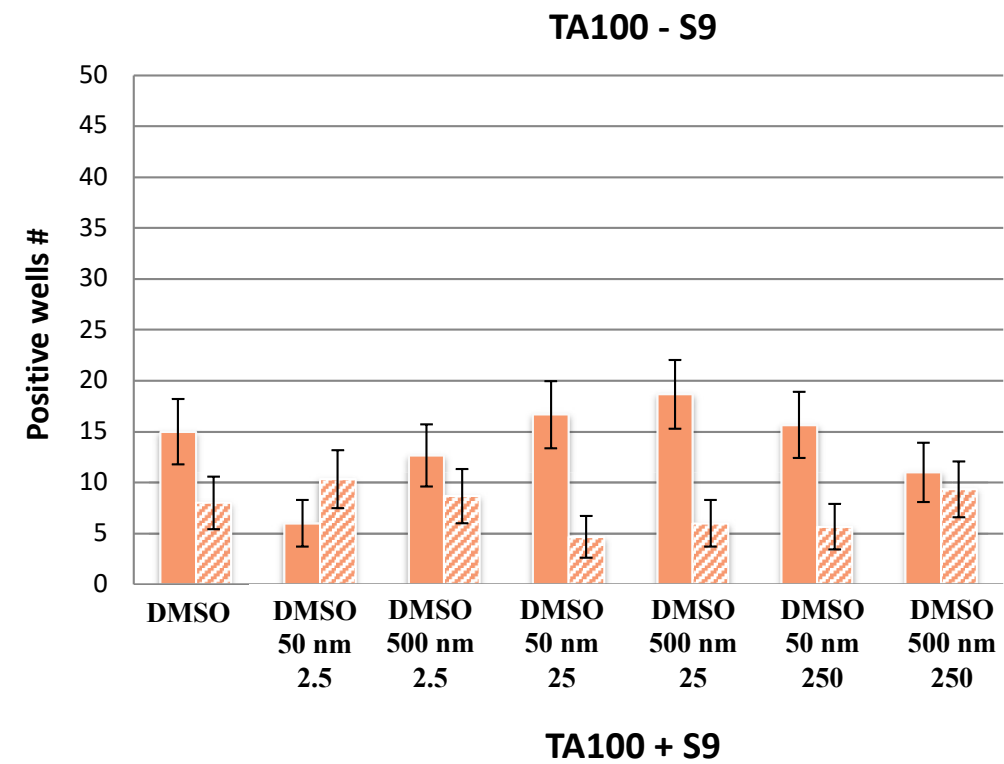

- TA100-S9 EXP1

2. TA100-S9 EXP2

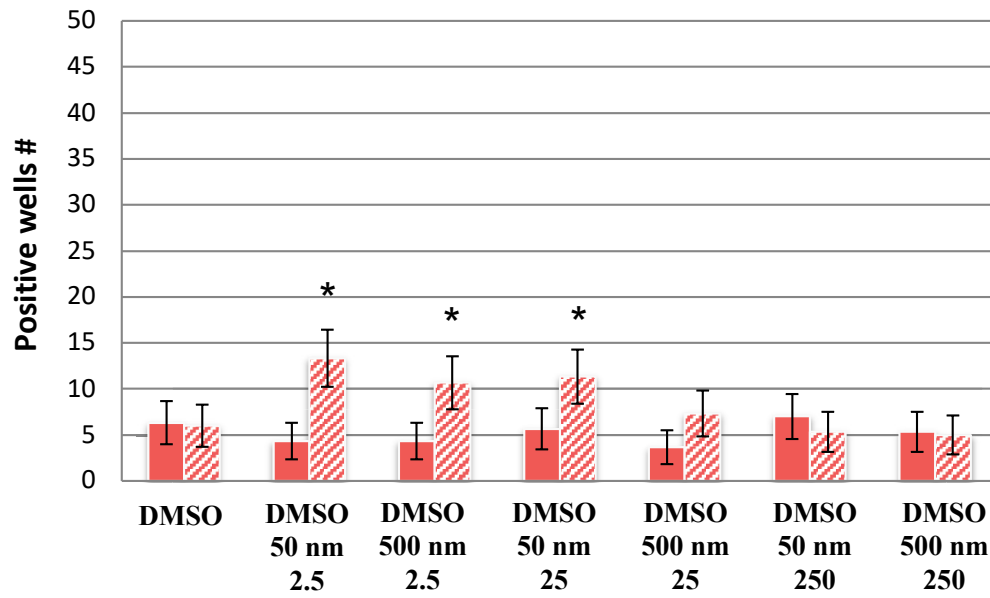

TA100+S9 EXP1 z TA100+S9 EXP2

Figure A6.4. Ames fluctuation test results with TA100-S9 (upper panel) and TA100+S9 (lower panel) in DMSO with increasing NP concentrations $(2.5,25$ and $250 \mu \mathrm{g} / \mathrm{l})$ for both experiments (EXP1 and EXP2). Bars represent average values of two experiments \pm standard deviations (n =3). Asterisks show significant differences between the treatments with NP concentrations and the DMSO matrix without NP in each of the experiments. 


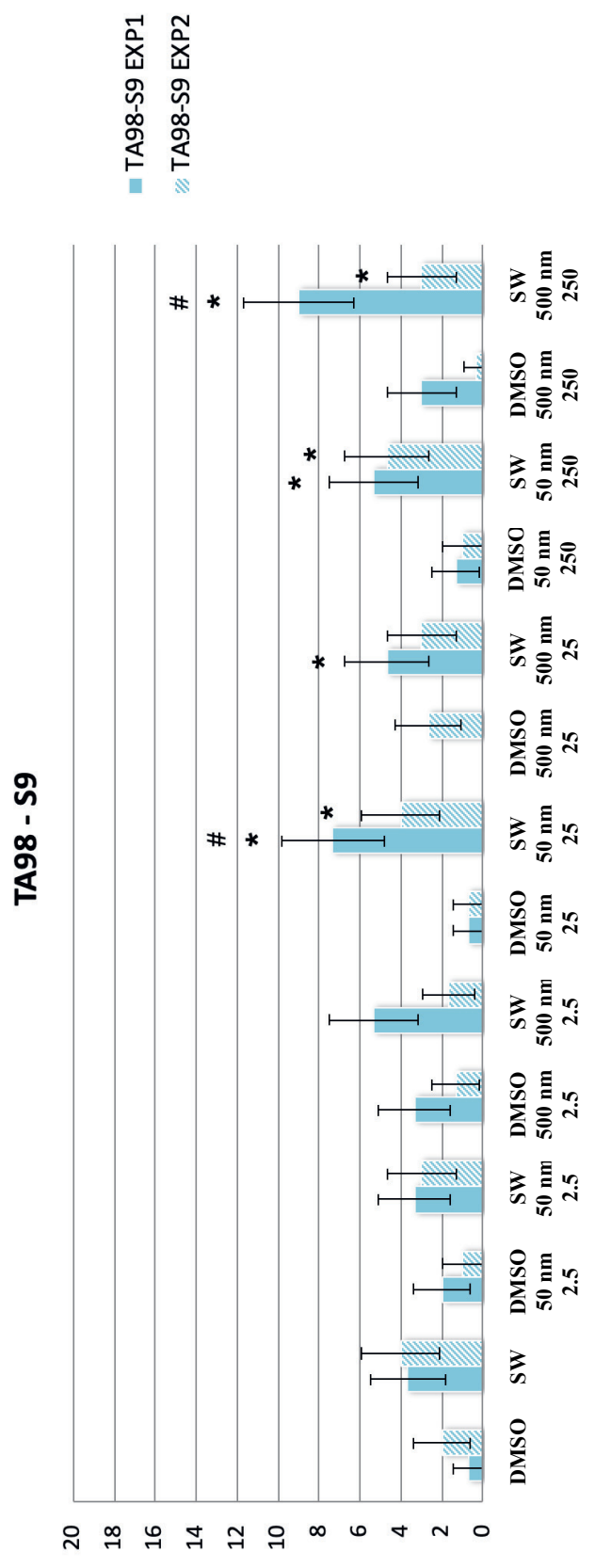

\# S||әM әм!?!SOd

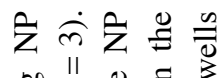

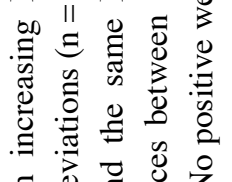

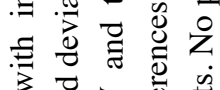

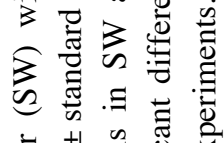

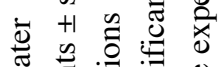

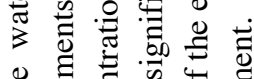

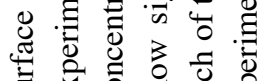

ज 范

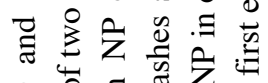

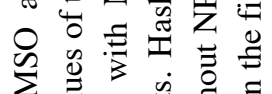

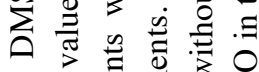

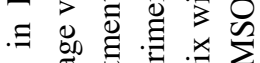

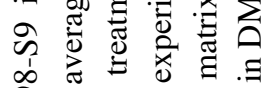

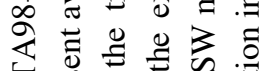

$\forall$ 造

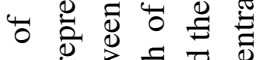

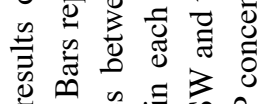

ڤ

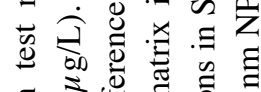

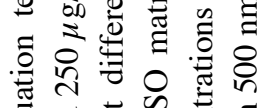

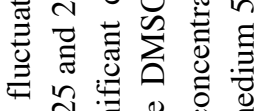

๙ิ

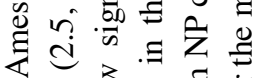

ம்

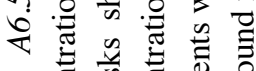

ऽ

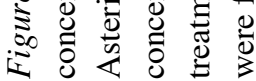




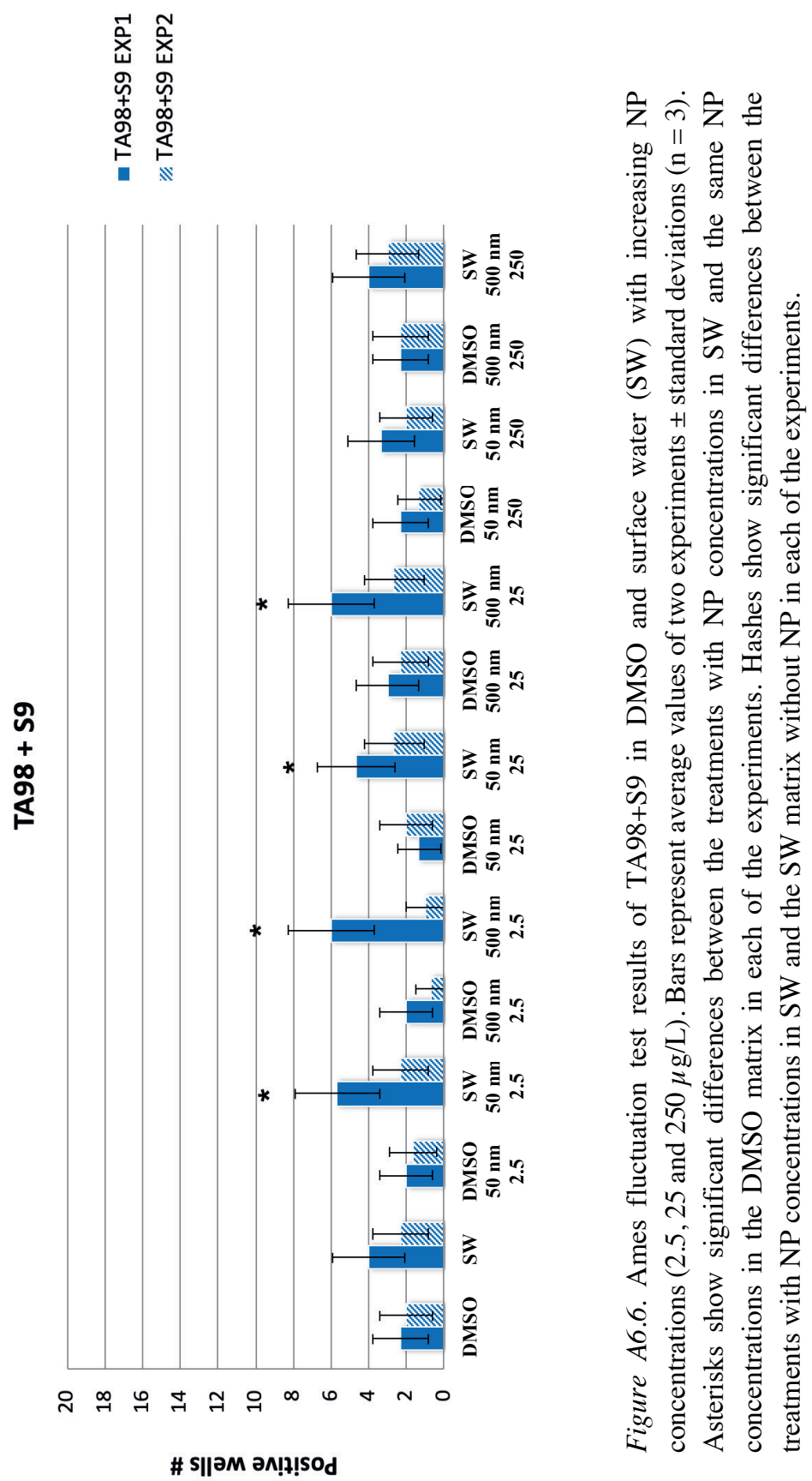




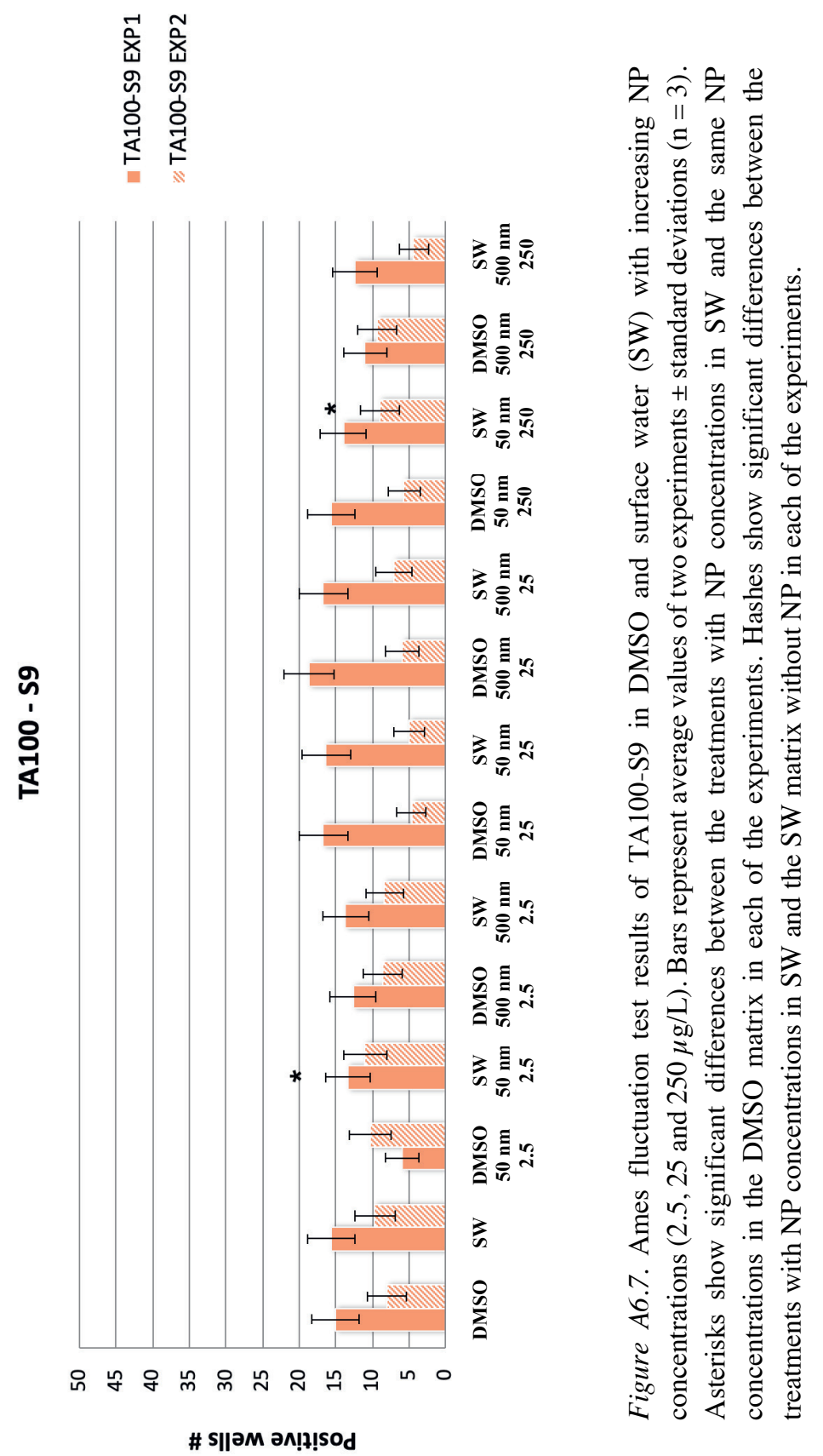




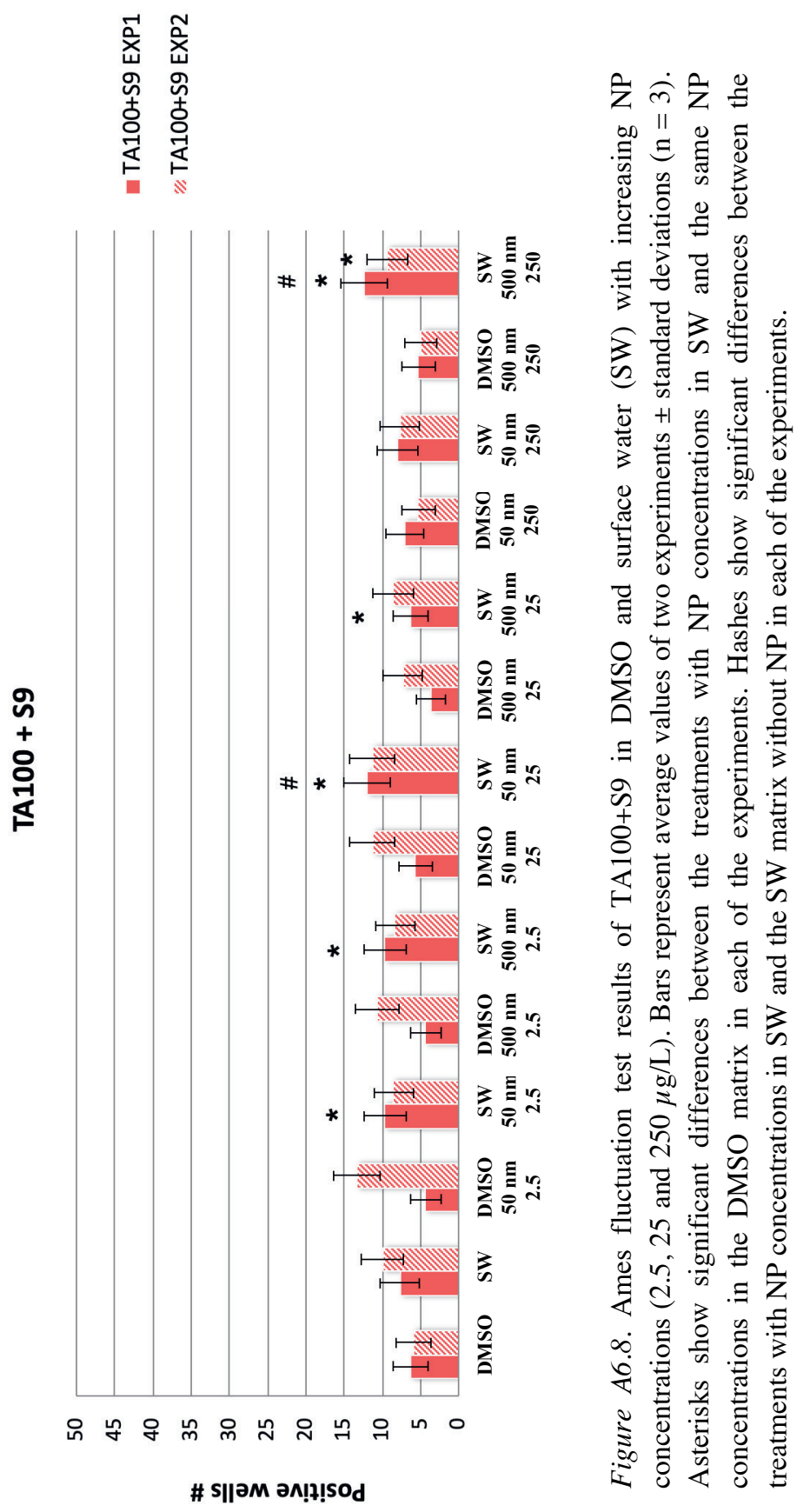




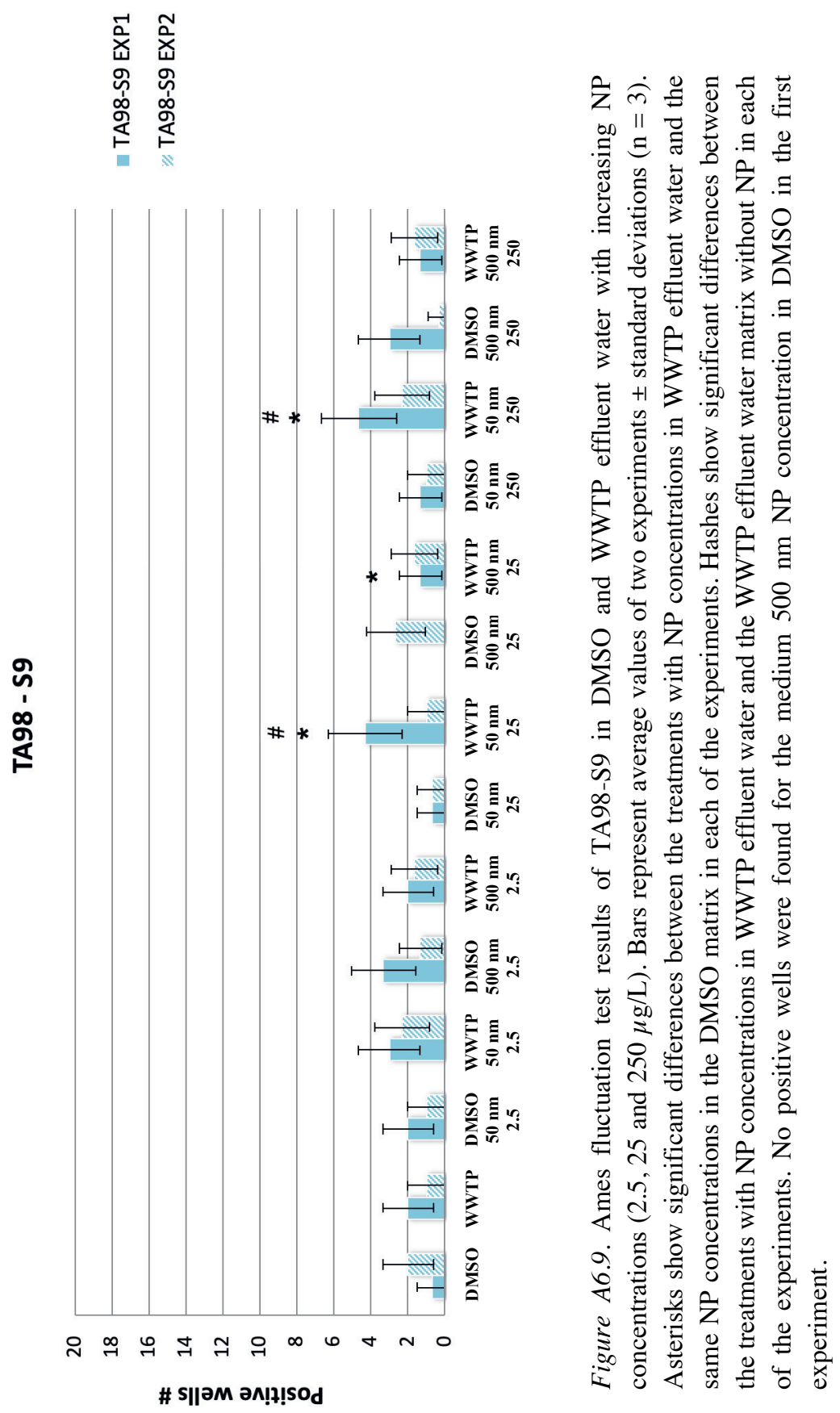




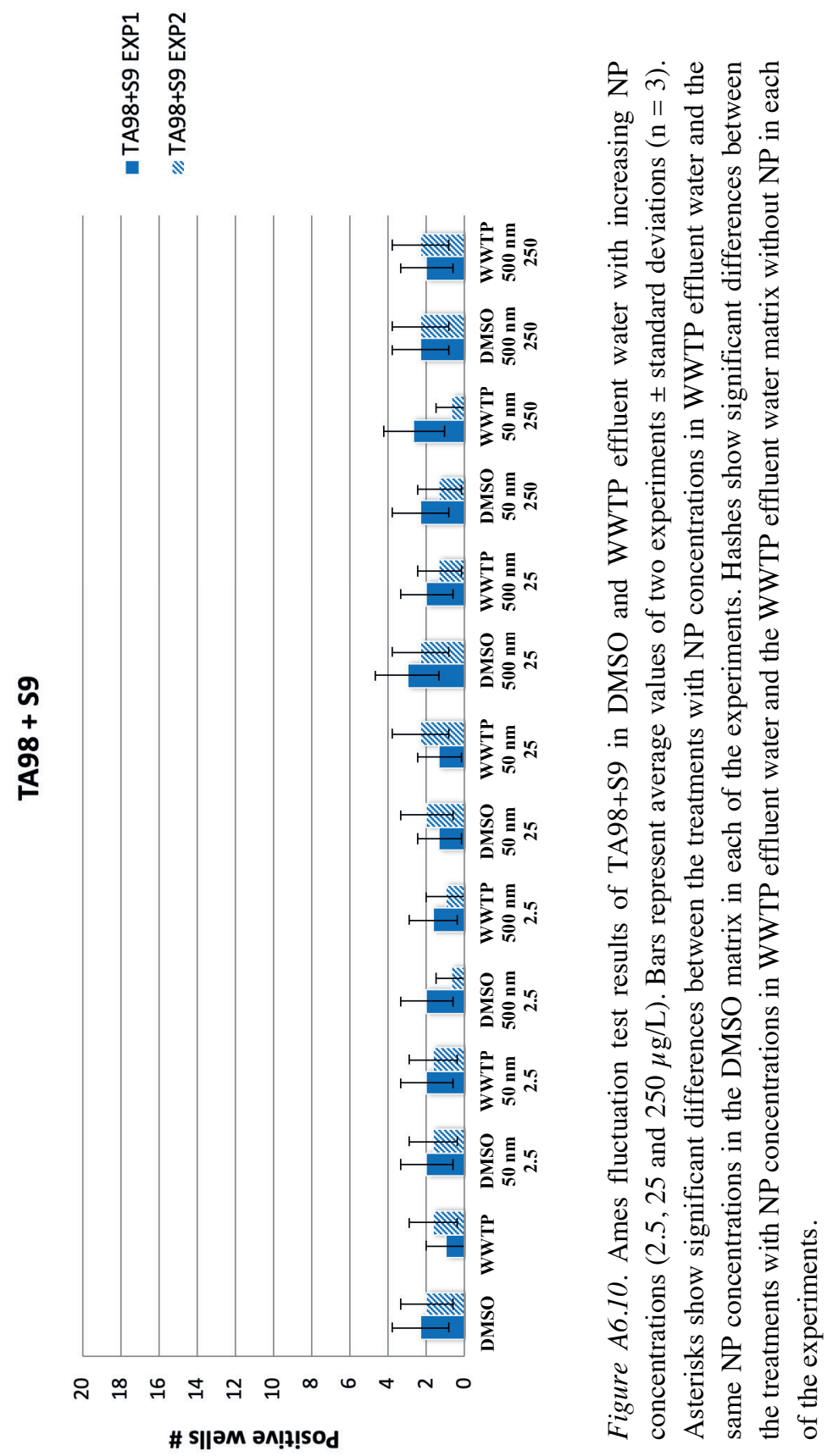




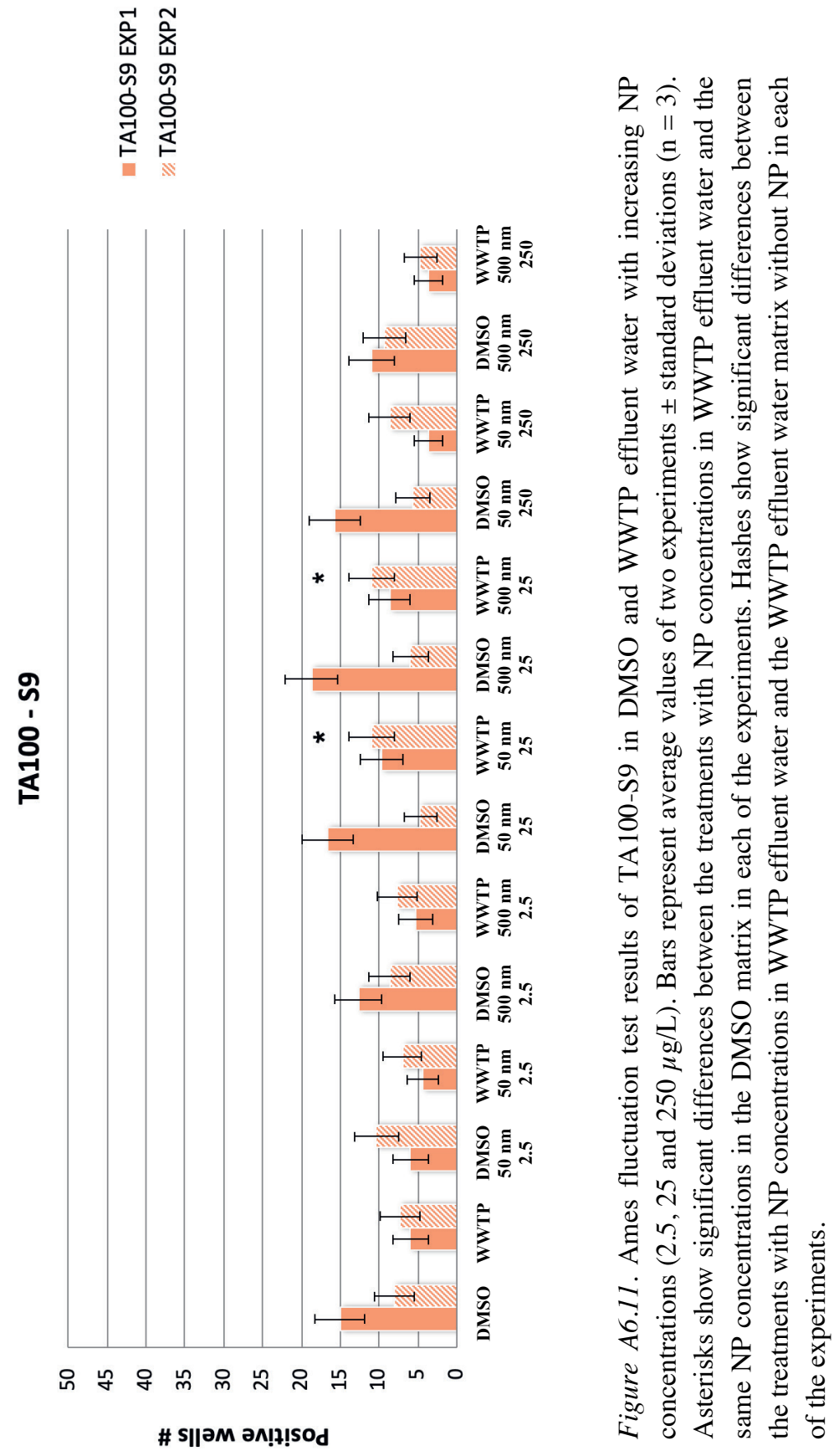




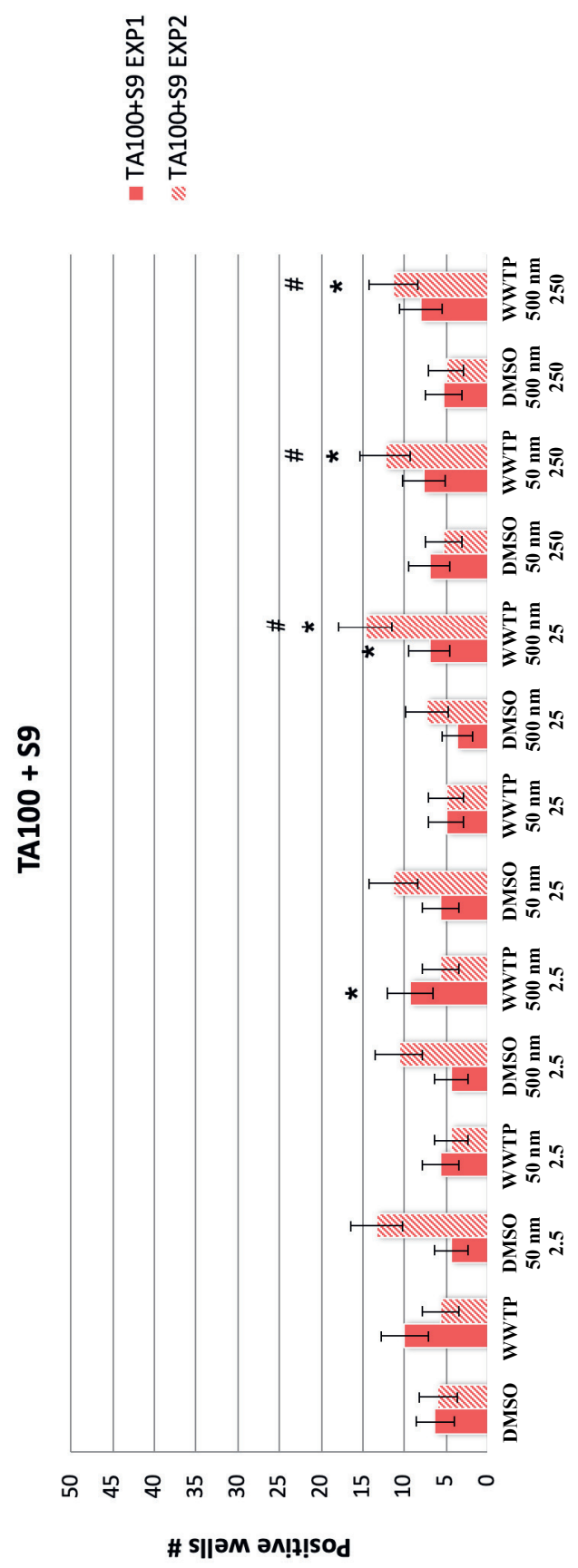

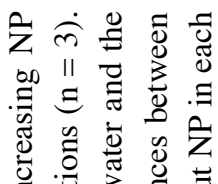

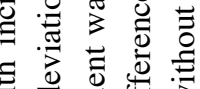

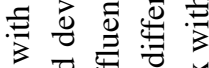

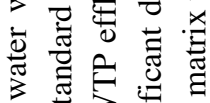

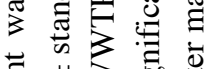

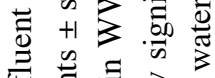

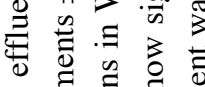

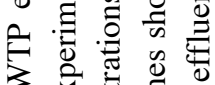

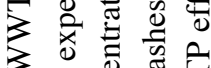

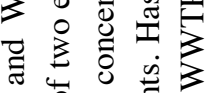

○ ठ

方吾青要

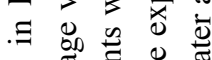

จे

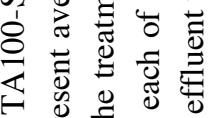

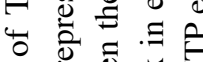

品

矛莕芯

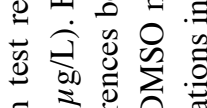

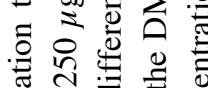

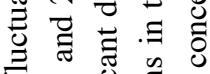

ธุ

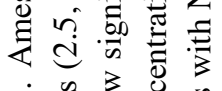

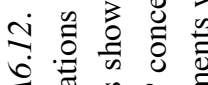

४

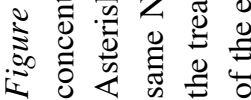




\section{Appendix of Chapter 7}

Methods continued - Detailed motivation for each criterion used in the quality evaluation.

Table A7.1. Study characteristics.

Table A7.2. Explanation of the quantitative scoring system proposed to evaluate the studies testing the effects of $M P$ on aquatic biota using the $(Q A / Q C)$ criteria.

Figure A7.1. Size ranges used in the scored studies. Lines represent the size range reported and data points represent the reported or calculated average size.

Figure A7.2. Exposure duration in hours for $n=105$ studies. 


\section{Methods continued - Detailed motivation for each criterion used in the quality evaluation.}

\section{Particle characterization}

Criterion 1. Particle size. Species-specific physiological and behavioural traits can strongly influence the relative size of particles ingested by an organism, including MP. ${ }^{46,53,186,303}$ Size selectivity depends on the morphology and feeding strategy of a species, which determines the upper size limit for the food they can ingest, as well as for the ingestible size of MP. ${ }^{297,379,380}$ For instance, in a study assessing the ingestion of MP by seven Cladocera species, the maximum size of MP ingested increased proportionally with the body size. ${ }^{379}$ The upper size limit will differ between species at varying trophic levels, but can also show significant variation within species depending on their developmental stage. ${ }^{46,83}$ Based on species traits, size preferences have been demonstrated for a few organisms, being some MP sizes ingested in higher quantities than others. ${ }^{186,380}$ Particle shape and polymer identity also affect the probability of MP to be encountered and ingested, thereby affecting the bioavailability of MP. ${ }^{46}$ Furthermore, the residence time of MP in the body of the organisms has also been related with the size of the particles. ${ }^{328}$ The relative relationship between the ingestion and retention of MP can result in decreased nutritional value and/or physical obstruction in the digestive tract, which have been proposed as two of the mechanisms underlying observed adverse effects for organisms exposed to MP. ${ }^{83,92,186,303,304}$ As the ingestion and effects of MP can be size-dependent, the size distribution of the MP selected in an effect study can directly influence the occurrence and severity of the effects observed and therefore requires analytical characterization. Consequently, studies that report the full particle size distribution of the tested MP are assigned a criterion value of 2 . The distribution, however, should be provided with sufficient resolution, ideally with 10 bins or more. If only one size is reported instead of a range, a study receives 2 points when the size reported is supported by analytical characterization and reported with a measurement error. MP sizes should ideally be characterized analytically using dynamic light scattering or laser diffraction methods or alternatively estimated using high resolution microscopy of the MP with a scale in combination with imaging analysis software. When the particle size/sizes are reported but not supported by analytical characterization, based on information provided in material safety data sheets or size separation using sieves, a study is assigned a criterion value of 1 . Finally, studies that did not report the size of the MP used in their experiments are assigned a criterion value of 0 .

Criterion 2. Particle shape. For several species, selective ingestion, gut retention, and effects of MP have been found to depend on their shape. ${ }^{83,327,328}$ For instance, fibres were more lethal than spheres for the amphipod Hyalella azteca. ${ }^{83}$ Authors report that fibres resulted in longer gut retention times, speculating that fibres may have aggregated in the gut. ${ }^{83}$ Additionally, Piarulli et al. (2020) showed that the MP analysed in six different benthic invertebrate species collected from salt marshes, were mostly fibres (98.5\%). MP fragments are also reported to be associated with longer gut retention times in the cladoceran Daphnia magna in comparison to spherical MP. ${ }^{327}$ It has been suggested that the rounded shape of spherical MP facilitates their transport through the digestive system of organisms, resulting in less severe effects than for other shapes of MP. ${ }^{83}$ Given several observations reporting on the relative influence of the shape of MP on effect 
endpoints, the evaluation criterion related to characterizing MP shape is seen as an important factor when interpreting ecotoxicological effects data. The shapes of MP have been defined in many ways, such as e.g., fragment, fibre, film, foam, pellet, sphere, line, bead, flake, sheet and granule. ${ }^{21,35,291}$ Different shape categories can be found even within these categories; for instance, MP fragments can be further characterized as rounded circular or edgy rectangular shapes. ${ }^{186}$ Further complicating shape characterization is the observation that the dimensions of MP vary along continuous scales and therefore do not lend themselves well to discrete categories of characterization. ${ }^{297}$ Consequently, we consider the term "irregular MP" as an ambiguous definition of the shape, as it includes the potential to reflect several shape categories. Moreover, for a complete characterization of the shape, it is necessary to include at least one high-resolution photo illustrating each of the shapes included in the MP tested. Therefore, studies that provide an image obtained from a high-resolution microscope of the MP tested are assigned a criterion value of 2 . Studies that limit the reporting of the shape of MP to the definitions of Rochman et al. (2019) or their synonyms (sphere vs. bead), based on the information obtained from material safety data sheets but without a visual confirmation by the authors are assigned a criterion value of 1. Finally, studies that do not report the shape of the MP used or reported shapes that did not fall within the definitions described by Rochman et al. (2019), are assigned a criterion value of 0 .

Criterion 3. Polymer type. The fate, bioavailability, uptake and thus potential effects of MP can be also influenced by the composition of the polymer representing the MP, which determines the density of the particles in aqueous systems. ${ }^{44,45}$ In a sterile system without potential biofouling of the particles and in the absence of agitation, positively buoyant MP will float on the water surface, while negatively buoyant MP will remain in the water column until they sink to the bottom of the system. ${ }^{44}$ The fate of the MP in the water column thus influences their bioavailability and therefore the polymer type, as a proxy for density, needs to be characterized and reported. Additionally, knowing the polymer type will allow comparisons with field data on the occurrence, abundance and physical properties of the same polymer type, and possibly linking it with certain products and product emissions. Currently, elaborate techniques for polymer identification are available and widely applied in MP research, such as ATR-FTIR, micro-FTIR, Raman spectroscopy, pyrolysis GC-MS or similar methods ${ }^{382}$. For studies that analytically characterize the polymer type using one of these methods, a criterion score of 2 is assigned. When the polymer type is reported following the information given in the material safety data sheet and not confirmed by the authors, the study is assigned a criterion value of 1 . Finally, studies that did not report the polymer type of the MP used are assigned a criterion value of 0 .

Criterion 4. Source of MP. Reporting the source of where the MP were obtained is essential in order to better interpret the data the MP relate to, and to strengthen data reproducibility in future studies. Some studies, for instance, use in-house manufactured MP, following ad-hoc procedures which may not lend themselves well to reproducibility. In these instances it is imperative that detailed descriptions of the protocol used in producing the MP is provided (e.g., Korez et al., 2019). Results of effect studies on MP published to date show a wide variety of responses for different organisms. ${ }^{96}$ Even for the same species, different results can be obtained, 
which could be attributed to differences in the source(s) of MP. ${ }^{135,186}$ Therefore, when MP are purchased from a commonly available supplier and where specifics of the provider is provided in the main text or in the Appendix, a study is assigned a criterion value of 2, as this scenario lends itself best to reproducibility. For those studies where MP are prepared in-house using commercially available plastic products, we also assign a criterion value of 2 when the name of that plastic product is provided as well as a detailed protocol for the preparation or extraction of the MP. For instance, Jemec Kokalj, Kunej and Skalar (2018), extracted MP from a facial cleanser and made MP from a plastic bag. Polymers were characterized using FTIR, particle size distributions were measured by laser diffraction, and images of the MP were taken with a field emission scanning electron microscope. However, they do not provide the name of the facial cleanser nor the precedence of the plastic bag. Consequently, when the information given on a MP source is incomplete and thus not fully reproducible, a criterion value of 1 is assigned. Finally, studies that do not provide any information on the source of the MP are assigned a criterion value of 0 .

Criterion 5. Data reporting. It is widely acknowledged that inconsistency in how concentrations are reported make it difficult to compare between effects studies. ${ }^{96,140,294}$ Concentrations of MP can be presented as a particle number concentration, like the number of MP particles per $\mathrm{L}$ or per $\mathrm{Kg}$ of sediment, food or weight of the organism; or mass concentration, like grams of MP per L or per Kg of sediment, food or weight of the organism. ${ }^{40}$ Some studies quantify the number of MP in a specific volume or weight using a haemocytometer, a flow cytometer or a coulter counter. ${ }^{80,90,213,250}$ Other studies estimate the number of MP manually using a stereomicroscope combined with image analysis software, applicable for MP. ${ }^{80,250,339}$ Moreover, some studies convert mass concentrations to number concentrations or vice versa based on assumptions that correlate the size of a particle to its volume, for which MP characteristics such as size distribution, shape and density are required. ${ }^{79,186,385} \mathrm{~A}$ few other studies make reference to the conversion provided by the supplier of the MP. ${ }^{303,386,387}$ Thus, the reporting and conversion of concentrations between particle number and mass concentration units can be done using a variety of methods, and should be clearly described in the study in order to facilitate comparisons across studies. Since the units of concentration represent a fundamental parameter to assess risk, which compares environmental concentrations to effect threshold concentrations, consistency in units is therefore of paramount importance. ${ }^{11,40}$ Studies that report concentrations in particle number as well as in mass concentrations are thus assigned a criterion value of 2 , as they provide the greatest opportunity to compare between studies and for use in assessing environmental risk. Studies that limit the reporting of concentrations to only either particle number or mass concentrations, are assigned a criterion value of 1 . Finally, studies where concentrations of MP are not reported receive a criterion value of 0 .

\section{Experimental design}

Criterion 6. Chemical purity. Studies that aimed to investigate the interactive effects of MP and chemicals are not included in this study but are reviewed elsewhere. ${ }^{96,388,389}$ Persistent organic pollutants (POPs), such as polychlorinated biphenyls (PCBs) and organochlorine 
pesticides are ubiquitous in the environment and will partition into any organic carbon, including MP. ${ }^{11}$ Experiments measuring the partitioning behaviour between MP and organic chemicals are relevant for determining sorption/desorption coefficients and/or sorption kinetics. However, from the perspective of assessing risk it is more relevant to evaluate the toxicity of plastic-associated chemicals in the absence of MP. ${ }^{11}$ Assessing the adverse effects of the chemical stressor in the absence of the MP individually first, can provide an effective strategy for developing more complex test systems aimed at assessing multiple chemical and nonchemical stressors, and help address the immediate challenges of assessing the environmental risks of MP themselves. ${ }^{11}$ This reasoning also applies for the diversity of chemical additives and plasticizers commonly associated with plastic. ${ }^{357}$ Moreover, disentangling the effect assessment associated with chemical stressors from the non-chemical particle stressor can strengthen overall understanding of the mechanisms that influence MP toxicity. For instance, studies by Martínez-Gómez et al. (2017) and Pikuda et al. (2019) have shown that the toxicity from leachates derived from additives are more harmful than the inert polymer material, highlighting the importance of washing MP before the start of an experiment, if insight regarding the effects associated with the particles themselves is the main objective. ${ }^{390,391}$ Otherwise, the chemical stressor overwhelms the effects that might be associated with the particles, preventing the ability to distinguish between the two. Additionally, the artificiality of an exposing test organisms to MP containing chemical additives within a closed system represents a worst-case scenario that is not representative of an environmentally relevant exposure. In the environment, organic chemicals, including POPs, chemical additives and plasticizers are widely dispersed as a consequence of their use in manufacturing and consumer products, and partition into all environmental media, resulting in various exposure pathways to exist. Consequently, assessing chemical exposure requires an understanding of the multimedia behaviour of organic chemicals, whereby exposure via MP likely represents a negligible pathway as compared to other sources. ${ }^{388,392}$ Therefore, in order to disentangle the effects associated with the particle stressor from confounding chemical effects, the toxicity of background chemicals should be minimized. This includes minimizing exposure to chemical additives and plasticizer that might be present in MP, but also chemicals associated with food particle surfactants (e.g., Tween) and markers (fluorescence). Minimizing chemical exposures in MP effects studies, however, represents a major challenge. For instance, a recent study by Cole et al. (2019) extensively measured chemicals in MP, and reports that a wide variety of unknown chemicals are used in MP, making it nearly impossible to confirm conclusively that all relevant chemicals have been assessed. ${ }^{347}$ Therefore, it is preferred to repeatedly wash the particles with an organic solvent(s) in an effort to minimize effects associated with a chemicalassociated contaminant. It is notable, however, that this could have the undesired effect of altering the properties of the particles themselves, consequently care is required with respect to which organic solvents are used as well as the conditions of cleaning. Alternatively, several studies have demonstrated that it is possible to minimize the influence of the chemical stressor by providing evidence that the mass of chemical in the test system is at an exposure that remains below a chemical toxicity (e.g., Bellingeri et al., 2019; van Weert et al., 2019; RedondoHasselerharm et al., 2020) (Chapter 5). ${ }^{220,226,239}$ In summary, studies that report the inclusion of methods to thoroughly clean MP by washing with an organic solvent are assigned a criterion value of 2 , since the observations of adverse effects could be more confidently allocated to a 
particle-associated effect. If a certificate from the manufacturer was used or measurements were taken to subsequently use a control for the chemicals or the toxicity of chemicals was calculated based on $\mathrm{LC}_{\mathrm{x}}$ or $\mathrm{EC}_{\mathrm{x}}$ from literature, the study is assigned a criterion value of 1 . Finally, studies that did not address the potential influence of a chemical stressor on observed adverse effects when testing MP are assigned a criterion value of 0 .

Criterion 7. Laboratory preparation. The importance of preventing contamination when testing MP is emphasized in several recent papers and critical reviews. ${ }^{31,35,314,315,393,394}$ Catarino et al. (2018), for instance, quantified atmospheric fall-out within households, which, when rescaled to the surface area of a representative experimental test system of e.g. $20 \times 25 \mathrm{~cm}^{2}$, would imply a flux of 8333 particles per test system per day. The amount of natural fall-out of MP likely differs between locations within a laboratory and among laboratories. Catarino et al. (2018), emphasized the need to account for atmospheric deposition during experiments, even in instances where relatively high concentrations are tested. ${ }^{113}$ We argue, therefore, that the uncertainty related to contamination with MP during MP effects studies, also requires care in mitigating the potential for deposition and with respect to characterizing and quantifying the nature of the contaminants. This is because the nature of the MP-contaminants may be significantly different than those used in the test system, in that they may contain chemical additives that can strongly influence observed effects, negating test results. This is particularly relevant to the control test-system, meant to have zero MP concentration, or very low dosed systems, for which greater sensitivity would be anticipated due to the influence of MPcontaminants. Some studies thoroughly report measures taken to prevent MP-contaminates, such as wearing cotton lab coats, rinsing of equipment, covering the test systems or avoiding the use of plastic materials during the experiment. ${ }^{224,236,343}$ Consequently, a criterion value of 2 is assigned for those studies adopting measures aimed at avoiding contamination from air, water and all materials used during the experiment. Studies adopting limited measures are assigned a criterion value of 1 . Finally, studies that do not report the use of any measure to prevent contamination are assigned a criterion value of 0 .

Criterion 8. Verification of background contamination. Whereas the previous criterion focuses on the measures taken to mitigate background MP-contaminants, the present criterion evaluates the extent to which studies verify that such measures are successful or alternatively that the adoption of taking no action to reduce background contamination is needed because the potential for MP-contaminants is demonstrated to be minimal. In this case, verification implies the use of methods that characterize and analytically measure MP concentrations in exposure systems. A study by Welden and Cowie (2016), for instance, observed a fibre in the foregut of one of their control animals, underlining the importance of including method verification in MP effects test studies. ${ }^{395} \mathrm{~A}$ few studies, on the other hand, have limited verification of background contamination to the reporting of visual observations. ${ }^{236,342,343}$ Visual inspection, however, is generally considered inaccurate, as there is a high probability of missing small and transparent MP. ${ }^{31,35}$ Moreover, reliance on the use of visual observations is susceptible to false positives. ${ }^{382}$ Based on these considerations, a criterion value of 2 is assigned to studies measuring background contamination with analytical detection methods, such as by FTIR or Raman. For studies that limit the verification of background MP-contaminants to a visual inspection, a 
criterion value of 1 is assigned. Finally, for studies that do not report on background contamination of MP, a criterion value of 0 is assigned.

Criterion 9. Verification of exposure. In order to obtain accurate dose-effect relationships, exposure concentrations in the test systems must be quantified. Test concentrations are typically prepared by adding particles to the test medium, occasionally followed by dilution and homogenization steps. There are several reasons why the actual exposure concentration can deviate from the nominal concentration estimated from the initial preparation. First, human error can occur in the initial calculations or laboratory manipulations of glass ware and equipment can lead to deviations in the concentration. Secondly, the test system itself can influence exposure, whereby particles can stick to container walls and/or become unevenly distributed across test systems when homogenization is insufficient. Actual concentrations can also be higher than nominal concentrations due to background MP-contaminants, as discussed in the previous criterion. ${ }^{113,396}$ These factors can propagate and substantially influence initial estimates of test concentrations. Furthermore, the dynamic behaviour of the particles themselves can cause significant changes in exposure during the test. While less important for sediment-test systems, the behaviour of particles in aqueous test systems can result in settling, floating or aggregation of the particles, changing the actual exposure conditions over time. ${ }^{27}$ Fundamentally, the exposure of the stressor in an ecotoxicity test system should be constant over time and reproducible for each test. Demonstrating consistency in the exposure concentrations for the duration of the test is thus important to develop accurate dose-effect relationships, and the quantification of the exposure concentration should therefore be verified. A criterion value of 2 is assigned to studies that verify the exposure concentration of MP and ensure that at least $80 \%$ of the nominal concentration is maintained throughout the test 397,398 . Studies that measure the exposure concentration, but without verifying that at least $80 \%$ of the nominal concentration is maintained throughout the test are assigned a criterion value of 1 . Studies that only report the nominal concentration or limit the verification of the concentration to the stock solution are assigned a criterion value of 0 .

Criterion 10. Homogeneity of exposure. The previous criterion evaluates the extent to which the exposure concentration is verified. However, unlike the fate of dissolved chemicals in ecotoxicological effect testing, solid particles are prone to inhomogeneity of exposure as they tend to settle or float depending on a variety of factors, such as the difference in their density compared to that of the medium they are dispersed in. ${ }^{67,123,141,399-403}$ Therefore, especially for aqueous test systems, MP that have a higher density than water may settle when the dispersion is not well mixed, whereas buoyant particles may tend to reside at the surface of the test system only. Presence of air pockets or biofilm layers may change over time and influence exposure as a result of settling or causing differences in particle-particle interactions and settling velocities as a function of time, thus questioning the assumption of exposure homogeneity. These inhomogeneities can strongly influence the bioavailability and thus the exposure of the particles, resulting in a lack of control and reproducibility of test results. Methods for addressing heterogeneity in test systems assessing particle stressors include the use of ultrasonic agitation, and other physical mixing techniques (circular, wrist action shaking, plankton wheels) prior or 
during exposure, or by simply reporting the absence of such problems based on visual observations. ${ }^{338,347,385,404-406}$

Natural sediments are comprised of a mixture of particles with densities spanning a wide range, as compared to that of the solid polymeric particles that have been tested. MP mixed in sediment are 'held' in the sediment matrix and progressively encapsulated when biofilms form and test particles form hetero-aggregates and - agglomerates with the natural particles in the sediment matrix. This implies that exposure in effects test systems of MP mixed in sediment are homogeneously distributed. Many studies have recognized the need for homogeneity and have described in detail how MP were mixed in the exposure medium and sometimes also how homogeneity of exposure was verified (Chapter 5). ${ }^{226,407}$ For aqueous exposures, a criterion value of 2 is assigned to studies that verify that MP were homogeneously distributed through the use of microscopy photos and/or apply analytical tools to demonstrate that the MP were well mixed or dispersed in the solution. In instances where the method used to generate a homogeneous exposure is described but not verified, a criterion value of 1 is assigned. Effect testing of MP in sediment test systems, for which the verification of homogeneity is deemed to be not crucial, results in a criterion score of 2 for all studies that describe the method by which the MP are homogeneously mixed with the sediment, in detail. Studies that do not address the issue of homogeneity, or that observed an inhomogeneous exposure, are assigned a criterion value of 0 .

Criterion 11. Exposure assessment of organisms. To be able to understand and interpret effect data, it is important to be able to causally link an observed effect to actual exposure data. The question 'what is an organism exposed to?' however can have different answers for different organisms, particles and/or test conditions. The metric used to quantify the effect should be ecologically relevant and should be the same as the one used to quantify exposure. ${ }^{11}$ Microplastics can have multiple of such environmentally relevant metrics (ERMs). They can be characterized on the basis of known species- and particle-specific effect mechanisms. Hence, it is the actual effect mechanism which defines how microplastic particles and test organisms interact and how actual exposure should be assessed. Exposure then can be seen as accumulation at the receptor site, i.e., where the interaction takes place, and which is considered as the target for the microplastic effect under consideration. We illustrate the principle with three examples. For instance, one of the more well understood effect mechanisms, is the deterioration of food quality due to the dilution of nutritious food particles caused by an elevated exposure to low-caloric, non-digestible MP that are co-ingested with food..$^{92,186}$ Therefore, for a study that would ascribe observed effects to this mechanism, demonstrating ingestion would be a crucial criterion. Instead, studies that ascribe sub-organismal effects to damage at the cell level should ideally demonstrate systemic uptake and/or penetration of MPs and should demonstrate that these cells are reached. ${ }^{338,346,408,409}$ As a final example, studies that explain growth inhibition in algal cultures from a decrease in photosynthesis, should verify the presence of MPs at or in between algal cells in the culture. ${ }^{47,49}$ A detailed overview and analysis of such reported effect mechanisms is provided in section 3.3 of his review. In the majority of instances, effects related to the ingestion of MP are reported as the most relevant exposure pathway, implying that the quality criteria to detect and quantify MP ingested by biota are of 
critical importance. Exposure due to translocation and cell penetration also requires detection and quantification of MP in biota tissue and is thus also important in defining the quality criteria. These criteria have been reported in a previous study, ${ }^{31}$ for which criteria related to tissue digestion, particle detection and polymer identification are all applicable. For adverse effects influenced by external exposure of MP, i.e., from MP just being present in water or sediment, as in the example for algae, criteria for the analysis and quantification of MP in water are most relevant. It is widely understood that visual sorting of MP is insufficient to detect the small and often light-coloured MP against a background of e.g., animal tissue. Therefore, following the QA/QC criteria suggested by Hermsen et al. (2018), a criterion value of 2 is assigned to studies that report the detection of MP quantitatively using e.g., FTIR or Raman imaging, to support statements of MP ingestion and/or penetration into cells of biological tissues that have been appropriately digested and filtered. ${ }^{31}$ Studies demonstrating exposure of organisms to MP based on qualitative or visual observation, or citing results from a separate experiment, or in the absence of a digestion step, are assigned a criterion value of 1 . Studies that do not report data on exposure, are assigned a criterion value of 0 .

Criterion 12. Replication. In every effect assessment, an adequate experimental design requires a sufficient number of replicates in order to ensure statistically reliable results. ${ }^{312,410}$ Studies should therefore clearly explain the degree of replication of each treatment. ${ }^{410}$ Some studies, however, fail to report on the use of replicates in their experimental design ${ }^{384,395}$ while other studies report the use of replicates, but which are not actual replicates but better characterized as pseudo-replicates. ${ }^{61,67,330}$ For instance, Jovanović et al. (2018) considered as replicates the 15 fish exposed to MP in the same tank. ${ }^{411}$ As each replicate should be an independent experimental unit, with the experimental unit here being the tank, the exposure of all fish via the same tank should thus be better defined as multiple measurements taken one experimental unit. ${ }^{412}$ In contrast to soluble chemicals, which can be homogenously distributed in the test system, the severity of the effects detected in MP studies can be attributed to the relative extent of bioavailability of the particles and the probability of encountering them in the test system. Therefore, in the case of MP, it is especially important to have several replicates to compensate for the uncertainties associated with the potential for inhomogeneous exposure associated with the test system. Studies were assigned a value of 2 when they included results from a minimum of three replicates. A criterion value of 1 is assigned to studies using only two replicates. Finally, studies that do not include any replicates or do not report the number of replicates used are assigned a criterion value of 0 .

\section{Applicable to risk assessment}

Criterion 13. Endpoints. Effect studies with MP use a wide variety of endpoints, sometimes even within studies. We argue that when data from such studies are to be used in ecological risk assessment, the ecological relevance of the selected endpoint represents an important criterion to consider. From a risk assessment perspective, endpoints such as survival, growth and reproduction are considered ecologically relevant, because these endpoints directly relate to a population-level effect. These endpoints are preferred over e.g. sub-organismal or behavioural endpoints, which are generally less relevant in assessing population-level responses, unless there is a clear demonstrated causal relationship between these responses and a higher level 
effect e.g. population effect. ${ }^{140,413}$ For instance, de Sá et al. (2015) speculated that reduced food intake caused by the ingestion of MPs adversely affects both the individual and populationlevel fitness of a species. ${ }^{414}$ The endpoints studied, however, are attributed to the predatory performance and efficiency of the species, which does not necessarily translate to an ecologically relevant population level effect. Whereas it has been suggested that sub-organismal endpoints such as biomarkers can be representative of early warning signals and are thus more sensitive indicators than the traditional endpoints used in risk assessment, ${ }^{343,415}$ they can also be perceived as being susceptible to type I and II error, due to under-replication and pseudoreplication in ecotoxicological bioassays, which could lead to false alarms or undetected effects. ${ }^{415,416}$ Moreover, there is no evidence that sub-organismal endpoints are more sensitive than endpoints taken at higher organismal level responses, particularly for MP effects studies. Additionally it is possible that effects seen at the sub-organismal level merely resemble reaction to decreased nutritional intake. ${ }^{302}$ Furthermore, endpoints at these sub-organismal levels are not likely to be useful predictors since they have complicated time- or dose-dependent responses, which makes it difficult to extrapolate correlations to higher levels of biological organization. ${ }^{415}$ Still, in carefully controlled studies e.g. biomarkers can be useful for elucidating mechanisms of toxic action. ${ }^{415}$ In summary, a criterion value of 2 is assigned to studies where endpoints at either community or individual level of biological organization (e.g. survival, growth, development or reproduction) are used. If sub-organismal endpoints are used, for which a causal relationship with effects on higher levels of biological organization is demonstrated, a criterion value of 1 is assigned to the study. Finally, studies that use endpoints that cannot be unambiguously linked to a threat at the individual or population level are assigned a criterion value of 0 .

Criterion 14. Presence of natural (food) particles. It is important to note that the natural environment is not free of particles and that organisms have adapted various species-specific traits in relation to strategies for interacting with particles. While MP are ubiquitous in the aquatic environment, the amount of natural particles is typically greater than the concentrations of MP that have been reported in the environment. ${ }^{293,388}$ Therefore, when designing an experiment meant to simulate natural conditions it is important to consider the response of organisms to both naturally occurring particles as well as a MP-stressor exposure. ${ }^{140,302}$ Exposure to naturally occurring particulates, for instance, can represent an important food source to an organism or may otherwise form part of their natural habitat, such as sediment or suspended solids (Chapters 2 and 5). ${ }^{186,226}$ The inclusion of food and other particulates is needed because ecotoxicological effects of MP on organisms has been demonstrated to be influenced by the presence of naturally occurring particulates. ${ }^{46,67,417,418}$ Observations that the co-exposure of both naturally occurring particulates and MP can mitigate toxicity implies the relative importance of a species ability to selectively feed and therefore reduce the risks associated with MP under environmentally relevant conditions. ${ }^{302} \mathrm{We}$ argue that without taking natural (food) particles into account, the observed adverse effects represent a system-dependent artefact that does not lend itself to risk assessment purposes. An exception, however, is made for algal studies, as their food source are nutrients and light, and therefore the addition of other naturally occurring particles is less likely to influence adverse effects. ${ }^{419}$ 
It is further noted that there are several studies that adopt standard test protocol guidelines for acute toxicity testing, which are applicable to soluble chemicals. ${ }^{78,79,384,420}$ In such experiments the test guidance is not to feed the organisms, which is logical when testing soluble chemicals as the food particles may influence the bioavailability of the test chemical and the presence of food does not represent a limiting factor due to the short duration of the acute study. However, this guidance is not applicable to experiments aimed at assessing the acute response of MP, because the adverse effects can also potentially be influenced by the presence of food particles 302. Therefore, when natural particles (at least food) are added to avoid an exposure that might be perceived as analogous to 'force feeding' the organisms with MP, a criterion value of 2 is assigned to the study. Studies that add food, but in which the food is not optimally available to the organisms are assigned a criterion value of 1 . Finally, studies that do not include any naturally occurring or food particles are assigned a criterion value of 0 .

Criterion 15. Reporting of effect thresholds. To date, the majority of effect studies report adverse effects for MP at a single or limited number of test concentrations. ${ }^{81,89,304,414,421}$ These observations are beneficial in demonstrating the potential adverse effects that MP can have on biota. It remains unclear, however, the threshold concentration above which the adverse effect initiates. For the purposes of risk assessment, where the ratio of exposure concentrations to that of effect threshold concentrations are derived, accurate estimates of effect threshold concentrations, such as derived from dose-response relationships in the form of $L(E) C_{x}$, (or the generally less preferred NOEC or LOEC), ${ }^{422-424}$ are required. Given the paucity of doseresponse threshold effects data for MP, the need for effect threshold concentrations to help inform the risk assessment process has been widely recognized. ${ }^{13,40,96}$ Therefore, given the relative importance of this criterion regarding applicability in risk assessment, effect studies aiming at reporting effect thresholds are assigned the greatest value. To be effective it is notable that effect threshold concentrations must be accompanied with estimates of error or uncertainty, in order to evaluate that differences in exposure concentrations are statistically meaningful. Based on this reasoning, we assign a criterion value of 2 to studies that report threshold effects data using $\mathrm{L}(\mathrm{E}) \mathrm{C}_{\mathrm{x}}$ derived from dose-response relationship modelling, with error data $(95 \%$ confidence interval, standard error or standard deviation). If other metrics like NOEC or LOEC are used, or when no error data are provided, the data are still considered useful and a criterion value of 1 is assigned. Studies that do not explicitly provide data on threshold concentrations for the reported effects are assigned a criterion value of 0 .

Criterion 16. Quality of the dose-response relationship - Effect threshold concentrations, such as $\mathrm{EC}_{50}$ or $\mathrm{LC}_{50}$, are typically obtained by fitting a logit or probit model to dose-response data, 159 in which $\mathrm{EC}_{50}$ or $\mathrm{LC}_{50}$ is a model parameter. This implies that the statistical significance of the resulting $\mathrm{EC}_{50}$ or $\mathrm{LC}_{50}$ value depends on the quality of the fit to the data, and on the number of parameters fitted, compared to the number of data points in the dose-response relationship. In standard ecotoxicity test systems it is generally suggested to assess effects using a minimum of six different exposure dose concentrations, including the control, to obtain an accurate $\mathrm{EC}_{50}$ or $\mathrm{LC}_{50}$ value. ${ }^{159}$ Ideally, the exposure concentrations used are representative of the full range of effects, i.e. from low effect to near-maximum effect, such that an $\mathrm{EC}_{50}$ or $\mathrm{LC}_{50}$ value can be derived without extrapolation. Intuitively, replication of test results at each exposure 
concentration will also contribute to more accurate $\mathrm{EC}_{50}$ or $\mathrm{LC}_{50}$ values. Since replication is already covered by criterion 12 , only the number of exposure concentrations used in an effect study is evaluated under this criterion. Studies that use the recommended minimum of six exposure dose concentrations or more, including a treatment control (zero microplastic concentration), are assigned a criterion value of 2 , and a criterion value of 1 if five different concentrations are used. For studies reporting dose-response relationships using less than five concentrations, a criterion value of 0 is assigned.

Criterion 17. Concentration range tested. Recent studies have drawn attention to the need to better define ecologically relevant concentration ranges for effect testing of MP. ${ }^{294,425}$ As previously discussed, studies reporting adverse effects for MP often use unrealistically high exposure concentrations, which has resulted in suggestions for future studies to assess effects using lower, more environmentally relevant, concentrations. ${ }^{11,425}$ However, if studies limit assessing effects to low concentrations, it is possible that derivation of effect threshold concentrations may not be possible. Consequently, we argue that studies must follow standard principles adopted in assessing the risks of chemicals, such as through the use of quantitative dose-effect relationships to obtain an assessment of effect threshold endpoints typical of ecotoxicology (i.e., $\mathrm{EC}_{50}$ or $\mathrm{LC}_{50}$ ) with sufficient quality. To meet this requirement, effect testing can include both high and low concentrations, as long as the results are used to quantitatively derive the appropriate threshold values. For example, if an effect observed in an ecotoxicity test system occurs only at concentrations that exceed environmentally relevant exposure concentrations by several orders of magnitude, the end result would be supportive of demonstrating low risk. Nevertheless, there can also be strong arguments that support the use of environmentally realistic, low concentrations in ecotoxicity effects tests. This is because the reported effects occurring at high concentrations may be linked to an effect associated with a decrease in food quality, resulting from either the ingestion of inert non-digestible particles or due to an overwhelming number of particles in the test system that results in a decreased potential for the test organisms to find food particles. This type of effects occurs with any type of particle of low nutritional value and may be perceived as an artefact of the test system design, not an effect that is intrinsic to the MP themselves ${ }^{140,385}$ and is therefore better understood as a non-specific particle effect. This exposure scenario typically results in the test organisms suffering from starvation prior to any other modes of action that the MP may cause effects that might occur at lower concentrations following a chronic exposure (Chapter 5). ${ }^{87,226}$ In other words, at environmentally relevant concentrations, it is unlikely that food dilution represents a mechanism of ecological significance, but that more subtle effect mechanisms (related to behaviour, avoidance, reproduction, particle toxicity) are likely of greater relevance to assess and for which long term chronic effects testing would be beneficial. For this reason, some studies intentionally assess the effects associated with lower test concentrations (Chapter 2). ${ }^{186,339}$ In summary, environmentally relevant concentrations should be given priority for effects testing of MP, which forms the basis of a legitimate criterion for the ecological relevance associated with chronic ecotoxicity test system design. Note that exposure duration is evaluated below, in a separate criterion, and only the ecological relevance of the concentration is evaluated under this criterion. Thus, studies that use two or more environmentally realistic concentrations in the exposure concentration doses tested, supported by credible literature data, 
are assigned a criterion value of 2 . If the test system uses only a single environmentally relevant concentration, supported by credible literature data, a criterion value of 1 is assigned. Studies that acknowledge that concentrations are far above environmentally relevant concentrations, or that do not evaluate their exposure concentrations with environmental monitoring data, are assigned a criterion value of 0 .

Criterion 18. Aging and biofouling. Under environmentally relevant conditions, MP undergo abiotic and biotic processes that alter their shape, size, structure and eventually their bioavailability. ${ }^{316}$ Vroom et al. (2017) demonstrate that the aging of MP promotes their ingestion by marine zooplankton. As the surface of MP functions as a substrate for biofilm to grow, ingestion of biofouled MP potentially represents an additional energy source for test organisms. ${ }^{426}$ This implies that ecotoxicity tests that assess pristine particles may potentially underestimate the ingestion rates that may occur in the environment, whereby the potential to ingest aged and biofouled particles may be higher. Since MP undergo both aging and biofouling in the environment, it would thus be beneficial to consider how such processes influence ecotoxicity results and would further strengthen aims directed at ecological relevance. Consequently, studies that include aging of MP to make them more environmentally realistic and also characterized the MP for aging and biofouling, for instance by scanning electron microscope (SEM), are assigned a criterion value of 2 . Studies that have only aged the MP but do not characterize them (e.g., Zettler, Mincer and Amaral-Zettler, 2013) are assigned a criterion value of 1 . Finally, studies that limit testing to only the use of pristine MP and/or conditions that prevent the formation of a biofilm are assigned a criterion value of 0 .

Criterion 19. Diversity of MP tested. To date, most studies assessing the effects of MP limit observations to a relatively small sub-set of all possible characteristics. For instance, studies testing MP based on a single or limited range of particle sizes, shapes and polymeric type may provide valuable information on how specific particle characteristics influence uptake and effects, but under ecologically relevant conditions, organisms will encounter a wide variety of characteristics, of which size, shape and density often are considered the most important properties influence the transport, fate and bioavailability of MP. ${ }^{13,20,297}$ Species-specific biological and behavioural traits can also play an important factor in determining which properties of MP found in the environment will most likely result in an exposure for the individuals of a species (Chapter 2). ${ }^{13,186}$ The ecotoxicological effects related to the properties of the relevant fraction of MP for a species, may also be influenced by the presence of either other MP or of naturally occurring particles. Simulating species-specific responses to exposures of environmentally relevant heterogeneous mixtures of both MP and naturally occurring particles represents a significant challenge in MP effects testing. Recently, Kooi and Koelmans (2019) reviewed the ranges and distributions of the characteristics of environmentally relevant MP and observed relative similarity across datasets taken from different locations, with respect to their physicochemical characteristics. ${ }^{297}$ Given the recent awareness associated with this criterion, we suggest that future studies adopt the use of distributions in physicochemical properties of MP as a standard approach to enable better environmental realism in MP effects testing. Consequently, studies that use MP with a range of sizes, shapes and densities in one mixture exposure, and which attempts to simulate the diversity of environmental MP, are 
assigned a criterion value of 2 . If the diversity related to only one or two of the physicochemical characteristics and/or a limited distribution, a criterion value of 1 is assigned. Studies that limited effect testing to a single type of MP were assigned a criterion value of 0 .

Criterion 20. Exposure time. Standard test protocol guidelines for the ecotoxicity testing of chemicals recommend the application of defined exposure times for each of the endpoints assessed. While these guidelines are also routinely adopted in the effects testing of MP, some studies highlight the need for longer exposure times, due to the detection of time-dependent effects (Chapter 5) ${ }^{75,79,87,226,329,330,428}$ For instance, the effects of MP on the freshwater coral Lophelia pertusa differed between exposure times of 7, 20 and 47 days. ${ }^{330}$ While the coral growth rate decreased over time, effects on capture prey and polyp activity disappeared after 47 days, revealing that both positive and adverse effects of MP can differ with time. ${ }^{330}$ Furthermore, observations for the marine mussel Mytilus edulis, report the formation of granulocytomas and the destabilization of the lysosomal membrane increased significantly with longer exposure times when exposed to MP. ${ }^{75}$ Moreover, adverse effects of MP on the growth of the cladoceran Daphnia magna were only found after 25-31 days of exposure. ${ }^{329}$ For $D$. magna, another study demonstrated that their immobilization increased over time when exposed to MP. ${ }^{79}$ Generational effects following exposure to MP have also been reported, as in the case of the copepod Tigriopus japonicus. ${ }^{428}$ Therefore, the importance of exposure duration, which can influence the detection of adverse effects that might differ between chemicals and MP is emphasized within this evaluation criterion. Exposure duration is of particular importance for endpoints that seem to be time-dependent, such as growth, reproduction and long term community effects (Chapter 5). ${ }^{226,310}$ Additionally, increasing the exposure time can be perceived as adding greater environmental relevance to the effect study, explaining the logic for why this criterion is in the ecological relevance category. Thus, for studies that include a minimum exposure time of 7 days for bacteria or phytoplankton, 21 days for zooplankton, 28 days for benthic invertebrates, macrophytes or fish larvae and 3 months for adult fish, the study is assigned a criterion value of 2 . For studies that use an exposure time between 1 and 7 days for bacteria or phytoplankton, between 4 and 21 days for zooplankton, between 7 and 28 days for benthic invertebrates, macrophytes or fish larvae and between 1 and 3 months for adult fish, a criterion value of 1 is assigned. Finally, studies that use substantially shorter exposure times, specifically $<1$ day for bacteria and phytoplankton, 4 days for zooplankton, 7 days for benthic invertebrates, macrophytes or fish larvae and 1 month for adult fish, are assigned a criterion value of 0 , except in instances where multigenerational studies are performed, where a criterion value of 1 is assigned. 


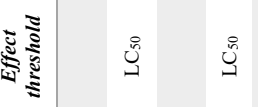

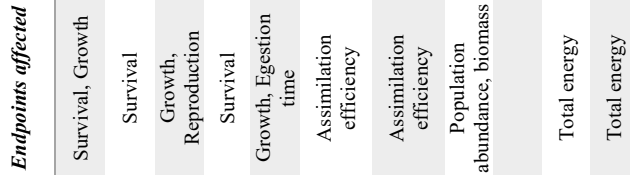

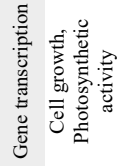

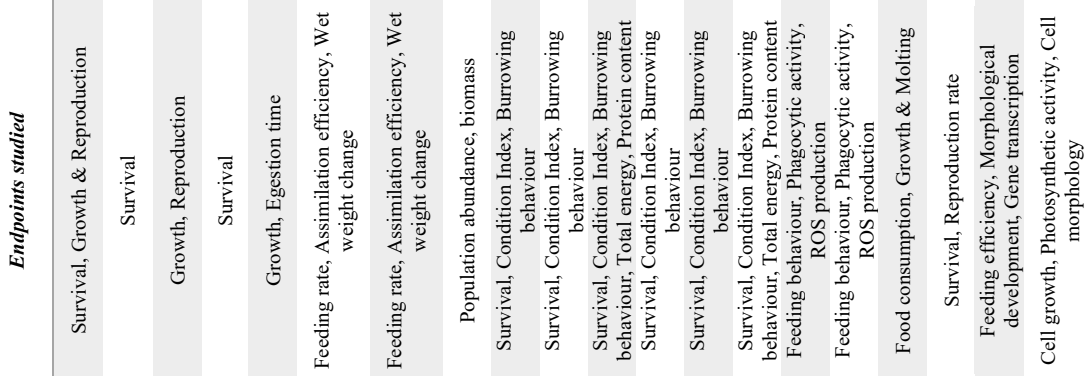

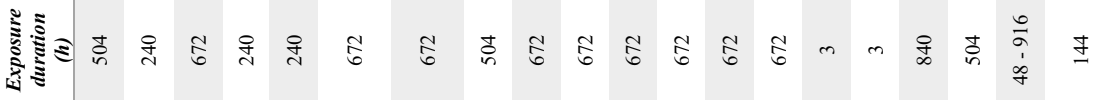

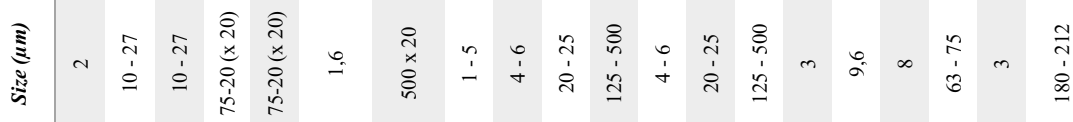

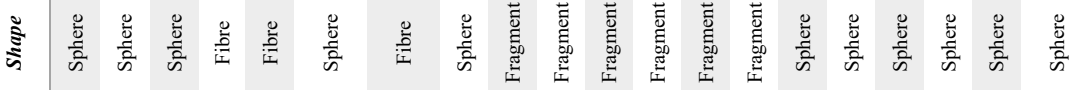

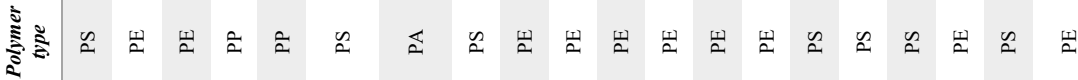

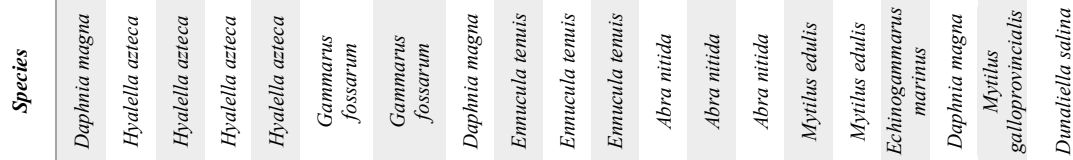

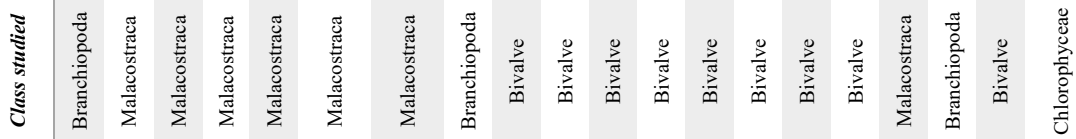

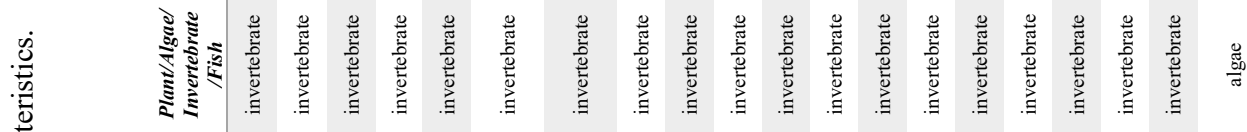

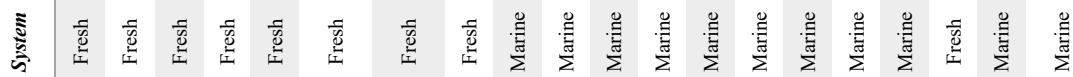

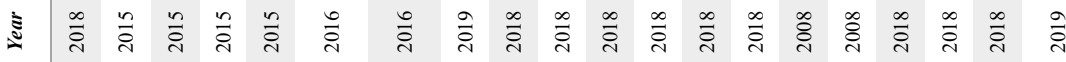

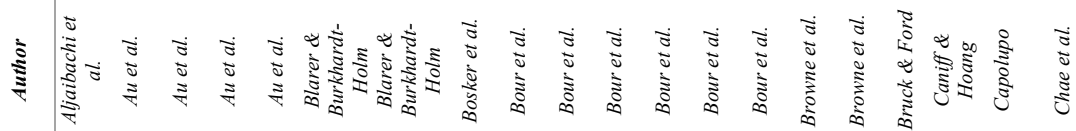




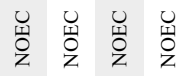

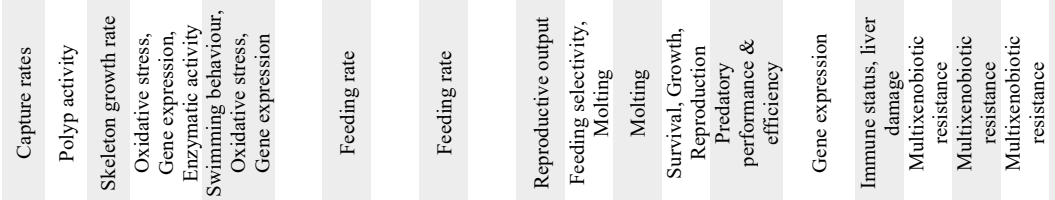

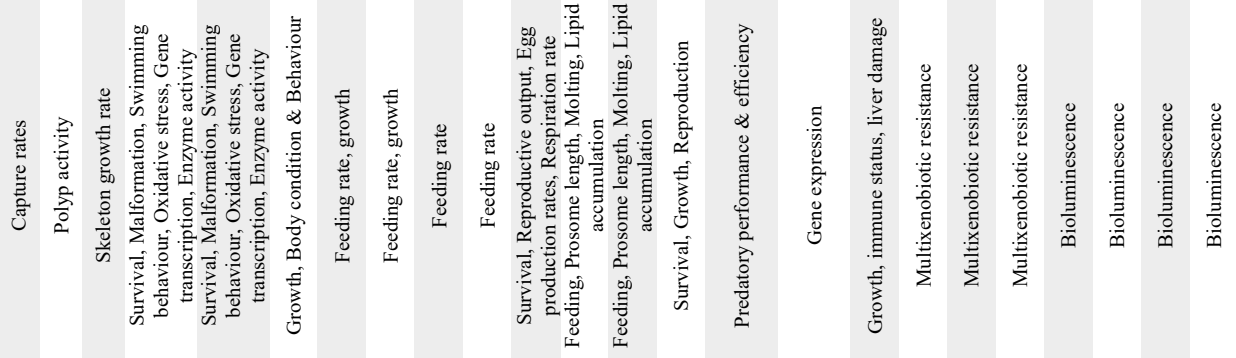

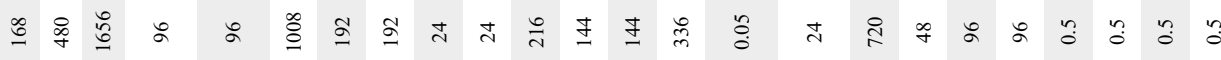

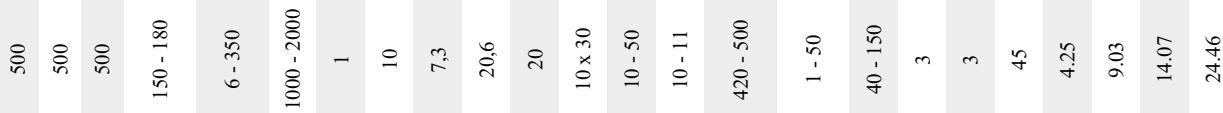

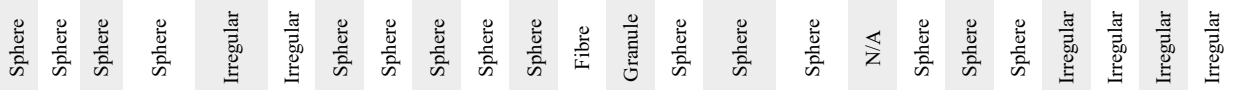

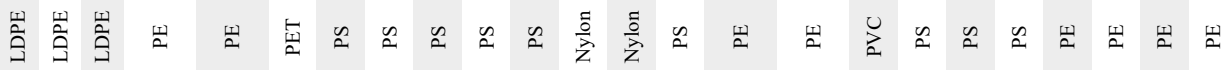

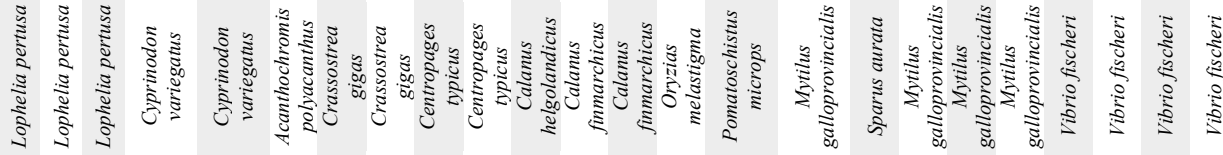

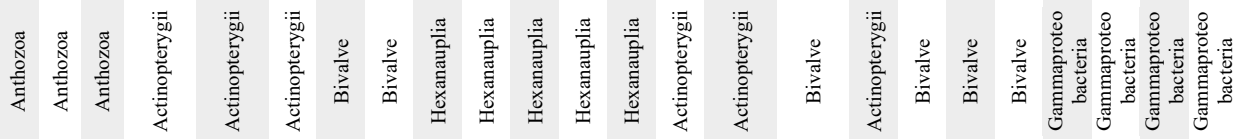

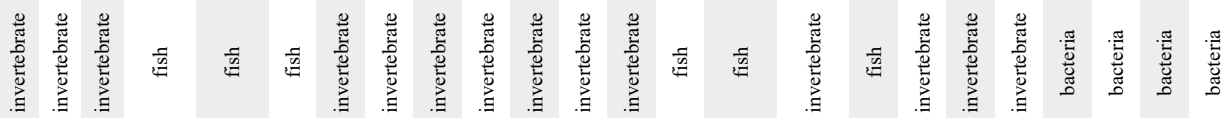

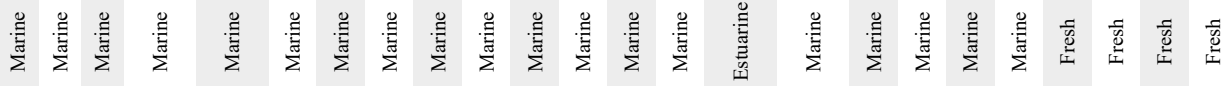

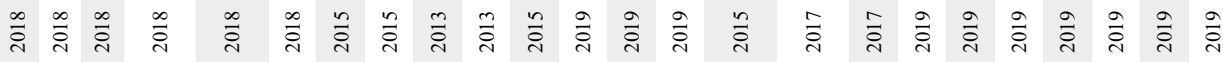

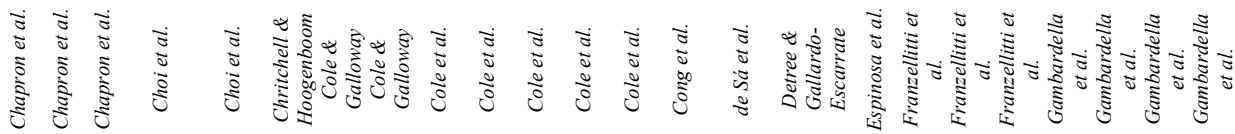




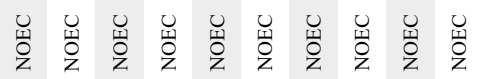
ษ̊ำ

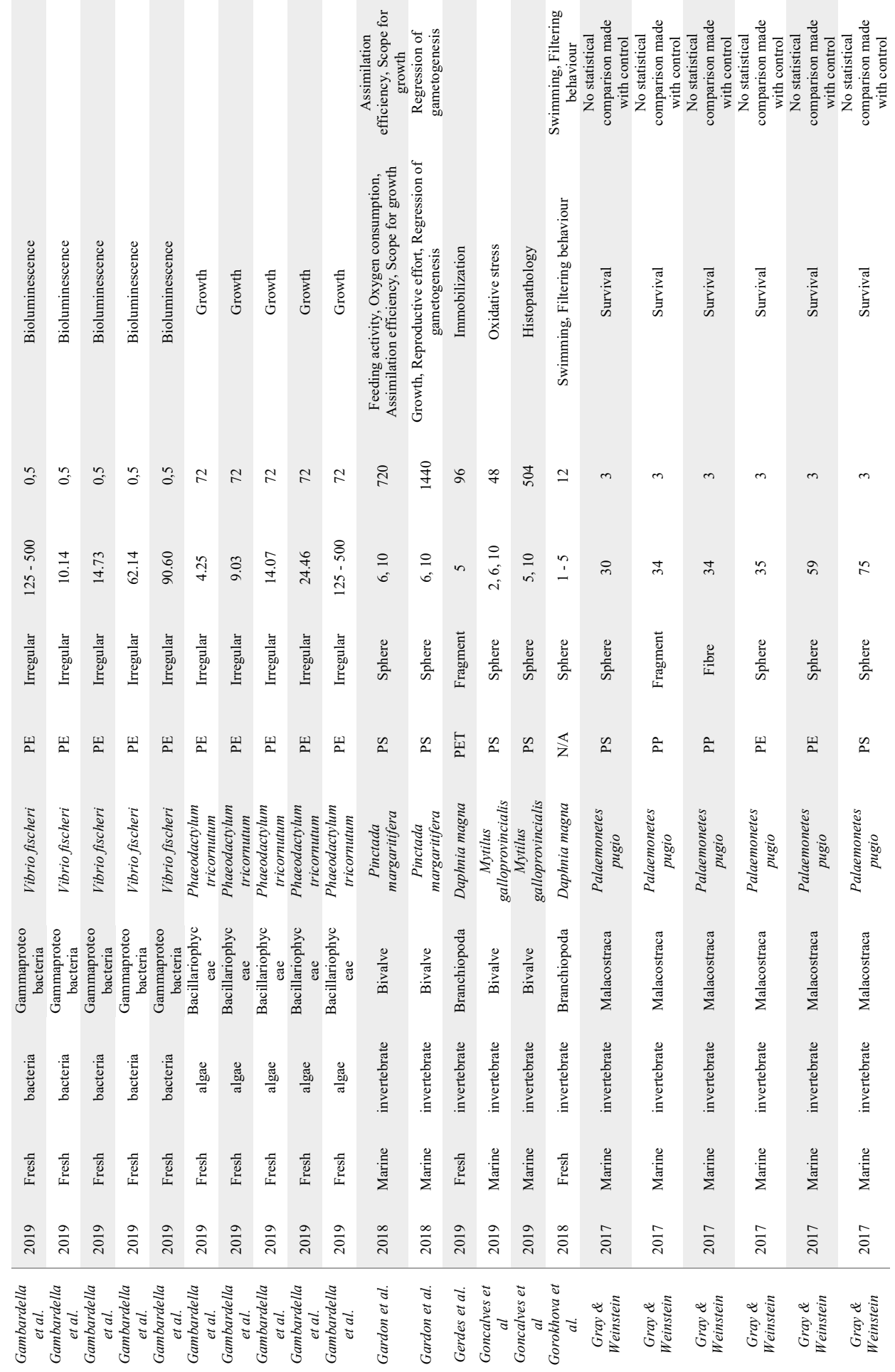




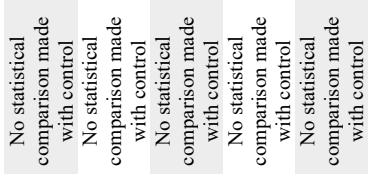
IIIIIMIIIII $\ln 11\|\|\|\|\|\| M \| M$

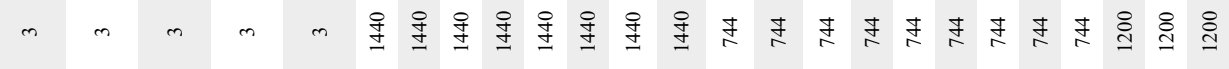

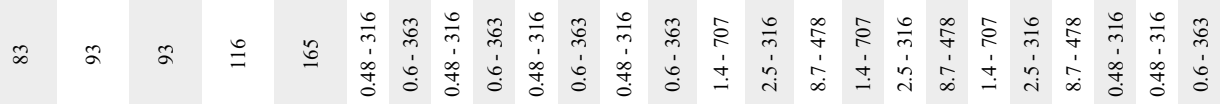

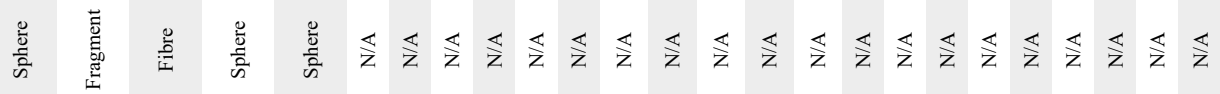

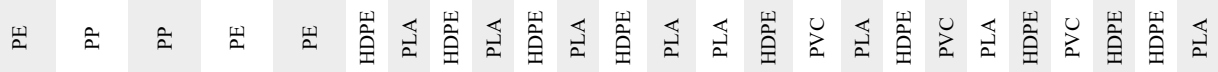

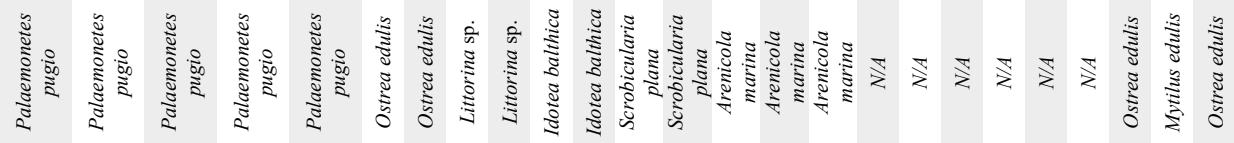

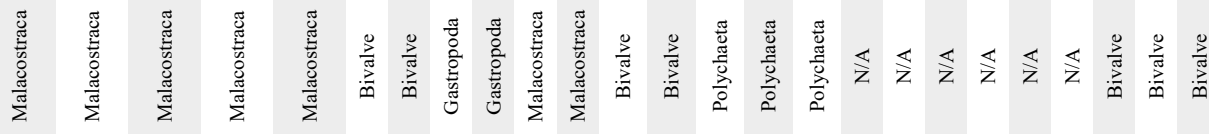

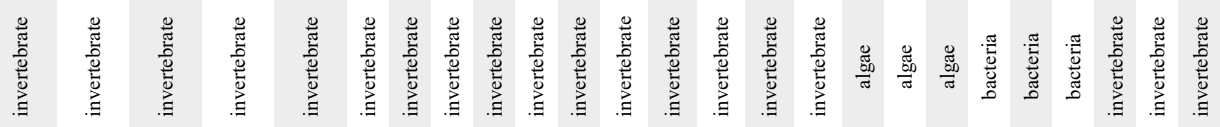

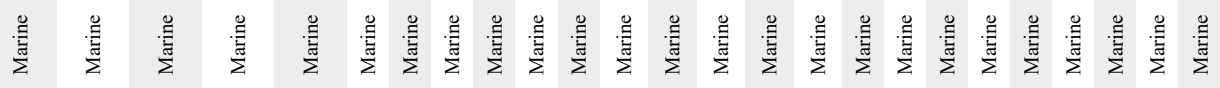

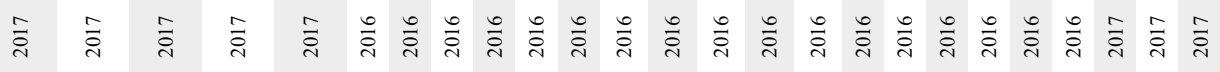
मำ 


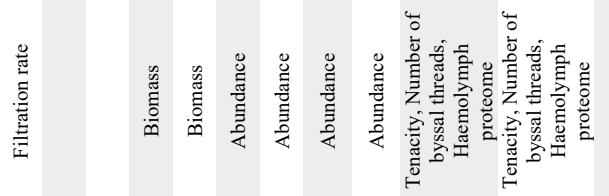

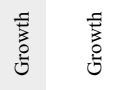

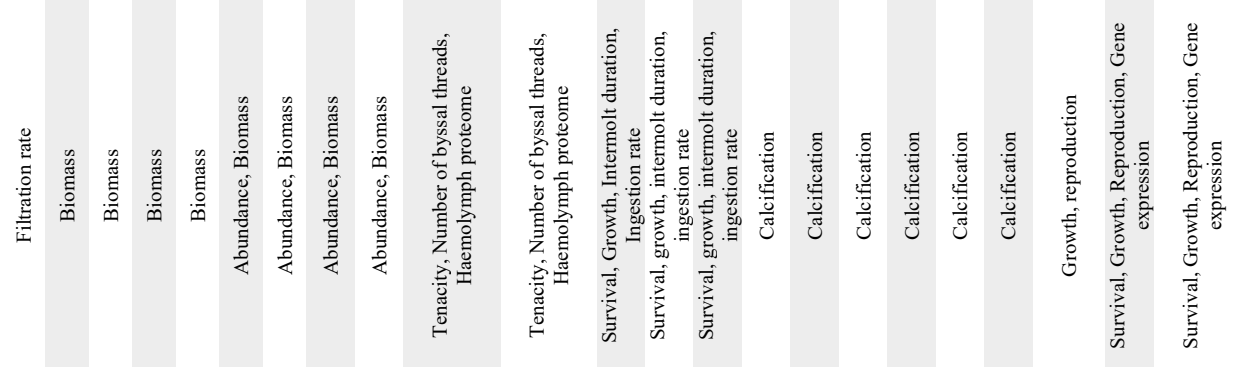

\&

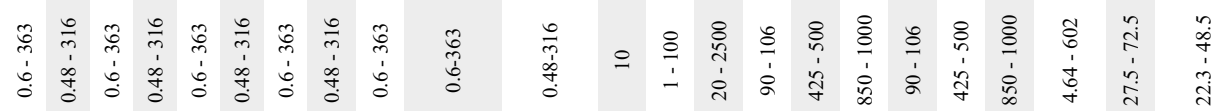

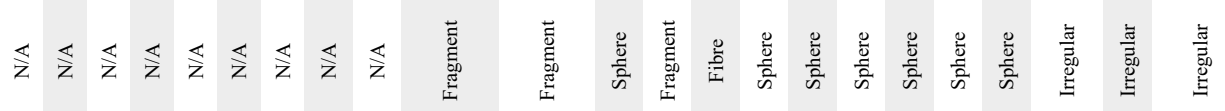

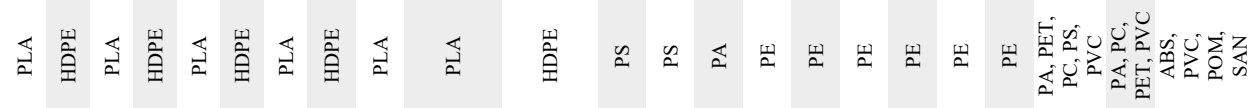

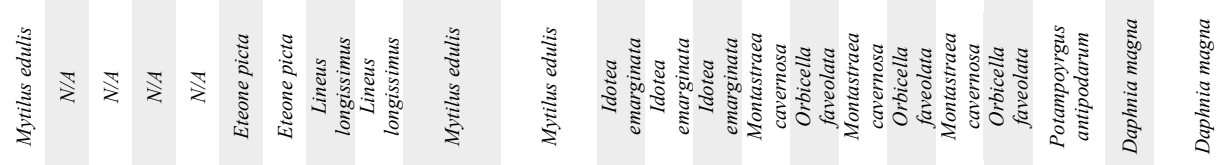

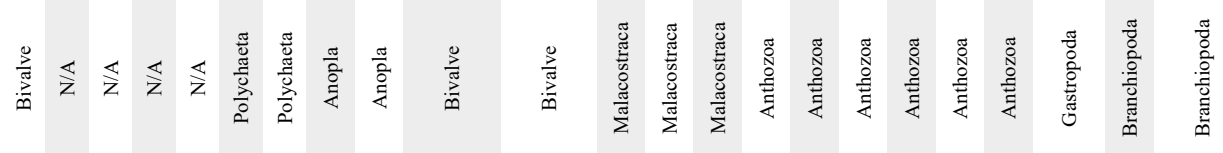

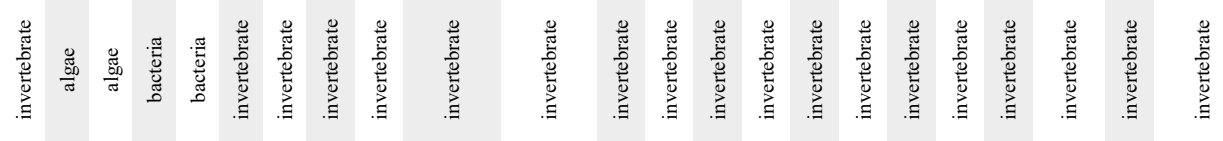

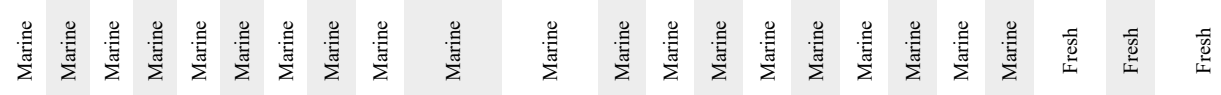

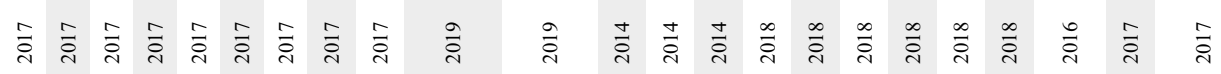

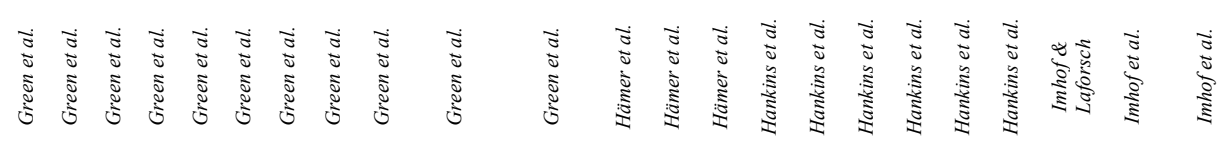




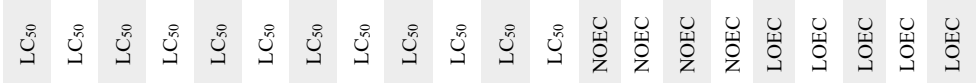

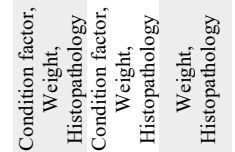

mHMm!n

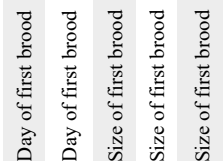

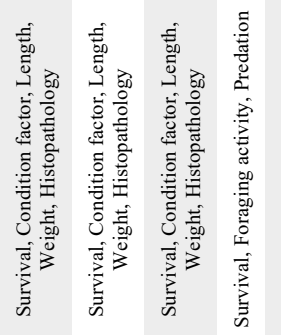

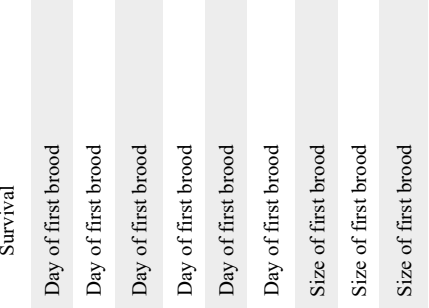

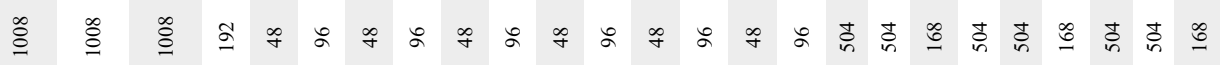

兽

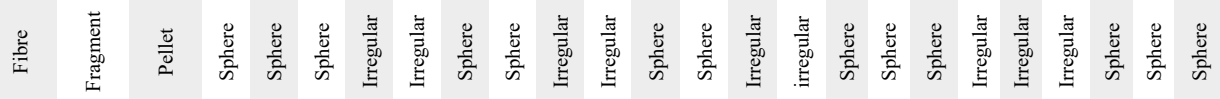

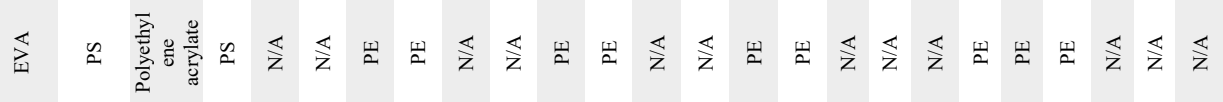

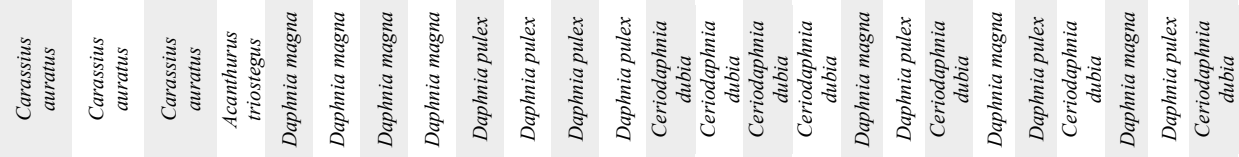

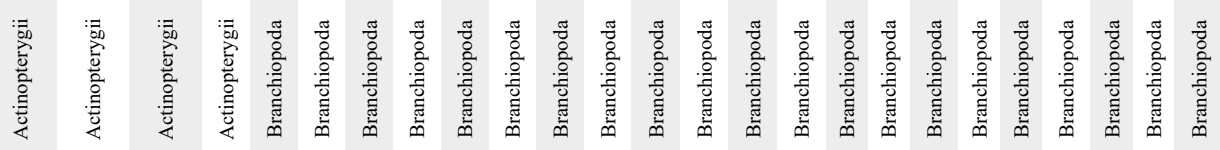

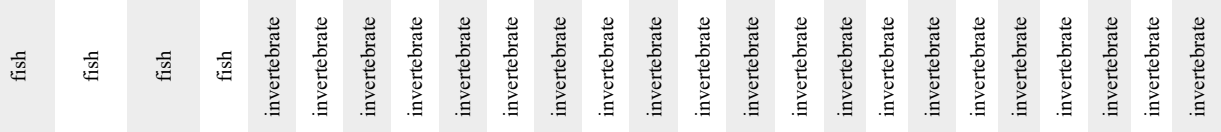

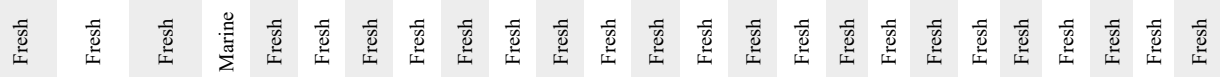

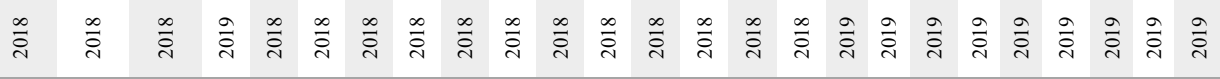

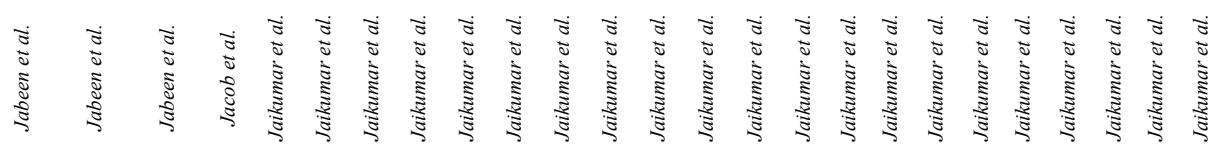




\section{Appendices}

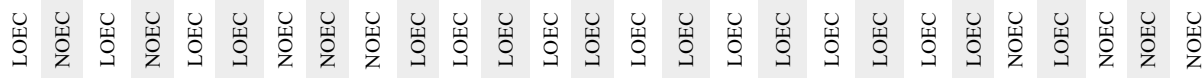

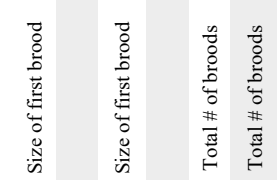

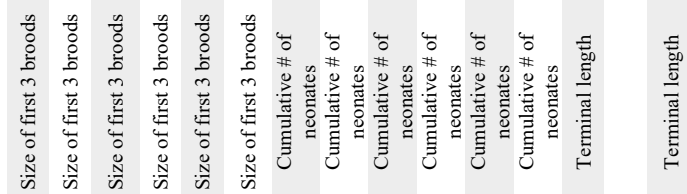

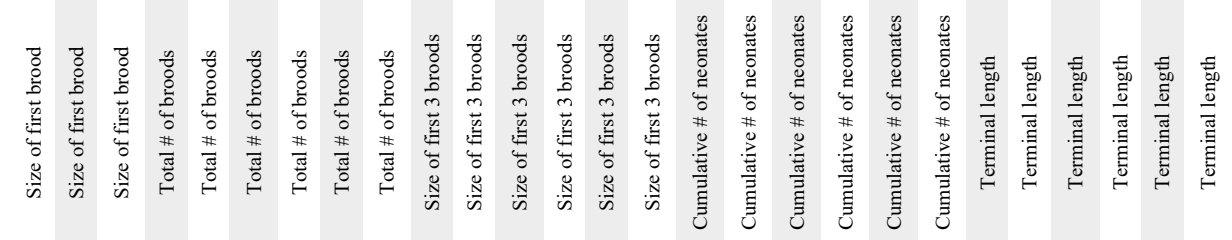

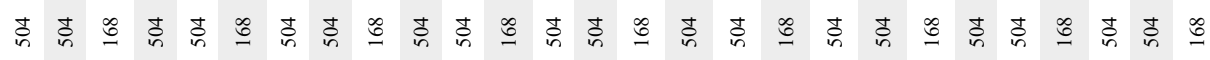

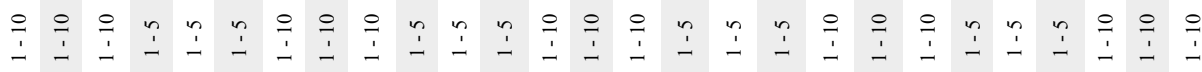

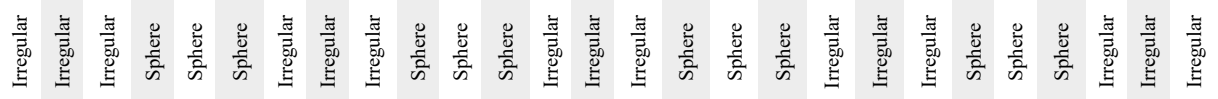

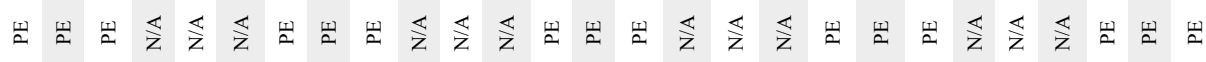

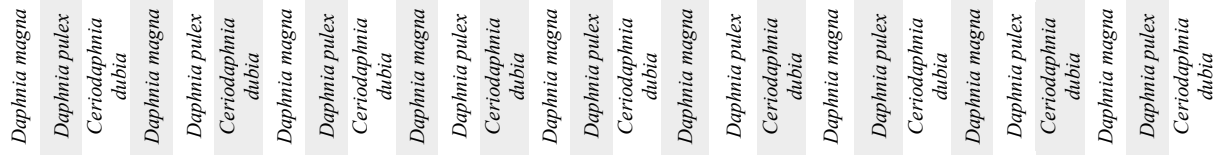

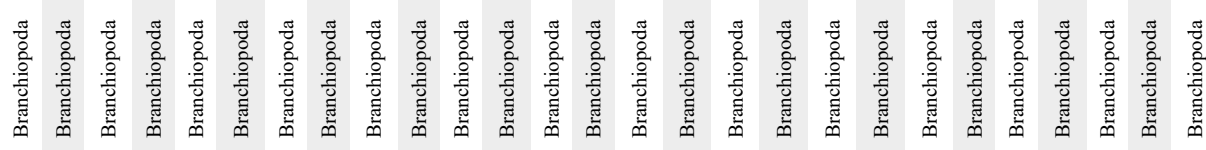

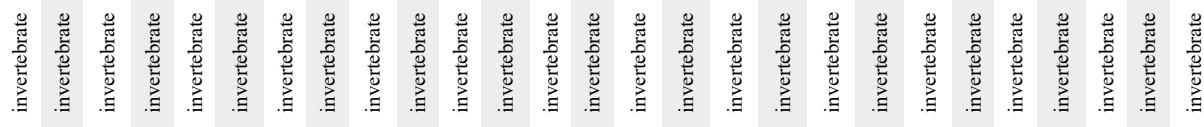

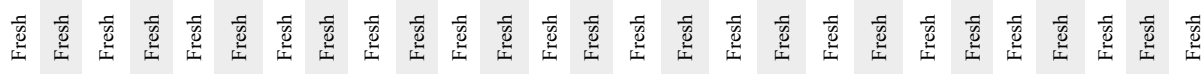

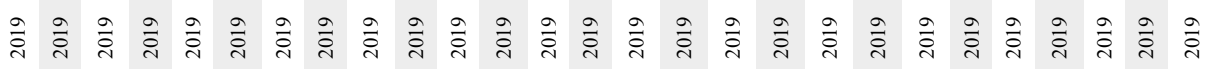

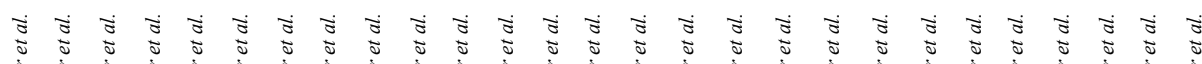
\|\|\|\|$\|H\| M\|M\| M \| M$ 


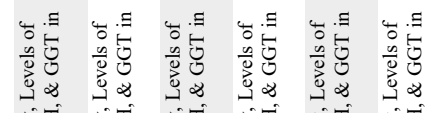

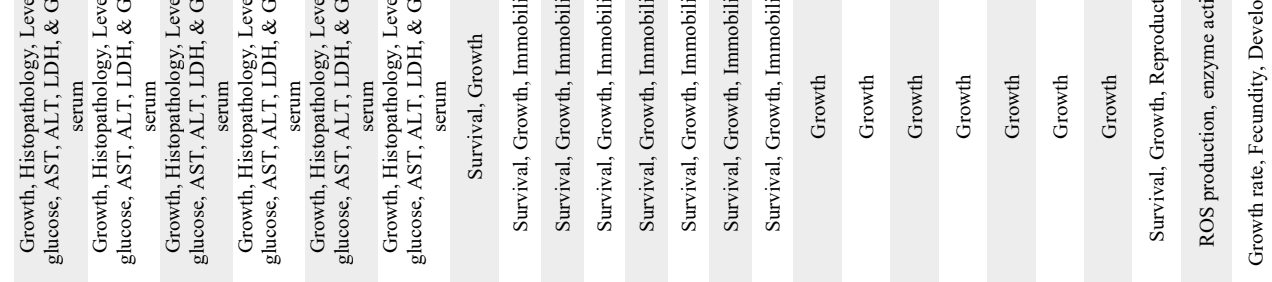

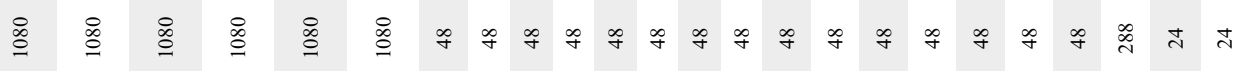

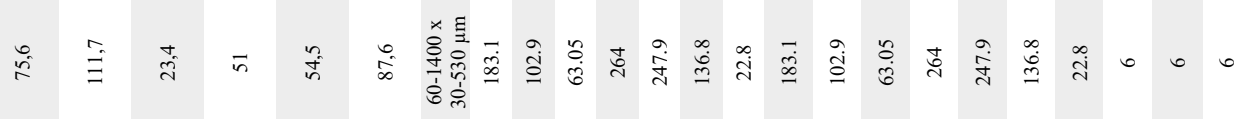

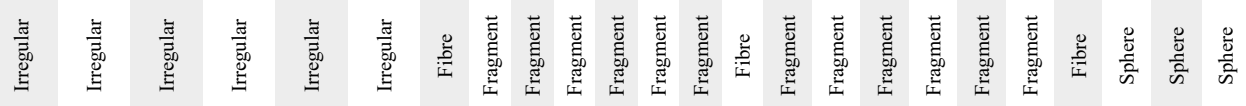

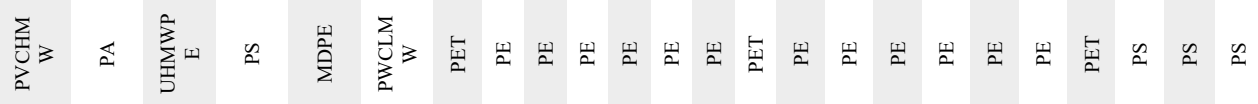

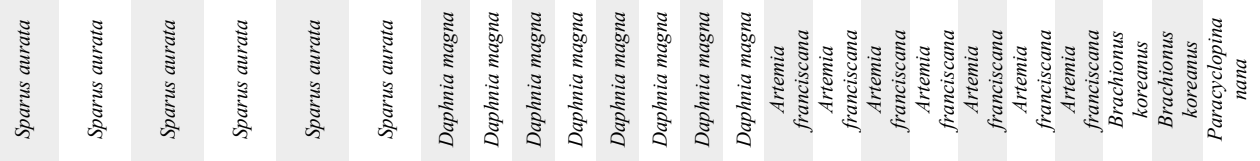

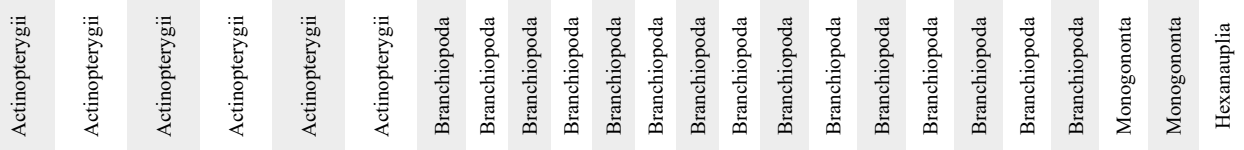

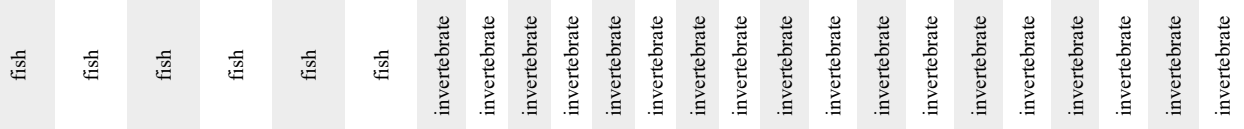

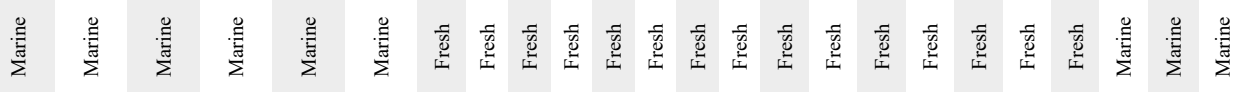

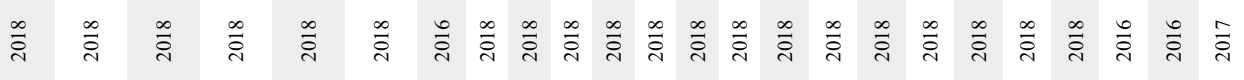

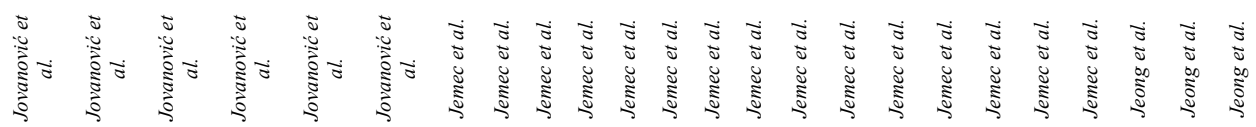




\section{胥 离}

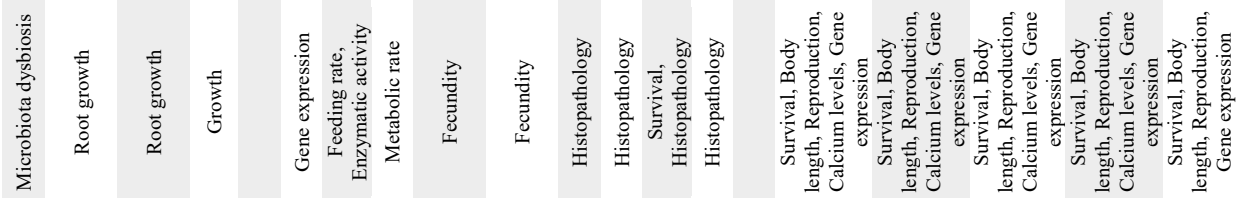

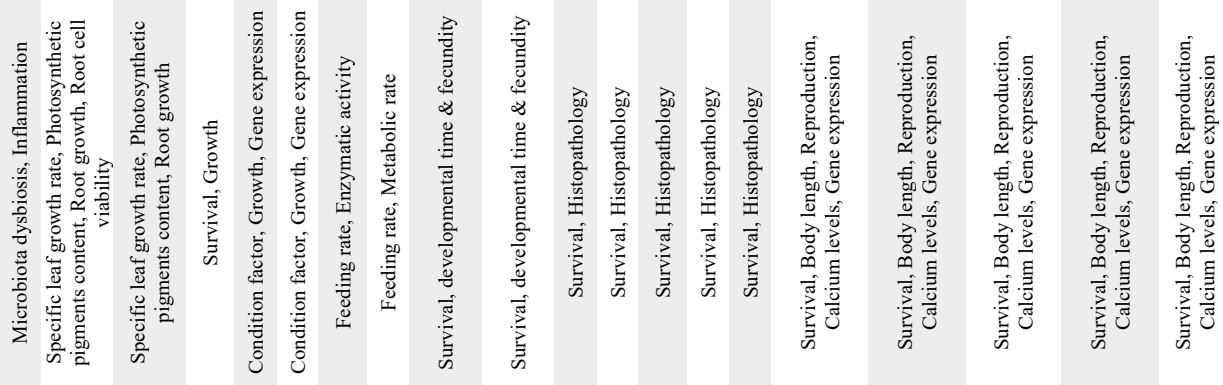

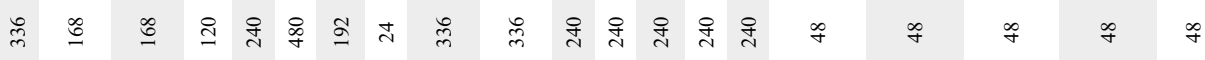

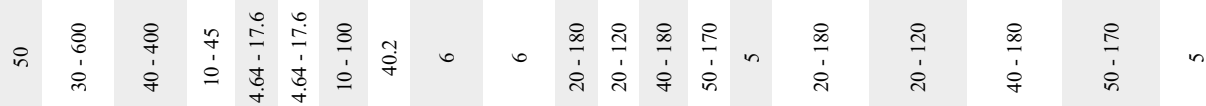

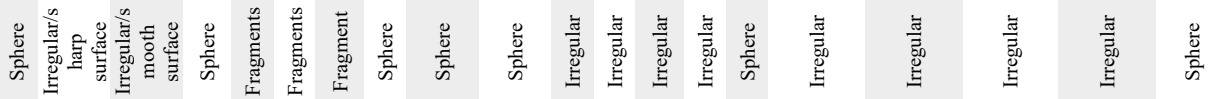

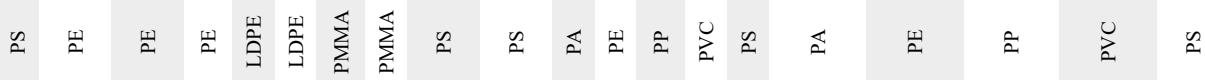

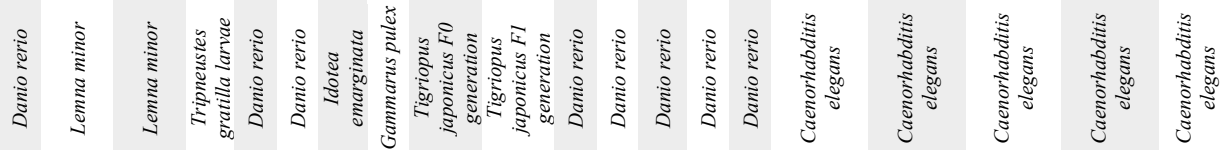

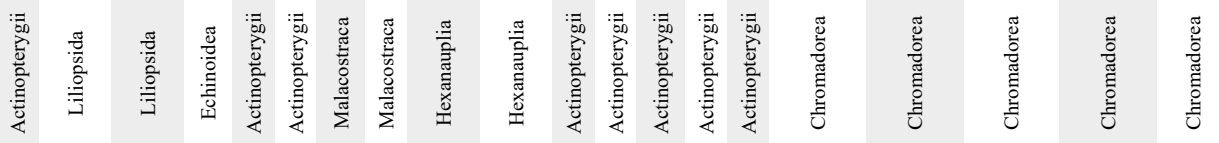

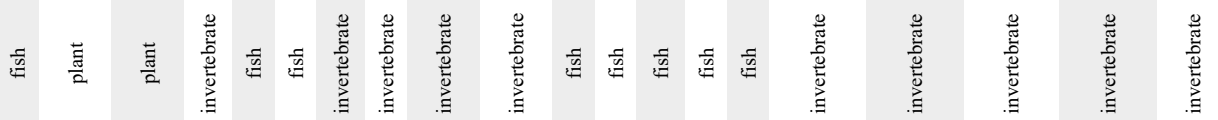

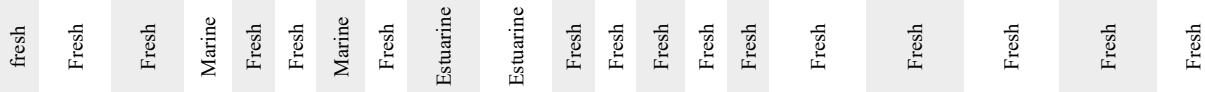

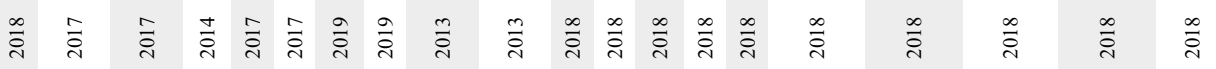

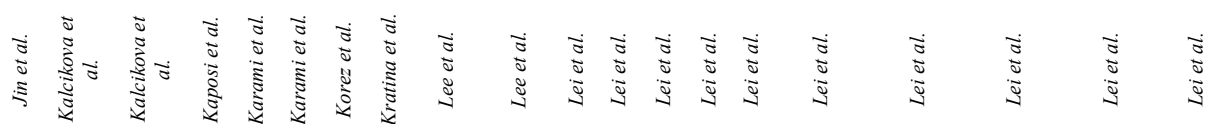




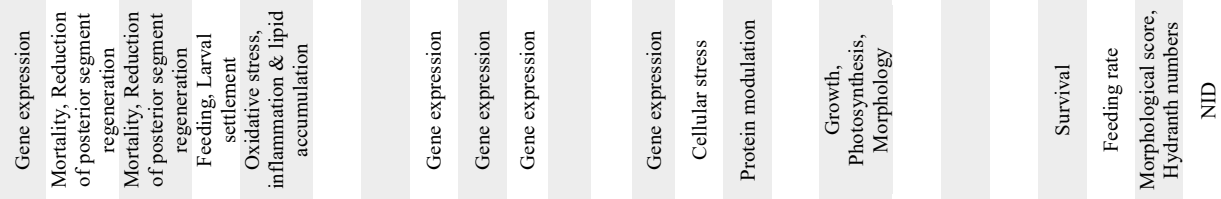

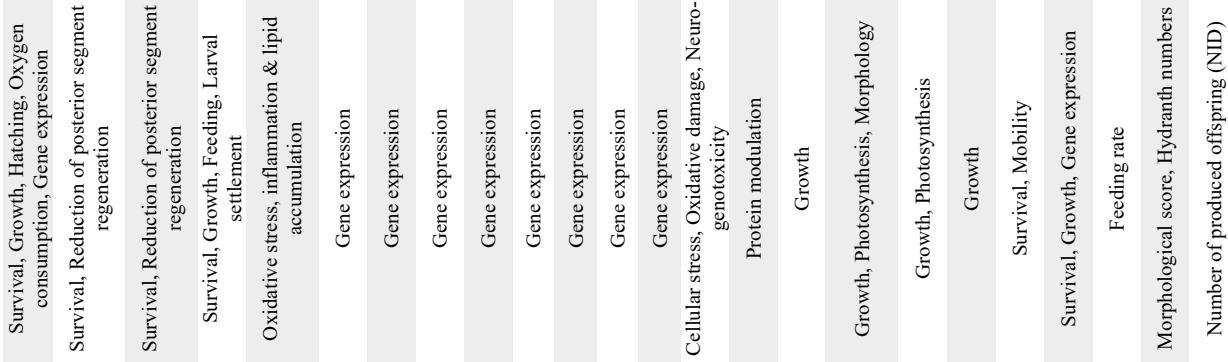

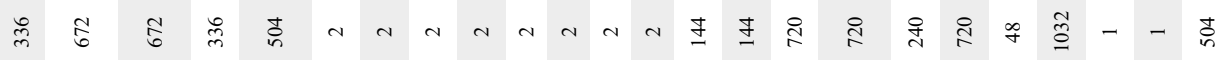

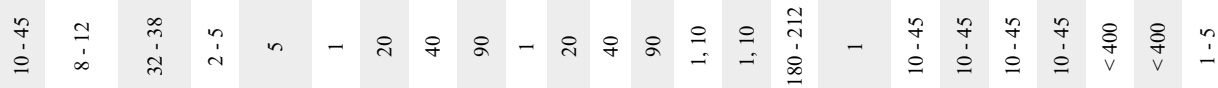

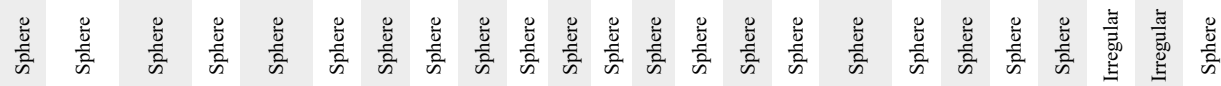

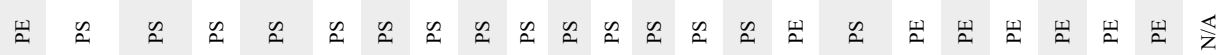

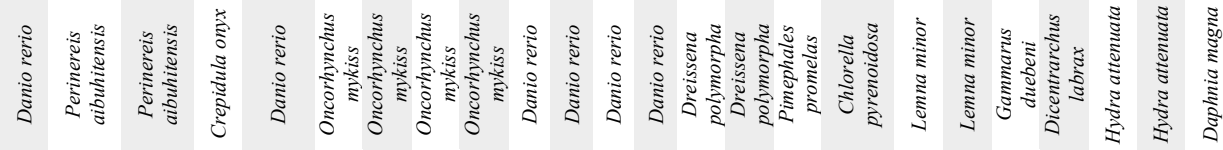

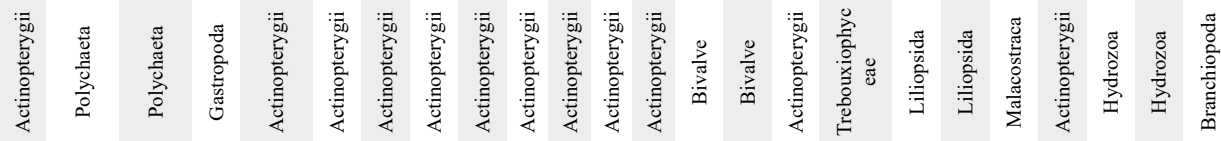

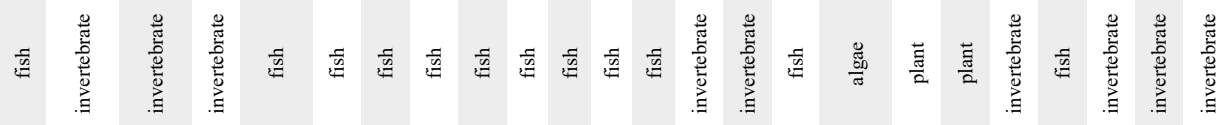

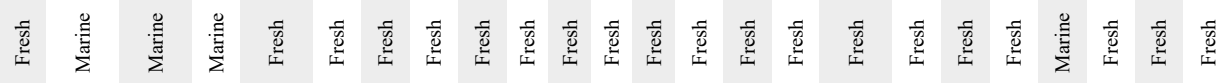

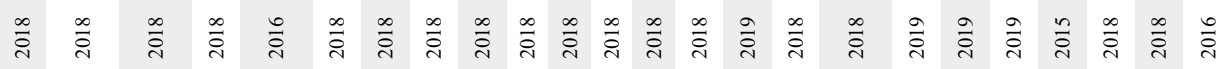

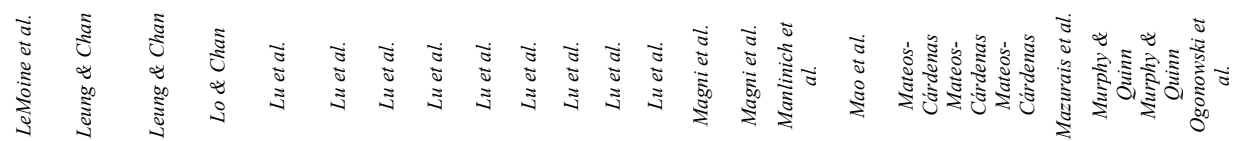




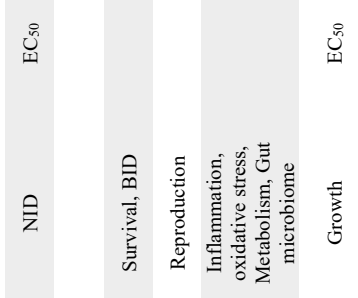

总

\|\|$\|$

MI!

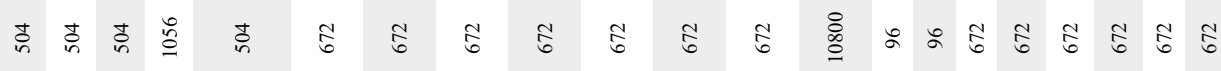

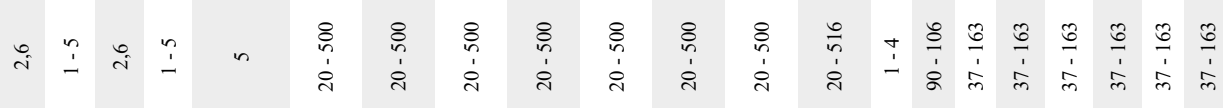
$\|H\|\|\|\|\| H\|H\|$

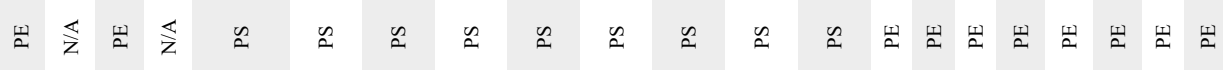

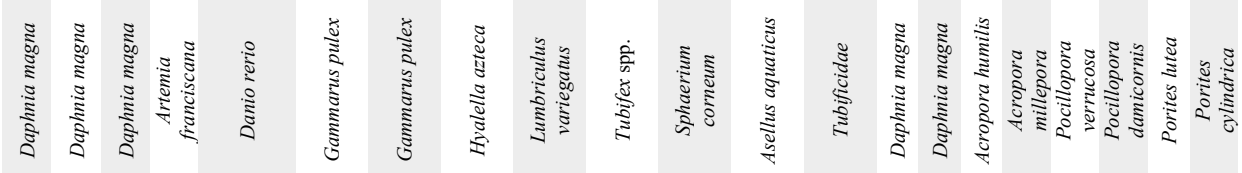
\|\|\|\|\|\|\|\|\|\|$\|$ \|\|$:\|\|\|\|\|\|\|\| \|$

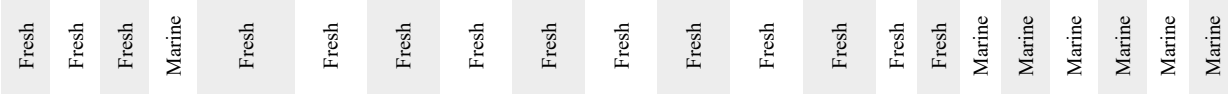

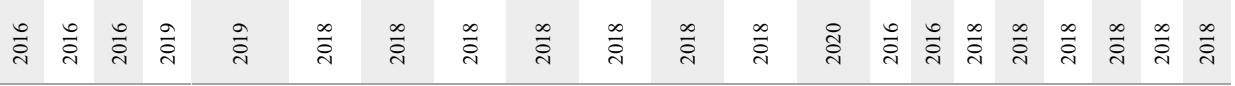

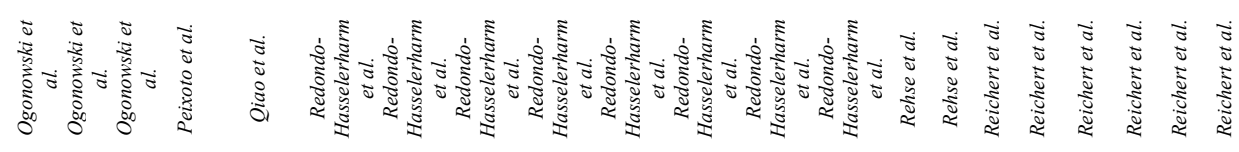




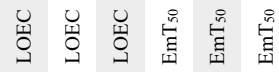

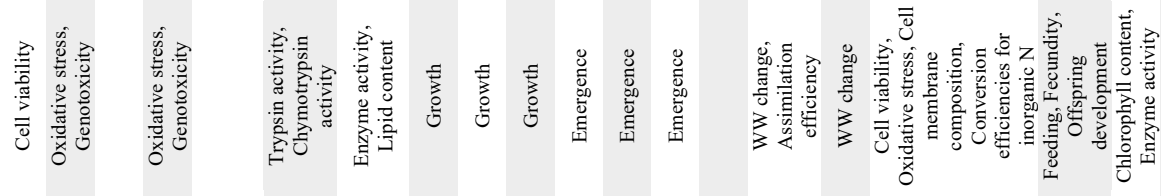

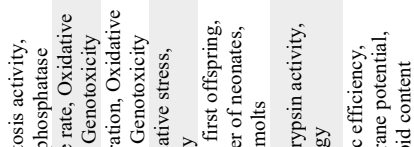

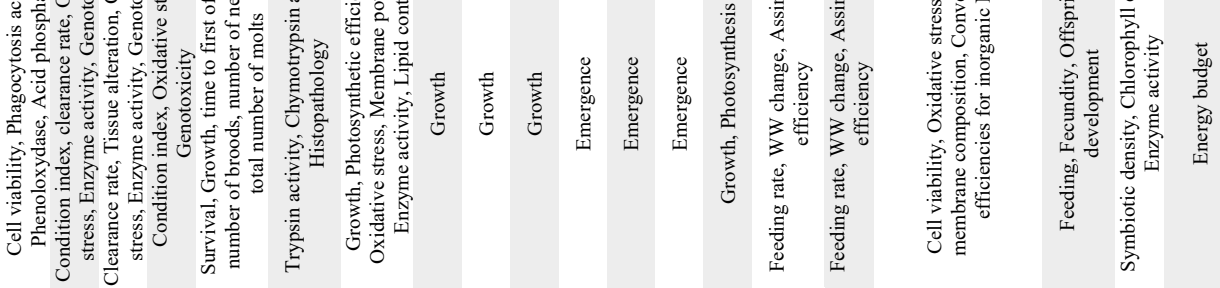

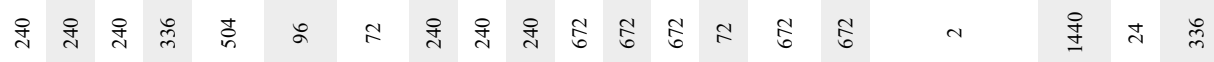

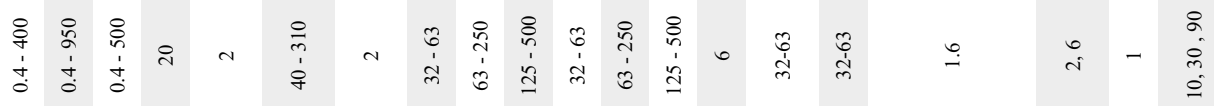

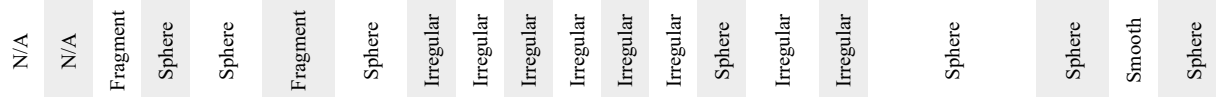

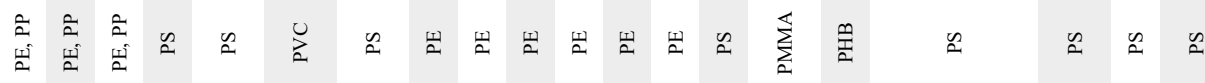

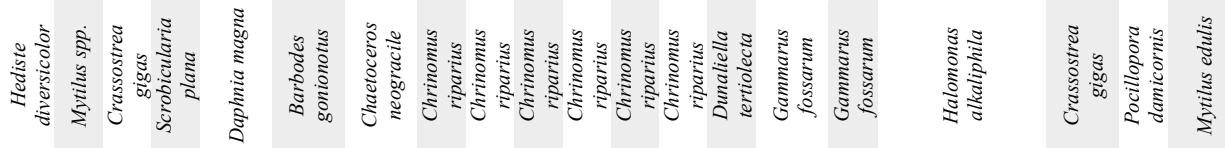

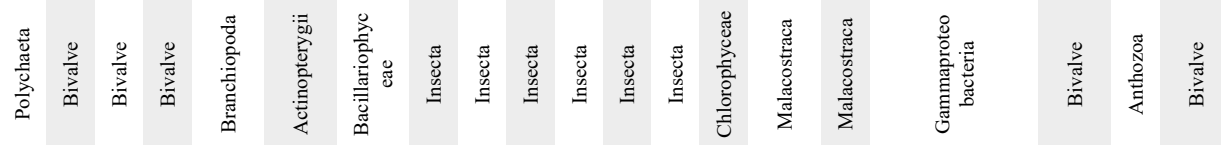

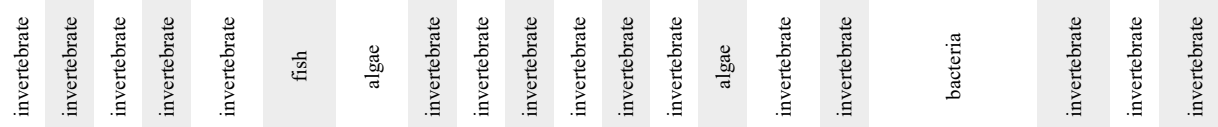

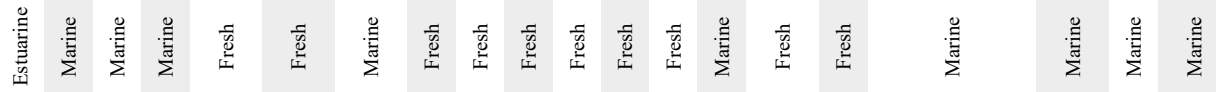

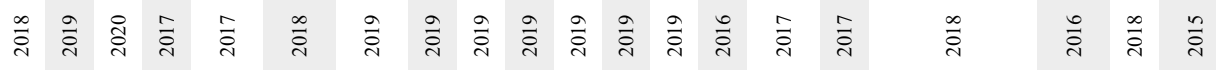

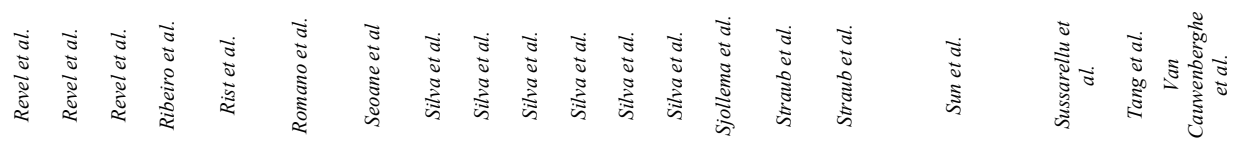




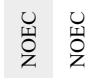

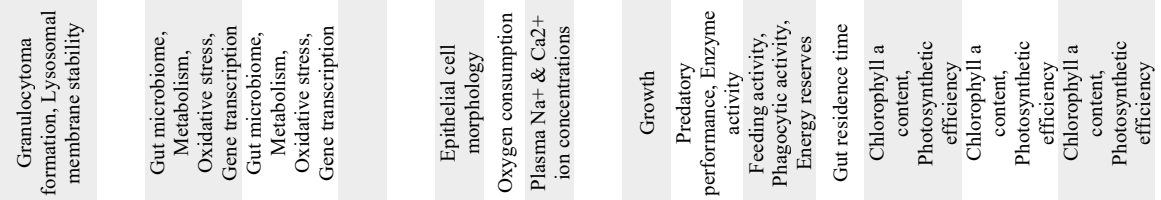

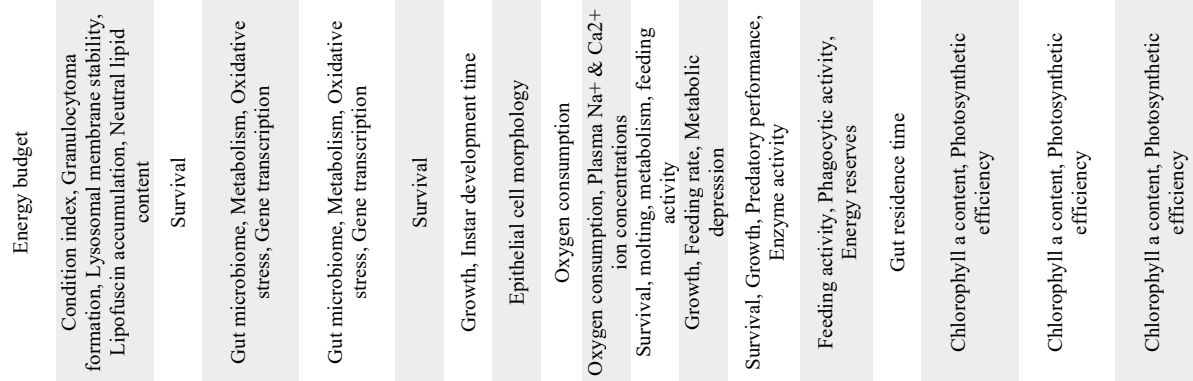

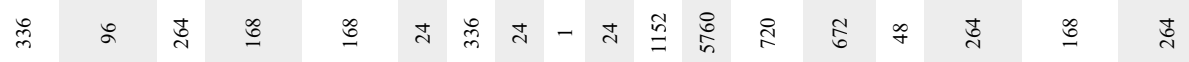

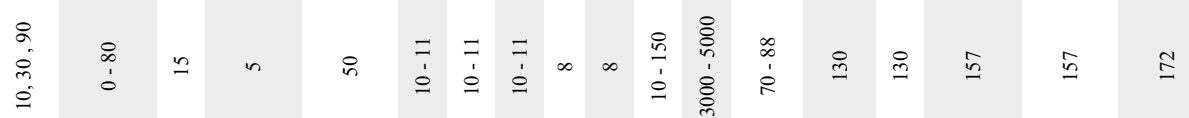

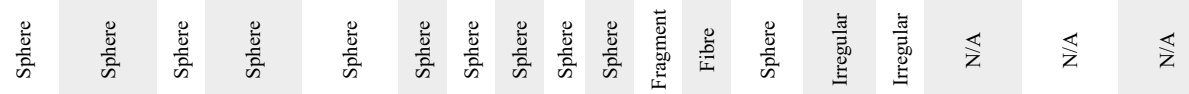

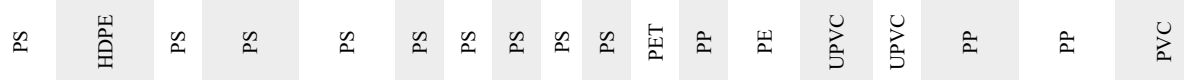

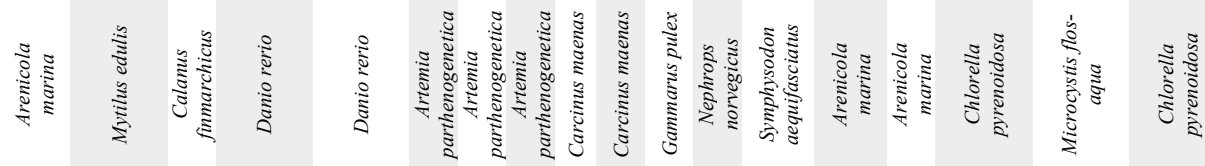

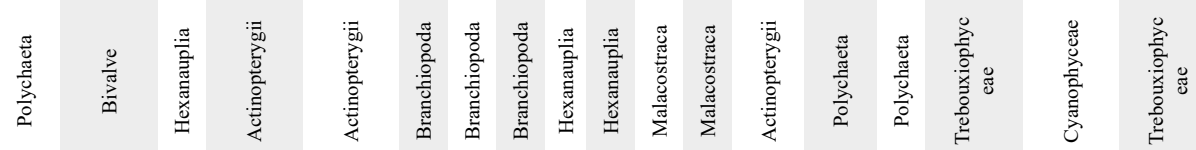

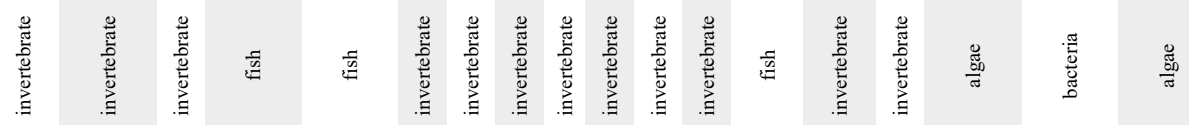

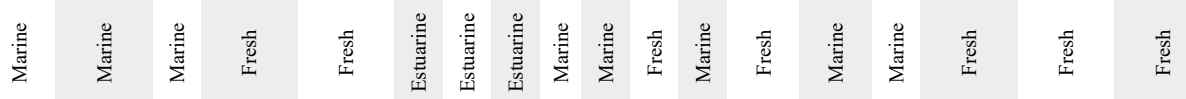

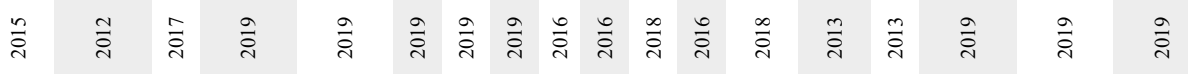

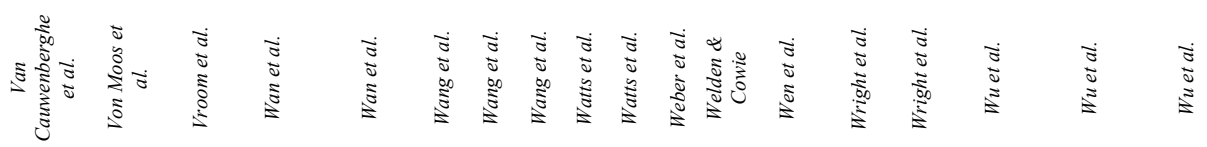




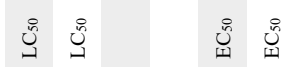

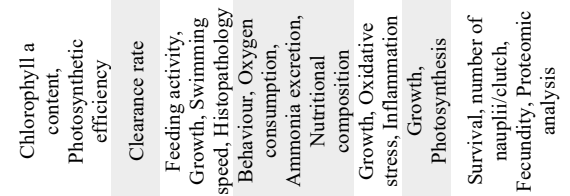

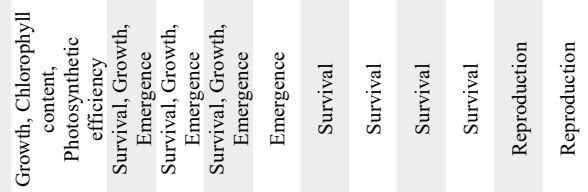

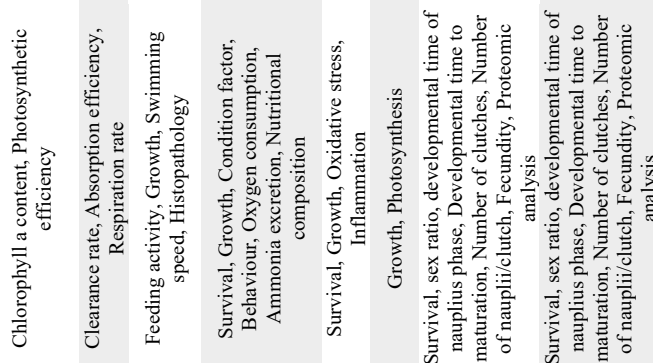

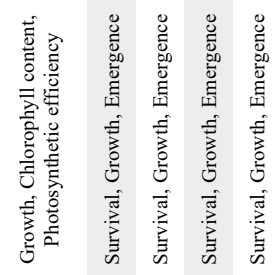

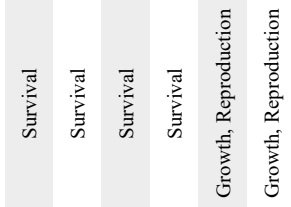

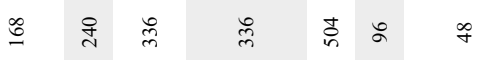

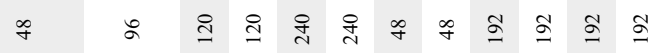

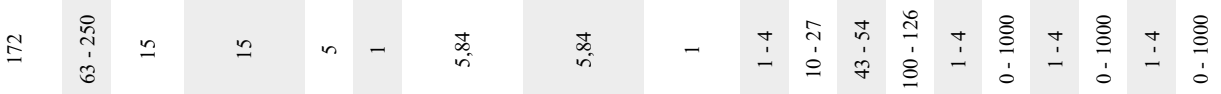

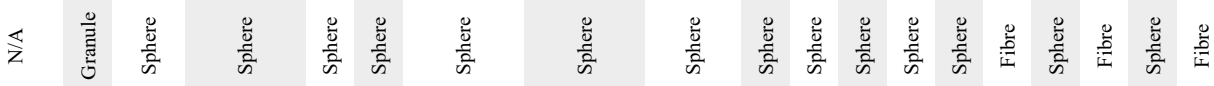

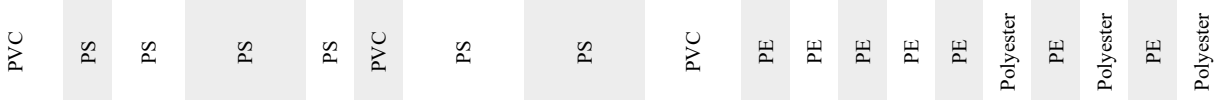

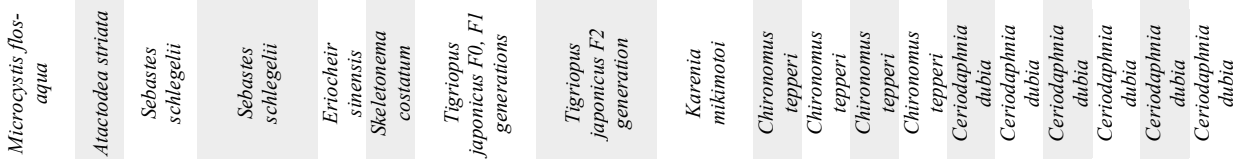

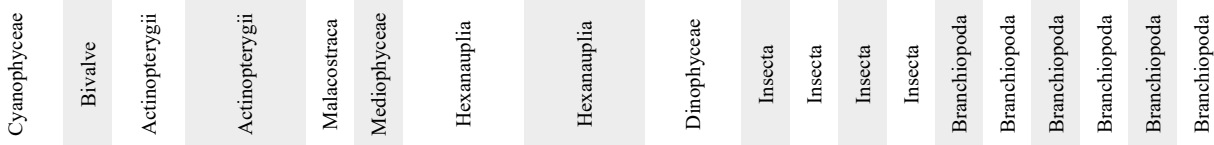

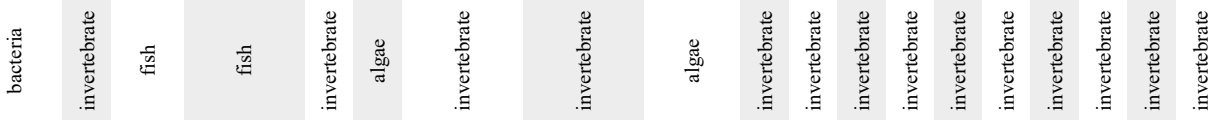

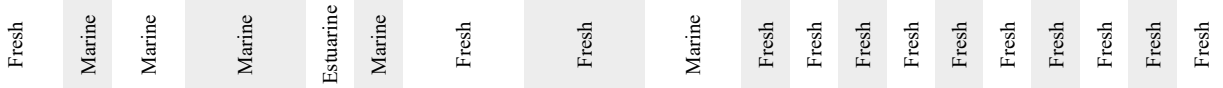

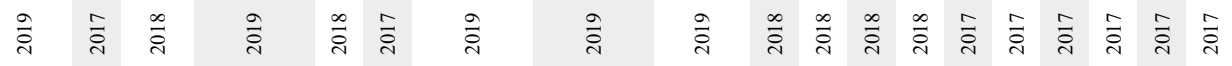

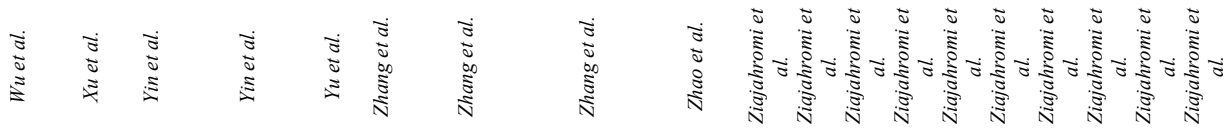




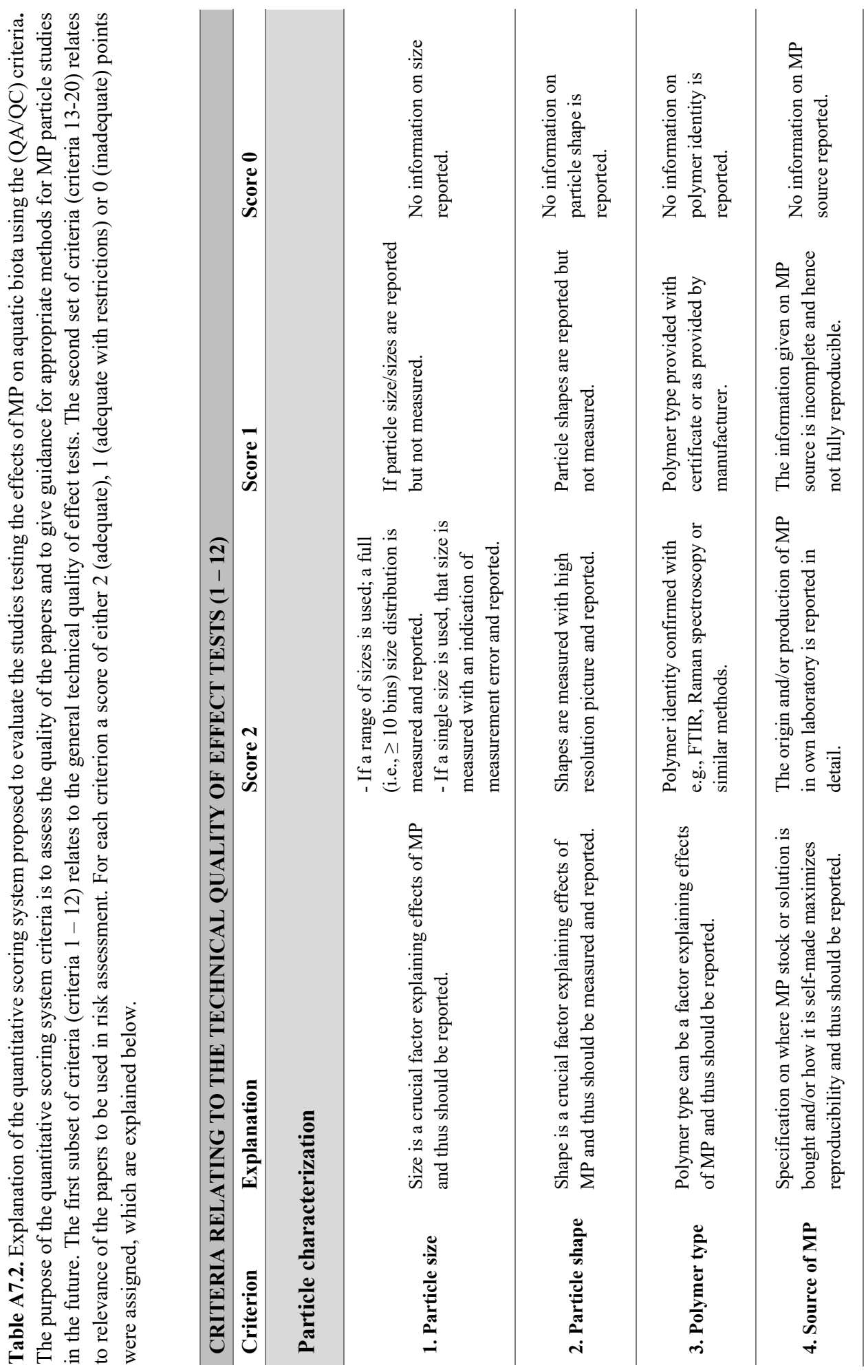




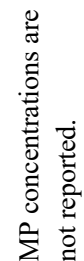

롬

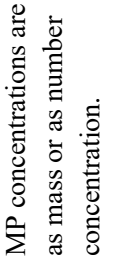

:

는

气ิ

:

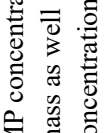

之

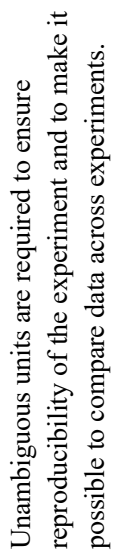

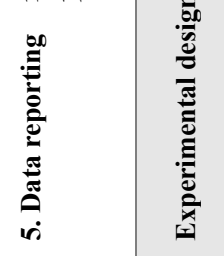

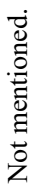
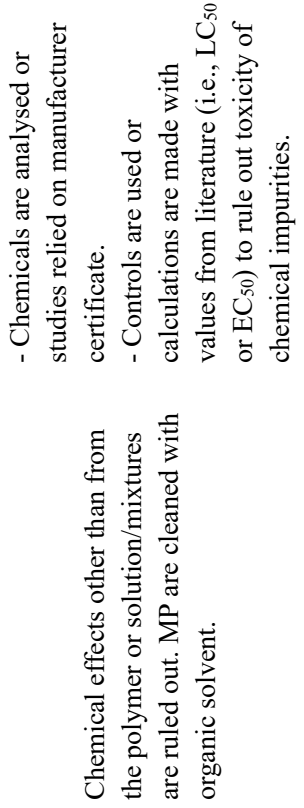

4 훙

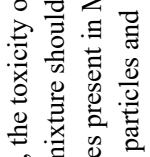

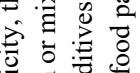

产 吾

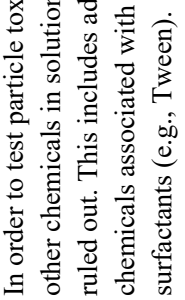

胥

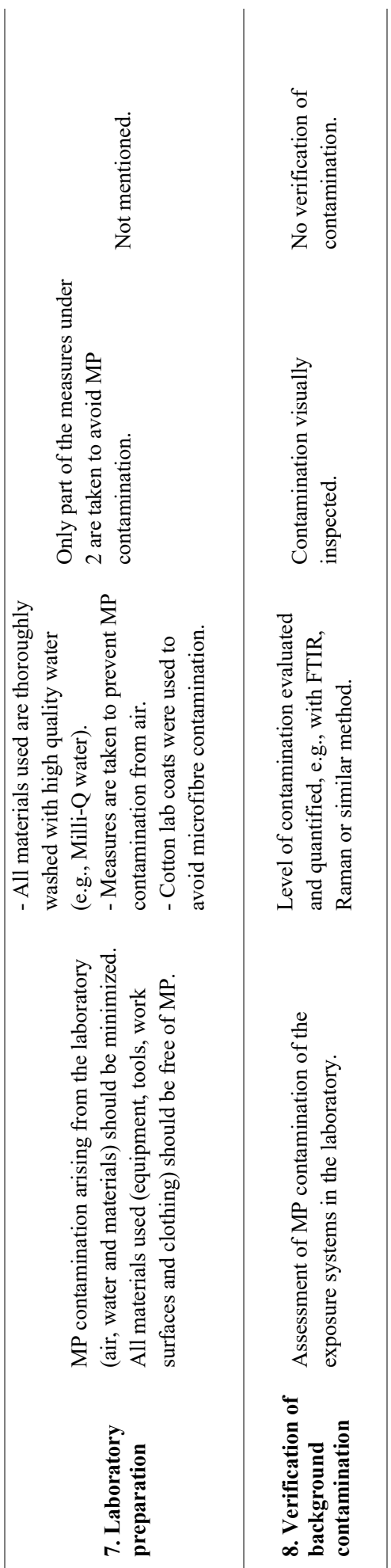




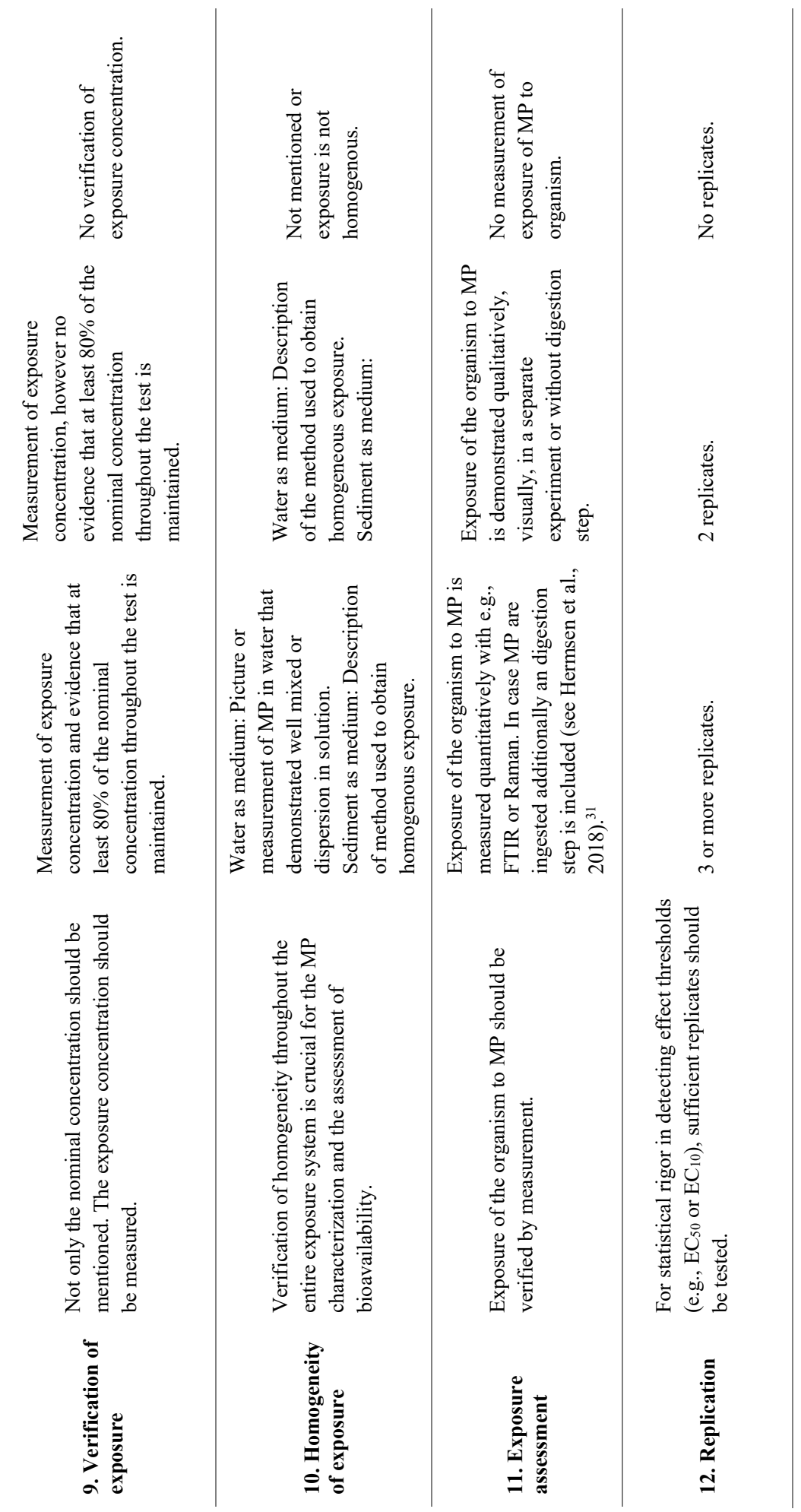




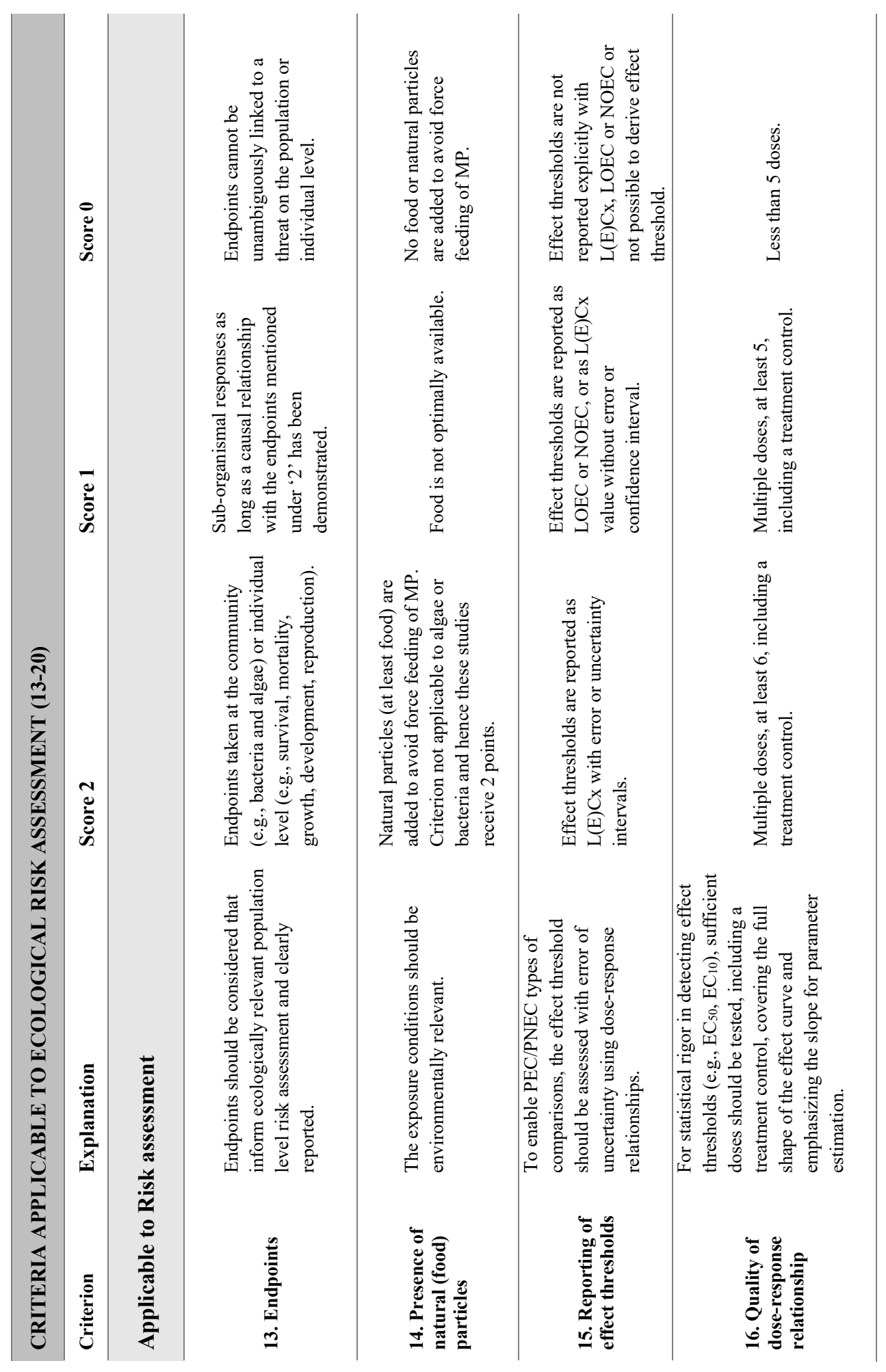




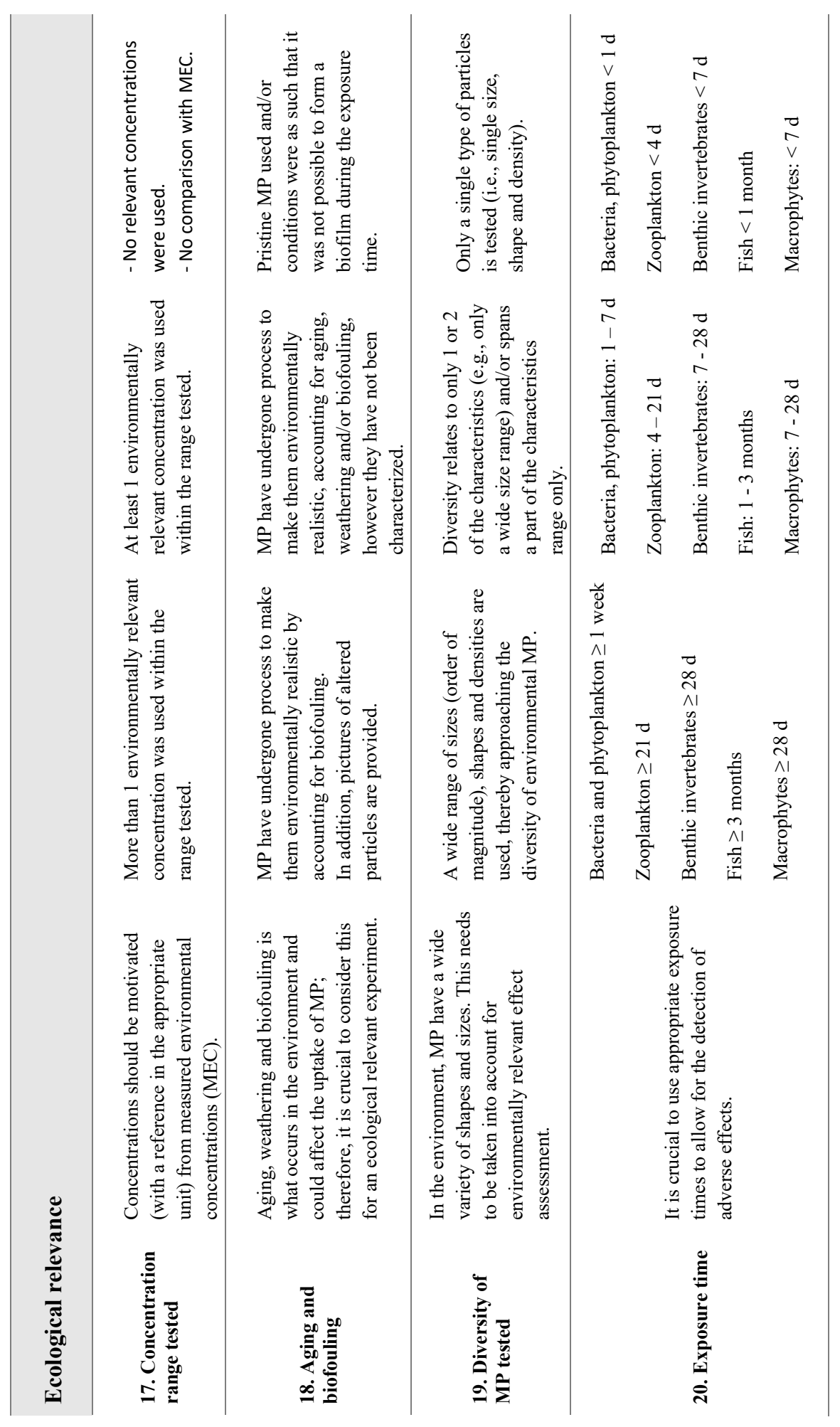




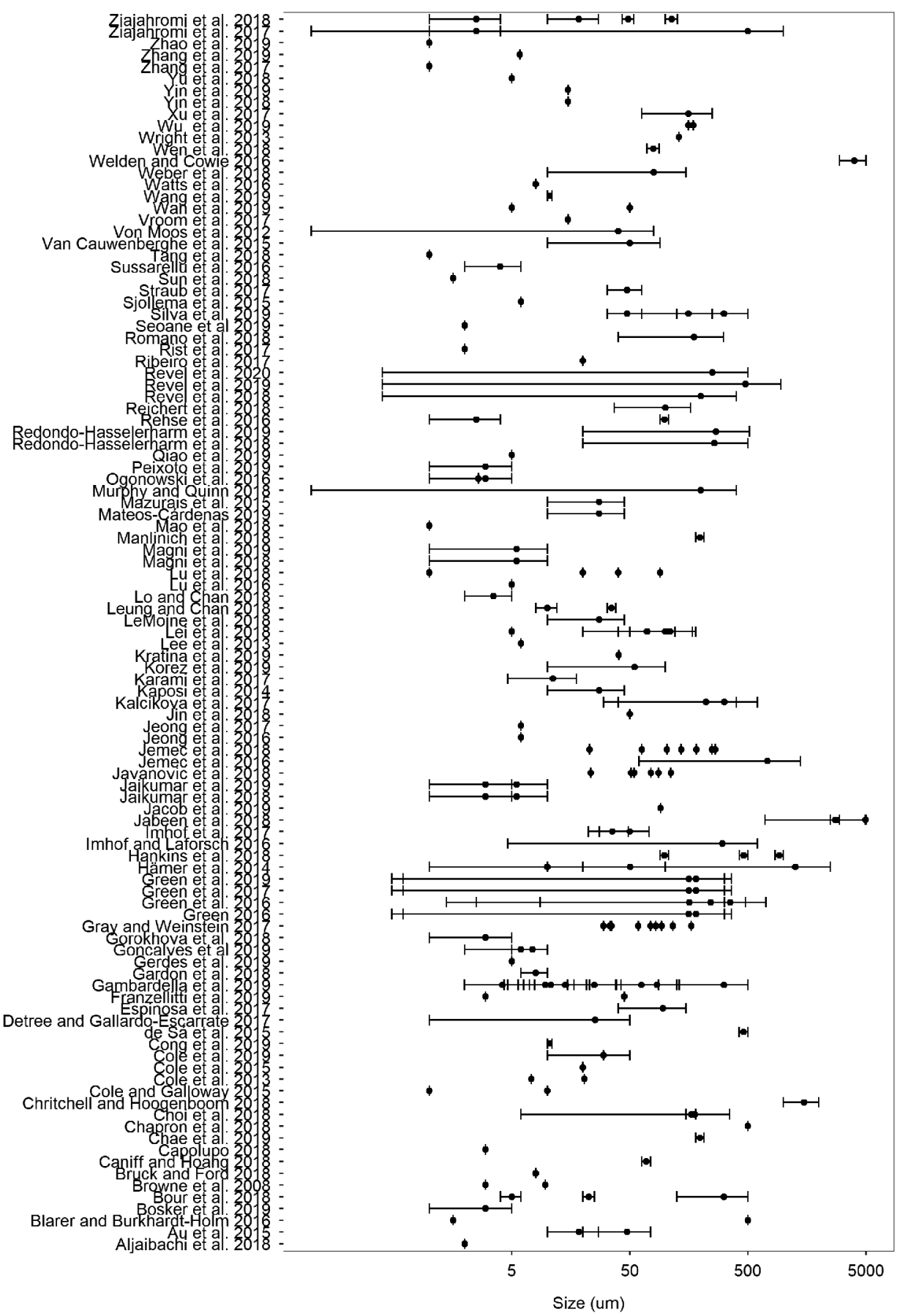

Figure A7.1. Size ranges used in the scored studies. Lines represent the size range reported and data points represent the reported or calculated average size. 


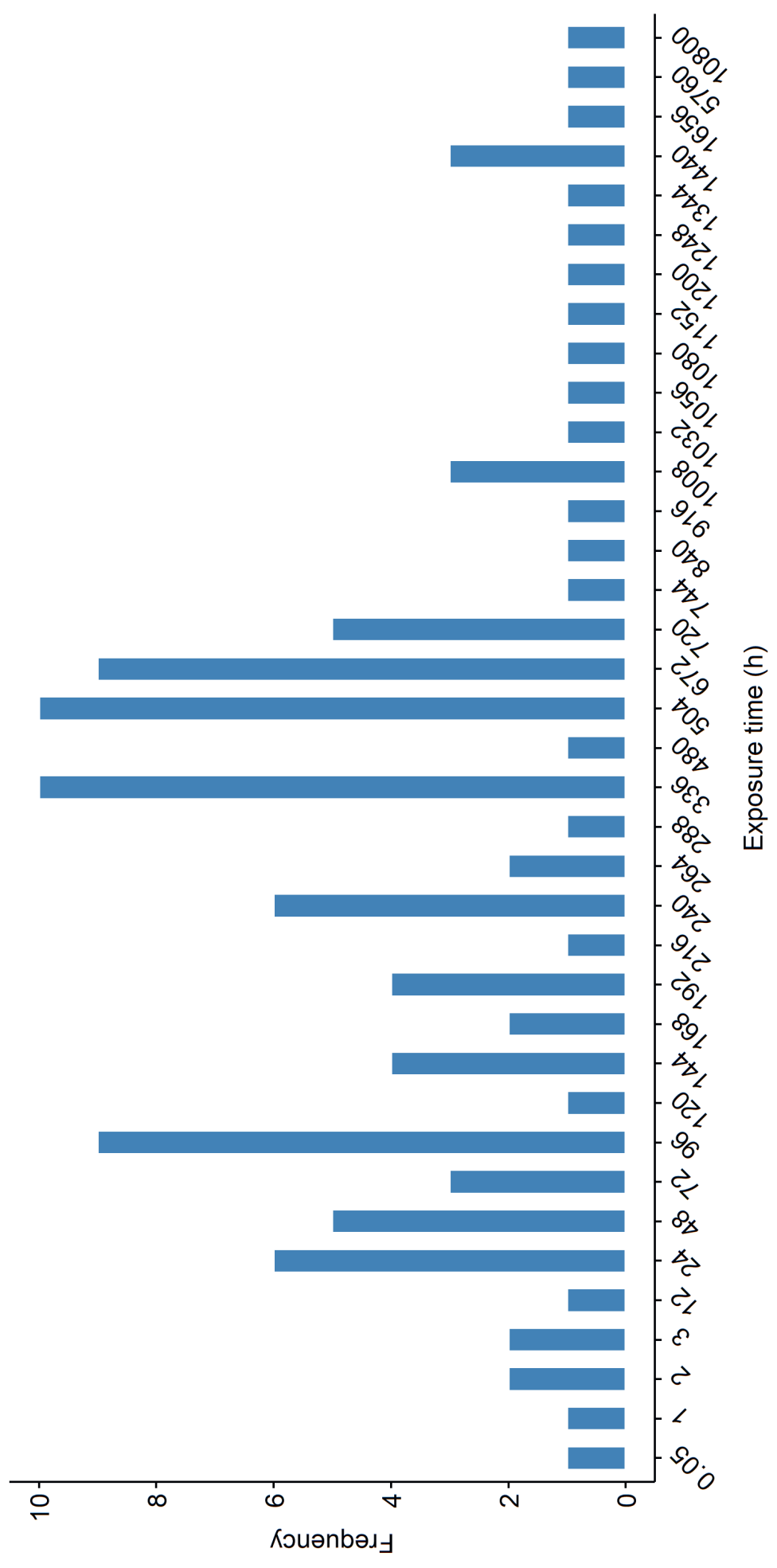

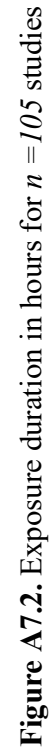




\section{Appendix of Chapter 8}

Table A8.1. Mean, minimum and maximum microplastic number concentrations (particles/kg of sediment $d w)$ and their size ranges reported for freshwater sediments in water bodies from Europe, Asia and Africa.

Table A8.2. Microplastic effect thresholds reported for benthic species using chronic single species tests with sediments.

Table A8.3. Maximum ingestible sizes for the freshwater benthic species used in the Species Sensitivity Distribution (SSD).

Figure A8.1. Cumulative frequency distribution of rescaled to 1 - $5000 \mu \mathrm{m}$ measured exposure concentrations in 20 water bodies based on the minimum, maximum and mean concentrations reported.

Figure A8.2. Cumulative frequency distribution of rescaled to 1 - $5000 \mu \mathrm{m}$ measured exposure concentrations (MECs) in 20 water bodies compared to the predicted no effect concentration (PNEC) obtained in tier 1 based on single species tests with freshwater benthic species.

Figure A8.3. Cumulative frequency distribution of rescaled to 1 - $5000 \mu \mathrm{m}$ measured exposure concentrations (MECs) in 20 water compared to the predicted no effect concentration (PNEC) obtained in tier 3 based on semi-field experiments with a freshwater benthic community. 


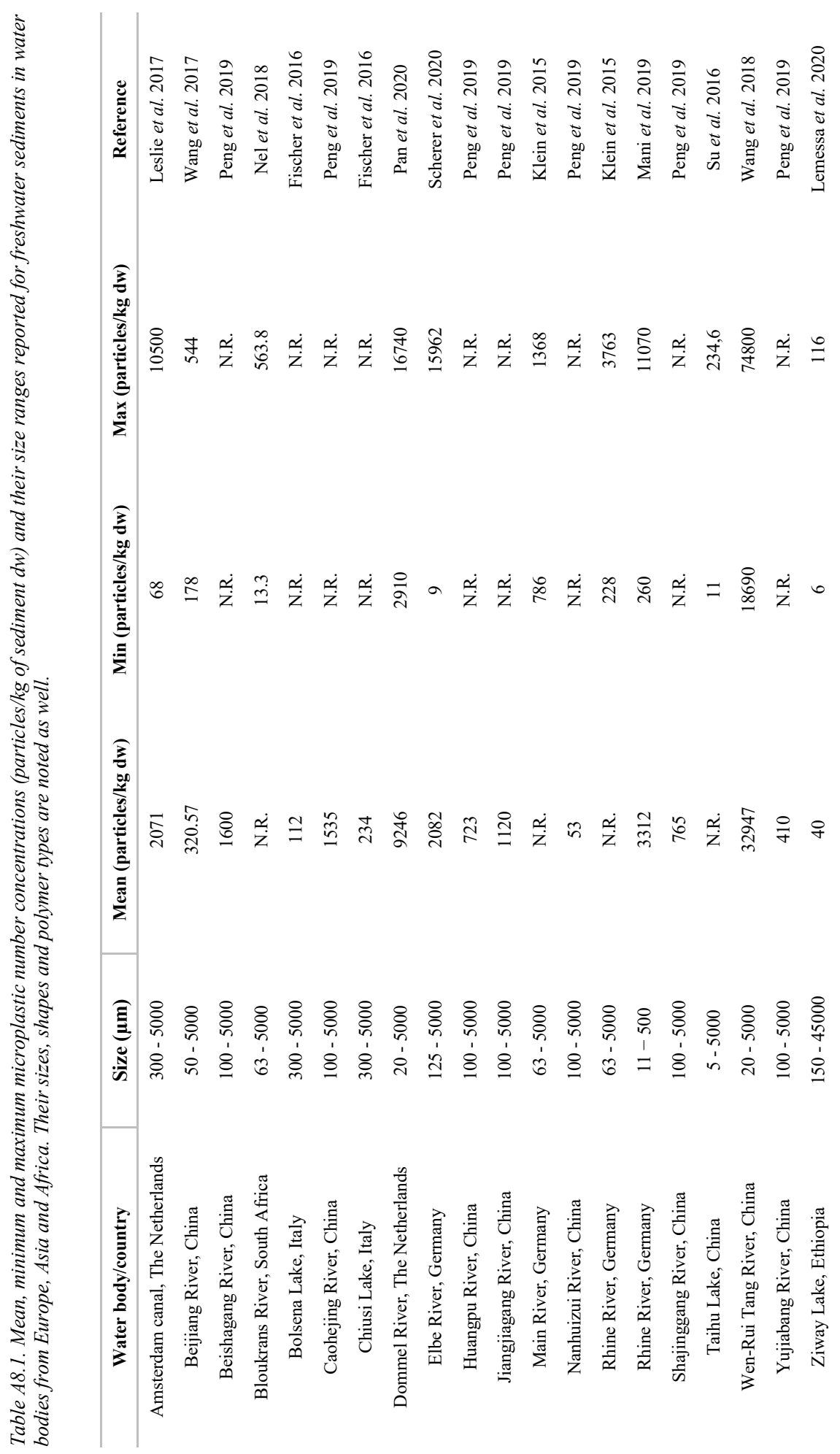




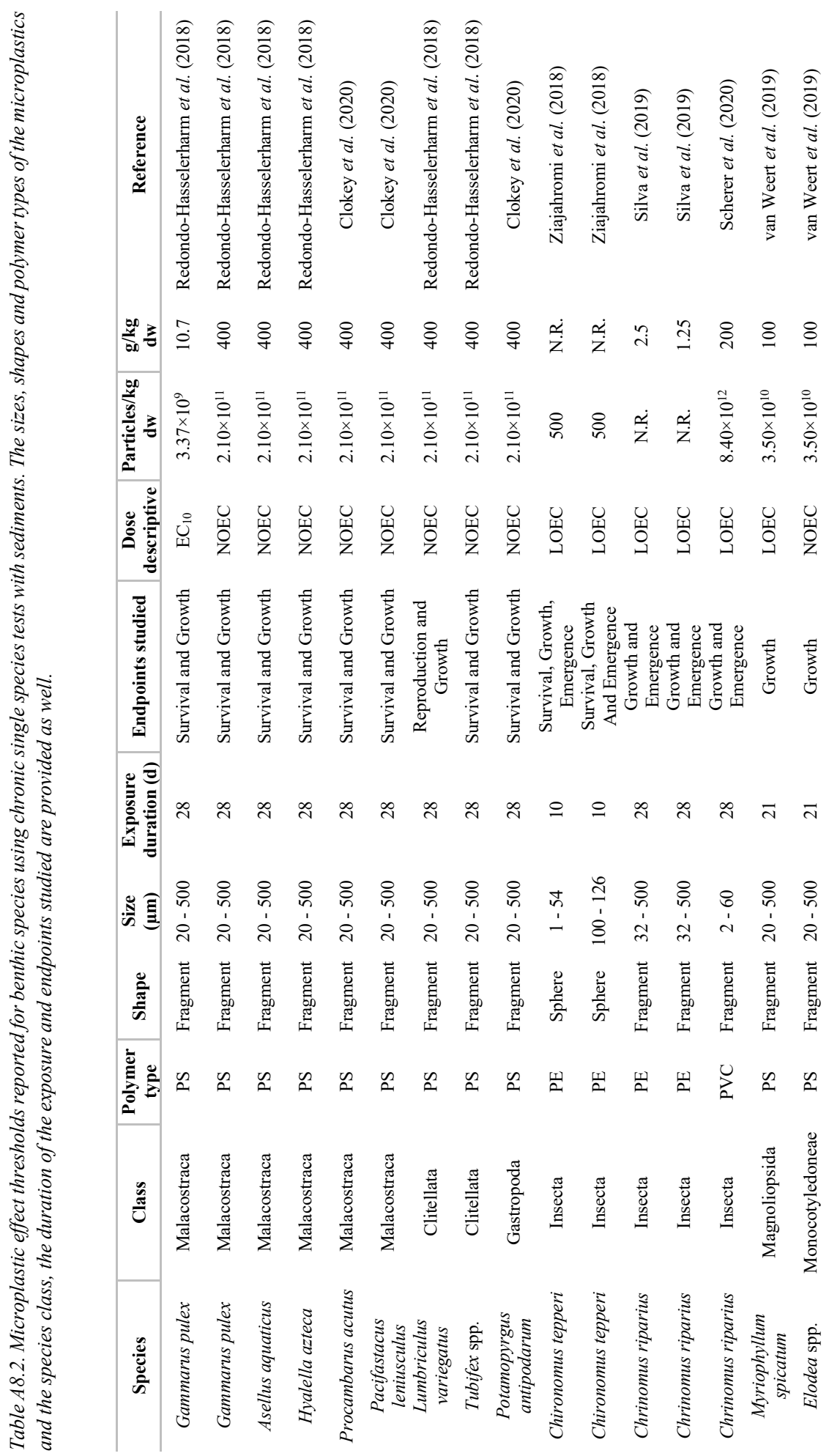




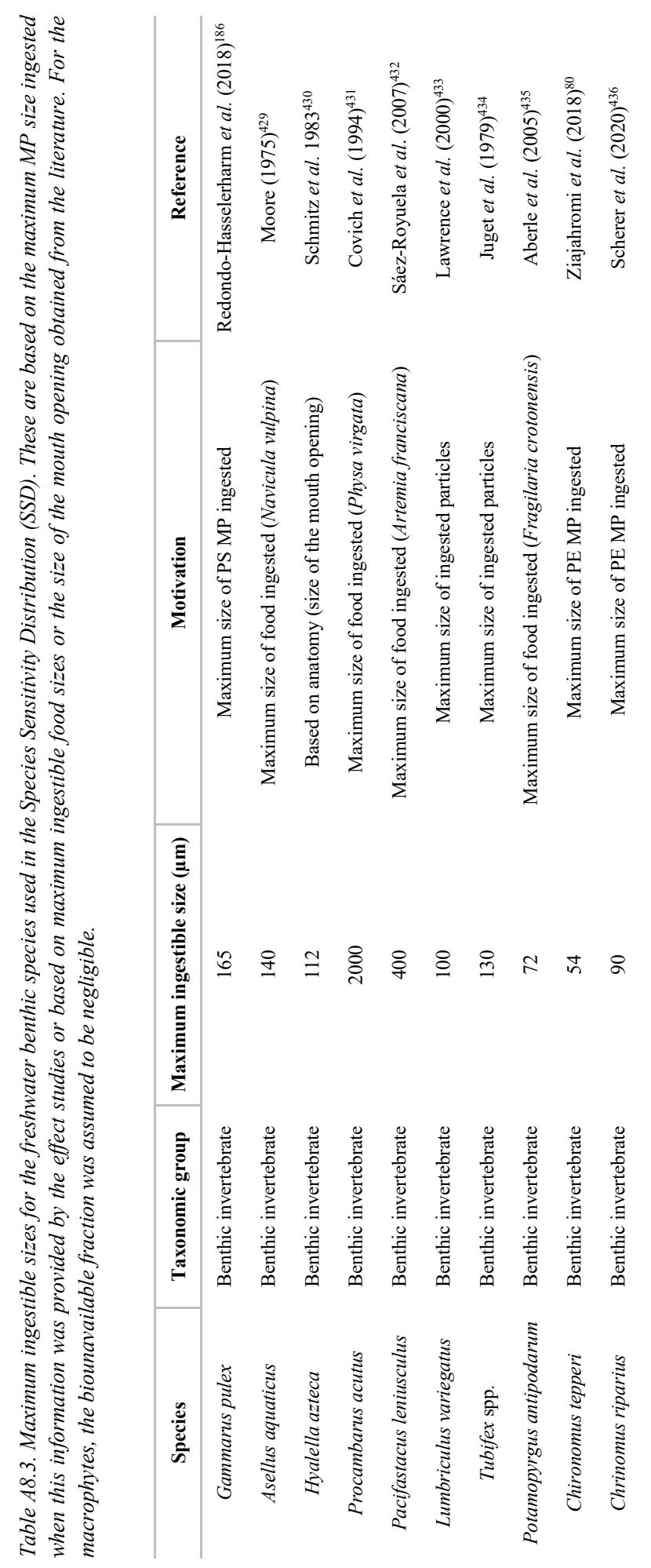




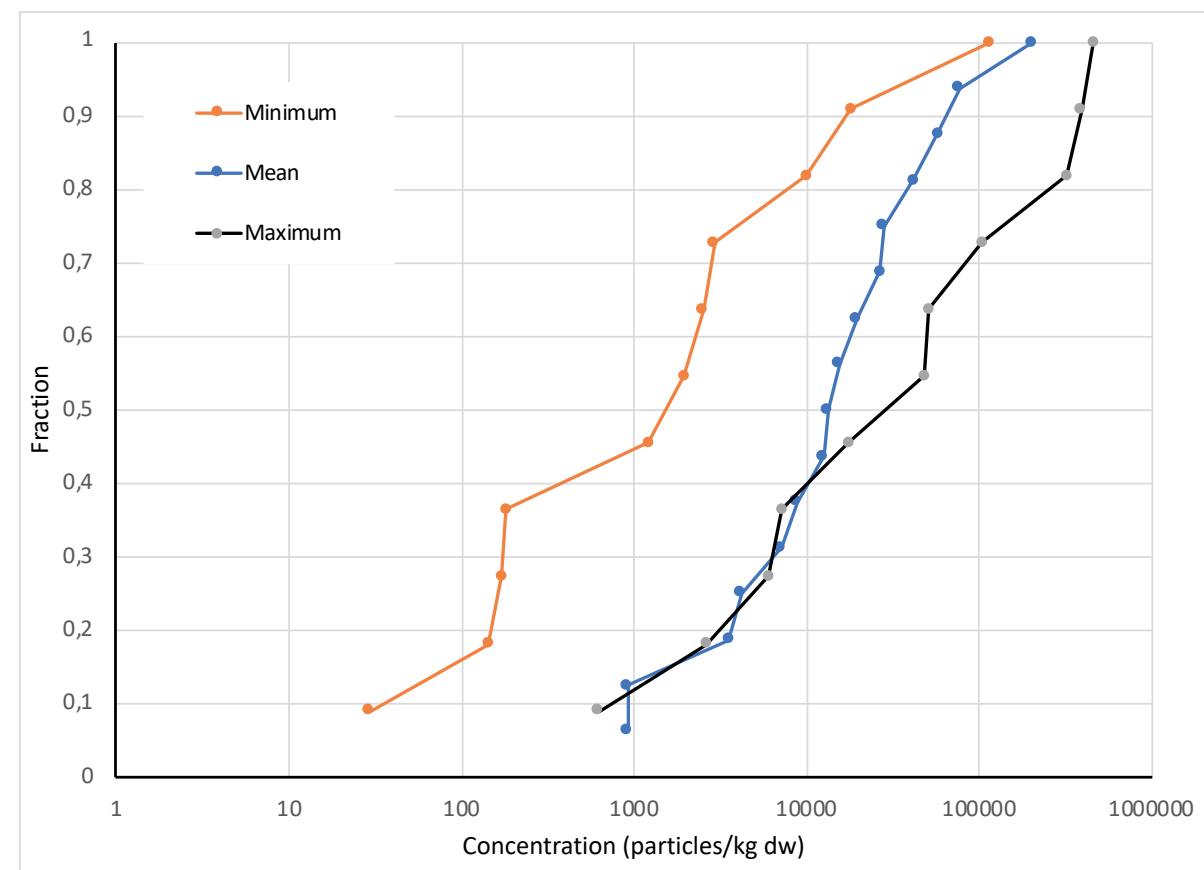

Figure A8.1. Cumulative frequency distribution of rescaled to 1 - $5000 \mu m$ measured exposure concentrations in 20 water bodies based on the minimum, maximum and mean concentrations reported. 


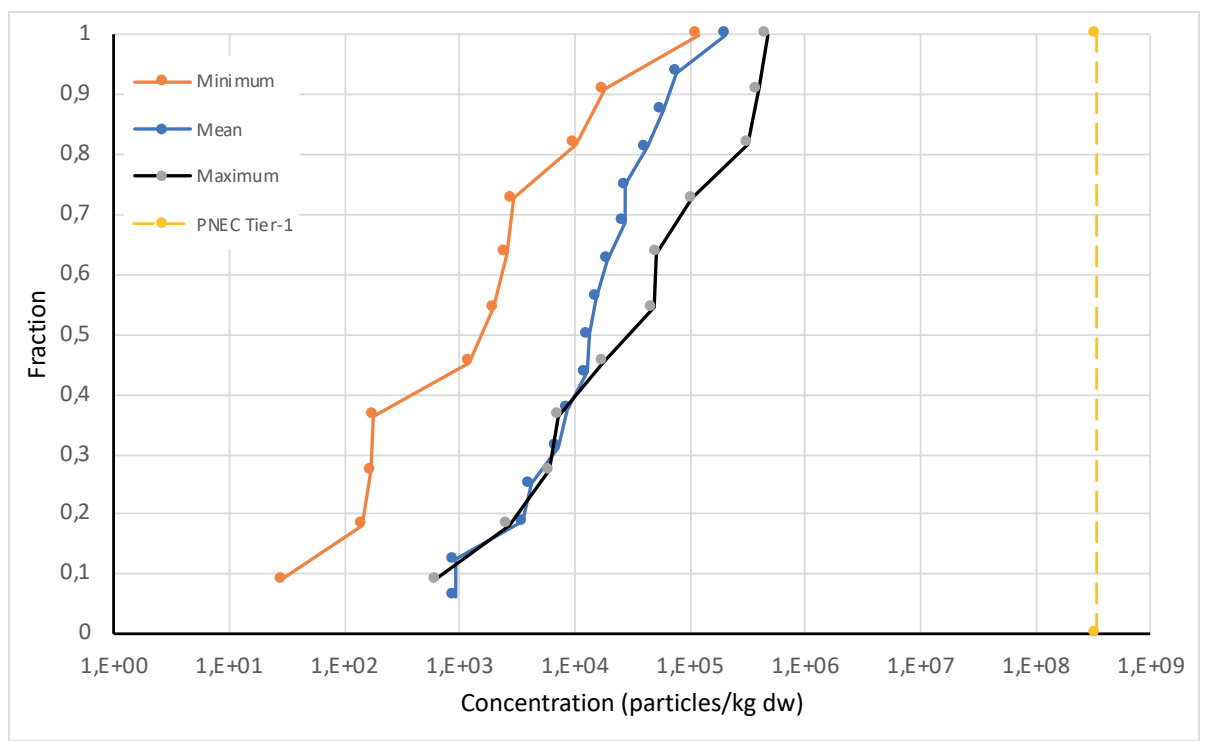

Figure A8.2. Cumulative frequency distribution of rescaled to 1 - $5000 \mu \mathrm{m}$ measured exposure concentrations (MECs) in 20 water bodies compared to the predicted no effect concentration (PNEC) obtained in tier 1 based on single species tests with freshwater benthic species. MECs are based on the minimum, maximum and mean concentrations reported. 


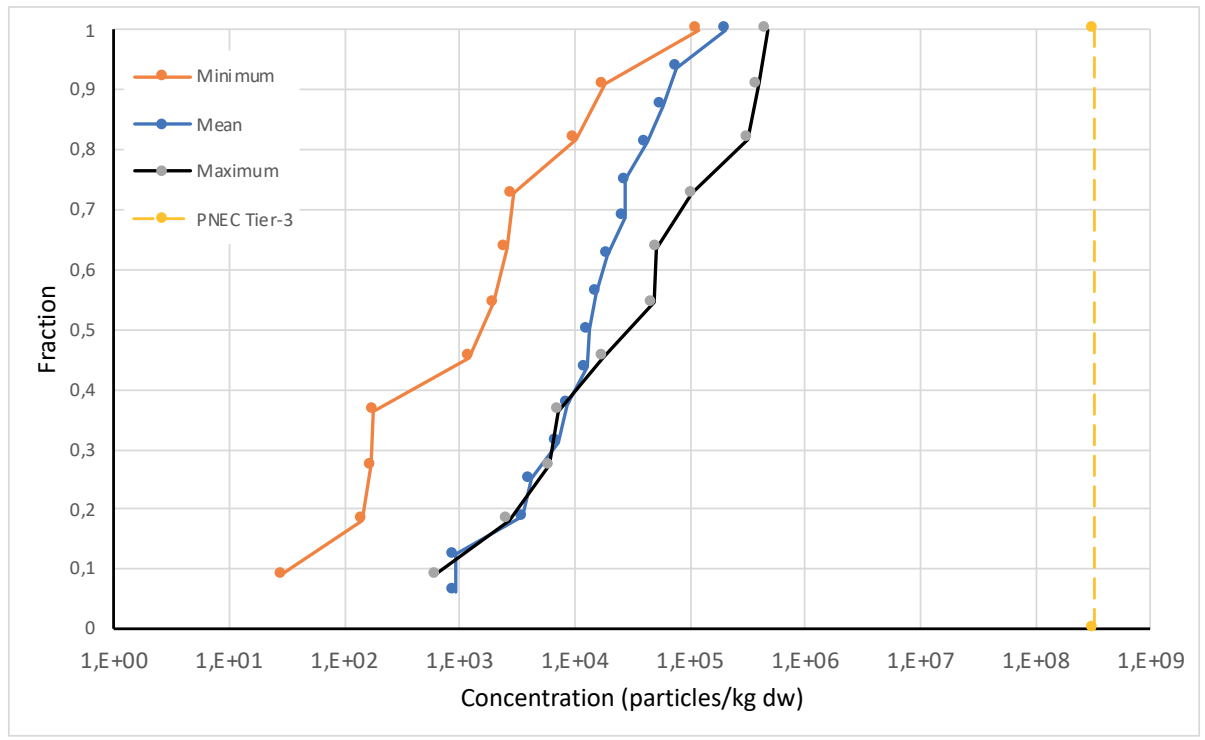

Figure A8.3. Cumulative frequency distribution of rescaled to 1 - $5000 \mu \mathrm{m}$ measured exposure concentrations (MECs) in 20 water compared to the predicted no effect concentration (PNEC) obtained in tier 3 based on semi-field experiments with a freshwater benthic community. MECs are based on the minimum, maximum and mean concentrations reported. 


\section{References}





\section{References}

(1) Andrady, A. L.; Neal, M. A. Applications and Societal Benefits of Plastics. Philos. Trans. R. Soc. B Biol. Sci. 2009, 364 (1526), 1977-1984. https://doi.org/10.1098/rstb.2008.0304.

(2) Ostle, C.; Thompson, R. C.; Broughton, D.; Gregory, L.; Wootton, M.; Johns, D. G. The Rise in Ocean Plastics Evidenced from a 60-Year Time Series. Nat. Commun. 2019, 10 (1), 8-13. https://doi.org/10.1038/s41467-019-09506-1.

(3) PlasticsEurope. Plastics - the Facts 2019. An analysis of European plastics production, demand and waste data. https://www.plasticseurope.org/.

(4) Barnes, D. K. A.; Galgani, F.; Thompson, R. C.; Barlaz, M. Accumulation and Fragmentation of Plastic Debris in Global Environments. Philos. Trans. R. Soc. B Biol. Sci. 2009, 364 (1526), 1985-1998. https://doi.org/10.1098/rstb.2008.0205.

(5) Geyer, R.; Jambeck, J. R.; Law, K. L. Production, Use, and Fate of All Plastics Ever Made. Sci. Adv. 2017, 3 (7), 25-29. https://doi.org/10.1126/sciadv.1700782.

(6) Derraik, J. G. B. The Pollution of the Marine Environment by Plastic Debris: A Review. Mar. Pollut. Bull. 2002, 44 (9), 842-852. https://doi.org/10.1016/S0025326X(02)00220-5.

(7) Thompson, R. C.; Moore, C. J.; Saal, F. S. V.; Swan, S. H. Plastics, the Environment and Human Health: Current Consensus and Future Trends. Philos. Trans. R. Soc. B Biol. Sci. 2009, 364 (1526), 2153-2166. https://doi.org/10.1098/rstb.2009.0053.

(8) Councell, T. B.; Duckenfield, K. U.; Landa, E. R.; Callender, E. Tire-Wear Particles as a Source of Zinc to the Environment. Env. Sci Technol 2004, 38 (15), 4206-4214. https://doi.org/https://doi.org/10.1021/es034631f.

(9) Moore, C. J. Synthetic Polymers in the Marine Environment: A Rapidly Increasing, Long-Term Threat. Environ. Res. 2008, 108 (2), 131-139. https://doi.org/10.1016/j.envres.2008.07.025.

(10) Ryan, P. G.; Moloney, C. L. Marine Litter Keeps Increasing. Nature 1993, 361 (6407), 23. https://doi.org/10.1038/361023a0.

(11) Koelmans, A. A.; Besseling, E.; Foekema, E.; Kooi, M.; Mintenig, S.; Ossendorp, B. C.; Redondo-Hasselerharm, P. E.; Verschoor, A.; Van Wezel, A. P.; Scheffer, M. Risks of Plastic Debris: Unravelling Fact, Opinion, Perception, and Belief. Environ. Sci. Technol. 2017, 51 (20), 11513-11519. https://doi.org/10.1021/acs.est.7b02219.

(12) Lehner, R.; Weder, C.; Petri-Fink, A.; Rothen-Rutishauser, B. Emergence of Nanoplastic in the Environment and Possible Impact on Human Health. Environ. Sci. Technol. 2019, 53 (4), 1748-1765. https://doi.org/10.1021/acs.est.8b05512.

(13) Science Advice for Policy by European Academies. A Scientific Perspective on Microplastics in Nature and Society; Berlin, 2019.

(14) Sundt, P.; Schulze, P.-E.; Syversen, F. Sources of Microplastic- Pollution to the Marine Environment Project Report; 2014.

(15) Banerjee, A.; Qi, J.; Gogoi, R.; Wong, J.; Mitragotri, S. Role of Nanoparticle Size, Shape and Surface Chemistry in Oral Drug Delivery. J. Control. Release 
2016, 238, 176-185. https://doi.org/10.1016/j.jconrel.2016.07.051.

(16) Hernandez, L. M.; Yousefi, N.; Tufenkji, N. Are There Nanoplastics in Your Personal Care Products? Environ. Sci. Technol. Lett. 2017, 4 (7), 280-285. https://doi.org/10.1021/acs.estlett.7b00187.

(17) Napper, I. E.; Bakir, A.; Rowland, S. J.; Thompson, R. C. Characterisation, Quantity and Sorptive Properties of Microplastics Extracted from Cosmetics. Mar. Pollut. Bull. 2015, 99, 178-185. https://doi.org/10.1016/j.marpolbul.2015.07.029.

(18) Dawson, A. L.; Kawaguchi, S.; King, C. K.; Townsend, K. A.; King, R.; Huston, W. M.; Bengtson Nash, S. M. Turning Microplastics into Nanoplastics through Digestive Fragmentation by Antarctic Krill. Nat. Commun. 2018, 9 (1), 1001. https://doi.org/10.1038/s41467-018-03465-9.

(19) Lambert, S.; Wagner, M. Characterisation of Nanoplastics during the Degradation of Polystyrene. Chemosphere 2016, 145, 265-268. https://doi.org/10.1016/j.chemosphere.2015.11.078.

(20) Andrady, A. L. Microplastics in the Marine Environment. Mar. Pollut. Bull. 2011, 62 (8), 1596-1605. https://doi.org/10.1016/j.marpolbul.2011.05.030.

(21) Hartmann, N. B.; Hüffer, T.; Thompson, R. C.; Hassellöv, M.; Verschoor, A.; Daugaard, A. E.; Rist, S.; Karlsson, T.; Brennholt, N.; Cole, M.; et al. Are We Speaking the Same Language? Recommendations for a Definition and Categorization Framework for Plastic Debris. Environ. Sci. Technol. 2019, 53 (3), 1039-1047. https://doi.org/10.1021/acs.est.8b05297.

(22) Van Sebille, E.; Aliani, S.; Law, K. L.; Maximenko, N.; Alsina, J. M.; Bagaev, A.; Bergmann, M.; Chapron, B.; Chubarenko, I.; Cózar, A.; et al. The Physical Oceanography of the Transport of Floating Marine Debris. Environ. Res. Lett. 2020, 15 (2). https://doi.org/10.1088/1748-9326/ab6d7d.

(23) Kooi, M.; Besseling, E.; Kroeze, C.; Van Wezel, A.; Koelmans, A. A. Modelling the Fate and Transport of Plastic Debris in Fresh Waters. Review and Guidance. In Freshwater microplastics. Emerging environmental contaminants?; Wagner, M., Lambert, S., Eds.; Springer, Hdb Env Chem 58, 2017. https://doi.org/10.1007/978-3-319-61615-5.

(24) Horton, A. A.; Walton, A.; Spurgeon, D. J.; Lahive, E.; Svendsen, C. Microplastics in Freshwater and Terrestrial Environments: Evaluating the Current Understanding to Identify the Knowledge Gaps and Future Research Priorities. Sci. Total Environ. 2017, 586, 127-141. https://doi.org/10.1016/j.scitotenv.2017.01.190.

(25) Lebreton, L. C. M.; Van Der Zwet, J.; Damsteeg, J. W.; Slat, B.; Andrady, A.; Reisser, J. River Plastic Emissions to the World's Oceans. Nat. Commun. 2017, 8, 15611. https://doi.org/10.1038/ncomms 15611 .

(26) Eerkes-Medrano, D.; Thompson, R. C.; Aldridge, D. C. Microplastics in Freshwater Systems: A Review of the Emerging Threats, Identification of Knowledge Gaps and Prioritisation of Research Needs. Water Res. 2015, 75, 6382. https://doi.org/10.1016/j.watres.2015.02.012.

(27) Besseling, E.; Quik, J. T. K.; Sun, M.; Koelmans, A. A. Fate of Nano- and Microplastic in Freshwater Systems: A Modeling Study. Environ. Pollut. 2017, 220, 540-548. https://doi.org/10.1016/j.envpol.2016.10.001. 
(28) Hurley, R. R.; Nizzetto, L. Fate and Occurrence of Micro(Nano)Plastics in Soils: Knowledge Gaps and Possible Risks. Curr. Opin. Environ. Sci. Heal. 2018, 1, 611. https://doi.org/10.1016/j.coesh.2017.10.006.

(29) Eriksen, M.; Maximenko, N.; Thiel, M.; Cummins, A.; Lattin, G.; Wilson, S.; Hafner, J.; Zellers, A.; Rifman, S. Plastic Pollution in the South Pacific Subtropical Gyre. Mar. Pollut. Bull. 2013, 68 (1-2), 71-76. https://doi.org/10.1016/j.marpolbul.2012.12.021.

(30) Zarfl, C.; Fleet, D.; Fries, E.; Galgani, F.; Gerdts, G.; Hanke, G.; Matthies, M. Microplastics in Oceans. Mar. Pollut. Bull. 2011, 62 (8), 1589-1591. https://doi.org/10.1016/j.marpolbul.2011.02.040.

(31) Hermsen, E.; Mintenig, S. M.; Besseling, E.; Koelmans, A. A. Quality Criteria for the Analysis of Microplastic in Biota Samples: A Critical Review. Environ. Sci. Technol. 2018, 52, 10230-10240. https://doi.org/10.1021/acs.est.8b01611.

(32) Mintenig, S. M.; Bäuerlein, P. S.; Koelmans, A. A.; Dekker, S. C.; Van Wezel, A. P. Closing the Gap between Small and Smaller: Towards a Framework to Analyse Nano- and Microplastics in Aqueous Environmental Samples. Environ. Sci. Nano 2018, 5 (7), 1640-1649. https://doi.org/10.1039/c8en00186c.

(33) Mintenig, S. M.; Kooi, M.; Erich, M. W.; Primpke, S.; Redondo- Hasselerharm, P. E.; Dekker, S. C.; Koelmans, A. A.; van Wezel, A. P. A Systems Approach to Understand Microplastic Occurrence and Variability in Dutch Riverine Surface Waters. Water Res. 2020, 176, 115723. https://doi.org/10.1016/j.watres.2020.115723.

(34) Environmental Protection Ageny. Microplastic in Danish Wastewater: Sources, Occurrences and Fate Fast DNA Sequencing for Optimization of Wastewater Treatment Plants View Project; 2017.

(35) Koelmans, A. A.; Mohamed Nor, N. H.; Hermsen, E.; Kooi, M.; Mintenig, S. M.; De France, J. Microplastics in Freshwaters and Drinking Water: Critical Review and Assessment of Data Quality. Water Res. 2019, 155, 410-422. https://doi.org/10.1016/j.watres.2019.02.054.

(36) Kapp, K. J.; Yeatman, E. Microplastic Hotspots in the Snake and Lower Columbia Rivers: A Journey from the Greater Yellowstone Ecosystem to the Pacific Ocean. Environ. Pollut. 2018, 241, 1082-1090. https://doi.org/10.1016/j.envpol.2018.06.033.

(37) Schell, T.; Rico, A.; Vighi, M. Occurrence, Fate and Fluxes of Plastics and Microplastics in Terrestrial and Freshwater Ecosystems. In Reviews of Environmental Contamination and Toxicology; Springer Nature Switzerland AG, 2020. https://doi.org/10.1007/398_2019_40.

(38) Wang, Z.; Su, B.; Xu, X.; Di, D.; Huang, H.; Mei, K.; Dahlgren, R. A.; Zhang, M.; Shang, X. Preferential Accumulation of Small $(<300 \mathrm{Mm})$ Microplastics in the Sediments of a Coastal Plain River Network in Eastern China. Water Res. 2018, 144, 393-401. https://doi.org/10.1016/j.watres.2018.07.050.

(39) Khordagui, H. K.; Abu- Hilal, A. H. Industrial Plastic on the Southern Beaches of the Arabian Gulf and the Western Beaches of the Gulf of Oman. Environ. Pollut. 1994, 84 (3), 325-327. https://doi.org/10.1016/0269-7491(94)90143-0.

(40) Besseling, E.; Redondo-Hasselerharm, P.; Foekema, E. M.; Koelmans, A. A. Quantifying Ecological Risks of Aquatic Micro- and Nanoplastic. Crit. Rev. 
Environ. Sci. Technol. 2019, 49 (1), 32-80.

https://doi.org/10.1080/10643389.2018.1531688.

(41) Claessens, M.; Meester, S. De; Landuyt, L. Van; Clerck, K. De; Janssen, C. R. Occurrence and Distribution of Microplastics in Marine Sediments along the Belgian Coast. Mar. Pollut. Bull. 2011, 62 (10), 2199-2204. https://doi.org/10.1016/j.marpolbul.2011.06.030.

(42) Ter Halle, A.; Jeanneau, L.; Martignac, M.; Jardé, E.; Pedrono, B.; Brach, L.; Gigault, J. Nanoplastic in the North Atlantic Subtropical Gyre. Environ. Sci. Technol. 2017, 51 (23), 13689-13697. https://doi.org/10.1021/acs.est.7b03667.

(43) Scherer, C.; Weber, A.; Lambert, S.; Wagner, M. Interactions of Microplastics with Freshwater Biota. In Handbook of Environmental Chemistry; Springer, Cham, 2018; Vol. 58, pp 153-180. https://doi.org/10.1007/978-3-319-61615-5_8.

(44) Kooi, M.; Van Nes, E. H.; Scheffer, M.; Koelmans, A. A. Ups and Downs in the Ocean: Effects of Biofouling on Vertical Transport of Microplastics. Environ. Sci. Technol. 2017, 51 (14), 7963-7971. https://doi.org/10.1021/acs.est.6b04702.

(45) O’Connor, J. D.; Mahon, A. M.; Ramsperger, A. F. R. M.; Trotter, B.; RedondoHasselerharm, P. E.; Koelmans, A. A.; Lally, H. T.; Murphy, S. Microplastics in Freshwater Biota: A Critical Review of Isolation, Characterization, and Assessment Methods. Glob. Challenges 2020, 4 (6), 1800118. https://doi.org/10.1002/gch2.201800118.

(46) Scherer, C.; Brennholt, N.; Reifferscheid, G.; Wagner, M. Feeding Type and Development Drive the Ingestion of Microplastics by Freshwater Invertebrates. Sci. Rep. 2017, 7, 17006. https://doi.org/10.1038/s41598-017-17191-7.

Zhang, C.; Chen, X.; Wang, J.; Tan, L. Toxic Effects of Microplastic on Marine Microalgae Skeletonema Costatum: Interactions between Microplastic and Algae. Environ. Pollut. 2017, 220, 1282-1288. https://doi.org/10.1016/j.envpol.2016.11.005.

(48) Long, M.; Paul-Pont, I.; Hégaret, H.; Moriceau, B.; Lambert, C.; Huvet, A.; Soudant, P. Interactions between Polystyrene Microplastics and Marine Phytoplankton Lead to Species-Specific Hetero-Aggregation. Environ. Pollut. 2017, 228, 454-463. https://doi.org/10.1016/J.ENVPOL.2017.05.047.

(49) Seoane, M.; González-Fernández, C.; Soudant, P.; Huvet, A.; Esperanza, M.; Cid, Á.; Paul-Pont, I. Polystyrene Microbeads Modulate the Energy Metabolism of the Marine Diatom Chaetoceros Neogracile. Environ. Pollut. 2019, 251, 363371. https://doi.org/10.1016/j.envpol.2019.04.142.

(50) Mateos-Cárdenas, A.; Scott, D. T.; Seitmaganbetova, G.; van Pelt, F. N. A. M.; O'Halloran, J.; Jansen, M. A. K. Polyethylene Microplastics Adhere to Lemna Minor (L.), yet Have No Effects on Plant Growth or Feeding by Gammarus Duebeni (Lillj.). Sci. Total Environ. 2019, 689, 413-421. https://doi.org/10.1016/j.scitotenv.2019.06.359.

(51) Dovidat, L. C.; Brinkmann, B. W.; Vijver, M. G.; Bosker, T. Plastic Particles Adsorb to the Roots of Freshwater Vascular Plant Spirodela Polyrhiza but Do Not Impair Growth. Limnol. Oceanogr. Lett. 2020, 5 (1), 37-45. https://doi.org/10.1002/lol2.10118.

(52) Murphy, F.; Quinn, B. The Effects of Microplastic on Freshwater Hydra Attenuata Feeding, Morphology \&amp; Reproduction. Environ. Pollut. 2018, 
234, 487-494. https://doi.org/10.1016/J.ENVPOL.2017.11.029.

(53) Cole, M.; Lindeque, P.; Fileman, E.; Halsband, C.; Goodhead, R.; Moger, J.; Galloway, T. S. Microplastic Ingestion by Zooplankton. Environ. Sci. Technol. 2013, 47 (12), 6646-6655. https://doi.org/10.1021/es400663f.

(54) Nelms, S. E.; Barnett, J.; Brownlow, A.; Davison, N. J.; Deaville, R.; Galloway, T. S.; Lindeque, P. K.; Santillo, D.; Godley, B. J. Microplastics in Marine Mammals Stranded around the British Coast: Ubiquitous but Transitory? Sci. Rep. 2019, 9, 1075. https://doi.org/10.1038/s41598-018-37428-3.

(55) Bravo Rebolledo, E. L.; Van Franeker, J. A.; Jansen, O. E.; Brasseur, S. M. J. M. Plastic Ingestion by Harbour Seals (Phoca Vitulina) in The Netherlands. Mar. Pollut. Bull. 2013, 67, 200-202. https://doi.org/10.1016/j.marpolbul.2012.11.035.

(56) Hu, L.; Chernick, M.; Hinton, D. E.; Shi, H. Microplastics in Small Waterbodies and Tadpoles from Yangtze River Delta, China. Environ. Sci. Technol. 2018, 52 (15), 8885-8893. https://doi.org/10.1021/acs.est.8b02279.

(57) Windsor, F. M.; Tilley, R. M.; Tyler, C. R.; Ormerod, S. J. Microplastic Ingestion by Riverine Macroinvertebrates. Sci. Total Environ. 2018, 646, 68-74. https://doi.org/10.1016/j.scitotenv.2018.07.271.

(58) McNeish, R. E.; Kim, L. H.; Barrett, H. A.; Mason, S. A.; Kelly, J. J.; Hoellein, T. J. Microplastic in Riverine Fish Is Connected to Species Traits. Sci. Rep. 2018, 8, 11639. https://doi.org/10.1038/s41598-018-29980-9.

(59) Duncan, E. M.; Broderick, A. C.; Fuller, W. J.; Galloway, T. S.; Godfrey, M. H.; Hamann, M.; Limpus, C. J.; Lindeque, P. K.; Mayes, A. G.; Omeyer, L. C. M.; et al. Microplastic Ingestion Ubiquitous in Marine Turtles. Glob. Chang. Biol. 2019, 25 (2), 744-752. https://doi.org/10.1111/gcb.14519.

(60) Kühn, S.; Bravo Rebolledo, E. L.; Van Franeker, J. A. Deleterious Effects of Litter on Marine Life. In Marine Anthropogenic Litter; Bergmann, M., Gutow, L., Klages, M., Eds.; Springer International Publishing, 2015; pp 75-116.

(61) Jovanović, B. Ingestion of Microplastics by Fish and Its Potential Consequences from a Physical Perspective. Integr. Environ. Assess. Manag. 2017, 13 (3), 510515. https://doi.org/10.1002/ieam.1913.

(62) Mattsson, K.; Johnson, E. V.; Malmendal, A.; Linse, S.; Hansson, L. A.; Cedervall, T. Brain Damage and Behavioural Disorders in Fish Induced by Plastic Nanoparticles Delivered through the Food Chain. Sci. Rep. 2017, 7, 11452. https://doi.org/10.1038/s41598-017-10813-0.

(63) Cedervall, T.; Hansson, L.-A. A.; Lard, M.; Frohm, B.; Linse, S. Food Chain Transport of Nanoparticles Affects Behaviour and Fat Metabolism in Fish. PLoS One 2012, 7 (2), 1-6. https://doi.org/10.1371/journal.pone.0032254.

(64) Botterell, Z. L. R.; Beaumont, N.; Dorrington, T.; Steinke, M.; Thompson, R. C.; Lindeque, P. K. Bioavailability and Effects of Microplastics on Marine Zooplankton: A Review. Environ. Pollut. 2019, 245, 98-110. https://doi.org/10.1016/J.ENVPOL.2018.10.065.

(65) Ribeiro, F.; O’Brien, J. W.; Galloway, T.; Thomas, K. V. Accumulation and Fate of Nano- and Micro-Plastics and Associated Contaminants in Organisms. TrAC Trends Anal. Chem. 2019, 111, 139-147.

https://doi.org/10.1016/j.trac.2018.12.010. 
(66) Booth, A. M.; Hansen, B. H.; Frenzel, M.; Johnsen, H.; Altin, D. Uptake and Toxicity of Methylmethacrylate-Based Nanoplastic Particles in Aquatic Organisms. Environ. Toxicol. Chem. 2016, 35 (7), 1641-1649. https://doi.org/10.1002/etc.3076.

(67) Rist, S.; Baun, A.; Hartmann, N. B. Ingestion of Micro- and Nanoplastics in Daphnia Magna - Quantification of Body Burdens and Assessment of Feeding Rates and Reproduction. Environ. Pollut. 2017, 228, 398-407. https://doi.org/10.1016/j.envpol.2017.05.048.

(68) Rosenkranz, P.; Chaudhry, Q.; Stone, V.; Fernandes, T. F. A Comparison of Nanoparticle and Fine Particle Uptake by Daphnia Magna. Environ. Toxicol. Chem. 2009, 28 (10), 2142-2149. https://doi.org/10.1897/08-559.1.

(69) Ribeiro, F.; Garcia, A. R.; Pereira, B. P.; Fonseca, M.; Mestre, N. C.; Fonseca, T. G.; Ilharco, L. M.; Bebianno, M. J. Microplastics Effects in Scrobicularia Plana. Mar. Pollut. Bull. 2017, 122, 379-391. https://doi.org/10.1016/j.marpolbul.2017.06.078.

(70) Lu, Y.; Zhang, Y.; Deng, Y.; Jiang, W.; Zhao, Y.; Geng, J.; Ding, L.; Ren, H. Uptake and Accumulation of Polystyrene Microplastics in Zebrafish (Danio Rerio) and Toxic Effects in Liver. Environ. Sci. Technol. 2016, 50 (7), 4054 4060. https://doi.org/10.1021/acs.est.6b00183.

(71) Jin, Y.; Lu, L.; Tu, W.; Luo, T.; Fu, Z. Impacts of Polystyrene Microplastic on the Gut Barrier, Microbiota and Metabolism of Mice. Sci. Total Environ. 2019, 649, 308-317. https://doi.org/10.1016/j.scitotenv.2018.08.353.

(72) Jin, Y.; Xia, J.; Pan, Z.; Yang, J.; Wang, W.; Fu, Z. Polystyrene Microplastics Induce Microbiota Dysbiosis and Inflammation in the Gut of Adult Zebrafish. Environ. Pollut. 2018, 235, 322-329. https://doi.org/10.1016/j.envpol.2017.12.088.

(73) Jeong, C. B.; Won, E. J.; Kang, H. M.; Lee, M. C.; Hwang, D. S.; Hwang, U. K.; Zhou, B.; Souissi, S.; Lee, S. J.; Lee, J. S. Microplastic Size-Dependent Toxicity, Oxidative Stress Induction, and p-JNK and p-P38 Activation in the Monogonont Rotifer (Brachionus Koreanus). Environ. Sci. Technol. 2016, 50 (16), 8849-8857. https://doi.org/10.1021/acs.est.6b01441.

(74) Espinosa, C.; Cuesta, A.; Esteban, M. Á. Effects of Dietary Polyvinylchloride Microparticles on General Health, Immune Status and Expression of Several Genes Related to Stress in Gilthead Seabream (Sparus Aurata L.). Fish Shellfish Immunol. 2017, 68, 251-259. https://doi.org/10.1016/j.fsi.2017.07.006.

(75) Von Moos, N.; Burkhardt-Holm, P.; Köhler, A. Uptake and Effects of Microplastics on Cells and Tissue of the Blue Mussel Mytilus Edulis L. after an Experimental Exposure. Environ. Sci. Technol. 2012, 46 (20), 11327-11335. https://doi.org/10.1021/es302332w.

(76) Green, D. S.; Boots, B.; Sigwart, J.; Jiang, S.; Rocha, C. Effects of Conventional and Biodegradable Microplastics on a Marine Ecosystem Engineer (Arenicola Marina) and Sediment Nutrient Cycling. Environ. Pollut. 2016, 208, 426-434. https://doi.org/10.1016/j.envpol.2015.10.010.

(77) Watts, A. J. R.; Urbina, M. A.; Goodhead, R.; Moger, J.; Lewis, C.; Galloway, T. S. Effect of Microplastic on the Gills of the Shore Crab Carcinus Maenas.

Environ. Sci. Technol. 2016, 50 (10), 5364-5369.

https://doi.org/10.1021/acs.est.6b01187. 
(78) Gambardella, C.; Morgana, S.; Ferrando, S.; Bramini, M.; Piazza, V.; Costa, E.; Garaventa, F.; Faimali, M. Effects of Polystyrene Microbeads in Marine Planktonic Crustaceans. Ecotoxicol. Environ. Saf. 2017, 145, 250-257. https://doi.org/10.1016/j.ecoenv.2017.07.036.

(79) Rehse, S.; Kloas, W.; Zarfl, C. Short-Term Exposure with High Concentrations of Pristine Microplastic Particles Leads to Immobilisation of Daphnia Magna. Chemosphere 2016, 153, 91-99. https://doi.org/10.1016/j.chemosphere.2016.02.133.

(80) Ziajahromi, S.; Kumar, A.; Neale, P. A.; Leusch, F. D. L. Environmentally Relevant Concentrations of Polyethylene Microplastics Negatively Impact the Survival, Growth and Emergence of Sediment-Dwelling Invertebrates. Environ. Pollut. 2018, 236, 425-431. https://doi.org/10.1016/j.envpol.2018.01.094.

(81) Kaposi, K. L.; Mos, B.; Kelaher, B. P.; Dworjanyn, S. A. Ingestion of Microplastic Has Limited Impact on a Marine Larva. Environ. Sci. Technol. 2014, 48 (3), 1638-1645. https://doi.org/10.1021/es404295e.

(82) Bergami, E.; Pugnalini, S.; Vannuccini, M. L.; Manfra, L.; Faleri, C.; Savorelli, F.; Dawson, K. A.; Corsi, I. Long-Term Toxicity of Surface-Charged Polystyrene Nanoplastics to Marine Planktonic Species Dunaliella Tertiolecta and Artemia Franciscana. Aquat. Toxicol. 2017, 189, 159-169. https://doi.org/10.1016/j.aquatox.2017.06.008.

(83) Au, S. Y.; Bruce, T. F.; Bridges, W. C.; Klaine, S. J. Responses of Hyalella Azteca to Acute and Chronic Microplastic Exposures. Environ. Toxicol. Chem. 2015, 34 (11), 2564-2572. https://doi.org/10.1002/etc.3093.

(84) Besseling, E.; Wang, B.; Lürling, M.; Koelmans, A. A. Nanoplastic Affects Growth of S. Obliquus and Reproduction of D. Magna. Environ. Sci. Technol. 2014, 48 (20), 12336-12343. https://doi.org/10.1021/es503001d.

(85) Wegner, A.; Besseling, E.; Foekema, E. M.; Kamermans, P.; Koelmans, A. A. Effects of Nanopolystyrene on the Feeding Behavior of the Blue Mussel (Mytilus Edulis L.). Environ. Toxicol. Chem. 2012, 31 (11), 2490-2497. https://doi.org/10.1002/etc.1984.

(86) Mazurais, D.; Ernande, B.; Quazuguel, P.; Severe, A.; Huelvan, C.; Madec, L.; Mouchel, O.; Soudant, P.; Robbens, J.; Huvet, A.; et al. Evaluation of the Impact of Polyethylene Microbeads Ingestion in European Sea Bass (Dicentrarchus Labrax) Larvae. Mar. Environ. Res. 2015, 112, 78-85. https://doi.org/10.1016/j.marenvres.2015.09.009.

(87) Bosker, T.; Olthof, G.; Vijver, M. G.; Baas, J.; Barmentlo, S. H. Significant Decline of Daphnia Magna Population Biomass Due to Microplastic Exposure. Environ. Pollut. 2019, 250, 669-675. https://doi.org/10.1016/j.envpol.2019.04.067.

(88) Green, D. S.; Boots, B.; O'Connor, N. E.; Thompson, R. Microplastics Affect the Ecological Functioning of an Important Biogenic Habitat. Environ. Sci. Technol. 2017, 51 (1), 68-77. https://doi.org/10.1021/acs.est.6b04496.

(89) Green, D. S. Effects of Microplastics on European Flat Oysters, Ostrea Edulis and Their Associated Benthic Communities. Environ. Pollut. 2016, 216, 95-103. https://doi.org/10.1016/J.ENVPOL.2016.05.043.

(90) Sussarellu, R.; Suquet, M.; Thomas, Y.; Lambert, C.; Fabioux, C.; Pernet, M. E. J.; Le Goïc, N.; Quillien, V.; Mingant, C.; Epelboin, Y.; et al. Oyster 
Reproduction Is Affected by Exposure to Polystyrene Microplastics. Proc. Natl. Acad. Sci. 2016, 113 (9), 2430-2435. https://doi.org/10.1073/pnas.1519019113.

(91) Nobre, C. R.; Santana, M. F. M.; Maluf, A.; Cortez, F. S.; Cesar, A.; Pereira, C. D. S.; Turra, A. Assessment of Microplastic Toxicity to Embryonic Development of the Sea Urchin Lytechinus Variegatus (Echinodermata: Echinoidea). Mar. Pollut. Bull. 2015, 92, 99-104. https://doi.org/10.1016/j.marpolbul.2014.12.050.

(92) Wright, S. L.; Rowe, D.; Thompson, R. C.; Galloway, T. S. Microplastic Ingestion Decreases Energy Reserves in Marine Worms. Curr. Biol. 2013, 23 (23), R1031-R1033. https://doi.org/10.1016/j.cub.2013.10.068.

(93) Diepens, N. J.; Koelmans, A. A.; Baveco, H.; van den Brink, P. J.; van den Heuvel-Greve, M. J.; Brock, T. C. M. Prospective Environmental Risk Assessment for Sediment-Bound Organic Chemicals: A Proposal for Tiered Effect Assessment. In Reviews of Environmental Contamination and Toxicology; de Voogt, P., Ed.; Springer, Cham 239, 2016; p 77.

https://doi.org/10.1007/398_2015_5004.

(94) Karman, C. C. The Role of Time in Environmental Risk Assessment. Spill Sci. Technol. Bull. 2000, 6, 159-164. https://doi.org/10.1016/s1353-2561(00)00071-2.

(95) Adam, V.; Yang, T.; Nowack, B. Toward an Ecotoxicological Risk Assessment of Microplastics: Comparison of Available Hazard and Exposure Data in Freshwaters. Environ. Toxicol. Chem. 2019, 38 (2), 436-447. https://doi.org/10.1002/etc.4323.

(96) Burns, E. E.; Boxall, A. B. A. Microplastics in the Aquatic Environment: Evidence for or against Adverse Impacts and Major Knowledge Gaps. Environ. Toxicol. Chem. 2018, 37 (11), 2776-2796. https://doi.org/10.1002/etc.4268.

(97) Everaert, G.; Van Cauwenberghe, L.; De Rijcke, M.; Koelmans, A. A.; Mees, J.; Vandegehuchte, M.; Janssen, C. R. Risk Assessment of Microplastics in the Ocean: Modelling Approach and First Conclusions. Environ. Pollut. 2018, 242, 1930-1938. https://doi.org/10.1016/J.ENVPOL.2018.07.069.

(98) Garner, K. L.; Suh, S.; Lenihan, H. S.; Keller, A. A. Species Sensitivity Distributions for Engineered Nanomaterials. Environ. Sci. Technol. 2015, 49 (9), 5753-5759. https://doi.org/10.1021/acs.est.5b00081.

(99) Wheeler, J. R.; Grist, E. P. M.; Leung, K. M. Y.; Morritt, D.; Crane, M. Species Sensitivity Distributions: Data and Model Choice. Mar. Pollut. Bull. 2002, 45 (112), 192-202. https://doi.org/10.1016/S0025-326X(01)00327-7.

(100) Revel, M.; Châtel, A.; Mouneyrac, C. Micro(Nano)Plastics: A Threat to Human Health? Curr. Opin. Environ. Sci. Heal. 2018, 1, 17-23.

https://doi.org/10.1016/j.coesh.2017.10.003.

(101) Karami, A.; Golieskardi, A.; Keong Choo, C.; Larat, V.; Galloway, T. S.; Salamatinia, B. The Presence of Microplastics in Commercial Salts from Different Countries. Sci. Rep. 2017, 7, 46173. https://doi.org/10.1038/srep46173.

(102) Liebezeit, G.; Liebezeit, E. Synthetic Particles as Contaminants in German Beers. Food Addit. Contam. - Part A Chem. Anal. Control. Expo. Risk Assess. 2014, 31 (9), 1574-1578. https://doi.org/10.1080/19440049.2014.945099.

(103) Liebezeit, G.; Liebezeit, E. Non-Pollen Particulates in Honey and Sugar. Food Addit. Contam. - Part A Chem. Anal. Control. Expo. Risk Assess. 2013, 30 (12), 2136-2140. https://doi.org/10.1080/19440049.2013.843025. 
(104) Huerta Lwanga, E.; Mendoza Vega, J.; Ku Quej, V.; Chi, J. de los A.; Sanchez del Cid, L.; Chi, C.; Escalona Segura, G.; Gertsen, H.; Salánki, T.; van der Ploeg, M.; et al. Field Evidence for Transfer of Plastic Debris along a Terrestrial Food Chain. Sci. Rep. 2017, 7, 14071. https://doi.org/10.1038/s41598-017-14588-2.

(105) Santillo, D.; Miller, K.; Johnston, P. Microplastics as Contaminants in Commercially Important Seafood Species. Integr. Environ. Assess. Manag. 2017, 13 (3), 516-521. https://doi.org/10.1002/ieam.1909.

(106) Ustabasi, G. S.; Baysal, A. Occurrence and Risk Assessment of Microplastics from Various Toothpastes. Env. Monit Assess 2019, 191, 438. https://doi.org/https://doi.org/10.1007/s10661-019-7574-1.

(107) Szentkuti, L. Light Microscopical Observations on Luminally Administered Dyes, Dextrans, Nanospheres and Microspheres in the Pre-Epithelial Mucus Gel Layer of the Rat Distal Colon. J. Control. Release 1997, 46 (3), 233-242. https://doi.org/10.1016/S0168-3659(96)01600-8.

(108) Jani, P.; Halbert, G. W.; Langridge, J.; Florence, A. T. Nanoparticle Uptake by the Rat Gastrointestinal Mucosa: Quantitation and Particle Size Dependency. $J$. Pharm. Pharmacol. 1990, 42, 821-826. https://doi.org/doi.org/10.1111/j.20427158.1990.tb07033.x.

(109) Eyles, J.; Alpar, O.; Field, W.; Lewis, D.; Keswick, M. The Transfer of Polystyrene Microspheres from the Gastrointestinal Tract to the Circulation after Oral Administration in the Rat. J. Pharm. Pharmacol. 1995, 47, 561-565.

(110) Sykes, E. A.; Dai, Q.; Tsoi, K. M.; Hwang, D. M.; Chan, W. C. W. Nanoparticle Exposure in Animals Can Be Visualized in the Skin and Analysed via Skin Biopsy. Nat. Commun. 2014, 5, 3796. https://doi.org/10.1038/ncomms4796

(111) Wagner, S.; Hüffer, T.; Klöckner, P.; Wehrhahn, M.; Hofmann, T.; Reemtsma, T. Tire Wear Particles in the Aquatic Environment - a Review on Generation, Analysis, Occurrence, Fate and Effects. Water Res. 2018, 139, 83-100. https://doi.org/10.1016/j.watres.2018.03.051.

(112) Dris, R.; Gasperi, J.; Saad, M.; Mirande, C.; Tassin, B. Synthetic Fibers in Atmospheric Fallout: A Source of Microplastics in the Environment? Mar. Pollut. Bull. 2016, 104, 290-293. https://doi.org/10.1016/j.marpolbul.2016.01.006.

(113) Catarino, A. I.; Macchia, V.; Sanderson, W. G.; Thompson, R. C.; Henry, T. B. Low Levels of Microplastics (MP) in Wild Mussels Indicate That MP Ingestion by Humans Is Minimal Compared to Exposure via Household Fibres Fallout during a Meal. Environ. Pollut. 2018, 237, 675-684. https://doi.org/10.1016/J.ENVPOL.2018.02.069.

(114) Vianello, A.; Jensen, R. L.; Liu, L.; Vollertsen, J. Simulating Human Exposure to Indoor Airborne Microplastics Using a Breathing Thermal Manikin. Sci. Rep. 2019, 9, 8670. https://doi.org/10.1038/s41598-019-45054-w.

(115) Wright, S. L.; Kelly, F. J. Plastic and Human Health: A Micro Issue? Environ. Sci. Technol. 2017, 51 (12), 6634-6647. https://doi.org/10.1021/acs.est.7b00423.

(116) Pauly, J. L.; Stegmeier, S. J.; Allaart, H. A.; Cheney, R. T.; Zhang, P. J.; Mayer, A. G.; Streck, R. J. Inhaled Cellulosic and Plastic Fibers Found in Human Lung Tissue. Cancer Epidemiol. Biomarkers Prev. 1998, 7 (5), 419-428.

(117) Mantecca, P.; Sancini, G.; Moschini, E.; Farina, F.; Gualtieri, M.; Rohr, A.; Miserocchi, G.; Palestini, P.; Camatini, M. Lung Toxicity Induced by 
Intratracheal Instillation of Size-Fractionated Tire Particles. Toxicol. Lett. 2009, 189 (3), 206-214. https://doi.org/10.1016/j.toxlet.2009.05.023.

(118) Geiser, M.; Rothen-Rutishauser, B.; Kapp, N.; Schürch, S.; Kreyling, W.; Schulz, H.; Semmler, M.; Im Hof, V.; Heyder, J.; Gehr, P. Ultrafine Particles Cross Cellular Membranes by Nonphagocytic Mechanisms in Lungs and in Cultured Cells. Environ. Health Perspect. 2005, 113 (11), 1555-1560. https://doi.org/10.1289/ehp.8006.

(119) Forte, M.; Iachetta, G.; Tussellino, M.; Carotenuto, R.; Prisco, M.; De Falco, M.; Laforgia, V.; Valiante, S. Polystyrene Nanoparticles Internalization in Human Gastric Adenocarcinoma Cells. Toxicol. Vitr. 2016, 31, 126-136. https://doi.org/10.1016/j.tiv.2015.11.006.

(120) Schirinzi, G. F.; Pérez-Pomeda, I.; Sanchís, J.; Rossini, C.; Farré, M.; Barceló, D. Cytotoxic Effects of Commonly Used Nanomaterials and Microplastics on Cerebral and Epithelial Human Cells. Environ. Res. 2017, 159, 579-587. https://doi.org/10.1016/j.envres.2017.08.043.

(121) Xu, H.; Hoet, P. H. M.; Nemery, B. In Vitro Toxicity Assessment of Polyvinyl Chloride Particles and Comparison of Six Cellular Systems. J. Toxicol. Environ. Heal. Part A. 2002, 65, 1141-1159. https://doi.org/10.1080/152873902760125372.

(122) Prata, J. C.; da Costa, J. P.; Lopes, I.; Duarte, A. C.; Rocha-Santos, T. Environmental Exposure to Microplastics: An Overview on Possible Human Health Effects. Sci. Total Environ. 2020, 702, 134455. https://doi.org/10.1016/j.scitotenv.2019.134455.

(123) Rist, S.; Carney Almroth, B.; Hartmann, N. B.; Karlsson, T. M. A Critical Perspective on Early Communications Concerning Human Health Aspects of Microplastics. Sci. Total Environ. 2018, 626, 720-726. https://doi.org/10.1016/j.scitotenv.2018.01.092.

(124) Cox, K. D.; Covernton, G. A.; Davies, H. L.; Dower, J. F.; Juanes, F.; Dudas, S. E. Human Consumption of Microplastics. Environ. Sci. Technol. 2019, 53 (12), 7068-7074. https://doi.org/10.1021/acs.est.9b01517.

(125) de Sá, L. C.; Oliveira, M.; Ribeiro, F.; Lopes Rocha, T.; Futter, M. N.; Barcelo, D.; de Sá, L. C.; Oliveira, M.; Ribeiro, F.; Rocha, T. L.; et al. Studies of the Effects of Microplastics on Aquatic Organisms: What Do We Know and Where Should We Focus Our Efforts in the Future? Sci. Total Environ. 2018, 645, 1029-1039. https://doi.org/10.1016/j.scitotenv.2018.07.207.

(126) Kögel, T.; Bjorøy, Ø.; Toto, B.; Bienfait, A. M.; Sanden, M. Micro- and Nanoplastic Toxicity on Aquatic Life: Determining Factors. Sci. Total Environ. 2020, 709 (5817), 136050. https://doi.org/10.1016/j.scitotenv.2019.136050.

(127) Catarino, A. I.; Frutos, A.; Henry, T. B. Use of Fluorescent-Labelled Nanoplastics (NPs) to Demonstrate NP Absorption Is Inconclusive without Adequate Controls. Sci. Total Environ. 2019, 670, 915-920. https://doi.org/10.1016/J.SCITOTENV.2019.03.194.

(128) Liu, L.; Fokkink, R.; Koelmans, A. A. Sorption of Polycyclic Aromatic Hydrocarbons to Polystyrene Nanoplastic. Environ. Toxicol. Chem. 2016, 35 (7), 1650-1655. https://doi.org/10.1002/etc.3311.

(129) Wagner, M.; Scherer, C.; Alvarez-Muñoz, D.; Brennholt, N.; Bourrain, X.; Buchinger, S.; Fries, E.; Grosbois, C.; Klasmeier, J.; Marti, T.; et al. 
Microplastics in Freshwater Ecosystems: What We Know and What We Need to Know. Environ. Sci. Eur. 2014, 26, 12. https://doi.org/10.1186/s12302-014-0012-7.

(130) Klein, S.; Worch, E.; Knepper, T. P. Occurrence and Spatial Distribution of Microplastics in River Shore Sediments of the Rhine-Main Area in Germany. Environ. Sci. Technol. 2015, 49 (10), 6070-6076.

https://doi.org/10.1021/acs.est.5b00492.

(131) Leslie, H. A.; Brandsma, S. H.; Van Velzen, M. J. M. M.; Vethaak, A. D. Microplastics En Route: Field Measurements in the Dutch River Delta and Amsterdam Canals, Wastewater Treatment Plants, North Sea Sediments and Biota. Environ. Int. 2017, 101, 133-142.

https://doi.org/10.1016/j.envint.2017.01.018.

(132) Imhof, H. K.; Ivleva, N. P.; Schmid, J.; Niessner, R.; Laforsch, C. Contamination of Beach Sediments of a Subalpine Lake with Microplastic Particles. Curr. Biol. 2013, 23 (19), R867-R868. https://doi.org/10.1016/j.cub.2013.09.001.

(133) Straub, S.; Hirsch, P. E.; Burkhardt-Holm, P. Biodegradable and PetroleumBased Microplastics Do Not Differ in Their Ingestion and Excretion but in Their Biological Effects in a Freshwater Invertebrate Gammarus Fossarum. Int. J. Environ. Res. Public Health 2017, 14 (7), 774. https://doi.org/10.3390/ijerph14070774.

(134) Hurley, R. R.; Woodward, J. C.; Rothwell, J. J. Ingestion of Microplastics by Freshwater Tubifex Worms. Environ. Sci. Technol. 2017, 51 (21), 12844-12851. https://doi.org/10.1021/acs.est.7b03567.

(135) Weber, A.; Scherer, C.; Brennholt, N.; Reifferscheid, G.; Wagner, M. PET Microplastics Do Not Negatively Affect the Survival, Development, Metabolism and Feeding Activity of the Freshwater Invertebrate Gammarus Pulex. Environ. Pollut. 2018, 234, 181-189. https://doi.org/10.1016/j.envpol.2017.11.014.

(136) Hämer, J.; Gutow, L.; Köhler, A.; Saborowski, R. Fate of Microplastics in the Marine Isopod Idotea Emarginata. Environ. Sci. Technol. 2014, 48 (22), 1345113458. https://doi.org/10.1021/es501385y.

(137) Besseling, E.; Wegner, A.; Foekema, E. M.; Van Den Heuvel-Greve, M. J.; Koelmans, A. A. Effects of Microplastic on Fitness and PCB Bioaccumulation by the Lugworm Arenicola Marina (L.). Environ. Sci. Technol. 2013, 47 (1), 593600. https://doi.org/10.1021/es302763x.

(138) Besseling, E.; Foekema, E. M. E. M.; Van den Heuvel-Greve, M. J. M. J.; Koelmans, A. A. A. A. The Effect of Microplastic on the Uptake of Chemicals by the Lugworm Arenicola Marina (L.) under Environmentally Relevant Exposure Conditions. Environ. Sci. Technol. 2017, 51 (15), 8795-8804. https://doi.org/10.1021/acs.est.7b02286.

(139) Lo, H. K. A.; Chan, K. Y. K. Negative Effects of Microplastic Exposure on Growth and Development of Crepidula Onyx. Environ. Pollut. 2018, 233, 588595. https://doi.org/10.1016/J.ENVPOL.2017.10.095.

(140) Connors, K. A.; Dyer, S. D.; Belanger, S. E. Advancing the Quality of Environmental Microplastic Research. Environ. Toxicol. Chem. 2017, 36 (7), 1697-1703. https://doi.org/10.1002/etc.3829.

(141) Karami, A. Gaps in Aquatic Toxicological Studies of Microplastics. Chemosphere 2017, 184, 841-848. https://doi.org/10.1016/j.chemosphere.2017.06.048. 
(142) Browne, M. A.; Niven, S. J.; Galloway, T. S.; Rowland, S. J.; Thompson, R. C. Microplastic Moves Pollutants and Additives to Worms, Reducing Functions Linked to Health and Biodiversity. Curr. Biol. 2013, 23 (23), 2388-2392. https://doi.org/10.1016/j.cub.2013.10.012.

(143) Maltby, L. Sensitivity of the Crustaceans Gammarus Pulex (L.) and Asellus Aquaticus (L.) to Short-Term Exposure to Hypoxia and Unionized Ammonia: Observations and Possible Mechanisms. Wat. Res 1995, 29, 781-787.

(144) Kupryianchyk, D.; Reichman, E. P.; Rakowska, M. I.; Peeters, E. T. H. M.; Grotenhuis, J. T. C.; Koelmans, A. A. Ecotoxicological Effects of Activated Carbon Amendments on Macroinvertebrates in Nonpolluted and Polluted Sediments. Environ. Sci. Technol. 2011, 45 (19), 8567-8574. https://doi.org/10.1021/es2014538.

(145) Rodriguez, P.; Martinez-Madrid, M.; Arrate, J. A.; Navarro, E. Selective Feeding by the Aquatic Oligochaete Tubifex Tubifex (Tubificidae, Clitellata). Hydrobiologia 2001, 463, 133-140.

(146) Sidney, L. A.; Diepens, N. J.; Guo, X.; Koelmans, A. A. Trait-Based Modelling of Bioaccumulation by Freshwater Benthic Invertebrates. Aquat. Toxicol. 2016, 176, 88-96. https://doi.org/10.1016/j.aquatox.2016.04.017.

(147) OECD. OECD Guidelines for the Testing of Chemicals. Test No. 225: SedimentWater Lumbriculus Toxicity Test Using Spiked Sediment. OECD Guidel. 2007.

(148) Ankley, G. T.; Schubauer-Berigan, M. K.; Monson, P. D. Influence of PH and Hardness on Toxicity of Ammonia to the Amphipod Hyalella Azteca. Can. J. Fish. Aquat. Sci. 1995, 52 (10), 2078-2083. https://doi.org/10.1139/f95-801.

(149) Schubaur-Berigan, M. K.; Monson, P. D.; West, C. W.; Ankley, G. T. Influence of PH on the Toxicity of Ammonia to Chironomus Tentans and Lumbriculus Variegatus. Environ. Toxicol. Chem. 1995, 14, 713-717. https://doi.org/10.1002/etc.5620140419.

(150) Arrate, J. Á.; Rodrigez, P.; Martinez-Madrid, M. Tubifex Tubifex Chronic Toxicity Test Using Artificial Sediment: Methodological Issues. Limnetica 2014, $23,25-36$.

(151) Wilhelm, F. M.; Lasenby, D. C. Seasonal Trends in the Head Capsule Length and Body Length/Weight Relationships of Two Amphipod Species. Crustaceana 1998, 71, 399-410.

(152) Maltby, L.; Clayton, S. A.; Wood, R. M.; McLoughlin, N. Evaluation of the Gammarus Pulex in Situ Feeding Assay as a Biomonitor of Water Quality: Robustness, Responsiveness, and Relevance. Environ. Toxicol. Chem. 2002, 21 (2), 361-368. https://doi.org/10.1002/etc.5620210219.

(153) Leppänen, M. T.; Kukkonen, J. V. K. Factors Affecting Feeding Rate, Reproduction and Growth of an Oligochaete Lumbriculus Variegatus (Müller). Hydrobiologia 1998, 377, 183-194.

(154) Rasmussen, J. B. Comparison of Gut Contents and Assimilation Efficiency of Fourth Instar Larvae of Two Coexisting Chironomids, Chironomus Riparius Meigen and Glyptotendipes Paripes (Edwards). Can. J. Zool. 1984, 62 (6), 10221026. https://doi.org/10.1139/z84-145.

(155) Claessens, M.; Van Cauwenberghe, L.; Vandegehuchte, M. B.; Janssen, C. R. New Techniques for the Detection of Microplastics in Sediments and Field 
Collected Organisms. Mar. Pollut. Bull. 2013, 70, 227-233.

https://doi.org/10.1016/j.marpolbul.2013.03.009.

(156) Polymer Database http://polymerdatabase.com/polymers/polystyrene.html.

(157) Franken, R. J. M.; Waluto, B.; Peeters, E. T. H. M.; Gardeniers, J. J. P.; Beijer, J. A. J.; Scheffer, M. Growth of Shredders on Leaf Litter Biofilms: The Effect of Light Intensity. Freshw. Biol. 2005, 50 (3), 459-466.

https://doi.org/10.1111/j.1365-2427.2005.01333.x.

(158) Kerr, D. R.; Meador, J. P. Modeling Dose Response Using Generalized Linear Models. Environ. Toxicol. Chem. 1996, 15 (3), 395-401.

https://doi.org/10.1002/etc.5620150325.

(159) Ritz, C. Toward a Unified Approach to Dose-Response Modeling in Ecotoxicology. Environ. Toxicol. Chem. 2010, 29 (1), 220-229. https://doi.org/10.1002/etc.7.

(160) Draper, N. R.; Smith, H. Applied Regression Analysis; Wiley, 1998.

(161) Vadher, A. N.; Stubbington, R.; Wood, P. J. Fine Sediment Reduces Vertical Migrations of Gammarus Pulex (Crustacea: Amphipoda) in Response to Surface Water Loss. Hydrobiologia 2015, 753 (1), 61-71. https://doi.org/10.1007/s10750-015-2193-5.

(162) Rochman, C. M.; Browne, M. A.; Halpern, B. S.; Hentschel, B. T.; Hoh, E.; Karapanagioti, H. K.; Rios-Mendoza, L. M.; Takada, H.; Teh, S.; Thompson, R. C. Policy: Classify Plastic Waste as Hazardous. Nature 2013, 494 (7436), 169171. https://doi.org/10.1038/494169a.

(163) Kelly, D. W.; Dick, J. T. A.; Montgomery, W. I. The Functional Role of Gammarus (Crustacea, Amphipoda): Shredders, Predators, or Both? Hydrobiologia 2002, 485, 199-203.

(164) Macneil, C.; A Dick, J. T.; Elwood, R. W. The Thropic Ecology of Freshwater Gammarus Spp. (Crustacea: Amphipoda): Problems and Perspectives Concerning the Functional Feeding Group Concept. Biol. Rev 1997, 72, 349-364.

(165) Kole, P. J.; Löhr, A. J.; Van Belleghem, F.; Ragas, A. Wear and Tear of Tyres: A Stealthy Source of Microplastics in the Environment. Int. J. Environ. Res. Public Health 2017, 14, 1265. https://doi.org/10.3390/ijerph14101265.

(166) Boucher, J.; Friot, D. Primary Microplastics in the Oceans: A Global Evaluation of Sources; IUCN: Gland, Switzerland, 2017.

https://doi.org/10.2305/iucn.ch.2017.01.en.

(167) Oomen, A.; de Groot, G. Evaluation of Health Risks of Playing Sports on Synthetic Turf Pitches with Rubber Granulate; Bilthoven, 2017.

(168) Cheng, H.; Hu, Y.; Reinhard, M. Environmental and Health Impacts of Artificial Turf: A Review. Environ. Sci. Technol. 2014, 48, 2114-2129. https://doi.org/10.1021/es4044193.

(169) Kreider, M. L.; Panko, J. M.; McAtee, B. L.; Sweet, L. I.; Finley, B. L. Physical and Chemical Characterization of Tire-Related Particles: Comparison of Particles Generated Using Different Methodologies. Sci. Total Environ. 2010, 408 (3), 652-659. https://doi.org/10.1016/j.scitotenv.2009.10.016.

(170) Rogge, W. F.; Hildemann, L. M.; Mazurek, M. A.; Cass, G. R.; Simoneit, B. R. T. Sources of Fine Organic Aerosol. 3. Road Dust, Tire Debris, and 
Organometallic Brake Lining Dust: Roads as Sources and Sinks. Environ. Sci. Technol. 1993, 27, 1892-1904. https://doi.org/10.1021/es00046a019.

(171) Wik, A.; Dave, G. Occurrence and Effects of Tire Wear Particles in the Environment - A Critical Review and an Initial Risk Assessment. Environ. Pollut. 2009, 157, 1-11. https://doi.org/10.1016/J.ENVPOL.2008.09.028.

(172) Gualtieri, M.; Andrioletti, M.; Vismara, C.; Milani, M.; Camatini, M. Toxicity of Tire Debris Leachates. 2005, 31, 723-730. https://doi.org/10.1016/j.envint.2005.02.001.

(173) Kumata, H.; Sanada; Takada, H.; Takashi. Historical Trends of N-Cyclohexyl-2Benzothiazolamine, 2-(4-Morpholinyl)Benzothiazole, and Other Anthropogenic Contaminants in the Urban Reservoir Sediment Core. Environ 2000, 34, 246253. https://doi.org/10.1021/ES990738K.

(174) Spies, R. B.; Andresen, B. D.; Rice Jr, D. W. Benzthiazoles in Estuarine Sediments as Indicators of Street Runoff. Nature 1987, 327, 697-699. https://doi.org/10.1038/327697a0.

(175) Reddy, C. M.; Quinn, J. G. Environmental Chemistry of Benzothiazoles Derived from Rubber. Env. Sci Technol 1997, 31, 2847-2853. https://doi.org/10.1021/ES9700780.

(176) Wik, A.; Lycken, J.; Dave, G. Sediment Quality Assessment of Road Runoff Detention Systems in Sweden and the Potential Contribution of Tire Wear. Water. Air. Soil Pollut. 2008, 194, 301-314. https://doi.org/10.1007/s11270-0089718-8.

(177) Ni, H.-G.; Lu, F.-H.; Luo, X.-L.; Tian, H.-Y.; Zeng, E. Y. Occurrence, Phase Distribution, and Mass Loadings of Benzothiazoles in Riverine Runoff of the Pearl River Delta, China. Environ. Sci. Technol. 2008, 42, 1892-1897. https://doi.org/10.1021/es071871c.

(178) Schuchardt, B.; Beilfuß, S.; Reincke, T.; Hofmann, O.; Ziebarth, N.; Liebezeit, G.; Dubaish, F. Müll in Der Nordsee - Pilotprojekt Zur Relevanz Des Eintragspfades Ästuar Am Beispiel Der Unterweser Studie Vor Dem Hintergrund Der Meeresstrategie-Rahmenrichtlinie (MSRL); Bremen, Germany, 2013.

(179) Day, K. E.; Holtze, K. E.; Metcalfe-Smith, J. L.; Bishop, C. T.; Dutka, B. J. Toxicity of Leachate from Automobile Tires to Aquatic Biota. Chemosphere 1993, 27, 665-675. https://doi.org/10.1016/0045-6535(93)90100-J.

(180) Stephensen, E.; Adolfsson-Erici, M.; Celander, M.; Hulander, M.; Parkkonen, J.; Hegelund, T.; Sturve, J.; Hasselberg, L.; Bengtsson, M.; Förlin, L. Biomarker Responses and Chemical Analyses in Fish Indicate Leakage of Polycyclic Aromatic Hydrocarbons and Other Compounds from Car Tire Rubber. Environ. Toxicol. Chem. 2003, 22, 2926. https://doi.org/10.1897/02-444.

(181) Marwood, C.; McAtee, B.; Kreider, M.; Ogle, R. S.; Finley, B.; Sweet, L.; Panko, J. Acute Aquatic Toxicity of Tire and Road Wear Particles to Alga, Daphnid, and Fish. Ecotoxicology 2011, 20, 2079-2089. https://doi.org/10.1007/s10646-011-0750-X.

(182) Villena, O. C.; Terry, I.; Iwata, K.; Landa, E. R.; LaDeau, S. L.; Leisnham, P. T. Effects of Tire Leachate on the Invasive Mosquito Aedes Albopictus and the Native Congener Aedes Triseriatus. PeerJ 2017, 5, e3756. https://doi.org/10.7717/peerj.3756. 
(183) Turner, A.; Rice, L. Toxicity of Tire Wear Particle Leachate to the Marine Macroalga, Ulva Lactuca. Environ. Pollut. 2010, 158 (12), 3650-3654. https://doi.org/10.1016/j.envpol.2010.08.001.

(184) Panko, J. M.; Kreider, M. L.; Mcatee, B. L.; Marwood, C. Chronic Toxicity of Tire and Road Wear Particles to Water-and Sediment-Dwelling Organisms. Ecotoxicology 2013, 22, 13-21. https://doi.org/10.1007/s10646-012-0998-9.

(185) Camponelli, K. M.; Casey, R. E.; Snodgrass, J. W.; Lev, S. M.; Landa, E. R. Impacts of Weathered Tire Debris on the Development of Rana Sylvatica Larvae. Chemosphere 2009, 74, 717-722. https://doi.org/10.1016/j.chemosphere.2008.09.056.

(186) Redondo-Hasselerharm, P. E.; Falahudin, D.; Peeters, E. T. H. M.; Koelmans, A. A. Microplastic Effect Thresholds for Freshwater Benthic Macroinvertebrates. Environ. Sci. Technol. 2018, 52 (4), 2278-2286. https://doi.org/10.1021/acs.est.7b05367.

(187) Januszewicz, K.; Klein, M.; Klugmann-Radziemska, E.; Kardas, D. Physicochemical Problems of Mineral Processing Thermogravimetric Analysis/Pyrolysis of Used Tyres and Waste Rubber. Physicochem. Probl. Miner. Process 2017, 53, 802-811. https://doi.org/10.5277/ppmp170211.

(188) Wagner, M. Thermal Analysis in Practice. Fundamental Aspects.; Hanser: München, 2009.

(189) Gueissaz, L.; Massonnet, G. Study on the Discrimination of Tires Using Chemical Profiles Obtained by Py-GC/MS. J. Anal. Appl. Pyrolysis 2017, 124, 704-718. https://doi.org/10.1016/j.jaap.2016.11.024.

(190) Tsuge, S.; Ohtani, H.; Watanabe, C. Pyrolysis - GC/MS Data Book of Synthetic Polymers: Pyrograms, Thermograms and MS of Pyrolyzates; Elsevier, 2011.

(191) Velzeboer, I.; Kupryianchyk, D.; Peeters, E. T. H. M.; Koelmans, A. A. Community Effects of Carbon Nanotubes in Aquatic Sediments. Environ. Int. 2011, 37 (6), 1126-1130. https://doi.org/10.1016/j.envint.2011.01.016.

(192) Kupryianchyk, D.; Peeters, E. T. H. M.; Rakowska, M. I.; Reichman, E. P.; Grotenhuis, J. T. C.; Koelmans, A. A. Long-Term Recovery of Benthic Communities in Sediments Amended with Activated Carbon. Environ. Sci. Technol. 2012, 46 (19), 10735-10742. https://doi.org/10.1021/es302285h.

(193) Huang, X.; Liang, P.; Qian, Y. Excess Sludge Reduction Induced by Tubifex Tubifex in a Recycled Sludge Reactor. J. Biotechnol. 2007, 127 (3), 443-451. https://doi.org/10.1016/J.JBIOTEC.2006.07.035.

(194) Sadiktsis, I.; Bergvall, C.; Johansson, C.; Westerholm, R. Automobile Tires-A Potential Source of Highly Carcinogenic Dibenzopyrenes to the Environment. Environ. Sci. Technol. 2012, 46, 3326-3334. https://doi.org/10.1021/es204257d.

(195) Horner, J. M. Environmental Health Implications of Heavy Metal Pollution from Car Tires. Rev. Environ. Health 1996, 11, 175-178. https://doi.org/10.1515/reveh.1996.11.4.175.

(196) Van Griethuysen, C.; Van Baren, J.; Peeters, E. T. H. M.; Koelmans, A. A. Trace Metal Availability and Effects on Benthic Community Structure in Floodplain Lakes. Environ. Toxicol. Chem. 2004, 23, 668. https://doi.org/10.1897/02-583.

(197) Katayama, A.; Bhula, R.; Burns, G. R.; Carazo, E.; Felsot, A.; Hamilton, D.; 
Harris, C.; Kim, Y.-H.; Kleter, G.; Koedel, W.; et al. Bioavailability of Xenobiotics in the Soil Environment. In Reviews of environmental contamination and toxicology; 2010; Vol. 203, pp 1-86. https://doi.org/10.1007/978-1-44191352-4_1.

(198) Organization for Economic Co-operation and Development. OECD 225 Guidelines for the Testing of Chemicals. Sediment-Water Lumbriculus Toxicity Test Using Spiked Sediment. Test 225 2007, No. October, 1-31.

(199) Löder, M. G. J.; Imhof, H. K.; Ladehoff, M.; Lö, L. A.; Lorenz, C.; Mintenig, S.; Piehl, S.; Primpke, S.; Schrank, I.; Laforsch, C.; et al. Enzymatic Purification of Microplastics in Environmental Samples. Env. Sci Technol 2017, 51, 14283-14292. https://doi.org/10.1021/acs.est.7b03055.

(200) Käppler, A.; Fischer, D.; Oberbeckmann, S.; Schernewski, G.; Labrenz, M.; Eichhorn, K.-J.; Voit, B. Analysis of Environmental Microplastics by Vibrational Microspectroscopy: FTIR, Raman or Both? Anal. Bioanal. Chem. 2016, 408 (29), 8377-8391. https://doi.org/10.1007/s00216-016-9956-3.

(201) Löder, M. G. J.; Gerdts, G. Methodology Used for the Detection and Identification of Microplastics - a Critical Appraisal. In Marine anthropogenic litter; Bergmann, M., Gutow, L., Klages, M., Eds.; Springer International Publishing, 2015; pp 201-227.

(202) Persson, B. N. J. Theory of Powdery Rubber Wear. J. Phys. Condens. Matter 2009, 21, 8. https://doi.org/10.1088/0953-8984/21/48/485001.

(203) Milani, M.; Pucillo, F. P.; Ballerini, M.; Camatini, M.; Gualtieri, M.; Martino, S. First Evidence of Tyre Debris Characterization at the Nanoscale by Focused Ion Beam. Mater. Charact. 2004, 52, 283-288. https://doi.org/10.1016/J.MATCHAR.2004.06.001.

(204) Back, H. Epidermal Uptake of $\mathrm{Pb}, \mathrm{Cd}$, and $\mathrm{Zn}$ in Tubificid Worms. Oecologia 1990, 85, 226-232. https://doi.org/10.1007/BF00319405.

(205) Phipps, G. L.; Mattson, V. R.; Ankley, G. T. Relative Sensitivity of Three Freshwater Benthic Macroinvertebrates to Ten Contaminants. Arch. Environ. Contam. Toxicol. 1995, 28, 281-286. https://doi.org/10.1007/BF00213103.

(206) Zakaria, M. P. 200. Distribution of Polycyclic Aromatic Hydrocarbons (PAHs) in Rivers and Estuaries in Malaysia. A widespread input petrogenic PAHs 2002, 36, 1907-1918. https://doi.org/10.1021/es011278.

(207) MacDonald, D. D.; Ingersoll, C. G.; Berger, T. A. Development and Evaluation of Consensus-Based Sediment Quality Guidelines for Freshwater Ecosystems. Arch. Environ. Contam. Toxicol. 2000, 39, 20-31. https://doi.org/10.1007/s002440010075.

(208) Verschoor, A.; de Poorter, L.; Dröge, R.; Kuenen, J.; de Valk, E. Emission of Microplastics and Potential Mitigation Measures; Bilthoven, 2016.

(209) DeForest, D. K.; Brix, K. V.; Adams, W. J. Assessing Metal Bioaccumulation in Aquatic Environments: The Inverse Relationship between Bioaccumulation Factors, Trophic Transfer Factors and Exposure Concentration. Aquat. Toxicol. 2007, 84, 236-246. https://doi.org/10.1016/J.AQUATOX.2007.02.022.

(210) Mattsson, K.; Hansson, L. A.; Cedervall, T. Nano-Plastics in the Aquatic Environment. Environ. Sci. Process. Impacts 2015, 17 (10), 1712-1721. https://doi.org/10.1039/c5em00227c. 
(211) Koelmans, A. A. Proxies for Nanoplastic. Nat. Nanotechnol. 2019, 14 (4), $307-$ 308. https://doi.org/10.1038/s41565-019-0416-z.

(212) Lambert, S.; Wagner, M. Formation of Microscopic Particles during the Degradation of Different Polymers. Chemosphere 2016, 161, 510-517. https://doi.org/10.1016/j.chemosphere.2016.07.042.

(213) Cole, M.; Galloway, T. S. Ingestion of Nanoplastics and Microplastics by Pacific Oyster Larvae. Environ. Sci. Technol. 2015, 49 (24), 14625-14632. https://doi.org/10.1021/acs.est.5b04099.

(214) Cui, R.; Kim, S. W.; An, Y. J. Polystyrene Nanoplastics Inhibit Reproduction and Induce Abnormal Embryonic Development in the Freshwater Crustacean Daphnia Galeata. Sci. Rep. 2017, 7, 12095. https://doi.org/10.1038/s41598-01712299-2.

(215) Schür, C.; Rist, S.; Baun, A.; Mayer, P.; Hartmann, N. B.; Wagner, M. When Fluorescence Is Not a Particle: The Tissue Translocation of Microplastics in Daphnia Magna Seems an Artifact. Environ. Toxicol. Chem. 2019, 38 (7), 14951503. https://doi.org/10.1002/etc.4436.

(216) Vicentini, D. S.; Nogueira, D. J.; Melegari, S. P.; Arl, M.; Köerich, J. S.; Cruz, L.; Justino, N. M.; Oscar, B. V.; Puerari, R. C.; da Silva, M. L. N.; et al. Toxicological Evaluation and Quantification of Ingested Metal-Core Nanoplastic by Daphnia Magna Through Fluorescence and Inductively Coupled Plasma-Mass Spectrometric Methods. Environ. Toxicol. Chem. 2019, 38 (10), 2101-2110. https://doi.org/10.1002/etc.4528.

(217) Frehland, S.; Kaegi, R.; Hufenus, R.; Mitrano, D. M. Long-Term Assessment of Nanoplastic Particle and Microplastic Fiber Flux through a Pilot Wastewater Treatment Plant Using Metal-Doped Plastics. Water Res. 2020, 182, 115860. https://doi.org/10.1016/j.watres.2020.115860.

(218) Keller, A. S.; Jimenez-Martínez, J.; Mitrano, D. M. Transport of Nano- and Microplastic through Unsaturated Porous Media from Sewage Sludge Application. Environ. Sci. Technol. 2020, 54, 911-920.

(219) González-Pleiter, M.; Tamayo-Belda, M.; Pulido-Reyes, G.; Amariei, G.; Leganés, F.; Rosal, R.; Fernández-Piñas, F. Secondary Nanoplastics Released from a Biodegradable Microplastic Severely Impact Freshwater Environments. Environ. Sci. Nano 2019, 6 (5), 1382-1392. https://doi.org/10.1039/c8en01427b.

(220) van Weert, S.; Redondo-Hasselerharm, P. E.; Diepens, N. J.; Koelmans, A. A. Effects of Nanoplastics and Microplastics on the Growth of Sediment-Rooted Macrophytes. Sci. Total Environ. 2019, 654, 1040-1047. https://doi.org/10.1016/j.scitotenv.2018.11.183.

(221) Greven, A.-C.; Merk, T.; Karagöz, F.; Mohr, K.; Klapper, M.; Jovanović, B.; Palić, D. Polycarbonate and Polystyrene Nanoplastic Particles Act as Stressors to the Innate Immune System of Fathead Minnow ( Pimephales Promelas ). Environ. Toxicol. Chem. 2016, 35 (12), 3093-3100. https://doi.org/10.1002/etc.3501.

(222) Liu, Z.; Cai, M.; Yu, P.; Chen, M.; Wu, D.; Zhang, M.; Zhao, Y. Age-Dependent Survival, Stress Defense, and AMPK in Daphnia Pulex after Short-Term Exposure to a Polystyrene Nanoplastic. Aquat. Toxicol. 2018, 204 (August), 1-8. https://doi.org/10.1016/j.aquatox.2018.08.017. 
(223) de Ruijter, V. N. V. N.; Redondo-Hasselerharm, P. E. P. E.; Gouin, T.; Koelmans, A. A. A. A. Quality Criteria for Microplastic Effect Studies in the Context of Risk Assessment: A Critical Review. Environ. Sci. Technol. 2020, 54 (19), 11692-11705. https://doi.org/10.1021/acs.est.0c03057.

(224) Revel, M.; Yakovenko, N.; Caley, T.; Guillet, C.; Châtel, A.; Mouneyrac, C. Accumulation and Immunotoxicity of Microplastics in the Estuarine Worm Hediste Diversicolor in Environmentally Relevant Conditions of Exposure. Environ. Sci. Pollut. Res. 2018, 27, 3574-3583. https://doi.org/10.1007/s11356018-3497-6.

(225) Heinlaan, M.; Kasemets, K.; Aruoja, V.; Blinova, I.; Bondarenko, O.; Lukjanova, A.; Khosrovyan, A.; Kurvet, I.; Pullerits, M.; Sihtmäe, M.; et al. Hazard Evaluation of Polystyrene Nanoplastic with Nine Bioassays Did Not Show Particle-Specific Acute Toxicity. Sci. Total Environ. 2020, 707, 136073. https://doi.org/10.1016/j.scitotenv.2019.136073.

(226) Redondo-Hasselerharm, P. E.; Gort, G.; Peeters, E. T. H. M.; Koelmans, A. A. Nano- And Microplastics Affect the Composition of Freshwater Benthic Communities in the Long Term. Sci. Adv. 2020, 6 (5). https://doi.org/10.1126/sciadv.aay4054.

(227) Mitrano, D. M.; Beltzung, A.; Frehland, S.; Schmiedgruber, M.; Cingolani, A.; Schmidt, F. Synthesis of Metal-Doped Nanoplastics and Their Utility to Investigate Fate and Behaviour in Complex Environmental Systems. Nat. Nanotechnol. 2019, 1. https://doi.org/10.1038/s41565-018-0360-3.

(228) ECHA. Styrene - Registration Dossier - ECHA https://echa.europa.eu/registration-dossier/-/registered-dossier/15565/6/2/5.

(229) ECHA. Acrylonitrile - Registration dossier - ECHA https://echa.europa.eu/es/registration-dossier/-/registered-dossier/15561.

(230) Redondo-Hasselerharm, P. E.; de Ruijter, V. N.; Mintenig, S. M.; Verschoor, A.; Koelmans, A. A. Ingestion and Chronic Effects of Car Tire Tread Particles on Freshwater Benthic Macroinvertebrates. Environ. Sci. Technol. 2018, 52, 13986-13994. https://doi.org/10.1021/acs.est.8b05035.

(231) Van Den Brink, N. W.; Jemec Kokalj, A.; Silva, P. V.; Lahive, E.; Norrfors, K.; Baccaro, M.; Khodaparast, Z.; Loureiro, S.; Drobne, D.; Cornelis, G.; et al. Tools and Rules for Modelling Uptake and Bioaccumulation of Nanomaterials in Invertebrate Organisms. Environ. Sci. Nano 2019, 6 (7), 1985-2001. https://doi.org/10.1039/c8en01122b.

(232) Ribeiro, F.; Van Gestel, C. A. M.; Pavlaki, M. D.; Azevedo, S.; Soares, A. M. V. M.; Loureiro, S. Bioaccumulation of Silver in Daphnia Magna: Waterborne and Dietary Exposure to Nanoparticles and Dissolved Silver. Sci. Total Environ. 2017, 574, 1633-1639. https://doi.org/10.1016/j.scitotenv.2016.08.204.

(233) Koelmans, A. A.; Redondo-Hasselerharm, P. E.; Mohamed Nor, N. H.; Kooi, M. Solving the Non-Alignment of Methods and Approaches Used in Microplastic Research in Order to Consistently Characterize Risk. Environ. Sci. Technol. 2020, 54 (19). https://doi.org/10.1021/acs.est.0c02982.

(234) Mattsson, K.; Jocic, S.; Doverbratt, I.; Hansson, L.A. Nanoplastics in the Aquatic Environment. In Microplastic Contamination in Aquatic Environments; Elsevier, 2018; pp 379-399. https://doi.org/10.1016/b978-0-12-813747-5.00013-8. 
(235) Rochman, C. M.; Browne, M. A.; Underwood, A. J.; Van Franeker, J. A.; Thompson, R. C.; Amaral-Zettler, L. A. The Ecological Impacts of Marine Debris: Unraveling the Demonstrated Evidence from What Is Perceived; 2016; Vol. 97. https://doi.org/10.1890/14-2070.1.

(236) Silva, C. J. M.; Silva, A. L. P.; Gravato, C.; Pestana, J. L. T. Ingestion of SmallSized and Irregularly Shaped Polyethylene Microplastics Affect Chironomus Riparius Life-History Traits. Sci. Total Environ. 2019, 672, 862-868. https://doi.org/10.1016/J.SCITOTENV.2019.04.017.

(237) Frost, T. M.; Carpenter, S. R.; Ives, A. R.; Kratz, T. K. Species Compensation and Complementarity in Ecosystem Function. In Linking Species \& Ecosystems; Springer US: Boston, MA, 1995; pp 224-239. https://doi.org/10.1007/978-14615-1773-3_22.

(238) Wallace, J. B.; Webster, J. R. The Role of Macroinvertebrates in Stream Ecosystem Function. Annual Review of Entomology. Anna Rev. Emomd 1996, 41 (131), 115-139. https://doi.org/10.1146/annurev.ento.41.1.115.

(239) Bellingeri, A.; Bergami, E.; Grassi, G.; Faleri, C.; Redondo-Hasselerharm, P.; Koelmans, A. A.; Corsi, I. Combined Effects of Nanoplastics and Copper on the Freshwater Alga Raphidocelis Subcapitata. Aquat. Toxicol. 2019, 210, 179-187. https://doi.org/10.1016/j.aquatox.2019.02.022.

(240) ECHA. Sodium dodecyl sulphate - Registration Dossier - ECHA https://echa.europa.eu/registration-dossier/-/registered-dossier/2126/6/2/4.

(241) Sighicelli, M.; Pietrelli, L.; Lecce, F.; Iannilli, V.; Falconieri, M.; Coscia, L.; Di Vito, S.; Nuglio, S.; Zampetti, G. Microplastic Pollution in the Surface Waters of Italian Subalpine Lakes. Environ. Pollut. 2018, 236, 645-651. https://doi.org/10.1016/J.ENVPOL.2018.02.008.

(242) Quik, J. T. K.; van De Meent, D.; Koelmans, A. A. Simplifying Modeling of Nanoparticle Aggregation-Sedimentation Behavior in Environmental Systems: A Theoretical Analysis. Water Res. 2014, 62, 193-201. https://doi.org/10.1016/J.WATRES.2014.05.048.

(243) Faravelli, T.; Pinciroli, M.; Pisano, F.; Bozzano, G.; Dente, M.; Ranzi, E. Thermal Degradation of Polystyrene. J. Anal. Appl. Pyrolysis 2001, 60 (1), 103121. https://doi.org/10.1016/S0165-2370(00)00159-5.

(244) Velzeboer, I.; Peeters, E. T. H. M.; Koelmans, A. A. Multiwalled Carbon Nanotubes at Environmentally Relevant Concentrations Affect the Composition of Benthic Communities. Environ. Sci. Technol. 2013, 47 (13), 7475-7482. https://doi.org/10.1021/es400777j.

(245) Timm, T.; Martin, P. J. Clitellata: Oligochaeta. Thorp Covich's Freshw. Invertebr. 2015, 529-549. https://doi.org/10.1016/B978-0-12-385026-3.00021-8.

(246) van Griethuysen, C.; de Lange, H. J.; van den Heuij, M.; de Bies, S. C.; Gillissen, F.; Koelmans, A. A. Temporal Dynamics of AVS and SEM in Sediment of Shallow Freshwater Floodplain Lakes. Appl. Geochemistry 2006, 21 (4), 632642. https://doi.org/10.1016/J.APGEOCHEM.2005.12.010.

(247) Koelmans, A. A.; Besseling, E.; Shim, W. J. Nanoplastics in the Aquatic Environment. Critical Review. In Marine Anthropogenic Litter; Bergmann, M., Gutow, L., Klages, M., Eds.; Springer International Publishing, 2015; pp 325340. https://doi.org/10.1007/978-3-319-16510-3_12. 
(248) Oriekhova, O.; Stoll, S. Heteroaggregation of Nanoplastic Particles in the Presence of Inorganic Colloids and Natural Organic Matter. Environ. Sci. Nano 2018. https://doi.org/10.1039/C7EN01119A.

(249) Velzeboer, I.; Quik, J. T. K.; van de Meent, D.; Koelmans, A. A. Rapid Settling of Nanoparticles Due to Heteroaggregation with Suspended Sediment. Environ. Toxicol. Chem. 2014, 33 (8), 1766-1773. https://doi.org/10.1002/etc.2611.

(250) Ogonowski, M.; Schür, C.; Jarsén, Å.; Gorokhova, E. The Effects of Natural and Anthropogenic Microparticles on Individual Fitness in Daphnia Magna. PLoS One 2016, 11 (5), e0155063. https://doi.org/10.1371/journal.pone.0155063.

(251) Ward, J. E.; Kach, D. J. Marine Aggregates Facilitate Ingestion of Nanoparticles by Suspension-Feeding Bivalves. Mar. Environ. Res. 2009, 68 (3), 137-142. https://doi.org/10.1016/j.marenvres.2009.05.002.

(252) Alimi, O. S.; Farner Budarz, J.; Hernandez, L. M.; Tufenkji, N. Microplastics and Nanoplastics in Aquatic Environments: Aggregation, Deposition, and Enhanced Contaminant Transport. Environ. Sci. Technol. 2018, 52 (4), 1704-1724. https://doi.org/10.1021/acs.est.7b05559.

(253) Strungaru, S.-A.; Jijie, R.; Nicoara, M.; Plavan, G.; Faggio, C. Micro- (Nano) Plastics in Freshwater Ecosystems: Abundance, Toxicological Impact and Quantification Methodology. Trends Anal. Chem. 2019, 110, 116-128. https://doi.org/10.1016/j.trac.2018.10.025.

(254) Gigault, J.; Halle, A. ter; Baudrimont, M.; Pascal, P. Y.; Gauffre, F.; Phi, T. L.; El Hadri, H.; Grassl, B.; Reynaud, S. Current Opinion: What Is a Nanoplastic? Environ. Pollut. 2018, 235, 1030-1034. https://doi.org/10.1016/j.envpol.2018.01.024.

(255) Wang, F.; Wong, C. S.; Chen, D.; Lu, X.; Wang, F.; Zeng, E. Y. Interaction of Toxic Chemicals with Microplastics: A Critical Review. Water Res. 2018, 139, 208-219. https://doi.org/10.1016/j.watres.2018.04.003.

(256) Raju, S.; Carbery, M.; Kuttykattil, A.; Senathirajah, K.; Subashchandrabose, S. R.; Evans, G.; Thavamani, P. Transport and Fate of Microplastics in Wastewater Treatment Plants: Implications to Environmental Health. Rev. Environ. Sci. Biotechnol. 2018, 17 (4), 637-653. https://doi.org/10.1007/s11157-018-9480-3.

(257) Koelmans, A. A.; Besseling, E.; Foekema, E. M. Leaching of Plastic Additives to Marine Organisms. Environ. Pollut. 2014, 187, 49-54. https://doi.org/10.1016/j.envpol.2013.12.013.

(258) Gouin, T.; Roche, N.; Lohmann, R.; Hodges, G. A Thermodynamic Approach for Assessing the Environmental Exposure of Chemicals Absorbed to Microplastic. Environ. Sci. Technol. 2011, 45, 1466-1472. https://doi.org/10.1021/es1032025.

(259) Velzeboer, I.; Kwadijk, C. J. A. F.; Koelmans, A. A. Strong Sorption of PCBs to Nanoplastics, Microplastics, Carbon Nanotubes, and Fullerenes. Environ. Sci. Technol. 2014, 48 (9), 4869-4876. https://doi.org/10.1021/es405721v.

(260) Lemoine, C. M. R.; Kelleher, B. M.; El Lagarde, R. €; Northam, C.; Elebute, O. O.; Cassone, B. J. Transcriptional Effects of Polyethylene Microplastics Ingestion in Developing Zebrafish (Danio Rerio). Environ. Pollut. 2018, 243, 591-600. https://doi.org/10.1016/j.envpol.2018.08.084.

(261) Yu, P.; Liu, Z.; Wu, D.; Chen, M.; Lv, W.; Zhao, Y. Accumulation of Polystyrene Microplastics in Juvenile Eriocheir Sinensis and Oxidative Stress 
Effects in the Liver. Aquat. Toxicol. 2018, 200, 28-36.

https://doi.org/10.1016/j.aquatox.2018.04.015.

(262) Rejman, J.; Oberle, V.; Zuhorn, I. S.; Hoekstra, D. Size-Dependent

Internalization of Particles via the Pathways of Clathrin- and Caveolae-Mediated Endocytosis. Biochem. J. 2004, 377, 159-169.

https://doi.org/10.1042/BJ20031253.

(263) Browne, M. A.; Dissanayake, A.; Galloway, T. S.; Lowe, D. M.; Thompson, R. C. Ingested Microscopic Plastic Translocates to the Circulatory System of the Mussel, Mytilus Edulis (L.). Environ. Sci. Technol. 2008, 42 (13), 5026-5031. https://doi.org/10.1021/es800249a.

(264) Chae, Y.; An, Y. J. Effects of Micro- and Nanoplastics on Aquatic Ecosystems: Current Research Trends and Perspectives. Mar. Pollut. Bull. 2017, 124 (2), 624632. https://doi.org/10.1016/j.marpolbul.2017.01.070.

(265) World Health Organization. Microplastics in Drinking-Water; Geneva, 2019.

(266) Ghallab, A. In Vitro Test Systems and Their Limitations. EXCLI J. 2013, 12, 1024-1026. https://doi.org/10.17877/DE290R-7558.

(267) Judson, R. S.; Houck, K. A.; Kavlock, R. J.; Knudsen, T. B.; Martin, M. T.; Mortensen, H. M.; Reif, D. M.; Rotroff, D. M.; Shah, I.; Richard, A. M.; et al. In Vitro Screening of Environmental Chemicals for Targeted Testing Prioritization: The Toxcast Project. Environ. Health Perspect. 2010, 118 (4), 485-492. https://doi.org/10.1289/ehp.0901392.

(268) Thubagere, A.; Reinhard, B. M. Nanoparticle-Induced Apoptosis Ropagates through Hydrogen-Peroxide-Mediated Bystander Killing: Insights from a Human Intestinal Epithelium in Vitro Model. ACS Nano 2010, 4 (7), 3611-3622. https://doi.org/10.1021/nn100389a.

(269) Hesler, M.; Aengenheister, L.; Ellinger, B.; Drexel, R.; Straskraba, S.; Jost, C.; Wagner, S.; Meier, F.; von Briesen, H.; Büchel, C.; et al. Multi-Endpoint Toxicological Assessment of Polystyrene Nano- and Microparticles in Different Biological Models in Vitro. Toxicol. Vitr. 2019, 61, 104610. https://doi.org/10.1016/j.tiv.2019.104610.

(270) Jiang, X.; Chen, H.; Liao, Y.; Ye, Z.; Li, M.; Klobučar, G. Ecotoxicity and Genotoxicity of Polystyrene Microplastics on Higher Plant Vicia Faba. Environ. Pollut. 2019, 831-838. https://doi.org/10.1016/j.envpol.2019.04.055.

(271) Paget, V.; Dekali, S.; Kortulewski, T.; Grall, R.; Gamez, C.; Blazy, K.; AguerreChariol, O.; Chevillard, S.; Braun, A.; Rat, P.; et al. Specific Uptake and Genotoxicity Induced by Polystyrene Nanobeads with Distinct Surface Chemistry on Human Lung Epithelial Cells and Macrophages. PLoS One 2015, 10 (4), e0123297. https://doi.org/10.1371/journal.pone.0123297.

(272) Poma, A.; Vecchiotti, G.; Colafarina, S.; Zarivi, O.; Aloisi, M.; Arrizza, L.; Chichiriccò, G.; Di Carlo, P. In Vitro Genotoxicity of Polystyrene Nanoparticles on the Human Fibroblast Hs27 Cell Line. Nanomaterials 2019, 9 (9), 1299. https://doi.org/10.3390/nano9091299.

(273) Mishra, P.; Vinayagam, S.; Duraisamy, K.; Patil, S. R.; Godbole, J.; Mohan, A.; Mukherjee, A.; Chandrasekaran, N. Distinctive Impact of Polystyrene NanoSpherules as an Emergent Pollutant toward the Environment. Environ. Sci. Pollut. Res. 2019, 26, 1537-1547. https://doi.org/10.1007/s11356-018-3698-z. 
(274) Tallec, K.; Huvet, A.; Di Poi, C.; González-Fernández, C.; Lambert, C.; Petton, B.; Le Goïc, N.; Berchel, M.; Soudant, P.; Paul-Pont, I. Nanoplastics Impaired Oyster Free Living Stages, Gametes and Embryos. Environ. Pollut. 2018, 242, 1226-1235. https://doi.org/10.1016/j.envpol.2018.08.020.

(275) Reifferscheid, G.; Maes, H. M.; Allner, B.; Badurova, J.; Belkin, S.; Bluhm, K.; Brauer, F.; Bressling, J.; Domeneghetti, S.; Elad, T.; et al. International RoundRobin Study on the Ames Fluctuation Test. Environ. Mol. Mutagen. 2012, 53 (3), 185-197. https://doi.org/10.1002/em.

(276) Park, C. G.; Cho, H. K.; Shin, H. J.; Park, K. H.; Lim, H. Bin. Comparison of Mutagenic Activities of Various Ultra-Fine Particles. Toxicol. Res. 2018, 34 (2), 163-172. https://doi.org/10.5487/TR.2018.34.2.163.

(277) Zhao, H. W.; Barger, M. W.; Ma, J. K. H.; Castranova, V.; Ma, J. Y. C. Effects of Exposure to Diesel Exhaust Particles (DEP) on Pulmonary Metabolic Activation of Mutagenic Agents. Mutat. Res. - Genet. Toxicol. Environ. Mutagen. 2004, 564, 103-113. https://doi.org/10.1016/j.mrgentox.2004.07.014.

(278) Kumar, A.; Pandey, A. K.; Singh, S. S.; Shanker, R.; Dhawan, A. Cellular Uptake and Mutagenic Potential of Metal Oxide Nanoparticles in Bacterial Cells. Chemosphere 2011, 83 (8), 1124-1132. https://doi.org/10.1016/j.chemosphere.2011.01.025.

(279) Buchner, E. M.; Happel, O.; Schmidt, C. K.; Scheurer, M.; Schmutz, B.; Kramer, M.; Knauer, M.; Gartiser, S.; Hollert, H. Approach for Analytical Characterization and Toxicological Assessment of Ozonation Products in Drinking Water on the Example of Acesulfame. Water Res. 2019, 153, 357-368. https://doi.org/10.1016/j.watres.2019.01.018.

(280) Albergamo, V.; Escher, B. I.; Schymanski, E. L.; Helmus, R.; Dingemans, M. M. L.; Cornelissen, E. R.; Kraak, M. H. S.; Hollender, J.; De Voogt, P. Evaluation of Reverse Osmosis Drinking Water Treatment of Riverbank Filtrate Using Bioanalytical Tools and Non-Target Screening. Environ. Sci. Water Res. Technol. 2020, 6 (1), 103-116. https://doi.org/10.1039/c9ew00741e.

(281) Heringa, M. B.; Harmsen, D. J. H.; Beerendonk, E. F.; Reus, A. A.; Krul, C. A. M.; Metz, D. H.; Ijpelaar, G. F. Formation and Removal of Genotoxic Activity during UV/H2O2-GAC Treatment of Drinking Water. Water Res. 2011, 45 (1), 366-374. https://doi.org/10.1016/j.watres.2010.08.008.

(282) Kolkman, A.; Schriks, M.; Brand, W.; Bäuerlein, P. S.; van der Kooi, M. M. E.; van Doorn, R. H.; Emke, E.; Reus, A. A.; van der Linden, S. C.; de Voogt, P.; et al. Sample Preparation for Combined Chemical Analysis and in Vitro Bioassay Application in Water Quality Assessment. Environ. Toxicol. Pharmacol. 2013, 36, 1291-1303. https://doi.org/10.1016/j.etap.2013.10.009.

(283) Riwa. Municipal Waste Water Treatment Plant (WWTP) Effluents. A Concise Overview of the Occurrence of Organic Substances; 2007.

(284) Kolkman, A.; Martijn, B. J.; Vughs, D.; Baken, K. A.; Van Wezel, A. P. Tracing Nitrogenous Disinfection Byproducts after Medium Pressure UV Water Treatment by Stable Isotope Labeling and High Resolution Mass Spectrometry. Environ. Sci. Technol. 2015, 49, 4458-4465. https://doi.org/10.1021/es506063h.

(285) ISO 11350:2012. Determination of the Genotoxicity of Water and Waste Water. Salmonella/Microsome Fluctuation Test (Ames Fluctuation Test). 2012, p 37. 
(286) Zwart, N.; Jonker, W.; Broek, R. ten; de Boer, J.; Somsen, G.; Kool, J.; Hamers, T.; Houtman, C. J.; Lamoree, M. H. Identification of Mutagenic and Endocrine Disrupting Compounds in Surface Water and Wastewater Treatment Plant Effluents Using High-Resolution Effect-Directed Analysis. Water Res. 2020, 168, 115204. https://doi.org/10.1016/j.watres.2019.115204.

(287) Clift, M. J. D.; Raemy, D. O.; Endes, C.; Ali, Z.; Lehmann, A. D.; Brandenberger, C.; Petri-Fink, A.; Wick, P.; Parak, W. J.; Gehr, P.; et al. Can the Ames Test Provide an Insight into Nano-Object Mutagenicity? Investigating the Interaction between Nano-Objects and Bacteria. Nanotoxicology 2013, 7 (8), 1373-1385. https://doi.org/10.3109/17435390.2012.741725.

(288) SAPEA. A Scientific Perspective on Microplastics in Nature and Society; Science Advice for Policy by European Academies, Berlin, 2019. https://doi.org/10.26356/microplastics.

(289) Arthur, C.; Baker, J.; Bamford, H.; Arthur, C.; Baker, J.; Bamford, H. Proceedings of the International Research Workshop on the Occurrence, Effects, and Fate of Microplastic Marine Debris. NOAA Mar. Debris Progr. 2009.

(290) Verschoor, A. Towards a Definition of Microplastics; Bilthoven, The Netherlands, 2015. https://doi.org/10.1080/0449010X.1964.10703070.

(291) Rochman, C. M.; Brookson, C.; Bikker, J.; Djuric, N.; Earn, A.; Bucci, K.; Athey, S.; Huntington, A.; McIlwraith, H.; Munno, K.; et al. Rethinking Microplastics as a Diverse Contaminant Suite. Environ. Toxicol. Chem. 2019, 38 (4), 703-711. https://doi.org/10.1002/etc.4371.

(292) ECHA. Annex XV Restriction Report Proposal for a Restriction; European Chemicals Agency, Helsinki, 2019.

(293) Triebskorn, R.; Braunbeck, T.; Grummt, T.; Hanslik, L.; Huppertsberg, S.; Jekel, M.; Knepper, T. P.; Krais, S.; Müller, Y. K.; Pittroff, M.; et al. Relevance of Nano- and Microplastics for Freshwater Ecosystems: A Critical Review. TrAC Trends Anal. Chem. 2019, 110, 375-392. https://doi.org/10.1016/J.TRAC.2018.11.023.

(294) Van Cauwenberghe, L.; Devriese, L.; Galgani, F.; Robbens, J.; Janssen, C. R. Microplastics in Sediments: A Review of Techniques, Occurrence and Effects. Mar. Environ. Res. 2015, 111, 5-17. https://doi.org/10.1016/j.marenvres.2015.06.007.

(295) Xu, S.; Ma, J.; Ji, R.; Pan, K.; Miao, A. J. Microplastics in Aquatic Environments: Occurrence, Accumulation, and Biological Effects. Sci. Total Environ. 2020, 703, 134699. https://doi.org/10.1016/j.scitotenv.2019.134699.

(296) Gouin, T. Towards Improved Understanding of the Ingestion and Trophic Transfer of Microplastic Particles - Critical Review and Implications for Future Research. Environ. Toxicol. Chem. 2020, 0-3. https://doi.org/10.1002/etc.4718.

(297) Kooi, M.; Koelmans, A. A. Simplifying Microplastic via Continuous Probability Distributions for Size, Shape, and Density. Environ. Sci. Technol. Lett. 2019, 6 (9), 551-557. https://doi.org/10.1021/acs.estlett.9b00379.

(298) Jambeck, J. R.; Geyer, R.; Wilcox, C.; Siegler, T. R.; Perryman, M.; Andrady, A.; Narayan, R.; Law, K. L. Plastic Waste Inputs from Land into the Ocean. Science (80-. ). 2015, 347, 768-771. https://doi.org/10.1126/science.1260352.

(299) Jabeen, K.; Li, B.; Chen, Q.; Su, L.; Wu, C.; Hollert, H.; Shi, H. Effects of Virgin 
Microplastics on Goldfish (Carassius Auratus). Chemosphere 2018, 213, 323332. https://doi.org/10.1016/j.chemosphere.2018.09.031.

(300) Jin, Y.; Lu, L.; Tu, W.; Luo, T.; Fu, Z. Impacts of Polystyrene Microplastic on the Gut Barrier, Microbiota and Metabolism of Mice. Sci. Total Environ. 2018, 649, 308-317. https://doi.org/10.1016/j.scitotenv.2018.08.353.

(301) Prokić, M. D.; Radovanović, T. B.; Gavrić, J. P.; Faggio, C. Ecotoxicological Effects of Microplastics: Examination of Biomarkers, Current State and Future Perspectives. TrAC Trends Anal. Chem. 2019, 111, 37-46. https://doi.org/10.1016/J.TRAC.2018.12.001.

(302) Ogonowski, M.; Gerdes, Z.; Gorokhova, E. What We Know and What We Think We Know about Microplastic Effects - A Critical Perspective. Curr. Opin. Environ. Sci. Heal. 2018, 1, 41-46. https://doi.org/10.1016/j.coesh.2017.09.001.

(303) Lee, K. W.; Shim, W. J.; Kwon, O. Y.; Kang, J. H. Size-Dependent Effects of Micro Polystyrene Particles in the Marine Copepod Tigriopus Japonicus.

Environ. Sci. Technol. 2013, 47, 11278-11283.

https://doi.org/10.1021/es401932b.

(304) Blarer, P.; Burkhardt-Holm, P. Microplastics Affect Assimilation Efficiency in the Freshwater Amphipod Gammarus Fossarum. Environ. Sci. Pollut. Res. 2016, 23, 23522-23532. https://doi.org/10.1007/s11356-016-7584-2.

(305) Imhof, H. K.; Rusek, J.; Thiel, M.; Wolinska, J.; Laforsch, C. Do Microplastic Particles Affect Daphnia Magna at the Morphological, Life History and Molecular Level? PLoS One 2017, 12 (11), e0187590. https://doi.org/10.1371/journal.pone.0187590.

(306) Gardon, T.; Reisser, C.; Soyez, C.; Quillien, V.; Le Moullac, G. Microplastics Affect Energy Balance and Gametogenesis in the Pearl Oyster Pinctada Margaritifera. Environ. Sci. Technol. 2018, 52 (9), 5277-5286. https://doi.org/10.1021/acs.est.8b00168.

(307) Leung, J.; Chan, K. Y. K. Microplastics Reduced Posterior Segment Regeneration Rate of the Polychaete Perinereis Aibuhitensis. Mar. Pollut. Bull. 2018, 129 (2), 782-786. https://doi.org/10.1016/J.MARPOLBUL.2017.10.072.

(308) ECETOC. An Evaluation of the Challenges and Limitations Associated with Aquatic Toxicity and Bioaccumulation Studies for Sparingly Soluble and Manufactured Particulate Substances. Tech. Rep. No. 132 2018, No. 13, 111.

(309) Bucci, K.; Tulio, M.; Rochman, C. M. What Is Known and Unknown about the Effects of Plastic Pollution: A Meta-Analysis and Systematic Review. Ecol. Appl. 2020, 30 (2), 1-16. https://doi.org/10.1002/eap.2044.

(310) Foley, C. J.; Feiner, Z. S.; Malinich, T. D.; Höök, T. O. A Meta-Analysis of the Effects of Exposure to Microplastics on Fish and Aquatic Invertebrates. Sci. Total Environ. 2018, 631-632, 550-559. https://doi.org/10.1016/J.SCITOTENV.2018.03.046.

(311) Klimisch, H. J.; Andreae, M.; Tillmann, U. A Systematic Approach for Evaluating the Quality of Experimental Toxicological and Ecotoxicological Data Regul. Toxicol. Pharmacol. 1997, 25, 1-5. https://doi.org/10.1006/rtph.1996.1076.

(312) Moermond, C. T. A.; Kase, R.; Korkaric, M.; Ågerstrand, M. CRED: Criteria for Reporting and Evaluating Ecotoxicity Data. Environ. Toxicol. Chem. 2016, 35 (5), 1297-1309. https://doi.org/10.1002/etc.3259. 
(313) SETAC. Recommended Minimum Reporting Information for Environmental Toxicity Studies. 2019, 1-3.

(314) Vandermeersch, G.; Van Cauwenberghe, L.; Janssen, C. R.; Marques, A.; Granby, K.; Fait, G.; Kotterman, M. J. J.; Bekaert, K.; Robbens, J.; Devriese, L. A Critical View on Microplastic Quantification in Aquatic Organisms. Environ. Res. 2015, 143, 46-55. https://doi.org/10.1016/J.ENVRES.2015.07.016.

(315) Wesch, C.; Bredimus, K.; Paulus, M.; Klein, R. Towards the Suitable Monitoring of Ingestion of Microplastics by Marine Biota: A Review. Environ. Pollut. 2016, 218, 1200-1208. https://doi.org/10.1016/J.ENVPOL.2016.08.076.

(316) Jahnke, A.; Arp, H. P. H.; Escher, B. I.; Gewert, B.; Gorokhova, E.; Kühnel, D.; Ogonowski, M.; Potthoff, A.; Rummel, C.; Schmitt-Jansen, M.; et al. Reducing Uncertainty and Confronting Ignorance about the Possible Impacts of Weathering Plastic in the Marine Environment. Environ. Sci. Technol. Lett. 2017, 4 (3), 8590. https://doi.org/10.1021/acs.estlett.7b00008.

(317) Rummel, C. D.; Jahnke, A.; Gorokhova, E.; Kühnel, D.; Schmitt-Jansen, M. Impacts of Biofilm Formation on the Fate and Potential Effects of Microplastic in the Aquatic Environment. Environ. Sci. Technol. Lett. 2017, 4 (7), 258-267. https://doi.org/10.1021/acs.estlett.7b00164.

(318) Paul-Pont, I.; Tallec, K.; Gonzalez-Fernandez, C.; Lambert, C.; Vincent, D.; Mazurais, D.; Zambonino-Infante, J.-L.; Brotons, G.; Lagarde, F.; Fabioux, C.; et al. Constraints and Priorities for Conducting Experimental Exposures of Marine Organisms to Microplastics. Front. Mar. Sci. 2018, 5 (July), 1-22. https://doi.org/10.3389/fmars.2018.00252.

(319) Markic, A.; Gaertner, J.-C. C.; Gaertner-Mazouni, N.; Koelmans, A. A. Plastic Ingestion by Marine Fish in the Wild. Crit. Rev. Environ. Sci. Technol. 2019, 0 (0), 1-41. https://doi.org/10.1080/10643389.2019.1631990.

(320) Feng, Z.; Zhang, T.; Li, Y.; He, X.; Wang, R.; Xu, J.; Gao, G. The Accumulation of Microplastics in Fish from an Important Fish Farm and Mariculture Area, Haizhou Bay, China. Sci. Total Environ. 2019, 696, 133948. https://doi.org/10.1016/j.scitotenv.2019.133948.

(321) Su, L.; Sharp, S. M.; Pettigrove, V. J.; Craig, N. J.; Nan, B.; Du, F.; Shi, H. Superimposed Microplastic Pollution in a Coastal Metropolis. Water Res. 2020, 168, 115140. https://doi.org/10.1016/j.watres.2019.115140.

(322) Su, L.; Nan, B.; Hassell, K. L.; Craig, N. J.; Pettigrove, V. Microplastics Biomonitoring in Australian Urban Wetlands Using a Common Noxious Fish (Gambusia Holbrooki). Chemosphere 2019, 228, 65-74. https://doi.org/10.1016/j.chemosphere.2019.04.114.

(323) Michida, Y.; Chavanich, S.; Cózar Cabañas, A.; Hagmann, P.; Hinata, H.; Isobe, A.; Kershaw, P.; Kozlovskii, N.; Li, D.; Lusher, A. L.; et al. Guidelines for Harmonizing Ocean Surface Microplastic Monitoring Methods. Ministry of the Environment Japan:; Tokyo, 2019.

(324) Ogonowski, M.; Wenman, V.; Barth, A.; Hamacher-Barth, E.; Danielsson, S.; Gorokhova, E. Microplastic Intake, Its Biotic Drivers, and Hydrophobic Organic Contaminant Levels in the Baltic Herring. Front. Environ. Sci. 2019, 7 (September), 1-11. https://doi.org/10.3389/fenvs.2019.00134.

(325) Slootmaekers, B.; Catarci Carteny, C.; Belpaire, C.; Saverwyns, S.; Fremout, W.; 
Blust, R.; Bervoets, L. Microplastic Contamination in Gudgeons (Gobio Gobio) from Flemish Rivers (Belgium). Environ. Pollut. 2019, 244 (2019), 675-684. https://doi.org/10.1016/j.envpol.2018.09.136.

(326) Harris, C. A.; Scott, A. P.; Johnson, A. C.; Panter, G. H.; Sheahan, D.; Roberts, M.; Sumpter, J. P. Principles of Sound Ecotoxicology. Environ. Sci. Technol. 2014, 48 (6), 3100-3111. https://doi.org/10.1021/es4047507.

(327) Frydkjær, C. K.; Iversen, N.; Roslev, P. Ingestion and Egestion of Microplastics by the Cladoceran Daphnia Magna: Effects of Regular and Irregular Shaped Plastic and Sorbed Phenanthrene. Bull. Environ. Contam. Toxicol. 2017, 99 (6), 655-661. https://doi.org/10.1007/s00128-017-2186-3.

(328) Gray, A. D.; Weinstein, J. E. Size- and Shape-Dependent Effects of Microplastic Particles on Adult Daggerblade Grass Shrimp ( Palaemonetes Pugio ). Environ. Toxicol. Chem. 2017, 36 (11), 3074-3080. https://doi.org/10.1002/etc.3881.

(329) Schrank, I.; Trotter, B.; Dummert, J.; Scholz-böttcher, B. M.; Löder, M. G. J.; Laforsch, C. Effects of Microplastic Particles and Leaching Additive on the Life History and Morphology of Daphnia Magna. Environ. Pollut. 2019, 113233. https://doi.org/10.1016/j.envpol.2019.113233.

(330) Chapron, L.; Peru, E.; Engler, A.; Ghiglione, J. F.; Meistertzheim, A. L.; Pruski, A. M.; Purser, A.; Vétion, G.; Galand, P. E.; Lartaud, F. Macro- and Microplastics Affect Cold-Water Corals Growth, Feeding and Behaviour. Sci. Rep. 2018, 8 (1), 15299. https://doi.org/10.1038/s41598-018-33683-6.

(331) Karami, A.; Groman, D. B.; Wilson, S. P.; Ismail, P.; Neela, V. K. Biomarker Responses in Zebrafish (Danio Rerio) Larvae Exposed to Pristine Low-Density Polyethylene Fragments. Environ. Pollut. 2017, 223, 466-475. https://doi.org/10.1016/j.envpol.2017.01.047.

(332) Romano, N.; Ashikin, M.; Teh, J. C.; Syukri, F.; Karami, A. Effects of Pristine Polyvinyl Chloride Fragments on Whole Body Histology and Protease Activity in Silver Barb Barbodes Gonionotus Fry. Environ. Pollut. 2018, 237, 1106-1111. https://doi.org/10.1016/j.envpol.2017.11.040.

(333) Imhof, H. K.; Laforsch, C. Hazardous or Not - Are Adult and Juvenile Individuals of Potamopyrgus Antipodarum Affected by Non-Buoyant Microplastic Particles? Environ. Pollut. 2016, 218, 383-391. https://doi.org/10.1016/j.envpol.2016.07.017.

(334) Eriksson Wiklund, A. Alterations in Swimming Behavior of Daphnia Exposed to Polymer and Mineral Particles: Towards Understanding Effects of Microplastics on Planktonic Filtrators. bioRxiv 2018, 406587. https://doi.org/10.1101/406587.

(335) Jeong, J.; Choi, J. Adverse Outcome Pathways Potentially Related to Hazard Identification of Microplastics Based on Toxicity Mechanisms. Chemosphere 2019, 231, 249-255. https://doi.org/10.1016/j.chemosphere.2019.05.003.

(336) Gouin, T.; Becker, R. A.; Collot, A. G.; Davis, J. W.; Howard, B.; Inawaka, K.; Lampi, M.; Ramon, B. S.; Shi, J.; Hopp, P. W. Toward the Development and Application of an Environmental Risk Assessment Framework for Microplastic. Environ. Toxicol. Chem. 2019, 38, 2087-2100. https://doi.org/10.1002/etc.4529.

(337) Kong, X.; Koelmans, A. A. Modeling Decreased Resilience of Shallow Lake Ecosystems toward Eutrophication Due to Microplastic Ingestion across the Food Web. Environ. Sci. Technol. 2019, 53 (23), 13822-13831. 
https://doi.org/10.1021/acs.est.9b03905.

(338) Qiao, R.; Sheng, C.; Lu, Y.; Zhang, Y.; Ren, H.; Lemos, B. Microplastics Induce Intestinal Inflammation, Oxidative Stress, and Disorders of Metabolome and Microbiome in Zebrafish. Sci. Total Environ. 2019, 662, 246-253.

https://doi.org/10.1016/j.scitotenv.2019.01.245.

(339) Wang, Y.; Zhang, D.; Zhang, M.; Mu, J.; Ding, G.; Mao, Z.; Cao, Y.; Jin, F.; Cong, Y.; Wang, L.; et al. Effects of Ingested Polystyrene Microplastics on Brine Shrimp, Artemia Parthenogenetica. Environ. Pollut. 2019, 244, 715-722. https://doi.org/10.1016/j.envpol.2018.10.024.

(340) Kalčíková, G.; Žgajnar Gotvajn, A.; Kladnik, A.; Jemec, A. Impact of Polyethylene Microbeads on the Floating Freshwater Plant Duckweed Lemna Minor. Environ. Pollut. 2017, 230, 1108-1115. https://doi.org/10.1016/J.ENVPOL.2017.07.050.

(341) Zhao, T.; Tan, L.; Huang, W.; Wang, J. The Interactions between Micro Polyvinyl Chloride (MPVC) and Marine Dinoflagellate Karenia Mikimotoi: The Inhibition of Growth, Chlorophyll and Photosynthetic Efficiency. Environ. Pollut. 2019, 247, 883-889. https://doi.org/10.1016/j.envpol.2019.01.114.

(342) Ziajahromi, S.; Kumar, A.; Neale, P. A.; Leusch, F. D. L. Impact of Microplastic Beads and Fibers on Waterflea (Ceriodaphnia Dubia) Survival, Growth, and Reproduction: Implications of Single and Mixture Exposures. Environ. Sci. Technol. 2017, 51 (22), 13397-13406. https://doi.org/10.1021/acs.est.7b03574.

(343) Revel, M.; Lagarde, F.; Perrein-Ettajani, H.; Bruneau, M.; Akcha, F.; Sussarellu, R.; Rouxel, J.; Costil, K.; Decottignies, P.; Cognie, B.; et al. Tissue-Specific Biomarker Responses in the Blue Mussel Mytilus Spp. Exposed to a Mixture of Microplastics at Environmentally Relevant Concentrations. Front. Environ. Sci. 2019, 7, 33. https://doi.org/10.3389/fenvs.2019.00033.

(344) Green, D. S.; Colgan, T. J.; Thompson, R. C.; Carolan, J. C. Exposure to Microplastics Reduces Attachment Strength and Alters the Haemolymph Proteome of Blue Mussels (Mytilus Edulis). Environ. Pollut. 2019, 246, 423434. https://doi.org/10.1016/j.envpol.2018.12.017.

(345) Wan, Z.; Wang, C.; Zhou, J.; Shen, M.; Wang, X.; Fu, Z.; Jin, Y. Effects of Polystyrene Microplastics on the Composition of the Microbiome and Metabolism in Larval Zebrafish. Chemosphere 2019, 646-658. https://doi.org/10.1016/j.chemosphere.2018.11.070.

(346) Détrée, C.; Gallardo-Escárate, C. Polyethylene Microbeads Induce Transcriptional Responses with Tissue-Dependent Patterns in the Mussel Mytilus Galloprovincialis. J. Molluscan Stud. 2017, 83 (2), 220-225. https://doi.org/10.1093/mollus/eyx005.

(347) Cole, M.; Coppock, R.; Lindeque, P. K.; Altin, D.; Reed, S.; Pond, D. W.; Sørensen, L.; Galloway, T. S.; Booth, A. M. Effects of Nylon Microplastic on Feeding, Lipid Accumulation, and Moulting in a Coldwater Copepod. Environ. Sci. Technol. 2019, 53 (12), 7075-7082. https://doi.org/10.1021/acs.est.9b01853.

(348) Koelmans, A. A.; Besseling, E.; Shim, W. J. Nanoplastics in the Aquatic Environment. Critical Review. In Marine Anthropogenic Litter; Bergmann, M., Gutow, L., Klages, M., Eds.; Springer International Publishing, 2015; pp 325340. https://doi.org/10.1007/978-3-319-16510-3_12. 
(349) Da Costa, J. P.; Santos, P. S. M. M.; Duarte, A. C.; Rocha-Santos, T.; Pinto Da Costa, J.; Santos, P. S. M. M.; Duarte, A. C.; Rocha-Santos, T. (Nano)Plastics in the Environment - Sources, Fates and Effects. Sci. Total Environ. 2016, 566567, 15-26. https://doi.org/https://doi.org/10.1016/j.scitotenv.2016.05.041.

(350) Moore, C. J.; Moore, S. L.; Leecaster, M. K.; Weisberg, S. B. A Comparison of Plastic and Plankton in the North Pacific Central Gyre. Mar. Pollut. Bull. 2001, 42 (12), 1297-1300. https://doi.org/10.1016/S0025-326X(01)00114-X.

(351) Yonkos, L. T.; Friedel, E. A.; Perez-Reyes, A. C.; Ghosal, S.; Arthur, C. D. Microplastics in Four Estuarine Rivers in the Chesapeake Bay, U.S.A. Environ. Sci. Technol. 2014, 48 (24), 14195-14202. https://doi.org/10.1021/es5036317.

(352) Merga, L. B.; Redondo-Hasselerharm, P. E.; Van den Brink, P. J.; Koelmans, A. A. Distribution of Microplastic and Small Macroplastic Particles across Four Fish Species and Sediment in an African Lake. Sci. Total Environ. 2020, 741, 140527. https://doi.org/10.1016/j.scitotenv.2020.140527.

(353) Koelmans, A. A.; Kooi, M.; Law, K. L.; Van Sebille, E. All Is Not Lost: Deriving a Top-down Mass Budget of Plastic at Sea. Environ. Res. Lett. 2017, 12 (11), 114028. https://doi.org/10.1088/1748-9326/aa9500.

(354) Jaikumar, G.; Brun, N. R.; Vijver, M. G.; Bosker, T. Reproductive Toxicity of Primary and Secondary Microplastics to Three Cladocerans during Chronic Exposure. Environ. Pollut. 2019, 249, 638-646. https://doi.org/10.1016/j.envpol.2019.03.085.

(355) Reichert, J.; Schellenberg, J.; Schubert, P.; Wilke, T. Responses of Reef Building Corals to Microplastic Exposure. Environ. Pollut. 2018, 237, 955-960. https://doi.org/10.1016/j.envpol.2017.11.006.

(356) Casado, M. P.; Macken, A.; Byrne, H. J. H. J. Ecotoxicological Assessment of Silica and Polystyrene Nanoparticles Assessed by a Multitrophic Test Battery. Environ. Int. 2013, 51, 97-105. https://doi.org/10.1016/j.envint.2012.11.001.

(357) Zimmermann, L.; Dierkes, G.; Ternes, T. A.; Völker, C.; Wagner, M. Benchmarking the in Vitro Toxicity and Chemical Composition of Plastic Consumer Products. Environ. Sci. Technol. 2019, acs.est.9b02293. https://doi.org/10.1021/acs.est.9b02293.

(358) Binelli, A.; Pietrelli, L.; Di Vito, S.; Coscia, L.; Sighicelli, M.; Torre, C. Della; Parenti, C. C.; Magni, S. Hazard Evaluation of Plastic Mixtures from Four Italian Subalpine Great Lakes on the Basis of Laboratory Exposures of Zebra Mussels. Sci. Total Environ. 2020, 699, 134366. https://doi.org/10.1016/j.scitotenv.2019.134366.

(359) Vroom, R. J. E.; Koelmans, A. A.; Besseling, E.; Halsband, C. Aging of Microplastics Promotes Their Ingestion by Marine Zooplankton. Environ. Pollut. 2017, 231, 987-996. https://doi.org/10.1016/J.ENVPOL.2017.08.088.

(360) Elizalde-Velázquez, A.; Carcano, A. M.; Crago, J.; Green, M. J.; Shah, S. A.; Cañas-Carrell, J. E. Translocation, Trophic Transfer, Accumulation and Depuration of Polystyrene Microplastics in Daphnia Magna and Pimephales Promelas. Environ. Pollut. 2020, 259, 113937. https://doi.org/10.1016/j.envpol.2020.113937.

(361) Redondo-Hasselerharm, P. E.; Mitrano, D. M.; Koelmans, A. A. Uptake and Effects of Metal-Doped Nanoplastics on Gammarus Pulex. In Prep. 2020. 
(362) Escher, B. I.; Stapleton, H. M.; Schymanski, E. L. Tracking Complex Mixtures of Chemicals in Our Changing Environment. Science (80-. ). 2020, 367 (6476), 388-392. https://doi.org/10.1126/science.aay6636.

(363) Di Sotto, A.; Chiaretti, M.; Carru, G. A.; Bellucci, S.; Mazzanti, G. Multi-Walled Carbon Nanotubes: Lack of Mutagenic Activity in the Bacterial Reverse Mutation Assay. Toxicol. Lett. 2009, 184 (3), 192-197. https://doi.org/10.1016/j.toxlet.2008.11.007.

(364) Pan, C.; Mintenig, S. M.; Redondo-Hasselerharm, P. E.; Nijenhuis, P.; Yu, K.-F.; Wang, Y.-H.; Koelmans, A. A. Automated MFTIR Imaging Demonstrates Taxon-Specific and Selective Uptake of Microplastic by Freshwater Invertebrates. In Prep. 2020.

(365) Li, Q.; Feng, Z.; Zhang, T.; Ma, C.; Shi, H. Microplastics in the Commercial Seaweed Nori. J. Hazard. Mater. 2020, 388 (December 2019), 122060. https://doi.org/10.1016/j.jhazmat.2020.122060.

(366) Sbrana, A.; Valente, T.; Scacco, U.; Bianchi, J.; Silvestri, C.; Palazzo, L.; de Lucia, G. A.; Valerani, C.; Ardizzone, G.; Matiddi, M. Spatial Variability and Influence of Biological Parameters on Microplastic Ingestion by Boops Boops (L.) along the Italian Coasts (Western Mediterranean Sea). Environ. Pollut. 2020, 263, 114429. https://doi.org/10.1016/j.envpol.2020.114429.

(367) Jacob, H.; Besson, M.; Swarzenski, P. W.; Lecchini, D.; Metian, M. Effects of Virgin Micro- and Nanoplastics on Fish: Trends, Meta-Analysis, and Perspectives. Environ. Sci. Technol. 2020, 54 (8), 4733-4745. https://doi.org/10.1021/acs.est.9b05995.

(368) EFSA. Guidance on Tiered Risk Assessment for Plant Protection Products for Aquatic Organisms in Edge-of-Field Surface Waters. EFSA J. 2013, 11 (7), 268p.

(369) Diepens, N. J.; Arts, G. H. P.; Brock, T. C. M.; Smidt, H.; Van Den Brink, P. J.; Van Den Heuvel-Greve, M. J.; Koelmans, A. A. Sediment Toxicity Testing of Organic Chemicals in the Context of Prospective Risk Assessment: A Review. Crit. Rev. Environ. Sci. Technol. 2014, 44 (3), 255-302. https://doi.org/10.1080/01496395.2012.718945.

(370) Clokey, J. E.; Redondo-Hasselerharm, P. E.; Roessink, I.; Peeters, E. T. H. M.; Koelmans, A. A. No Effects of Microplastics to Three Benthic Invertebrate Species up to a Concentration of $40 \%$ Dry Weight in Sediment. In Prep.

(371) Echa. Guidance on Information Requirements and Chmical Safety Assessment. Chapter R.10: Characterisation of Dose [Concentration]-Response for Environment. Eur. Chem. Agency 2008, No. May, 1-65.

(372) Kryger, J.; Riisgard, H. U. Filtration Rate Capacities in 6 Species of European Freshwater Bivalve. Int. Assoc. Ecol. 1988, 77, 34-38.

(373) Scherer, C.; Weber, A.; Stock, F.; Vurusic, S.; Egerci, H.; Kochleus, C.; Arendt, N.; Foeldi, C.; Dierkes, G.; Wagner, M.; et al. Comparative Assessment of Microplastics in Water and Sediment of a Large European River. Sci. Total Environ. 2020, 738, 139866. https://doi.org/10.1016/j.scitotenv.2020.139866.

(374) VKM; Skåre, J. U.; Alexander, J.; Haave, M.; Jakubowicz, I.; Knutsen, H. K.; Lusher, A.; Ogonowski, M.; Rakkestad, K. E.; Skaar, I.; et al. Microplastics; Occurrence, Levels and Implications for Environment and Human Health Related to Food. Scientific Opinion of the Scientific Steering Committee of the 
Norwegian Scientific Committee for Food and Environment.; Oslo, 2019.

(375) European Union. CIS-WFD Guidance Document No. 27. Technical Guidance for Deriving Environmental Quality Standards. Technical Report-2011-055; 2011.

(376) Dong, M.; Luo, Z.; Jiang, Q.; Xing, X.; Zhang, Q.; Sun, Y. The Rapid Increases in Microplastics in Urban Lake Sediments. Sci. Rep. 2020, 10, 848. https://doi.org/10.1038/s41598-020-57933-8.

(377) Lijzen, J. P. A.; Baars, A. J.; Otte, P. F.; Rikken, M. G. J.; Swartjes, F. A.; Verbruggen, E. M. J.; Van Wezel, A. P. RIVM Report 711701 023. Technical Evaluation of the Intervention Values for Soil/Sediment and Groundwater Human and Ecotoxicological Risk Assessment and Derivation of Risk Limits for Soil, Aquatic Sediment and Groundwater; 2001.

(378) Jones-Hughes, T.; Turner, A. Sorption of Ionic Surfactants to Estuarin Sediment and Their Influence on the Sequestration of Phenanthrene. Environ. Sci. Technol. 2005, 39 (6), 1688-1697. https://doi.org/10.1021/es040077d.

(379) Burns, C. W. The Relationship Between Body Size of Filter-Feeding Cladocera and the Maximum Size of Particle Ingested. Limnol. Oceanogr. 1968, 13 (4), 675-678. https://doi.org/10.4319/lo.1968.13.4.0675.

(380) Jâms, I. B.; Windsor, F. M.; Poudevigne-Durance, T.; Ormerod, S. J.; Durance, I. Estimating the Size Distribution of Plastics Ingested by Animals. Nat. Commun. 2020, 11 (1), 1-7. https://doi.org/10.1038/s41467-020-15406-6.

(381) Piarulli, S.; Vanhove, B.; Comandini, P.; Scapinello, S.; Moens, T.; Vrielinck, H.; Sciutto, G.; Prati, S.; Mazzeo, R.; Booth, A. M.; et al. Do Different Habits Affect Microplastics Contents in Organisms? A Trait-Based Analysis on Salt Marsh Species. Mar. Pollut. Bull. 2020, 153, 110983. https://doi.org/10.1016/j.marpolbul.2020.110983.

(382) Shim, W. J.; Hong, S. H.; Eo, S. E. Identification Methods in Microplastic Analysis: A Review. Anal. Methods 2017, 9 (9), 1384-1391. https://doi.org/10.1039/c6ay02558g.

(383) Korez, Š.; Gutow, L.; Saborowski, R. Feeding and Digestion of the Marine Isopod Idotea Emarginata Challenged by Poor Food Quality and Microplastics. Comp. Biochem. Physiol. Part C Toxicol. Pharmacol. 2019, 226, 108586. https://doi.org/10.1016/j.cbpc.2019.108586.

(384) Jemec, A.; Kunej, U.; Skalar, T. Screening Study of Four Environmentally Relevant Microplastic Pollutants: Uptake and Effects on Daphnia Magna and Artemia Franciscana. Chemosphere 2018, 208, 522-529. https://doi.org/10.1016/j.chemosphere.2018.05.172.

(385) Gerdes, Z.; Hermann, M.; Ogonowski, M.; Gorokhova, E. A Novel Method for Assessing Microplastic Effect in Suspension through Mixing Test and Reference Materials. Sci. Rep. 2019, 9, 10695. https://doi.org/10.1038/s41598-019-47160-1.

(386) Sjollema, S. B.; Redondo-Hasselerharm, P. E.; Leslie, H. A.; Kraak, M. H. S.; Vethaak, A. D. Do Plastic Particles Affect Microalgal Photosynthesis and Growth? Aquat. Toxicol. 2016, 170, 259-261. https://doi.org/10.1016/j.aquatox.2015.12.002.

(387) Jeong, C. B.; Kang, H. M.; Lee, M. C.; Kim, D. H.; Han, J.; Hwang, D. S.; Souissi, S.; Lee, S. J.; Shin, K. H.; Park, H. G.; et al. Adverse Effects of Microplastics and Oxidative Stress-Induced MAPK/Nrf2 Pathway-Mediated 
Defense Mechanisms in the Marine Copepod Paracyclopina Nana. Sci. Rep. 2017, 7, 41323. https://doi.org/10.1038/srep41323.

(388) Koelmans, A. A.; Bakir, A.; Burton, G. A. A.; Janssen, C. R. C. R. Microplastic as a Vector for Chemicals in the Aquatic Environment: Critical Review and Model-Supported Reinterpretation of Empirical Studies. Environ. Sci. Technol. 2016, 50 (7), 3315-3326. https://doi.org/10.1021/acs.est.5b06069.

(389) Ziccardi, L. M.; Edgington, A.; Hentz, K.; Kulacki, K. J.; Kane Driscoll, S. Microplastics as Vectors for Bioaccumulation of Hydrophobic Organic Chemicals in the Marine Environment: A State-of-the-Science Review. Environ. Toxicol. Chem. 2016, 35 (7), 1667-1676. https://doi.org/10.1002/etc.3461.

(390) Pikuda, O.; Xu, E. G.; Berk, D.; Tufenkji, N. Toxicity Assessments of Microand Nanoplastics Can Be Confounded by Preservatives in Commercial Formulations. Environ. Sci. Technol. Lett. 2019, 6 (1), 21-25. https://doi.org/10.1021/acs.estlett.8b00614.

(391) Martínez-Gómez, C.; León, V. M.; Calles, S.; Gomáriz-Olcina, M.; Vethaak, A. D. The Adverse Effects of Virgin Microplastics on the Fertilization and Larval Development of Sea Urchins. Mar. Environ. Res. 2017, 130, 69-76. https://doi.org/10.1016/j.marenvres.2017.06.016.

(392) Bakir, A.; O’Connor, I. A.; Rowland, S. J.; Hendriks, A. J.; Thompson, R. C. Relative Importance of Microplastics as a Pathway for the Transfer of Hydrophobic Organic Chemicals to Marine Life. Environ. Pollut. 2016, 219, 5665. https://doi.org/10.1016/j.envpol.2016.09.046.

(393) Prata, J. C.; da Costa, J. P.; Duarte, A. C.; Rocha-Santos, T. Methods for Sampling and Detection of Microplastics in Water and Sediment: A Critical Review. TrAC - Trends Anal. Chem. 2019, 110, 150-159. https://doi.org/10.1016/j.trac.2018.10.029.

(394) Hanvey, J. S.; Lewis, P. J.; Lavers, J. L.; Crosbie, N. D.; Pozo, K.; Clarke, B. O. A Review of Analytical Techniques for Quantifying Microplastics in Sediments. Anal. Methods 2017, 9 (9), 1369-1383. https://doi.org/10.1039/c6ay02707e.

(395) Welden, N. A. C.; Cowie, P. R. Long-Term Microplastic Retention Causes Reduced Body Condition in the Langoustine, Nephrops Norvegicus. Environ. Pollut. 2016, 218, 895-900. https://doi.org/10.1016/j.envpol.2016.08.020.

(396) Foekema, E. M.; De Gruijter, C.; Mergia, M. T.; Van Franeker, J. A.; Murk, A. J.; Koelmans, A. A. Plastic in North Sea Fish. Environ. Sci. Technol. 2013, 47 (15), 8818-8824. https://doi.org/10.1021/es400931b.

(397) Benfenati, E.; Clook, M.; Fryday, S.; Hart, A. QSARs for Regulatory Purposes: The Case for Pesticide Authorization. In Quantitative Structure-Activity Relationships (QSAR) for Pesticide Regulatory Purposes; Benfenati, E., Ed.; Elsevier: Amesterdam, 2007.

(398) OECD. Test No. 239: Water-Sediment Myriophyllum Spicatum Toxicity Test. In Guidelines for the Testing of Chemicals; OECD: Paris, 2014.

(399) Abdolahpur Monikh, F.; Praetorius, A.; Schmid, A.; Kozin, P.; Meisterjahn, B.; Makarova, E.; Hofmann, T.; von der Kammer, F. Scientific Rationale for the Development of an OECD Test Guideline on Engineered Nanomaterial Stability. NanoImpact 2018, 11, 42-50. https://doi.org/10.1016/j.impact.2018.01.003.

(400) Galloway, T. S.; Dogra, Y.; Garrett, N.; Rowe, D.; Tyler, C. R.; Moger, J.; 
Lammer, E.; Landsiedel, R.; Sauer, U. G.; Scherer, G.; et al. Ecotoxicological Assessment of Nanoparticle-Containing Acrylic Copolymer Dispersions in Fairy Shrimp and Zebrafish Embryos. Environ. Sci. Nano 2017, 4 (10), 1981-1997. https://doi.org/10.1039/c7en00385d.

(401) Hartmann, N. B.; Jensen, K. A.; Baun, A.; Rasmussen, K.; Rauscher, H.; Tantra, R.; Cupi, D.; Gilliland, D.; Pianella, F.; Riego Sintes, J. M. Techniques and Protocols for Dispersing Nanoparticle Powders in Aqueous Media - Is There a Rationale for Harmonization? J. Toxicol. Environ. Heal. - Part B Crit. Rev. 2015, 18 (6), 299-326. https://doi.org/10.1080/10937404.2015.1074969.

(402) Hjorth, R.; Skjolding, L. M.; Sørensen, S. N.; Baun, A. Regulatory Adequacy of Aquatic Ecotoxicity Testing of Nanomaterials. NanoImpact 2017, 8, $28-37$. https://doi.org/10.1016/j.impact.2017.07.003.

(403) Petersen, E. J.; Henry, T. B.; Zhao, J.; MacCuspie, R. I.; Kirschling, T. L.; Dobrovolskaia, M. A.; Hackley, V.; Xing, B.; White, J. C. Identification and Avoidance of Potential Artifacts and Misinterpretations in Nanomaterial Ecotoxicity Measurements. Environ. Sci. Technol. 2014, 48 (8), 4226-4246. https://doi.org/10.1021/es4052999.

(404) Gambardella, C.; Piazza, V.; Albentosa, M.; Bebianno, M. J.; Cardoso, C.; Faimali, M.; Garaventa, F.; Garrido, S.; González, S.; Pérez, S.; et al. Microplastics Do Not Affect Standard Ecotoxicological Endpoints in Marine Unicellular Organisms. Mar. Pollut. Bull. 2019, 143, 140-143. https://doi.org/10.1016/j.marpolbul.2019.04.055.

(405) Magni, S.; Della Torre, C.; Garrone, G.; D’Amato, A.; Parenti, C. C.; Binelli, A. First Evidence of Protein Modulation by Polystyrene Microplastics in a Freshwater Biological Model. Environ. Pollut. 2019, 250, 407-415. https://doi.org/10.1016/j.envpol.2019.04.088.

(406) Tang, J.; Ni, X.; Zhou, Z.; Wang, L.; Lin, S. Acute Microplastic Exposure Raises Stress Response and Suppresses Detoxification and Immune Capacities in the Scleractinian Coral Pocillopora Damicornis. Environ. Pollut. 2018, 243, 66-74. https://doi.org/10.1016/j.envpol.2018.08.045.

(407) Bour, A.; Haarr, A.; Keiter, S.; Hylland, K. Environmentally Relevant Microplastic Exposure Affects Sediment-Dwelling Bivalves. Environ. Pollut. 2018, 236, 652-660. https://doi.org/10.1016/j.envpol.2018.02.006.

(408) Gonçalves, C.; Martins, M.; Sobral, P.; Costa, P. M.; Costa, M. H. An Assessment of the Ability to Ingest and Excrete Microplastics by Filter-Feeders: A Case Study with the Mediterranean Mussel. Environ. Pollut. 2019, 245, 600606. https://doi.org/10.1016/j.envpol.2018.11.038.

(409) Franzellitti, S.; Capolupo, M.; Wathsala, R. H. G. R.; Valbonesi, P.; Fabbri, E. The Multixenobiotic Resistance System as a Possible Protective Response Triggered by Microplastic Ingestion in Mediterranean Mussels (Mytilus Galloprovincialis): Larvae and Adult Stages. Comp. Biochem. Physiol. Toxicol. Pharmacol. 2019, 219, 50-58. https://doi.org/10.1016/j.cbpc.2019.02.005.

(410) Hanson, M. L.; Wolff, B. A.; Green, J. W.; Kivi, M.; Panter, G. H.; Warne, M. S. J.; Ågerstrand, M.; Sumpter, J. P. How We Can Make Ecotoxicology More Valuable to Environmental Protection. Sci. Total Environ. 2017, 578, 228-235. https://doi.org/10.1016/j.scitotenv.2016.07.160. 
(411) Jovanović, B.; Gökdağ, K.; Güven, O.; Emre, Y.; Whitley, E. M.; Kideys, A. E. Virgin Microplastics Are Not Causing Imminent Harm to Fish after Dietary Exposure. Mar. Pollut. Bull. 2018, 130, 123-131. https://doi.org/10.1016/j.marpolbul.2018.03.016.

(412) Welham, S. J.; Gezan, S. A.; Clarck, S. J.; Mead, A. Design and Analysis of Experiments and Regression; CRC Press: Florida, 2015.

(413) Solomon, K.; Tackacs, P. Species Sensitivity Distributions in Ecotoxicology; Leo Posthuma, Glenn W. Suter II, and T. P. T., Ed.; Lewis Publishers: Boca Raton, London, New York, Washington, D.C., 2001.

(414) de Sá, L. C.; Luís, L. G.; Guilhermino, L. Effects of Microplastics on Juveniles of the Common Goby (Pomatoschistus Microps): Confusion with Prey, Reduction of the Predatory Performance and Efficiency, and Possible Influence of Developmental Conditions. Environ. Pollut. 2015, 196, 359-362. https://doi.org/10.1016/j.envpol.2014.10.026.

(415) Forbes, V. E.; Palmqvist, A.; Bach, L. The Use and Misuse of Biomarkers in Ecotoxicology. Environ. Toxicol. Chem. 2006, 25 (1), 272-280. https://doi.org/10.1897/05-257R.1.

(416) Tincani, F. H.; Galvan, G. L.; Marques, A. E. M. L.; Santos, G. S.; Pereira, L. S.; da Silva, T. A.; Silva de Assis, H. C.; Barbosa, R. V.; Cestari, M. M.

Pseudoreplication and the Usage of Biomarkers in Ecotoxicological Bioassays. Environ. Toxicol. Chem. 2017, 36, 2868-2874. https://doi.org/10.1002/etc.3823.

(417) Aljaibachi, R.; Callaghan, A. Impact of Polystyrene Microplastics on Daphnia Magna Mortality and Reproduction in Relation to Food Availability. PeerJ 2018, 2018 (4), e4601. https://doi.org/10.7717/peerj.4601.

(418) Critchell, K.; Hoogenboom, M. O. Effects of Microplastic Exposure on the Body Condition and Behaviour of Planktivorous Reef Fish (Acanthochromis Polyacanthus). PLoS One 2018, 13 (3), e0193308. https://doi.org/10.1371/journal.pone.0193308.

(419) Wu, Y.; Guo, P.; Zhang, X.; Zhang, Y.; Xie, S.; Deng, J. Effect of Microplastics Exposure on the Photosynthesis System of Freshwater Algae. J. Hazard. Mater. 2019, 374, 219-227. https://doi.org/10.1016/j.jhazmat.2019.04.039.

(420) Jemec, A.; Horvat, P.; Kunej, U.; Bele, M.; Kržan, A. Uptake and Effects of Microplastic Textile Fibers on Freshwater Crustacean Daphnia Magna. Environ. Pollut. 2016, 219, 201-209. https://doi.org/10.1016/j.envpol.2016.10.037.

(421) Cole, M.; Lindeque, P.; Fileman, E.; Halsband, C.; Galloway, T. S. The Impact of Polystyrene Microplastics on Feeding, Function and Fecundity in the Marine Copepod Calanus Helgolandicus. Environ. Sci. Technol. 2015, 49 (2), 1130 1137. https://doi.org/10.1021/es504525u.

(422) Jager, T. Bad Habits Die Hard: The NOEC's Persistence Reflects Poorly on Ecotoxicology. Environ. Toxicol. Chem. 2012, 31 (2), 228-229. https://doi.org/10.1002/etc.746.

(423) Green, J. W.; Springer, T. A.; Staveley, J. P. The Drive to Ban the NOEC/LOEC in Favor of ECx Is Misguided and Misinformed. Integr. Environ. Assess. Manag. 2013, 9 (1), 12-16. https://doi.org/10.1002/ieam.1367.

(424) Landis, W. G.; Chapman, P. M. Well Past Time to Stop Using NOELs and LOELs. Integr. Environ. Assess. Manag. 2011, 7 (4), 7-9. 
https://doi.org/10.1002/ieam.249.

(425) Lenz, R.; Enders, K.; Gissel, T. Microplastic Exposure Studies Should Be Environmentally Realistic. Proc. Natl. Acad. Sci. U. S. A. 2016, 113 (29), 2-3. https://doi.org/10.1073/pnas.1606615113.

(426) Canniff, P. M.; Hoang, T. C. Microplastic Ingestion by Daphnia Magna and Its Enhancement on Algal Growth. Sci. Total Environ. 2018, 633, 500-507. https://doi.org/10.1016/J.SCITOTENV.2018.03.176.

(427) Zettler, E. R.; Mincer, T. J.; Amaral-Zettler, L. A. Life in the "Plastisphere": Microbial Communities on Plastic Marine Debris. Environ. Sci. Technol. 2013, 47 (13), 7137-7146. https://doi.org/10.1021/es401288x.

(428) Zhang, C.; Jeong, C. B.; Lee, J. S.; Wang, D.; Wang, M. Transgenerational Proteome Plasticity in Resilience of a Marine Copepod in Response to Environmentally Relevant Concentrations of Microplastics. Environ. Sci. Technol. 2019, 53 (14), 8426-8436. https://doi.org/10.1021/acs.est.9b02525.

(429) Moore, J. W. The Role of Algae in the Diet of Asellus Aquaticus L. and Gammarus Pulex L. Author (s): J. W. Moore Source: Journal of Animal Ecology, Vol. 44, No. 3 (Oct., 1975), Pp. 719-730 Published by: British Ecological Society Stable. Soc. Br. Ecol. 1975, 44 (3), 719-730.

(430) Schmitz, E. H.; Scherrey, P. M. Digestive Anatomy of Halella Azteca (Crustacea, Amphipoda). J. Morphol. 1983, 175, 91-100. https://doi.org/10.1002/jmor.1051750109.

(431) Covich, A. P.; Crowl, T. A.; Alexander, J. E. .; Vaughn, C. C. PredatorAvoidance Responses in Freshwater Decapod-Gastropod Interactions Mediated by Chemical Stimuli. J. North Am. Benthol. Soc. 1994, 13 (2), 283-290.

(432) Sáez-Royuela, M.; Carral, J. M.; Celada, J. D.; Pérez, J. R.; González, A. Live Feed as Supplement from the Onset of External Feeding of Juvenile Signal Crayfish (Pacifastacus Leniusculus Dana. Astacidae) under Controlled Conditions. Aquaculture 2007, 269 (1-4), 321-327. https://doi.org/10.1016/j.aquaculture.2007.04.053.

(433) Voparil, I. M.; Burgess, R. M.; Mayer, L. M.; Tien, R.; Cantwell, M. G.; Ryba, S. A. Digestive Bioavailability to a Deposit Feeder (Arenicola Marina) of Polycyclic Aromatic Hydrocarbons Associated with Anthropogenic Particles. Environ. Toxicol. Chem. 2004, 23 (11), 2618. https://doi.org/10.1897/03-357.

(434) Juget, J. La Texture Granulometrique Des Sediments et Le Regime Alimentaire Des Oligochetes Limnicoles. Hydrobiologia 1979, 65 (2), 145-154. https://doi.org/10.1007/BF00017420.

(435) Aberle, N.; Hillebrand, H.; Grey, J.; Wiltshire, K. H. Selectivity and Competitive Interactions between Two Benthic Invertebrate Grazers (Asellus Aquaticus and Potamopyrgus Antipodarum): An Experimental Study Using 13C- and 15NLabelled Diatoms. Freshw. Biol. 2005, 50 (2), 369-379. https://doi.org/10.1111/j.1365-2427.2004.01325.x.

(436) Scherer, C.; Wolf, R.; Völker, J.; Stock, F.; Brennhold, N.; Reifferscheid, G.; Wagner, M. Toxicity of Microplastics and Natural Particles in the Freshwater Dipteran Chironomus Riparius: Same Same but Different? Sci. Total Environ. 2020, 711, 134604. https://doi.org/10.1016/j.scitotenv.2019.134604. 


Summary 



\section{Summary}

After the general introduction in Chapter 1, Chapters 2 - 4 focus on the development of standard single species tests to detect individual effect thresholds of environmentally relevant NP and MP. Then, in Chapter 5, we measure the effect thresholds of NP and MP at the level of community using an ecologically relevant outdoor experimental system. Chapter 6 aims at evaluating the suitability of in vitro tests commonly used for chemical screening in drinking and WWTP effluent waters to detect the mutagenicity of NP and MP alone and in combination chemicals extracted from surface and WWTP effluent waters. In Chapter 7, we provide a guidance protocol for effect testing, identify knowledge gaps with respect to study characteristics and define the weight of evidence for demonstrated effects and effect mechanisms. Finally, in Chapter $\mathbf{8}$ we answer the research questions formulated in the introduction, develop a tiered approach risk assessment using the data generated in this thesis and literature data and we provide recommendations for future research. Detailed summaries of the research approaches used to address the above research questions can be found below:

In Chapter 2, we assess the effects of MP on six freshwater benthic macroinvertebrates with different living and feeding strategies with the aim of measuring their individual effect thresholds. For this, standard 28-days single species tests were performed using pre-washed PS MP fragments with sizes between 20 and $500 \mu \mathrm{m}$ at concentrations ranging from 0 to $40 \% \mathrm{MP}$ in sediment $\mathrm{dw}$, including one field measured concentration. Species selected were the amphipods Gammarus pulex and Hyalella azteca, the isopod Asellus aquaticus, the worms Lumbriculus variegatus and Tubifex spp. and the bivalve Sphaerium corneum. Endpoints assessed included survival, growth and feeding activity of G. pulex, H. Azteca, A. aquaticus and Tubifex spp., reproduction, growth and feeding activity of $L$. variegatus and survival and growth of $S$. corneum. In addition, we analysed the ingestion and egestion of MP of G. pulex and H. azteca using $\mu$-Fourier Transformed Spectroscopy and provide trophic transfer factors (TTF) for G. pulex. Finally, we compare the effect thresholds obtained with MP environmental concentrations measured in freshwater sediments.

Similarly, in Chapter 3 we provide individual effect thresholds for a battery of benthic macroinvertebrates, this time exposed to MP released from car tires. As mentioned before, car tire particles seem to greatly contribute to plastic pollution in aquatic systems, ${ }^{14,165}$ causing their risk potentially to be higher due to their chemical composition, as they contain high concentrations of sulphur and zinc. Therefore, MP were made by scraping the first $2 \mathrm{~cm}$ of five second-hand tires and grinding them until obtaining a size distribution of 10 to $585 \mu \mathrm{m}$. Main constituents of the MP were quantified using thermogravimetric analysis (TGA) and gas chromatography - mass spectrometry (GCMS). Using standard 28-days single species tests we assessed the effects of six MP concentrations ranging from 0 to $10 \%$ in sediment dw on the survival and growth of $G$. pulex. A. aquaticus, L. variegatus and Tubifex spp.. Moreover, ingestion and egestion of TP were investigated for G. pulex. To this aim, we developed a method to quantify MP 
released from car tires in organisms and faeces of G. pulex, which included testing the resistance of these MP to the digestion's fluids used. Finally, we calculated the TTF for G. pulex and compared them with the ones obtained in Chapter 2, and we evaluated the risks of TP by comparing them with field measured concentrations in sediments.

In Chapter 4, we evaluated the effects of $228 \mathrm{~nm}$ raspberry shaped palladium-doped NP on the survival and growth of the freshwater amphipod G. pulex. Organisms were exposed to the NP via natural sediment at six concentrations $(0,0.03,0.1,0.3,1$ and $3 \%$ sediment $\mathrm{dw}$ ) under 28-day laboratory standardized single species toxicity tests. In addition, NP concentrations were quantified based on palladium $(\mathrm{Pd})$ concentrations measured with inductively coupled plasma mass spectrometry (ICP-MS) in the body of the exposed organisms and in the faecal pellets excreted during a 24 hours post-exposure depuration period. In addition, palladium-doped NP concentrations were measured in sediments and water to demonstrate the accuracy of the dosing and to quantify the resuspension of NP from the sediment. Moreover, TTF were calculated for the body and gut of G. pulex using a biodynamic model. For comparison, MP accumulation data obtained in Chapter 2 were modelled as well.

Then, in Chapter 5, we evaluate the effects of a range of NP or MP concentrations on a freshwater benthic macroinvertebrate community after 3-and 15-months of exposure via natural sediment using an outdoor ditch as experimental system. For this, trays containing sediment and NP or MP at five concentrations between 0 and $5 \%$ plastic in sediment $\mathrm{dw}$, including two environmentally realistic concentrations, were embedded at the bottom of the ditch containing a stable donor community. Spherical PS NP with an average size of $96 \mathrm{~nm}$ and PS MP fragments with sizes ranging from 20 to $516 \mu \mathrm{m}$ were used for the NP and MP treatments, respectively. The donor community was allowed to colonize the trays and after 3 and 15 months, trays were retrieved, and species were identified and counted. Effects were assessed on the community composition, population sizes and species diversity. In this chapter, we provide the first long-term community effect thresholds for freshwater benthic macroinvertebrates exposed to NP and MP and compare them with environmental concentrations measured in freshwater sediments.

In Chapter 6, we explore the potential use of in vitro toxicity tests to inform the assessment of the risks of NP and MP for human health, in this case with and without chemical mixtures originating from WWTP effluent and surface water samples. For this, we evaluated the genotoxicity of spherical PS NP with an average size of $50 \mathrm{~nm}$ and spherical PS MP with an average size of $500 \mathrm{~nm}$ at four environmentally relevant concentrations $(0,2.5,25$ and $250 \mu \mathrm{g} / \mathrm{l})$ in three matrices using the Ames fluctuation test, which has the purpose of detecting base-pair and frameshift mutations in the genome of Salmonella typhimurium with and without metabolic activation. We thereby assess the genotoxicity of the environmental matrices, NP or MP alone, NP or MP in the presence of chemicals extracted from surface water, and NP or MP in the presence of chemicals extracted from WWTP effluent. Finally, we provide recommendations to increase the relevance of in vitro tests for the assessment of NP and MP risks for human health. 
Following recently developed quality assessment methods for studies reporting abundance of MP in biota and water samples, ${ }^{31,35}$ in Chapter 7 we critically review 105 papers reporting MP effects on aquatic biota. For this, 20 Quality assurance/Quality control (QA/QC) criteria were defined within four main categories: particle characterization, experimental design, applicability for risk assessment and ecological relevance. Based on our analysis and practical experiences from Chapters 2, 3, and 4, a guidance protocol for testing ecotoxicological effects of MP for aquatic species is consequently provided. In addition, with the aim of detecting knowledge gaps within effect studies with MP, we provide an overview of the study characteristics of the reviewed studies with respect to the size, shape and polymer type of the MP used, the tested species, the duration of the exposure, the endpoints studied and use or not of effect thresholds to report the results. Moreover, demonstrated and suggested effects and effect mechanisms reported in the reviewed papers are summarized and discussed, with the results of the quality evaluation applied as a method to assess the overall weight of evidence regarding probable ecologically relevant effects.

In Chapter 8 we bring all the answers to the research questions together and develop a tiered approach ecological risk assessment for MP using the data generated in Chapters $\mathbf{2 , 3}$ and $\mathbf{5}$ and data taken from the literature. Following the tier approach, we first compare MP effect thresholds obtained in Chapters $\mathbf{2}$ and $\mathbf{3}$ with environmental concentrations of the corresponding MP types measured in freshwater sediments (tier 1). Then, we create two Species Sensitivity Distributions (SSDs), one for water exposure data and one for sediment exposure data using the effect thresholds obtained in Chapters $\mathbf{2}$ and $\mathbf{3}$ and MP effect thresholds taken from the literature (tier 2). Here, a $\mathrm{HC}_{50}$ is calculated. For tier 3 , we use the community effect thresholds obtained in Chapter 5 and compare them with field measured concentrations. Finally, we discuss the implications of the results obtained in this thesis and provide recommendations for future research. 

Acknowledgements 



\section{Acknowledgements}

I would first like to thank the two most important people in my life: my parents, Gonny and Paco. Thanks to you, today I am able to enjoy what I do for a living, which makes me extremely happy. Next, I want to deeply thank my supervisors, Bart and Edwin, for giving me the opportunity to live this bittersweet experience, which is doing a $\mathrm{PhD}$. You have taught me an incredible number of things, which made me grow scientifically and personally. I would also like to thank my two amazing project colleagues, Svenja and Merel, who were always there to provide help, motivation and, most importantly, good times. Many thanks also to the rest of the people who helped me making this thesis possible: Frits, John, Marlies, Ellen, Ivo, Marie-Claire, Dick, Vera, Arianna, Annemarie, Noël, Kirsten, Patrick, Leonie, Gerrit, Gerlinde, Denise, Joe, Dede, Changgui, Paula, and Baiq. I very much enjoyed working with you all. I would also like to acknowledge the AEW team for making the work environment "heel gezellig": Maira, Pablo, Hazi, Sanne, Irene, Nancy, Sebastian, Bernardo, Ariadna, Jacqueline, Jelle, Wendy, Jeroen, Michael, Marten, Els, Ingrid, Lemessa, Goraw, Georgiana. It was a great pleasure meeting you all.

I also wish to thank the people that have accompanied me during the thesis. Special thanks to Mireia, who was my pillar in the NL and who will always be my best memory of those years; to Oma, who was the strongest woman I knew and who believed more in me than myself; to Jesús and his parents, with whom I shared many special moments; to my aunt Miranda and the rest of the Hasselerharm family, who made me feel at home; and to Ari, who always found some time to drink a beer with me. Many thanks also to my Spanish friends who were always ready for chat, even though we were miles away from each other: Alvaro (who made the cover of this thesis and the logo of the TRAMP project), Olivia, Sofi, Dani, Marina, Dora, Rocío, Paula, Steffy and Nacho. Thanks also to the friends that kept me motivated in the final stretch of the thesis: Andrea, Javi, Máher, Mar and Cristina. And last but not least, I would like to thank Namaskar for their delicious Indian food and Hema for their coffee and cake offer.

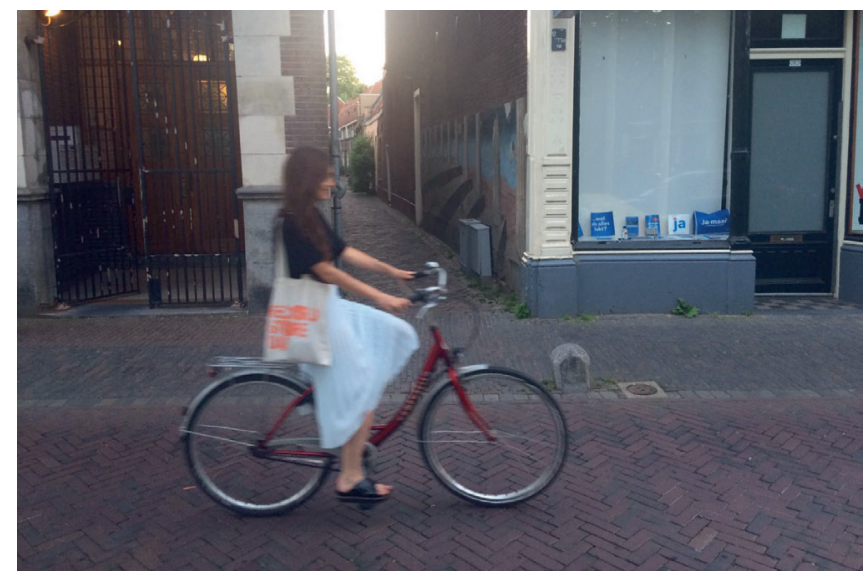



Curriculum vitae 

Paula E. Redondo Hasselerharm was born in Benalmádena, Málaga, in 1989. At the age of 18 she moved to Madrid to study a 5-year BSc degree in Biology at the Autonomous University of Madrid. During these studies, she enjoyed the subject of Limnology the most, which made her choose the specialization of aquatic ecology. For her BSc thesis, she did an internship at the Water Institute of Granada to evaluate the bacterioplankton responses to global change factors in lakes from the Mediterranean region. In the last year of these studies, she did an internship at the University of Amsterdam to assess the effects of nano- and microplastics on the photosynthetic efficiency and growth of freshwater and marine microalgae. Then, she coursed a MSc in Water Quality Sciences and Techniques at the University of Granada. As part of the MSc, she did an internship at the Regional Government of Granada, where she wrote a LIFE project proposal on mobile groundwater biofilm-denitrification technology and nitrate pollution prevention in small communities. After finishing her thesis, Paula started her PhD at the Aquatic Ecology and Water Quality Management Group (AEW) at Wageningen University \& Research. Her PhD was part of the project "Technologies for the Risk Assessment of Microplastics", where Merel Kooi and Svenja Mintenig were also involved as PhDs. Together they would develop a risk assessment for microplastics in Dutch freshwater systems. During this period, she cosupervised seven BSc and MSc students and assisted on three courses. In addition, she conducted experiments at KWR Water Research Institute, and contributed scientifically to the project "Microplastic Effect Thresholds for Aquatic Species". She presented the outcomes of her PhD at SETAC Europe in Brussels, the MICRO Conferences of 2016/18 in Lanzarote and the 2018 Water Science for Impact Conference in Wageningen. Paula is currently working at the Barcelona Global Health Institute on the "AiguaBCN" project, where she is measuring emerging contaminants in drinking water.

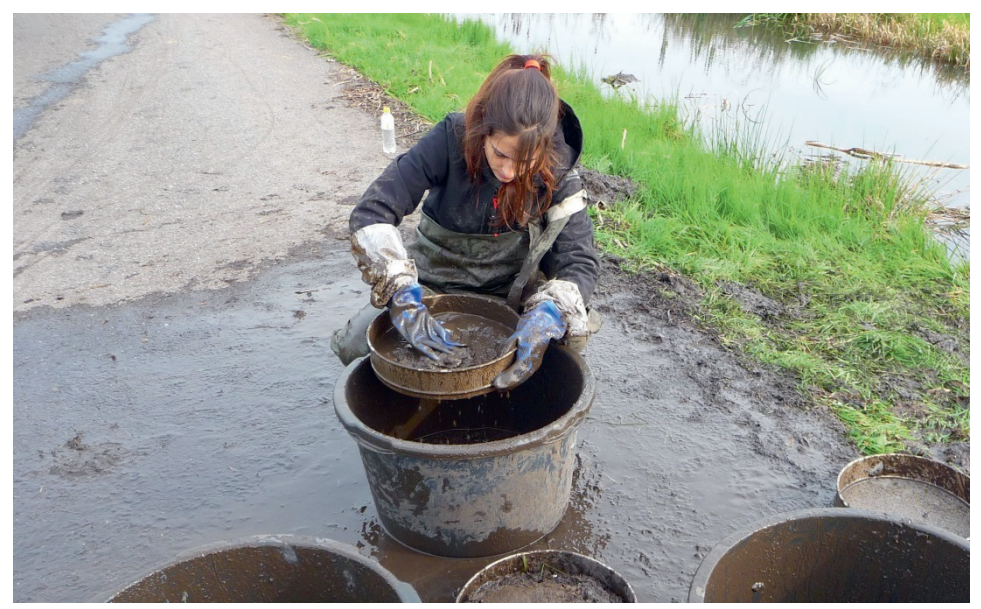



Scientific publications 

Redondo-Hasselerharm, P.E.; Mitrano, D.; Koelmans, A.A. Uptake and effects of metal-doped nanoplastics on Gammarus pulex. In preparation.

Pan, C.; Mintenig, S.M.; Redondo-Hasselerharm, P.E.; Yu, K.; Wang, Y; Koelmans, A.A.; Taxon-specific ingestion of microplastics by freshwater benthic invertebrates in the Dommel River of the Netherlands. Environ. Sci. Technol. Under review.

Redondo-Hasselerharm, P.E.; Baken, K.A.; Pap, L.; Mintenig, S.M.; Bäuerlein, P.S.; van Wezel, A.P.; Koelmans, A.A. Assessing the toxicity of nanoplastics using in vitro bioassays with relevance for human health. Environ. Tox. \& Chem. Under review.

Koelmans, A.A.; Redondo-Hasselerharm, P.E.; Mohamed Nor, N.H.; Kooi, M. Solving the non-alignment of methods and approaches used in microplastic research in order to consistently characterize risk. Environ. Sci. Technol., 54: 12307-12315 (2020).

De Ruijter, V.; Redondo-Hasselerharm, P.E., Gouin, T.; Koelmans, A.A. Quality criteria for microplastic effect studies in the context of risk assessment: A critical review. Environ. Sci. Technol., 54: 11692-11705 (2020).

Merga, L.; Redondo-Hasselerharm, P.E; van den Brink, P. Koelmans, A.A. Distribution of microplastic and small macroplastic particles across four fish species and sediment in an African lake. Sci. Total Environ., 741: 140527 (2020).

Mintenig, S.M.; Kooi, M.; Erich, M.W.; Primpke, S.; Redondo-Hasselerharm, P.E.; Dekker, S.C.; Koelmans, A.A.; van Wezel, A.P. A systems approach to understand microplastic occurrence and variability in Dutch riverine surface waters. Wat. Res., 176: 115723 (2020).

Redondo-Hasselerharm, P.E.; Gort, G.; Peeters, E.T.H.M.; Koelmans, A.A. Nano- and microplastics affect the composition of freshwater benthic communities in the long term. Sci. Adv., 6: eaay4054 (2020).

Besseling, E.; Redondo Hasselerharm, P. E.; Foekema, E.M.; Koelmans, A. A. Quantifying ecological risks of aquatic micro- and nanoplastic. Critical Reviews in Environmental Science and Technology, 49: 32-80 (2019).

O'Connor, J.D.; Mahon, A.M.; Ramsperger, A.F.R.M.; Trotter, B.; Redondo Hasselerharm, P. E.; Koelmans, A.A.; Lally, H.T.; Murphy, S. Microplastics in Freshwater Biota: A Critical Review of Isolation, Characterization, and Assessment Methods. Global Challenges 1800118 (2019). 
Bellingeri, A.; Bergami, E.; Grassi, G.; Faleri, C.; Redondo Hasselerharm, P. E.; Koelmans, A.A.; Corsi, 1. Combined effects of nanoplastics and copper on the freshwater alga Raphidocelis subcapitata. Aquat. Tox., 210: 179-187 (2019).

Van Weert, S.; Redondo-Hasselerharm, P.E.; Diepens, N.; Koelmans, A.A. Effects of nanoplastics and microplastics on the growth of sediment-rooted macrophytes. Sci. Total Environ., 654, 1040-1047 (2019).

Redondo-Hasselerharm, P.E.; De Ruijter, V.N.; Mintenig S.M.; Verschoor, A.; Koelmans, A.A. Ingestion and Chronic Effects of Car Tire Tread Particles on Freshwater Benthic Macroinvertebrates. Environ. Sci. Technol., 52: 13986-13994 (2018).

Redondo-Hasselerharm, P.E.; Falahudin, D.; Peeters, E.T.H.M.; Koelmans, A.A. Microplastic Effect Thresholds for Freshwater Benthic Macroinvertebrates. Environ. Sci. Technol. 52: 2278-2286 (2018).

Koelmans, A. A., Besseling, E.; Foekema, E.; Kooi, M.; Mintenig, S.; Ossendorp, B. C.; Redondo Hasselerharm, P. E.; Verschoor, A.; van Wezel, A. P.; Scheffer, M. Risks of Plastic Debris: Unravelling Fact, Opinion, Perception, and Belief. Environ. Sci. Technol., 51: 11513-11519 (2017).

Sjollema, S.; Redondo-Hasselerharm, P.E.; Kraak, M.; Leslie, H.; Vethaak, D. Do plastic particles affect microalgal photosynthesis and growth? Aquat. Tox., 170: 259-261 (2016). 



\title{
SENSE
}

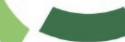

Netherlands Research School for the

Socio-Economic and Natural Sciences of the Environment

\section{I P L O M A}

for specialised PhD training

The Netherlands research school for the Socio-Economic and Natural Sciences of the Environment (SENSE) declares that

\section{Paula Elisa}

\section{Redondo Hasselerharm}

\author{
born on 12 April 1989 in Benalmádena, Spain \\ has successfully fulfilled all requirements of the \\ educational PhD programme of SENSE.
}

Wageningen, 16 December 2020
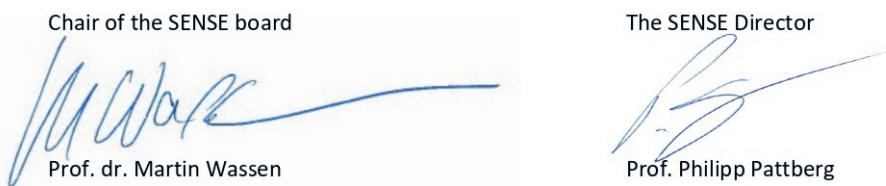

The SENSE Research School has been accredited by the Royal Netherlands Academy of Arts and Sciences (KNAW)

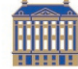

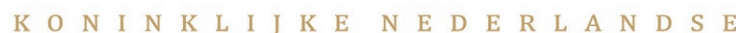

A K A D E M I E V A N W E T E N S C H A P P E N 


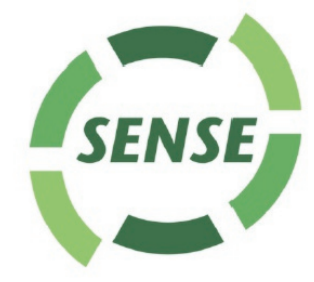

The SENSE Research School declares that Paula Elisa Redondo Hasselerharm has successfully fulfilled all requirements of the educational $\mathrm{PhD}$ programme of SENSE with a work load of $50.0 \mathrm{EC}$, including the following activities:

\section{SENSE PhD Courses}

- Environmental research in context (2016)

- Research in context activity: 'Title of A2 SENSE' (2015)

\section{Other PhD and Advanced MSc Courses}

- Multivariate Analysis, Wageningen University (2016)

- The Essentials of Scientific Writing and Presenting, Wageningen Graduate Schools (2016)

- Techniques for Writing and Presenting Scientific Papers, Wageningen Graduate Schools (2016)

- Reviewing a Scientific Paper, Wageningen Graduate Schools (2017)

- Ecotoxicology, Utrecht University \& VU Amsterdam (2017)

- Risk Assessment, Wageningen University (2018)

- Basic Statistics, Wageningen University (2018)

External training at a foreign research institute

- Effect-based in vitro tests, KWR Water Research Institute, Netherlands (2017)

\section{Management and Didactic Skills Training}

- Supervising one BSc student and six MSc students with thesis (2016-2020)

- Assisting practicals of the MSc courses 'Environmental Risk Assessment of Chemicals Course' and 'Aquatic ecology and water quality'(2017-2019)

- Teaching in the MSc course 'Water quality' (2018-2019)

\section{Oral Presentations}

- Chronic effects of polystyrene microplastics on freshwater benthic macroinvertebrates. SETAC Europe, 7-11 May 2017, Brussels, Belgium

- Microplastic effect thresholds for freshwater benthic macroinvertebrates. Water Science for Impact, 16-18 October 2018, Wageningen, The Netherlands

- Long-term community effects of nano-and microplastics on freshwater benthic macroinvertebrates. MICRO, 19 - 23 November 2018, Lanzarote, Spain

SENSE coordinator PhD education

Dr. ir. Peter Vermeulen

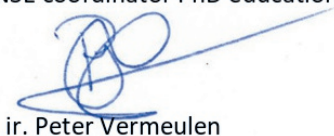


This work was funded by the Dutch Technology Foundation TTW, project no. 13940. We acknowledge additional support from KWR; WMR; NVWA; RIKILT; the Dutch Ministry of Infrastructure and the Environment; the Dutch Ministry of Health, Welfare and Sport; Wageningen Food \& Biobased Research; STOWA; RIWA; and water boards Hoogheemraadschap van Delfland, Zuiderzeeland, Rijn en IJssel, Vechtstromen, Scheldestromen, Aa en Maas, de Dommel, and Rivierenland.

Cover design by Unbuentipo (Álvaro Fernández).

Printed by Proefschriftmaken. 



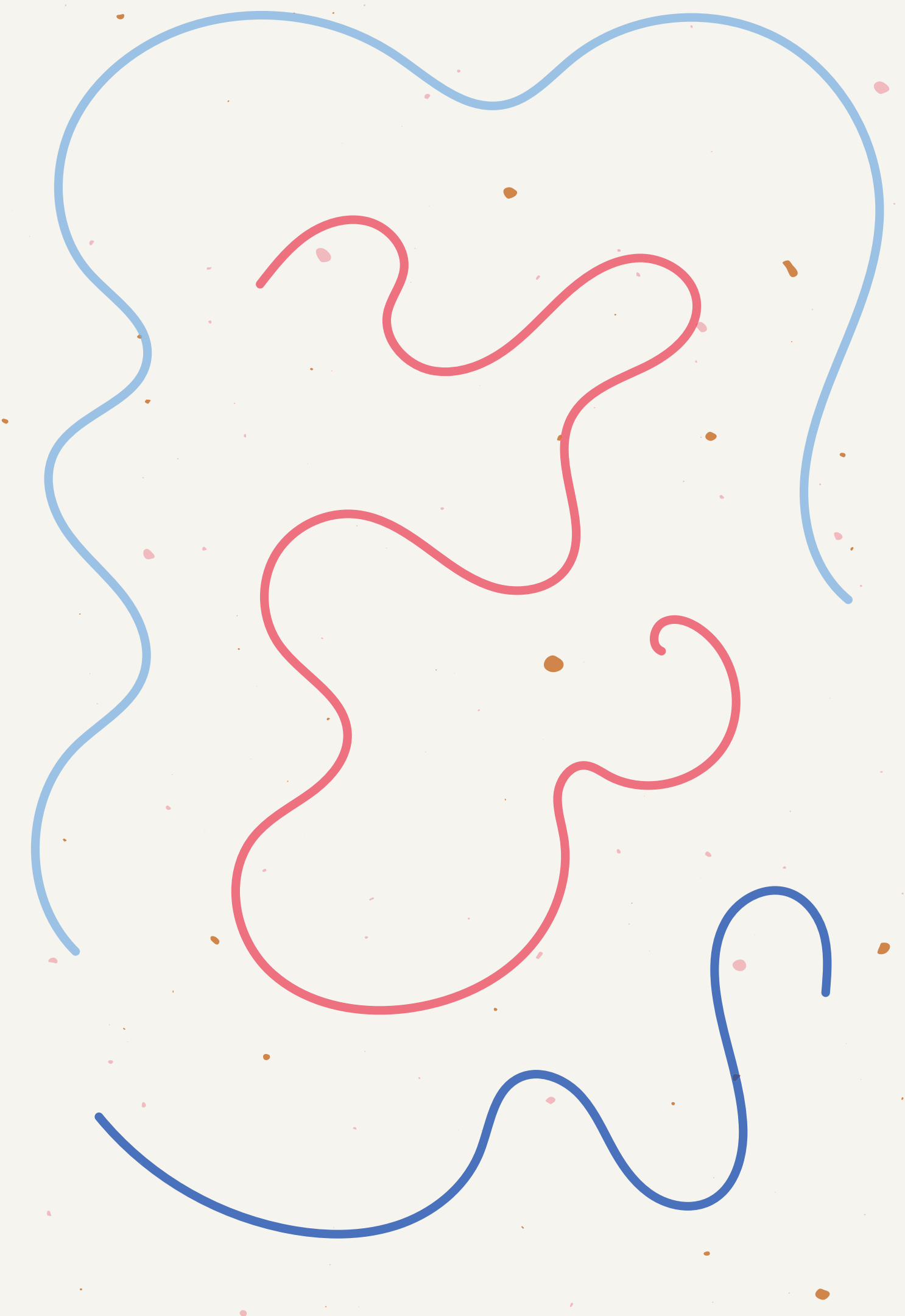

